\title{
Development of real time roof geology detection system using drilling parameters during roof bolting operation
}

Xianguang Tang

West Virginia University

Follow this and additional works at: https://researchrepository.wvu.edu/etd

\section{Recommended Citation}

Tang, Xianguang, "Development of real time roof geology detection system using drilling parameters during roof bolting operation" (2006). Graduate Theses, Dissertations, and Problem Reports. 2710. https://researchrepository.wvu.edu/etd/2710

This Dissertation is protected by copyright and/or related rights. It has been brought to you by the The Research Repository @ WVU with permission from the rights-holder(s). You are free to use this Dissertation in any way that is permitted by the copyright and related rights legislation that applies to your use. For other uses you must obtain permission from the rights-holder(s) directly, unless additional rights are indicated by a Creative Commons license in the record and/ or on the work itself. This Dissertation has been accepted for inclusion in WVU Graduate Theses, Dissertations, and Problem Reports collection by an authorized administrator of The Research Repository @ WVU.

For more information, please contact researchrepository@mail.wvu.edu. 


\title{
Development of Real Time Roof Geology Detection System Using Drilling Parameters During Roof Bolting Operation
}

\author{
Xianguang Tang \\ Dissertation Submitted to the \\ College of Engineering and Mineral Resources \\ At West Virginia University \\ In Partial Fulfillment of the Requirements \\ for the Degree of \\ Doctor of Philosophy \\ In \\ Engineering \\ Syd Peng, Ph.D., Chair \\ Gregory Elmes, Ph.D. \\ Gerald Finfinger, Ph.D. \\ Felicia Peng, Ph.D. \\ Yi Luo, Ph.D. \\ Department of Mining Engineering \\ Morgantown, West Virginia \\ 2006
}

Keywords: Ground Control, Roof Support, Roof Bolting, Drilling, Uniaxial Compressive Strength, Rock Void, Drill Bits, Rock Strength

Copyright 2006 Xianguang Tang 


\title{
ABSTRACT \\ Development of Real Time Roof Geology Detection System Using Drilling Parameters During Roof Bolting Operation
}

\author{
Xianguang Tang
}

Roof bolting is the most popular method for underground openings in the mining industry, especially in the bedded deposits such as coal. In fact, all U.S. underground coal mine entries are roof-bolted as required by law.

However, roof falls still occur frequently in the roof bolted entries. The two possible reasons are: the lack of knowledge of and technology to detect the roof geological conditions in advance of mining, and lack of roof bolting design criteria for modern roof bolting systems.

This research is to develop a method for predicting the roof geology and stability condition in real time during roof bolting operation.

For the prediction of roof geology and stability condition in real time, a micro processor was used and a program developed to monitor and record the drilling parameters of roof bolter. These parameters include feed pressure, feed flow (penetration rate), rotation pressure, rotation rate, vacuum pressure, oil temperature of hydraulic circuit, and signals for controlling machine. From the results of a series of laboratory and underground tests so far, feed pressure is found to be a good indicator for identifying the voids/fractures and estimating the roof rock strength. The method for determining quantitatively the location and the size of void/fracture and estimating the roof rock strength from the drilling parameters of roof bolter was developed. Also, a set of computational rules has been developed for in-mine roof using measured roof drilling parameters and implemented in MRGIS (Mine Roof Geology Information System), a software package developed to allow mine engineers to make use of the large amount of roof drilling parameters for predicting roof geology properties automatically. 


\section{ACKNOWLEDGEMENTS}

I wish to express my sincere appreciation to dedicated and remarkable individuals from WVU, J.H. Fletcher and Co., NIOSH, Cervis, and a series of coal mines. They have contributed significantly to my tests, research and the completion of my dissertation. The individuals who provided enormous help include, Dr. Syd Peng, WVU, Dr. Yi Luo, WVU, Dr. Felicia Peng, WVU, Dr. Gerald Finfinger, NIOSH, Dr. Gregory Elmes, WVU, Dr. Takashi Sasaoka, WVU, Mr. Gene Wilson and Mr. Craig Collins, J.H. Fletcher and Co., and Mr. Bob Thomas, Cervis. Thanks also go to fellow graduate students Mr. Benjamin T. Mirabile and Mr. Jishen Han for their assistance in experiments. 


\section{TABLE OF CONTENTS}

ABSTRACT...................................................................... ii

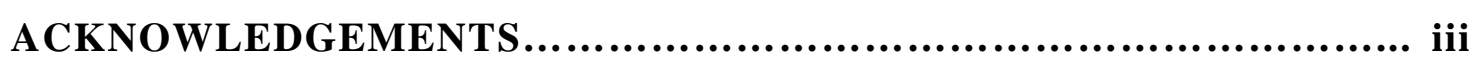

TABLE OF CONTENTS....................................................... iv

LIST OF FIGURES.............................................................. vii

LIST OF TABLES.................................................................. xiii

NOMENCLATURE.................................................. xiv

Chapter 1: INTRODUCTIION.............................................. 1

Chapter 2: LITTERATURE REVIEW........................................ 3

2.1 Specific Energy Developed by Teale.......................................................... 3

2.2 Research by WVU.............................................................. 5

2.3 Research Conducted by Itakura et al...................................................... 19

Chapter 3: OBJECTTIVES, SCOPE, AND METHODOLOGY .................. 24

3.1 Research Objectives........................................................................... 24

3.2 Scope of Research ............................................................................... 24

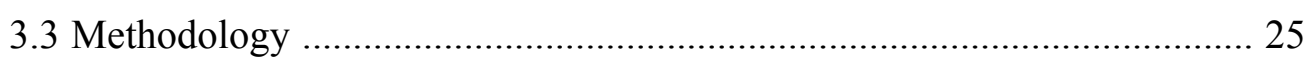

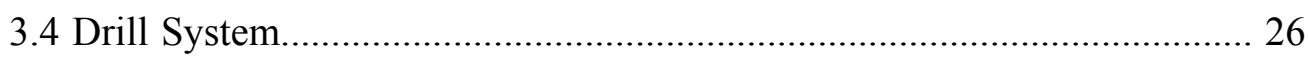

3.5 Drilling Algorithm (WVU Control Mode)............................................. 27

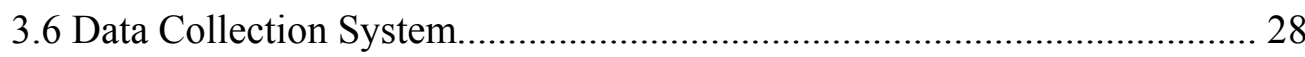

Chapter 4: DRILLING EXPERIMENTS....................................... 31

4.1 Laboratory Tests Using Manufactured Roof Rock Blocks........................ 32

4.1.1 Manufactured Blocks..................................................................... 33

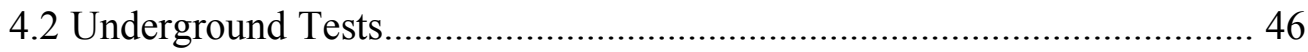

4.2.1 Design of Underground Tests....................................................... 46

4.2.2 Overview of Underground Tests................................................... 47

\section{Chapter 5: DEVELOPMENT OF DATA INTERPRETATION}

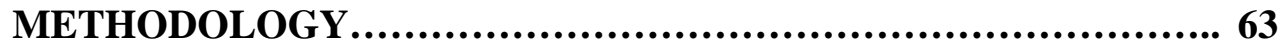

5.1 Concept of Data Analysis and Rule Generation........................................ 63

5.2 Effect of Drilling Settings and Machine/Drilling Conditions on Drilling Parameters for Compensation Runs............................................ 64

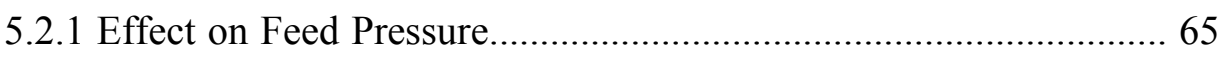

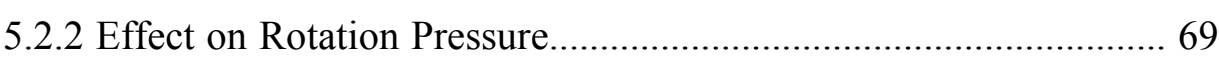




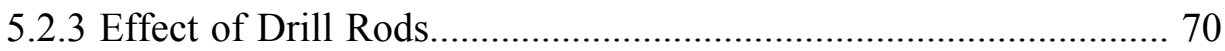

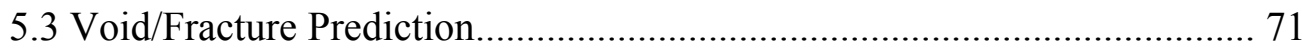

5.3.1 Determination of Relevant Drilling Parameters for the Voids/Fractures.

5.3.2 Mechanism of Rock Fragmentation When a Void/Fracture is Encountered. 72

5.3.3 Criterion for Void/Fracture Prediction........................................... 75

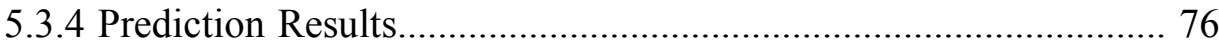

5.3.5 Prediction of the Size of Void/Fracture......................................... 76

5.3.6 Field Verification of Void/Fracture Prediction................................ 78

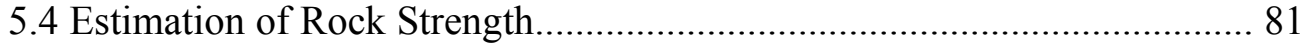

5.4.1 Impact of Different Rock Strengths on Feed Pressure and

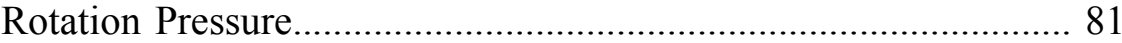

5.4.2 Effect of Penetration Rate on Feed Pressure................................... 83

5.4.3 Effect of Rotation Rate on Feed Pressure....................................... 96

5.4.4 Determination of the Boundary Planes for Estimating Roof Rock Strength.......................................................................... 97

5.4.5 Verification of the Boundary Planes for Estimating

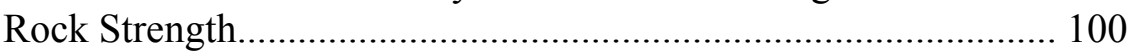

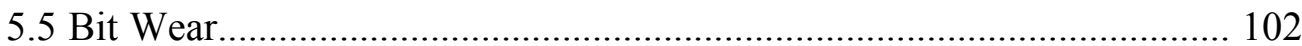

5.5.1 Characteristics and Effect of Bit Wear on Drilling Parameters......102

5.5.2 Effect of Bit Wear on Roof Geology Prediction................................108

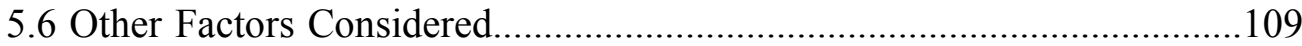

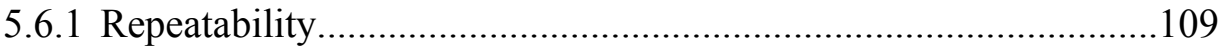

5.6.2 Effect of Control Mode (Feedback System Mode) ....................... 110

5.6.3 Effect of Thrust Cap...................................................................112

5.6.4 Drilling Conditions under a Large Bite Depth.............................. 113

Chapter 6: DATA VISUALIZATION AND DATA BASE SOFTWARE ......118

6.1 Requirements and Capabilities for MRGIS..........................................118

6.2 Data Import and Data Cleaning.......................................................... 120

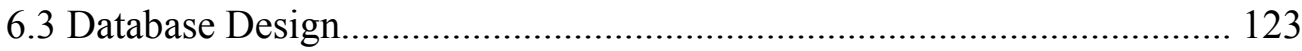

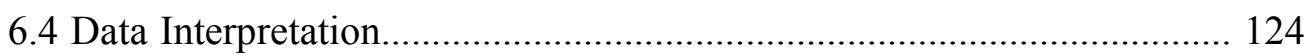

6.5 Roof Geology Visualization.................................................................125

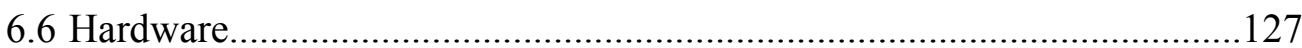


Chapter 7: CONCLUSIONS................................................. 129

Chapter 8: RECOMMENDATIONS FORFUTURE RESEARCH.............. 131

REFERENCES.............................................................. 132

APPNDIX A CONVERSION OF DRILLING PARAMETERS...................... 135

APPNDIX B DRILLING PARAMETERS PLOTS............................. 137

APPNDIX C PHOTOS AND DRILLING PARAMETERS PLOTS

OF BIT WEAR TESTS........................................... 305

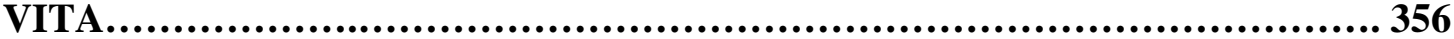




\section{LIST OF FIGURES}

Page

Figure 2.1 Abnormality indexes drilling in solid concrete block.................... 10

Figure 2.2 Bit-rock interaction in rotary drilling......................................... 11

Figure 2.3 Thrust and torque relationship for two materials........................... 19

Figure 2.4 Log pattern of mechanical data dependent on discontinuities...... 20

Figure 2.5 Mechanical data logs at boundary slope angle of 0 degree and 5 mm space.

Figure $3.1 \quad$ J.H. Fletcher \& Co.'s HDDR dual head roof bolter......................... 26

Figure $3.2 \quad$ Drill control unit (DCU) _..................................................... 27

Figure $3.3 \quad$ Data collecting system................................................................. 29

Figure $3.4 \quad$ Software for displaying drilling parameters................................. 30

Figure 4.1 Drill bits used for the tests........................................................ 31

Figure 4.2 Test scene of laboratory test (J.H. Fletcher \& Co.'s facility, Huntington, WV) ..................................................................... 33

Figure $4.3 \quad$ Schematic of solid concrete block................................................ 34

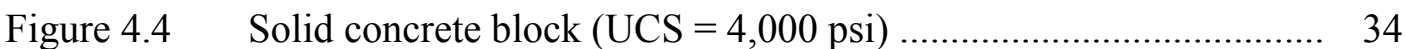

Figure 4.5 Schematic of layered block \#1 ................................................... 35

Figure 4.6 Schematic of layered block \#2 \#................................................ 36

Figure 4.7 Schematic of layered block \#3 _................................................. 36

Figure $4.8 \quad$ Schematic of layered block \#4................................................... 37

Figure $4.9 \quad$ Schematic of layered block \#5 .................................................... 37

Figure 4.10 Schematic of layered block \#6....................................................... 38

Figure 4.11 Schematic of fractured block..................................................... 42

Figure 4.12 Assembling fractured block....................................................... 42

Figure 4.13 Schematic of manufactured block with large bedding separations 
Figure 4.14 Schematic of manufactured block to simulate with horizontal

Figure 4.15 Schematic of manufactured block with angled cardboard lavers..... 46

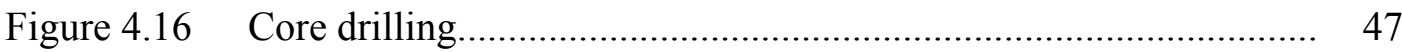

Figure $4.17 \quad$ Borehole scope system........................................................ 47

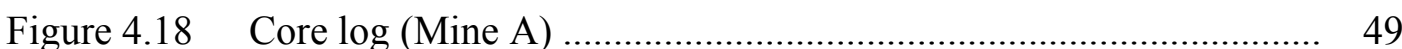

Figure 4.19 Result of borehole scoping (Mine B) .......................................... 49

Figure 4.20 Diagram of test site (Mine C) …................................................ 50

Figure 4.21 Core $\log ($ Mine $C)$............................................................... 50

Figure 4.22 Diagram of test site (Mine D) .................................................... 51

Figure $4.23 \quad$ Core logs (Mine D) …............................................................... 52

Figure 4.24 Angle drilling with roof bolter (Mine E) ..................................... 53

Figure 4.25 Diagram of test site (Mine E) .................................................... 54

Figure 4.26 Core logs (Mine E) ............................................................. 54

Figure 4.27 Diagram of test site (Mine F) ................................................... 56

Figure $4.28 \quad$ Core and core $\log ($ Mine F) ..................................................... 57

Figure 4.29 Diagram of test site (Mine G) ….............................................. $\quad 59$

Figure $4.30 \quad$ Core and core $\log ($ Mine $G)$...................................................... 60

Figure 4.31 Diagram of test site (Mine H) ..................................................... 61

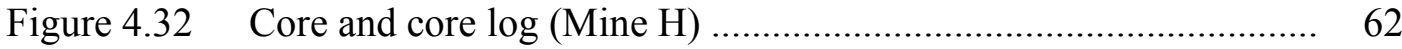

Figure $5.1 \quad$ Flowchart for generating rules................................................. 64

Figure 5.2 Relationships between feed pressure /carriage position/mast position and elapsed time (P.R. $=0.4 \mathrm{in} / \mathrm{sec}$, R.R. $=600 \mathrm{rpm}) \ldots \ldots .65$

Figure $5.3 \quad$ Carriage and mast stages................................................................. 65

Figure 5.4 Relationship between feed pressure and net bit position (P.R. $=0.4 \mathrm{in} / \mathrm{sec}$, R.R. $=600 \mathrm{rpm})$ 
Figure 5.5 Effect of drilling settings on the magnitude of feed pressure for compensation run.

Figure 5.6 Effect of oil temperature on the magnitude of feed pressure for compensation run (P.R. $=2.1 \mathrm{in} / \mathrm{sec}$, R.R. $=600 \mathrm{rpm})$

Figure 5.7 Approximate curve for the relationship between feed pressure and net bit position.

Figure 5.8 Feed pressure-net bit position curves at the beginning of test......... 68

Figure 5.9 Approximate curves for different test dates................................. 68

Figure 5.10 Effect of drilling settings on the magnitude of rotation pressure for compensation run................................................................ 57

Figure 5.11 Effect of oil temperature on rotation pressure for compensation run and drilling rock (P.R. $=1.1 \mathrm{in} / \mathrm{sec} \&$ R.R. $=600 \mathrm{rpm}) \ldots \ldots \ldots \ldots . .70$

Figure 5.12 Effect of drill rod on drilling parameters (P.R. $=2.1 \mathrm{in} / \mathrm{sec}$, R.R. $=600 \mathrm{rpm})$ 71

Figure 5.13 Drilling parameters. 73

Figure 5.14 Rock fragmentation when drilling close to a rock void 74

Figure 5.15 Conditions of both surfaces of void

Figure 5.16 Relationship between the width of feed pressure valley and the size of void.

Figure 5.17 Feed pressure curves and results of borehole scoping (Mine F, roof rock: shale)

Figure 5.18 Feed pressure curves and results of borehole scoping (Mine G, roof rock: sandstone)

Figure 5.19 Drilling parameters and results of borehole scoping (Mine G) ...... 80

Figure 5.20 Effect of rock strength on feed pressure/rotation pressure (P.R. $=1.5 \mathrm{in} / \mathrm{sec} \&$ R.R. $=600 \mathrm{rpm}$, Oil Temp $=126$ (sandstone) and 128 (shale) output units)

Figure 5.21 Effect of rock strength on feed pressure/rotation pressure and core $\log ($ Mine D, P.R. $=1.5 \mathrm{in} / \mathrm{sec} \&$ R.R. $=$ free $)$

Figure 5.22 Effect of penetration rate on feed pressure (R.R. $=600 \mathrm{rpm})$ 
Figure 5.23 Relationship between penetration rates calculated by heavily filtered method and feed pressure (R.R. $=600 \mathrm{rpm}$ )

Figure 5.24 Correlation between penetration rate ${ }_{(\mathrm{HF})}$ and feed pressure (P.R. $=0.4 \mathrm{in} / \mathrm{sec}$ and R.R. $=600 \mathrm{rpm})$

Figure 5.25 Correlation between penetration rate ${ }_{(10 \mathrm{BP})} /$ penetration rate $_{(\mathrm{FF})}$ and feed pressure (P.R. $=0.4 \mathrm{in} / \mathrm{sec}$ and R.R. $=600 \mathrm{rpm})$

Figure 5.26 Relationship between penetration rates and feed pressure (R.R. $=600 \mathrm{rpm}$, sandstone)

Figure 5.27 Relationship between the bit positions calculated from the data of two position sensors/feed flow and elapsed time $(\mathrm{P} . \mathrm{R} .=0.4 \mathrm{in} / \mathrm{sec}, \mathrm{R} . \mathrm{R} .=500 \mathrm{rpm})$

Figure 5.28 Relationship between the bit positions calculated from the data of two position sensors/feed flow and elapsed time under different penetration rates.

Figure 5.29 Relationship between bit position/penetration $\operatorname{rate}_{(\mathrm{FF})}$ and elapsed time for compensation run (P.R. $=2.1 \mathrm{in} / \mathrm{sec}$, R.R. $=500 \mathrm{rpm}$ )

Figure 5.30 Relationship between oil leakage factor and oil temperature at the carriage stage.

Figure 5.31 Relationship between the bit positions calculated by different methods and elapsed time.

Figure 5.32 Relationship between the bit positions calculated by different methods and elapsed time.

Figure 5.33 Relationship between feed pressure and penetration rate (R.R. $=600 \mathrm{rpm}$ )

Figure 5.34 Relationship between penetration rates calculated by different methods and feed pressure (R.R. $=600 \mathrm{rpm})$

Figure 5.35 Effect of rock strength on the relationship between net feed pressure and penetration rate (R.R. $=600 \mathrm{rpm})$

Figure 5.36 Effect of rotation rate on feed pressure (P.R=1.3 in $/ \mathrm{sec})$

Figure 5.37 Approximation curves for each setting for different rotation rates and different strengths of rocks.

Figure 5.38 Distributions of both sets of data points (Mine F and Mine G) ....... 
Figure 5.39 Upper and lower boundary planes for distributions of both data points (Mine $\mathrm{F}$ and Mine $\mathrm{G}$ )

Figure 5.40 Boundary planes for estimating rock strength.

Figure 5.41 Boundary planes for estimating rock strength and distributions of three sets of data points.

Figure 5.42 Drill bit (left, new bit: right, after having drilled 17 holes, shale)

Figure 5.43 Effect of bit wear on feed pressure \& rotation pressure (shale, P.R. $=1.7 \mathrm{in} / \mathrm{sec} \&$ R.R. $=600 \mathrm{rpm}$ )

Figure 5.44 Drill bit (left, new bit \& right, after 7 holes drilled) (sandstone, P.R. $=1.1 \mathrm{in} / \mathrm{sec}$, R.R. $=650 \mathrm{rpm}$ )

Figure 5.45 Drill bit (left, new: center, R.R. $=400$ rpm: right, R.R. $=650$ $\mathrm{rpm}$ ) (sandstone, P.R. $=1.1 \mathrm{in} / \mathrm{sec}$, number of holes drilled $=5)$

Figure 5.46 Magnitude of feed pressure/rotation pressure for each drill hole (bit wear test, sandstone, P.R. $=1.1 \mathrm{in} / \mathrm{sec} \&$ R.R. $=400 \mathrm{rpm}$ )

Figure 5.47 Magnitude of feed pressure/rotation pressure for each drill hole (bit wear test, sandstone, P.R. $=1.1 \mathrm{in} / \mathrm{sec} \&$ R.R. $=650 \mathrm{rpm}$ )

Figure 5.48 Drill bit (left, new: center, P.R. $=0.4 \mathrm{in} / \mathrm{sec}$ : right, P.R. $=1.5$ $\mathrm{in} / \mathrm{sec}$ )(sandstone, R.R. $=650 \mathrm{rpm}$, number of holes drilled $=3)$

Figure 5.49 Magnitude of feed pressure/rotation pressure for each drill hole (bit wear test, sandstone, P.R. $=0.4 \mathrm{in} / \mathrm{sec} \&$ R.R. $=650 \mathrm{rpm}$ )

Figure 5.50 Magnitude of feed pressure/rotation pressure for each drill hole (bit wear test, sandstone, P.R. $=1.5 \mathrm{in} / \mathrm{sec} \&$ R.R. $=650 \mathrm{rpm}$ )

Figure 5.51 Magnitude of feed pressure for each drill hole and the result of borehole scoping (bit wear test, sandstone, P.R. $=1.1 \mathrm{in} / \mathrm{sec}$ and R.R. $=650 \mathrm{pm})$

Figure 5.52 Confirmation of repeatability (P.R. $=1.1 \mathrm{in} / \mathrm{sec}$, R.R. $=600$ rpm)

Figure 5.53 Distribution of drilling data points and boundary planes for estimating rock strength (Mine $\mathrm{G}$, feedback system mode)

Figure 5.54 Drilling parameters for drilling in layered block \# 5 (P.R. $=0.4$ 
in/sec, R.R. $=500 \mathrm{rpm})$

Figure 5.55 Drilling parameters for drilling in sandstone (P.R. $=2.1 \mathrm{in} / \mathrm{sec}$, R.R. $=400 \mathrm{rpm})$

Figure 5.56 Relationship between net feed pressure and penetration rate ......... 114

Figure 5.57 Bit geometry (new bit) ............................................................. 115

Figure 5.58 Drill bit (R.R. $=400 \mathrm{rpm}$, number of holes drilled = 1) $\ldots \ldots \ldots \ldots \ldots \ldots \ldots . . . .116$

Figure 5.59 Drill bit (R.R. $=600 \mathrm{rpm}$, number of holes drilled =1) ............... 117

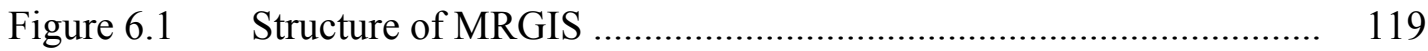

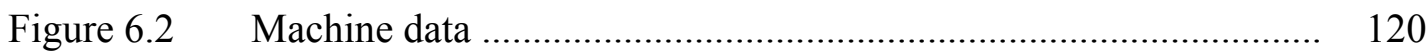

Figure 6.3 Interface for data importing ................................................... 122

Figure 6.4 Roof geology visualization: 2D + 1D .................................... 126

Figure 6.5 Roof geology visualization: 2D + 2D ...................................... 128

Figure 6.6 Roof geology visualization: 2D + 3D ...................................... 128 


\section{LIST OF TABLES}

$\underline{\text { Table \# }}$

Page

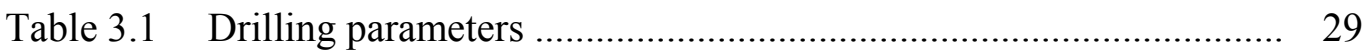

Table 4.1 Physical properties of solid concrete blocks .................................. 34

Table 4.2 Physical properties of the embedded rocks and the concrete used in the layered blocks .......................................................... $\quad 39$

Table 4.3 Physical properties of core samples (Mine G) .............................. 58

Table 4.4 Physical properties of core samples (Mine H) ............................. 61

Table 5.1 Statistics of valley bottoms of feed pressure …............................. 75

Table $5.2 \quad$ Results of void prediction ......................................................... 76

Table 5.3 Size of the plateau at the bottom of feed pressure valley ............... 77 


\title{
NOMENCLATURE
}

\section{Symbol Description}

\author{
A Area of hole \\ $A_{n} \quad$ Normal contact area \\ $\mathrm{A}_{\mathrm{i}} \quad$ Contact area between the bit and the drilled rock \\ b Bite depth \\ BTS Brazilian tensile strength \\ $\mathrm{BP}_{\text {net }} \quad$ Net Bit Position \\ c Factor by considering the geometry of the bit \\ D Diameter of bit \\ $\mathrm{d}_{\mathrm{i}} \quad$ Distance drilled from point $\mathrm{i}-1$ to $\mathrm{i}$ \\ DH Drilling hardness \\ e $\quad$ Specific energy \\ $e_{t} \quad$ Specific energy thrust component \\ $\mathrm{e}_{\mathrm{r}} \quad$ Specific energy rotary component \\ E Young's modulus \\ $\mathrm{E}_{50} \quad$ Young's modulus at the $50 \%$ stress level of the maximum stress \\ $\mathrm{f}_{\mathrm{i}} \quad$ Frictional coefficient between the front part of the drill bit and rock at \\ position $\mathrm{i}$ \\ $\mathrm{F}_{\mathrm{w}} \quad$ Size of the void \\ F Thrust \\ $\mathrm{F}_{\mathrm{L}} \quad$ Oil leakage factor \\ $\mathrm{FP}_{\mathrm{C}} \quad$ Feed pressure for compensation run \\ $\mathrm{F}_{\mathrm{i}} \quad$ measured thrust at point $\mathrm{i}$ \\ $\mathrm{FP}_{\mathrm{DR}} \quad$ Feed pressure when drilling in rock
}




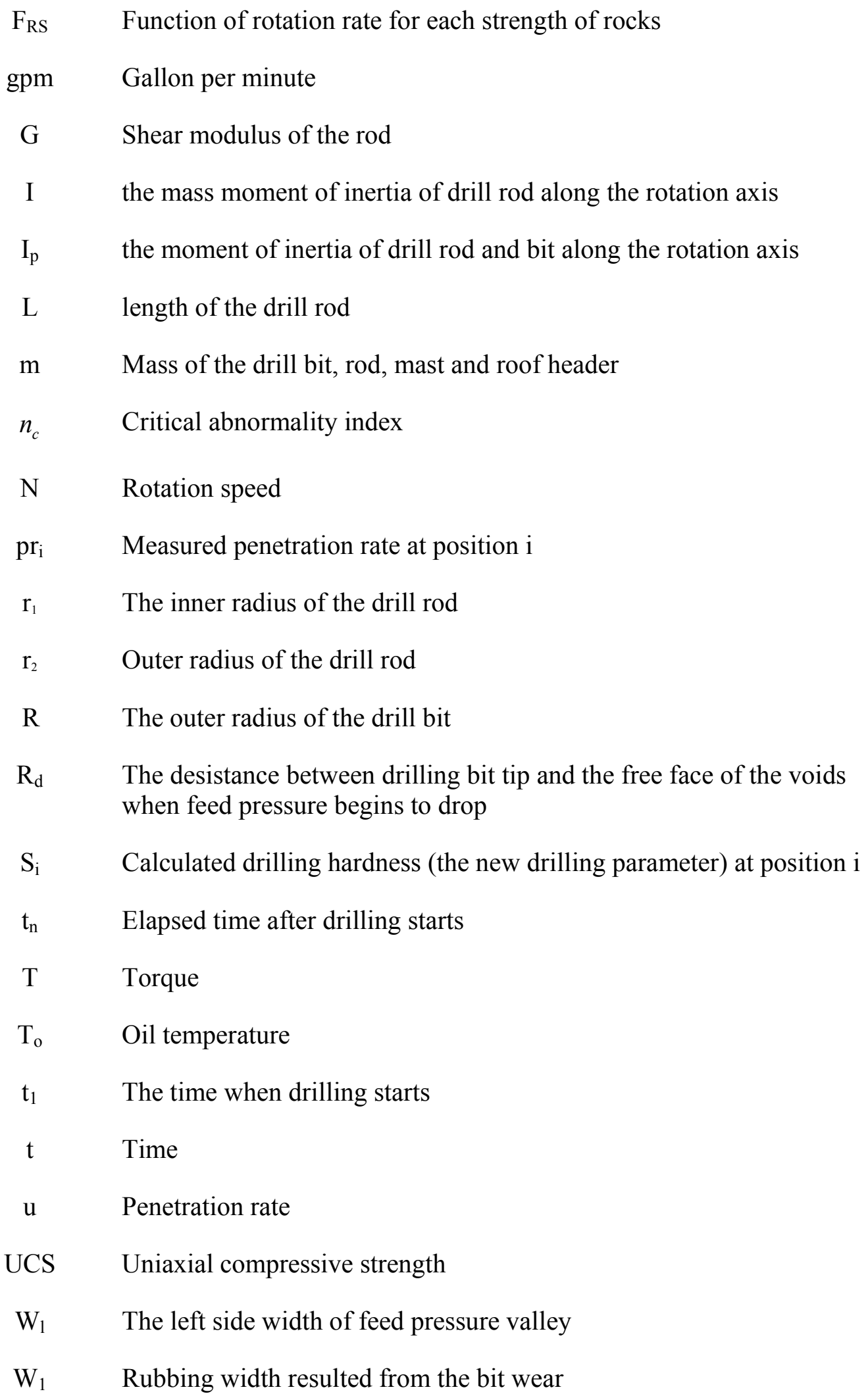




$\begin{array}{ll}\mathrm{W}_{2} & \text { Normal contact width } \\ \mathrm{X} & \text { Measured thrust valley width } \\ \mathrm{Y} & \text { Predicted void size } \\ \varphi & \text { Internal friction angle of the material } \\ v & \text { Poisson's Ratio } \\ \rho & \text { Unit weight } \\ \alpha & \text { Calculated rotational acceleration at a data point } \mathrm{i} \\ \alpha & \text { Angle of the increment of confined compressive stress } \\ \bar{\alpha} & \text { Average rotational acceleration for a drill hole } \\ \alpha \mathrm{i} & \text { Angular acceleration at point } \mathrm{i} \\ \sigma_{0} & \text { Uniaxial compressive strength } \\ \sigma_{\alpha} & \text { Standard deviation rotational acceleration for a drill hole } \\ \tau & \text { Shear Stress } \\ \tau \mathrm{i} & \text { measured torque at point } \\ \theta \mathrm{i} & \text { rotated angle from point } \mathrm{i}-1 \text { to } \mathrm{i} \\ & \text { angular velocity at point } \mathrm{i}\end{array}$




\section{CHAPTER 1}

\section{INTRODUCTION}

USA is one of the largest coal producers in the world. In 2003, 1316 coal mines in USA produced a total of 1,073.6 million short tons of coal ${ }^{(1),(2)}$. All U.S. underground coal mines use roof bolting as a major roof supporting method.

Since all underground coal mine entries are supported by roof bolts and the design and selection of roof bolts are based on the knowledge of roof geology, it is of utmost importance that the roof geology and its variation over the immediate operation areas be known in advance so that a proper roof bolting system can be designed and/or selected.

The current method of using surface borehole loggings, which is normally spaced more than $1,000 \mathrm{ft}$ apart, to determine the immediate roof is awfully inadequate. Roof falls that caused injuries/fatalities and/or production delays are mostly localized even though some massive roof falls have been reported. For localized roof falls, the major reason is change in geology. Obviously a selected roof bolting system must match a certain geological features (rock type and stratigraphic sequence). But when this geological feature change and differ considerably from the original one, the selected roof bolting system may not work and roof falls occur.

How can a roof control engineer know that geological features change at certain location? In fact in order to prevent roof falls the roof geology within the bolted horizon must be known in advance from bolt row to bolt row. Only with this knowledge, a roof control engineer can determine if a change in the current roof bolting system is needed. In order to achieve this objective, a detailed roof geology map depicting geological changes from bolt row to bolt row must be available. In this respect, if a roof bolter's drilling parameters can be monitored and correlated with the geological features, all changes in geological features can be mapped from bolthole to bolthole when the roof bolts are being installed and the roof bolting system will stay compatible with the roof geological features.

Against this background, a project sponsored by the US Department of Energy

under the Industry of Future (Mining) program was initiated five years ago. In this 
project a patented drill control unit (DCU) installed in the J.H. Flecher \& Co's roof bolter was used to record the drilling parameter for experiments conducted in the mines and laboratory. The objective of the project is to develop a set of algorithms for determining the geological features of mine roof rocks based on the drilling data measured during routine roof bolt drilling operations and a software package which enable mining engineers and miners to easily and conveniently utilize roof drilling data in the design of roof support.

A series of laboratory and mine site experiments are conducted. Finally, more than 1,000 roof bolt holes have been drilled in and drilling parameters recorded from 10 concrete and simulated blocks in the laboratory and 8 underground mines. The main characteristics of interest are rock strength and the identification of fractures/voids. A software package, Mine Roof Geological Information System (MRGIS), for drilling data collection, store, analysis and display are developed. The results of the research will help the mining industry improve production and safety. 


\section{CHAPTER 2}

\section{LITTERATURE REVIEW}

As geologic features play an important role in coal mine roof support design, many researches have been done in order to develop the methodology for detecting these geologic features. Using drilling parameters to identify geologic features is believed as promising therefore many researchers have invested their efforts in the study of this field.

\subsection{Specific Energy developed by Teale}

Teale ${ }^{(3)}$ conducted a theoretical study to examine the energy balance of rotary non-percussive actual drilling operations. Rotary drilling can be considered as a combination of two components: indentation and rotation cutting. Indentation forces the cutting bit into the rock. Simultaneously, the bit is moving laterally by rotation to break out fragments of rock. Teale proposed the concept of specific energy to describe the work done per unit volume excavated.

Teale defined specific energy as the follows:

$$
e=\frac{F}{A}+\frac{2 \pi N T}{A u} i n . l b / i n^{3}
$$

where: $\quad \mathrm{e}=$ specific energy

$\mathrm{F}=$ thrust, $1 \mathrm{bs}$

$\mathrm{A}=$ the area of the hole, in2

$\mathrm{N}=$ rotation speed, rev/min

$\mathrm{T}=$ torque, in- $\mathrm{lb}$ in

$\mathrm{u}=$ penetration rate, $\mathrm{in} / \mathrm{min}$

Specific energy can be divided into two components: the thrust component et and rotary component er.

$$
\begin{aligned}
& e_{t}=\frac{F}{A} \mathrm{in} . \mathrm{lb} / \mathrm{in}^{3} \\
& e_{r}=\frac{2 \pi N T}{A u} \mathrm{in} . \mathrm{lb} / \mathrm{in}^{3}
\end{aligned}
$$


According to Teale, et is ALWAYS small, sometimes negligible. Therefore he focused more on studying the rotary component of specific energy in his research.

Teale tried to study the relationships between specific energy and thrust, torque, rotation speed, penetration speed, and crushing force. If the area of the hole and rotation speed are constant, er is proportional to $\mathrm{T} / \mathrm{u}$. T/u curves are approximately straight lines through the origin over a relatively large range. T/u should be constant because it is the slope of straight curve lines. It can be further concluded that $\mathrm{e}_{\mathrm{r}}$ and therefore e itself should be approximately constant at large particle size. Another way Teale proposed is to check the relationship between the torque and the penetration per revolution as the follows:

$$
e_{r}=\left(\frac{2 \pi}{A}\right)\left(\frac{T}{p}\right) i n \cdot l b / i n^{3}
$$

where: $\mathrm{p}=\mathrm{u} / \mathrm{N}$ in $/ \mathrm{rev}$, the penetration per revolution.

For brittle materials like rock, the rate at which the required energy for breaking may not affect its amount. Thus rotation speed may not affect the amount of $\mathrm{T} / \mathrm{p}$ for a given rock type.

Teale believed that a certain theoretically attainable minimum quantity of energy will be needed to excavate a given volume of rock. Its amount will depend entirely on the nature of the rock. When the thrust is low, the specific energy will be very high. The penetration of the bit will close to zero if very low thrust can not provide enough force to push the bit into the rock. But a certain amount of energy is still consumed by friction. Also the size of the broken particles will be smaller. As the thrust decreases towards zero, the specific energy increases towards to infinity. As the thrust increase, the size of the broken particles will increase and the specific energy will decrease until it reaches a value beyond which it either will reduce slowly or will increase again. This minimum specific energy means the maximum drilling efficiency has been achieved for a given bit when drilling a given rock type and can be correlated with the crushing strength of the rock. After this point or stage, the drilling efficiency will decrease, which results in the rise of the specific energy. 
Teale used the data from different sources to examine his specific energy theory. It was found that in all cases the curves of the specific energy vs. the thrust show similar patterns: the specific energy reduces to the minimum value as the thrust increases, but only few of them rise again.

\section{Application and limitation:}

1. More work needs to be done to establish the relationship between specific energy and uniaxial compressive strength. Mining engineers can not directly use specific energy in their roof support designs

2. $\quad$ Further evidences are needed to prove that there is one and only one SED that can represent a given rock.

\subsection{Research by WVU}

Since 1999, a research team of West Virginia University, cooperating with J. H. Fletcher \& Co., has conducted a research on identifying roof geological properties using roof bolter drilling parameters. This research is the major part of a research project, Evaluation of Roof Bolting Requirements Based on In-mine Roof Bolter. The project was sponsored by U.S. Department of Energy under its Energy Industry of Future (mining) program. Dr. Syd S. Peng, Chair and C. T. Holland Professor of Department of West Virginia University, was the major investigator of the project. Gerald L. Finfinger, Yi Luo, Quanzhong Gu, David Xianguang Tang, Benjamin T. Mirabile, Takashi Sasaoka successively participated in the research. The achieved results of the research have been published over the past years ${ }^{(4-11)}$.

In the study, a J.H. Fletcher HDDR Walk-thru type dual head roof bolter was used in the research. This roof bolter is equipped with a feed back control and drilling parameters collecting system. The feed back control and drilling parameters collecting system was originally designed for controlling the roof bolter automatically so that overall drilling and bolting consistency can be improved ${ }^{(12)}$. It consists of a set of sensors and a data control unit (DCU) installed on the roof bolter. One side of the machine has standard hydraulic controls while the other side is fitted with the Fletcher Feedback Control System. This system allows the operator to pre-set the penetration rate, rotation rate, and maximum thrust of the machine. Once the parameters are set, the 
machine drills without additional operator input. A data logger allows drilling data to be monitored and analyzed.

The drilling parameters collecting system can collect 16 hydraulic drilling parameters. Feed pressure measures the hydraulic pressure inside the cylinders applying the axial load and can be used to determine thrust. The rotation pressure records the hydraulic pressure in the hydraulic motor that provides rotational force. RPM-Counts is measured using an electronic tachometer attached directly to the drill mast and can be converted into rotational velocity.

The drilling parameters are collected in terms of sensor output (voltages) and then converted to dimensionless numbers ranging from 0 to 255 since the resolution of the $\mathrm{A} / \mathrm{D}$ converter is only 8 bit. These dimensionless numbers are referred to as machine data and can be converted to engineering units based on different sets of conversion factors for different drilling parameters if necessary. In the tests, all drilling parameters are collected into an ASCII file and stored on floppy disks, and then copied to a desktop PC for data analysis. The ASCII file containing machine data is referred as machine data file. A machine data file has two headlines and 17 column data, with different columns representing different drilling parameters. The equations for converting machine data into engineering unit are as follows:

$$
\begin{aligned}
& \text { Thrust }=12.25 \times \pi \times \mathrm{D}^{2} \div 4 \times \text { Data_} 2 \text {, lbs } \\
& \text { where D is cylinder diameter, } 3 \text { in } \\
& \text { Penetration rate }=0.02691 \times \text { Data_ } 3, \text { in } / \mathrm{sec} \\
& \text { Torque }=18.01 \times 10.5 \times 0.91 \times \text { Data_ } 4 /(2 \times \pi), \text { lbs-in } \\
& \text { Rotation velocity }=6.977 \times \text { Data_ } 6, \mathrm{rpm}
\end{aligned}
$$

A series of lab and underground tests were conducted. A series of manufactured roof rock blocks, designed and constructed by the Spokane Research Laboratory of NIOSH, were used in lab tests. Different blocks were designed to simulate different geological properties, such as a sequence of rock layers with different strength, the 
bedding separations of varying dimensions and orientations, fractures and joints, or massive rock like limestone and sandstone.

The blocks were embedded with different type of rock in low-strength concrete. The physical properties of the concrete and the embedded rocks were measured according to ASTM standards.

\subsubsection{Finfinger's work}

As a member of the research team of WVU, Finfinger participated in a series of lab and underground tests and co-authored a series of papers ${ }^{(4-7)}$.

\section{- $\quad$ The prediction of the locations and sizes of the discontinuities}

Based on measured drilling data from the fractured blocks, Finfinger (2003) proposed that the thrust valley be used as identifier for the presence and the sizes of the discontinuities, such as fractures, joints, and voids in the rock. When a constant penetration rate is preset in the feedback drilling system, the thrust should decrease rapidly to slow down the penetration if the voids are met by the drill bit. After the drill bit goes through the voids, the thrust will increase rapidly to bring the penetration rate back to its preset level. Thus a thrust valley is formed. The thrust valleys that drop more 50 percent can be associated with encountering the discontinuous features.

The distance between one side of the thrust valley to the other can be used to determine the size of the discontinuity. The author proposed two models for calculating the size of the discontinuity. One is a liner model with the following equation:

$$
\mathrm{Y}=1.0278^{*} \mathrm{x}-0.8706
$$

Another is a logistic model with the following equation:

$$
\mathrm{Y}=\mathrm{a} /(1+\mathrm{b} * \exp (-\mathrm{cx}))
$$

where: $\mathrm{Y}$ - predicted void size

$\mathrm{x}-$ measured thrust valley width

$a=5.9165$

$b=82.0543$

$\mathrm{c}=1.3159$

Application and limitation: 
1. The author is the first who proposed thrust valley as the indicator of void/fracture. But the author did not give quantitative rules that can be implemented in computer program, such as the rules for determining if there is thrust valley in a set of measured data points. This limits its application.

2. The author proposed a thrust valley with at least $50 \%$ drop of thrust level as the criteria for determining voids/fractures. This conclusion was derived from the test results on fracture block which were constructed using high strength concrete (over 12,000 psi compressive strength). To extend this conclusion to roof rocks with lower compressive strength, for example shale, may result in high misprediction rate because thrust drop may be less than $50 \%$ when encountering a void. In addition, different drilling settings also have a large impact on the thrust level. If a high rotation rate and a low penetration rate are used when drilling a given rock, thrust level will be low. Therefore a void in the rock may not be predicted because the thrust drop resulted from the void is smaller than $50 \%$.

3. The author created two equations to calculate the size of void based on that of thrust valley. The accuracy of proposed method may be low when used in prediction for small voids because the right side of thrust valley is not related to the size of void.

\section{- The prediction of the rock layer interfaces}

Finfinger also proposed that the rotational acceleration be used for determining the boundaries or interfaces which are between the rock layers with different mechanical strengths. The author assumed that the rotation rate will change significantly when the drill bit penetrates through the boundaries between the rock layers with different strengths. To determine the significance of the changes in the rotational acceleration, the following equations are used:

$$
n=\left\{\begin{array}{cc}
0 & \text { if } \quad\left|\frac{\alpha-\bar{\alpha}}{\sigma_{\alpha}}\right|<n_{c} \\
\frac{\alpha-\bar{\alpha}}{\sigma_{\alpha}} & \text { if } \quad\left|\frac{\alpha-\bar{\alpha}}{\sigma_{\alpha}}\right| \geq n_{c}
\end{array}\right.
$$


where: $\alpha$ - calculated rotational acceleration at a data point i, $\alpha=5^{*}\left(\mathrm{RPM}_{\mathrm{i}+1^{-}}\right.$ $\left.\mathrm{RPM}_{\mathrm{i}-1}\right) / 60$

$\bar{\alpha}$ - average rotational acceleration for a drill hole

$\sigma_{\alpha}$-standard deviation rotational acceleration for a drill hole

$n_{c}$ - critical abnormality index, $n_{c}=1.3$

Based on the results of the lab tests drilled in the layered blocks, $n_{c}$ is determined as 1.3.

When the layer blocks (blocks 1-3) were used to evaluate the accuracy of the method for identifying the rock layer interfaces, overall $70 \%$ of the interfaces were predicted. The distances of $57 \%$ of the predicted interfaces from the actual locations were less than 2 inches.

Application and limitation:

1. According to the author, all the lab drilling tests were conducted under constant rotational rates. Under this kind of control mode, the feedback system will provide a constant rotation flow to the hydraulic motor in order to keep the rotation rate constant. This implies that the drill bit will rotate at a relative constant speed regardless whatever materials it meets unless a rotation stall occurs. Therefore the reliability of using the rotational acceleration as the indicator for the interfaces is questionable.

2. When the abnormality indexes were closely spaced, only the location of the first abnormality index was selected as an interface. This is only feasible for analyzing the results of the lab tests because the locations of the interfaces are known in advance. The author did not define how much the distance between the abnormality indexes should be when this rule is applicable. Thus its usefulness will be limited when it is applied in a production operation.

3. $\quad$ The author believed "A steady state operation is necessary to obtain a meaningful acceleration determination." The results of all the lab and field tests show that the hydraulic and data measuring system in use can not achieve an ideal steady state because of different reasons, such as the inhomogeneity of the 
rock and the adjustment of the hydraulic system. Therefore it is possible to predict the interfaces even this interface identifying methodology is applied on a massive rock which has no interfaces. For example, the data collected during the drilling in the solid concrete block is used to access the usefulness of the method (Figure 2.1). It can be seen that at least two interfaces can be predicted although no interface exists in the block.

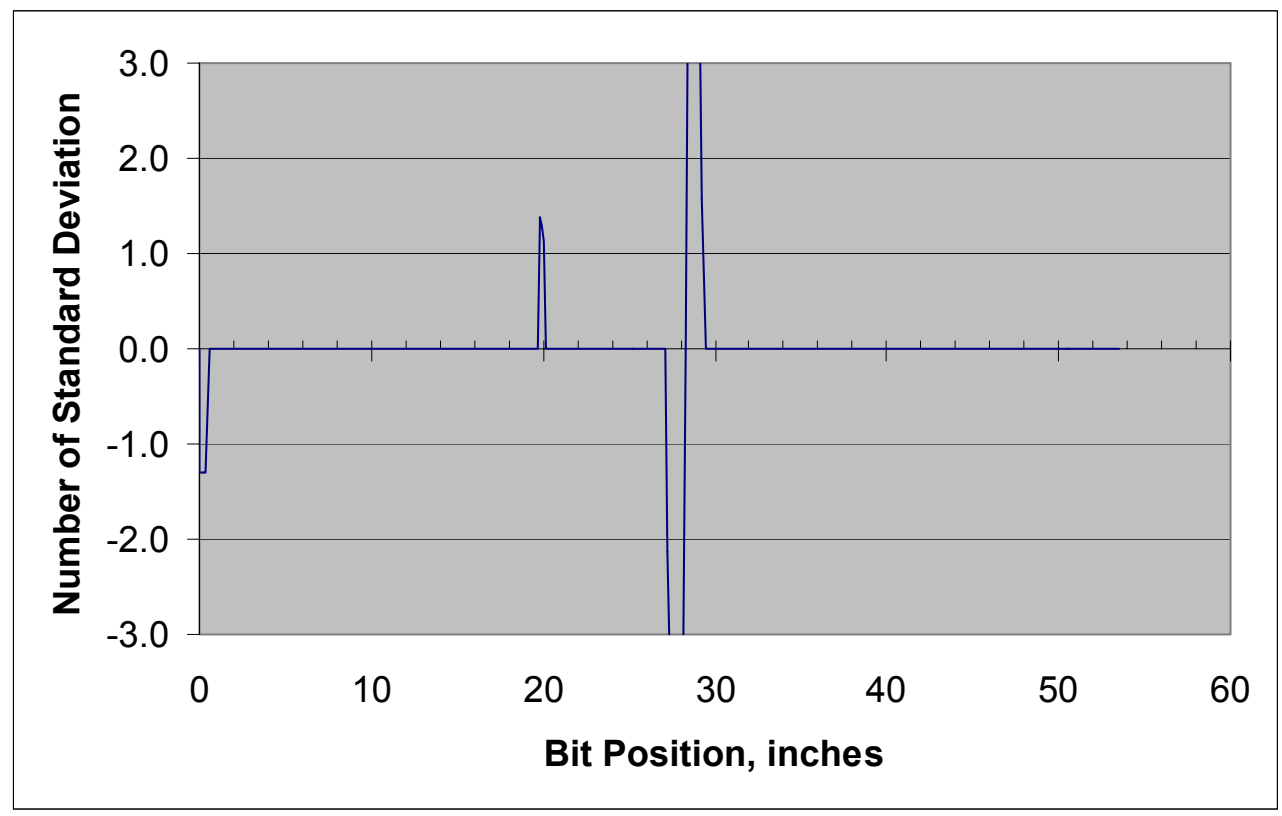

Figure 2.1_Abnormality indexes drilling in solid concrete block

\section{- The determination of the rock strength}

A mathematical model was proposed for determining the strength of the rock using the measured drilling parameters. This model was developed based on the following assumptions:

1. The drilling operation is in its steady state, that is, the bite depth defined as penetration per revolution $(b)$ is constant with no sudden changes in either penetration rate or rotation rate.

2. A sufficient length of the drill bit has penetrated in one type of rock that is free of fractures and voids. 
3. Thrust $(F)$ is used to overcome the compressive strength of the rock $\left(\sigma_{0}\right)$ over the normal contact area $\left(A_{n}\right)$ between the rock and drill bit along the axial direction.

4. Torque $(T)$ is used to overcome the moments generated by: (1) the frictional resistance at the front tip of the drill bit, and (2) the shear strength $(\tau 0)$ over the shear contact area $\left(A_{s}\right)$ in the tangential direction.

A core assumption that the authors made but did not mention explicitly is that the bit depth is equal to the bite depth (Figure 2.2). The bit depth is the length the drill bit immerses into the rock while the bite depth is the bit penetration per revolution. Mainly based on this assumption, the normal and shear contact areas were derived. The equations for determining the normal contact area are discussed later on in this chapter. The shear contact area is the product of the bite depth and the diameter of the bit.

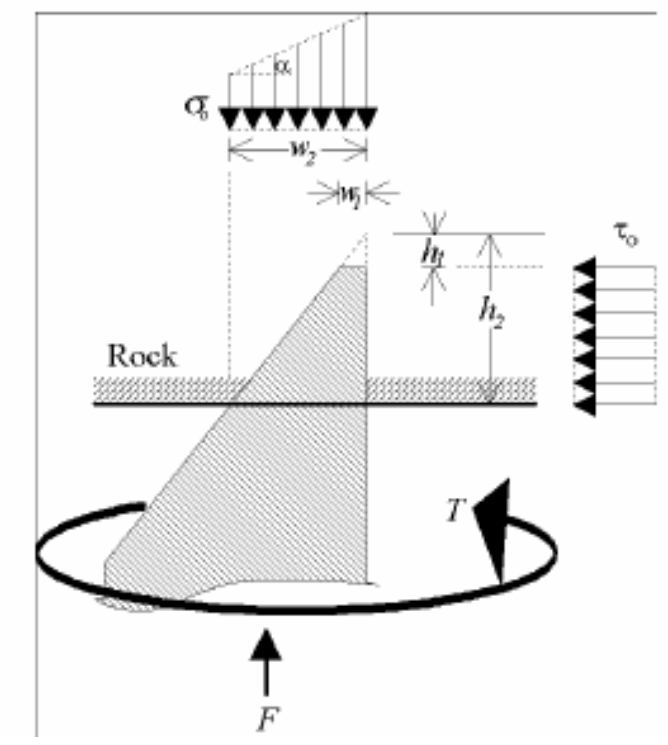

Figure 2.2 Bit-rock interaction in rotary drilling

The following equations were proposed to determine the unconfined compressive and shear strengths using the drilling parameters.

$$
\sigma_{0}=\frac{F}{D \times W_{2} \times\left(1+\left(W_{2} \times \tan \alpha\right) \div 2\right)}
$$




$$
\tau_{0}=\frac{4}{b \times D^{2}} \times\left[T-\frac{0.85}{4} \times \sigma_{0} \times W_{1} \times D^{2} \times\left(1+\frac{2 W_{2}-W_{1}}{2} \times \tan \alpha\right)\right]
$$

where: $\sigma_{0}$ - unconfined compressive strength

$\tau_{0}$ - shear strength

$\mathrm{W}_{1}$ - rubbing width resulted from the bit wear

$\mathrm{W}_{2}$ - normal contact width

$\alpha$ - angle of the increment of confined compressive stress

F - thrust

$\mathrm{T}$ - torque

$\mathrm{D}$ - the diameter of the bit

B - bite depth

\section{Application and limitation:}

1. The bit wear does have an effect on drilling parameters but is difficult to be considered in a routine production operation until an automatic bit wear measuring system is available.

2. The results of latest tests show that the two ends of the bit tip suffer more wear than the center of the bit tip. This is different from author's assumption made for the calculation of normal contact area.

\section{Friction is from not only the top but also the side of bit}

\subsubsection{Gu's work}

Gu proposed a method for determining the locations of the interfaces between two layers and the discontinuities which is defined as the lack of continuity within one rock layer, such as fractures, cracks, etc ${ }^{(8)}$. The box plot detecting outlier method is used to detect the outliers of the slopes of the drilling hardness (DH) which are mainly derived from the calculated thrusts of a single drill hole. Then the locations of these outliers are classified the location of the interfaces and discontinuities of the hole. 
Gu believed that the work done by thrust can be divided into components: kinetic energy, potential energy, the work used to overcome the frictional resistance between the front of the bit and rock in the axial direction, and work done to break the cementing bond of rock.

The author defined the stress used to break the cementing bond of or penetrate into the drilled rock as drilling hardness (DH). DH can be calculated as the follows based on the energy equilibrium.

$$
S_{i}=\frac{F_{i} \cdot d_{i}\left(1-c \cdot f_{i}\right)-0.5 \cdot m \cdot\left(\left(p r_{i}\right)^{2}-\left(p r_{i-1}\right)^{2}\right)-m g \cdot d_{i}}{d_{i} \cdot A_{i}}
$$

where $\mathrm{S}_{\mathrm{i}}-$ calculated drilling hardness (the new drilling parameter) at position $\mathrm{i}$, psi

$F_{i}-$ measured thrust at point $i$, lbs

$\mathrm{m}$ - mass of the drill bit, rod, mast and roof header

$\mathrm{pr}_{\mathrm{i}}-$ measured penetration rate at position $\mathrm{i}, \mathrm{in} / \mathrm{sec}$

$\mathrm{d}_{\mathrm{i}}-$ distance drilled from point $\mathrm{i}-1$ to $\mathrm{i}$

$\mathrm{A}_{\mathrm{i}}$ - contact area between the bit and the drilled rock, in ${ }^{2}$

$f_{i}-$ frictional coefficient between the front part of the drill bit and rock at position i

$F_{i} \cdot d_{i}-$ work done by thrust from point $\mathrm{i}-1$ to $\mathrm{i}$

$\mathrm{c}$ - factor by considering the geometry of the bit

$\mathrm{m} \cdot\left(\left(\mathrm{pr}_{\mathrm{i}}\right)^{2}-\left(\mathrm{pr}_{\mathrm{i}-1}\right)^{2}\right) / 2$ - change of kinetic energy from point $\mathrm{i}-1$ to point $\mathrm{i}$

$c \cdot F_{i} \cdot f_{i} \cdot d_{i}-$ work used to overcome the frictional resistance in drilling

(axial) direction

$\mathrm{mg} \cdot \mathrm{d}_{\mathrm{i}}-$ potential energy from point $\mathrm{i}-1$ to $\mathrm{i}$

$S_{i} \cdot A_{i} \cdot d_{i}-$ work used to overcome the resistance of rock 
$\mathrm{Gu}$ did not think that the shear strength of the rock has any influence on the energy consumed by rock drilling. The work done by the torque can be divided into three parts: kinetic energy, torsion energy, and the frictional resistance of rock in the tangential direction. Thus friction coefficient used in equation (2.14) can be calculated as the follows based on the energy equilibrium.

$$
f_{i}=\frac{\tau_{i} \cdot \theta_{i}-\frac{1}{2} I \cdot \omega_{i}^{2}+\frac{1}{2} I \cdot \omega_{i-1}^{2}+\frac{\tau_{i}^{2} \cdot L}{G \cdot I_{p}}}{F_{i} \cdot R \cdot \theta_{i}}
$$

where $\mathrm{fi}$ - the frictional coefficient at point $\mathrm{i}$

$$
\begin{aligned}
& \theta \mathrm{i}-\text { rotated angle from point } \mathrm{i}-1 \text { to } \mathrm{i}, \quad, \theta=\omega_{i-1} \cdot t+\frac{1}{2} a_{i} \cdot t^{2} \\
& \alpha \mathrm{i}-\text { angular acceleration at point } \mathrm{i}, \mathrm{rad} / \mathrm{sec}^{2}, \quad \alpha_{i}=\frac{2 \pi}{60 \cdot t} \cdot\left(R P M_{i}-R P M_{i-1}\right)
\end{aligned}
$$

$\omega \mathrm{i}$ - angular velocity at point $\mathrm{i}, \mathrm{rad} / \mathrm{s}, \quad \omega_{i}=\frac{R P M_{i}}{60} \cdot 2 \pi$

$\tau \mathrm{i}$ - measured torque at point $\mathrm{i}$

$\mathrm{L}-$ length of the drill rod, 62 inch

$\mathrm{G}$ - shear modulus of the rod, $11.5 \times 106, \mathrm{lb} / \mathrm{in} 2$

I - the mass moment of inertia of drill rod along the rotation axis)

$$
I=0.083 \cdot m\left(r_{1}^{2}+r_{2}^{2}\right), 1 \mathrm{~b}-\mathrm{in}^{2}
$$

$\mathrm{m}$ - mass of the rod

Ip - the moment of inertia of drill rod and bit along the rotation axis

$$
I_{p}=1.9246\left(r_{2}^{4}-r_{1}^{4}\right)
$$

$\mathrm{r} 1, \mathrm{r} 2-$ the inner and outer radius of the drill rod, $\mathrm{r} 1=0.271$ inch, $\mathrm{r}_{2}=$ 0.396 inch

$\mathrm{R}$ - the outer radius of the drill bit, $0.6875 \mathrm{inch}$ (1 $3 / 8$ bit)

$\mathrm{Fi}-$ measured thrust at point $\mathrm{i}, \mathrm{lbs}$ equation

After calculating the $\mathrm{DH}$, the slope of $\mathrm{DH}$ can be obtained using the following 


$$
\text { slope }(i)=\frac{n \cdot \sum_{j=1}^{n} x_{j} \cdot y_{j}-\left(\sum_{j=1}^{n} x_{j}\right)\left(\sum_{j=1}^{n} y_{j}\right)}{n \sum_{j=1}^{n} x_{j}^{2}-\left(\sum_{j=1}^{n} x_{j}\right)^{2}}
$$

where slope(i) - the slope of drilling hardness at point $\mathrm{i}$

$\mathrm{n}$ - the number of data points used to calculate the slope at point $\mathrm{i}$

$\mathrm{xj}$ - the drilling distance from hole mouth at point $\mathrm{i}-\mathrm{j}$ or $\mathrm{i}+\mathrm{j}$

$y j-$ the value of drilling hardness at point $i-j$ or $i+j$

Box plot method is used to detect the outliers of DH slopes.

Highly suspect outliers (the slopes of DH beyond the outer fences) are determined as interface. The positive suspect outliers (the slopes of DH between the upper inner and upper outer fence) are sublayers. The negative suspect outliers (the slopes of DH between the lower inner and lower outer fence) are discontinuities. The detected interfaces within 4 inches (counting from the bottom to the top) should be averaged. This averaged location is the location of the interface in the area.

Both the lab test data and field test data were used to verify the DH slope outlier method. When calculating the accuracy, the author ignored the unexpected results of the prediction. $75 \%$ of the interfaces drilled through the block 6 were detected using the method. The accuracy of the prediction for the block 2,3 , and 4 is $27.8 \%$. For fractured block, 38.1\%. When the method was applied to the test result for solid block, at least one discontinuity was predicted in each drilled hole.

\section{Application and limitation:}

1. In the calculation of the friction coefficient, the author assumed that the work done by the torque is consisted of only three parts: kinetic energy, torsion energy, and the energy to overcome the frictional resistance of rock in the tangential direction. From this assumption, it can be concluded that the rotation of the bit does not involve in the breakage of rock. Therefore the 
thrust energy is independent on the rotary energy. For a given rock and a given feed rate, the rotation speed should not affect the magnitude of the thrust. Our testing data can not support this conclusion.

2. Although not giving the definition of a sublayer, the author classified a positive slope between the outer and inner fences as a sublayer. On the other side, the author believed that a negative slope between the outer and inner fences as a discontinuity.

3. The author neither correlated the drilling hardness to any widely accepted rock hardness index such as UCS (Uniaxial Compressive Strength), shear strength, tensile strength, Point load or RQD (Rock Quality Designation), nor proven $D H$ is unique for a given type of rock. This limits its usefulness in roof support design.

4. DH is not zero when drill bit passes through a void/ fracture. This means that air also has a drilling hardness

5. The author did not prove that $D H$ is an intrinsic property of a rock. Different parameters presetting will produce different DH for a given rock.

6. The criteria for interface and discontinuity has no theoretical and experimental basis. There is no evidence support the rule for determining the location of the discontinuity because the fracture slope may be larger than interface slope. For example, hole 3 drilled in the fractured block ( $p r=1.5$, rpm=300)(p162) negative slope value reaches more than -20,000, while hole 3 drilled in layer block 6 minimum slope value is less than $-6,000$.

7. $\quad$ Positive slope is not necessarily an interface because the right side of a thrust valley caused by a void also has a positive slope. The author used 4 inch interval to avoid this which has no experimental evidence

8. Using neural network to classify rock needs to know how many layers in advance 


\section{- Contact area difference between Finfinger and Gu}

Both Finfinger and Gu proposed the concept of contact area in their algorithms of determining geological features using roof bolter drilling parameters. Gu described the contact area $\mathrm{A}_{\mathrm{i}}$ as the area between the front part of the bit and the corresponding rock while the drill bit is at point $i$. Finfinger divided the contact area into the normal contact area $A_{n}$ and shear contact area $A_{s}$.

None of authors gave the details as to how a contact area was derived or measured. Both Finfinger and $\mathrm{Gu}$ believed that the contact area is the function of penetration per revolution (bite depth). They all assumed that the bite depth is the depth which a drill bit immerses into the rock. Since Gu used the contact area Ai in the calculation of the work done to overcome the resistance of rock by the thrust, it can be derived that the contact area Ai proposed by $\mathrm{Gu}$ is the same as the normal contact area An proposed by Finfinger.

For the two sizes of bits used the test, Finfinger derived empirical equations for normal contact area as the follows:

For 1-3/8" bit

$$
\mathrm{A}_{\mathrm{n}}= \begin{cases}0.01085+4.1025 \times b & \mathrm{~b} \prec 0.16 \mathrm{in} / \mathrm{rev} \\ 0.60677+0.3750 \times b & \mathrm{~b} \geq 0.16 \mathrm{in} / \mathrm{rev}\end{cases}
$$

For $1-1 / 32$-in bit

$$
A_{n}=\left\{\begin{array}{cc}
0.01+2.4083 \times b & \mathrm{~b} \prec 0.12 \mathrm{in} / \mathrm{rev} \\
0.4562+0.4265 \times b-0.003 \div b^{2} & \mathrm{~b} \geq 0.12 \mathrm{in} / \mathrm{rev}
\end{array}\right.
$$

where: An - normal contact area, in2

$\mathrm{b}$ - bite depth, in/rev area:

Gu suggested that the following equations could be used to calculate the contact

For $1-3 / 8$-in bit

$$
\mathrm{A}_{\mathrm{i}}=\left\{\begin{array}{cc}
1.1518 \times b & \mathrm{~b} \leq 0.125 \mathrm{in} / \mathrm{rev} \\
1.1518 \times b+18.04762 \times(b-0.125)^{2} & 0.28125 \mathrm{in} / \mathrm{rev} \geq \mathrm{b} \geq 0.125 \mathrm{in} / \mathrm{rev}
\end{array}\right.
$$


For $1-1 / 32$-in bit

$$
\mathrm{A}_{\mathrm{i}}=\left\{\begin{array}{cc}
1.0607 \times b & \mathrm{~b} \leq 0.125 \mathrm{in} / \mathrm{rev} \\
0.13258 \times b+1.32583 \times(b-0.125)^{2} & 0.28125 \mathrm{in} / \mathrm{rev} \geq \mathrm{b} \geq 0.125 \mathrm{in} / \mathrm{rev}
\end{array}\right.
$$

where: Ai - contact area, in2

b - bite depth, in/rev

Comparing Finfinger's equations with that of $\mathrm{Gu}$, it can be seen that there is a big difference between them even they for the same type and size of bit. For example, when bite depth is $0.125 \mathrm{in} / \mathrm{rev}$ and the bit size is $1-3 / 8$-in, the contact area resulted from Gu's equation is 0.143975 while that from Finfinger's equation is 0.5236625 . The methods for deriving the equations may be different, but $72.5 \%$ of difference between the results from different equations seems to be unreasonable for a same size and type of bit. Therefore the concept of contact area and the method for calculating it need to be further explored and validated.

\subsubsection{Mirabile’s work}

Mirabile proposed to use shear stress/normal stress ratio as the basis for the interpretation of drilling data ${ }^{(9-11)}$. He defined normal stress as the ratio of thrust to normal contact area and shear stress as the ratio of shear force to shear contact area. Dividing torque by bit diameter can get shear force while normal and shear contact areas can be obtained using Figfinger's equations.

The author used Figure 2.3 to explain the thrust and torque relationship for two materials. Suppose that the bit geometry and thrust applied on the bit for drilling hard and soft materials are same. Then penetration distance in hard material will be smaller than that in soft material, which results in a smaller torque required for drilling in hard material. Therefore the torque/thrust ratio for hard material is smaller. The shear stress/normal stress ratio should have a similar relationship as torque/thrust ratio.

After calculating shear stress/normal stress ratio, the slope of the ratio can be calculated. Then box plot method is used to detect the outliers of shear stress/normal stress ratio slopes. 
Material A

Hard Material

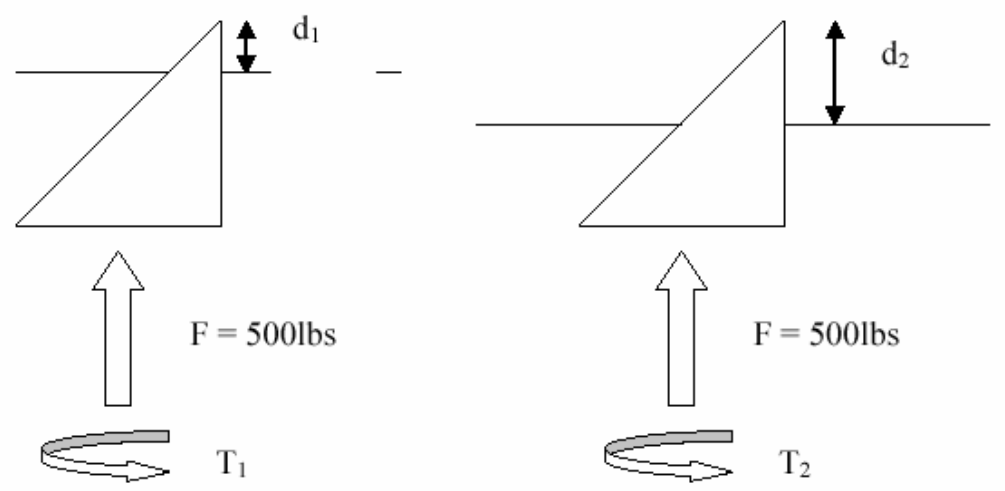

Material B

Soft Material

Figure 2.3 Thrust and torque relationship for two materials

\section{Application and limitation:}

1. In fact, shear stress/normal stress ratio is equal to torque/thrust ratio times a constant because the bit diameter and normal and shear contact areas derived from bit geometry should be constant for a given bit. So far no actual physical meaning of shear stress/normal stress ratio has been found out. Compared with torquelthrust ratio, the calculation of shear stress/normal stress ratio takes more time but achieves no improvement in geological feature prediction

2. The author did not suggest a way to distinguish cracks from layer interfaces

\subsection{Research Conducted by Itakura et al.,}

Itakur, et al, developed a roof logging system by rock bolt drilling which can

measure rock bolter drilling data such as torque, thrust, revolution and stroke $\mathrm{e}^{(13-14)}$. An Australian-made portable roof bolter which was popular in Japanese coal mines was chosen for the tests in the lab and the field. Different from the data collection system installed on J. H. Fletcher roof bolter which measures feed pressure and rotation pressure, the system used strain gauges attached to the surface of the drilling rod to measure torque 
and thrust. In the tests, both penetration rate and rotation rate were maintained at constant levels.

The authors conducted a series of lab tests in order to determine the relationship between rock drilling data and the following geostructural features:

1. rock type

2. rock structure

3. distribution of discontinuities (cracks, boundary of layers, separation of layers)

4. characterization of discontinuities (slope angles, crack width, orientations)

5. compressive strength of rock

Sandstone, sandyshale, and coal specimens were used in lab tests with compressive strength being 10,385, 8,600, and 3,597 psi (71.6, 59.3, and 24.8 MPa) respectively. The specimens with three different slope angles $(0,30,60$ degree) and two kinds of rock boundaries with the separation of layers being 0 and $5 \mathrm{~mm}$ respectively were constructed.

\section{LOG PATTERNS OF MECHANICAL DATA}

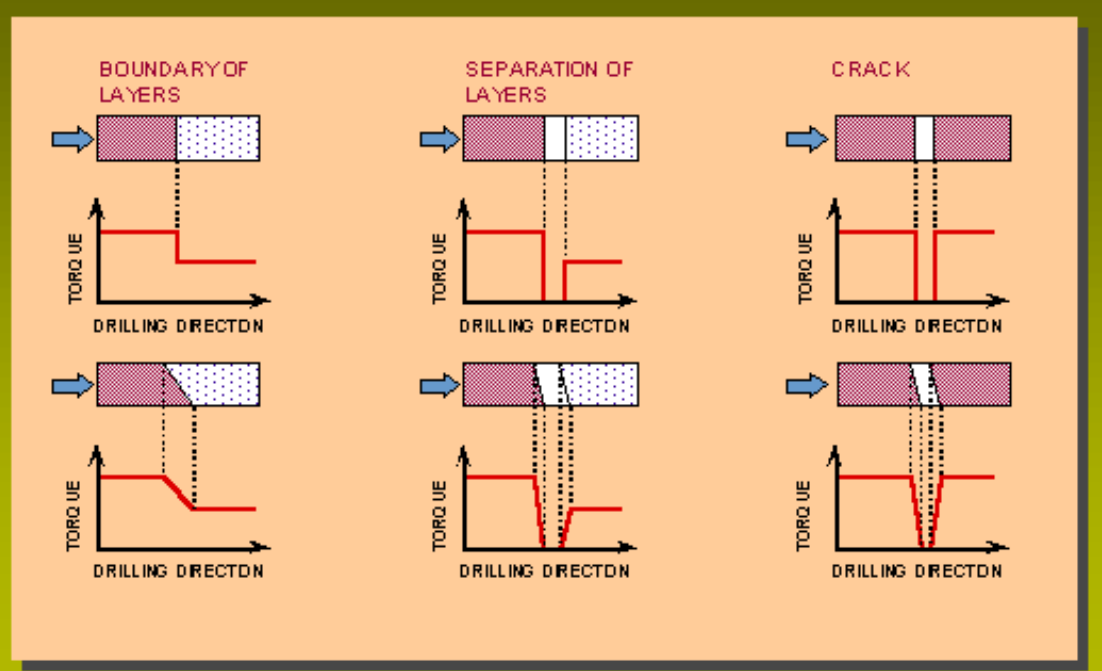

Figure 2.4 Log pattern of mechanical data dependent on discontinuities 
Based on the results of experiments, the authors concluded that the average value of torque and/or thrust could be used to determine the rock type. Also log patterns of thrust or torque were proposed by the authors. The angle and the spaces of discontinuity could be estimated using these torque or thrust patterns but the estimation error was large because the drilling mechanism near the discontinuity would change dramatically.

After data conversion and occasionally data smoothing to eliminate noise from eccentric rotation, the measured drilling data was analyzed using neural networks to automatically obtain the pattern of discontinuities. Two algorithms of neural networks were employed. As the first step, ART2 (adaptive resonance theory 2) was employed to classify rough patterns. One of the advantages of this algorithm is that it can classify patterns conveniently without teaching. However, it is too flexible to obtain precise pattern and difficult to tune because several parameters have to preset before inputting the data. Therefore this algorithm was used only for roughly classifying the patterns by setting the vigilance parameter to a high value. In the second step, the simple backpropagation network (BPN) was used, in which four patterns extracted by ART2 were used as learning data and ten series of log data were inputted for pattern extraction.

According to the authors, pattern recognition using neural networks could not tell the difference between cracks and layer boundaries. Therefore averages of torque/pressure between predicted discontinuities were calculated and compared. A predicted discontinuity was classified as a crack if the difference of both averages of before and after this discontinuity point was smaller than a threshold.

Application and limitation:

1. The log patterns proposed by the authors need to be improved. Take crack patters for example, the authors believed that torque would not drop until the drill bit reached the free face of crack and that once torque began to drop because of a crack, torque curve would have a slope angle of 0 degree if the boundary slope angle of the crack is 0 degree. This may not be true. Torque will start to decrease even before the drill bit arrives at the edge of the crack because brittle rock will break under the thrust force. It will take some time 
for torque to reduce to its lowest magnitude. During this period of time, the drill bit will keep moving at a preset penetration rate therefore the slope angle of torque curve will not be 0 degree even though the angle of the rock boundary at the other side of the crack is 0 . In summary, the shape of torque curve when encountering a crack will not be the same as that of the crack. This can be seen very clearly from the author's log data (Fig 2.5). Using improper patters in the analysis of drilling data may be one of the reasons for high estimation error rate although the authors thought it as a result of the significant change of drilling mechanism near the discontinuity.
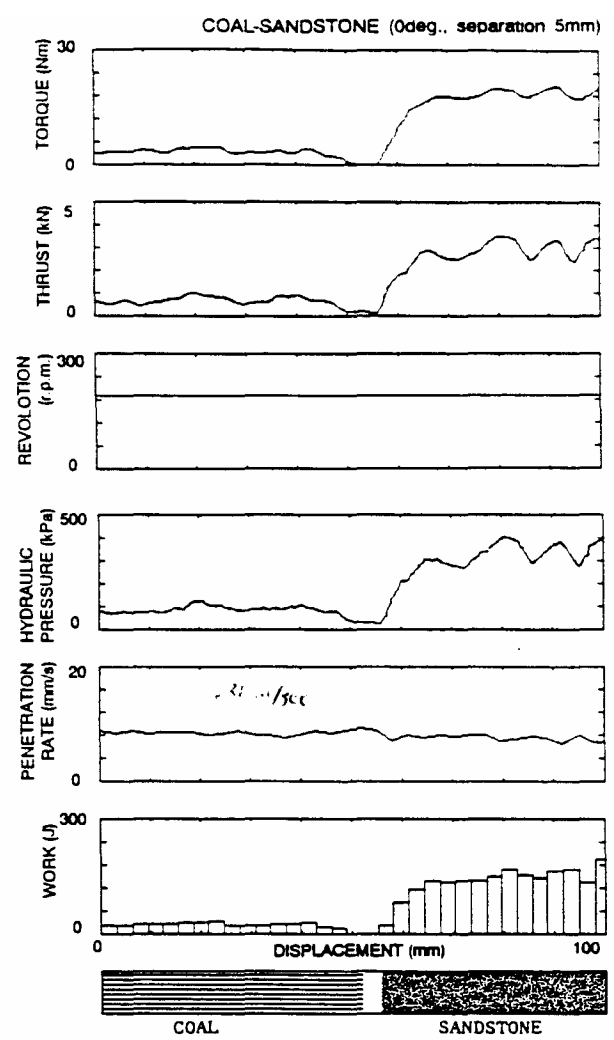

Figure 2.5 Mechanical data logs at boundary slope angle of 0 degree and $5 \mathrm{~mm}$ space

2. The authors did not mention how to determine the threshold which is the criteria for distinguishing the crack from a layer boundary based on the magnitude of the difference of the averages before and after a predicted 
discontinuity. This threshold has a huge impact on the accuracy rate of discontinuity determination. 


\section{CHAPTER 3}

\section{OBJECTIVES, SCOPE, AND METHODOLOGY}

\subsection{Research Objectives}

The major objectives of this research are:

1. Develop a set of algorithms or rules for determining the geological features of mine roof rocks based on the drilling data measured during routine roof bolt drilling operations. The geological features of interest are the location of fractures/voids and rock strength (UCS).

2. Develop a software package which enables mining engineers and miners to easily and conveniently utilize roof drilling data in the design of roof support.

\subsection{Scope of Research}

This research will focus on the following areas:

1. Determine the location of void/fracture using drilling parameters. Many researchers have tried to develop methods to determine the locations of discontinuities. A widely accepted definition about discontinuity is that it includes void and layer interface/boundary. There are two concerns associated with this research approach. First, there is no good way that has been developed to distinguish the voids from layer interfaces after determining the location of discontinuities. Second, there is no clear definition of layer interface in term of drilling parameters analysis. Finfinger defined the boundary interfaces as those with a significant change in physical properties and not necessarily a change in rock type. Because the strength of specific rock types ranges widely and roof drilling parameters can reflect the changes of hardness or strength of rock, it is difficult to use drilling parameters for identifying the rock types which are classified mainly based on the composition of rock, especially when the strengths of different types of rocks are closed to each other. But the questions still to be answered is how big change in rock strengths should be defined as a layer 
interface and if this change can be detected by using drilling parameters. Therefore only the location of rock void/fracture will be studied.

2. Estimating rock strength using drilling parameters. The strength of roof rock is a key factor in deciding a roof support plan. There is no doubt that being able to use roof drilling parameters to estimate roof rock strength will provide mining industry a faster and more cost effective option compared with other available methods for determining rock strength.

3. Develop a software package that implements the algorithms developed in this research.

\subsection{Methodology Used in This Research}

1. Use J. H. Fletcher roof bolter and DCU

2. Use WVU control mode

3. Select coal mine entry roof as test sites to simulate actual coal mining conditions

4. Conduct more compensation runs

5. Drill core to get roof rock sample

6. Using borehole scope device to verify the drilling data

7. Do lab tests to measure UCS of cores obtained from coal mine roof

8. Develop monitoring software to check drilling data on site

9. Select suitable drilling parameters to avoid thrust cap

10. Select suitable drilling location and sequence to observe the effect of oil temperature

11. Use raw data to analysis without converting into engineering unit.

12. Use Windows as platform and use VB, MS ACCESS, MapObject, OpenGL to develop software package.

13. Conduct bit wear tests 


\subsection{Drill System}

The research was conducted using a series of laboratory and underground tests for developing the theoretical methodology for roof geology prediction. All laboratory and underground tests were conducted using a J.H. Fletcher \& Co.'s model HDDR walk-thru type dual head roof bolter as shown in Figure 3.1. The drilling system consists of a set of sensors and a drill control unit (DCU) installed on the machine (Figure 3.2). One side of the machine has standard hydraulic controls while the other side is fitted with the patented Fletcher Feedback Control System ${ }^{(13)}$. This system allows the operator to preset the penetration rate, rotation rate, and the maximum feed pressure (= thrust cap) of the machine. Once the parameters are set, the machine drills without additional operator input. A data logger allows drilling data to be monitored and analyzed. Note the roof bolter is a mast feed type consisting of two stages: carriage and mast.

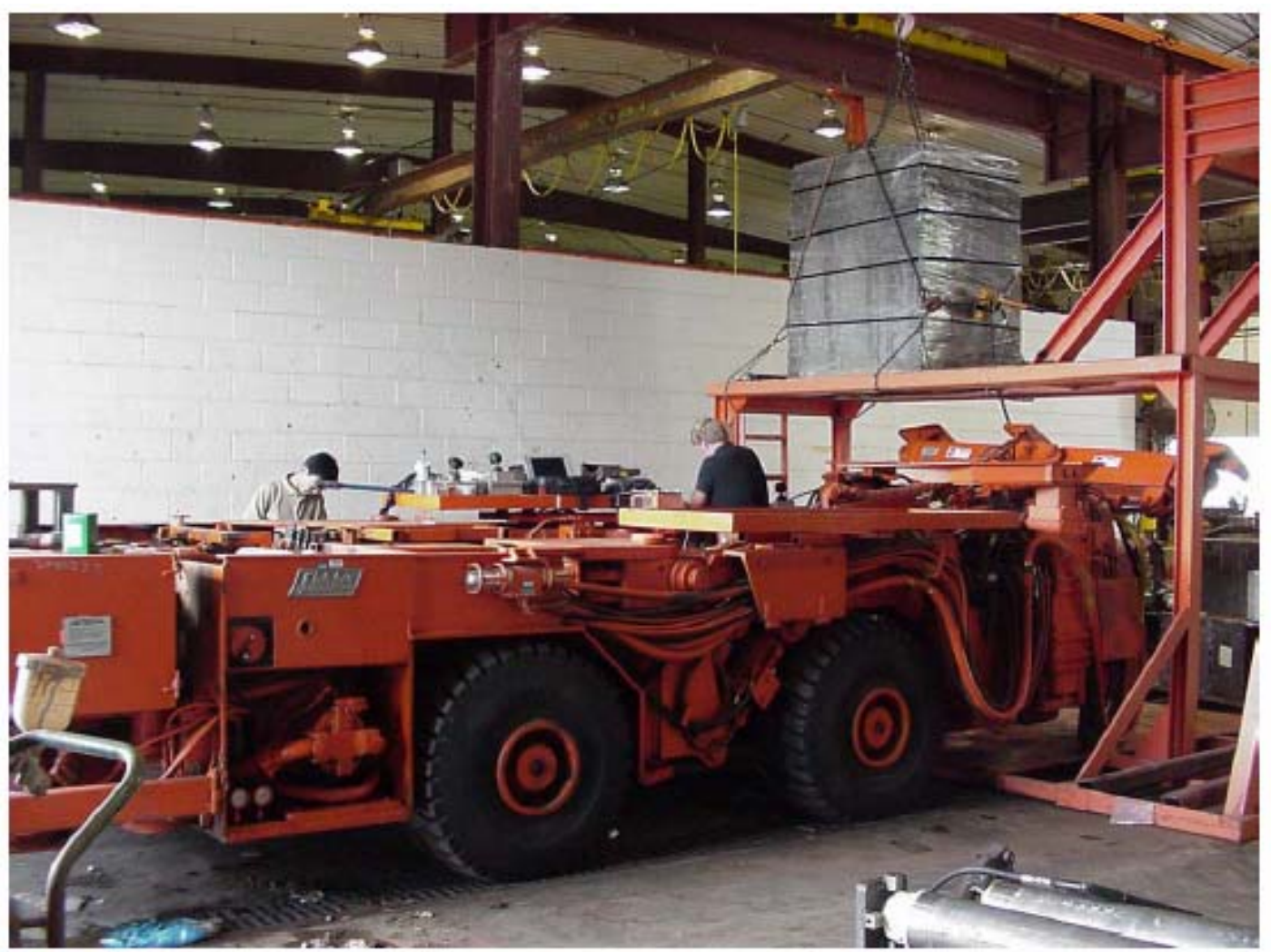

Figure 3.1 J.H. Fletcher \& Co.'s HDDR dual head roof bolter 


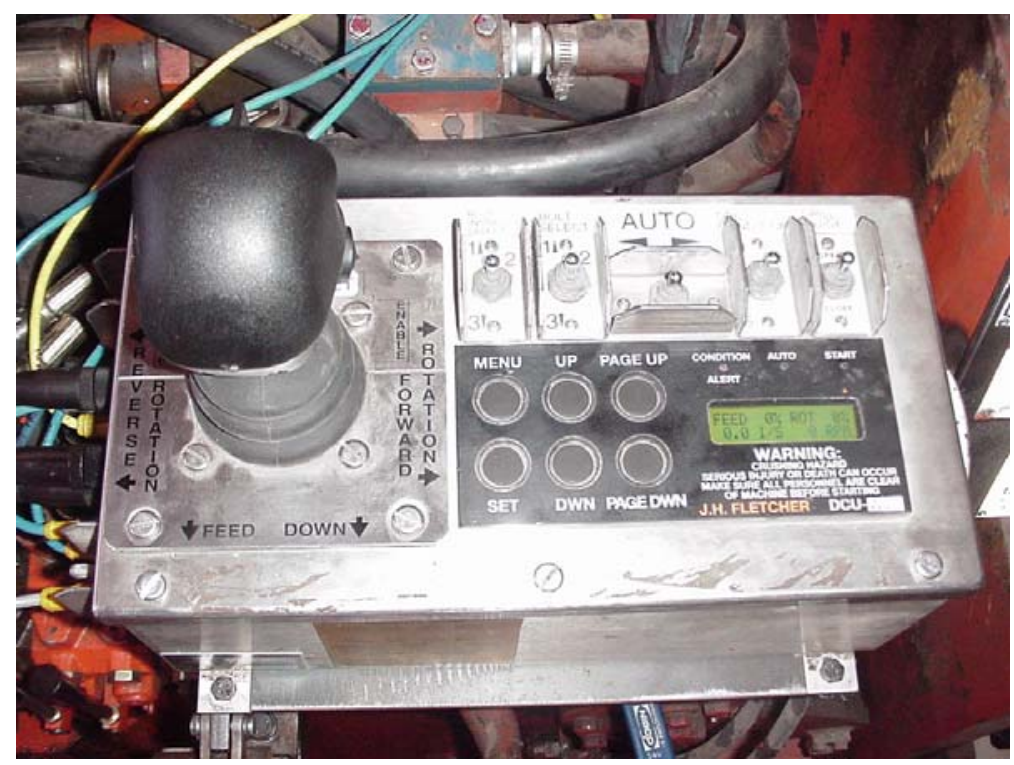

Figure 3.2 Drill control unit (DCU)

\subsection{Drilling Algorithm (WVU Control Mode)}

The drilling algorithms used in this project allow us to drill at both a pre-set penetration rate and a pre-set rotation rate. This control mode was designed for this project and called "WVU Control Mode". Using this mode, the effect of different drilling setting (penetration rate and rotation rate) on drilling parameters and drilling conditions can be determined. In other words, this control mode helps us develop the methodology for predicting roof geology that work at any penetration rate and rotation rate and determine the optimum drilling setting.

When the WVU control mode is set on, the penetration and rotation rates are controlled separately by the closed loop feed back system. When a specific penetration rate is keyed in before drilling, this value becomes the drill feed up target. The system analyzes only the current velocity and adjusts the electrical solenoid either up or down to achieve the preset target penetration rate. It does this without considering what other drilling parameters are. To drill the same velocity, the feed pressure will have to be higher while drilling through a harder material than a softer material. Likewise, the preset rotation rate is controlled based on the current rpm. System does this without considering other drilling parameters. At a constant drill penetration and rotation rates, the feed and rotation pressures should be higher when drilling through a harder material. 
Penetration rate and rotation rate are controlled independently by adjusting two 4way valves through pulse width modulation (PWM) control.

In a series of underground tests, the feedback control mode in which drilling is controlled based on both horsepower curve and bite curves selected was also tested.

\subsection{Data Collection System}

The drilling parameters collecting system was originally designed for controlling roof bolter automatically so that overall drilling and bolting consistency can be improved. Drilling parameters are recorded every 100 milliseconds so a 54-in long hole will have 250 to 850 records, depending on the penetration rate and the condition of roof geology.

The drilling parameters collecting system is designed to collect 17 or 15 drilling parameters as shown in Table 3.1. The feed pressure measures the hydraulic pressure inside the cylinders applying the axial load. The rotation pressure records the hydraulic pressure in the hydraulic motor that provides rotational force. RPM-counts is measured using an electronic tachometer attached directly to the drill mast and can be converted into rotational rate.

These drilling parameters are collected in terms of sensor output in voltages and then converted to dimensionless numbers ranging from 0 to 4095 for feed and rotation flow, and from 0 to 255 for others since the resolutions of A/D converter are 12 bits and 8 bits, respectively. Note before the underground test on May 2004, channel 3 and 5 (feed and rotation flows) are the upper 8 bits of the measurement of the feed/rotation flow transducers and the lower 4 bits of them are put into channel 14 and 15 as feed/rot flow LSB's. So, the data recorded before and after 2004 have 17 and 15 columns (channels), respectively. These dimensionless numbers are referred to as machine data and can be converted to engineering units based on the conversion factors if necessary. In the current experimental set up, all drilling parameters are collected and stored by a notebook PC into ASCII files. The ASCII file containing machine data is referred to as machine data file (Figure 3.3). A machine data file has 17 or 15 columns (channels) data, with different columns representing different drilling parameters. 
A software for displaying all the drilling parameters was developed as shown in Figure 3.4, so the drilling data and machine conditions including control condition can be checked at the test site immediately.

Table 3.1 Drilling parameters

(a)

\begin{tabular}{|c|c|}
\hline Column & Drilling Parameter \\
\hline Data 1 & Status Byte \\
\hline Data 2 & Feed Pressure \\
\hline Data 3 & Feed Flow \\
\hline Data 4 & Rotation Pressure \\
\hline Data 5 & Rotation Flow \\
\hline Data 6 & RPM Counts \\
\hline Data 7 & Rotation Torque \\
\hline Data 8 & Mast Position \\
\hline Data 9 & Carriage Position \\
\hline Data 10 & Vacuum \\
\hline Data 11 & Temperature \\
\hline Data 12 & Spare Channel \\
\hline Data 13 & Message Counter \\
\hline Data 14* & Feed Flow LSB's* \\
\hline Data 15* & Rot Flow LSB's* \\
\hline Data 16 & Feed PWM CMD \\
\hline Data 17 & Flow PWM CMD \\
\hline
\end{tabular}

(b)

\begin{tabular}{|c|c|}
\hline Column & Drilling Parameter \\
\hline Data 1 & Status Byte \\
\hline Data 2 & Feed Pressure \\
\hline Data 3 & Feed Flow \\
\hline Data 4 & Rotation Pressure \\
\hline Data 5 & Rotation Flow \\
\hline Data 6 & RPM Counts \\
\hline Data 7 & Rotation Torque \\
\hline Data 8 & Mast Position \\
\hline Data 9 & Carriage Position \\
\hline Data 10 & Vacuum \\
\hline Data 11 & Temperature \\
\hline Data 12 & Spare Channel \\
\hline Data 13 & Message Counter \\
\hline Data 14* & Feed PWM CMD \\
\hline Data 15* & Flow PWM CMD \\
\hline
\end{tabular}

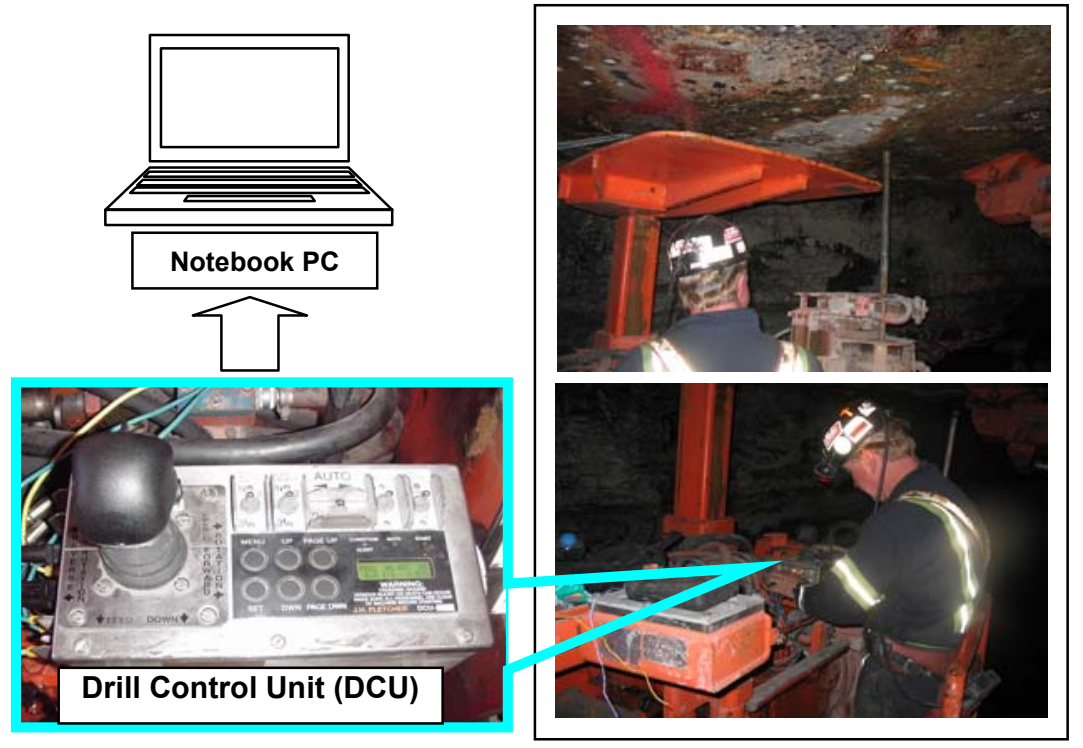

Figure 3.3 Data collecting system 


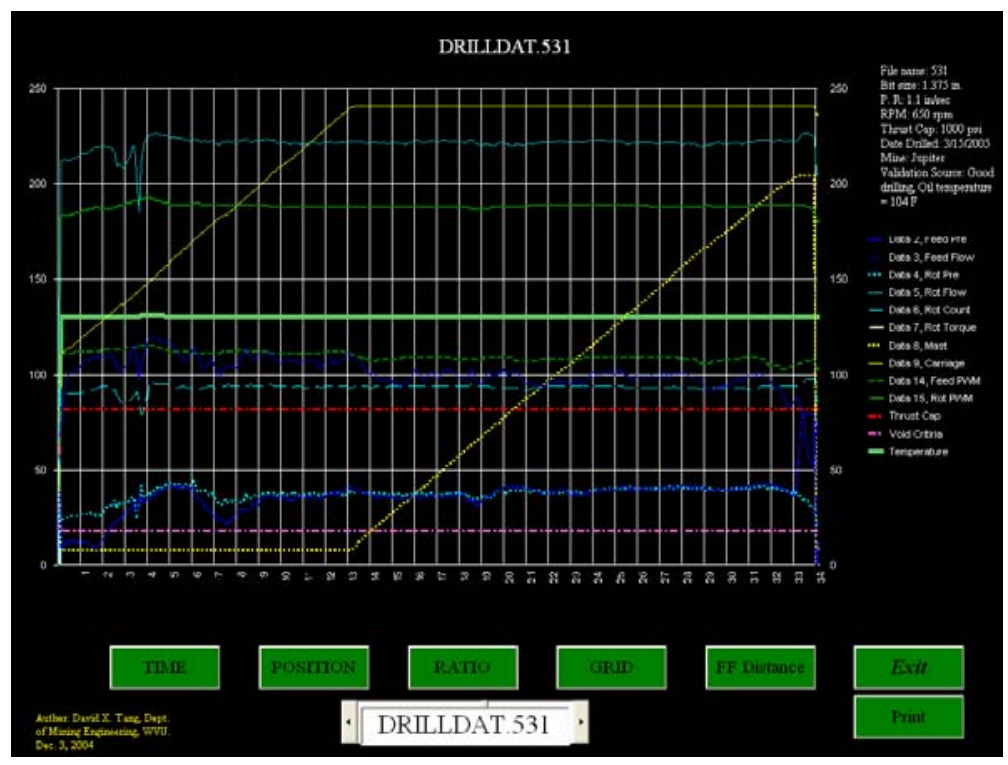

Figure 3.4 Software for displaying drilling parameters

In this research, all drilling parameters except the data of two mast feed position sensors and rotation count are not converted from dimensionless machine data to engineering units for the following reasons:

- The intention of this research is to develop a set of computational rules for predicting roof geology based on the collected drilling parameters and implement the rules in MRGIS. What the end users of MRGIS are interested in is the roof geological properties determined by using drilling parameters. If the rules for predicting roof geology are derived directly from machine data and implemented in MRGIS without conversation, a lot of computational time will be saved when displaying prediction results in MRGIS. This is especially meaningful when multiple holes are displayed at the same time.

- It is easier to plot and display all drilling parameters together for comparing their roles in reflecting the variation in roof geology properties.

- A lot of time is saved when analyzing drilling parameters. 


\section{CHAPTER 4}

\section{DRILLING EXPERIMENTS}

In this project, a series of laboratory and underground tests was conducted to develop the theoretical methodology for predicting roof geology based on the drilling parameters of roof bolter. A patented drill control unit (DCU) installed in the J.H. Fletcher \& Co.'s HDDR dual head roof bolter was used to control drilling and collect the drilling parameters for all experiments. More than 1,000 roof bolt holes have been drilled in, and drilling parameters recorded from, 13 concrete and simulated blocks in the laboratory and 8 underground coal mines.

As mentioned in Chapter 3, the drill control unit (DCU) allows the parameters of penetration rate, rotation rate and maximum feed pressure (thrust cap) to be set by the operators. Several drilling settings were tested in order to determine the effect of both penetration rate and rotation rate on drilling parameters and/or drilling conditions. In this series of tests, the role of thrust cap was only for safety. So, the thrust cap was basically set at the maximum value $(=1,000 \mathrm{psi})$ in order to eliminate its effect on drilling test as much as possible. In some holes, thrust cap was set at lower level as to observe the behavior of the drilling parameters and the machine including the response of DCU when feed pressure rises beyond the level of thrust cap.

All the holes were drilled using standard roof bolt drilling bit designed for underground coal mines (Kennametal's Dust Hog design bit). Figure 4.1 shows the bits used for this series of tests. The bits are fairly inexpensive carbide insert drag bits that are designed primarily for coal-bearing sequence rocks, and the sizes and designs were selected because of their wide-spread use in the underground coal mining industry. The majority of the holes were drilled using

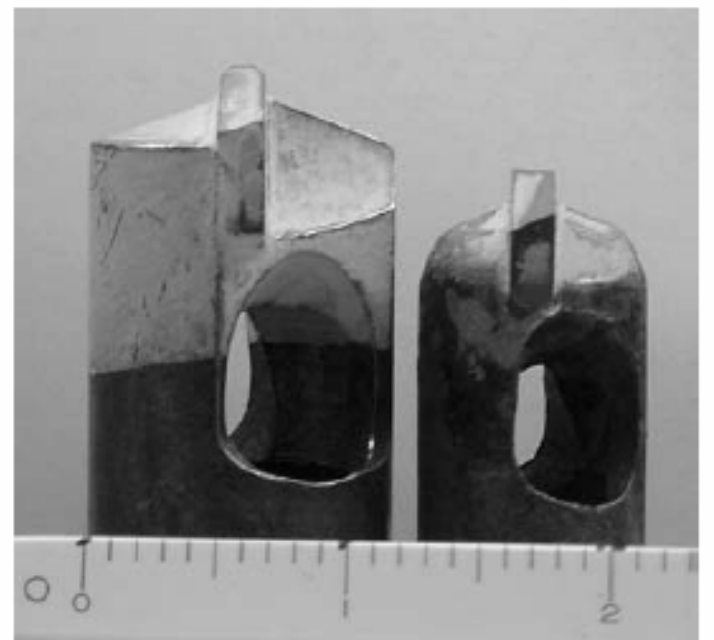

Figure 4.1 Drill bits used for the tests (left: $1-3 / 8$ in bit, right: $1-1 / 32$ in bit) 
the $1-3 / 8$ in diameter bits but several holes were drilled using the smaller size bit $(1-1 / 32$ in) which the mining industry uses for specialized bolting applications. A new bit was used for every hole so as to minimize the effect of bit wear on drilling parameters and conditions.

The compensation run, i.e. drilling in the air without steel rod and bit, was conducted before every drilling to check the impact of drilling settings and machine conditions on drilling parameters for running the machine itself. When comparing it with drilling data, the amount of feed pressure and rotation pressure consumed for drilling rock can be determined. It can also be distinguished whether the change of drilling parameters is caused by geology change or by the machine characteristics including control algorithm.

\subsection{Laboratory Tests Using Manufactured Roof Rock Blocks}

A series of manufactured rock blocks was constructed for the purpose of simulating various roof rock lithologies. Four blocks were designed to simulate massive rock units, 6 blocks were designed to simulate a sequence of rock layers of various physical properties, three blocks were designed to simulate bedding separations of varying dimensions and orientations, and one block was constructed to simulate different sizes of fractures. All were designed for laboratory testing using different combinations of drilling parameters. Figure 4.2 shows the test scene of laboratory test.

Each of the rock layers and the concrete used for constructing the manufactured rock blocks were tested to determine the physical properties. Each type was tested to measure unconfined compressive strength, Brazilian tensile strength, shear strength, Young's modulus and unit weight. Depending on the size of samples available, the number of tests per rock varied. The test procedures adhered to ASTM standards and were conducted on cores of 2- and 1-in in diameter. Basically, 2-in cores were used when available. 

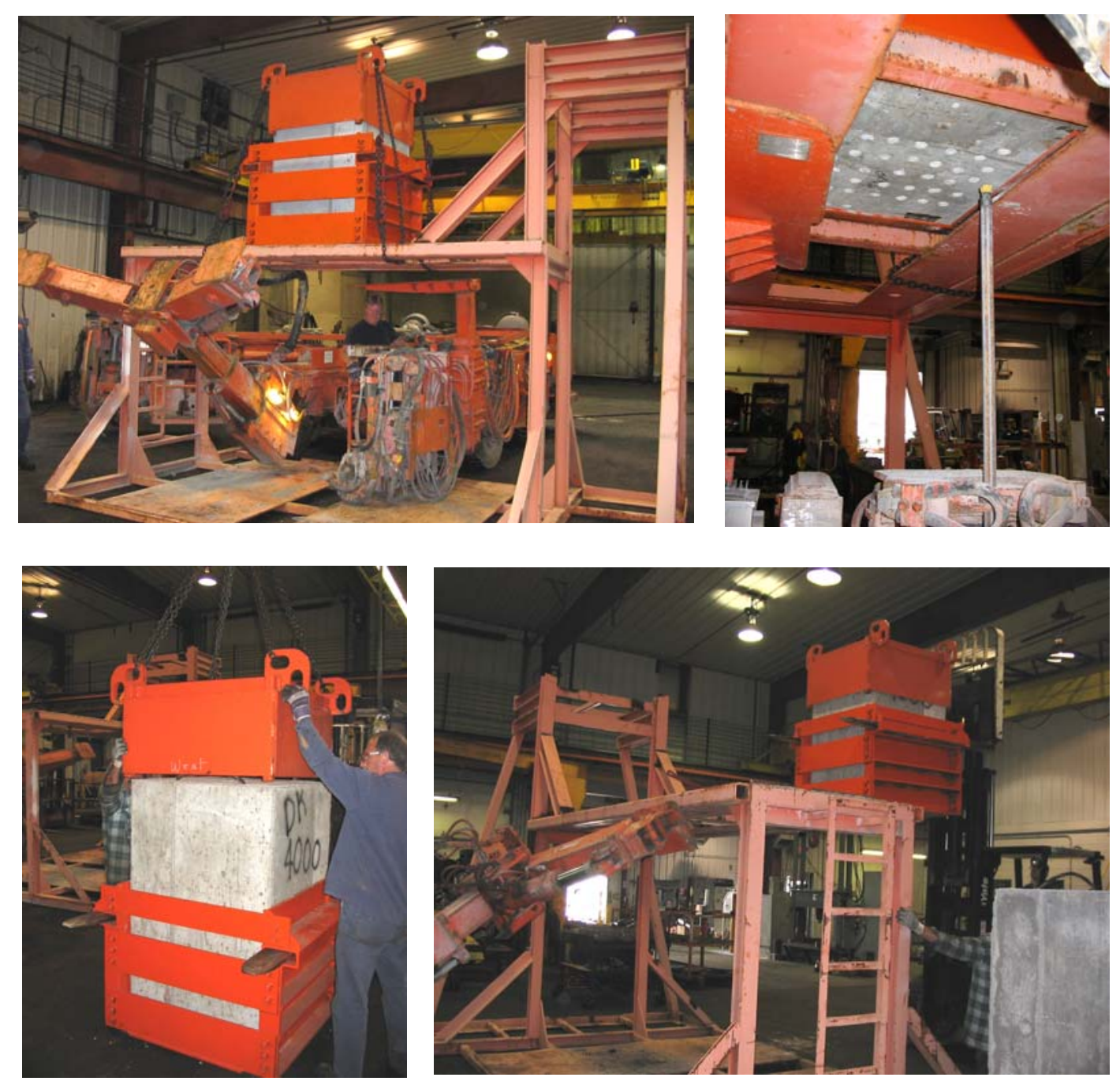

Figure 4.2 Test scene of laboratory test (J.H. Fletcher \& Co.'s facility, Huntington, WV)

\subsubsection{Manufactured Blocks}

\subsubsection{Solid Concrete Block}

As to check the consistency of the drilling parameters with a single rock type and the impact of different strengths of roof rocks on drilling parameters, three solid concrete blocks were constructed (Figures 4.3 and 4.4). Their dimensions were approximately $3 \mathrm{ft}$ $\times 4 \mathrm{ft} \times 5 \mathrm{ft}$. The blocks were constructed using the concretes with an unconfined compressive strength of 4,000 psi, 8,000 psi, and 12,000 psi, respectively (designed). Table 4.1 shows their physical properties.

$<$ Drilling Settings $>$ 
- DCU control mode: WVU mode = both penetration rate and rotation rate were controlled or kept constant.

- Penetration rate: $0.4,0.6,0.8,1.1,1.3,1.5,1.7,1.9$ and $2.1 \mathrm{in} / \mathrm{sec}$

- Rotation rate: $300,350,400,450,500,600,650 \mathrm{rpm}$ and free*

- Thrust cap: 1,000 psi

- Bit size: $1-1 / 32$ and $1-3 / 8$ in

- Number of drill holes: 150 holes

* Free means the maximum allowable value on the DCU.

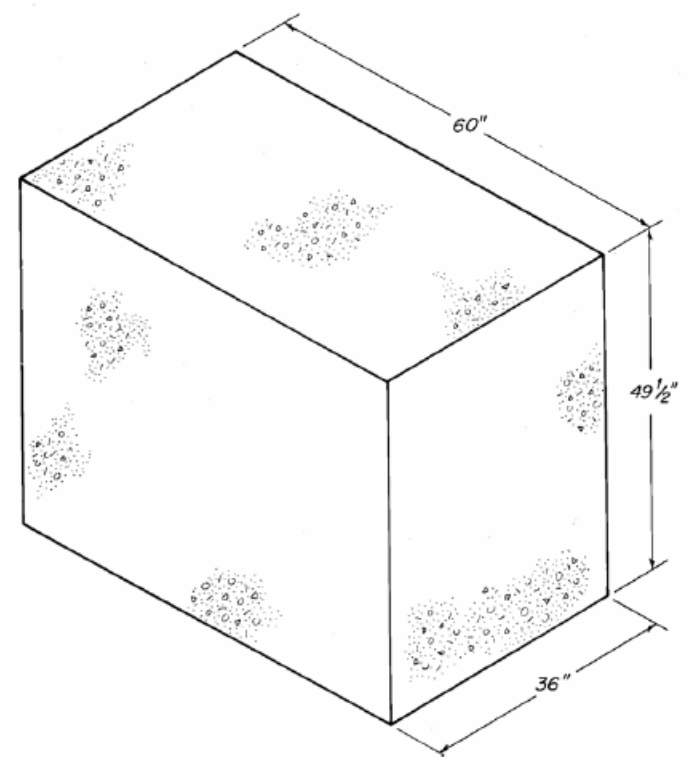

Figure 4.3 Schematic of solid concrete block

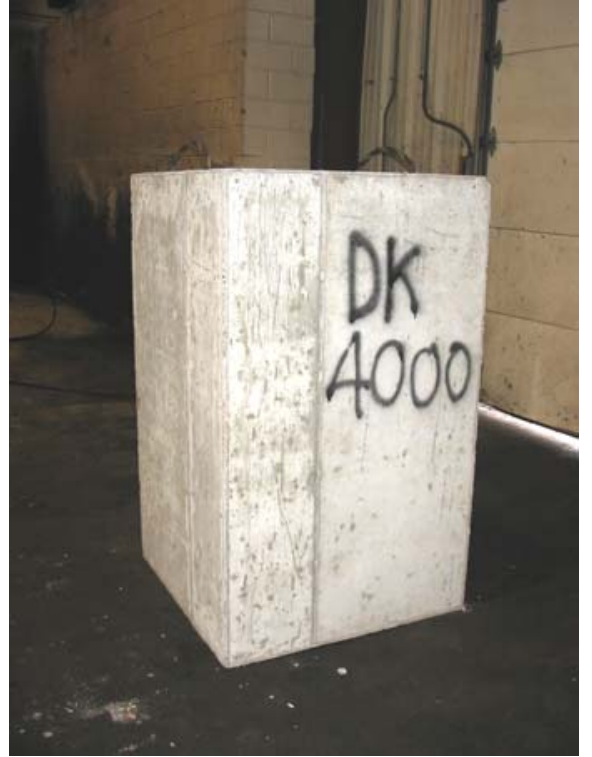

Figure 4.4 Solid concrete block $(\mathrm{UCS}=4,000 \mathrm{psi})$

Table 4.1 Physical properties of solid concrete blocks

\begin{tabular}{|c|c|c|c|c|}
\hline $\begin{array}{c}\text { Concrete Block } \\
(\text { designed })\end{array}$ & $\begin{array}{c}\text { UCS } \\
(\mathrm{psi})\end{array}$ & $\begin{array}{c}\text { BTS } \\
(\mathrm{psi})\end{array}$ & $\begin{array}{c}\mathrm{E}_{50} \\
\left(\times 10^{6} \mathrm{psi}\right)\end{array}$ & $\begin{array}{c}\text { Unit } \\
\text { Weight } \\
\left(\mathrm{lbs} / \mathrm{ft}^{3}\right)\end{array}$ \\
\hline The 4,000 psi block & 5,710 & 391.6 & 1.643 & 137.6 \\
\hline The 8,000 psi block & 6,070 & 492.7 & 1.550 & 137.4 \\
\hline
\end{tabular}

Where, $\hat{\mathrm{UCS}}=$ standardized unconfined compressive strength, $\mathrm{BTS}=$

Brazilian tensile strength, $\mathrm{E}_{50}=$ tangent Young's modulus at the $50 \%$ stress level of the maximum stress. 


\subsubsection{Layered Block}

Six different layered blocks were designed and constructed using a variety of quarried rocks that were embedded in poured concrete structures. The schematics of the blocks are shown in Figures $4.5-4.10$. Their dimensions were $2 \mathrm{ft} \times 2 \mathrm{ft} \times 6-1 / 2 \mathrm{ft}$ and each had four separate rock layers embedded. The rock units were cut to a finished size as indicated in the figures and in general were approximately $1 \mathrm{ft} \times 1 \mathrm{ft} \times 1 \mathrm{ft}$. The rocks were secured in place using steel banding attached to 3/4-in rebar, then encased with a standard concrete mix. Including the concrete that was poured to encase the rocks, each block had nine different layers (four rock layers and five concrete layers). The drilling tests were conducted along the long axis of the blocks which provided a maximum drilling length of $6-1 / 2 \mathrm{ft}$.

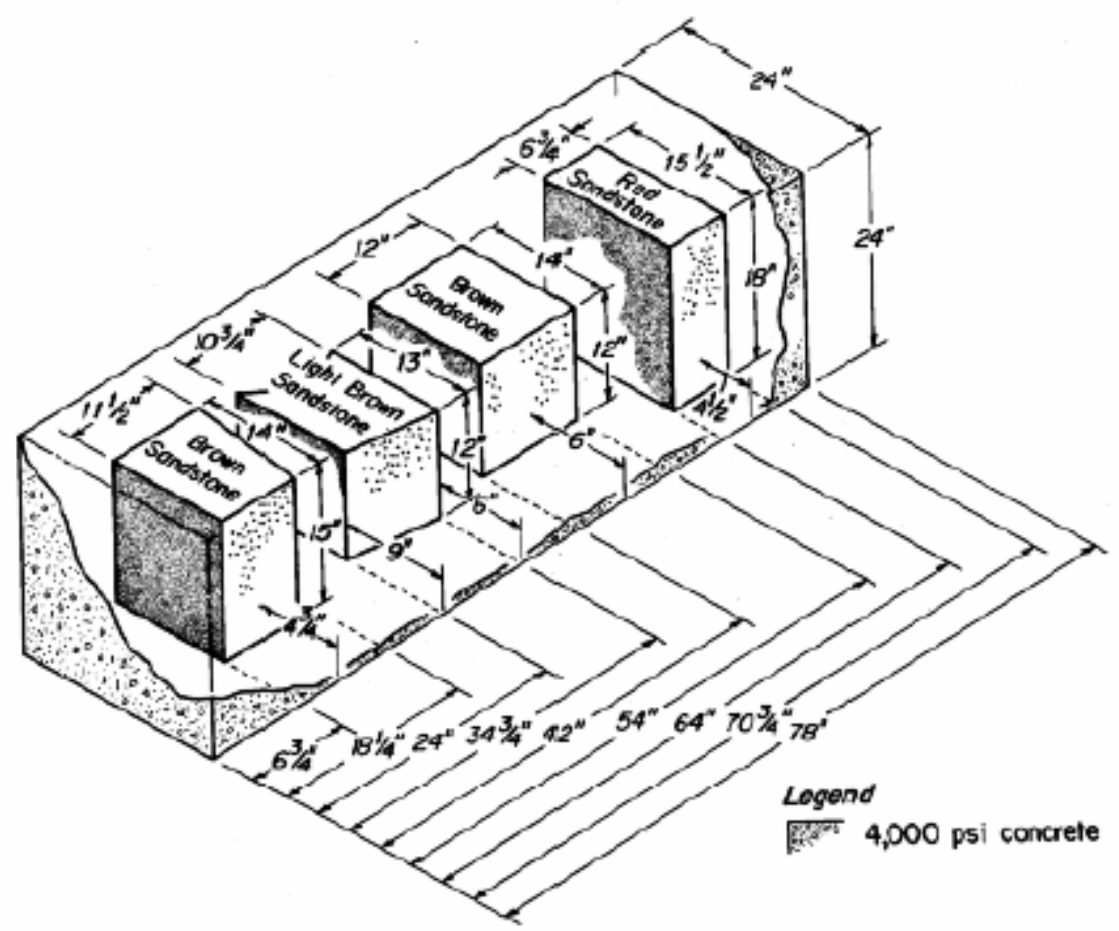

Figure 4.5 Schematic of layered block \#1 


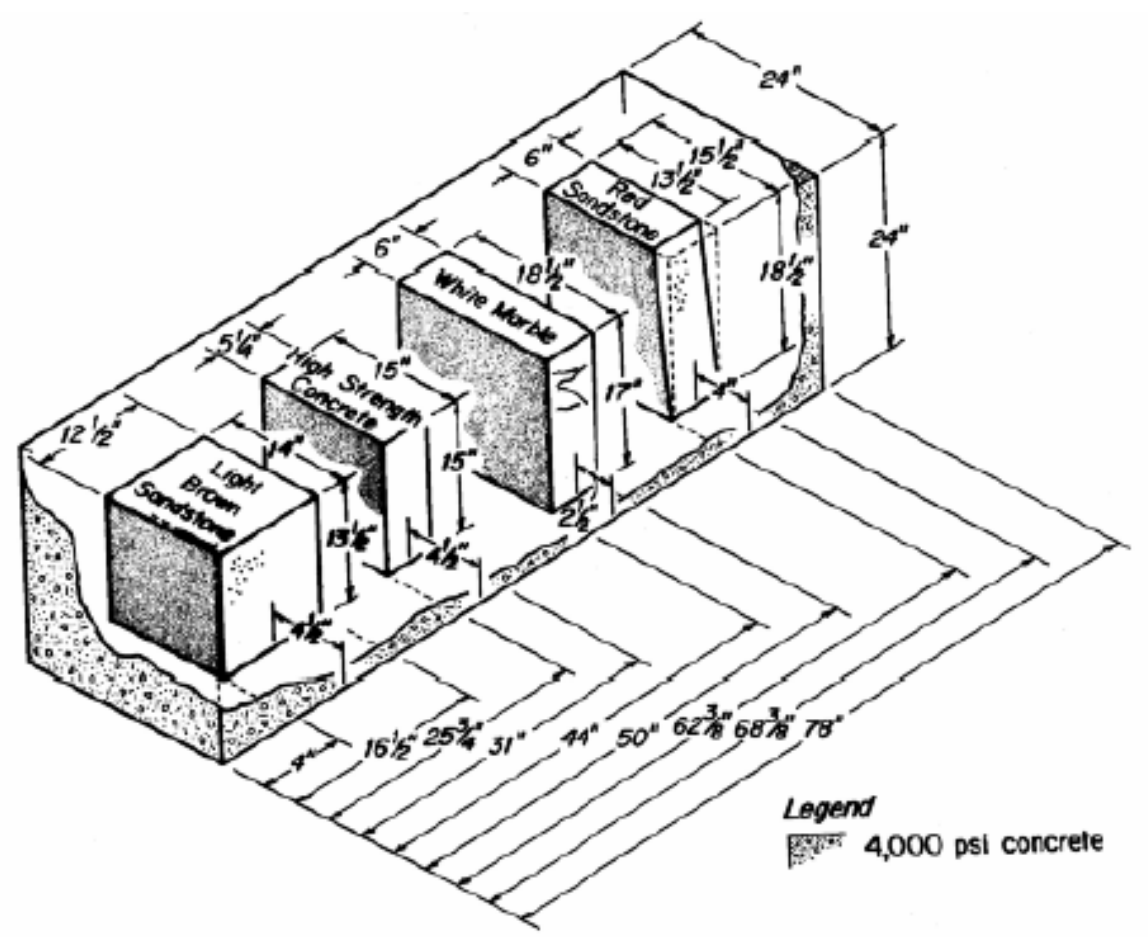

Figure 4.6 Schematic of layered block \#2

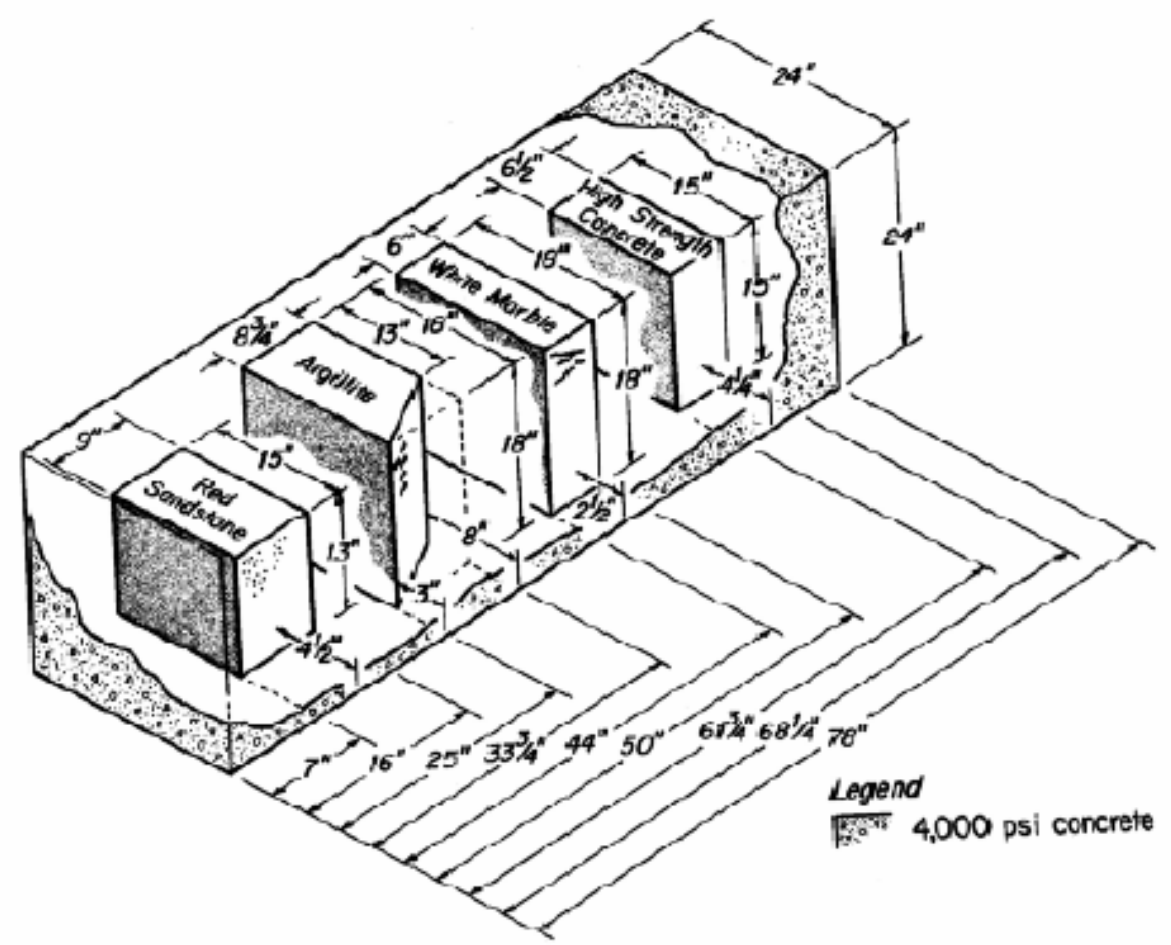

Figure 4.7 Schematic of layered block \#3 


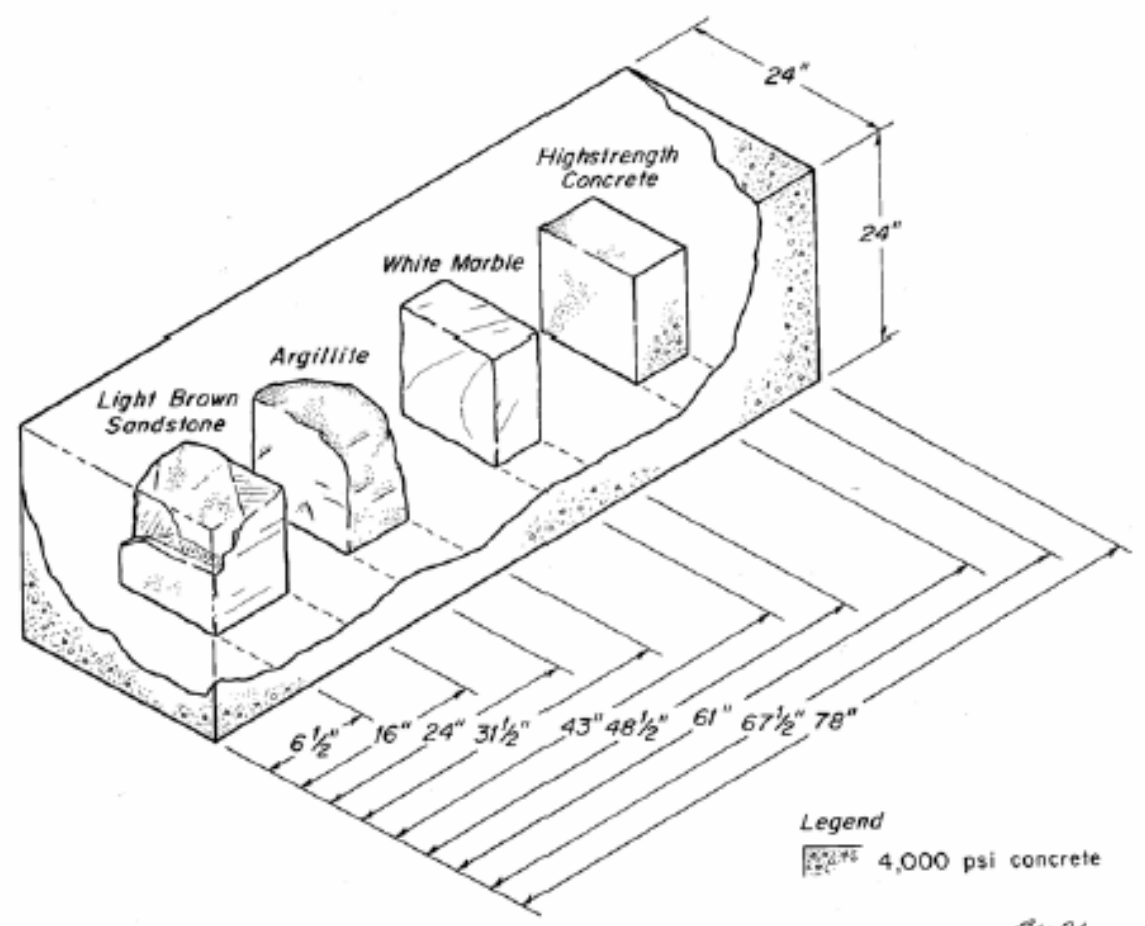

Figure 4.8 Schematic of layered block \#4

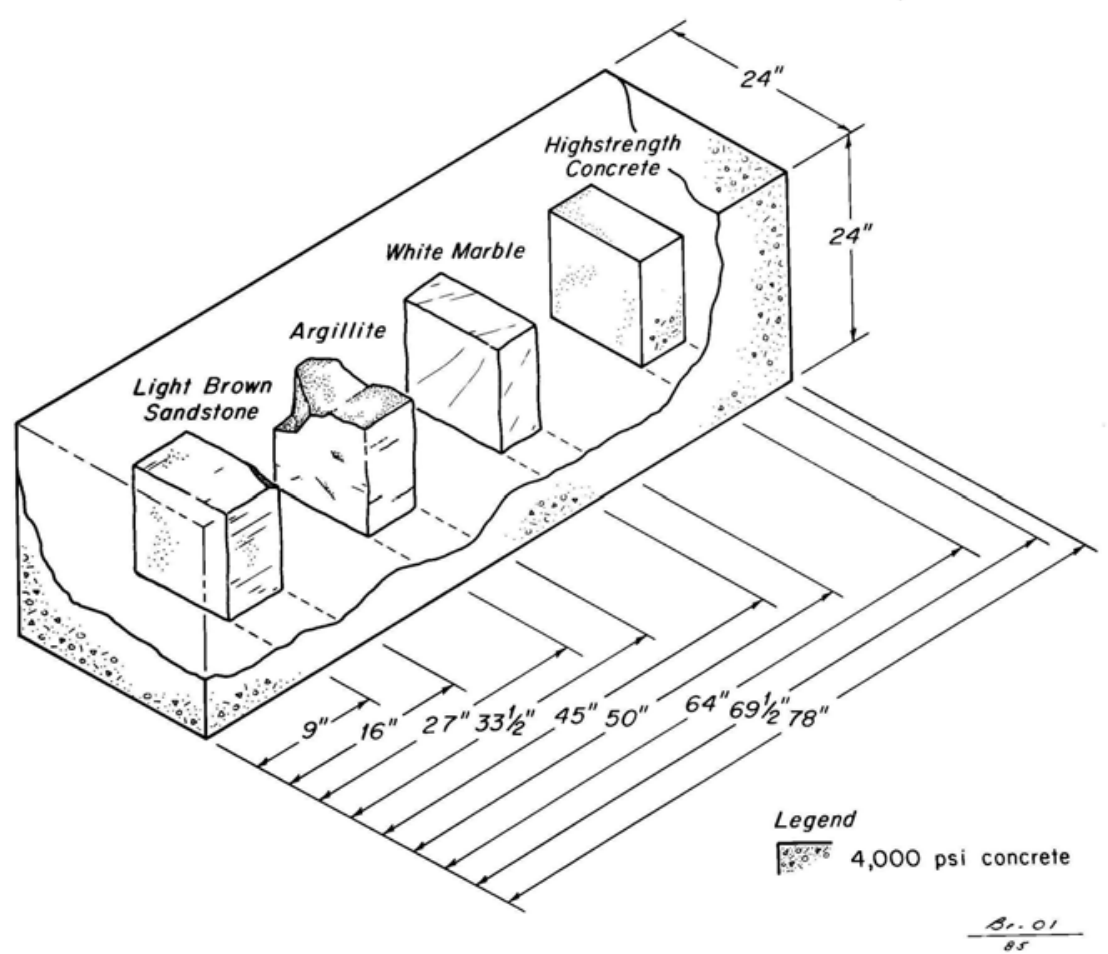

Figure 4.9 Schematic of layered block \#5 


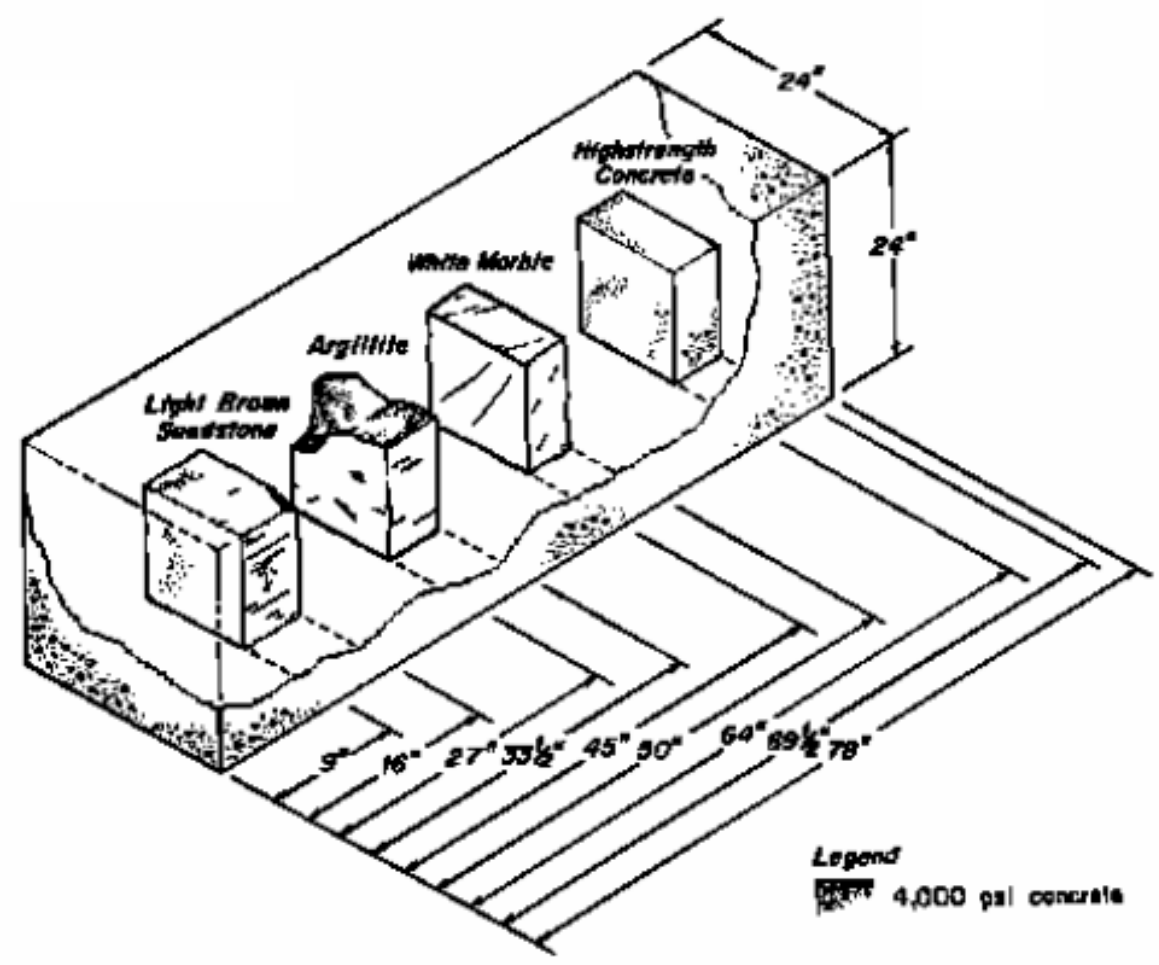

Figure 4.10 Schematic of layered block \#6

The physical properties of the rock units, including the concrete used in the manufactured roof blocks are shown in Table 4.2. The average unconfined compressive strengths of the rocks varied from about 7,000 to 27,000 psi. The three kinds of sandstone, designated red, brown and light brown, were very consistent with no laminations or apparent bedding planes. All three sandstones are fine to medium-grained. The white marble is vuggy with voids and discontinuities throughout. The argillite, weakly metamorphosed shale, has zones of healed and open fractures and is generally discontinuous. The high-strength concrete that was embedded as separate layers in layered blocks \#2-\#4 had a compressive strength less than 3,000 psi. While initially intended to be a high-strength concrete, these layers are the lowest strength units in the manufactured blocks. Samples of this concrete layer crumbled easily when handled probably due to an improper mix. The concrete used for embedding the rock layers had a compressive strength of 4,020 psi. Overall, the rock units and concrete layers in provided a series of drilling encounters with transition zones 
from weak-to-strong and strong-to-weak rocks. The drilling settings and number of drill holes for each layered block were as follows:

Table 4.2 Physical properties of the embedded rocks and the concrete used in the layered blocks

\begin{tabular}{|c|c|c|c|c|c|}
\hline Rock Type & $\begin{array}{c}\text { UCS } \\
(\mathrm{psi})\end{array}$ & $\begin{array}{c}\text { BTS } \\
(\mathrm{psi})\end{array}$ & $\begin{array}{c}\text { Shear } \\
\text { Strength } \\
(\mathrm{psi})\end{array}$ & $\begin{array}{c}\mathrm{E}_{50} \\
\left(\times 10^{6} \mathrm{psi}\right)\end{array}$ & $\begin{array}{c}\text { Unit } \\
\text { Weight } \\
\left(\mathrm{lbs} / \mathrm{ft}^{3}\right)\end{array}$ \\
\hline Red Sandstone & 6,986 & 1,053 & N/A & 2.77 & 149 \\
\hline Brown Sandstone & 17,104 & 930 & N/A & 1.94 & 160 \\
\hline Lt Brown Sandstone & 27,359 & 1,934 & 2,846 & 2.34 & 155 \\
\hline White Marble & 17,418 & 1,371 & 1,010 & 2.48 & 166 \\
\hline Argillite & 20,473 & 1,044 & 1,395 & 4.24 & 168 \\
\hline Soft Concrete & 2,864 & N/A & N/A & N/A & N/A \\
\hline Embedding Concrete & 4,021 & 474 & N/A & 0.65 & 132 \\
\hline High-strength Concrete & 12,340 & 430 & N/A & 2.07 & 159 \\
\hline
\end{tabular}

\section{Layered Block \#1}

$<$ Drilling Settings $>$

- DCU control mode: WVU mode $=$ both penetration rate and rotation rate were controlled or kept constant.

- Penetration rate: 0.6 and $1.1 \mathrm{in} / \mathrm{sec}$

- Rotation rate: $150,200,300,400$ and $500 \mathrm{rpm}$

- Thrust cap: 1,000 psi

- Bit size: $1-1 / 32$ and $1-3 / 8$ in

- Number of drill holes: 8 holes

\section{Layered Block \#2}

$<$ Drilling Settings $>$

- DCU control mode: WVU mode = both penetration rate and rotation rate were controlled or kept constant.

- Penetration rate: $0.6,1.1$ and $1.5 \mathrm{in} / \mathrm{sec}$

- Rotation rate: $150,200,300,400$ and $500 \mathrm{rpm}$

- Thrust cap: 1,000 psi

- Bit size: $1-1 / 32$ and $1-3 / 8$ in 
- Number of drill holes: 11 holes

\section{Layered Block \#3}

$<$ Drilling Settings $>$

- DCU control mode: WVU mode = both penetration rate and rotation rate were controlled or kept constant.

- Penetration rate: $0.6,1.1$ and $1.5 \mathrm{in} / \mathrm{sec}$

- Rotation rate: $150,200,300,400,500 \mathrm{rpm}$ and free

- Thrust cap: 1,000 psi

- Bit size: $1-1 / 32$ and $1-3 / 8$ in

- Number of drill holes: 10 holes

\section{Layered Block \#4}

$<$ Drilling Settings $>$

- DCU control mode: WVU mode = both penetration rate and rotation rate were controlled or kept constant.

- Penetration rate: 1.1 and $1.5 \mathrm{in} / \mathrm{sec}$

- Rotation rate: $300 \mathrm{rpm}$

- Thrust cap: 1,000 psi

- Bit size: $1-1 / 32$ and $1-3 / 8$ in

- Number of drill holes: 8 holes

\section{Layered Block \#5}

$<$ Drilling Settings $>$

- DCU control mode: WVU mode = both penetration rate and rotation rate were controlled or kept constant.

- Penetration rate: $0.4,0.6,1.1,1.5 \mathrm{in} / \mathrm{sec}$ and free

- Rotation rate: $150,400,500 \mathrm{rpm}$ and free

- Thrust cap: 1,000 psi

- Bit size: $1-1 / 32$ and 1-3/8 in 
- Number of drill holes: 15 holes

\section{Layered Block \#6}

$<$ Drilling Settings $>$

- DCU control mode: WVU mode = both penetration rate and rotation rate were controlled or kept constant.

- Penetration rate: $0.6,1.1,1.5 \mathrm{in} / \mathrm{sec}$ and free

- Rotation rate: $300,400,500 \mathrm{rpm}$ and free

- Thrust cap: 750 and 1,000 psi

- Bit size: $1-1 / 32$ and $1-3 / 8$ in

- Number of drill holes: 19 holes

\subsubsection{Fractured Block}

This block was constructed to simulate small voids/fractures (Figures 4.11). This concrete block was also constructed of high strength concrete mix with an unconfined compressive strength of about $12,300 \mathrm{psi}$ on average. The block for this test consists of four individual layers of concrete which were 15-in thick and constructed parallel faces. In between each concrete layers, a void was formed by inserting a narrow steel plate around the block perimeters as shown in Figures $4.12-4.14$. The thickness of 3 steel plates were 1/16-, 1/8-, and 3/8-in, respectively to simulate voids/fractures with different sizes. The layers were bolted together and in a steel frame so the block would act as one single unit. The final block dimensions were approximately $3 \mathrm{ft} \times 4 \mathrm{ft} \times 5 \mathrm{ft}$. The 1/16-, 1/8-, and 3/8-in voids were located at 15, 30 and 45 in, respectively.

\section{$<$ Drilling Settings $>$}

- DCU control mode: WVU mode = both penetration rate and rotation rate were controlled or kept constant.

- Penetration rate: $0.4,0.8,1.1$ and $1.5 \mathrm{in} / \mathrm{sec}$

- Rotation rate: $300,400,500 \mathrm{rpm}$ and free

- Thrust cap: $600,700,850$, and 1,000 psi

- Bit size: $1-1 / 32$ and $1-3 / 8$ in

- Number of drill holes: 37 holes 


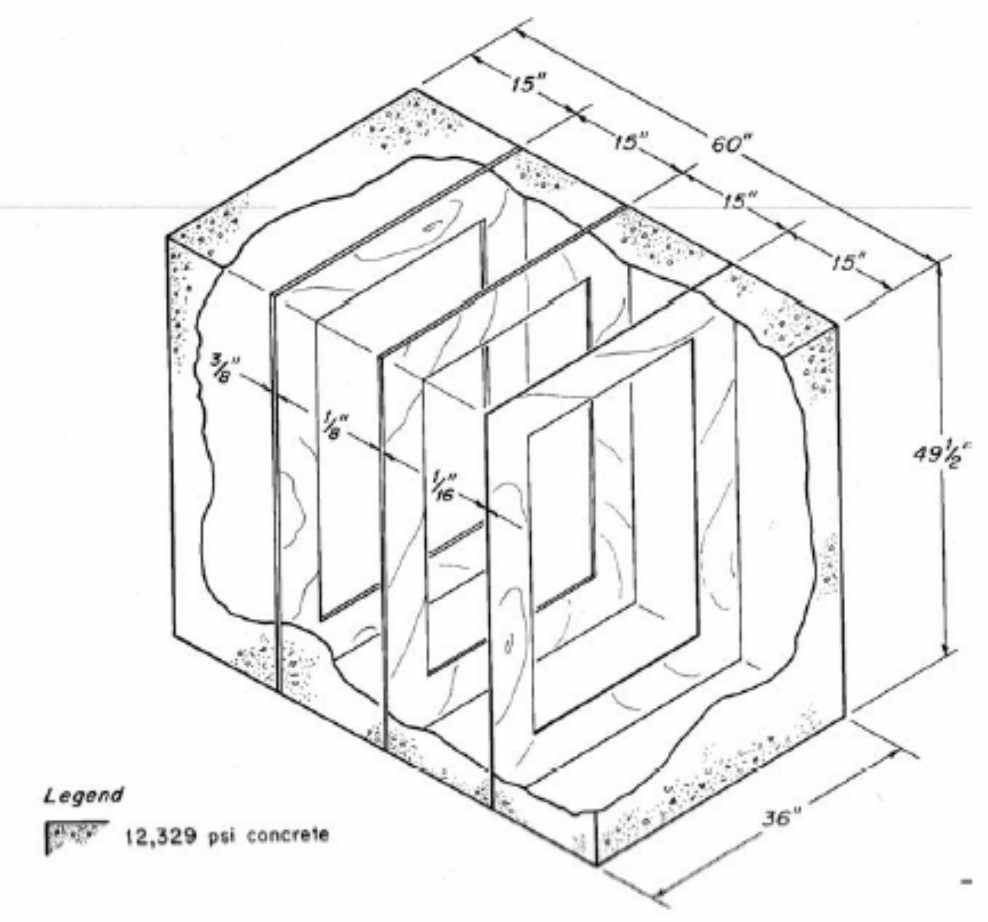

Figure 4.11 Schematic of fractured block
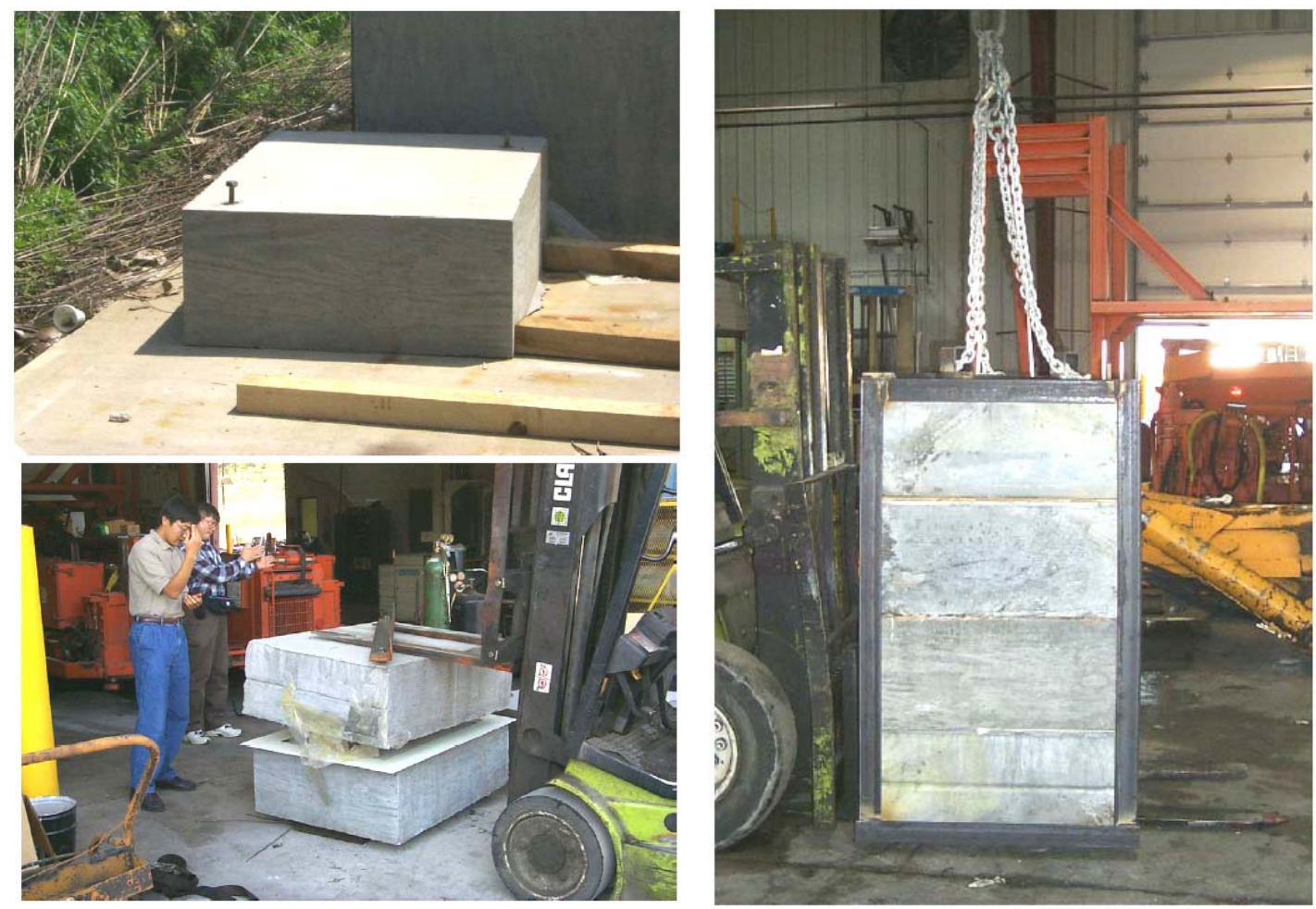

Figure 4.12 Assembling fractured block 


\subsubsection{Bedding Separations Block}

Three blocks were designed and constructed to simulate bedding separations in rock layers. Different block designs were constructed to simulate different types of bedding separations that could be encountered in roof rock sequences. The designs included different sizes of horizontal bedding separations and angled separations. The drilling settings and number of drill holes for each layered block were as follows:

\section{Large-size Bedding Separations Block}

This block was constructed using embedded foam sheets to simulate large-size bedding separations (Figure 4.13). Its dimensions were $2 \mathrm{ft} \times 2 \mathrm{ft} \times 6-1 / 2 \mathrm{ft}$. These were four separation layers composed of embedded foam sheets. The foam layers were 1-, 2-, 3-, and 4-in in thickness, respectively. The concrete for embedding the foam layers was a standard mix with an unconfined compressive strength of 4,020 psi.

$<$ Drilling Settings $>$

- DCU control mode: WVU mode = both penetration rate and rotation rate were controlled or kept constant.

- Penetration rate: 0.6 and $1.1 \mathrm{in} / \mathrm{sec}$

- Rotation rate: 150,400 and $500 \mathrm{rpm}$

- Thrust cap: 1,000 psi

- Bit size: $1-1 / 32$ and $1-3 / 8$ in

- Number of drill holes: 5 holes 


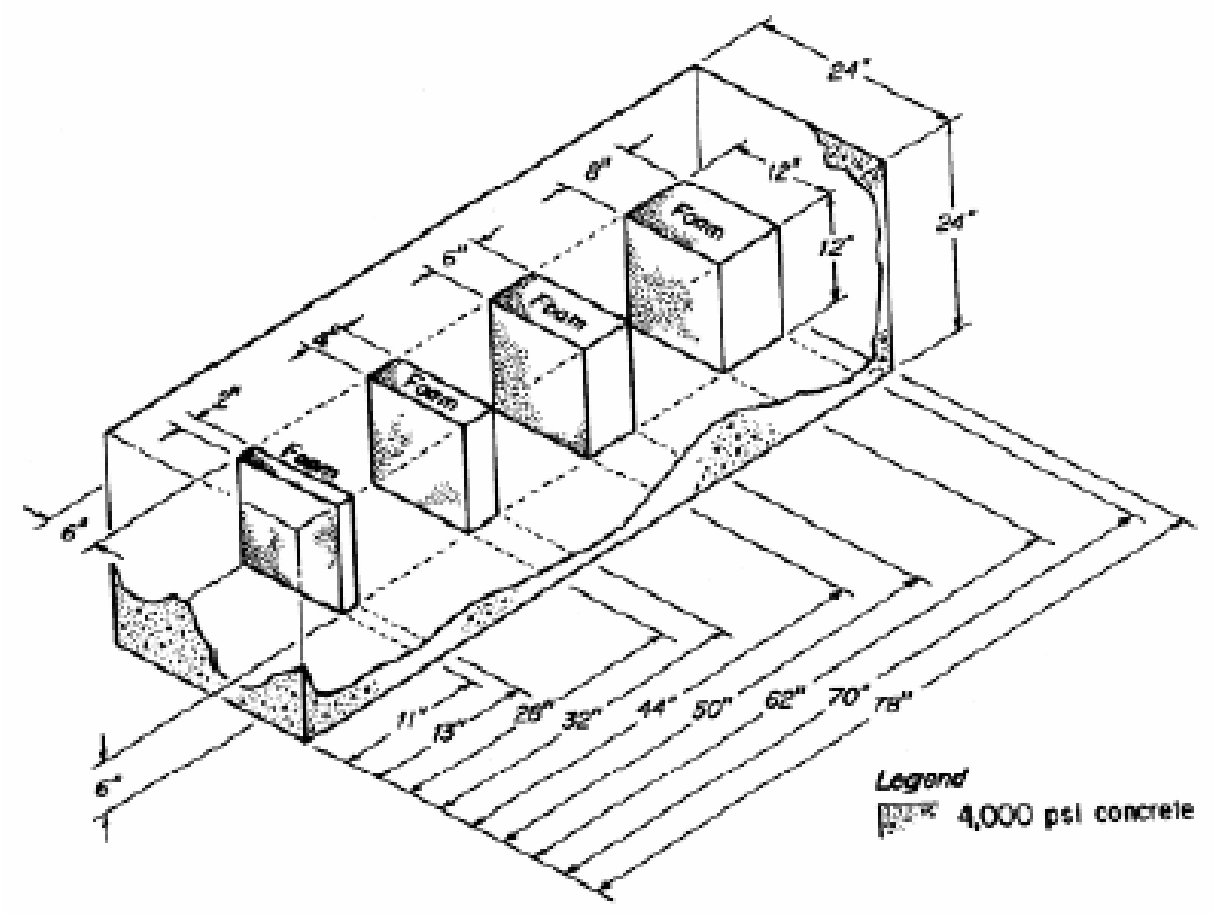

Figure 4.13 Schematic of manufactured block with large bedding separations

\section{Small-size Bedding Plane Separations Block}

This block was constructed using horizontal cardboard inserts to simulate smaller bedding separations (Figure 4.14). This block had three separate cardboard layer inserts constructed of 1/8-in sheets of poster board. The thickness of cardboard layers were 1/8-, 1/4- and 3/8-in, respectively. The concern to embed the cardboard layers was a special high-strength mix with an unconfined compressive strength of about 12,000 psi. Its dimensions were $3 \mathrm{ft} \times 4 \mathrm{ft} \times 5 \mathrm{ft}$.

$<$ Drilling Settings $>$

- DCU control mode: WVU mode = both penetration rate and rotation rate were controlled or kept constant.

- Penetration rate: 0.6 and $1.1 \mathrm{in} / \mathrm{sec}$

- Rotation rate: 300,400 and $500 \mathrm{rpm}$

- Thrust cap: 1,000 psi

- Bit size: 1-3/8 in

- Number of drill holes: 5 holes 


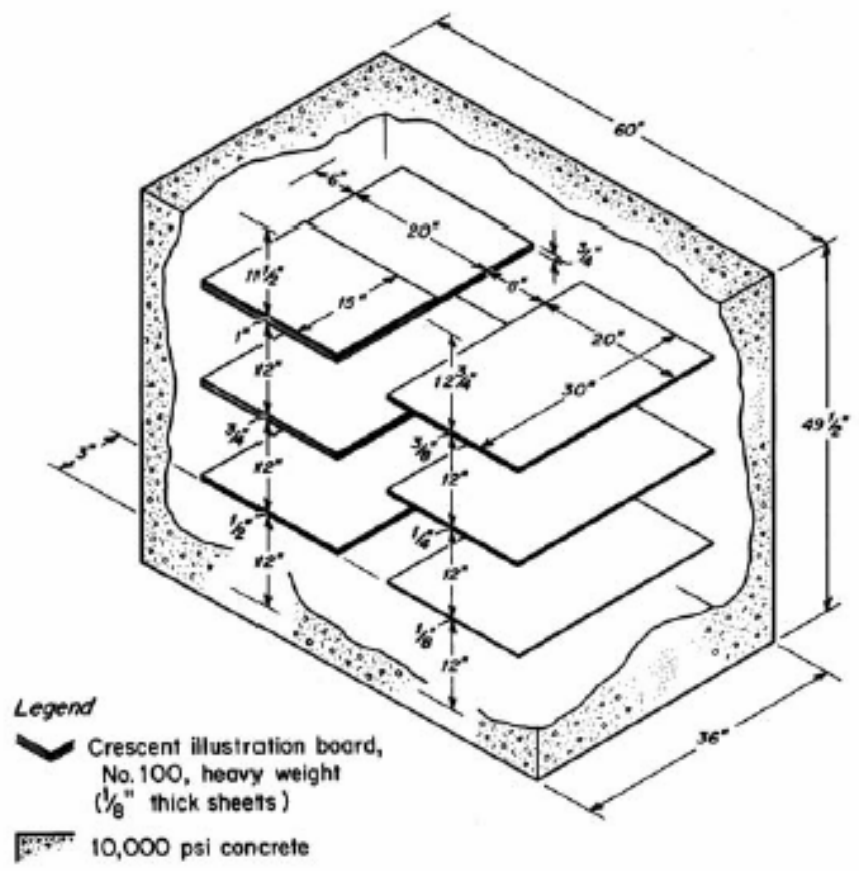

Figure 4.14 Schematic of manufactured block to simulate with horizontal cardboard

\section{Angled Bedding Separations Block}

This block was designed and constructed to simulate bedding separations that are intercepted by a drill bit at an angle as opposed to perpendicular (Figure 4.15). This block was constructed using the 1/8-in sheets of poster board embedded in the high strength concrete. The angled layers permitted a variety of drilling opportunities as the block was rotated so the layers could be intercepted at angles of 15, 30, 60 and 75 degreed. Its dimensions were $3 \mathrm{ft} \times 4 \mathrm{ft} \times 5 \mathrm{ft}$.

$<$ Drilling Settings $>$

- DCU control mode: WVU mode = both penetration rate and rotation rate were controlled or kept constant.

- Penetration rate: $0.6,1.1$ and $1.5 \mathrm{in} / \mathrm{sec}$

- Rotation rate: 200, 300, 400 and 500 rpm

- Thrust cap: 1,000 psi

- Bit size: 1-3/8 in

- Number of drill holes: 14 holes 


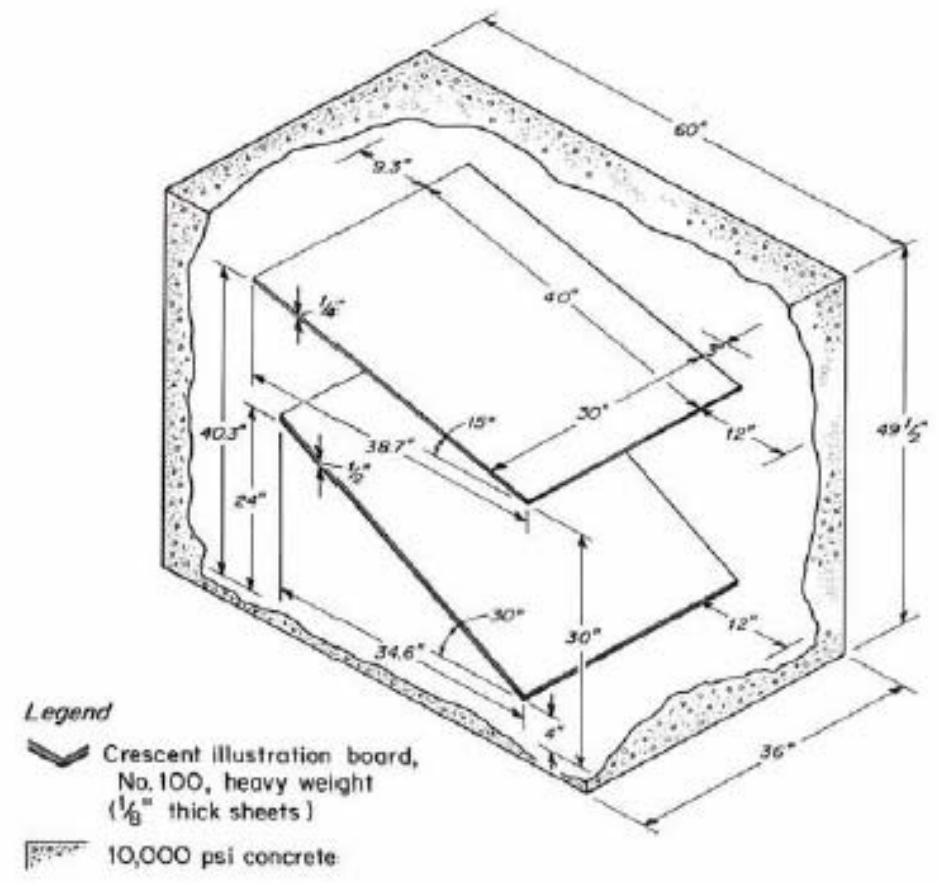

Figure 4.15 Schematic of manufactured block with angled cardboard lavers

\subsection{Underground Tests}

\subsubsection{Design of Underground Tests}

In order to observe the behaviors of drilling parameters when drilling in different strengths of rocks and to develop the criteria for estimating the strength of roof rock, underground tests were conducted in different coal mines. The criteria for void/fracture prediction developed from the results of laboratory tests were also checked and verified. In addition, bit wear tests were conducted so as to observe the impact of rock strength on the magnitude of bit wear and its impact on drilling parameters. Core sampling and borehole scoping were always conducted at each test site to verify the roof geology and determine the physical properties of each roof rock.

\subsubsection{Core Sampling}

A number of core samples were collected at each test site. These cores were used to determine the roof geology of their test area and to measure the physical properties of roof rocks. Cores were collected using a core barrel with a diamond bit as shown in 
Figure 4.16. Water was used to flush cuttings from the core barrel and to cool the drill bit. The diameter of cores obtained from the barrel was 2 in.

\subsubsection{Borehole Scoping}

Although the cores can be used to define the roof geology of test sites, geology can often change place to place. Moreover, it is difficult to determine the actual size and condition of void/fracture. Thus, borehole scoping was also conducted at each test site to verify the roof geology. Figure 4.17 shows the borehole scope system. The probe of the borehole scope contains a camera, light and mirror system. The digital camcorder records the image data to a micro tape.

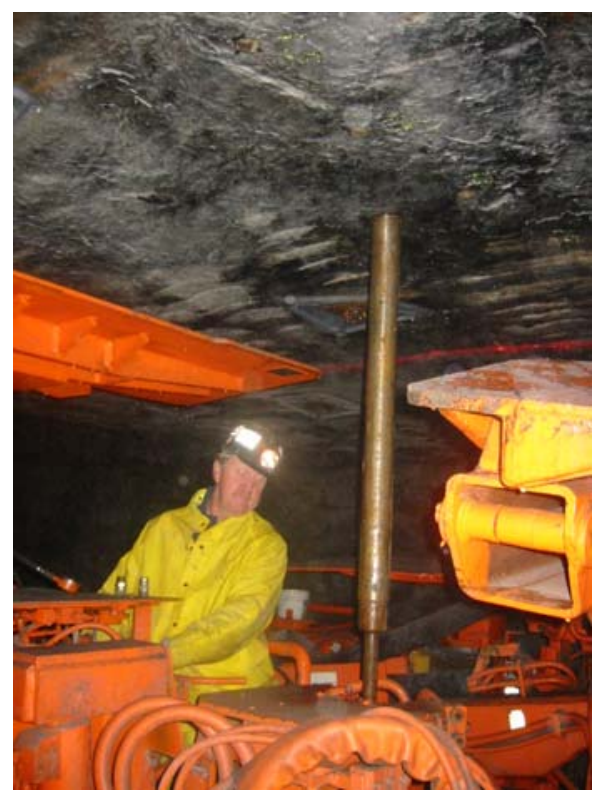

Figure 4.16 Core drilling

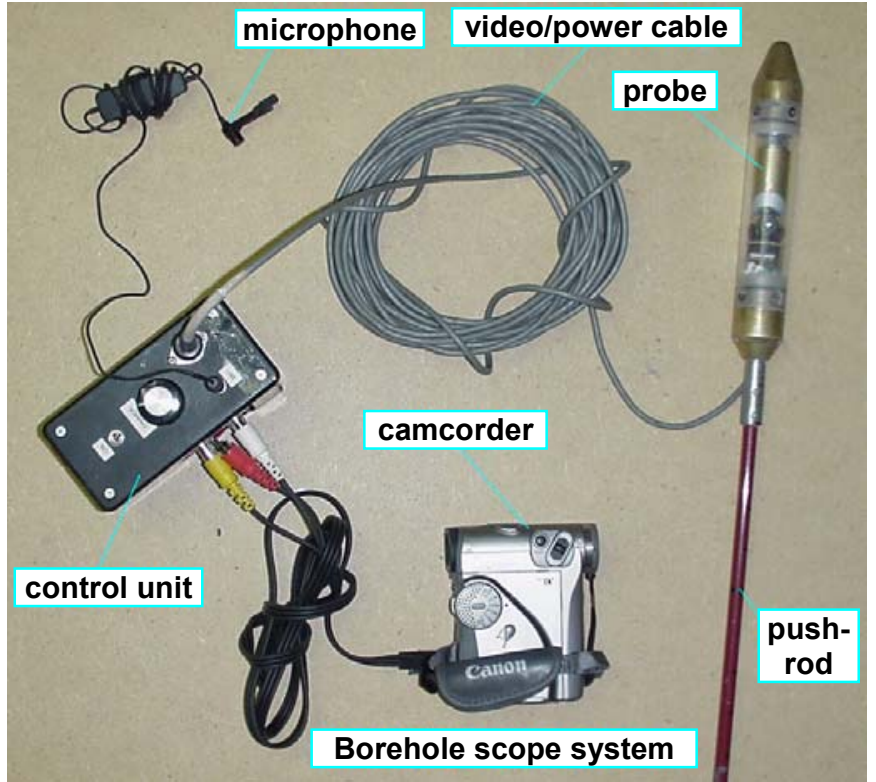

Figure 4.17 Borehole scope system

\subsubsection{Overview of Underground Tests}

The roof geology and drilling settings of each test site are mentioned as follows:

\subsubsection{Mine A Underground Test}

Mine A was located in western Colorado. The roof of this test site consisted of mostly shale and mudstone. There was one small layer of competent sandstone that was around 40 in deep from the roof line. Figure 4.18 shows the core log. 


\section{$<$ Drilling Settings $>$}

- DCU control mode: WVU mode = both penetration rate and rotation rate were controlled or kept constant.

- Penetration rate: $0.4,0.6,1.1,1.3$ and $1.5 \mathrm{in} / \mathrm{sec}$

- Rotation rate: 200, 300, 400 and $500 \mathrm{rpm}$

- Thrust cap: 1,000 psi

- Bit size: $1-1 / 32$ and $1-3 / 8$ in

- Number of drill holes: 34 holes

\subsubsection{Mine B Underground Test}

Mine B was located in eastern Kentucky. The roof of this mine consisted of approximately 20 -30-in of shale overlain by sandstone member. Figure 4.19 shows the result of borehole scoping. However, as the core drilling apparatus was broken, the core sample could not be obtained.

\section{$<$ Drilling Settings $>$}

- DCU control mode: WVU mode = both penetration rate and rotation rate were controlled or kept constant.

- Penetration rate: $0.8,1.1 \mathrm{in} / \mathrm{sec}$ and Free

- Rotation rate: 300, 400, $500 \mathrm{rpm}$ and Free

- Thrust cap: 400, 650 and 800 psi

- Bit size: $1-1 / 32$ and $1-3 / 8$ in

- Number of drill holes: 53 holes 


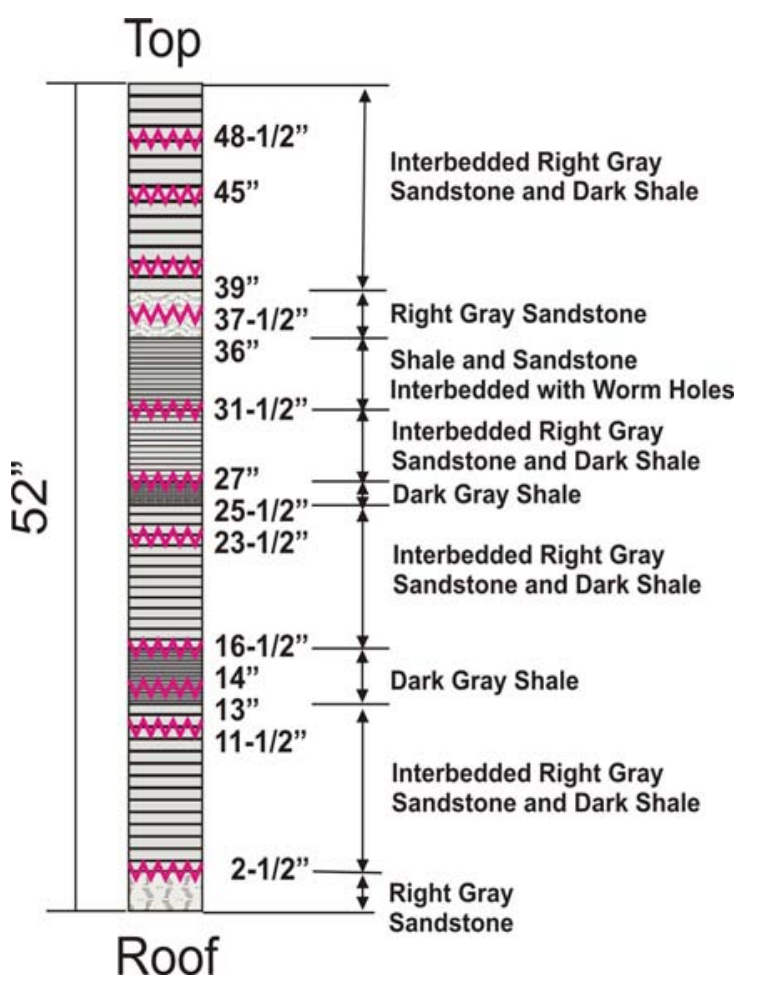

Fractures or Breaks

Figure 4.18 Core log (Mine A)

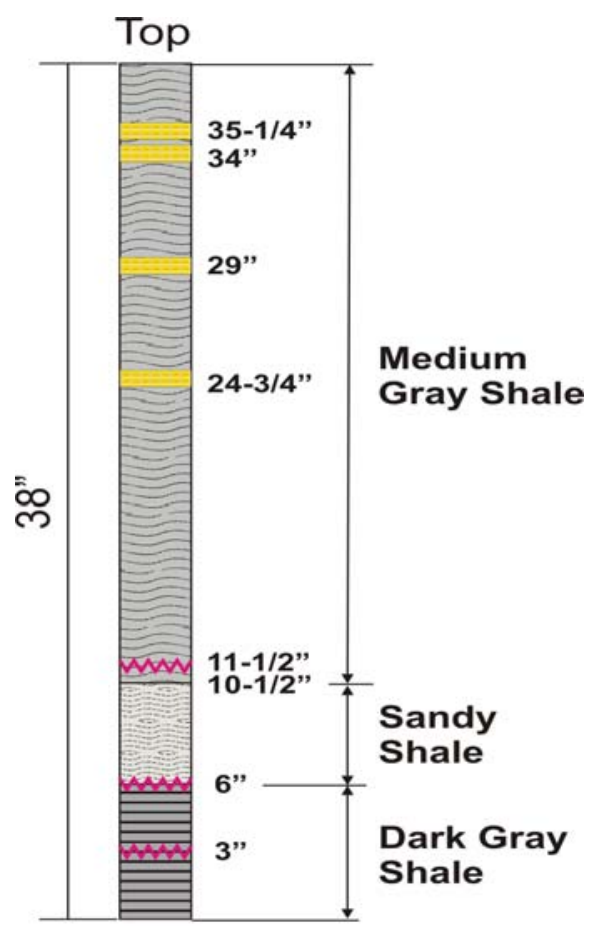

Roof

wn Fractures Iron Stains

Figure 4.19 Result of borehole scoping (Mine B)

\subsubsection{Mine C Underground Test}

Mine $\mathrm{C}$ was located in southern West Virginia. A diagram of the test area and the core $\log$ are shown in Figures 4.20 and 4.21, respectively. As shown in Figure 4.21, the roof of the test area consists of about 35 in shale overlain by sandstone layer that extends at least 35 in above the shale.

$<$ Drilling Settings $>$

- DCU control mode: WVU mode = both penetration rate and rotation rate were controlled or kept constant.

- Penetration rate: $0.8,1.1,1.5,5.1 \mathrm{in} / \mathrm{sec}$ and free

- Rotation rate: $400,500 \mathrm{rpm}$ and free 
- Thrust cap: 200, 400, 650 psi

- Bit size: $1-1 / 32$ and $1-3 / 8$ in

- Number of drill holes: 36 holes

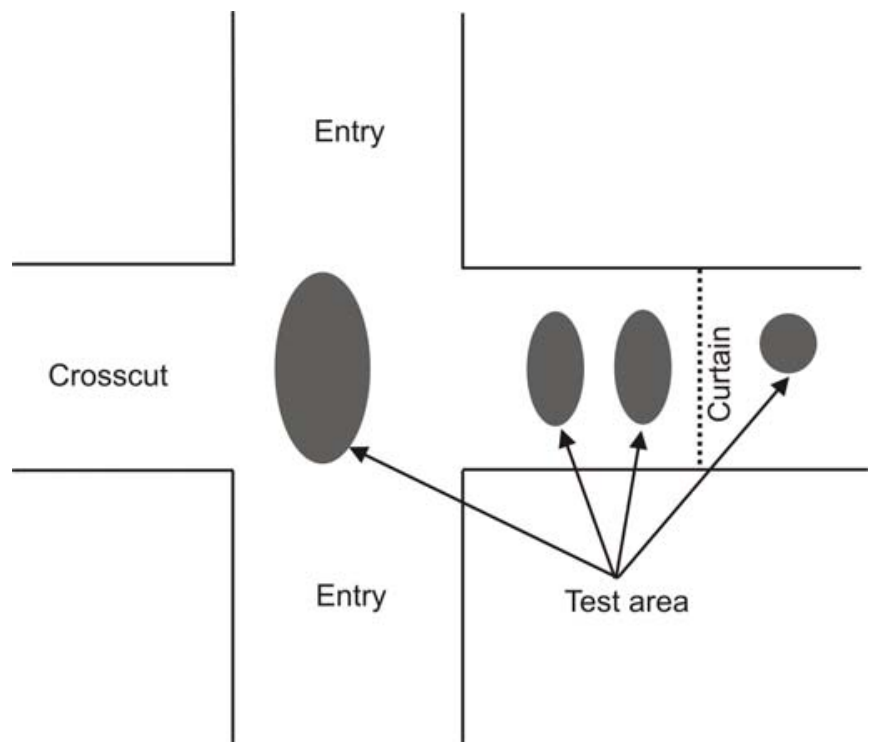

Figure 4.20 Diagram of test site (Mine C)

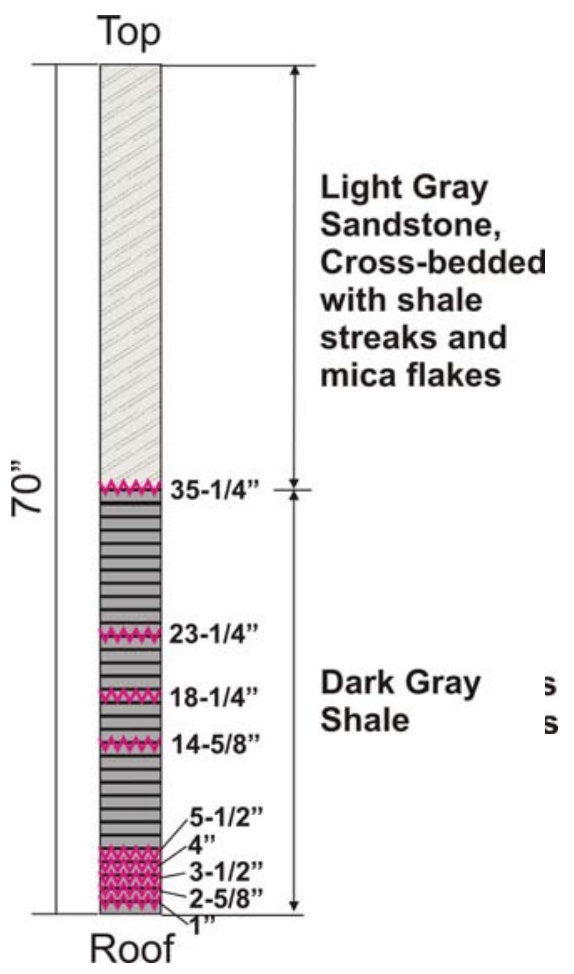

Figure 4.21 Core log (Mine C)

\subsubsection{Mine D Underground Test}

Mine D was located in southern West Virginia. A sandstone channel was observed. Drilling tests were focused in and around this sandstone channel. Because the edges of the channel had a steep slope, data could be collected from three different geologic conditions within close proximity. Drilling occurred in the sandstone channel yielding drilling data that intercepted only sandstone. Drilling was also conducted outside the sandstone channel, but near its edge, yielding drilling data the intercepted shale and sandstone, respectively. Finally, drilling was conducted at some distance from the 
channel, yielding drilling data that only intercepted shale. Figures 4.22 and 4.23 show a diagram of the test area and core logs, respectively.

$<$ Drilling Settings $>$

- DCU control mode: WVU mode $=$ both penetration rate and rotation rate were controlled or kept constant.

- Penetration rate: $0.8,1.1,1.5$ and $1.7 \mathrm{in} / \mathrm{sec}$

- Rotation rate: free

- Thrust cap: 650 and 800 psi

- Bit size: $1-3 / 8$ in

- Number of drill holes: 27 holes

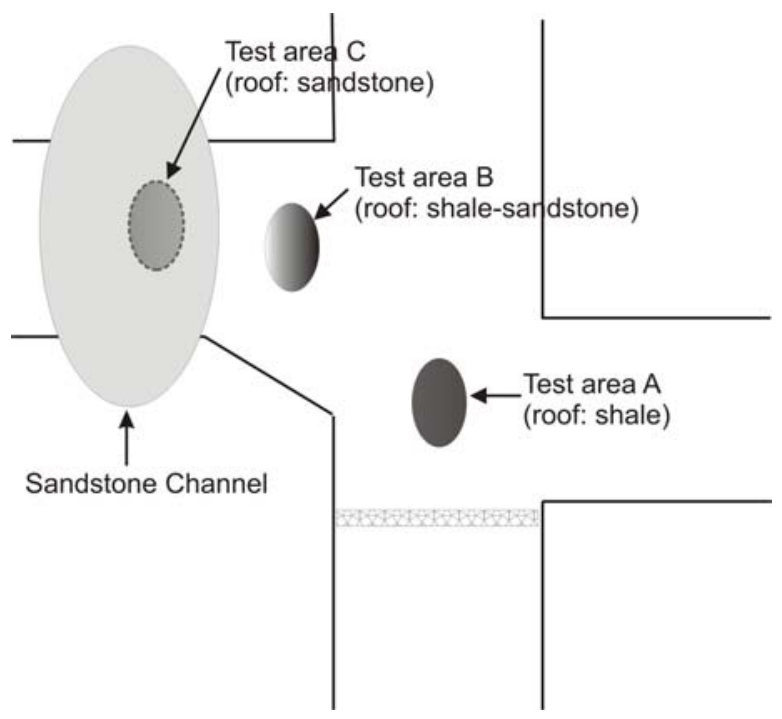

Figure 4.22 Diagram of test site (Mine D) 


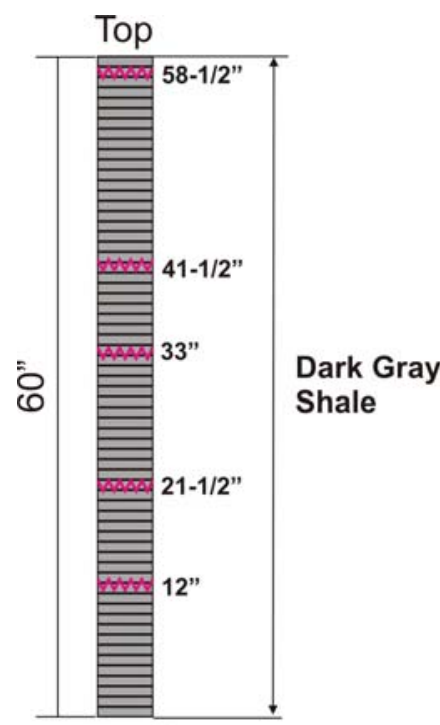

Roof

(a) Test area $\mathrm{A}$ (roof: shale)

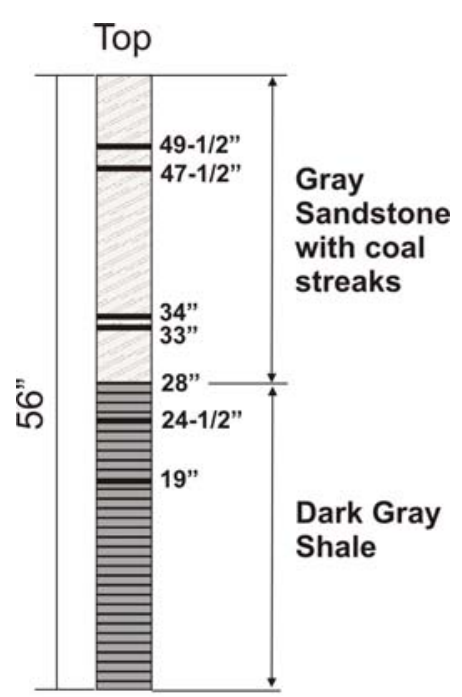

Roof

(b) Test area B (roof: shale-sandstone)

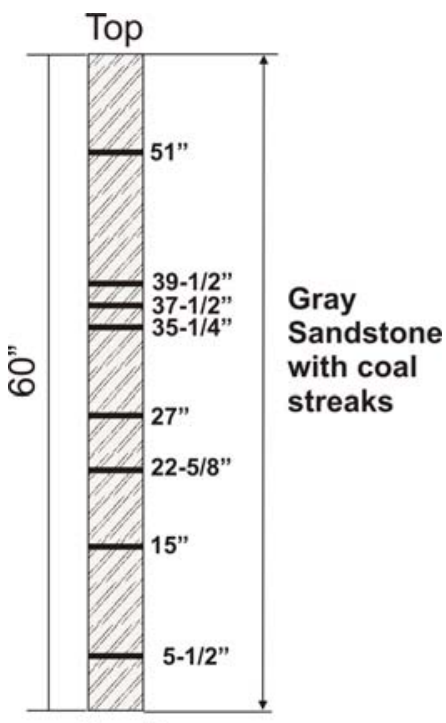

Roof

(c) Test area $\mathrm{C}$ (roof: sandstone)

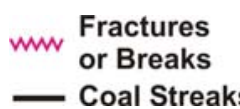

Figure 4.23 Core logs (Mine D)

\subsubsection{Mine E Underground Test}

Mine E was located in southern West Virginia. The roof at the test site was a consistent sandstone layer within the range of drilling of the roof bolter. Vertical holes were drilling into the sandstone roof. Despite the consistent roof strata, three different geological conditions were drilled using the angle drilling features of the roof bolter (Figure 4.24). The angle drilling feature of the roof bolter allows holes to be drilled at any angle from the vertical up to 90 degrees. Two geologic features were intercepted using this feature. A high angle (approximately 75 degrees from horizontal) mining-induced fracture was present in the roof. 4 holes were drilled to intercept this feature using the angle drilling feature of the roof bolter. The angle drilling feature was also used to begin drilling in the coal rib of the mine. As drilling proceeded, the drill bit intercepted the sandstone roof some distance into the hole. This procedure enabled the data collection 
system to monitor drilling through two distinct layers of strata in one hole. Figures 4.25 and 4.26 show a diagram of the test area and core logs, respectively.

\section{$<$ Drilling Settings $>$}

- DCU control mode: WVU mode = both penetration rate and rotation rate were controlled or kept constant.

- Penetration rate: $1.5 \mathrm{in} / \mathrm{sec}$

- Rotation rate: free

- Thrust cap: $800,1,000$ and 1,100 psi

- Bit size: $1-3 / 8$ in

- Number of drill holes: 18 holes

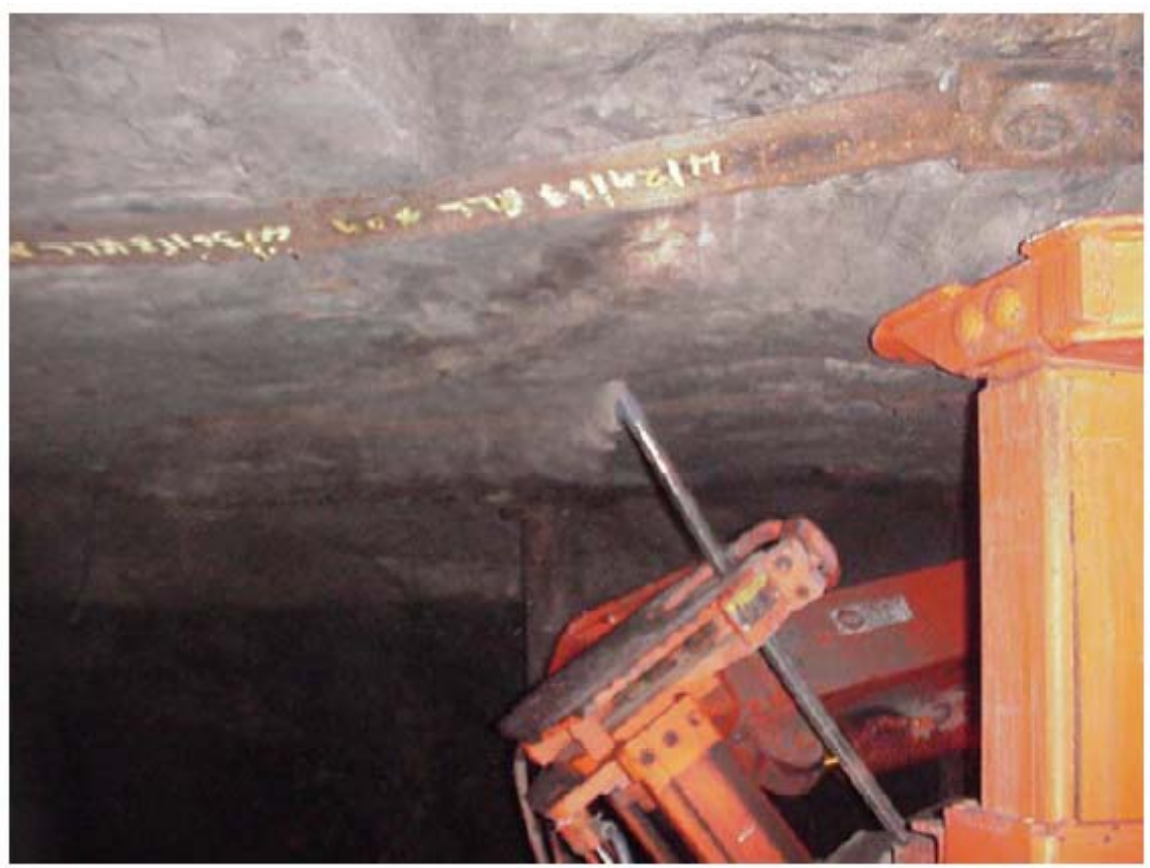

Figure 4.24 Angle drilling with roof bolter (Mine E) 


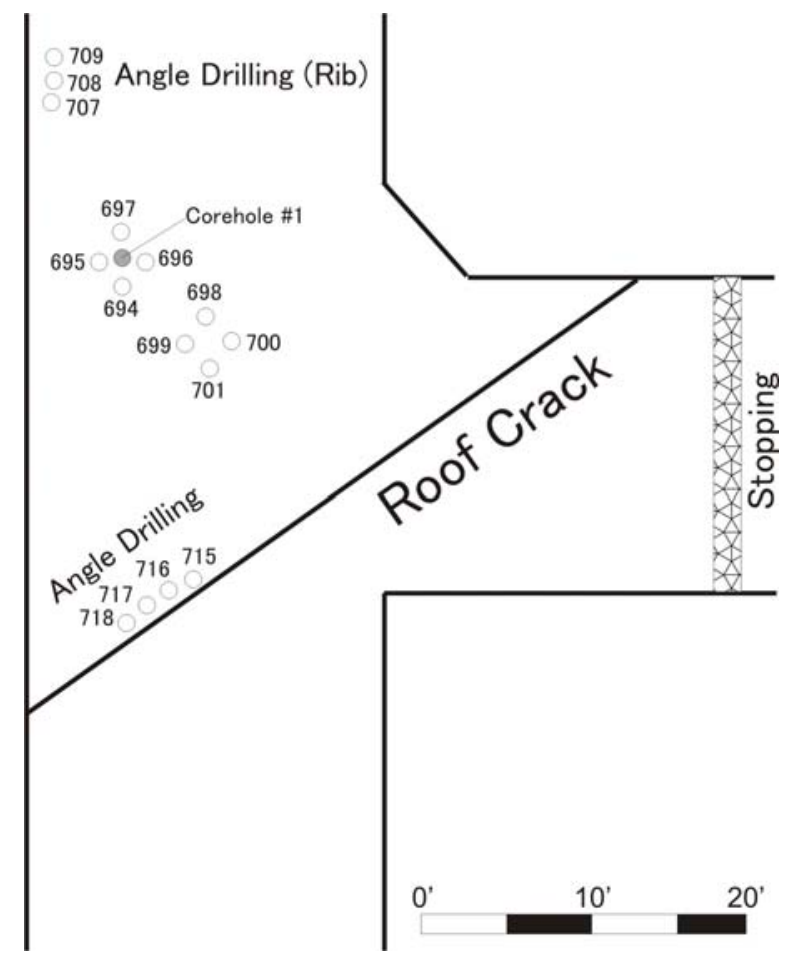

Figure 4.25 Diagram of test site (Mine E)

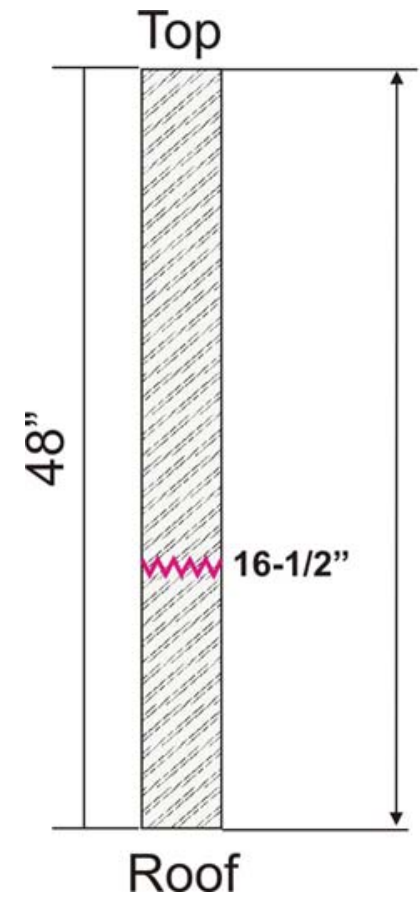

(a) Roof

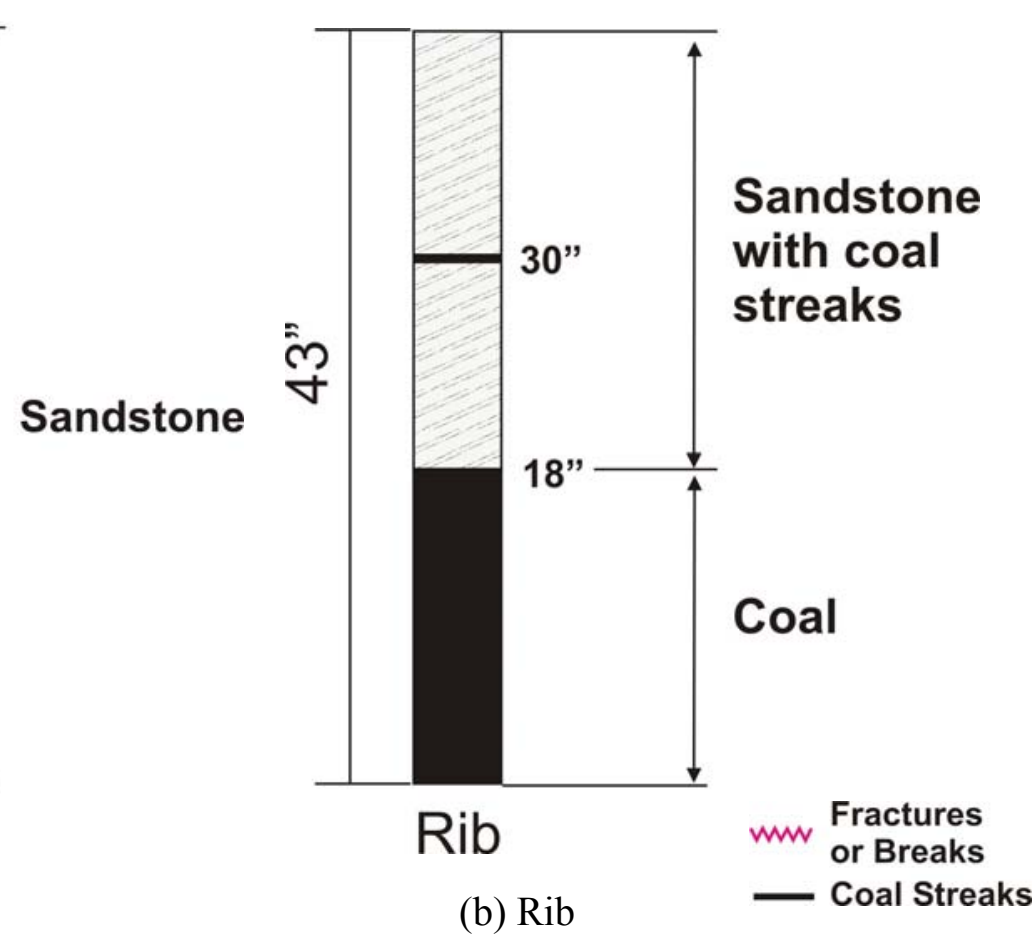

Figure 4.26 Core logs (Mine E) 


\subsubsection{Mine F Underground Test}

Mine F was located in southern West Virginia. In the testing area, soft shale strata were in the roof bolting horizon. Figure 4.27 shows a diagram of the test site. Figure 4.28 shows the core and core log. A bit wear test was also conducted during this test. As the rock sample was too soft to collect the specimen suitable for standard unconfined compressive strength testing, point load test was conducted to estimate the strength of the roof rock. The unconfined compressive strength of the rock unit was estimated at 3,400 psi or less.

$<$ Drilling Settings $>$

- DCU control mode: WVU mode = both penetration rate and rotation rate were controlled or kept constant.

- Penetration rate: $0.4,0.8,1.1,1.3,1.5,1.7$ and $2.1 \mathrm{in} / \mathrm{sec}$

- Rotation rate: $400,500,600,650$ and $700 \mathrm{rpm}$

- Thrust cap: 1,000 psi

- Bit size: $1-3 / 8$ in

- Number of drill holes: 103 holes 


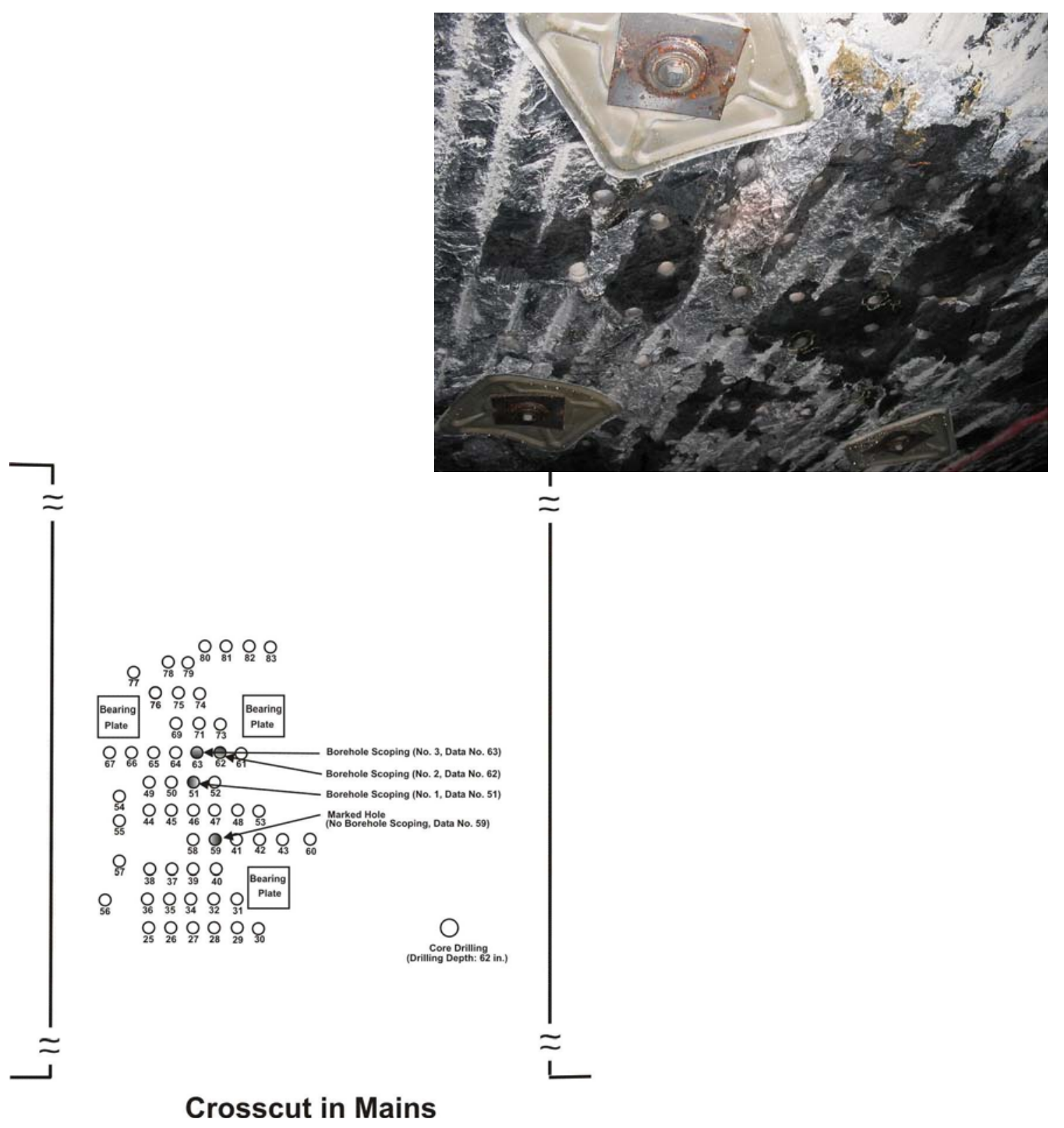

Figure 4.27 Diagram of test site (Mine F) 

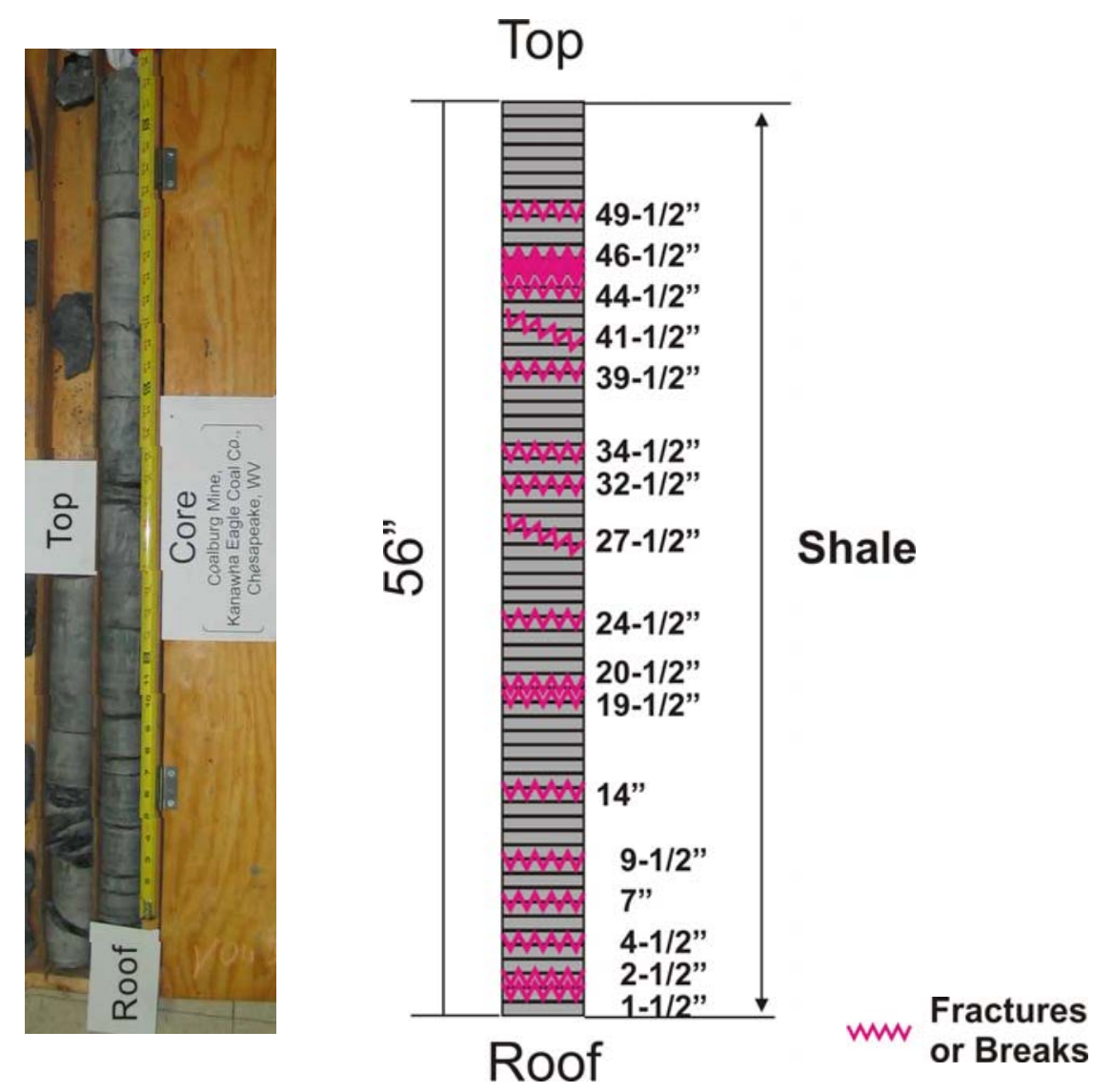

Figure 4.28 Core and core log (Mine F)

\subsubsection{Mine G Underground Test}

Mine $\mathrm{G}$ was located in southern West Virginia. In the testing site, hard sandstone roof strata were in the roof bolting horizon. Figure 4.29 show a diagram of the test site. Figure 4.30 shows the core and core log. Bit wear test was also conducted. Some holes were drilled by feedback system mode (horsepower curve and bite curve setting). From the results of rock property test, the uniaxial compressive strength of roof rock is about 9,400 psi on average, ranging from 8,200 to 10,400 psi. Table 4.3 shows the result of rock property test. 
$<$ Drilling Settings $>$

- DCU control mode: WVU mode = both penetration rate and rotation rate were controlled or kept constant, and feedback system mode $=$ penetration rate and rotation rate were controlled

- Drilling parameters

(a)WVU mode

o Penetration rate: $0.4,0.6,0.8,0.9,1.1,1.3,1.5,1.7$ and $2.1 \mathrm{in} / \mathrm{sec}$

o Rotation rate: $400,500,600,650 \mathrm{rpm}$ and free

(b)Bit wear test

o Penetration rate: $1.1 \mathrm{in} / \mathrm{sec}$

o Rotation rate: 400 and $650 \mathrm{rpm}$

o Number of drill holes with one bit: 3,5 and $7^{* *}$ holes

** After the $7^{\text {th }}$ hole was completed, the bit broke.

- Thrust cap: 1,000 psi

- Bit size: 1-3/8 in

- Number of drill holes: 268 holes

Table 4.3 Physical properties of core samples (Mine G)

\begin{tabular}{|c|c|c|c|c|}
\hline Rock Type & $\begin{array}{c}\text { UCS } \\
(\mathrm{psi})\end{array}$ & $\begin{array}{c}\text { BTS } \\
(\mathrm{psi})\end{array}$ & $\begin{array}{c}\mathrm{E}_{50} \\
\left(\times 10^{6} \mathrm{psi}\right)\end{array}$ & $\begin{array}{c}\text { Unit Weight } \\
\left(\mathrm{lbs} / \mathrm{ft}^{3}\right)\end{array}$ \\
\hline Sandstone & 9,392 & 589.6 & 2.082 & 150.9 \\
\hline
\end{tabular}




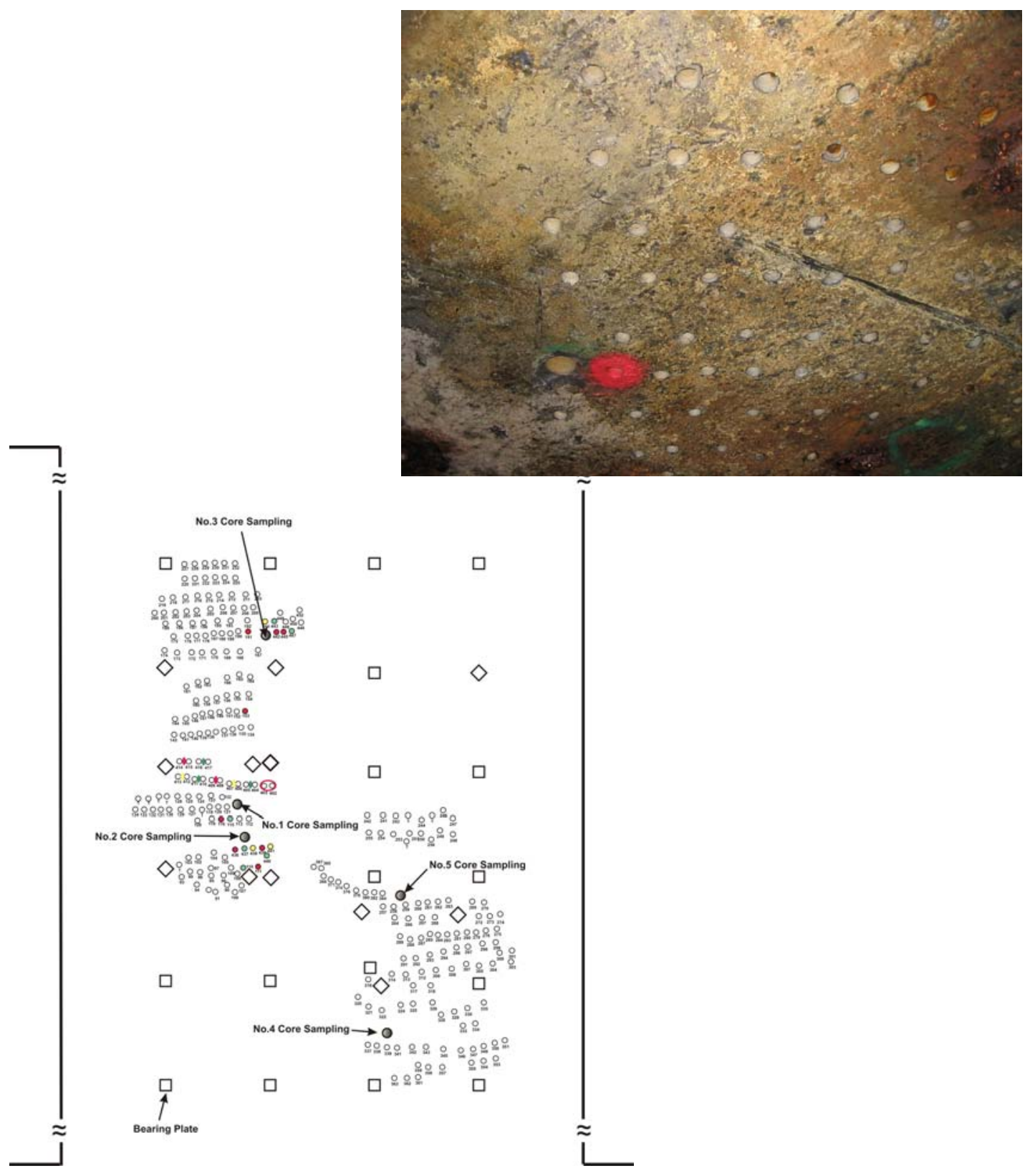

Figure 4.29 Diagram of test site (Mine G) 

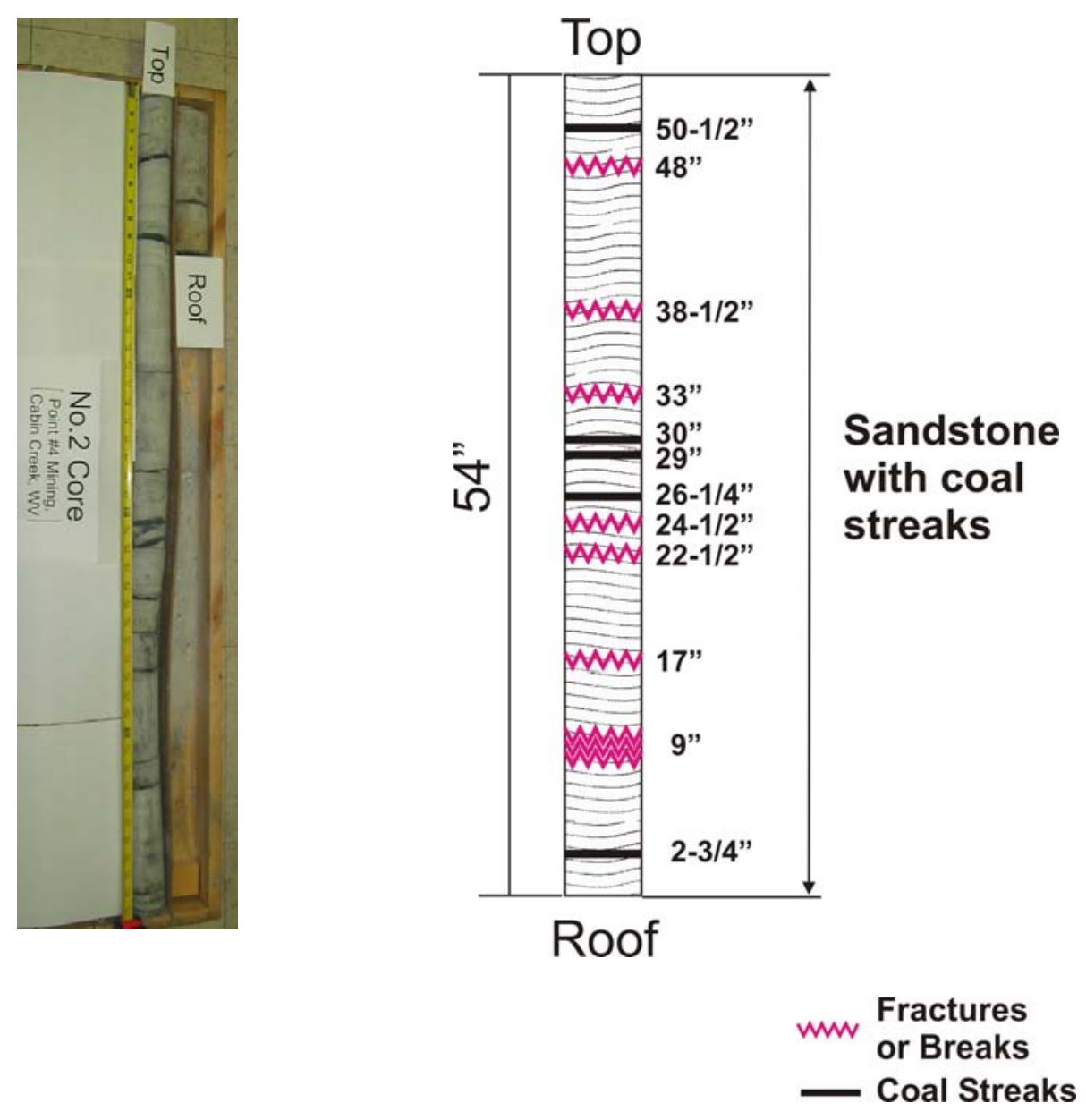

Figure 4.30 Core and core log (Mine G)

\subsubsection{Mine H Underground Test}

Mine $\mathrm{H}$ was located in southern West Virginia. In the testing area, hard sandstone roof strata were in the roof bolting horizon. Figure 4.31 shows a diagram of the test site. Figure 4.32 shows the core and the core log. From the results of the rock property test, the unconfined compressive strength of roof rock is about 9,700 psi on average, ranging from 8,900 to 10,300 psi. Table 4.4 shows the result of rock property test.

$<$ Drilling Settings $>$

- DCU control mode: WVU mode = both penetration rate and rotation rate were controlled or kept constant. 
- Penetration rate: $0.4,0.8,1.1,1.3,1.5,1.7$ and $2.1 \mathrm{in} / \mathrm{sec}$

- Rotation rate: $400,500,600 \mathrm{rpm}$ and free

- Thrust cap: 1,000 psi

- Bit size: 1-3/8 in

- Number of drill holes: 56 holes

Table 4.4 Physical properties of core samples (Mine H)

\begin{tabular}{|c|c|c|c|c|}
\hline Rock Type & $\begin{array}{c}\text { UCS } \\
(\mathrm{psi})\end{array}$ & $\begin{array}{c}\text { BTS } \\
(\mathrm{psi})\end{array}$ & $\begin{array}{c}\mathrm{E}_{50} \\
\left(\times 10^{6} \mathrm{psi}\right)\end{array}$ & $\begin{array}{c}\text { Unit Weight } \\
\left(\mathrm{lbs} / \mathrm{ft}^{3}\right)\end{array}$ \\
\hline Sandstone & 9,770 & 521.3 & 1.649 & 151.9 \\
\hline
\end{tabular}

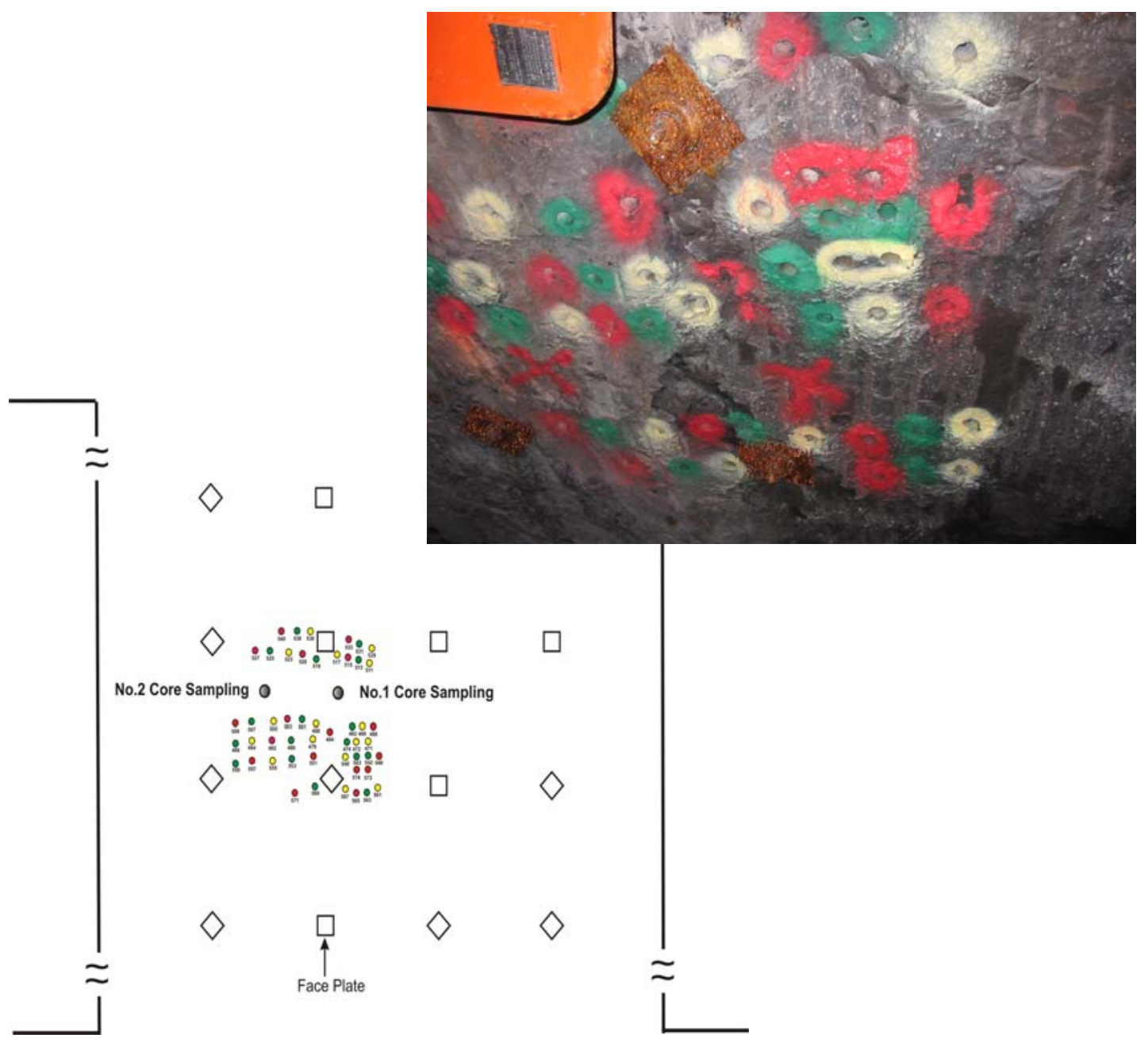

Figure 4.31 Diagram of test site (Mine $\mathrm{H}$ ) 

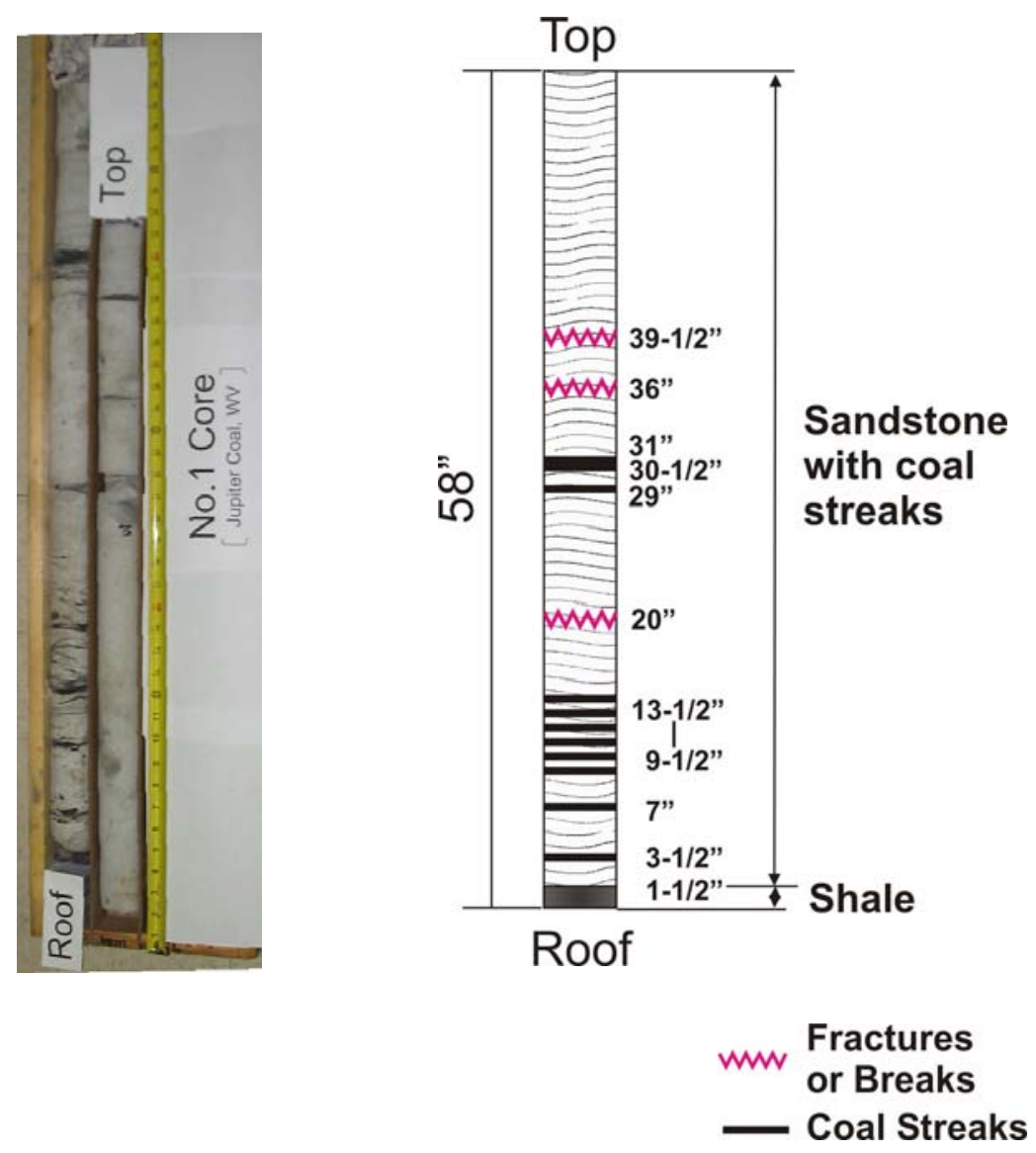

Figure 4.32 Core and core log (Mine H) 


\section{CHAPTER 5}

\section{DEVELOPMENT OF DATA INTERPRETATION METHODOLOGY}

In designing a proper roof support system, one must know the features of roof geology in advance of mining. These geological features include: rock type, rock strength, rock layer interfaces, voids, cracks, and bed separations. The drilling parameters obtained during the normal roof bolt installation cycle can provide a large amount of information on roof geology when properly interpreted. It is also an economic way for identifying the geological features of the roof. One of the main tasks of this project is to develop a method for predicting roof geology based on the drilling parameters obtained during normal roof bolting operation. In this project, experimental studies were conducted to determine the relationship between the drilling parameters and geological features based on the results of a series of laboratory and underground tests as mentioned in Chapter 4 . Study about the characteristics of the machine and control system used in this project was also conducted to eliminate their effects on drilling parameters. Because, as the drilling was controlled by DCU in this test (WVU control mode), it is necessary to distinguish the changes of the drilling parameters that were caused by geological change and that by the machine characteristics/control algorithm. Based on the above results, an empirical model for roof geology prediction has been developed.

This chapter describes the results including the methods for determining quantitatively the location and size of voids/fractures and estimation of roof rock strength from the recorded drilling parameters of roof bolter.

\subsection{Concept of Data Analysis and Rule Generation}

The basic assumptions for this research are as follows:

- Variation in geological properties of roof strata will result in the change of some of the measured drilling parameters. Those drilling parameters that obviously reflect the change of roof geological properties are referred as relevant drilling parameters. 
- Different roof geological properties are statistically independent. This independence could be a result of their different physical properties, for example, their different compressive strengths and shear strengths.

- Each relevant drilling parameter has a Gaussian or normal distribution for each roof geological property, but with different means and variances.

These assumptions provide the foundation for using a statistical model called finite mixtures in this research. In this case, a mixture is a set of $\mathrm{k}$ probability distributions, representing $\mathrm{k}$ geological properties, that governs the drilling parameter values for members of that geological property. In other words, each distribution gives the probability that a particular data point would have a certain set of drilling parameters values if it were known to be a member of that geology property. Each geological property has a different distribution. Any particular data

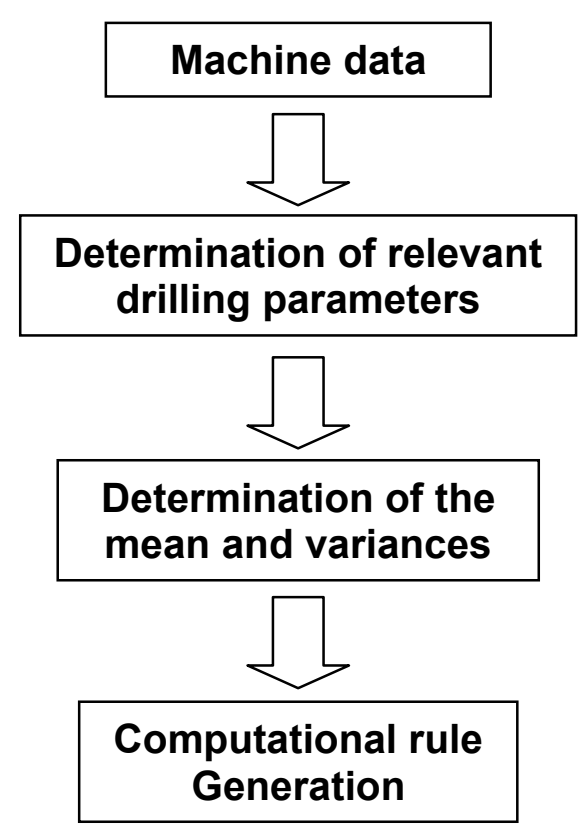

Figure 5.1 Flowchart for generating rules point really belongs to one and only one of the geological properties. Roof geological properties are not likely to be equal: there is some probability distribution that reflects their relative populations.

Based on the above mentioned assumptions, the following procedures are taken for generating the rules of predicting the voids in roof rock by using the roof drilling parameters as shown in Figure 5.1.

\subsection{Effect of Drilling Settings and Machine/Drilling Conditions on Drilling Parameters for Compensation Runs}

As the data collection system used in this series of research was designed not for prediction of roof geology but for control drilling, the drilling parameters which can be measured are the parameters in the hydraulic system. This means that the drilling 
parameters contain not only for drilling rock but also for running machine itself. First of all, one needs to know how much the drilling parameters, especially feed pressure / rotation pressure, consumed for running the machine itself and how much impact different drilling settings and machine conditions (i.e. oil temperature in the hydraulic system) have on them. So, the first set of experiments was performed by drilling nothing to determine the consistency of the drilling parameters in the air. These data collected when drilling in the air are referred as compensation run data. Besides, compensation run was always conducted before every drilling to determine the machine and control conditions.

\subsubsection{Effect on Feed Pressure}

Figure 5.2 shows the feed pressure, carriage position and mast position curves for compensation run. It can be seen that the magnitude of feed pressure consumed for running the machine itself changes. This is caused by the change from carriage stage to mast stage. This machine can drill a $52-54$ in deep hole in one cycle by two stages as shown in Figure 5.3. So, it was considered that feed pressure consumed for running the machine depends on the net bit position not on the bit position from the roof line. Here, the difference between bit position and net bit position is shown as follows:

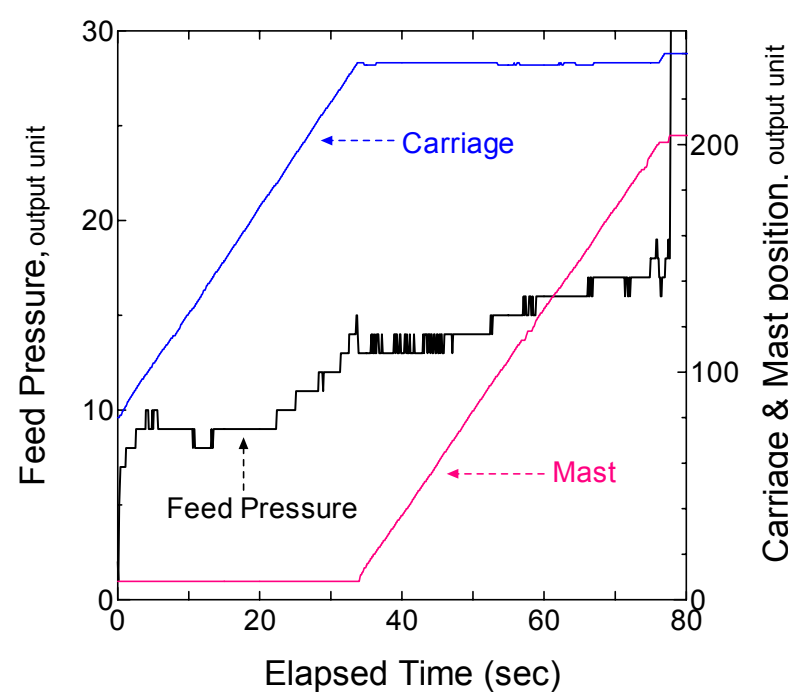

Figure 5.2 Relationships between feed pressure /carriage position/mast position and elapsed time (P.R. $=0.4 \mathrm{in} / \mathrm{sec}$, R.R. $=600 \mathrm{rpm})$

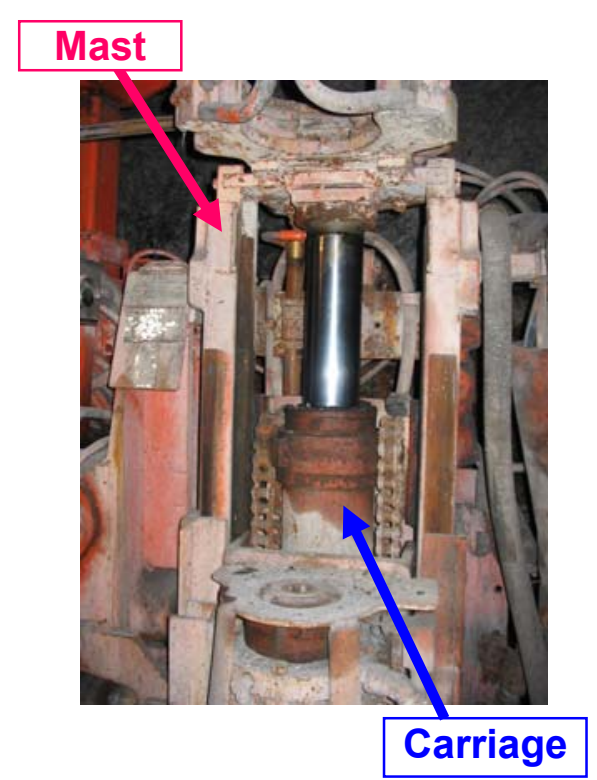

Figure 5.3 Carriage and mast stages 


$$
\begin{aligned}
\operatorname{BitPosition}(t)(\text { in })= & 0.1485 \times \text { MastPosition }(t)+0.1593 \times \text { CarriagePosition }(t)-\operatorname{BitPosition}\left(t_{1}\right) \\
= & 0.1485 \times \text { MastPosition }(t)+0.1593 \times \text { CarriagePosition }(t) \\
& \quad-0.1485 \times \text { MastPosition }\left(t_{1}\right)-0.1593 \times \text { CarriagePosition }\left(t_{1}\right)
\end{aligned}
$$

$\operatorname{NetBitPosition}(t)($ in $)=0.1485 \times$ MastPosition $(t)+0.1593 \times$ CarriagePosition $(t)$

where, $t_{1}=$ the time when drilling starts, mast position $(\mathrm{t})=$ output count of mast position sensor and carriage position $(t)=$ output count of carriage position sensor. The conversion factors of mast and carriage position sensors are $0.1593 \mathrm{in} /$ count and $0.1485 \mathrm{in} /$ count, respectively. Note bit position refers to the distance from roof line including initial startup adjustments while net bit position refers to the actual bit drilling distance.

There is about 7-10 output units difference between feed pressure in carriage

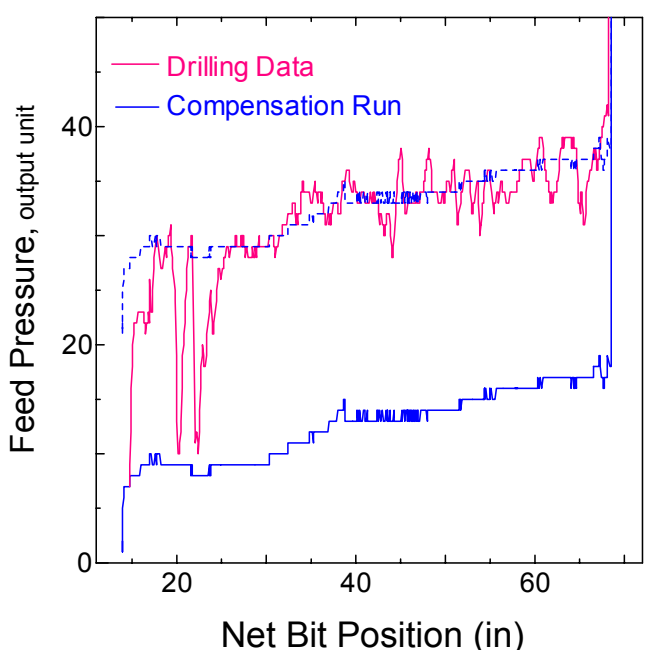

Figure 5.4 Relationship between feed pressure and net bit position (P.R. $=0.4 \mathrm{in} / \mathrm{sec}, \mathrm{R} . \mathrm{R} .=600 \mathrm{rpm})$ stage and that in mast stage. This means the magnitude of feed pressure is different with the different stages even drilling in a homogeneous rock. As shown in Figure 5.4, this feed pressure change was caused by the machine itself.

Figures 5.5 and 5.6 show the relationship between feed pressure and net bit position for compensation runs under different controlled settings and oil temperatures, respectively. It can be seen that drilling settings and oil temperature have no obvious impact on both the magnitude and trend of feed pressure-net bit position curve for compensation run. Hence, it was concluded that feed pressure for compensation run can be defined as a function of net bit position only in this machine and the machine effect on the feed pressure can be eliminated from the drilling data once feed pressure-bit position curve for compensation run has been recorded at any settings. From the compensation run data obtained so far, the approximate curve for the relationship between feed pressure and net bit position is defined by the following equation (Figure 5.7): 


$$
\begin{aligned}
F P_{C}\left(B P_{n e t}\left(t_{n}\right)\right) & =-5.49 \times 10^{2}+1.21 \times 10^{2} \times B P_{n e t}\left(t_{n}\right)-1.08 \times 10 \times\left\{B P_{n e t}\left(t_{n}\right)\right\}^{2}+5.08 \times 10^{-1} \times\left\{B P_{n e t}\left(t_{n}\right)\right\}^{\beta} \\
& -1.37 \times 10^{-2} \times\left\{B P_{n e t}\left(t_{n}\right)\right\}^{4}+2.14 \times 10^{-4} \times\left\{B P_{n e t}\left(t_{n}\right)\right\}^{5}-1.78 \times 10^{-6} \times\left\{B P_{n e t}\left(t_{n}\right)\right\}^{6^{6}} \\
& +6.20 \times 10^{-9} \times\left\{B P_{n e t}\left(t_{n}\right)\right\}^{7}
\end{aligned}
$$

$($ Number of data points $=27,626$, variance $=0.7163$, correlation coefficient $=0.945)$ where, $\mathrm{BP}_{\text {net }}\left(\mathrm{t}_{\mathrm{n}}\right)=$ net bit position and $\mathrm{t}_{\mathrm{n}}=$ elapsed time after drilling starts

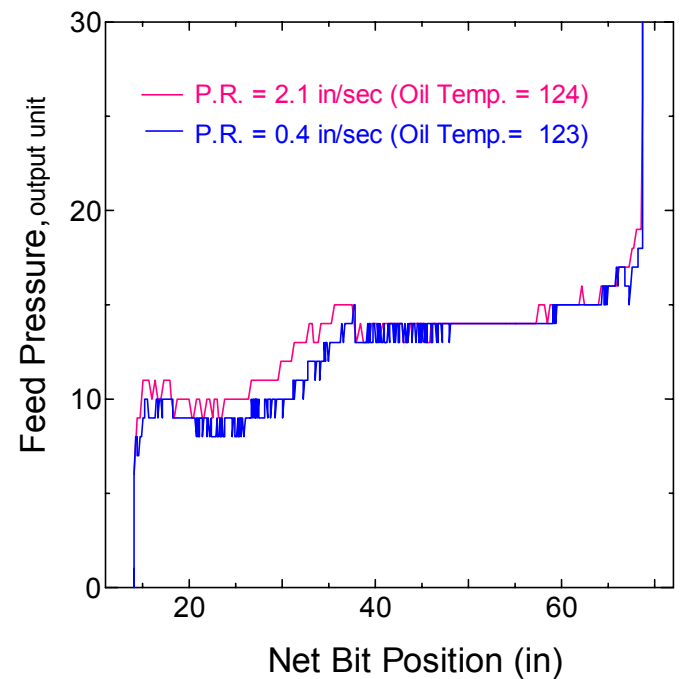

(a) Penetration Rate (R.R. $=600 \mathrm{rpm})$

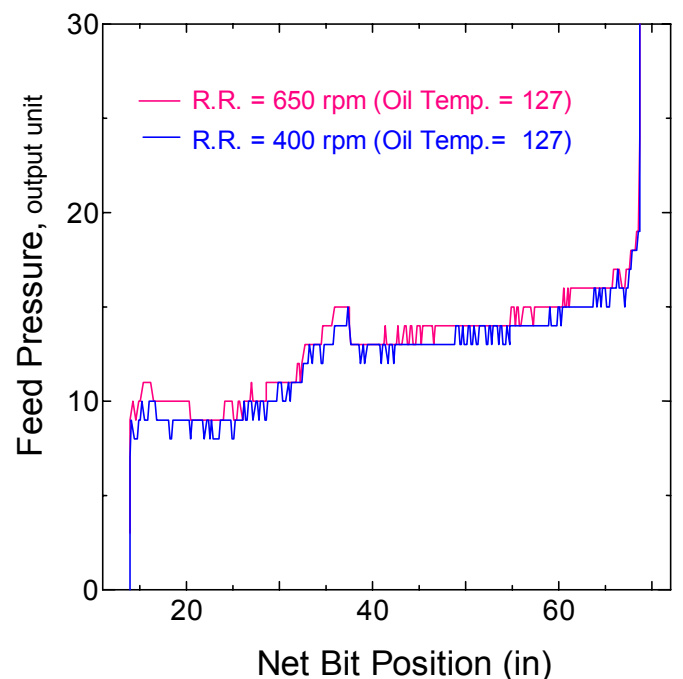

(b) Rotation Rate (P.R. = $1.1 \mathrm{in} / \mathrm{sec}$ )

Figure 5.5 Effect of drilling settings on the magnitude of feed pressure for compensation run

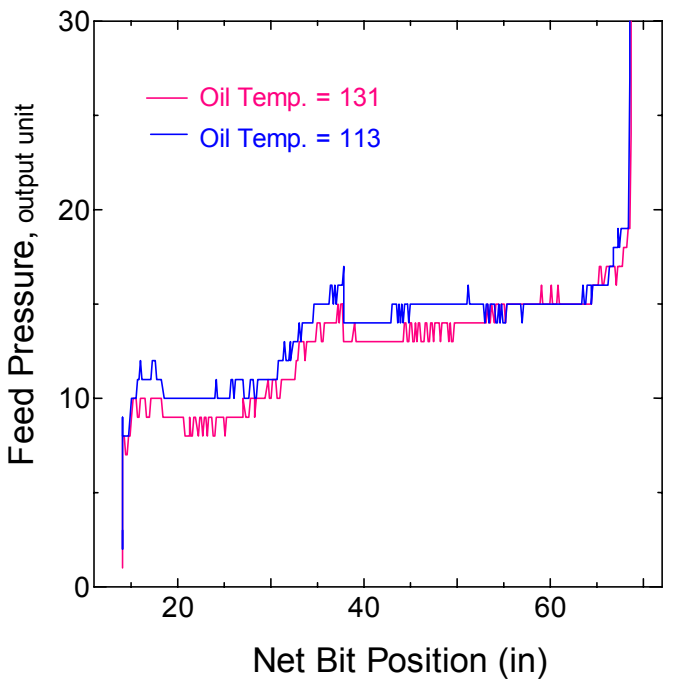

Figure 5.6 Effect of oil temperature on the magnitude of feed pressure for compensation run $(P . R .=2.1 \mathrm{in} / \mathrm{sec}$, R.R. $=600 \mathrm{rpm})$

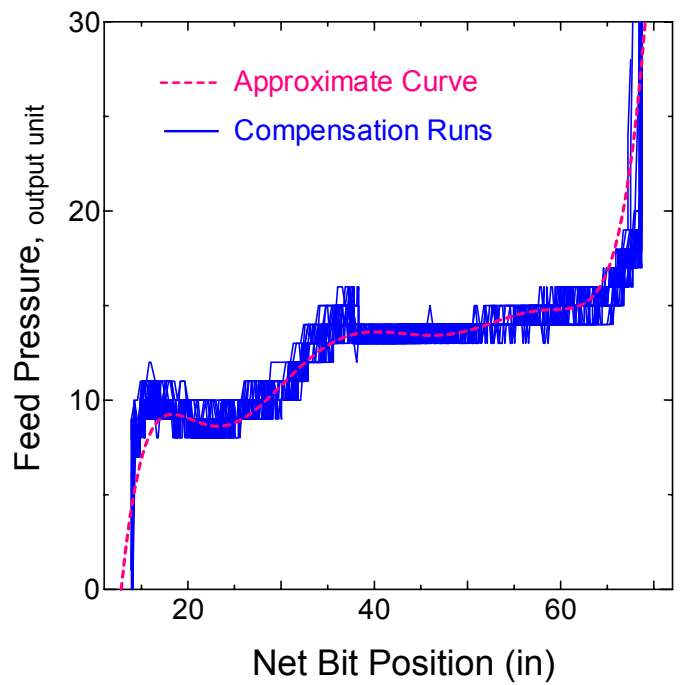

Figure 5.7 Approximate curve for the relationship between feed pressure and net bit position 
As the feed pressure for compensation runs defined by equation (5.3) is subtracted from the feed pressure when drilling rock, the machine effect can be eliminated and how much feed pressure consumed only for drilling rock can be determined. This value is called the 'net feed pressure'. The net feed pressure is defined by the following equation:

$$
\text { Net Feed Pr essure }\left(B P_{n e t}\left(t_{n}\right)\right)=F P_{D R}\left(B P_{\text {net }}\left(t_{n}\right)\right)-F P_{C}\left(B P_{\text {net }}\left(t_{n}\right)\right)
$$

where, $\mathrm{FP}_{\mathrm{DR}}=$ feed pressure when drilling in rock.

Most likely, as mentioned above, different drilling settings and machine conditions (i.e. oil temperature) have no obvious impact on feed pressure-bit position curve for compensation run. However, as shown in Figure 5.8, the magnitude of feed pressure is always slightly higher at the beginning of test. This point should be taken into account when the feed pressure-bit position curve for compensation runs is approximated. Moreover, from Figure 5.9, it can also be seen the difference among the drilling data for different date, it seems that the machine condition also has a small impact on the magnitude of feed pressure-bit position curve for compensation run. From these results, it was suggested that compensation runs should be conducted at the beginning and the end of each shift to check the machine condition and minimize the errors when calculating the net feed pressure. Besides, the rule for determining the approximate curve should be developed in order to apply it for practical use.

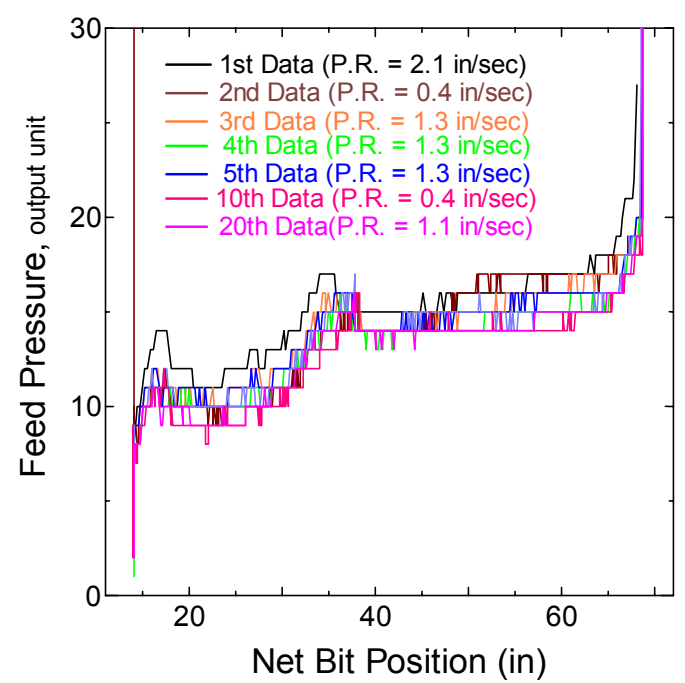

Figure 5.8 Feed pressure-net bit position curves at the beginning of test

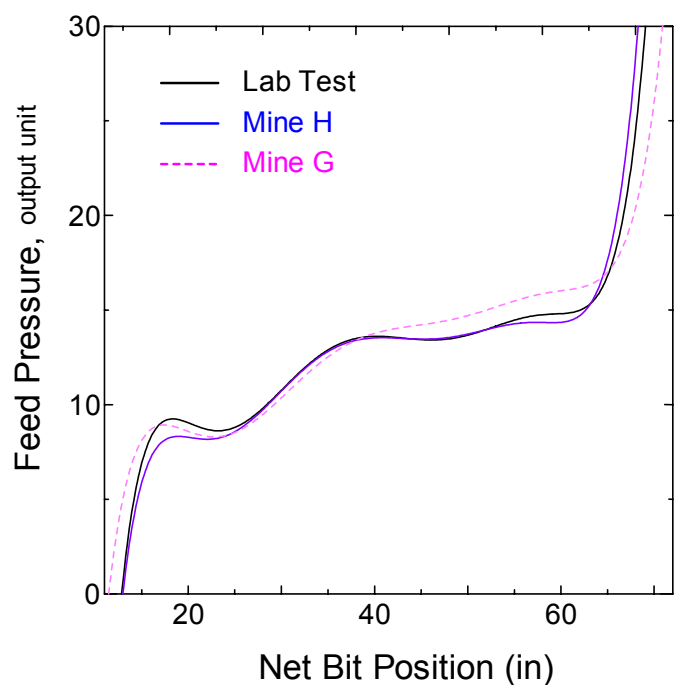

Figure 5.9 Approximate curves for different test dates 


\subsubsection{Effect on Rotation Pressure}

Figure 5.10 shows the relationship between rotation pressure and net bit position for compensation runs under different controlled settings. It can be seen that different rotation rates have obvious impact on the magnitude of rotation pressure. This is because of the increasing friction from bearings, gears and oil flow in the pipe. Figure 5.11 shows the relationship between rotation pressure and bit position for compensation runs and drilling rock under different oil temperatures. From this figure, it can be recognized that different oil temperatures also have obvious impact on the magnitude of rotation pressure and the impact of oil temperature when drilling rock is the same as that for compensation run. Since the locations of both drill holes are close to each other, the change of rotation pressure is not caused by the change of roof geology but by the change of oil temperature. This phenomenon may have been caused by the change of viscosity of hydraulic oil. Generally speaking, the viscosity of hydraulic fluid is altered by its temperature. The higher the oil temperature, the lower the viscosity of the hydraulic fluid. It changes significantly at low oil temperature. Accordingly, one question arises: why the effect of oil temperature was recognized only in rotation pressure but not in feed pressure? One conceivable reason is the difference between the magnitudes of oil flows. The feed flow varied from 0.857 to $4.820 \mathrm{gpm}$ when penetration rate was set from 0.4 to $2.1 \mathrm{in} / \mathrm{sec}$. On the other hand, rotation flow varied from 21.5 to $30.8 \mathrm{gpm}$ when rotation rate was set from 400 to $600 \mathrm{rpm}$. But this point has not been confirmed yet. Besides, it can also be seen from Figure 5.11 that the effects of rotation rate and oil temperature on rotation pressure are too large to be ignored comparing with that in drilling rock. Hence, in order to estimate roof rock strength based on the magnitude of rotation pressure, a model or formula for eliminating these effects needs to be developed. On the other hand, in order to develop a model or formula to eliminate these effects, there are still many unknown factors. In addition, their effects seem to be complicated. For example, even if the same hydraulic oil is used, its characteristics change with conditions (new or old, polluted, etc). 


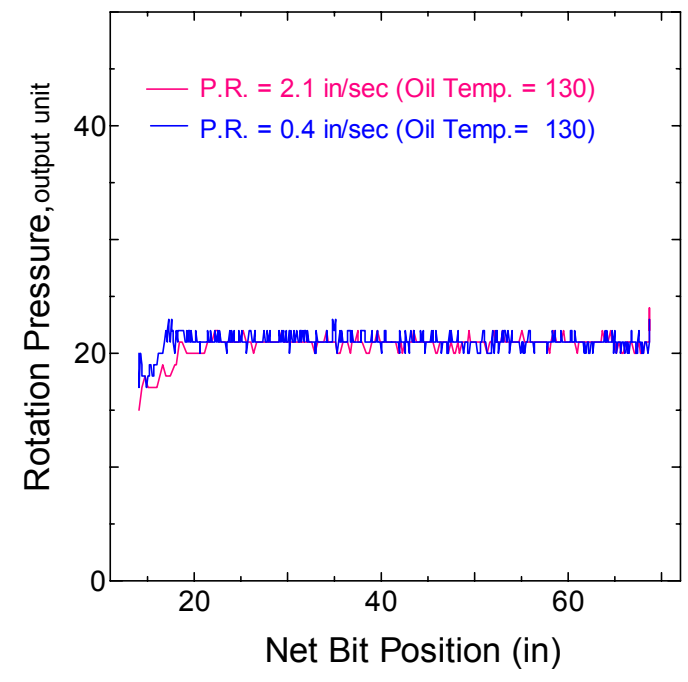

(a) Penetration Rate (R.R. $=600 \mathrm{rpm})$

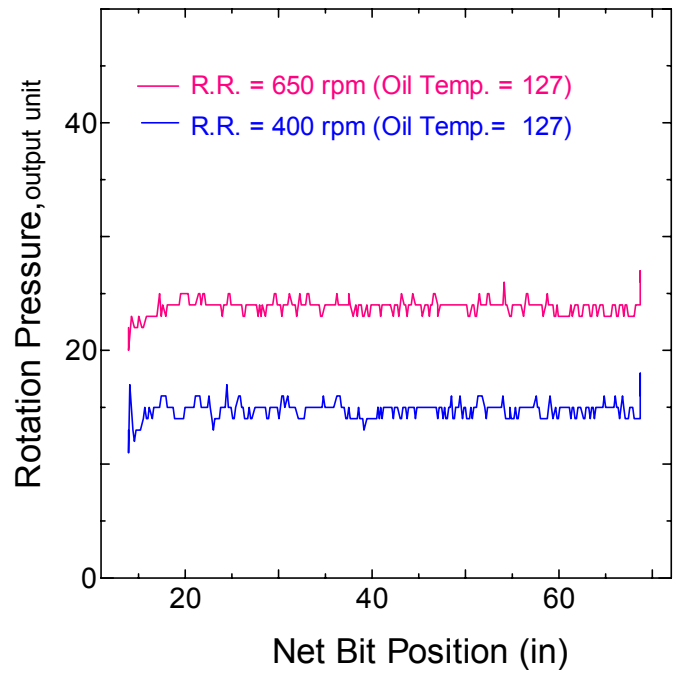

(b) Rotation Rate (P.R. = $1.1 \mathrm{in} / \mathrm{sec}$ )

Figure 5.10 Effect of drilling settings on the magnitude of rotation pressure for compensation run

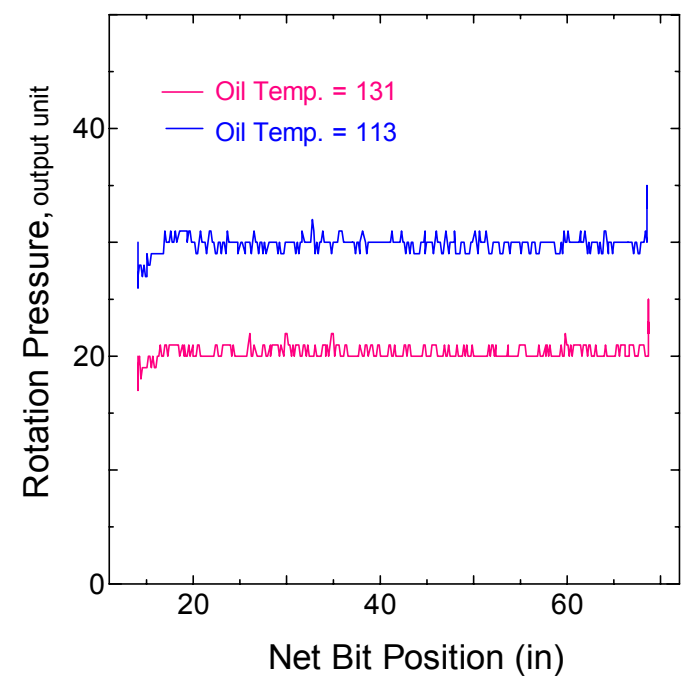

(a) Compensation Run

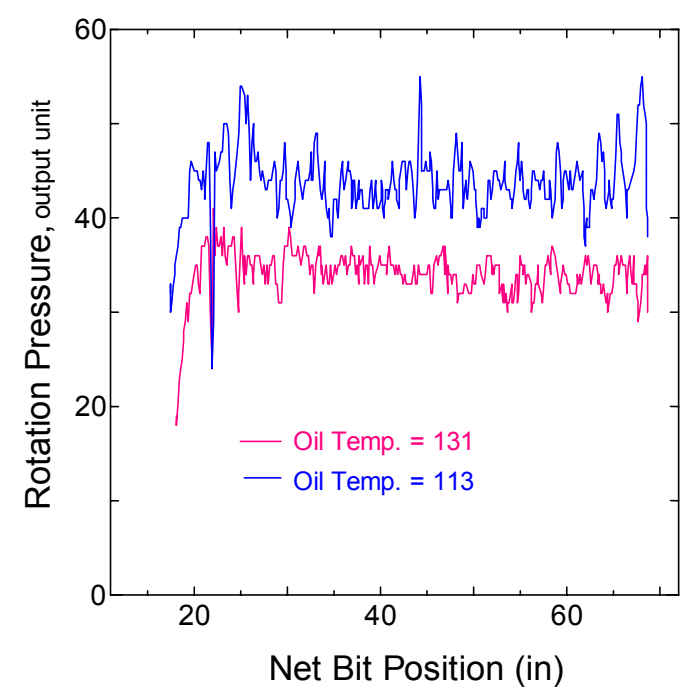

(b) Drilling Rock

Figure 5.11 Effect of oil temperature on rotation pressure for compensation run and drilling rock (P.R. $=1.1 \mathrm{in} / \mathrm{sec} \&$ R.R. $=600 \mathrm{rpm})$

\subsubsection{Effect of Drill Rods}

Basically, compensation runs were conducted without a drill rod and bit. So, in order to check the effect of drill rod on drilling parameters, compensation runs with drill rod were also conducted. Drilled holes were used for this test. As shown in Figure 5.12 
(a) and (b), even though there was some frictional resistance between bit/rod and the hole surface, the existence of drill rod has no obvious impact on drilling parameters. The effect of drill rod on drilling parameters is so small in comparison with the feed pressure and rotation pressure consumed for running the machine and drilling rock. It is therefore concluded that it is not necessary to take into account the effect of drill rod on drilling parameters.

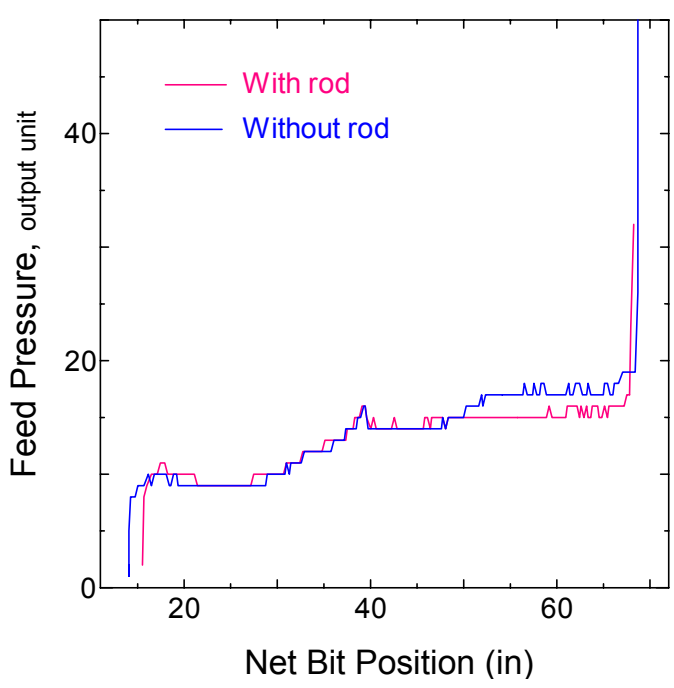

(a) Feed Pressure

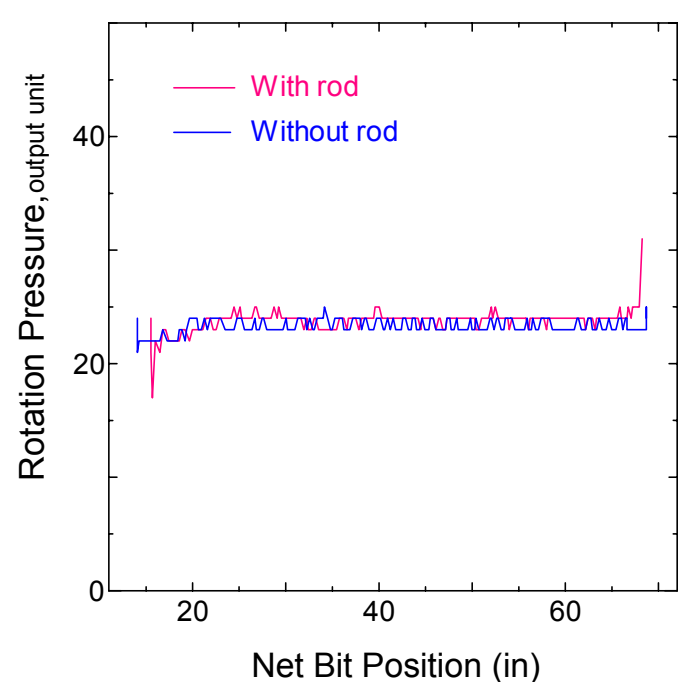

(b) Rotation Pressure

Figure 5.12 Effect of drill rod on drilling parameters (P.R. $=2.1 \mathrm{in} / \mathrm{sec}$, R.R. $=600 \mathrm{rpm})$

\subsection{Void / Fracture Prediction}

\subsubsection{Determination of Relevant Drilling Parameters for the Voids/Fractures}

The first step of data analysis is to find the most appropriate drilling parameters for void/fracture prediction. So, all the measured drilling data for a hole are plotted on a figure for comparison. Examples of the plotted data for holes drilled in the air, solid concrete and fractured blocks are shown in Figures 5.13 (a)-(c), respectively. All data are expressed in output counts of sensors. The basic principle for the drilling parameter selection is to look for highly discriminative drilling parameters regarding the rock voids and keep the number of selected drilling parameters as small as possible. Comparing these three figures, it can be seen that the feed pressure changes dramatically to form valleys around the locations of 15,30 and 45 in where the voids are located. It is also 
observed that rotation pressure has some kind of change around the locations of voids but the magnitude of the change is much smaller than that of feed pressure. The other drilling parameters do not change at the presence of voids. Therefore, it can be concluded that feed pressure is the most relevant drilling parameter for the existence of voids/fractures.

\subsubsection{Mechanism of Rock Fragmentation When a Void/Fracture is Encountered}

The phenomenon that a void in rock induces a valley in feed pressure can be explained by the mechanism of rock fragmentation. Feed pressure which to some extent represent the thrust force that the roof bolter provides for drilling in rock. According to the theory of rock fragmentation due to cutting, cracks will be created and propagate under a thrust force applied to by a drilling bit ${ }^{(17),(18),(19)}$. Once the cracks reach the free face of rock void, the whole pieces of the remaining rocks between drilling bit and free face of rock void will begin to break into small chips as shown in Figure 5.14. As the drill bit keeps advancing, some of these chips will further be broken into even smaller chips by the rotation of the drill bit and sucked out by the dry dust collector through the drill rod while others will be pushed into the void. Air will fill the space left by the removed rock chips. Therefore, the resistance that the drill bit needs to overcome will rapidly drop. Consequently, this result in a rapid drop of the force needed to keep the drill bit advancing and rotating because the penetration rate is maintained at a pre-set level for each hole. Since the void is filled with the air, the feed pressure should drop to the level when drilling in the air. Once drill bit encounters rock again after going through the void, the feed pressure will rapidly climb back to the level when drilling in rock. This is how the valleys in feed pressure curve are formed. If the size of the void is large enough, there should be a plateau at the valley bottom of feed pressure curve as the drill bit penetrates through the void. It seems that the width of the plateau can be used to estimate the size of void. Figure 5.15 shows the concrete blocks with a simulated void that the drilling test was conducted. From the chipping off nature of the void edge, the mechanism of rock fragmentation that is mentioned above can be verified. 


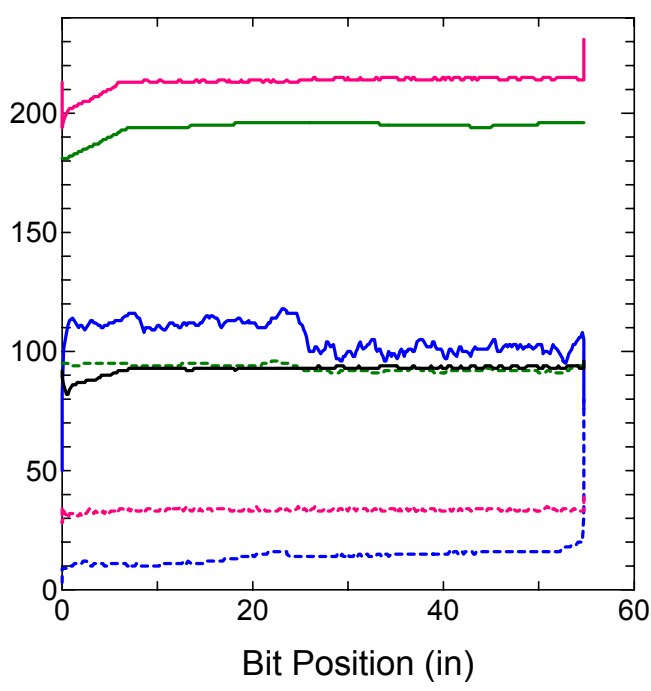

(a) Compensation Run

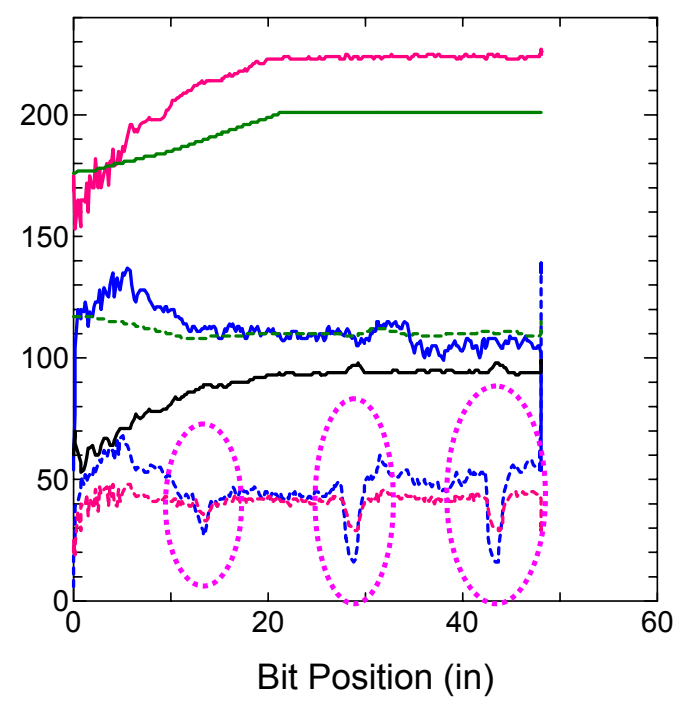

(c) Fractured Block

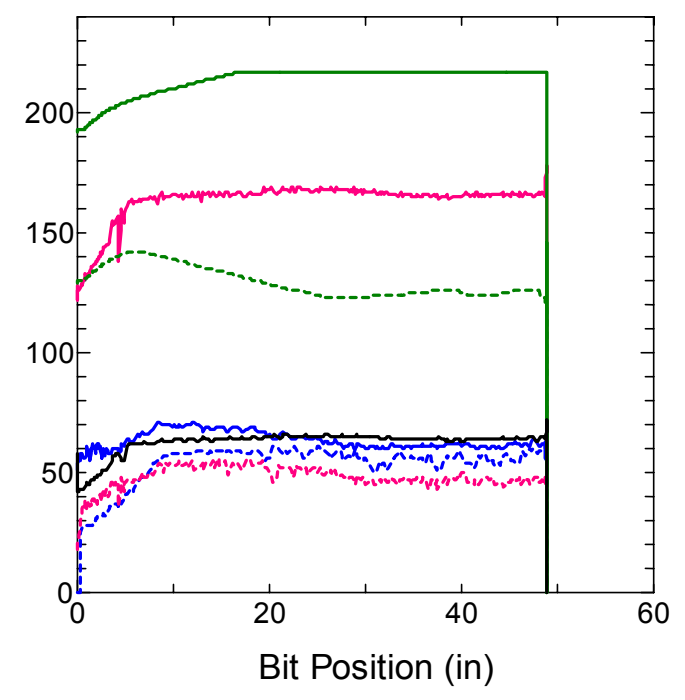

(b) Solid Concrete Block

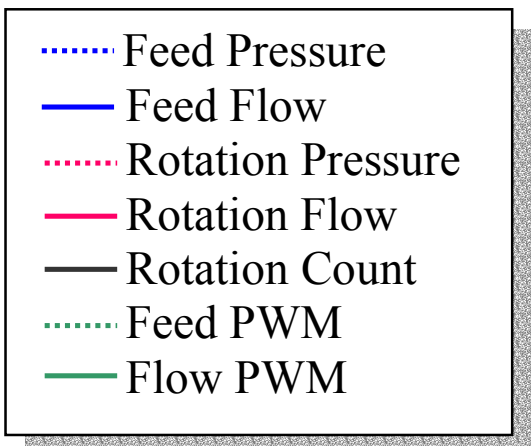

Figure 5.13 Drilling parameters 

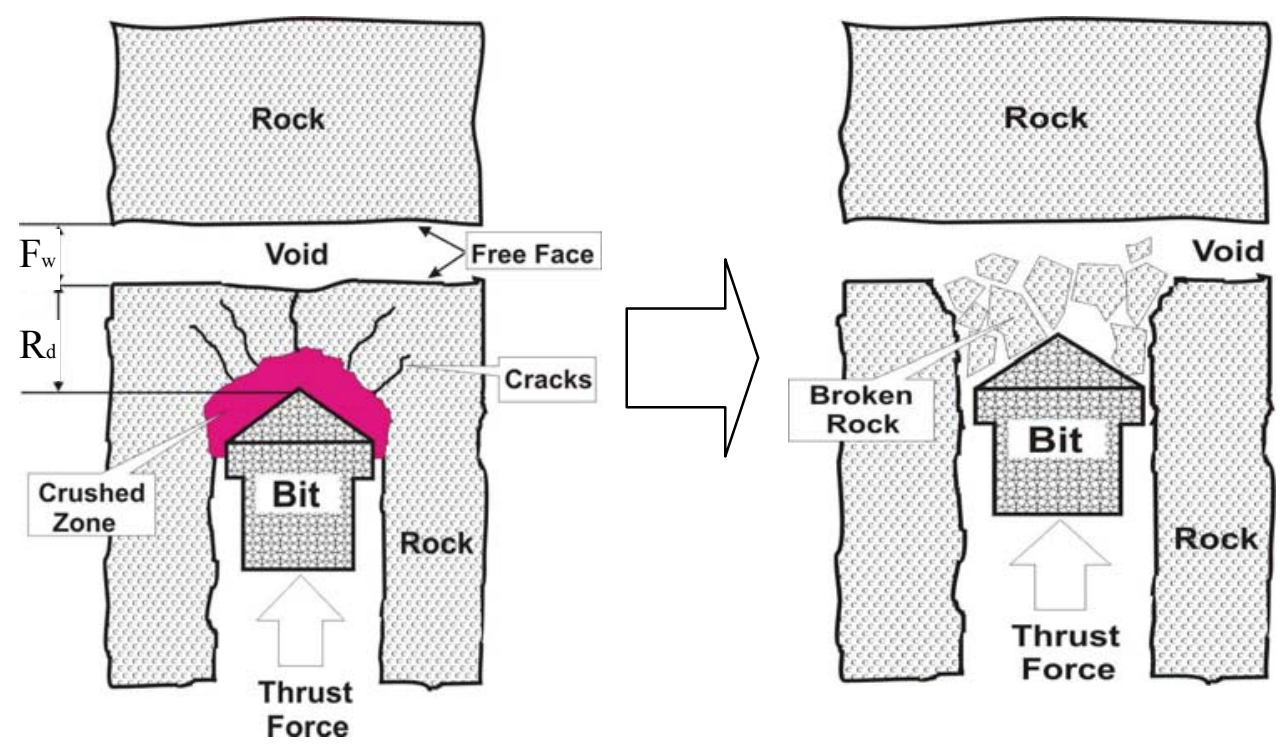

Figure 5.14 Rock fragmentation when drilling close to a rock void

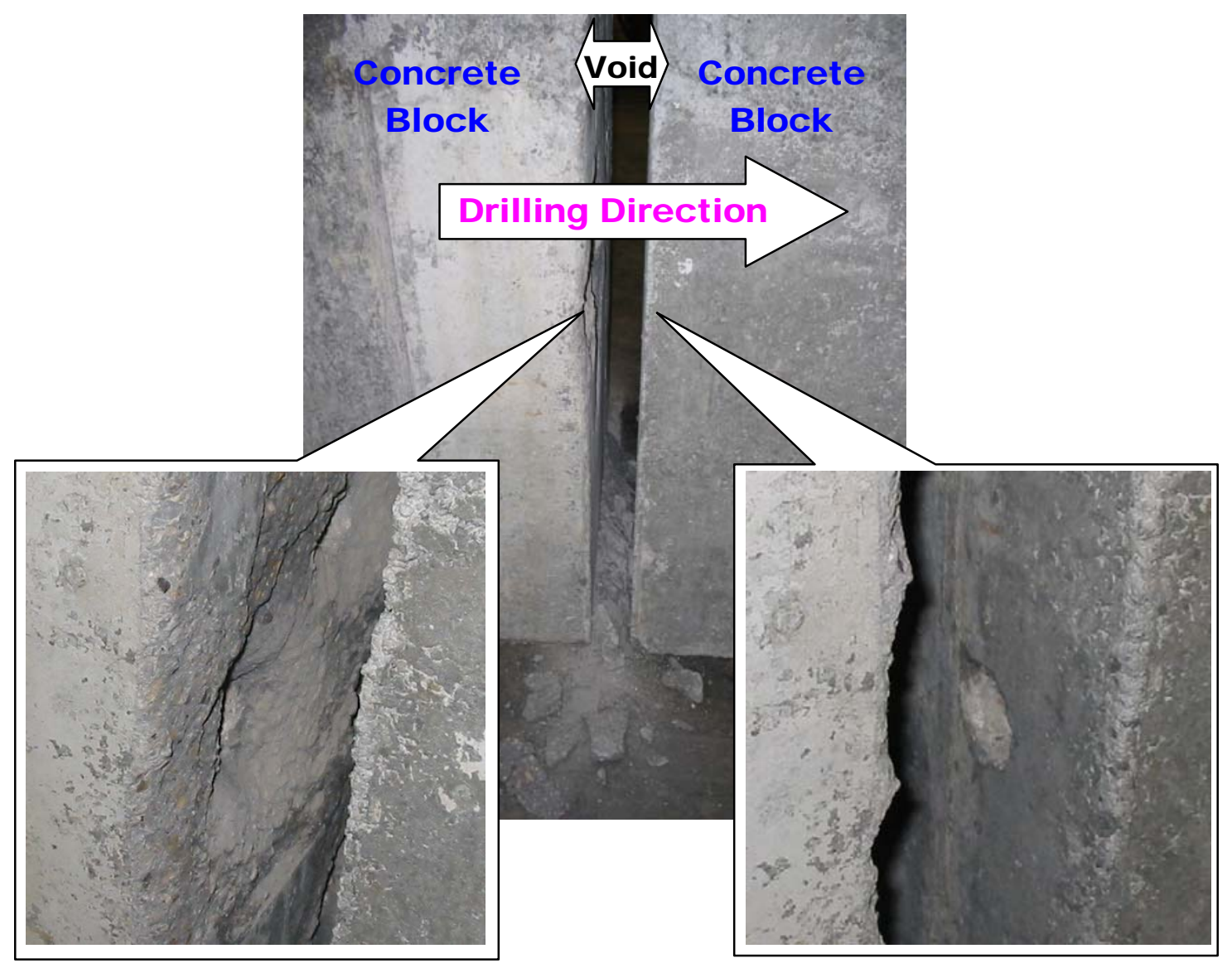

Figure 5.15 Conditions of both surfaces of void. 
Although the feed pressure tends to reduce the level when drilling in the air if a void is encountered, it is observed that the valley bottoms of all feed pressure curves do not reach the level when drilling in the air. Besides measurement errors, the size of the void plays the most important role in this phenomenon. The smaller the size of void is, the less possible it is for the feed pressure to fall into the range when drilling in the air (Table 5.1). Two possible reasons can be considered for this trend: One is that rough surfaces of concrete layers may make the actual void size smaller than the designed one, or close to zero. Another reason is the small void does not provide enough space for broken rock chips to move in although cracks have already propagated to the void. Consequently, these rock chips are still confined to their original locations before they are further broken into smaller chips and removed by the dust collector, as normal drilling does. In this situation, the magnitude of feed pressure is much higher than that for drilling in the air. Therefore, the valley bottoms of feed pressure will be very shallow or there is no valley at all.

Table 5.1 Statistics of valley bottoms of feed pressure

\begin{tabular}{|c|c|c|}
\hline $\begin{array}{c}\text { Void } \\
\text { Size } \\
\text { (in) }\end{array}$ & $\begin{array}{c}\text { Number of } \\
\text { holes with } \\
\text { available data }\end{array}$ & $\begin{array}{c}\text { Number of valley bottoms } \\
\text { of feed pressure reaching } \\
\text { the level in the air }\end{array}$ \\
\hline $1 / 16$ & 22 & 5 \\
\hline $1 / 8$ & 22 & 19 \\
\hline $3 / 8$ & 19 & 15 \\
\hline
\end{tabular}

\subsubsection{Criterion for Void/Fracture Prediction}

The major criterion for void prediction is developed based on the fact that feed pressure should drop to the level of drilling in the air when a void in rock is encountered. Here, as mentioned in 5.2.1, different drilling settings and machine conditions have no obvious impact on both the magnitude and trend of feed pressure-bit position curve when drilling in the air. So, this criterion is not affected by any drilling setting and machine conditions. Hence, once the compensation run is conducted and the feed pressure-bit position curve is recorded, this major criterion can be determined. Moreover, to help increase the correct prediction ratio, especially at a narrow void/fracture, a set of 
supplementary prediction rules are also developed considering not only the magnitude of feed pressure but also the shape of feed pressure valley.

In this research, it is assumed that the change in roof geological properties results in the change in roof drilling parameters. But this does not mean that all the changes in the collected drilling parameters are caused by roof geology change. Those that are not caused by roof geology changes are referred to as noise data. These noise data may come from the drilling system, the data collecting system or some other unknown sources. In order to distinguish the noise data from the normal ones, it needs in-depth information about the roof bolter, the data collecting devices and roof bolter operating procedures. Therefore, a set of rules for cleaning up the noise data are also created based on current knowledge about the machine used in this research in order to eliminate the effect of noise data on prediction as much as possible.

\subsubsection{Prediction Results}

The measured drilling parameters of 22 holes drilled in the fractured block were used to check the criteria for void/fracture prediction. The results are shown in Table 5.2. The prediction results show that a very high prediction percentage have been achieved for the $1 / 8$ in and $3 / 8$ in voids. But the $1 / 16$ in void does not cause an obvious change in not only feed pressure but also all other drilling parameters. It seems that there is a limitation of the void size that can be detected by the current system.

Table 5.2 Results of void prediction

\begin{tabular}{|c|c|c|c|c|c|}
\hline $\begin{array}{c}\text { Void } \\
\begin{array}{c}\text { Size } \\
\text { (in) }\end{array}\end{array}$ & $\begin{array}{c}\text { Actual } \\
\text { Location } \\
\text { (in) }\end{array}$ & $\begin{array}{c}\text { Number of } \\
\text { holes with } \\
\text { available } \\
\text { data }\end{array}$ & $\begin{array}{c}\text { Number } \\
\text { of correct } \\
\text { prediction }\end{array}$ & $\begin{array}{c}\text { Percentage } \\
\text { of correct } \\
\text { prediction } \\
\text { (\%) }\end{array}$ & $\begin{array}{c}\text { Average } \\
\text { predicted } \\
\text { location } \\
\text { (in) }\end{array}$ \\
\hline $1 / 16$ & 15 & 22 & 13 & 59.09 & 14.036 \\
\hline $1 / 8$ & 30 & 22 & 22 & 100 & 29.222 \\
\hline $3 / 8$ & 45 & 19 & 18 & 94.73 & 44.494 \\
\hline
\end{tabular}

\subsubsection{Prediction of the Size of Void/Fracture}

Mining engineers are interested in not only the void location but also its size when designing roof supports. The relationship between feed pressure valley and the size of 
void is modeled and shown in Figure 5.16. The width of the left side of the feed pressure valley can be expressed as follows (See Figures 5.14 and 5.16):

$$
W_{l}=\left\{\begin{array}{cc}
R_{d}+F_{w} & \text { If there is no plateau at the valley bottom } \\
R_{d} & \text { If there is a plateau at the valley bottom }
\end{array}\right.
$$

where $\mathrm{W}_{1}=$ the left side width of feed pressure valley, $\mathrm{R}_{\mathrm{d}}=$ the distance between drilling bit tip and the free face of the void when feed pressure begins to drop and $F_{w}=$ the size of the void.

On the other hand, the right side of a feed pressure valley has nothing to do with the size of void. It mainly depends on the strength of the rock since it represents the distance for a drill bit to start drilling in rock until the feed pressure reaches the level in rock.

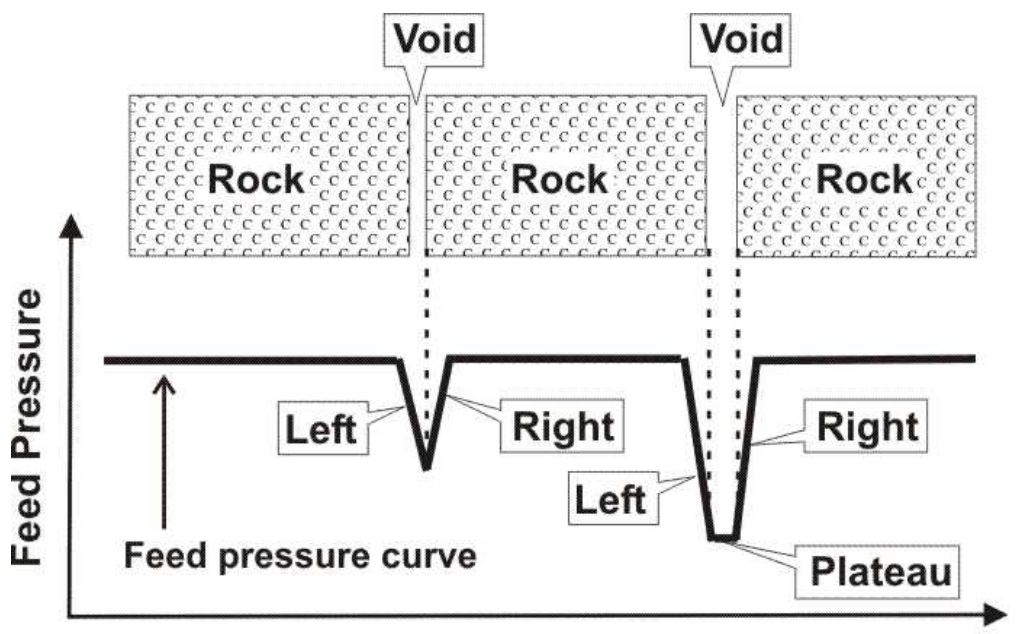

Bit Position

Figure 5.16 Relationship between the width of feed pressure valley and the size of void

Table 5.3 Size of the plateau at the bottom of feed pressure valley

\begin{tabular}{|c|c|c|c|c|c|}
\hline $\begin{array}{c}\text { Void } \\
\text { Size } \\
\text { (in) }\end{array}$ & $\begin{array}{c}\text { Number of } \\
\text { holes with } \\
\text { available data }\end{array}$ & $\begin{array}{c}\text { Number of } \\
\text { holes with } \\
\text { plateau }\end{array}$ & $\begin{array}{c}\text { Max. } \\
\text { plateau } \\
\text { width (in) }\end{array}$ & $\begin{array}{c}\text { Min. } \\
\text { plateau } \\
\text { width (in) }\end{array}$ & $\begin{array}{c}\text { Ave. } \\
\text { plateau } \\
\text { width (in) }\end{array}$ \\
\hline $1 / 16$ & 22 & 5 & 0.3186 & 0.1593 & 0.223 \\
\hline $1 / 8$ & 22 & 13 & 0.5940 & 0.1485 & 0.3084 \\
\hline $3 / 8$ & 19 & 13 & 0.9958 & 0.1593 & 0.4043 \\
\hline
\end{tabular}


Hence, it can be said that the width of the plateau at the bottom of feed pressure valley is much closer to the size of the void. Table 5.3 shows that the relationship between the width of plateau of feed pressure valley and the size of the void. It can be said that the larger the size of the void/fracture, the more possible it is to form a plateau at the bottom of feed pressure valley and the more precisely it can be predicted. On the other hand, it seems to be difficult to determine the size of a small void by using the current data collecting system. This is because of the resolution of the bit position sensors. The conversion factors for mast and carriage position sensors are 0.1485 in and 0.1593 in, respectively. This means that the minimum measurable plateau width is $0.1485 \mathrm{in}$, this value is much larger than 1/16-in. Considering measurement errors, the error rate of any prediction will be considerable. Moreover, the plateau may begin to form even before a drill bit reaches the void if broken rock chips are removed fast. This can explain why the maximum plateau size is almost three times of a 3/8-in void.

\subsubsection{Field Verification of Void/Fracture Prediction}

Figures 5.17 and 5.18 show the feed pressure curve and the result of borehole scoping in Mine F (Roof Rock: Shale) and Mine G (Roof Rock: Sandstone), respectively. It can be seen that the locations of feed pressure valleys and their bottom that drops down to the level of compensation run match well with the actual void locations. On the other hand, Figure 5.18 shows the trend that the feed pressure did not drop to the level of that obtained in compensation run under the high penetration rate drill settings. It seems that the higher the penetration rate, the higher the bottom level of the feed pressure valley is. This may be because the drill bit has already reached the other free face of the void before the feed pressure drops down to the level of drilling in the air. This result also indicates that supplemental prediction criteria considered the shape of feed pressure valley is needed to enhance the prediction accuracy. From the results of a series of underground tests, the criterion for void/fracture prediction developed from the results of laboratory tests was verified. 

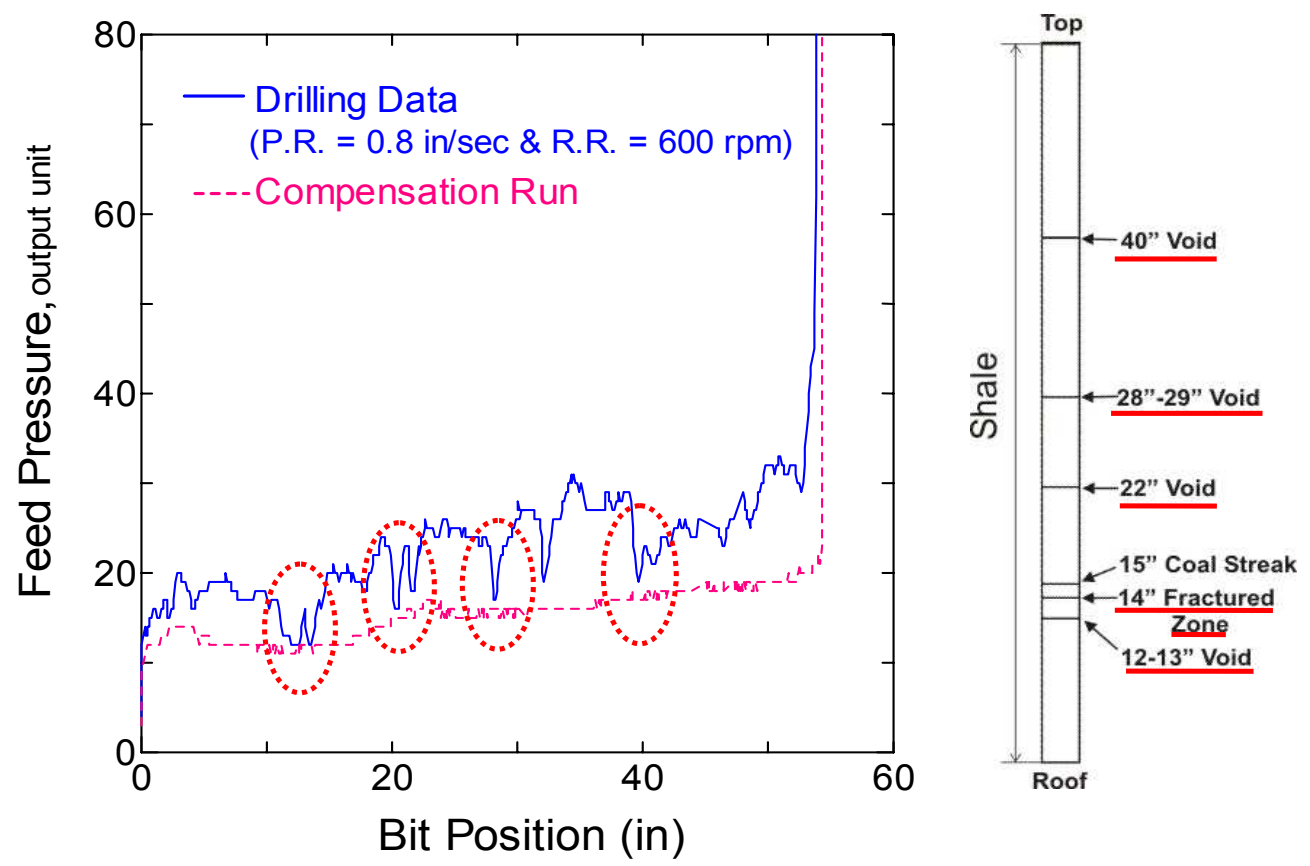

Figure 5.17 Feed pressure curves and results of borehole scoping (Mine F, roof rock: shale)
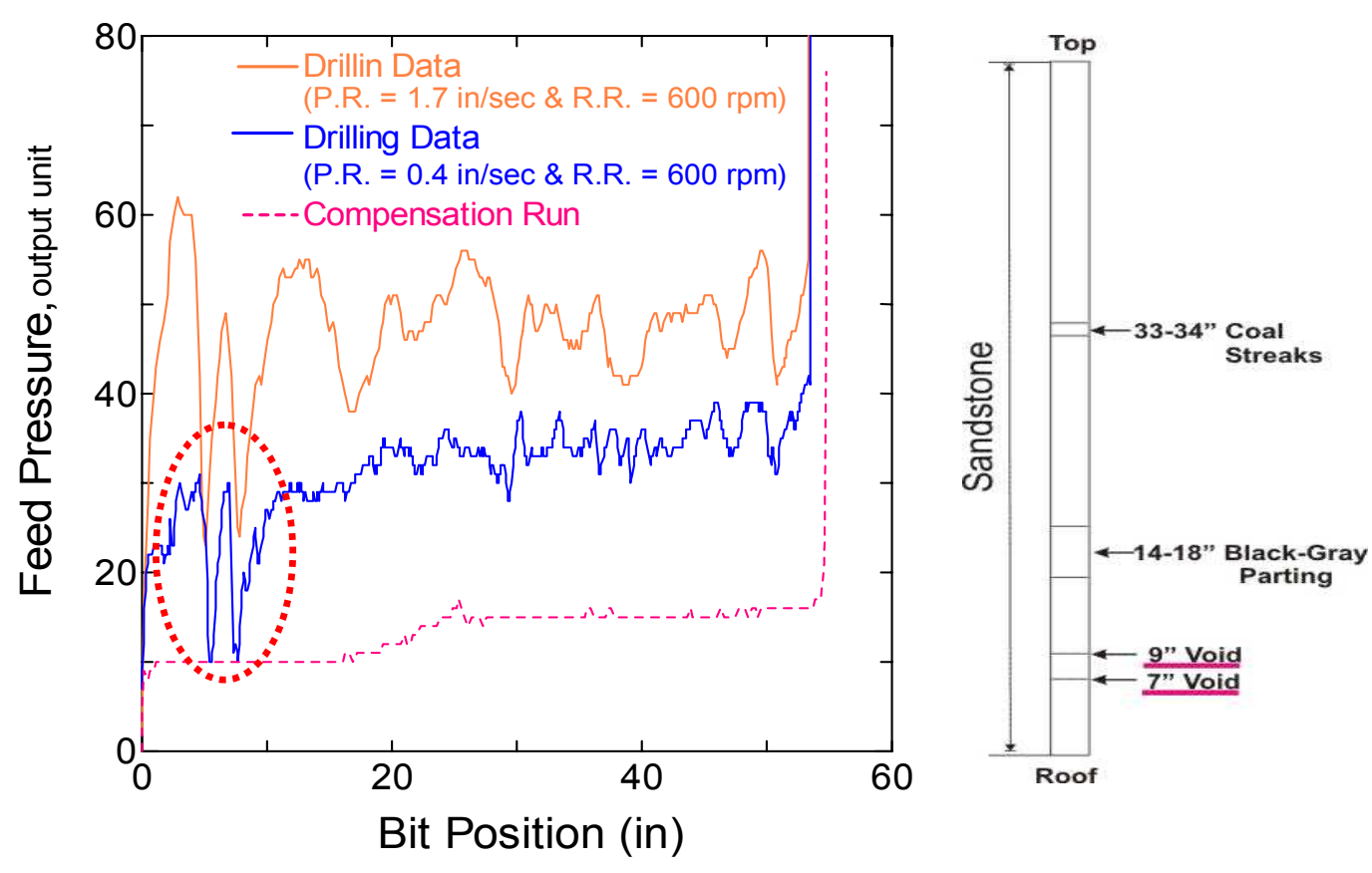

Figure 5.18 Feed pressure curves and results of borehole scoping (Mine G, roof rock: sandstone) 

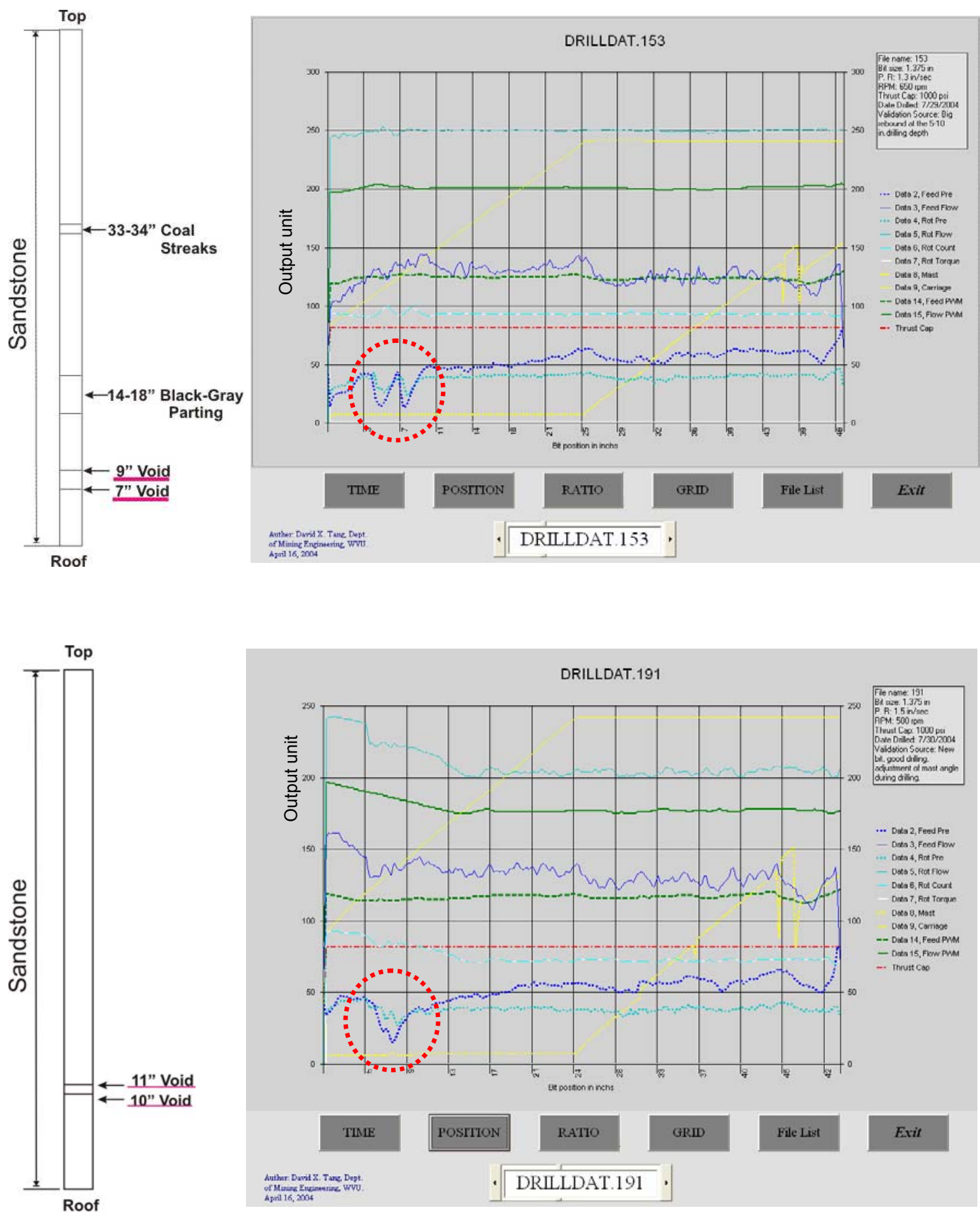

Figure 5.19 Drilling parameters and results of borehole scoping (Mine G)

On the other hand, the following two trends can also be observed. One is that the actual void location from the roof line was a little deeper than the location of feed 
pressure valley on the drilling data. This is because of the following drill procedures. At first, the drill bit was penetrated a few inches deep manually. And then, drilling controlled by DCU \& data recording start. As for roof mapping, to record the relative locations of voids is considered to be one of the important factor in the near future. Another is that even two voids exist in close proximity, only a single wide valley is observed in the feed pressure curve (Figure 5.19). This trend seems to depend on conditions such as intervals between voids, rock strength, existence of filling materials, etc.

\subsection{Estimation of the Rock Strength}

The same way as the development of void/fracture prediction methodology, the most appropriate drilling parameters for estimating rock strength and developing the criterion for rock classification can be found based on the behaviors of drilling parameters when drilling in different strengths of rocks. Note the parameters which can be measured are the parameters in hydraulic system. So, the drilling parameters contain not only for drilling rock but also for running machine itself. Therefore, the results that are indicated in Section 5.2 have to be taken into account for discussion.

\subsubsection{Impact of Different Rock Strengths on Feed Pressure and Rotation Pressure}

Figures 5.20 (a) and (b) show the impact of rock strength on feed pressure and rotation pressure at P.R. $=1.5 \mathrm{in} / \mathrm{sec}$ and R.R. $=600 \mathrm{rpm}$. It can be seen obviously that the harder the roof rock is the larger the feed pressure is. On the other hand, even though the impact of rock strength on the magnitude of rotation pressure can be recognized, it is not so clear cut. Figure 5.21 shows the same trend within one hole. Besides, the trend and magnitude of feed pressure for compensation run is not affected by any drilling settings and machine conditions (i.e. oil temperature) as mentioned in Section 5.2, the difference between their magnitudes of feed pressure represents the difference between their consumption for drilling rocks of different strength directly. On the other hand, the different rotation rate and machine conditions (i.e. oil temperature) have obvious impact on the rotation pressure for running the machine itself, and the magnitude of their impacts are too large to be ignored comparing with that for drilling rock. It also seems to be difficult to develop a model or formula to eliminate these effects on rotation pressure. 
Hence, it would be true that it is difficult to evaluate the roof rock strength by using the magnitude of rotation pressure under the current system.

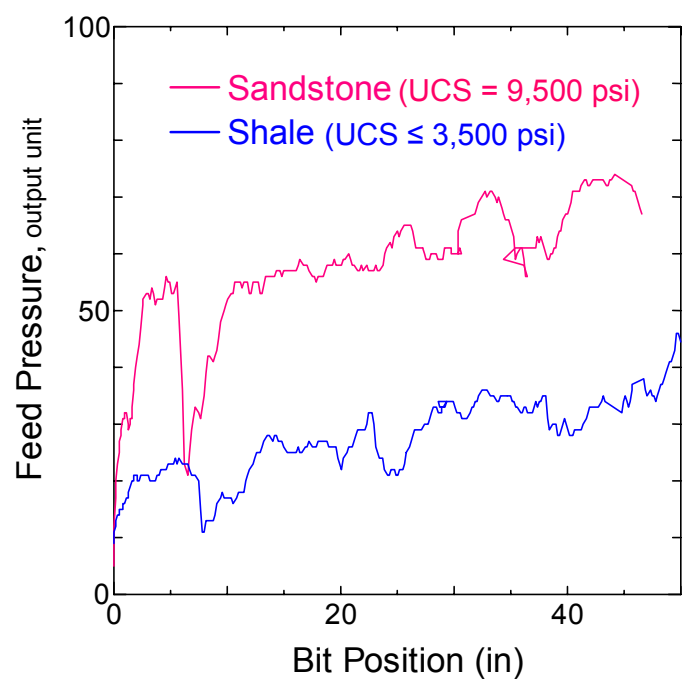

(a) Feed Pressure

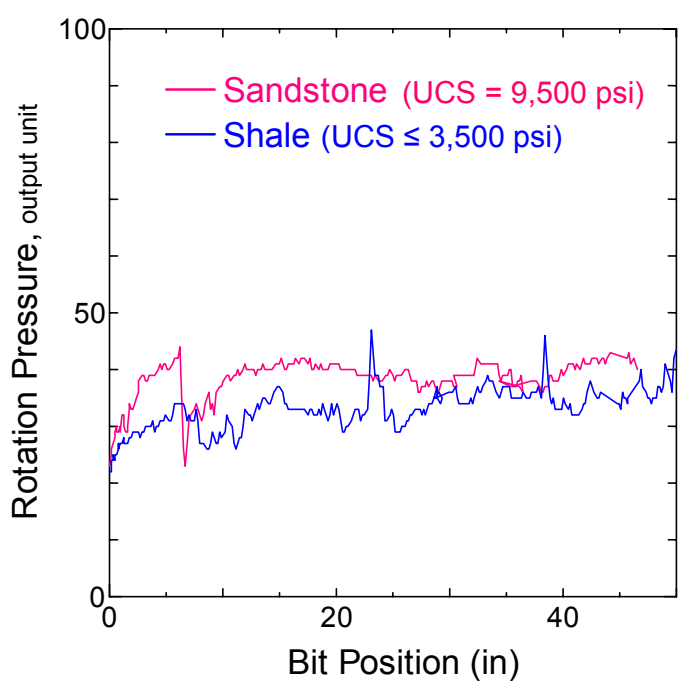

(b) Rotation Pressure

Figure 5.20 Effect of rock strength on feed pressure/rotation pressure (P.R. $=1.5 \mathrm{in} / \mathrm{sec} \&$ R.R. $=600 \mathrm{rpm}$, Oil Temp $=126$ (sandstone) and 128 (shale) output units)

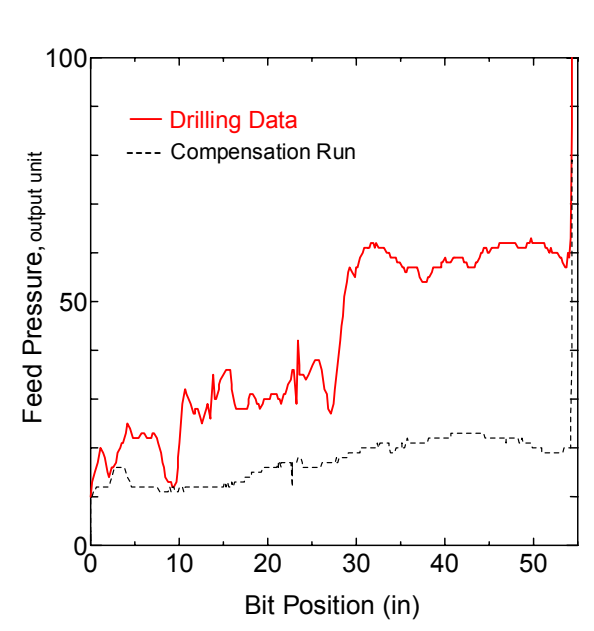

(a) Feed pressure

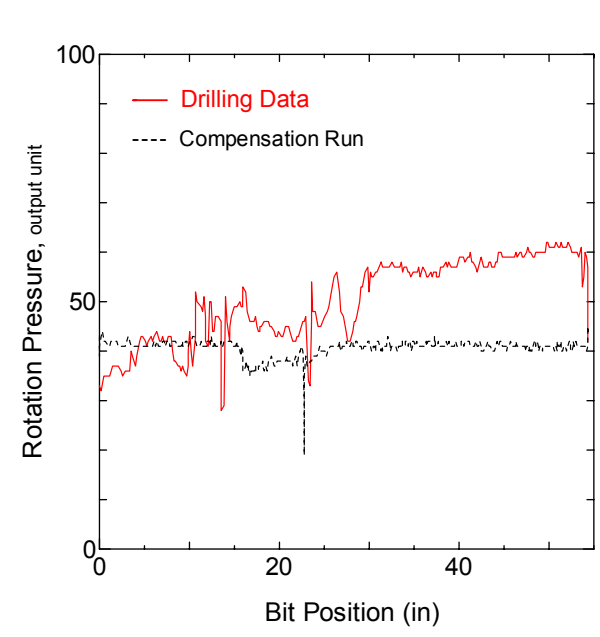

(b) Rotation pressure

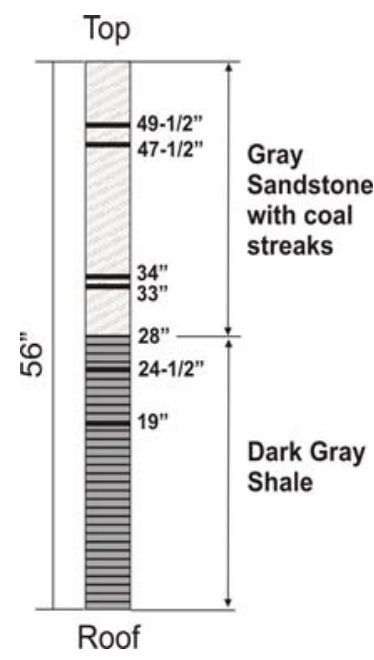

Figure 5.21 Effect of rock strength on feed pressure/rotation pressure and core log (Mine D, P.R. = $1.5 \mathrm{in} / \mathrm{sec} \&$ R.R. $=$ free)

Therefore, it can be concluded that feed pressure is the most sensitive and reliable parameter for estimating the strength of roof rock under the current system. 


\subsubsection{Effect of Penetration Rate on Feed Pressure}

Figures 5.22 (a) and (b) show the relationship between feed pressure and bit position under different penetration rates in shale and sandstone, respectively. It can be seen obviously that different penetration rates have obvious impact on the magnitude of feed pressure. The higher the penetration rate is the larger the magnitude of feed pressure is. Moreover, it was also observed that the harder the roof rock is the larger the impact of penetration rate on the trend of feed pressure-penetration rate curve is. So, it seems that not only the magnitude of feed pressure but also the slope of feed pressure-penetration rate curve is related to rock strength. Accordingly, the relationship between feed pressure and penetration rate under different rock strengths is analyzed and discussed. Here, following assumptions were made for simplification.

a. Drilling data for locations within 5 in from the beginning and the end of drilling were not used.

b. Each roof rock was assumed to be almost homogeneous.

c. The actual rotation rate can be kept constant as a target speed.

d. The effect of bit wear on feed pressure is not considered.

e. Compressibility of the hydraulic oil is not considered.

First of all, the penetration rate was calculated by using the heavily filtered method. This method was applied for the penetration rate calculation in DCU. By using this value, the DCU attempts to control the feed flow as to make the penetration rate close to the target speed. This method is shown as follows:

- Heavily Filtered Method

$$
\begin{aligned}
& \text { PenetrationRate }\left(t_{n}\right)=\frac{1}{\Delta t}\left\{\operatorname{BitPosition}\left(t_{n}\right)-\operatorname{BitPosition}\left(t_{n}-\Delta t\right)\right\} \\
& \text { PenetrationRate }_{(H F)}\left(t_{n}\right)=\frac{1}{4}\left\{\text { PenetrationRate }\left(t_{n}\right) \times 2+\text { PenetraitonRate }\left(t_{n-1}\right)\right. \\
& \left.+ \text { PenetrationRate }\left(t_{n-2}\right)\right\}
\end{aligned}
$$

Where, $\mathrm{t}=$ time, $\Delta \mathrm{t}=100 \mathrm{msec}$ 


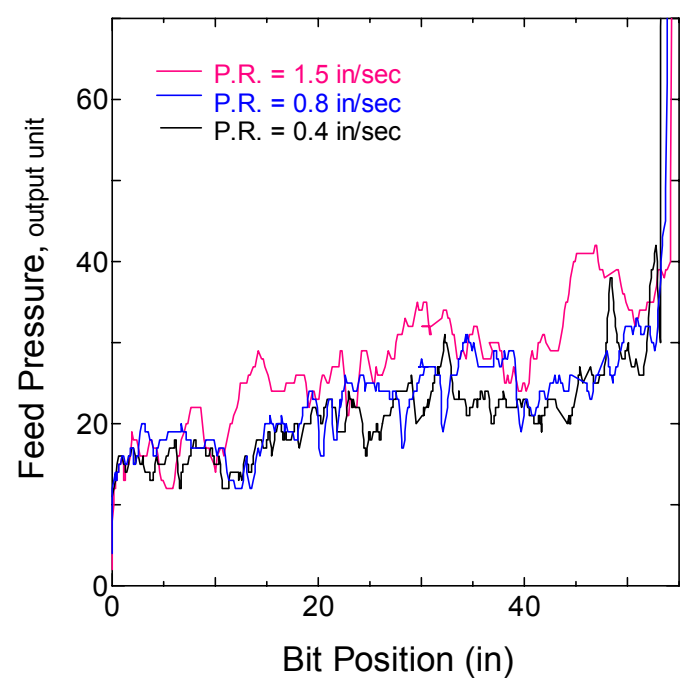

(a) Shale (UCS $\leq 3,400 \mathrm{psi})$

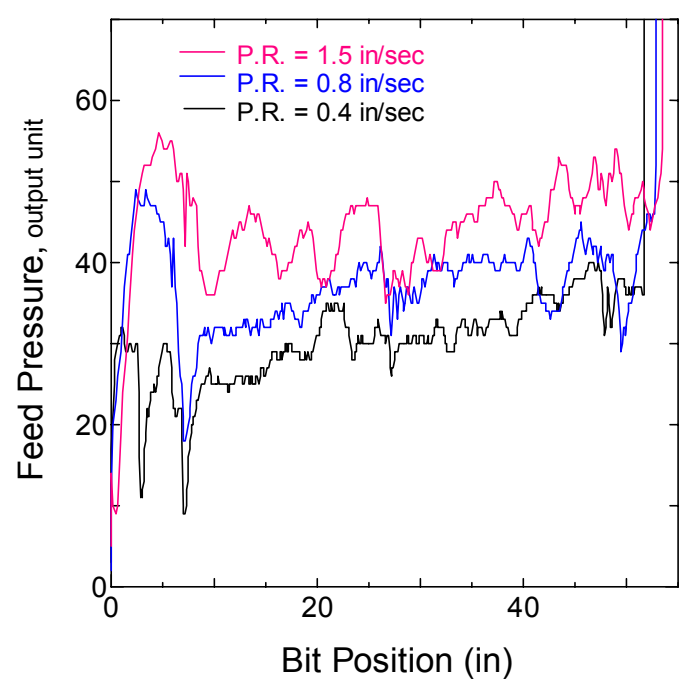

(b) Sandstone (UCS $=9,500 \mathrm{psi}$ )

Figure 5.22 Effect of penetration rate on feed pressure (R.R. $=600 \mathrm{rpm})$

Figure 5.23 shows the relationship between feed pressure and penetration rate for different strength of rocks. The blue and red data points indicate drilling data for Mine $\mathrm{F}$ where the roof is shale and those for Mine $\mathrm{G}$ where the roof is sandstone, respectively. Two trends can be recognized from this figure. One is that the harder the roof rock is, the higher the magnitude of feed pressure is while the other is that the slope of feed pressure-penetration rate curve is different for different strengths of rocks. The harder the roof rock is the steeper the slope of feed pressure-penetration rate curve is. However,

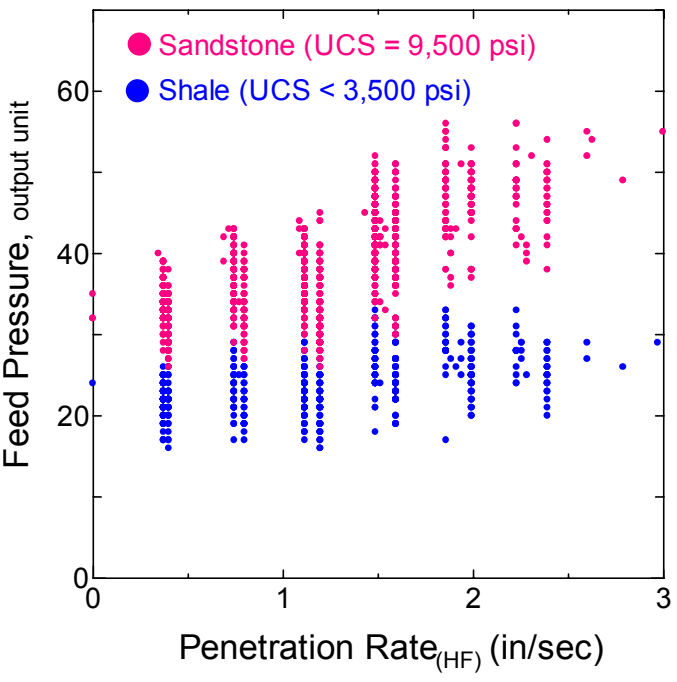

Figure 5.23 Relationship between penetration rates calculated by heavily filtered method and feed pressure (R.R. $=600 \mathrm{rpm})$ as distributions of both sets of data points vary widely, their trends and the boundary between them can not be clearly defined even if there is a difference in UCS of more than 5,000 psi. In regard to this point, the causes for their wide variation may be attributed to: 
(1) The roof rock was not homogeneous.

(2) The penetration rate calculated by the heavily filtered method varies widely because of the resolution of two position sensors as shown in Figure 5.23 (Carriage Position Sensor 0.1485 in/count and Mast Position Sensor 0.1593 in/count).

(3) The actual rotation rate is not kept constant as a target speed.

(4) The magnitude of feed pressure consumed for running the roof bolter itself changes with each stage of mast feed as mentioned in Section 5.2.

(5) The effect of bit wear

The items we can discuss and evaluate quantitatively are items (2), (3) and (4). Here, items (2) and (4) are discussed as to eliminate both effects on the relationship between penetration rate and feed pressure for different strengths of rocks.

\subsubsection{Effect of the Method of Penetration Rate Calculation}

As shown in Figure 5.24, the penetration rate calculated by the heavily filtered method (penetration rate $(\mathrm{HF})$ ) has wide variances. However, this trend and value are not for the actual penetration rate. This trend is because of the insufficient resolution of two position sensors. In other words, this variance is caused by the calculation method for penetration rate. In order to make the difference clear in the trend and distribution of both sets of data points of different strengths of rocks, one of the important key is how to calculate the penetration rate more exactly under the current system. So, first of all, in order to eliminate the effect of resolution on penetration rate calculation, the penetration rate was calculated from 10 backward points average (= penetration rate $\left.{ }_{(10 \mathrm{BP})}\right)$ instead of heavily filleted method. This formula is defined as follows:

$$
\begin{gathered}
\text { Penetration rate }_{(10 B P)}\left(t_{n}\right)=\frac{1}{10}\left\{\text { penetration rate }\left(t_{n}\right)+\text { penetraiton rate }\left(t_{n-1}\right)+\right. \\
\left.\cdots \cdots+\text { penetration rate }\left(t_{n-9}\right)\right\}
\end{gathered}
$$

Here, it is noted that speed is controlled by the amount of flow in the hydraulic system. Besides, pressure is the resistance of oil flow in the hydraulic circuit and pressure is also induced by the flow. So, it can be assumed that there are strong correlation between the actual penetration rate/feed pressure and feed flow. Consequently, the 
penetration rate can be calculated from the feed flow (= penetration rate $(\mathrm{FF})$ by using the following equation:

$$
\text { Penetration rate }_{(F F)}\left(t_{n}\right)=\left\{\text { feed flow }\left(t_{n}\right)-819\right\} \times 0.00305 \times 231 / 7.07 / 60
$$

where the conversion factor from output unit to actual hydraulic volume is $0.00305,1$ gallon $=231 \mathrm{in}^{3}$, cross section of the cylinder $=7.07 \mathrm{in}^{2}$ and both cylinder diameters in each stage are assumed to be the same.

Figure 5.25 shows the correlation between two penetration rates calculated by different methods and feed pressure. It can be seen that the penetration rate curve calculated from the 10 backward points average still has a variance. This calculation method has weak points in that the current calculation speed still reflects the information of previous locations and, in the same way, a current change is not reflected on the current speed directly. So, there is still difference in the trend between the penetration rate curve calculated from the 10 backward points average and feed pressure curve. On the other hand, as comparing with the penetration rate curve calculated from the feed flow, it can be seen that the change of feed pressure curve coincides with that of the penetration rate curve calculated from the feed flow except the data around the void location. Both the feed pressure curve and the penetration rate curve change simultaneously. Moreover, it can be distinguished by using the penetration rate curve calculated from the feed flow whether the feed pressure change is caused by geology change or DCU. For example, the change of feed pressure around No.1 in Figure 5.25 was caused by geology because the feed flow did not change. In fact, there were voids around this location. On the other hand, the change of feed pressure around No.2 was caused by DCU because the feed flow itself changed and both curves changed simultaneously. 


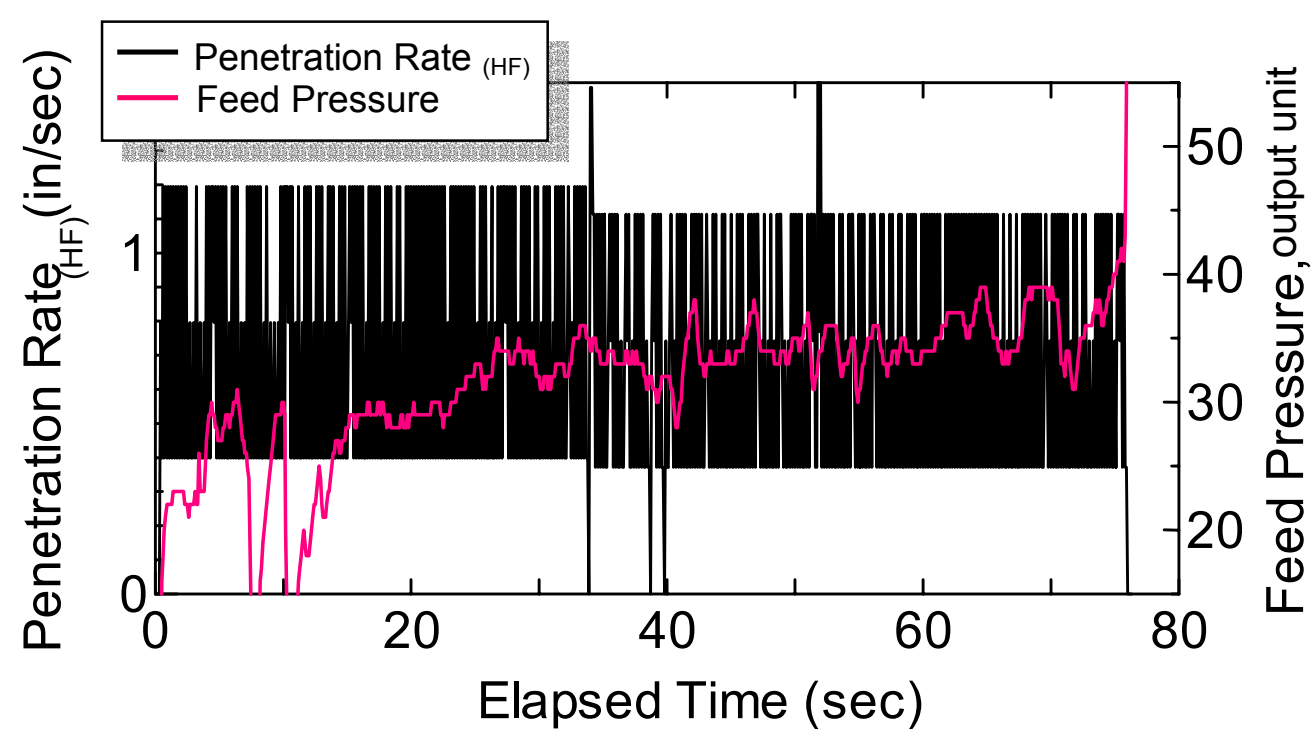

Figure 5.24 Correlation between penetration rate ${ }_{(\mathrm{HF})}$ and feed pressure (P.R. $=0.4 \mathrm{in} / \mathrm{sec}$ and R.R. $=600 \mathrm{rpm})$

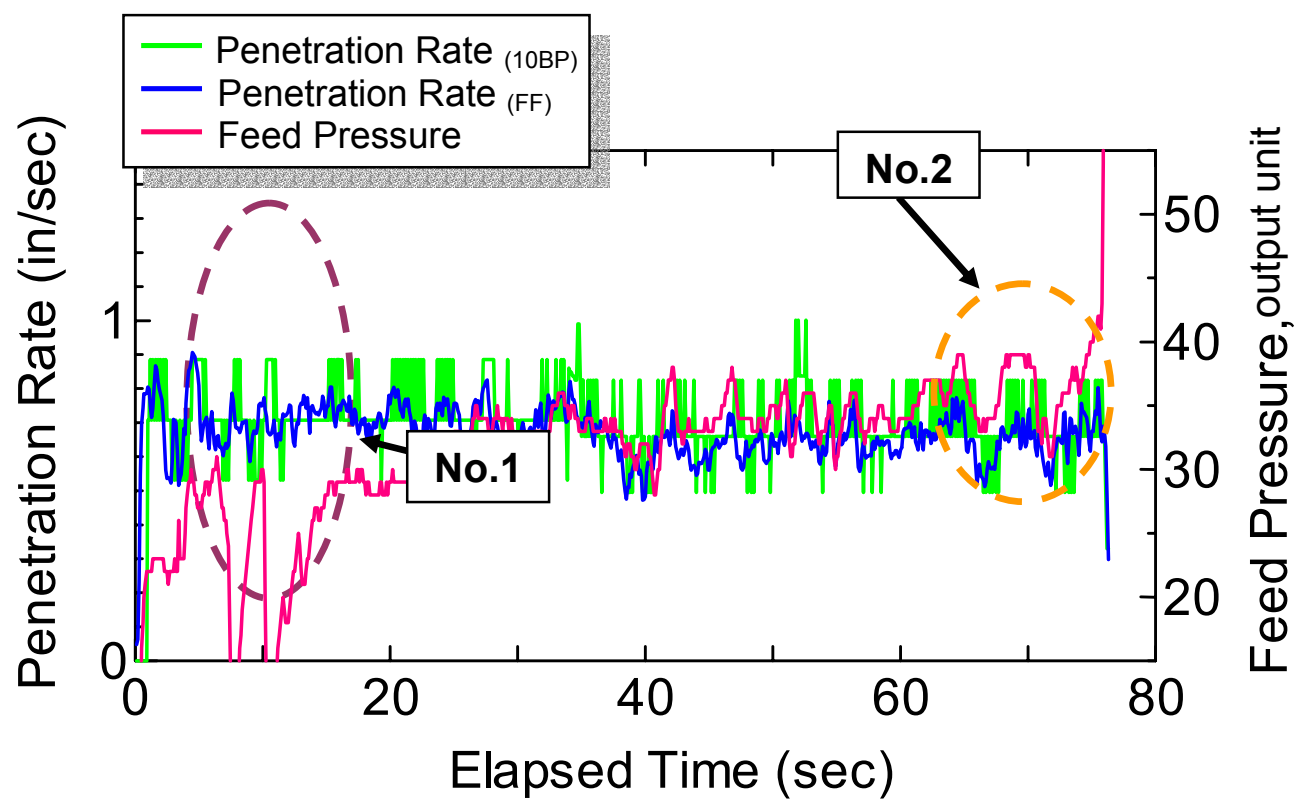

Figure 5.25 Correlation between penetration rate ${ }_{(10 \mathrm{BP})} /$ penetration rate $_{(\mathrm{FF})}$ and feed pressure (P.R. $=0.4 \mathrm{in} / \mathrm{sec}$ and R.R. $=600 \mathrm{rpm})$

Figures 5.26 (a)-(c) show the relationships between feed pressure and penetration rates calculated by different methods. From these figures, by applying feed flow for the penetration rate calculation, the distribution of data points becomes narrower and its trend is made clearer. Therefore it can be concluded that feed flow should be applied for the 
penetration rate calculation instead of the data of two position sensors in the current system. Hereafter, "penetration rate" means that calculated from the feed flow.

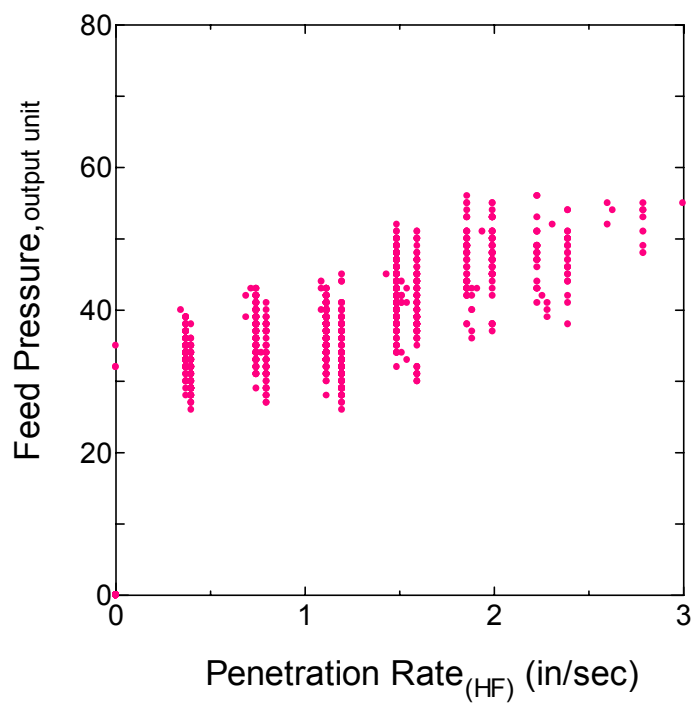

(a) Heavily Filtered Method

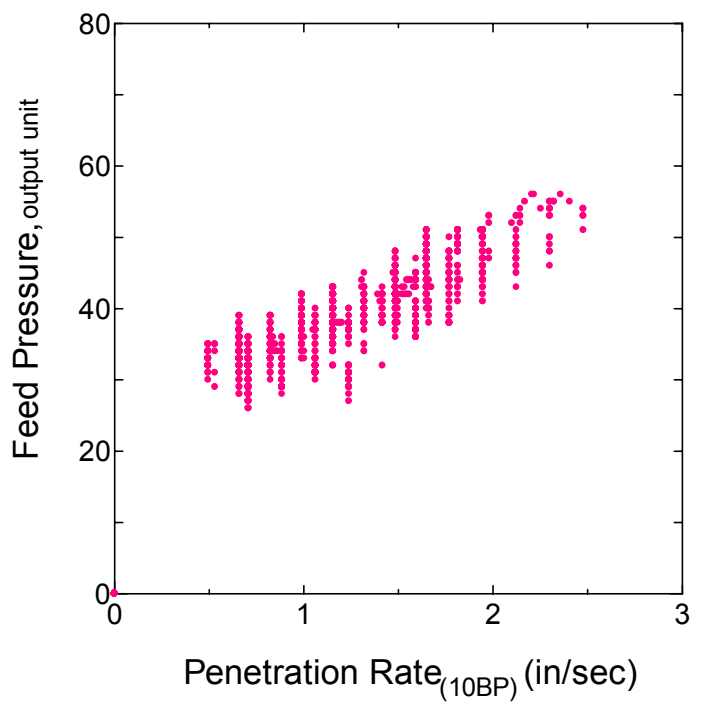

(b) 10 Backward Points Average

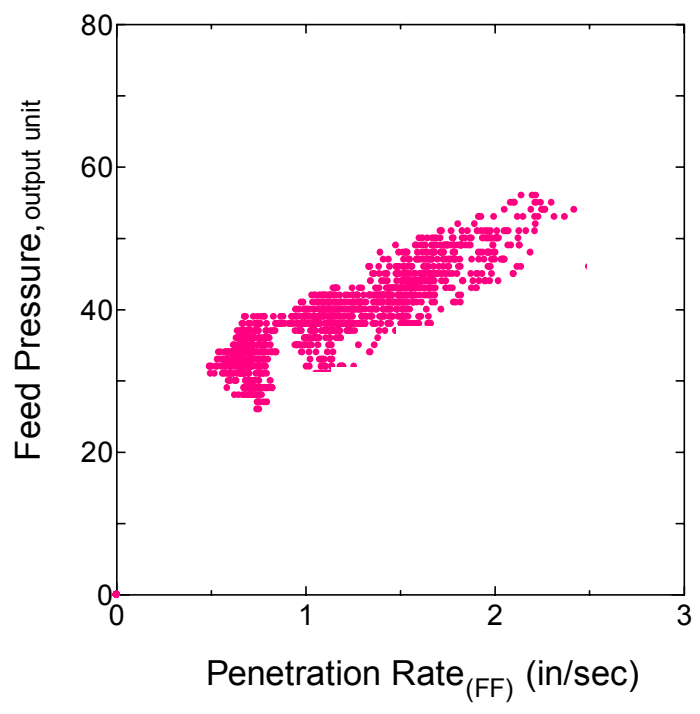

(c) Feed Flow

Figure 5.26 Relationship between penetration rates and feed pressure (R.R. $=600 \mathrm{rpm}$, sandstone)

5.4.2.2 Elimination of the Effect of Oil Temperature and Cylinder Diameter on Penetration Rate Calculation 
As mentioned above, from the hydraulic system and resolutions of two position sensors points of view, the penetration rate calculated from the feed flow is more reliable for calculating the penetration rate more exactly and determining the impact of rock strength on the relationship between feed pressure and the penetration rate under the current system. Therefore it can also be guessed that different oil temperatures have impact on the penetration rate calculation. In order to check if it is necessary to modify equation (5.9), the actual bit position calculated from the data of two position sensors and the bit position calculated from the feed flow are compared. The bit position was calculated from the feed flow by using formulas (5.10) and (5.11):

$$
\begin{gathered}
\text { Penetration rate }(t)(\text { in } / \mathrm{sec})=\{\text { feed flow }(t)-819\} \times 0.00305 \times 231 / 7.07 / 60 \\
\text { Bit position }(t)(\text { in })=\text { bit position }(t-1)+\text { penetration rate }(t-1) \times 0.1
\end{gathered}
$$

where, $\mathrm{t}=$ time $(\mathrm{sec})$

Figures 5.27 (a) and (b) show the relationships between elapsed time from the start of drilling and the bit position calculated from the data of two position sensors (= actual bit position)/feed flow under the different oil temperatures. It can be seen that the lower the oil temperature is, the larger the difference between actual bit position and the bit position calculated from feed flow is. This difference may be caused by oil leakage in the hydraulic circuits. The lower the oil temperature is, the more the oil leaks. This result indicates that oil leakage has to be taken into account when penetration rate is calculated from feed flow, especially when oil temperature is low. Figure 5.28 (a) and (b) show the relationship between the actual bit position/the bit position calculated from feed flow and elapsed time under different penetration rate settings. It seems that the difference between both bit positions is dependent on the time and there is no effect of penetration rate setting on the magnitude of their difference. In other words, the amount of oil leakage seems to be dependent on the oil temperature only and penetration rate setting has no obvious impact on it. From Figure 5.29, even though there is no obvious change 


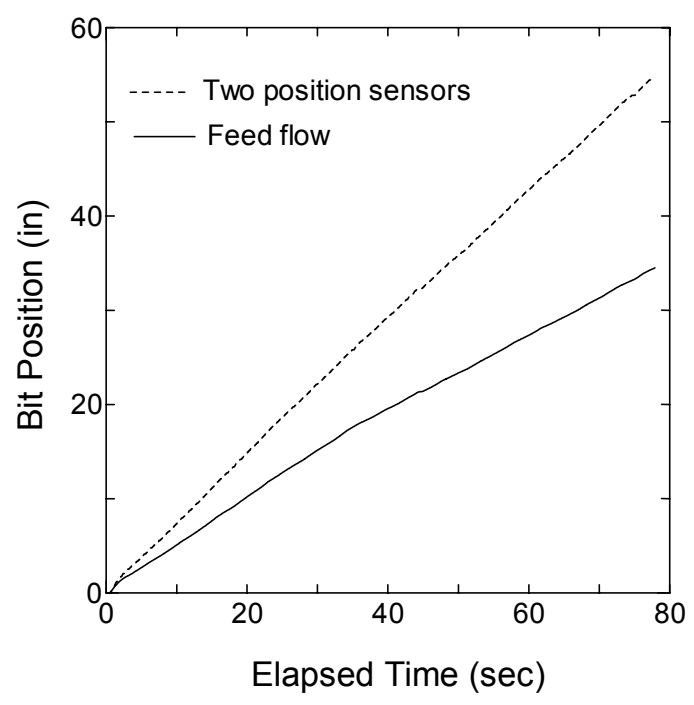

(a) Oil Temp $=108$ output units

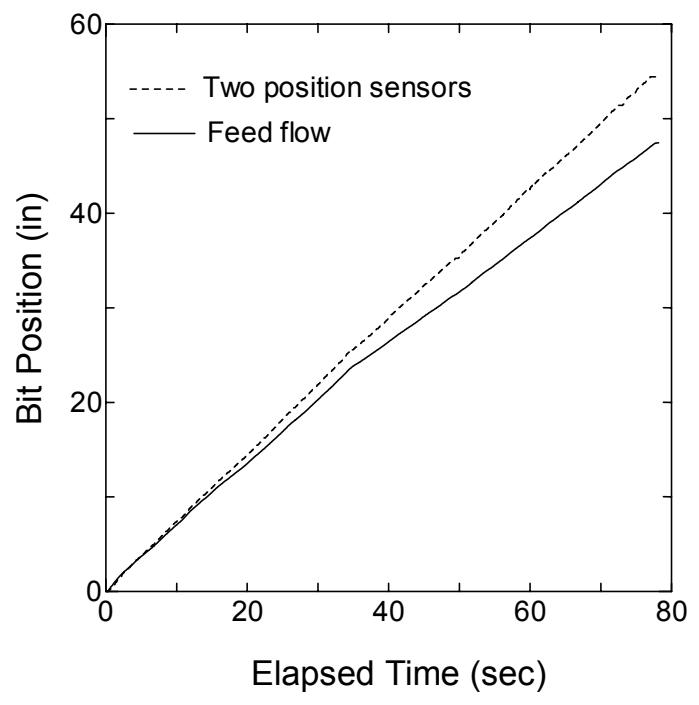

(b) Oil Temp $=130$ output units

Figure 5.27 Relationship between the bit positions calculated from the data of two position sensors/feed flow and elapsed time (P.R. $=0.4 \mathrm{in} / \mathrm{sec}$, R.R. $=500 \mathrm{rpm}$ )

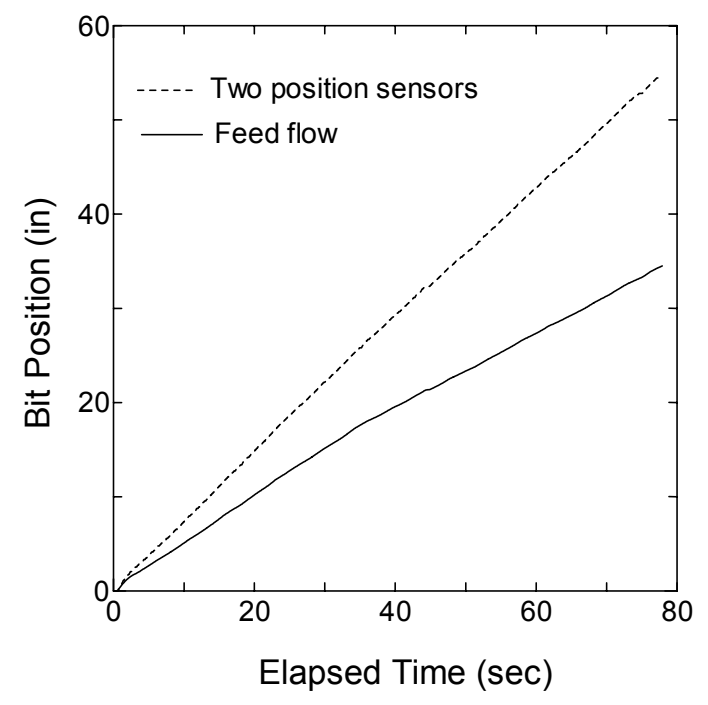

(a) P.R. $=0.4$ in $/ \mathrm{sec}$, Oil Temp $=108$ output units

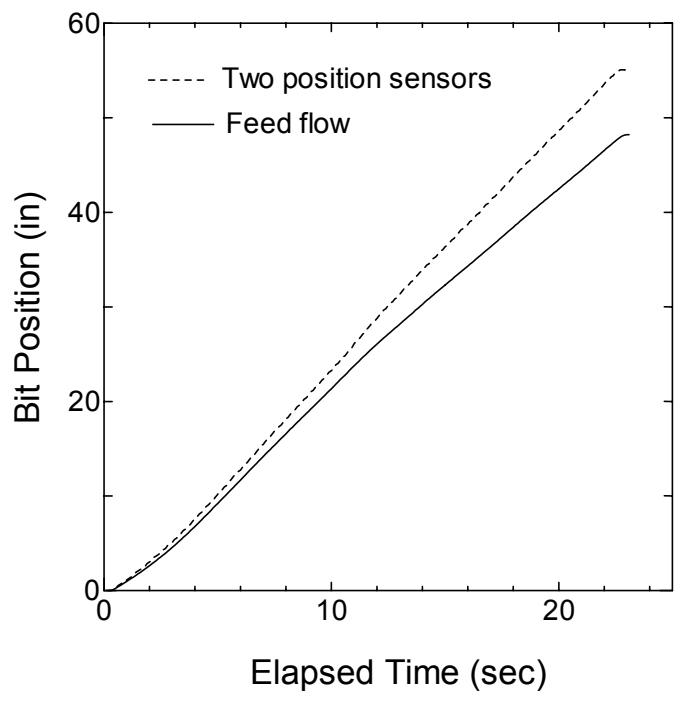

(b) P.R. $=2.1 \mathrm{in} / \mathrm{sec}$, Oil Temp $=111$ output units

Figure 5.28 Relationship between the bit positions calculated from the data of two position sensors/feed flow and elapsed time under different penetration rates 
about the trend of bit position curve, the magnitude of feed flow was different with different stages. It seems that the conversion factor (cylinder diameter, etc) and/or the amount of oil leakage at the mast stage are also different with those at the carriage stage. From these points of view, an empirical formula for the oil leakage factor was developed in order to calculate more exactly the penetration rate from the feed flow.

The following assumptions are used for trials:

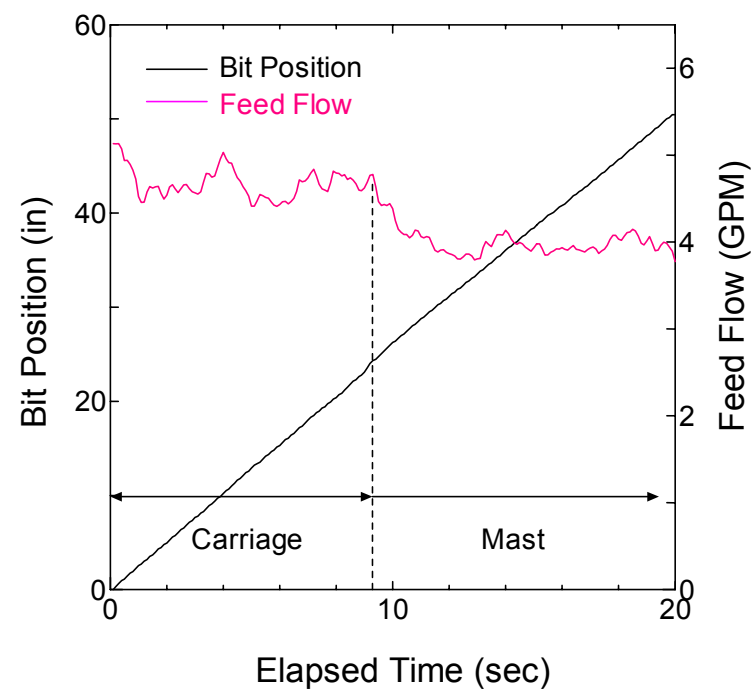

Figure 5.29 Relationship between bit position/penetration $\operatorname{rate}_{(\mathrm{FF})}$ and elapsed time for compensation run (P.R. $=2.1$ in $/ \mathrm{sec}$, R.R. $=500 \mathrm{rpm}$ )

a. Drilling settings have no obvious impact on oil leakage

b. The amount of oil leakage is only dependent on the oil temperature.

c. The magnitudes of oil leakage in both mast and carriage extension stage are the same.

d. Effective cylinder diameters of mast and carriage stage are different.

e. Compressibility of the oil fluid is not taken into account.

Based on the above assumptions, the calculation formula for penetration rate at each stage can be defined as follows:

$$
\begin{aligned}
& \text { Penetration rate }_{C}(t)=\left\{\text { feed flow }(t)+F_{L}\left(T_{o}\right)-819\right\} \times 0.00305 \times 231 / 7.07 / 60 \\
& \text { Penetration rate }_{M}(t)=a \times\left\{\text { feed flow }(t)+F_{L}\left(T_{o}\right)-819\right\} \times 0.00305 \times 231 / 7.07 / 60
\end{aligned}
$$

where, penetration $\operatorname{rate}_{C}(t)=$ penetration rate at the carriage stage, penetration rate $_{M}(t)=$ penetration rate at the mast stage, $\mathrm{F}_{\mathrm{L}}(\mathrm{T})=$ oil leakage factor, $\mathrm{T}_{\mathrm{o}}=$ oil temperature and $\mathrm{a}=$ constant. 
At first, an attempt was made to determine the oil leakage factor from compensation run data at the carriage stage. Figure 5.30 shows the relationship between oil leakage factor and oil temperature at the carriage stage. It can be seen that the effect of oil leakage decreases with increase of oil temperature and the effect of oil leakage can be ignored in penetration rate calculation when oil temperature is larger than about 135 in output units. Then, an approximation curve, which represents the

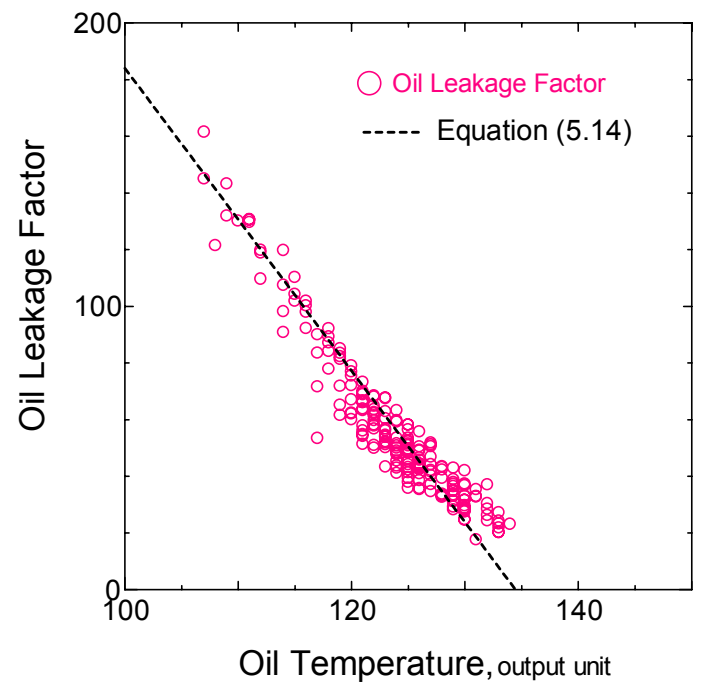

Figure 5.30 Relationship between oil leakage factor and oil temperature at the carriage stage

relationship between oil leakage factor and oil temperature, is determined. The oil leakage factor is defined as follows:

$$
F_{L}(t)=-5.34 \times T(t)+718
$$

Next, ' $a$ ' in equation (5.13) is determined by the data at the mast stage as follows:

$$
\mathrm{a}=1.09
$$

From the equations (5.12)-(5.15), the empirical formula for penetration rate calculation by the feed flow can be defined as follows:

i) Oil Temperature $<135$ output units

\section{Carriage stage}

$$
\text { Penetration rate }_{C}(t)=\{\text { feed flow }(t)-5.34 \times T(t)-101\} \times 1.66 \times 10^{-3}
$$

\section{Mast stage}

$$
\text { Penetration rate }{ }_{M}(t)=\{\text { feed flow }(t)-5.34 \times T(t)-101\} \times 1.83 \times 10^{-3}
$$

ii) Oil Temperature $\geq 135$ output units 


\section{$\underline{\text { Carriage stage }}$}

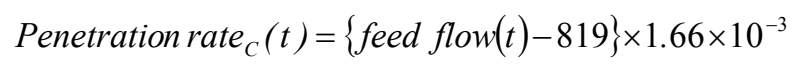

Mast stage

$$
\text { Penetration rate }_{M}(t)=\{\text { feed flow }(t)-819\} \times 1.83 \times 10^{-3}
$$

Figure 5.31 (a) and (b) show the examples of the revised results. The bit positions calculated by equations (5.16)-(5.19) fit the actual bit position well. Moreover, in order to verify these equations, they are applied to another set of independent data. From Figure 5.32, it can also be seen that bit position calculated by equation (5.16)-(5.18) fit the actual bit position well. From these results, it was concluded that the effect of oil temperature can be eliminated by applying equations (5.16)-(5.18).

Figure 5.33 shows the relationship between feed pressure and penetration rates calculated by the feed flow (= penetration rate $(\mathrm{FF})$ ). It can be seen that the revision of an oil leakage effect makes the data at low temperature shift to the right and the variance of distribution of the data points narrower.

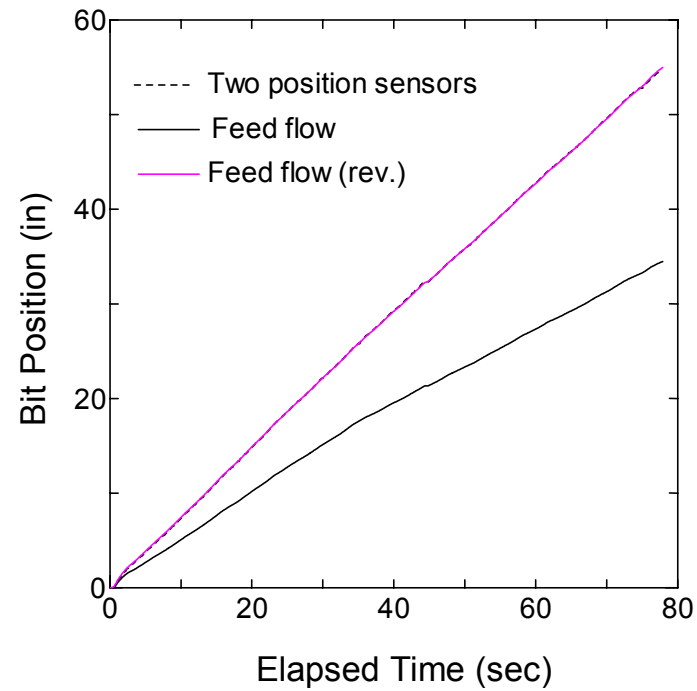

(a) P.R. $=0.4 \mathrm{in} / \mathrm{sec}$, Oil Temp $=$ 108 output units

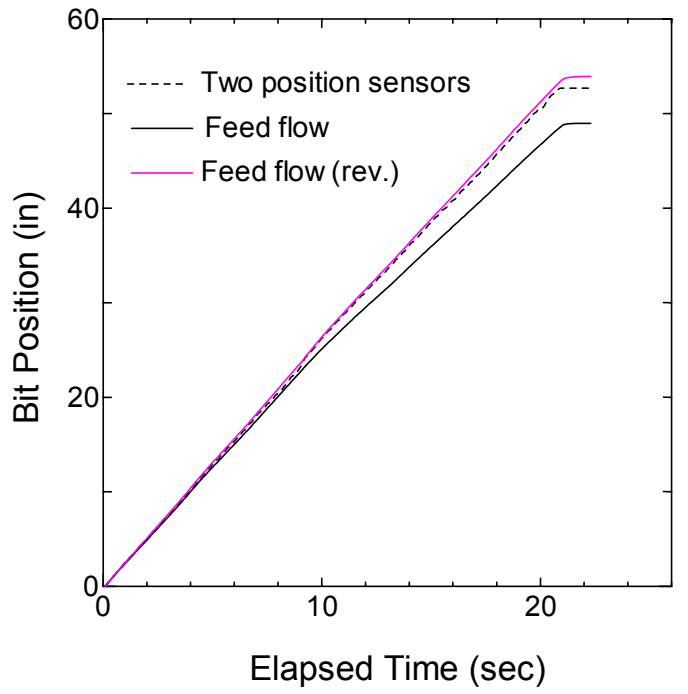

(b) P.R. $=2.1 \mathrm{in} / \mathrm{sec}$, Oil Temp = 123 output unit

Figure 5.31 Relationship between the bit positions calculated by different methods and elapsed time 


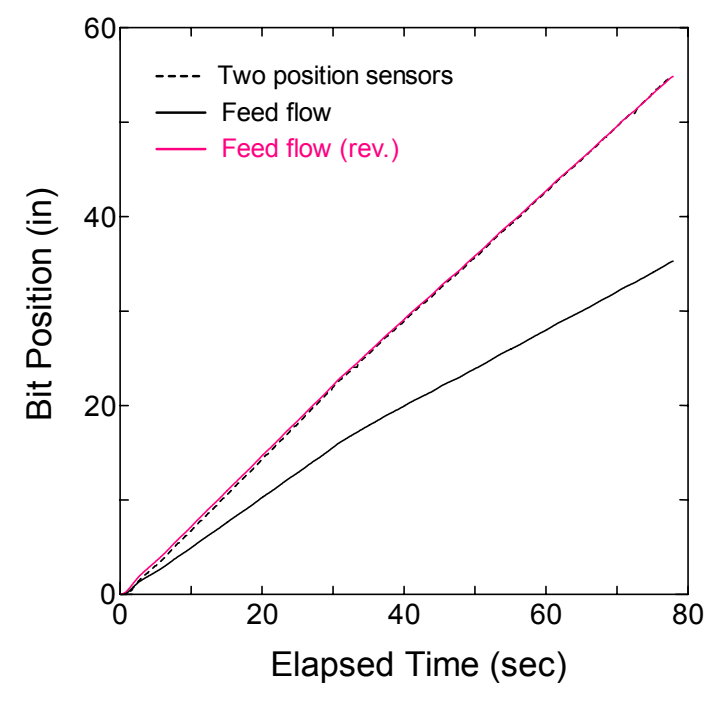

(a) P.R. $=0.4 \mathrm{in} / \mathrm{sec}$, R.R. $=600 \mathrm{rpm}$, Oil Temp $=109$ output units

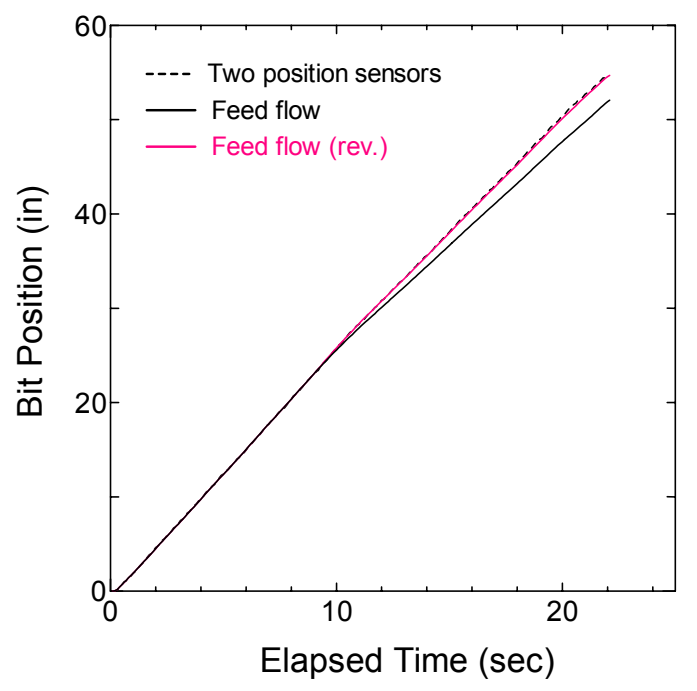

(b) P.R. $=2.1 \mathrm{in} / \mathrm{sec}$, R.R. $=650 \mathrm{rpm}$, Oil Temp $=136$ output unit

Figure 5.32 Relationship between the bit positions calculated by different methods and elapsed time

Figure 5.34 (a) and (b) show the relationships between penetration rate calculated by different methods and feed pressure for different strengths of rocks. From these figures, by applying feed flow for penetration rate calculation, distribution of both sets of data points become narrower, and differences in both trends and the boundary of both distributions were made clear.

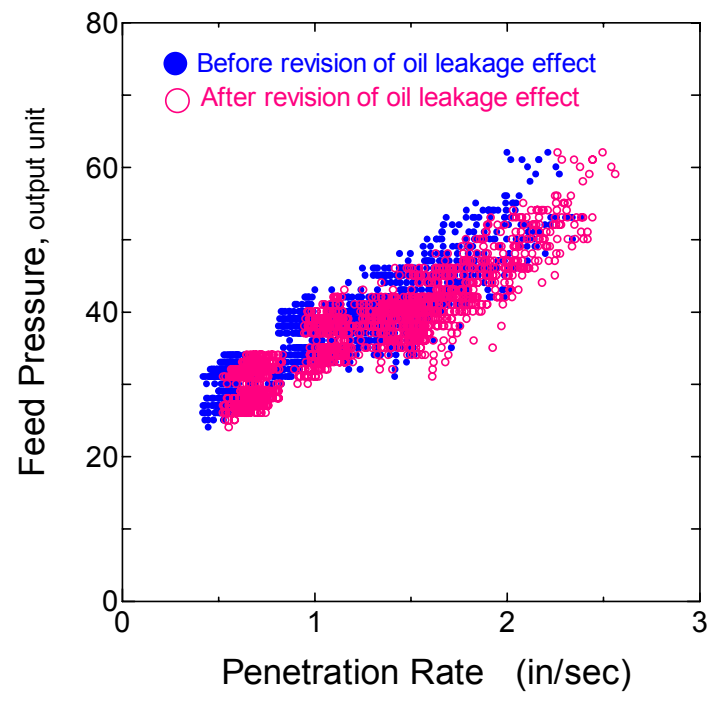

Figure 5.33 Relationship between feed pressure and penetration rate (R.R. $=600 \mathrm{rpm}$ ) 


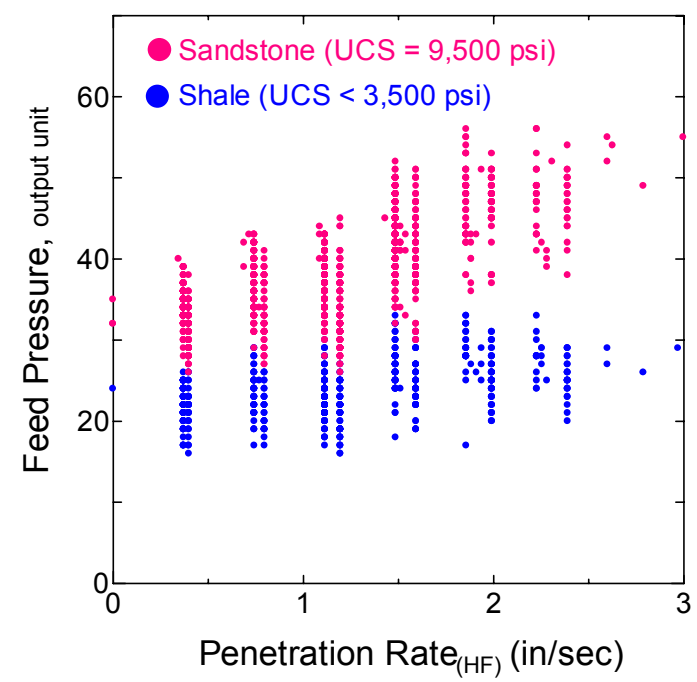

(a) Heavily Filtered Method

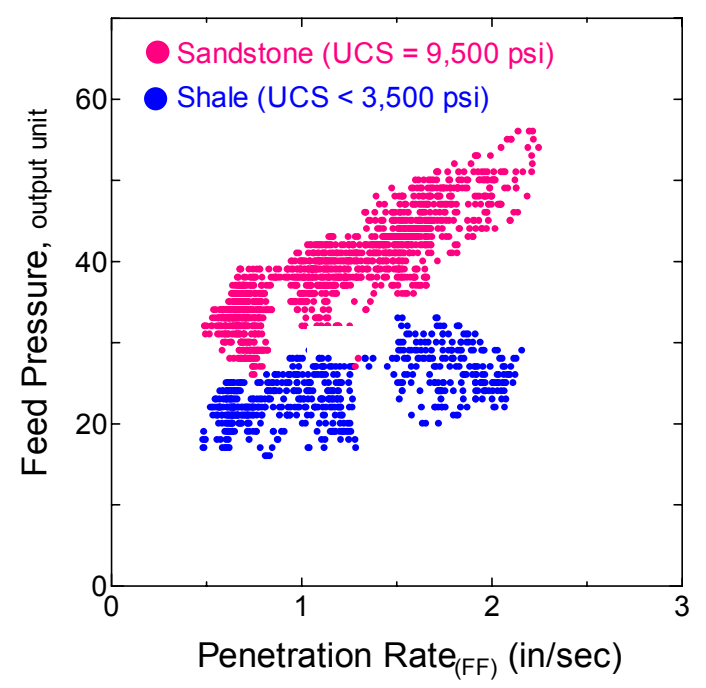

(b) Feed Flow

Figure 5.34 Relationship between penetration rates calculated by different methods and feed pressure (R.R. $=600 \mathrm{rpm})$

\subsubsection{Elimination of Machine Effect on Feed Pressure}

As mentioned in Section 5.2, the magnitude of feed pressure consumed for running the roof bolter itself changes and there is about 7-10 output units difference between feed pressure in carriage stage and that in mast stage. This means the magnitude of feed pressure is different with different stage even drilling in a homogeneous rock. This effect causes 7-10 output units variance in the distribution of feed pressure-penetration rate data points. On the other hand, drilling settings and machine conditions have no obvious impact on both the magnitude and trend of feed pressure-net bit position curve. Besides, the error for all the compensation run data is less than 3 output units. Therefore, the effect of machine itself on drilling

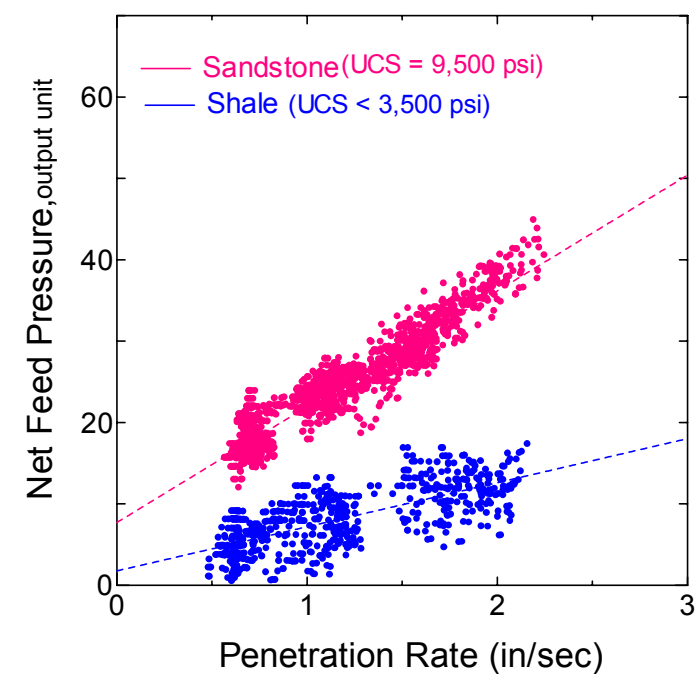

Figure 5.35 Effect of rock strength on the relationship between net feed pressure and penetration rate (R.R. $=600 \mathrm{rpm})$ 
parameters when drilling rock can be eliminated once the compensation run is conducted under any settings. For this reason, the net feed pressure is used for rock strength estimation instead of feed pressure. The net feed pressure was calculated by equation (5.3) and (5.4).

Figure 5.35 show the relationship between feed pressure/net feed pressure and penetration rate. Comparing with Figure 5.34 the variances of distribution of both sets of data points become even narrower, and both the trends of feed pressure-penetration rate curve and the boundary between both distributions of data points can be seen more clearly. Therefore, this presentation procedure enhances the correlation between net feed pressure and the rock strength / the slope of net feed pressure-penetration rate curve.

\subsubsection{Effect of Rotation Rate on Feed Pressure}

Figures 5.36 (a) and (b) show the relationship between feed pressure and bit position under different rotation rates when drilling in shale and sandstone, respectively. It can be seen that different rotation rates also have obvious impact on the magnitude of feed pressure. The magnitude of feed pressure decreases with the increasing rotation rate. This result indicates that the rotation rate helps feed pressure in the drilling process. Besides, it was also observed that the harder the roof rock is the larger the impact of rotation rate on

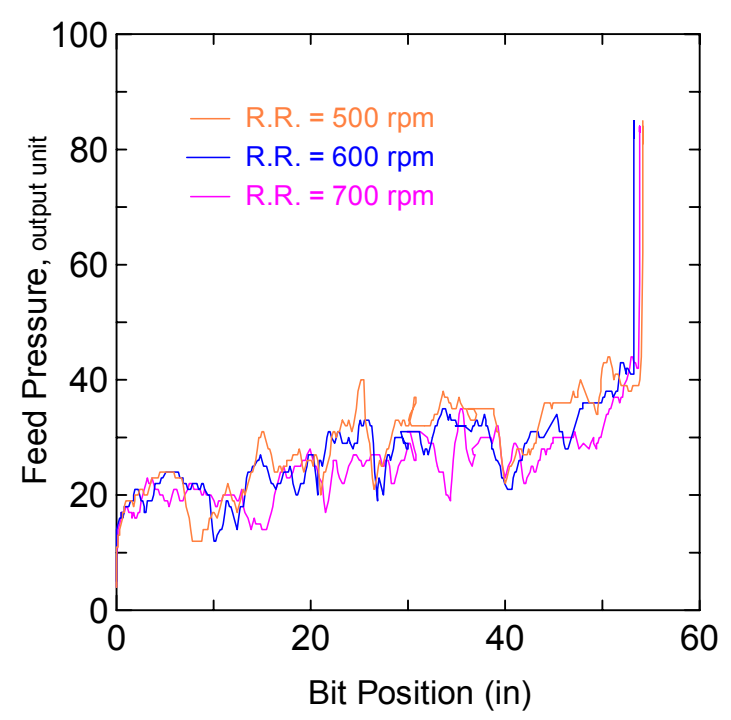

(a) Shale (UCS $<3,500$ psi)

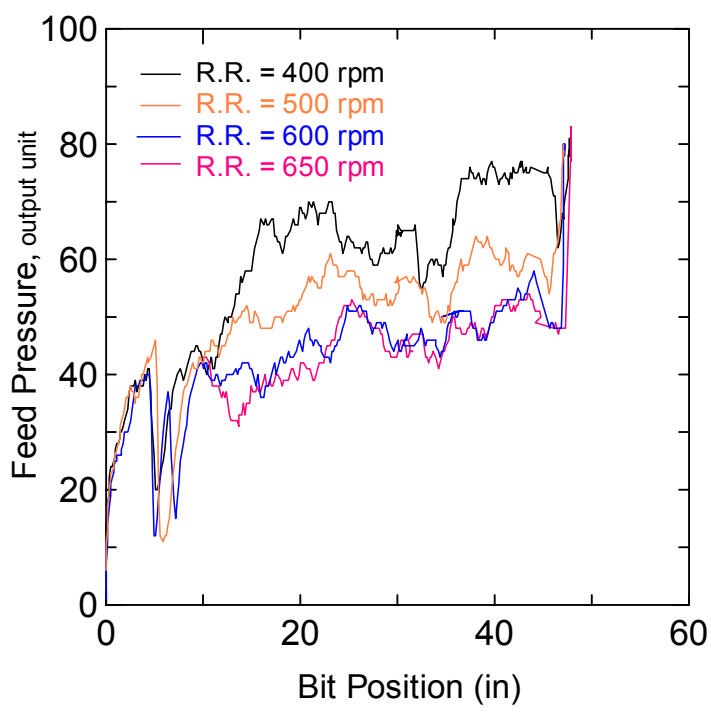

(b) Sandstone (UCS $=9,500 \mathrm{psi}$ )

Figure 5.36 Effect of rotation rate on feed pressure (P.R=1.3 in $/ \mathrm{sec})$ 
the magnitude of feed pressure is. Next, the effect of rotation rate on the trend of net feed pressure-penetration rate curve is determined. The approximate curves for each rotation rate setting vs. the strength of rock are defined as linear functions for simplification. Figure 5.37 shows the approximate curves under different strengths of rocks and rotation rates. It can be seen that the higher the rotation rate is the more gentle the slope of net feed pressurepenetration rate curve is. Moreover, the harder the roof rock is the larger the impact

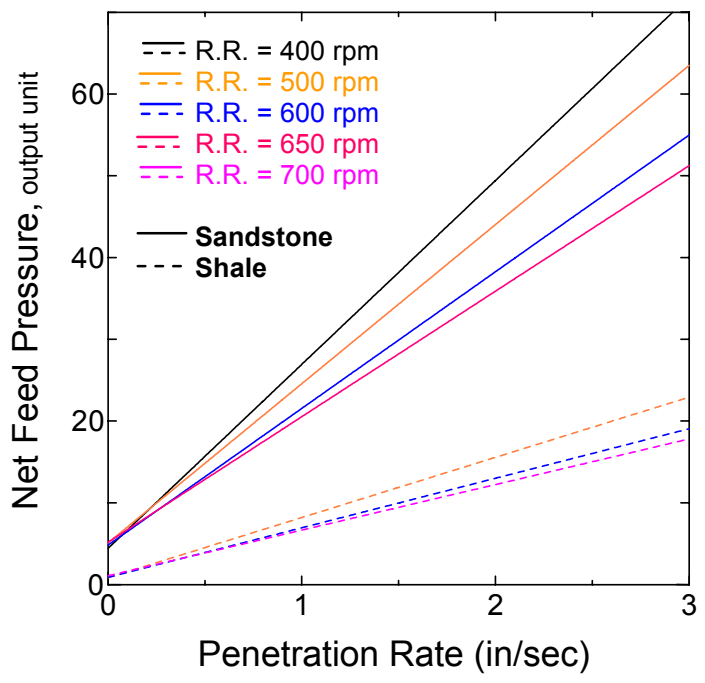

Figure 5.37 Approximation curves for each setting for different rotation rates and different strengths of rocks

of rotation rate on the slope of net feed pressure-penetration rate is. Therefore, it is concluded that the impact of rotation rate on the magnitude of net feed pressure is also related to rock strength.

\subsubsection{Determination of the Boundary Planes for Estimating Roof Rock Strength}

The results obtained so far clearly show that the magnitude of net feed pressure correlates well with the rock strength and both penetration rate and rotation rate have an obvious impact on the magnitude of net feed pressure. Therefore both parameters have to be considered when it comes to roof geology prediction using the magnitude of net feed pressure. From Figure 5.37, the relationship among net feed pressure, penetration rate and rotation rate can be represented by the following equation:

$$
\text { Net Feed Pr essure }\left(t_{n}\right)=F_{R S}\left(\text { Rotation Rate }\left(t_{n}\right)\right) \times \text { Penetration Rate }\left(t_{n}\right)+C_{0}
$$

where, $F_{R S}=$ function of rotation rate for each strength of rocks, $C_{0}=$ constant, and $t_{n}=$ elapsed time after drilling starts. 
Figure 5.38 shows the distributions of data points in Mine F and Mine G on the net feed pressure-penetration rate-rotation rate system. The roof rock in Mine F is shale (UCS $<3,500 \mathrm{psi}$ ) and that in Mine $\mathrm{G}$ is sandstone ranging from 8,400 to 10,400 psi with an average UCS of 9,500 psi. In order to classify the roof rock from the strength point of view, the upper and lower boundaries of each data set distribution are determined. Based on the trend of data set distributions and for simplicity, $F_{R S}$ is assumed to be a linear function of rotation rate. Each boundary plane shown in Figure 5.39 is defined as:

Sandstone (UCS $\approx$ Ave. 9,400 psi)

o The upper boundary plane (UCS $\approx 10,500 \mathrm{psi}$ )

Net feed pressure $\left(t_{n}\right)=\left\{35.84-0.02853 \times\right.$ rotation rate $\left.\left(t_{n}\right)\right\} \times$ penetration rate $\left(t_{n}\right)+9$

o The lower boundary plane $(\mathrm{UCS} \approx 8,500 \mathrm{psi})$

Net feed pressure $\left(t_{n}\right)=\left\{32.84-0.02753 \times\right.$ rotation rate $\left.\left(t_{n}\right)\right\} \times$ penetration rate $\left(t_{n}\right)-1$

Shale (UCS $<3,500$ psi)

o The upper boundary plane (UCS $\approx 3,500 \mathrm{psi}$ )

Net feed pressure $\left(t_{n}\right)=\left\{14-0.01 \times\right.$ rotation rate $\left.\left(t_{n}\right)\right\} \times$ penetration rate $\left(t_{n}\right)+5$

o The lower boundary plane

Net feed pressure $\left(t_{n}\right)=\left\{12-0.01 \times\right.$ rotation rate $\left.\left(t_{n}\right)\right\} \times$ penetration rate $\left(t_{n}\right)-2$

Where, $\mathrm{t}_{\mathrm{n}}=$ elapsed time after drilling start

The 5,500 psi boundary plane is also determined similarly using the set of drilling data in concrete block. This boundary is represented by the following equation:

Net feed pressure $\left(t_{n}\right)=\left\{24.50-0.01833 \times\right.$ rotation rate $\left.\left(t_{n}\right)\right\} \times$ penetration rate $\left(t_{n}\right)-2$ 


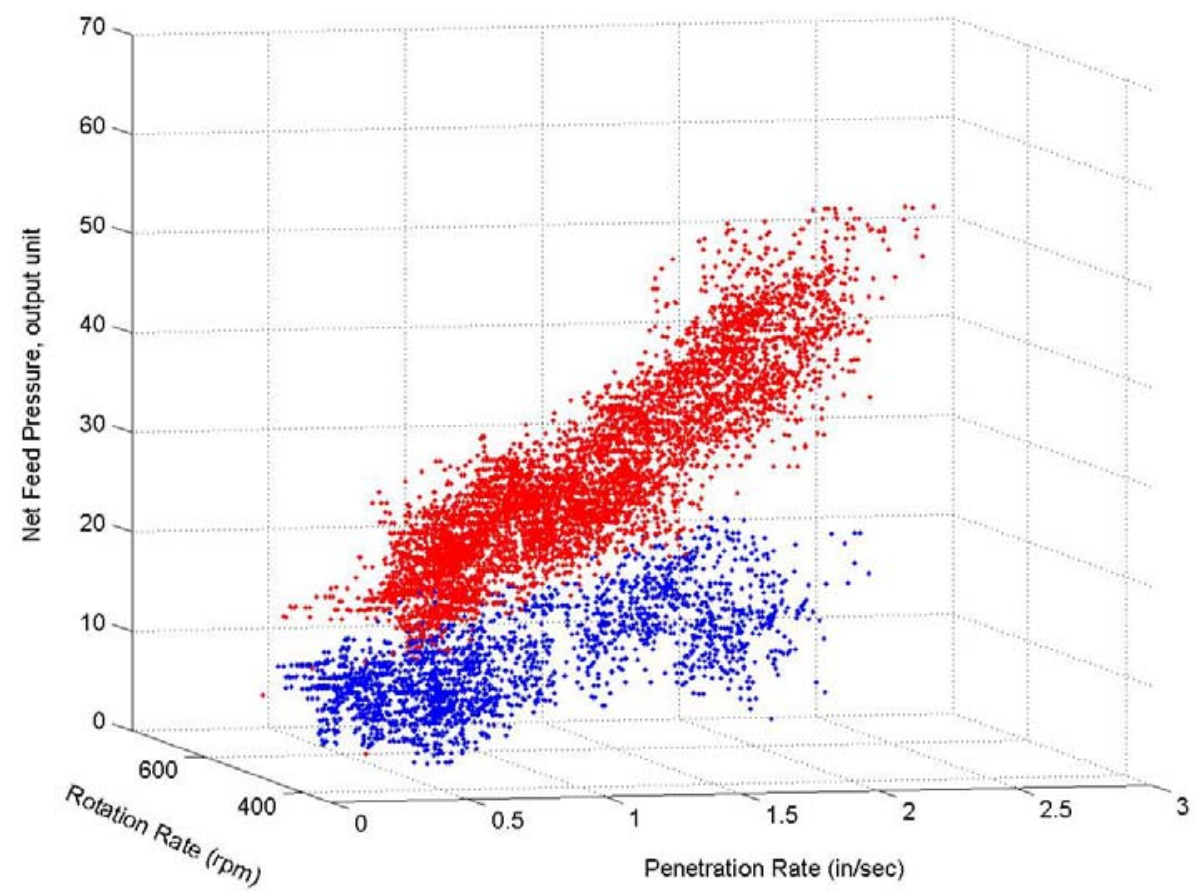

Figure 5.38 Distributions of both sets of data points (Mine F and Mine G)

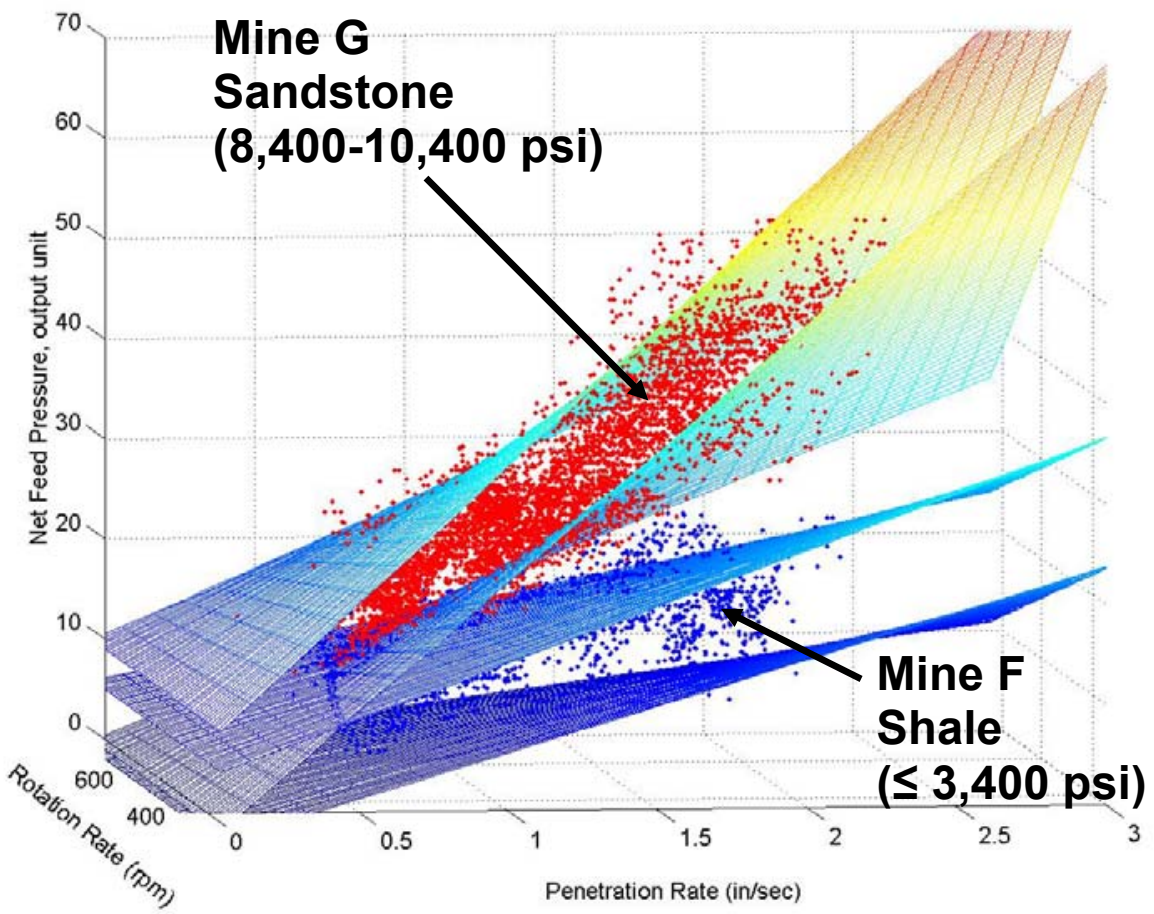

Figure 5.39 Upper and lower boundary planes for distributions of both data points (Mine $\mathrm{F}$ and Mine $\mathrm{G}$ ) 
The boundary planes defined so far are shown in Figure 5.40.

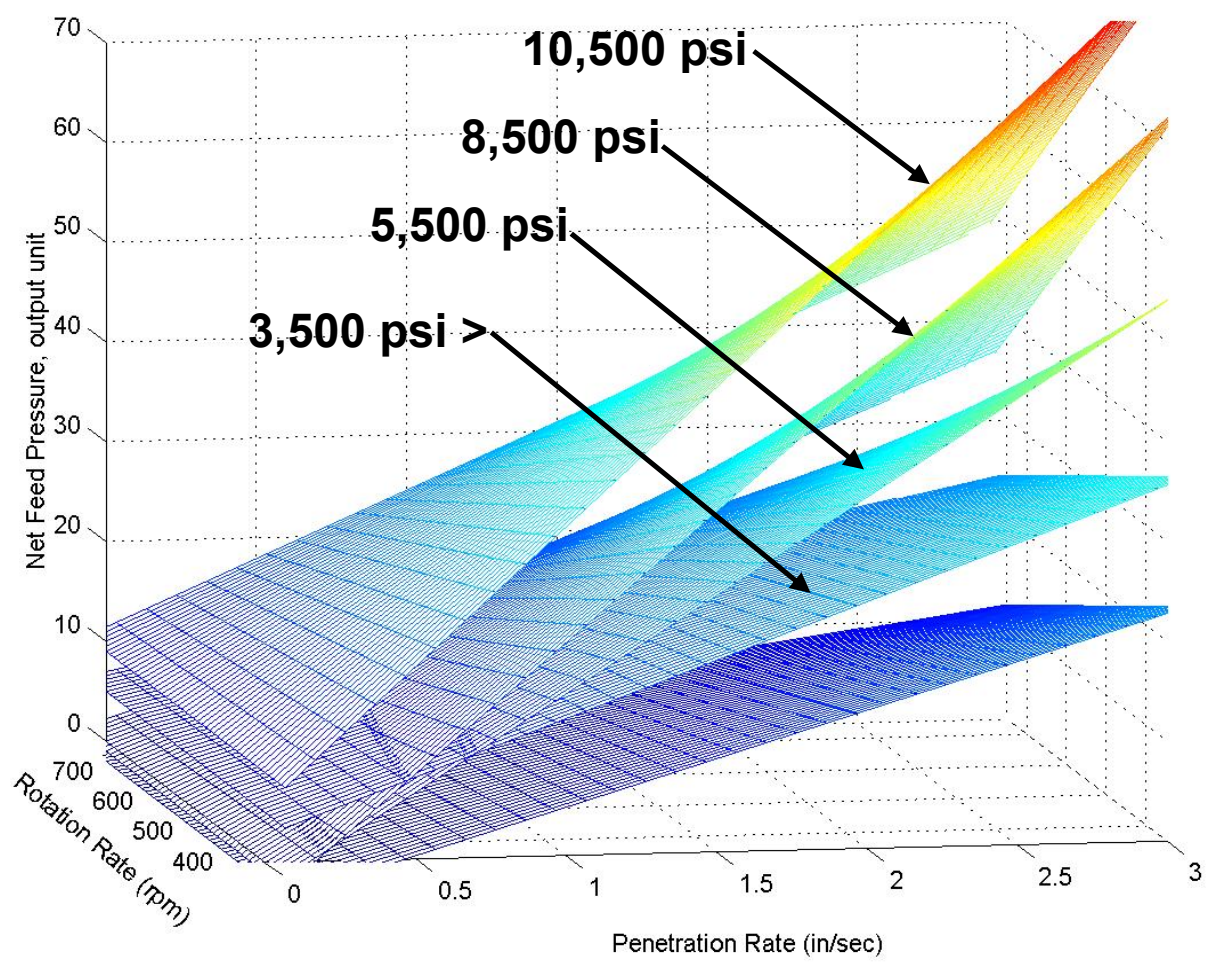

Figure 5.40 Boundary planes for estimating rock strength

\subsubsection{Verification of the Boundary Planes for Estimating Rock Strength}

In order to verify the above boundaries, another set of independent data has been selected and plotted on Figure 5.40. From the results of the lab tests, the data from two kinds of concrete blocks were used. one is 12,340 psi high strength concrete block and the other is 4,000 psi concrete. These two different strengths of concretes data plotted on the area of the approximate boundary planes are shown in Figure 5.41. It can be seen that the drilling data for the 12,340 psi concrete block is distributed above the 10,500 psi boundary plane. On the other hand, those of the 4,000 psi concrete block are distributed around the 3,500 psi boundary plane. These results indicate that roof rocks can be classified by these boundary planes defined in terms of rock strength. 
Moreover, the strength of roof rock in Mine H (UCS Ave. $\approx 9,700$ psi, ranging from 9,000 to $10,300 \mathrm{psi}$ ) is almost the same as that in Mine G (Ave. UCS $\approx 9,500$ psi). So, the data in Mine $\mathrm{H}$ was also plotted on the net feed pressure-penetration rate-rotation rate system (Figure 5.40). It can be seen that the distribution of drilling data in Mine $\mathrm{H}$ is distributed between the 8,500 psi and 10,500 psi boundary planes. This result verifies that if the strengths of roof rocks are almost the same, the distributions of their drilling data in net feed pressure-penetration rate-rotation rate system are also the same.

From the above results, it can be seen that roof rock can be classified based on the magnitude of net feed pressure because it takes both the effects of penetration rate and rotation rate into account. In other words, the relationship among net feed pressure, penetration rate and rotation rate is a good indicator for estimating the strength of roof rock. The strength of roof rock can be determined and/or classified based on the location of data point in the net feed pressure-penetration rate-rotation rate system.

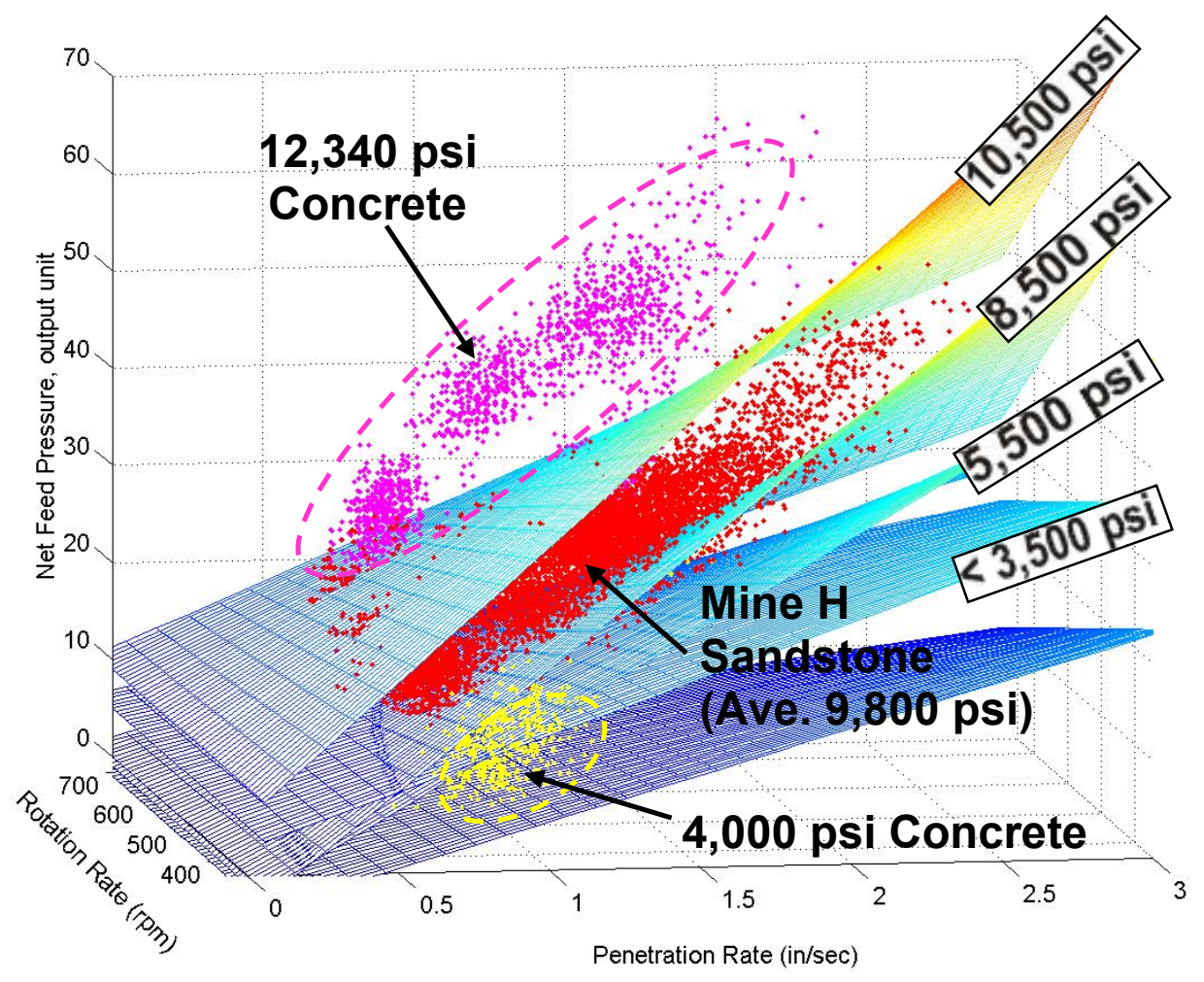

Figure 5.41 Boundary planes for estimating rock strength and distributions of three sets of data points 


\subsection{Bit Wear}

One of the important factors that would affect the drilling performance is bit wear. So, bit wear tests were also conducted in a series of underground tests in order to observe the impact of rock strength on the magnitude of bit wear and its impact on drilling parameters.

\subsubsection{Characteristics and Effect of Bit Wear on Drilling Parameters}

\subsubsection{Drilling in Soft Rock (Mine F, UCS $<3,500$ psi)}

Figure 5.42 shows a new bit and a drill bit after 17 full-length holes were drilled under several drilling settings. From the observation of old and new bits, bit wear was not recognizable. Figure 5.43 shows the relationship between feed pressure/rotation pressure and bit position before and after replacing a drill bit. Before replacing a bit, 17 full-length holes were drilled by using the same drill bit.
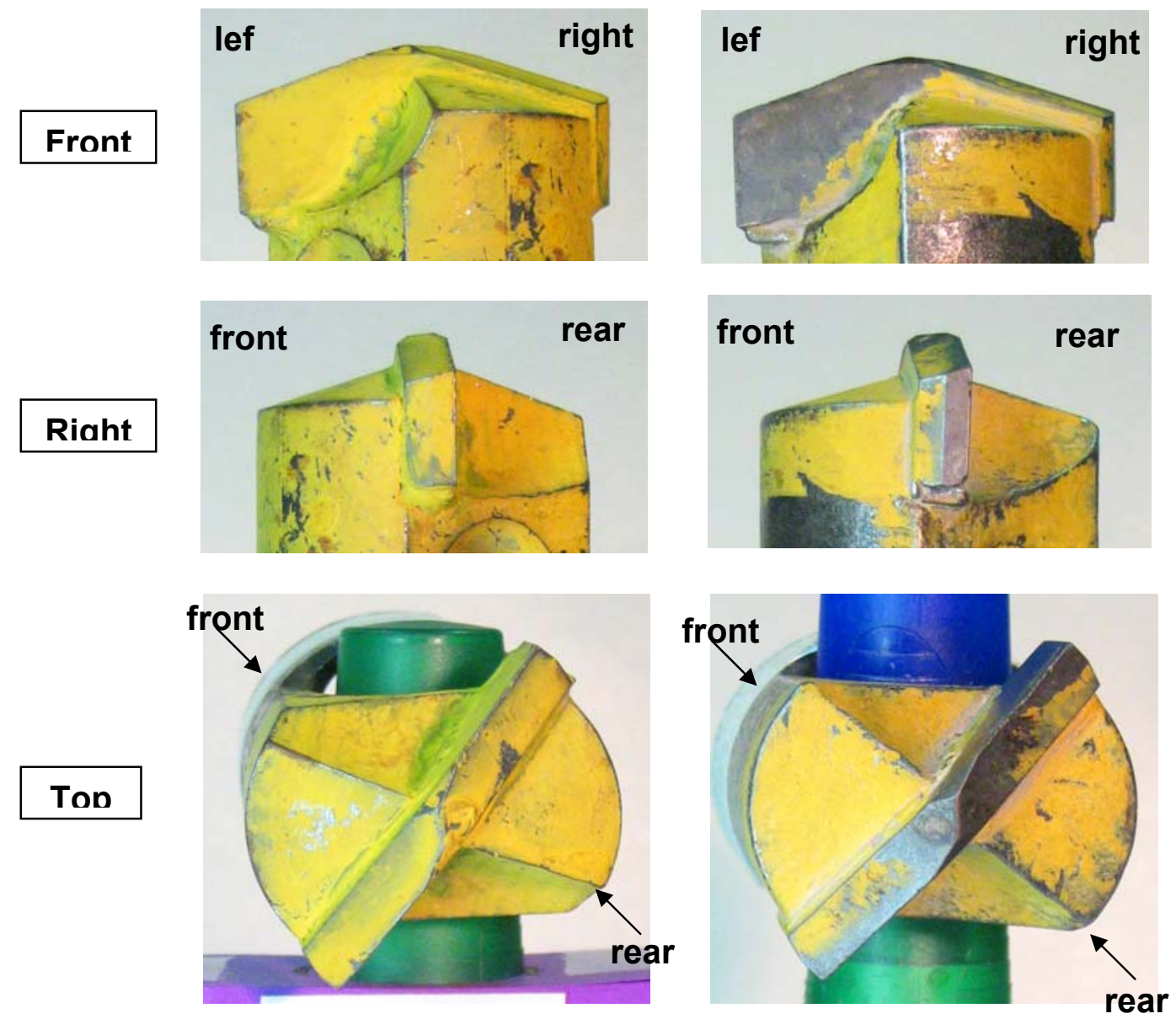

Figure 5.42 Drill bit (left, new bit: right, after having drilled 17 holes, shale) 
By comparing the two data, it shows that there was also no change in both feed pressure and rotation pressure curves. Therefore, it can be concluded that bit wear does not need to be taken into account in analyzing drilling data when drilling in soft rock. Incidentally, each drill bit was replaced by a new bit after 50 drilled holes at Mine F. By extrapolation one may further states that the magnitude of bit wear is not so big even after 50 holes have been drilled in the soft roof rock.

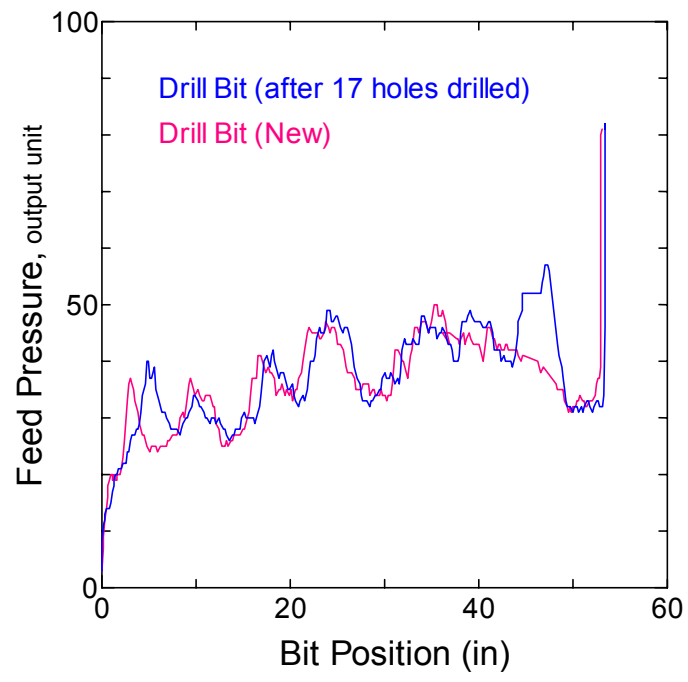

(a) Feed Pressure

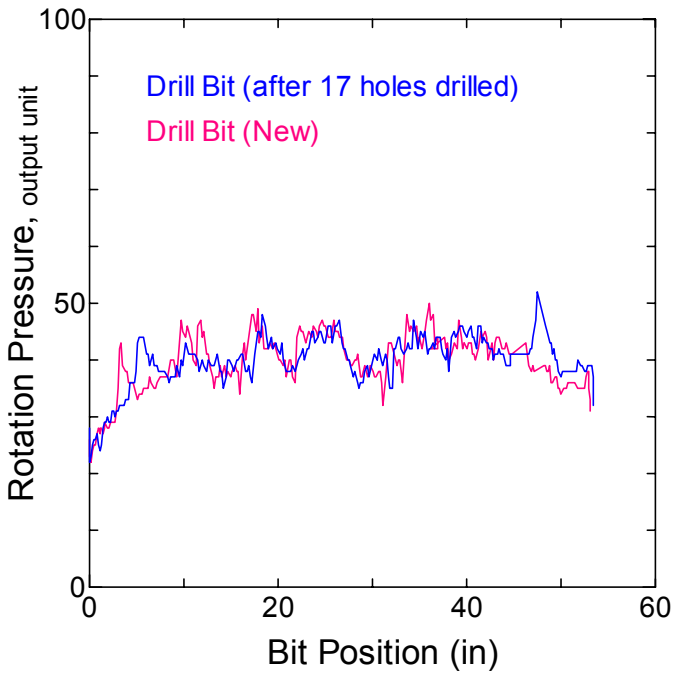

(b) Rotation Pressure

Figure 5.43 Effect of bit wear on feed pressure \& rotation pressure (shale, P.R. $=1.7 \mathrm{in} / \mathrm{sec} \&$ R.R. $=600 \mathrm{rpm}$ )

\subsubsection{Drilling in Hard Rock (Mine G, UCS $\approx 9,500$ psi)}

At first, it was planned to drill 10 holes with one bit under the controlled setting of P.R. $=1.1 \mathrm{in} / \mathrm{sec}$ and R.R. $=650 \mathrm{rpm}$. However, after completing the $7^{\text {th }}$ hole, the drill bit broke as shown in Figure 5.44 and the magnitude of bit wear could not be measured. So, the maximum number of drill holes with one bit was fixed at 5 and two different settings of controlled parameters were tested; P.R. $=1.1 \mathrm{in} / \mathrm{sec} \&$ R.R. $=400 \mathrm{rpm}$ and P.R. $=1.1 \mathrm{in} / \mathrm{sec} \&$ R.R. $=650 \mathrm{rpm}$ in order to study the effect of rotation rate on bit wear. Figure 5.45 shows a drill bit after 5 holes were drilled with different rotation rates (P.R. $=$ $1.1 \mathrm{in} / \mathrm{sec}$, R.R. $=400$ and $650 \mathrm{rpm})$. The relationship between feed pressure/rotation pressure and bit position are shown in Figures 5.46 and 5.47. Both the magnitude of feed pressure and rotation pressure increase with increase of bit wear and the higher the 
rotation rate is the more the bit wear is. As an example, comparing the drilling data for the $1^{\text {st }}$ hole to that of the $3^{\text {rd }}$ hole, the difference at R.R. $=650 \mathrm{rpm}$ is about 20 output units in feed pressure and about 10 output units in rotation pressure. On the other hand, the difference at R.R. $=400 \mathrm{rpm}$ is about 15 output units in feed pressure and about 7 output units in rotation pressure.
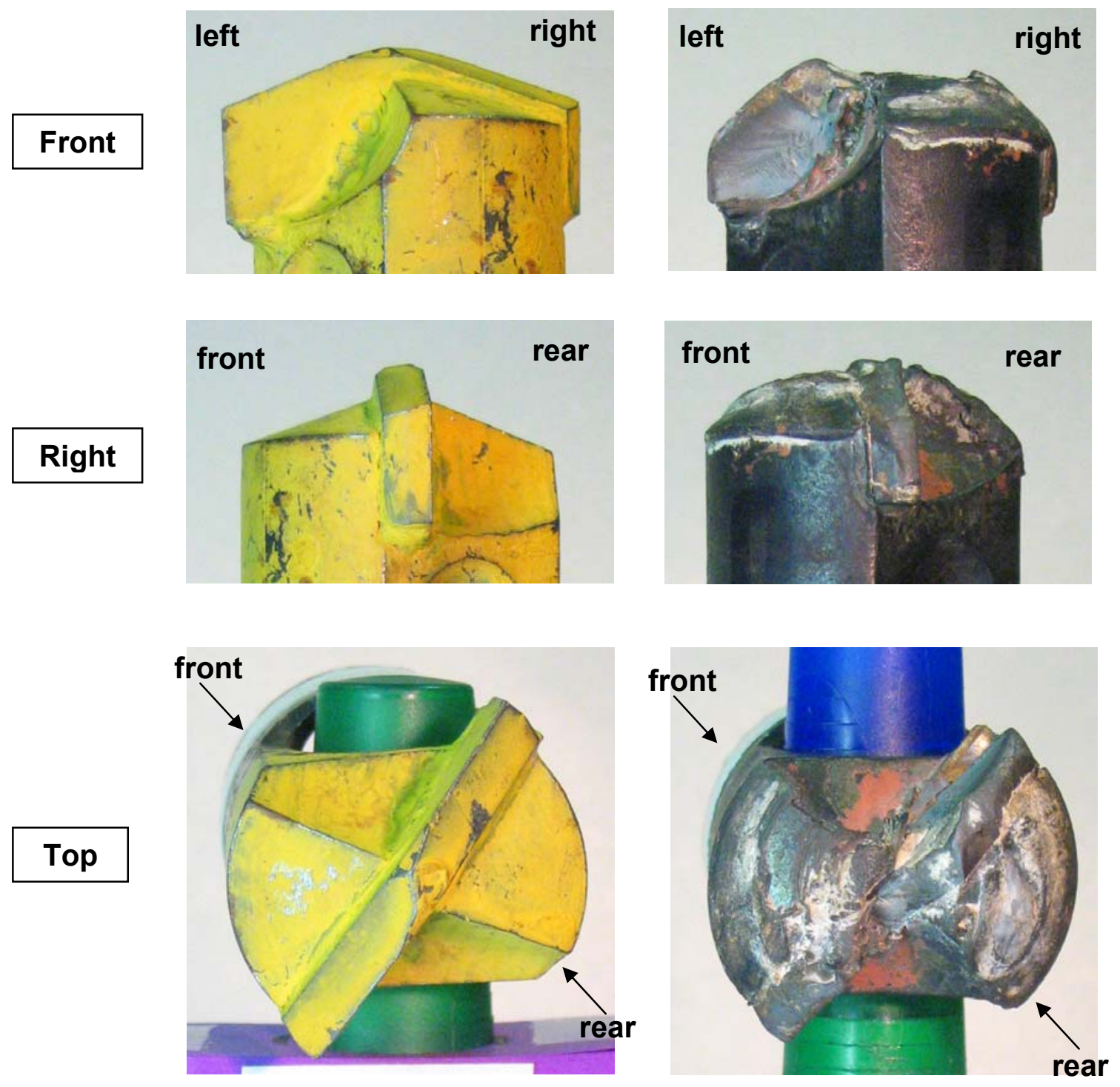

Figure 5.44 Drill bit (left, new bit \& right, after 7 holes drilled) (sandstone, P.R. $=1.1 \mathrm{in} / \mathrm{sec}$ and R.R. $=650 \mathrm{rpm}$ ) 
Next, the effect of penetration rate on the magnitude of bit wear was analyzed. Figure 5.48 shows a drill bit after 3 holes were drilled under different penetration rates (P.R. $=0.4 \mathrm{in} / \mathrm{sec}$ and $1.5 \mathrm{in} / \mathrm{sec}$, R.R. $=650 \mathrm{rpm})$. Figures 5.49 and 5.50 show the relationship between feed pressure/rotation pressure and bit position under different penetration rates. From Figure 5.48, it is observed that the magnitude of bit wear for P.R. $=0.4 \mathrm{in} / \mathrm{sec}$ is larger than that for P.R. $=1.5 \mathrm{in} / \mathrm{sec}$. From Figures 5.49 and 5.50, even both the magnitudes of feed pressure and rotation pressure increase with increase of bit wear, there is no big difference between the magnitude of feed pressure/rotation pressure for the $1^{\text {st }}$ hole and those for the $3^{\text {rd }}$ hole. Compared with the results of different rotation rates, bit wear seems to be more affected by rotation rate than by penetration rate.

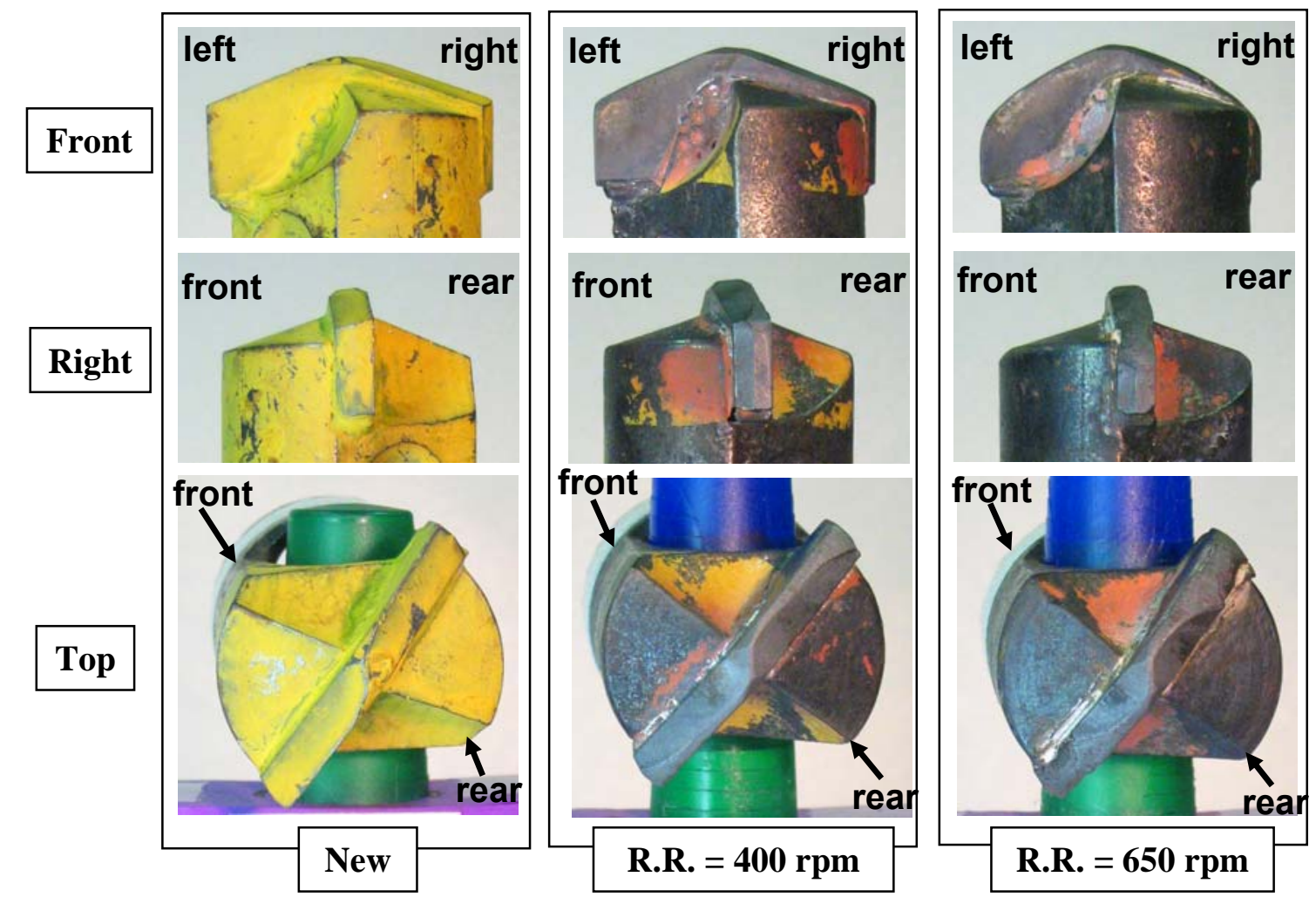

Figure 5.45 Drill bit (left, new: center, R.R. $=400 \mathrm{rpm}$ : right, R.R. $=650 \mathrm{rpm}$ ) (sandstone, P.R. $=1.1 \mathrm{in} / \mathrm{sec}$, number of holes drilled $=5$ ) 


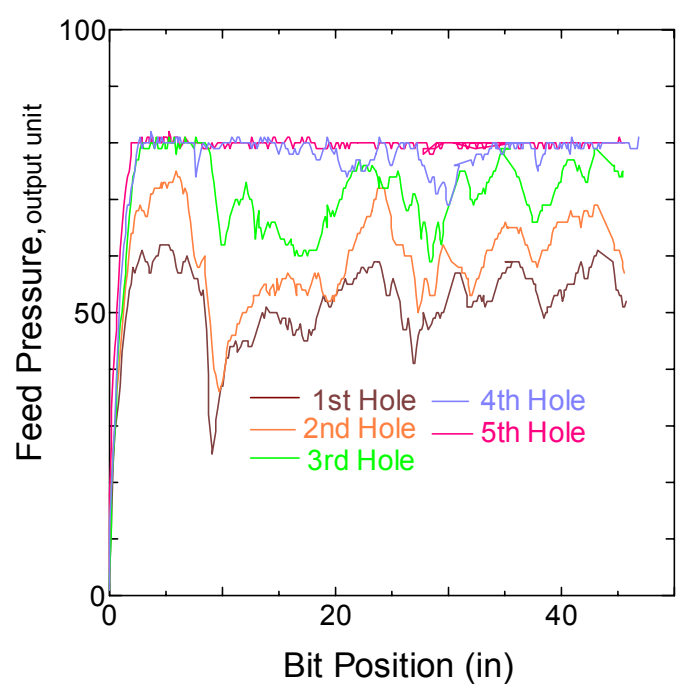

(a) Feed Pressure

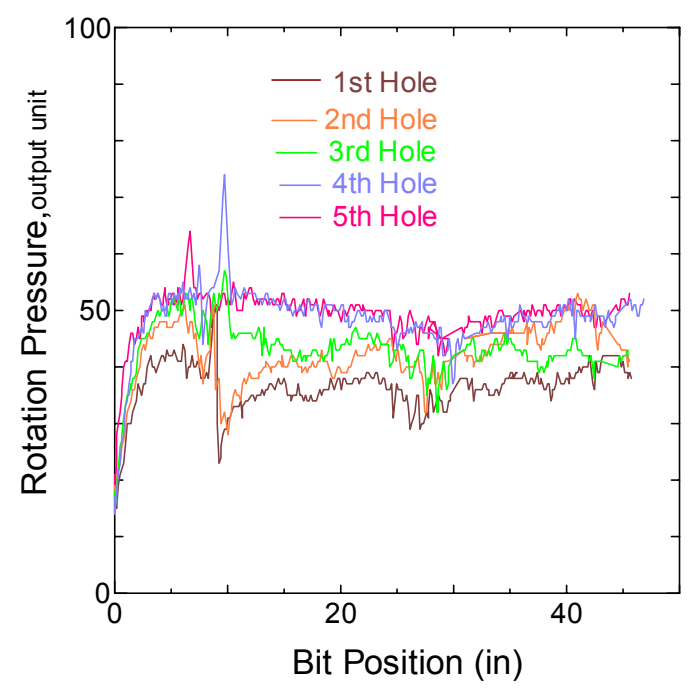

(b) Rotation Pressure

Figure 5.46 Magnitude of feed pressure/rotation pressure for each drill hole (bit wear test, sandstone, P.R. $=1.1 \mathrm{in} / \mathrm{sec}$ and R.R. $=400 \mathrm{rpm}$ )

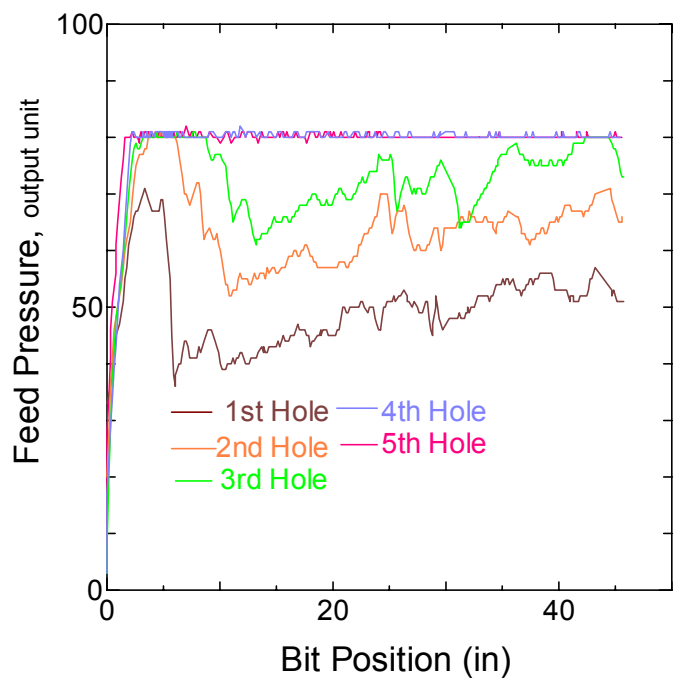

(a) Feed Pressure

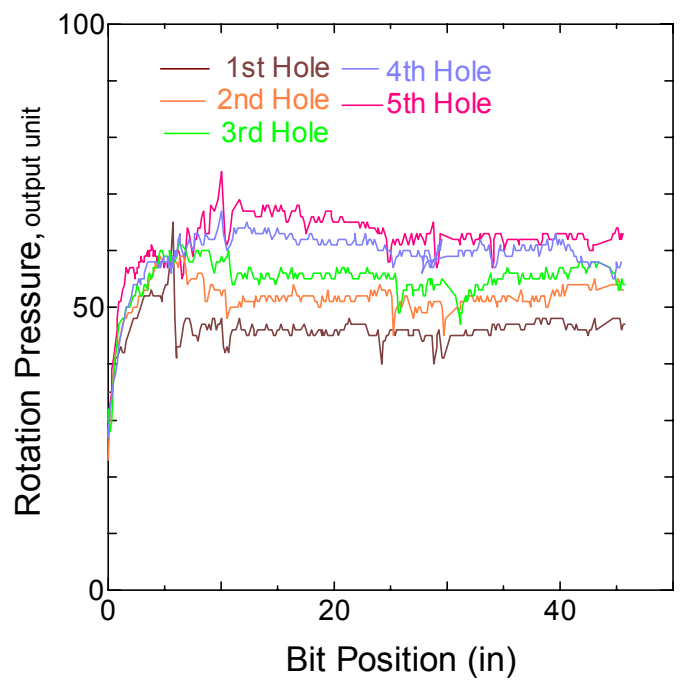

(b) Rotation Pressure

Figure 5.47 Magnitude of feed pressure/rotation pressure for each drill hole (bit wear test, sandstone, P.R. $=1.1 \mathrm{in} / \mathrm{sec}$ and R.R. $=650 \mathrm{rpm}$ ) 

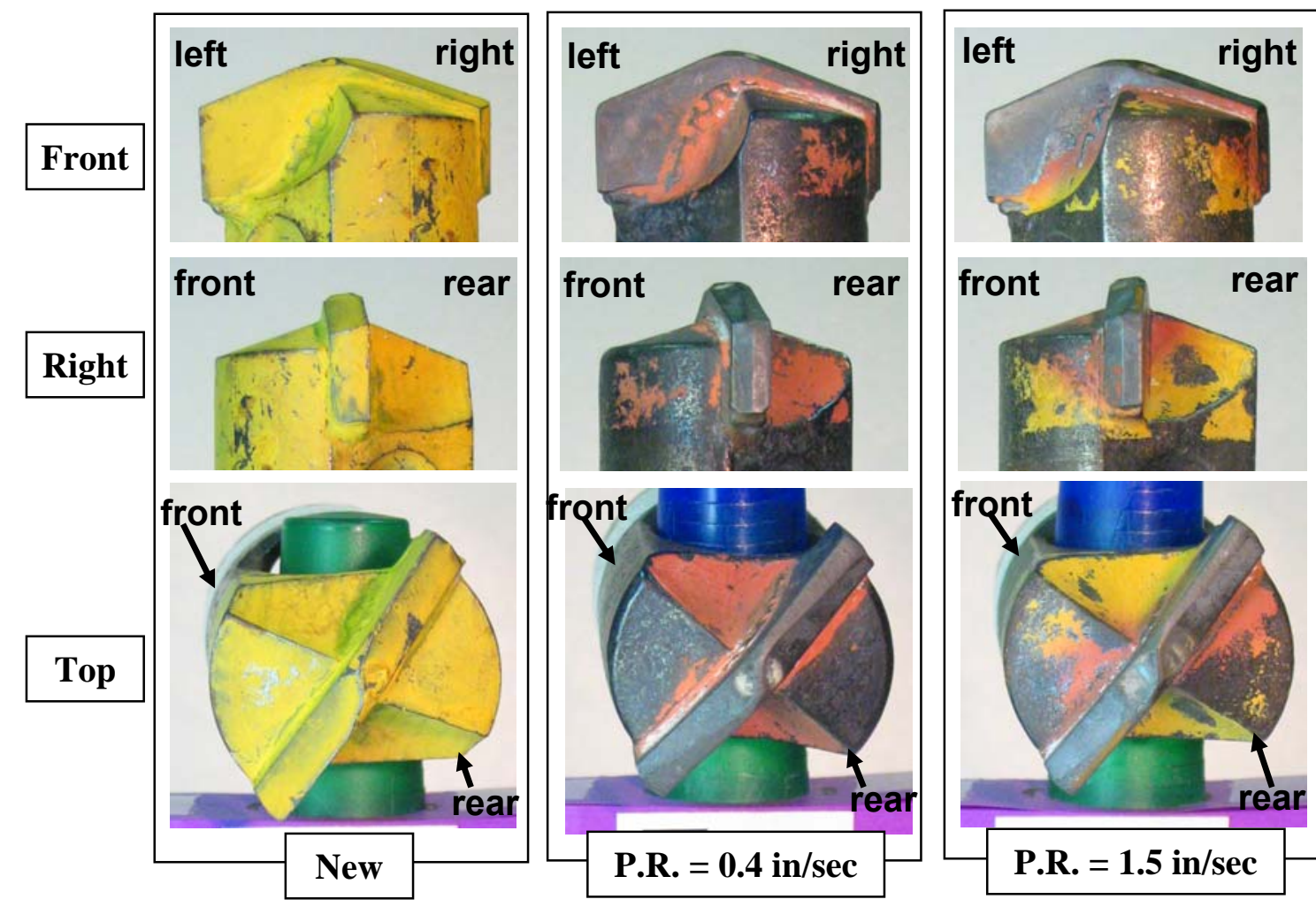

Figure 5.48 Drill bit (left, new: center, P.R. $=0.4$ in $/ \mathrm{sec}$ : right, P.R. $=1.5 \mathrm{in} / \mathrm{sec}$ ) (sandstone, R.R. $=650 \mathrm{rpm}$, number of holes drilled $=3$ )

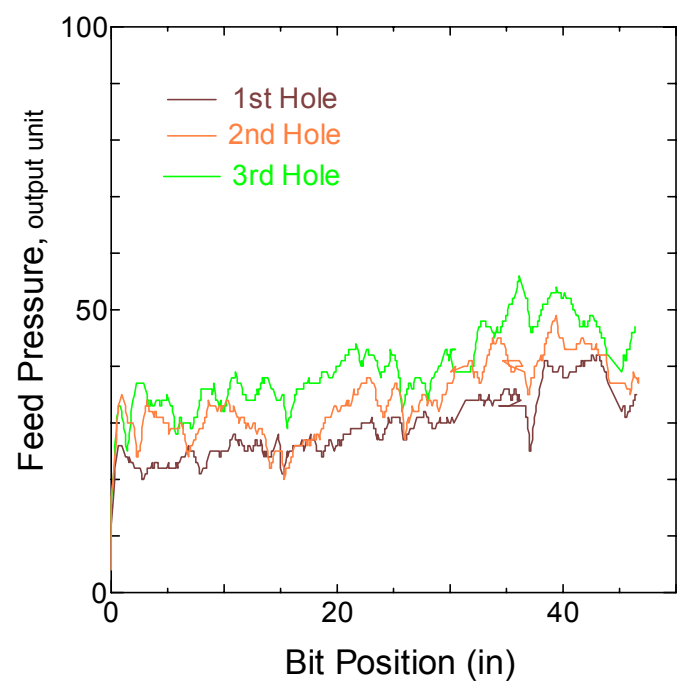

(a) Feed Pressure

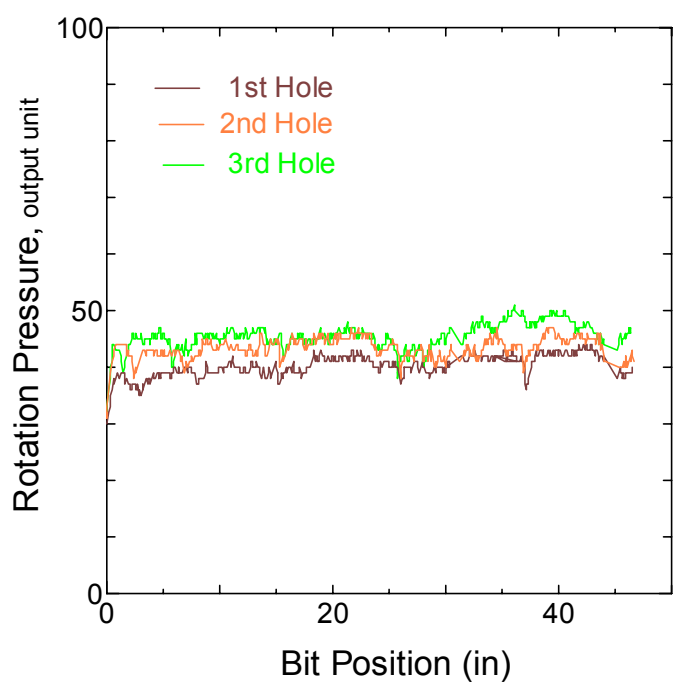

(b) Rotation Pressure

Figure 5.49 Magnitude of feed pressure/rotation pressure for each drill hole (bit wear test, sandstone, P.R. $=0.4 \mathrm{in} / \mathrm{sec}$ and R.R. $=650 \mathrm{rpm}$ ) 


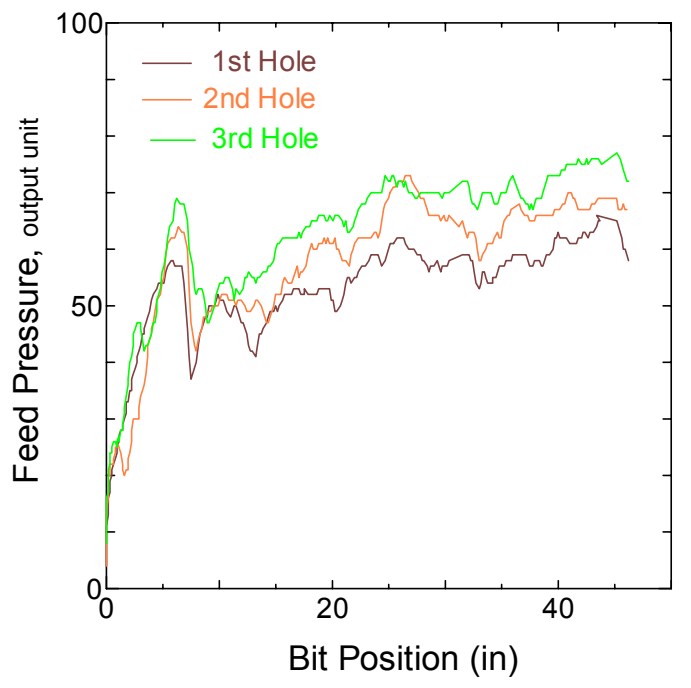

(a) Feed Pressure

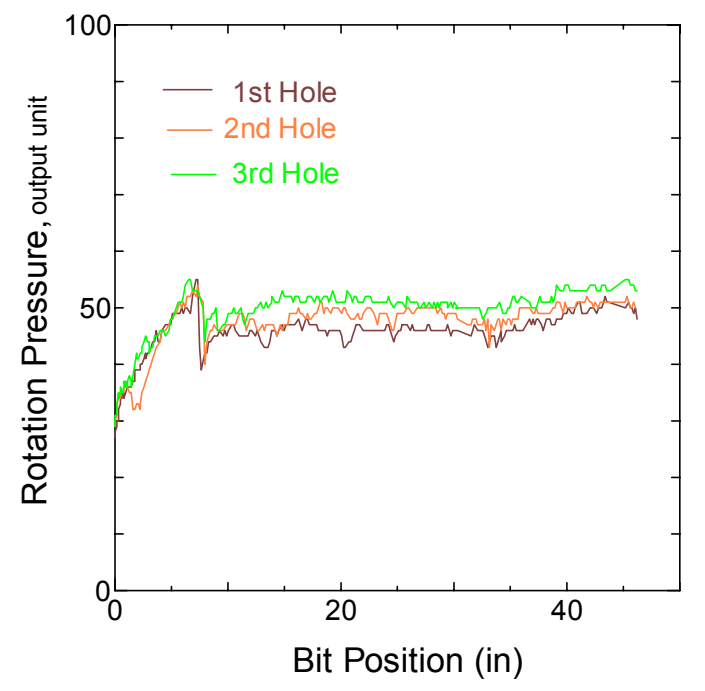

(b) Rotation Pressure

Figure 5.50 Magnitude of feed pressure/rotation pressure for each drill hole (bit wear test, sandstone, P.R. $=1.5 \mathrm{in} / \mathrm{sec}$ and R.R. $=650 \mathrm{rpm}$ )

Incidentally, from the observation of drill bits, it was noticed that the magnitude of wear on the front tip was different from the other parts. Moreover, wear of bit was seen clearly not only on the front tip but also on the sides of it. These points are illustrated in Figures 5.45 and 5.48.

\subsubsection{Effect of Bit Wear on Roof Geology Prediction}

\subsubsection{Effect on Void/Fracture Prediction}

Figure 5.51 show the impact of bit wear on the relationship between feed pressure and bit position under different penetration rates. A fracture existed around 6-7 in deep from the roof line in the drilling area. From these figures, it can be seen that even though the magnitude of feed pressure increases with increase of bit wear, bit wear has no obvious impact on the trend and the level of the bottom of feed pressure valley. Therefore, it was concluded that bit wear does not affect the void/fracture prediction. 

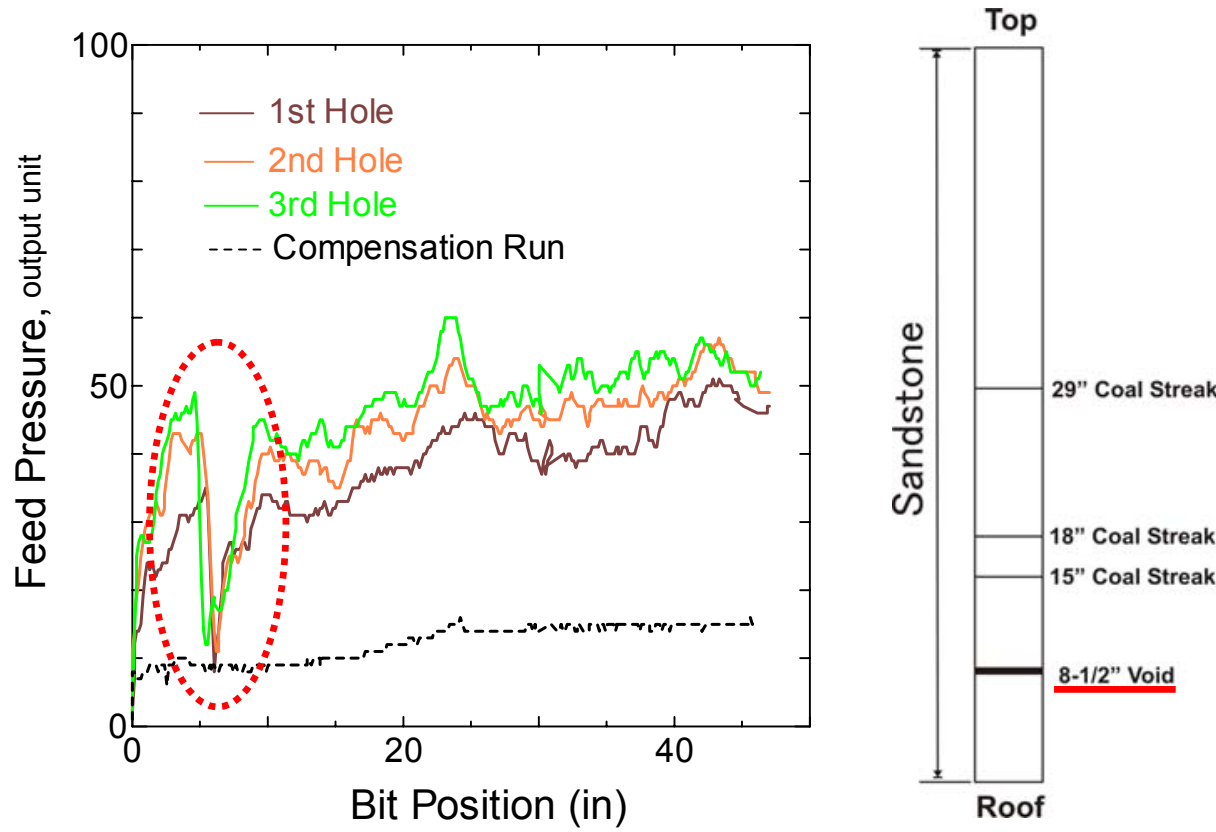

Figure 5.51 Magnitude of feed pressure for each drill hole and the result of borehole scoping (bit wear test, sandstone, P.R. $=1.1 \mathrm{in} / \mathrm{sec}$ and R.R. $=650 \mathrm{rpm}$ )

\subsubsection{Effect on Estimation of Rock Strength}

From the above results, as bit wear and its effect on feed pressure and rotation pressure was not recognizable in soft rock, the effect of bit wear may not be considered when drilling in soft rock. However, when drilling in hard rock, bit wear was recognizable clearly and had obvious impact on the magnitude of feed pressure and rotation pressure. Moreover, different controlled settings of drilling parameters, especially different rotation rates, have obvious impact on the magnitude of bit wear. Therefore, when applying the methodology for estimating rock strength developed so far for production, the effect of bit wear on the magnitude of feed pressure and rotation pressure should be taken into account. This point needs to be explored further in order to apply to this system for normal operation.

\subsection{Other Factors Considered}

\subsubsection{Repeatability}

Repeatability means that drilling parameters are in the same magnitude and trend if drilling is conducted under the same drilling setting and roof geology. In this test, two 
holes were drilled close each other at each setting. Figure 5.52 (a) and (b) show the relationship between feed pressure/rotation pressure and bit position. It can be seen that the drilling data for $1^{\text {st }}$ and $2^{\text {nd }}$ holes are in good agreement with each other. It was therefore confirmed that the data are repeatable.

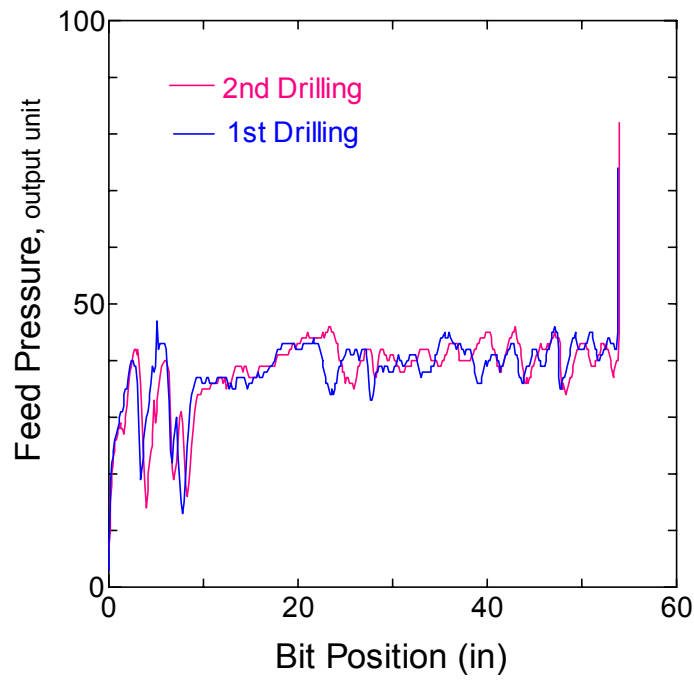

(a) Feed Pressure

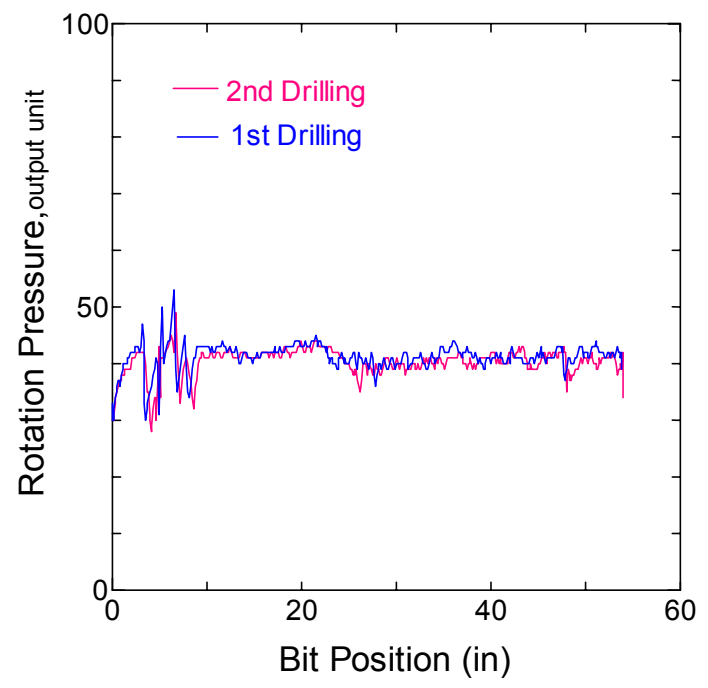

(b) Rotation Pressure

Figure 5.52 Confirmation of repeatability (P.R. $=1.1 \mathrm{in} / \mathrm{sec}$, R.R. $=600 \mathrm{rpm})$

\subsubsection{Effect of Control Mode (Feedback System Mode)}

As mentioned above, drilling was not conducted manually but controlled by DCU in this research. One of the main keywords is "automatically". WVU control mode, in which both penetration rate and rotation rate are controlled or kept constant, was mainly used in a series of underground and laboratory tests in order to determine the effect of penetration rate and rotation rate settings on drilling conditions and drilling parameters. Another control mode, the feedback system mode was also tested. Feedback system mode was developed and suggested by J.H. Fletcher \& Co to be applied for normal drilling operation. In this mode, drilling is controlled by both horsepower curve and bite curve selected by the operator. Depend on the two curves and current condition (feed pressure), penetration rate and rotation rate are changed and controlled. It can be estimated that different control modes have different drilling behaviors and responses include drilling parameters. So it is necessary to determine, based on the drilling data, whether or not the interpretation methodology for roof geology prediction developed so far (based on the 
data for drilling under the WVU control mode) can be applied for the interpretation of the drilling data controlled by feedback system mode.

A drilling test controlled by feedback system mode was conducted in Mine G where the roof is sandstone ranging from 8,400 to 10,400 psi with an average of 9,400 psi in UCS. Figure 5.53 shows the distribution of drilling data points and the boundary planes defined so far. From this figure, it can be seen that the drilling data in which drilling was conducted by feedback system mode are also distributed between the 8,500 psi and 10,500 psi boundary planes. It seems that the boundary planes can also be applied for interpretation of drilling data controlled by feedback system mode. However, the number of drill holes drilled by feedback system mode was only 12 holes and all of them were in only one type of roof rock. In addition, as there were no obvious voids/fractures in and around the drilled holes, it can not verify the void/fracture prediction criteria. Hence, in order to verify the applicability of the data interpretation methodology developed so far for the drilling data controlled by feedback system mode, more drilling test have to be conducted under several drilling settings and in different strengths of roof rocks with voids/fractures.

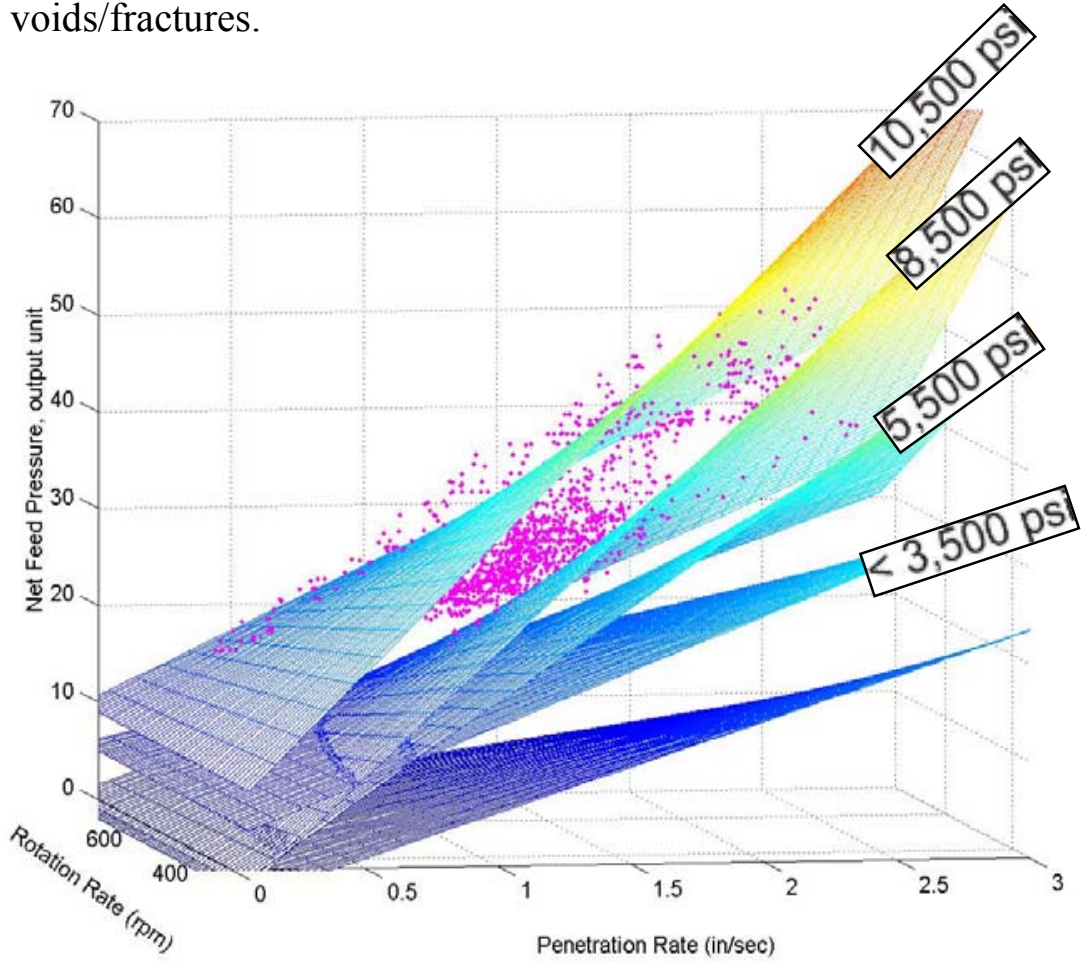

Figure 5.53 Distribution of drilling data points and boundary planes for estimating rock strength (Mine G. feedback svstem mode drilling) 


\subsubsection{Effect of Thrust Cap}

The thrust cap can be implemented by two ways in the machine used for this project. One is by setting the relieve valve while another is by setting the DCU. If the setting of DCU is smaller than that of relieve valve, the behavior of the feed pressure will be controlled by the algorithm of DCU once the feed pressure reaches beyond the preset value in DCU. In such a case, the feed pressure curve will be highly fluctuated because DCU tries to adjust the feed flow to prevent the feed pressure from exceeding the preset thrust cap (Figure 5.54). It can be seen from the feed PWM curve. So, this change is not caused by the change of roof geology but caused by DCU (control algorithm). On the other hand, if the setting of DCU is larger than that of the relieve valve, the behavior of the feed pressure will be controlled by the relieve valve when the feed pressure reaches beyond the preset value in the relieve valve. In this case, feed pressure curve is so smooth. In addition, as the DCU does not realized that the valve is relieved, the feed PWM curve keeps increasing to reach the target speed (Figure 5.55).

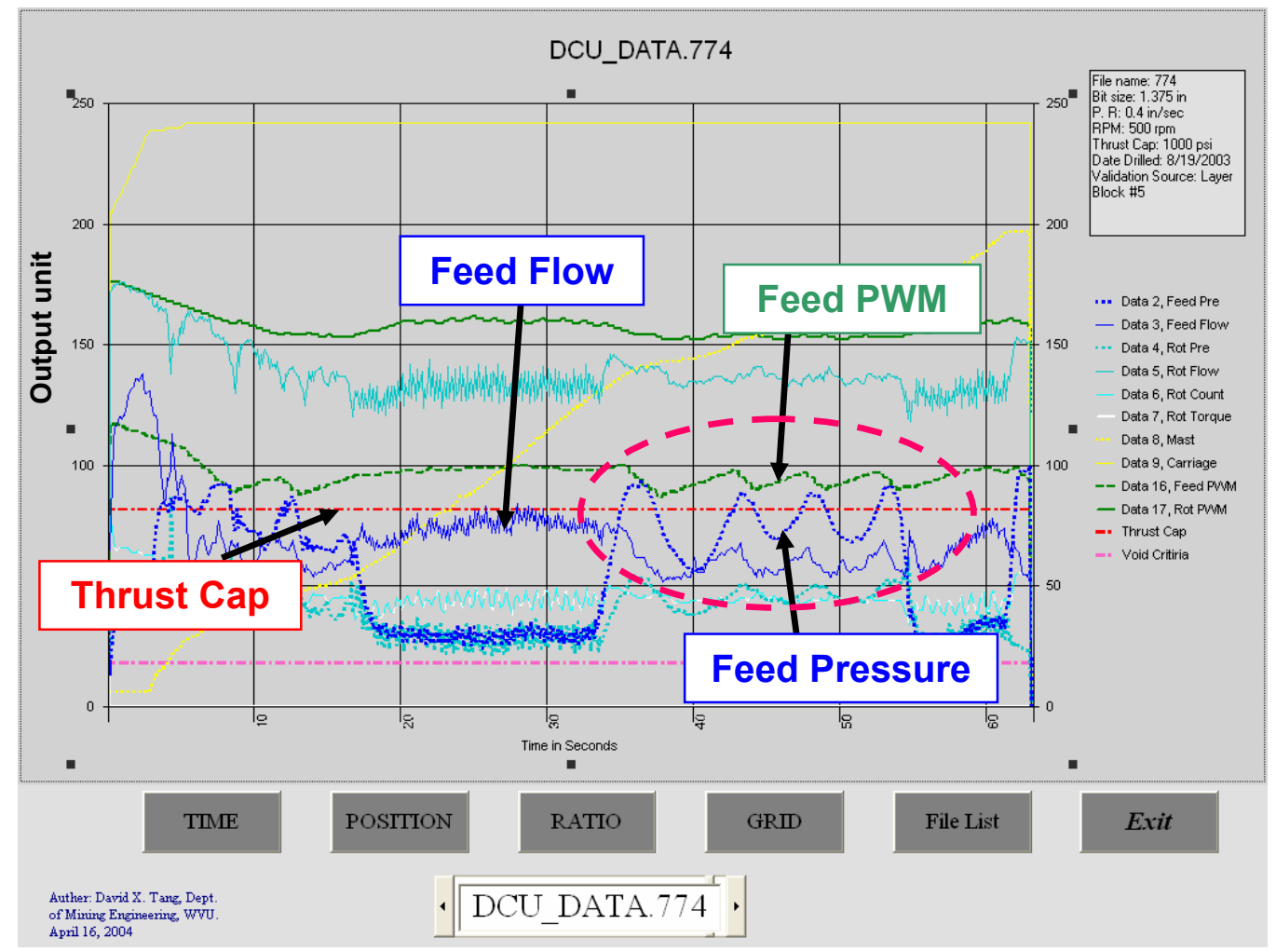

Figure 5.54 Drilling parameters for drilling in layered block \# 5 (P.R. $=0.4$ in $/ \mathrm{sec}$, R.R. $=500$ ) 


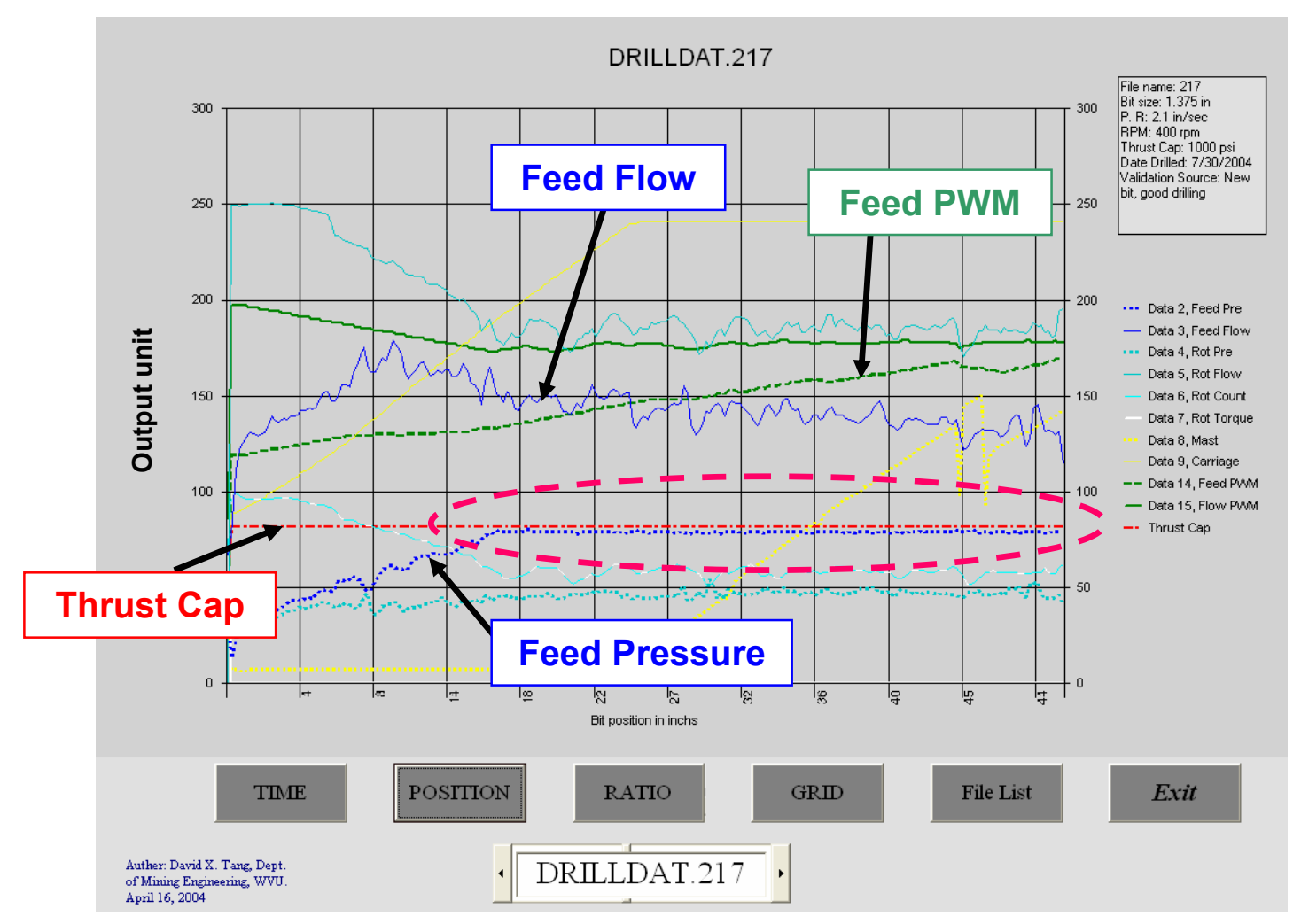

Figure 5.55 Drilling parameters for drilling in sandstone (P.R. $=2.1 \mathrm{in} / \mathrm{sec}$, R.R. $=400 \mathrm{rpm})$

From these results, it can be concluded that the drilling behavior when the feed pressure reaches beyond the preset level of thrust cap is different from that when the feed pressure is below it. Besides, the drilling behavior when the feed pressure reaches beyond the level of thrust cap is different for different thrust caps. Hence, the methodology for prediction of roof geology when the feed pressure reaches beyond the level of thrust cap also needs to be explored further.

\subsubsection{Drilling Conditions under a Large Bite Depth}

Figures 5.56 (a) and (b) show the relationship between net feed pressure and penetration rate under R.R. $=400 \mathrm{rpm}$ and $600 \mathrm{rpm}$, respectively. From these figures, as mentioned above, it can be seen that different rotation rate have obvious impact on the slope of the net feed pressure-penetration rate curve and the higher the rotation rate is the lower the magnitude of net feed pressure is. Moreover, it was recognized that the slope of net feed pressure-penetration rate curve also changes under high penetration rates and 
low rotation rates, i.e. large bite depth. For example, when the rotation rate was set at 400 $\mathrm{rpm}$, the slope becomes steep when penetration rate is larger than about $1.5 \mathrm{in} / \mathrm{sec}$.

As for this trend, there are two possible reasons. One is the capacity of vacuum pump while the other is the bit geometry. As shown in Figure 5.57, the height of the front tip of the bit is $0.16 \mathrm{in}$. Once the bite depth is larger than this value, the drilling mechanism between bit and rock seems to have changed. Once there is an interaction between bit body and rock, changes in drilling parameters occur and the coats of paint on the top of the bit body may be wiped out entirely. As every bit was used to drill only one hole, the slope of net feed pressure-penetration rate curve and the condition of paint on the top of the bit body was compared. As an example, when the rotation rate was set at $400 \mathrm{rpm}$, from Figure 5.56 (a), the slope of the net feed pressure-penetration rate curve seems to have changed and become steeper when penetration rate is larger than $1.3 \mathrm{in} / \mathrm{sec}$. On the other hand, from Figure 5.58, it can be seen clearly the paint on the top of bit body was wiped off when penetration rate was larger than $1.3 \mathrm{in} / \mathrm{sec}$. Similarly, when rotation rate was set at $600 \mathrm{rpm}$, changes in slope and condition of the pant were both observed when the penetration rate was around $2.1 \mathrm{in} / \mathrm{sec}$ (Figures 5.59). From these results, it

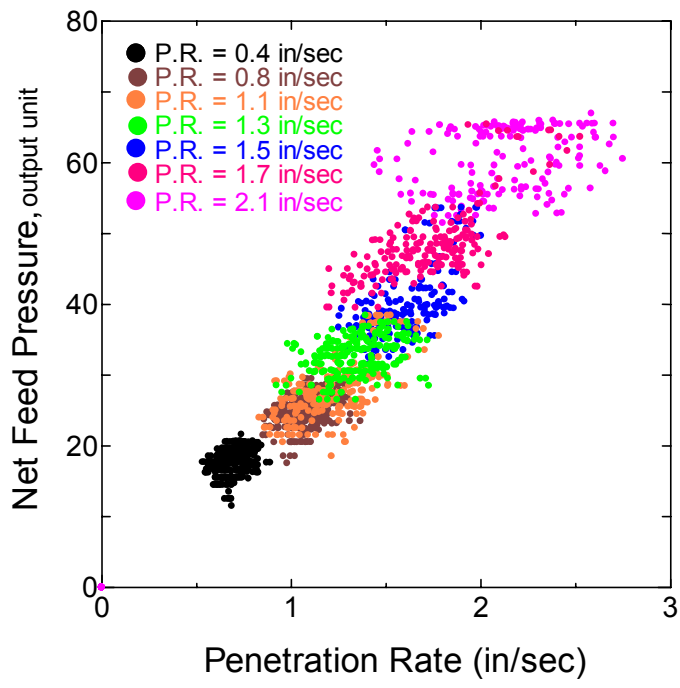

(a) R.R. $=400 \mathrm{rpm}$

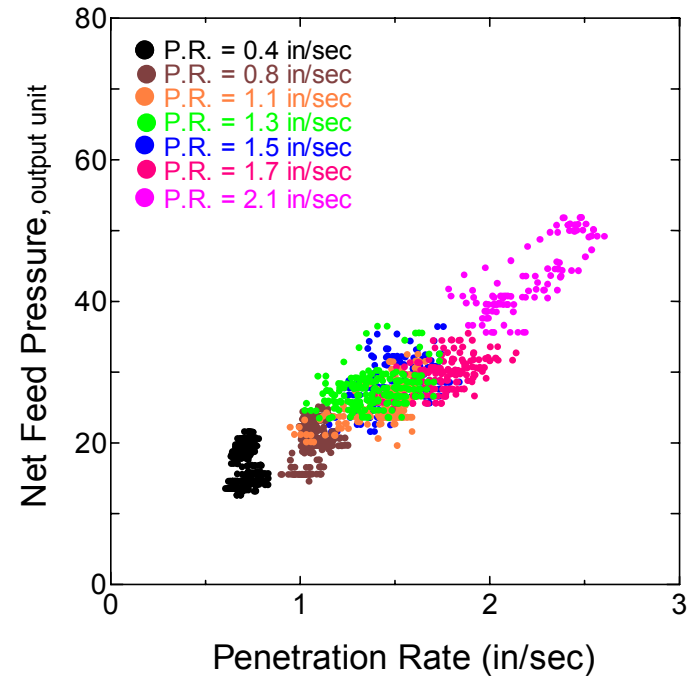

(b) R.R. $=600 \mathrm{rpm}$

Figure 5.56 Relationship between net feed pressure and penetration rate 

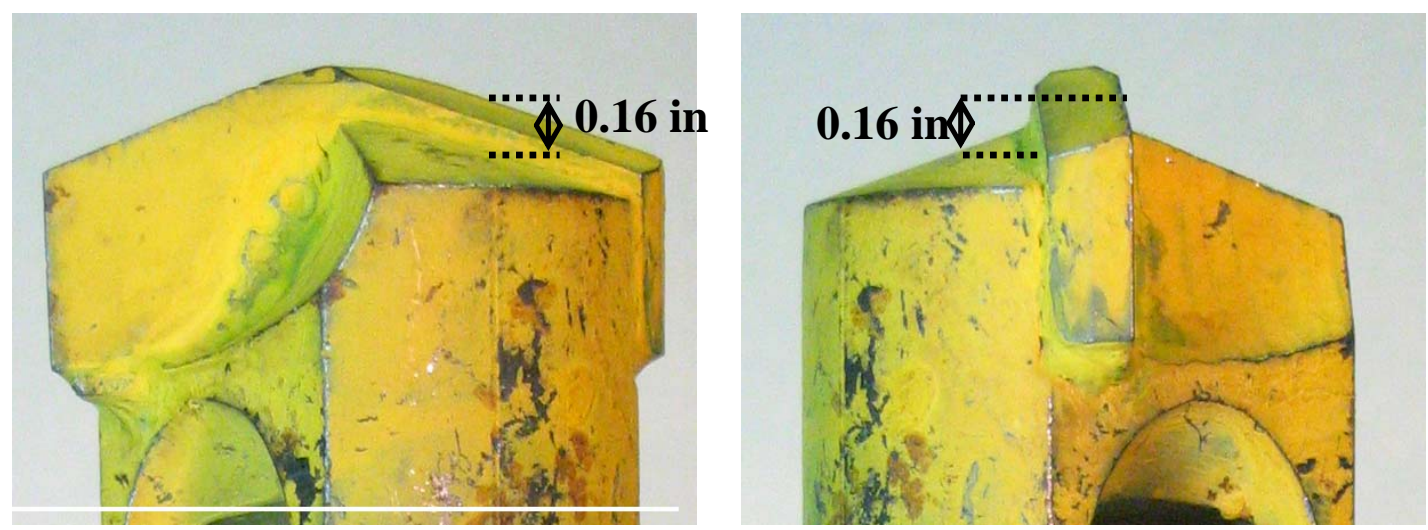

Figure 5.57 Bit geometry (new bit)

seems that drilling mechanism may change when the bite depth is larger than $0.20 \mathrm{in} / \mathrm{rev}$. which is larger than $0.16 \mathrm{in} / \mathrm{rev}$. This means that the roof rock is broken as the bit is penetrated.

Therefore, it can be concluded that bit geometry and/or the capacity of vacuum pump affects drilling parameters and drilling mechanism may change when the bite depth (penetration rate/rotation rate) is large. Hence, the model or equations developed so far may need to be modified at high penetration rates \& low rotation rates. This point also needs to be explored further. 

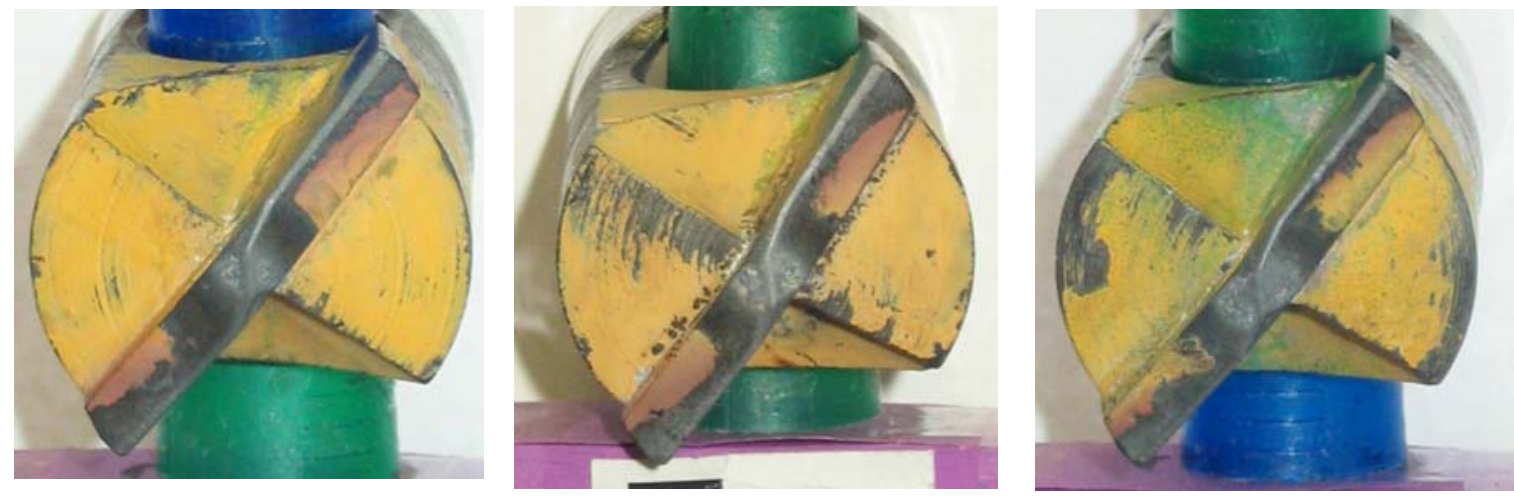

\section{P.R. $=0.4 \mathrm{in} / \mathrm{sec}$}

$$
\text { P.R. }=0.8 \mathrm{in} / \mathrm{sec}
$$

$$
\text { P.R. }=1.1 \mathrm{in} / \mathrm{sec}
$$
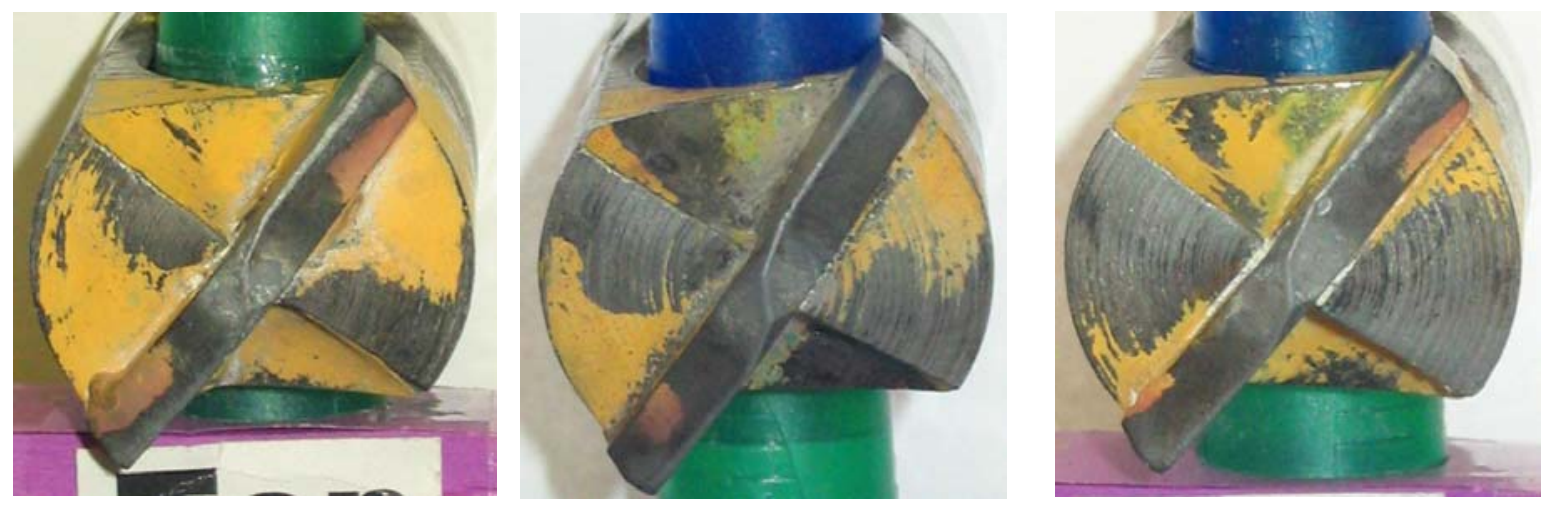

$$
\text { P.R. }=1.3 \mathrm{in} / \mathrm{sec}
$$

$$
\text { P.R. }=1.5 \mathrm{in} / \mathrm{sec}
$$

$$
\text { P.R. }=1.7 \mathrm{in} / \mathrm{sec}
$$

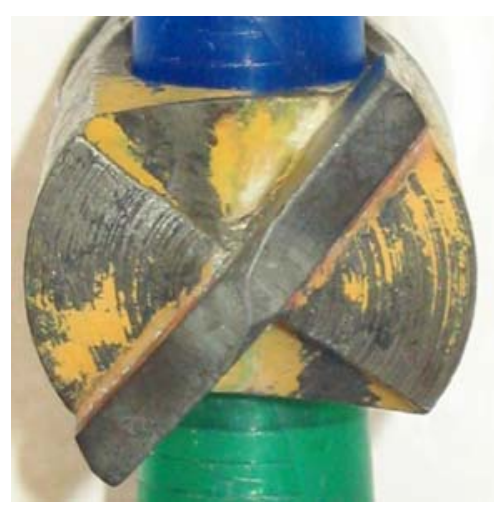

$$
\text { P.R. = } 2.1 \mathrm{in} / \mathrm{sec}
$$

Figure 5.58 Drill bit (R.R. $=400 \mathrm{rpm}$, number of holes drilled = 1) 


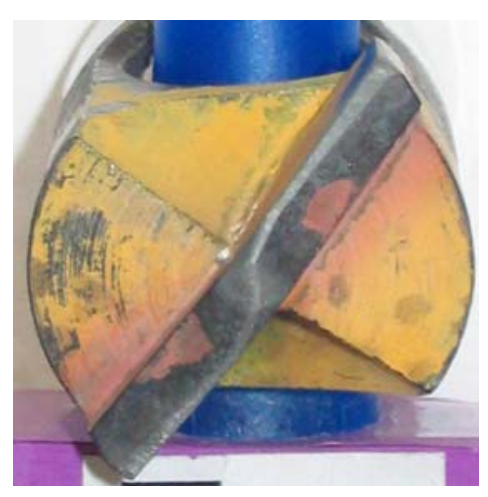

P.R. = $0.4 \mathrm{in} / \mathrm{sec}$

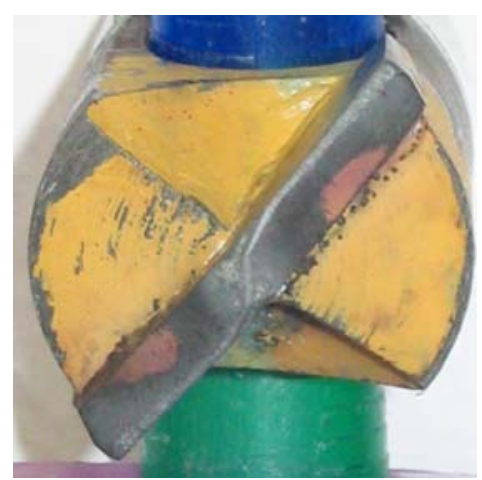

P.R. = $1.3 \mathrm{in} / \mathrm{sec}$
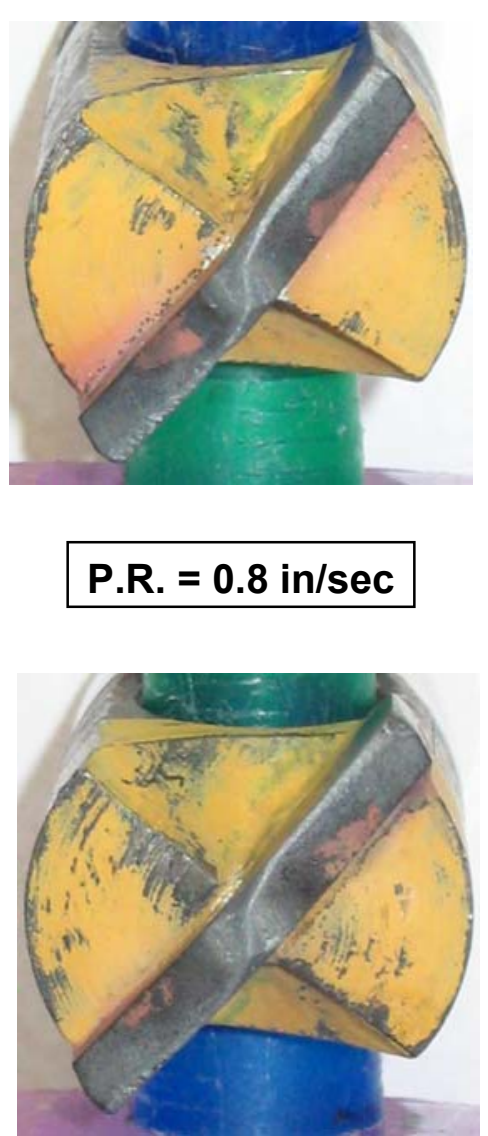

$$
\text { P.R. }=1.5 \mathrm{in} / \mathrm{sec}
$$

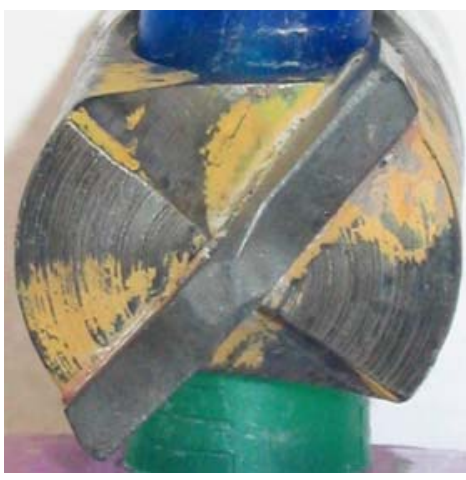

$$
\text { P.R. = } 2.1 \mathrm{in} / \mathrm{sec}
$$

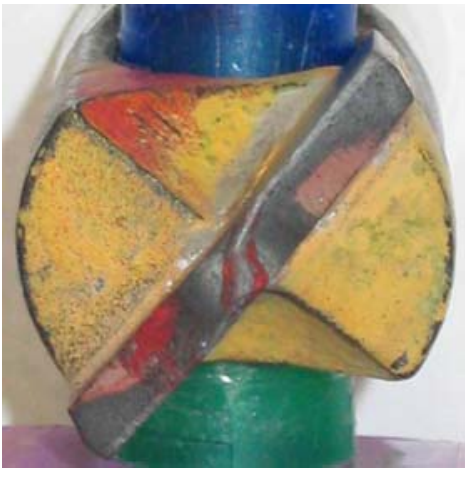

$$
\text { P.R. = } 1.1 \mathrm{in} / \mathrm{sec}
$$

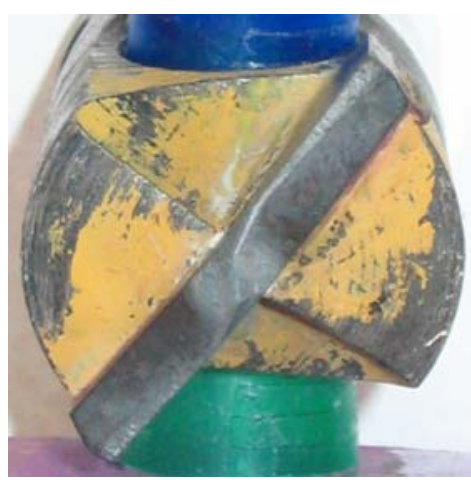

$$
\text { P.R. = } 1.7 \mathrm{in} / \mathrm{sec}
$$

Figure 5.59 Drill bit $($ R.R. $=600 \mathrm{rpm}$, number of holes drilled $=1)$ 


\section{CHAPTER 6}

\section{DATA VISUALIZATION AND DATA BASE SOFTWARE}

\subsection{Requirements and Capabilities for MRGIS}

In a production environment, manual interpretation and management of the collected drilling parameters is not practical since hundreds of holes will be drilled a production day. A new software package, MRGIS (Mine Roof Geology Information System), has been developed to allow mine engineers to make use of the large amount of roof drilling parameters for roof support design.

The basic requirements and goal for MRGIS include:

- A stand alone software

- Run on PCs under Windows 9.x, Me and XP

- Flexible development environments

- Inexpensive distribution cost

- Important machine data from an ASCII file which contains drilling parameters recorded during drilling a roof hole

- Conduct data clearing for removal of possible errors and useless data when importing machine data into MRGIS automatically

- Store cleaned machine data in a database

- Allow the user to manage the information about drilling holes

- Allow the user to enter or select conversion factors

- Allow the user to enter bit parameters

- Allow the user to enter or derive the location of the collar of a hole

- Import and display current CAD format mine map (.DXF format file)

- Match the drilling hole map to imported mine map

- Track mouse movement with northing and westing coordinate 
- Provide a routine to identify roof fractures based on drilling parameters and their locations using the rules developed by WVU

- Provide map tools (Zoom in, Zoom out, Pan, and Full extent) to allow user to search the specific area

- Allow the user to add a new mine map and corresponding hole location map

- Allow the user to remove all layers

- Allow the user to select any drilling hole on map

- Display roof fractures and their locations

Considering the distribution cost for hundreds of potential users, Visual Basic, Map Objects and OpenGl are selected as the development environments. This choice provides a flexible and powerful prototype and development environment. MS Access is selected as database engine.

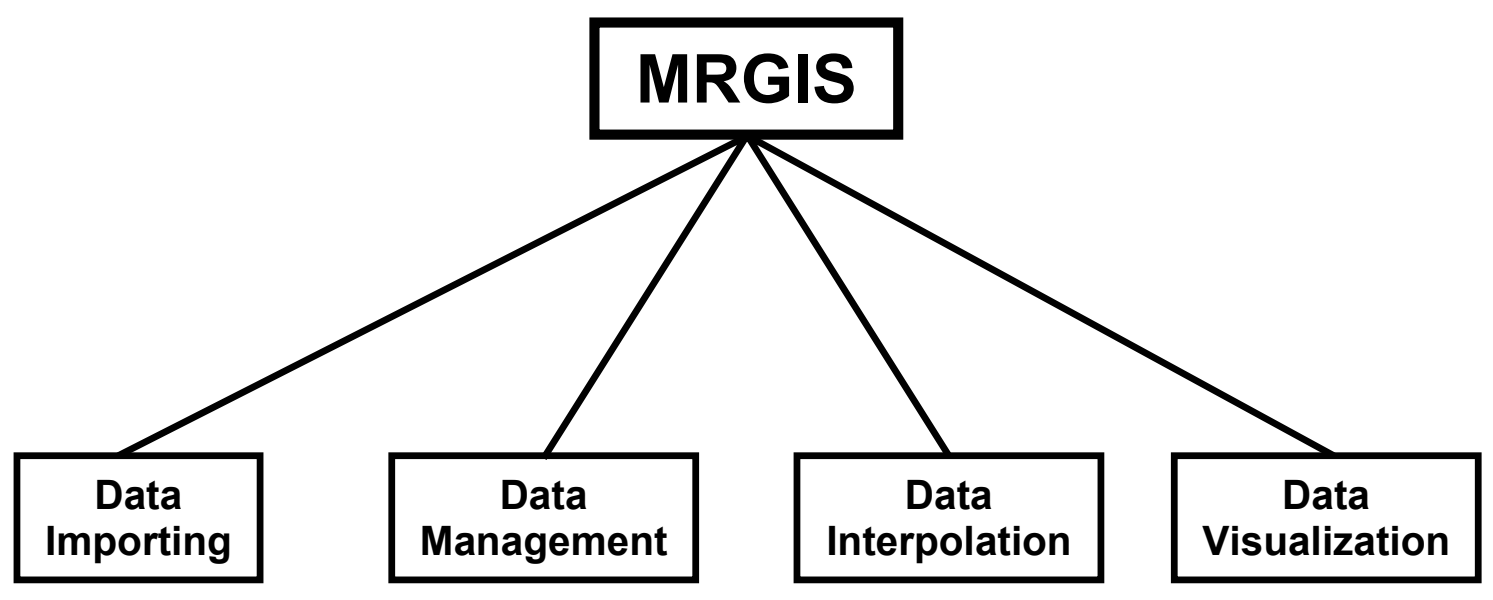

Figure 6.1 Structure of MRGIS

MRGIS consists of four modules developed for separate tasks (Figure 6.1):

- Data importing and cleaning

- Data management

- Data interpretation

- Data visualization 


\subsection{Data Import and Data Cleaning}

When a J.H. Fletcher \& Co.'s twin-boom roof bolter is drilling a hole into the roof, drilling parameters are collected into an ASCII file with a specialized microprocessor. Drilling parameters data recorded by roof bolter are referred to as machine data and the ASCII file containing machine data is referred to as machine data file. A machine data file has two headlines and 17 column data (before 2004) or one headline and 15 column data (after 2004) as shown in Figure 6.2. 8 columns represent feed pressure, feed flow, rotation pressure, rotation flow, rotation rate, mast position, carriage position, and oil temperature, respectively. Other columns represent the information such as vacuum pressure, control signal and status of the electronics that indicate control condition but are not used for the interpolation of roof geology directly in this research. The drilling parameters are recorded every 0.1 second so a 54 in long hole will have 250 to 850 records, depending on the penetration rate and roof geology. Every hole drilled by the roof bolter will have such a file.

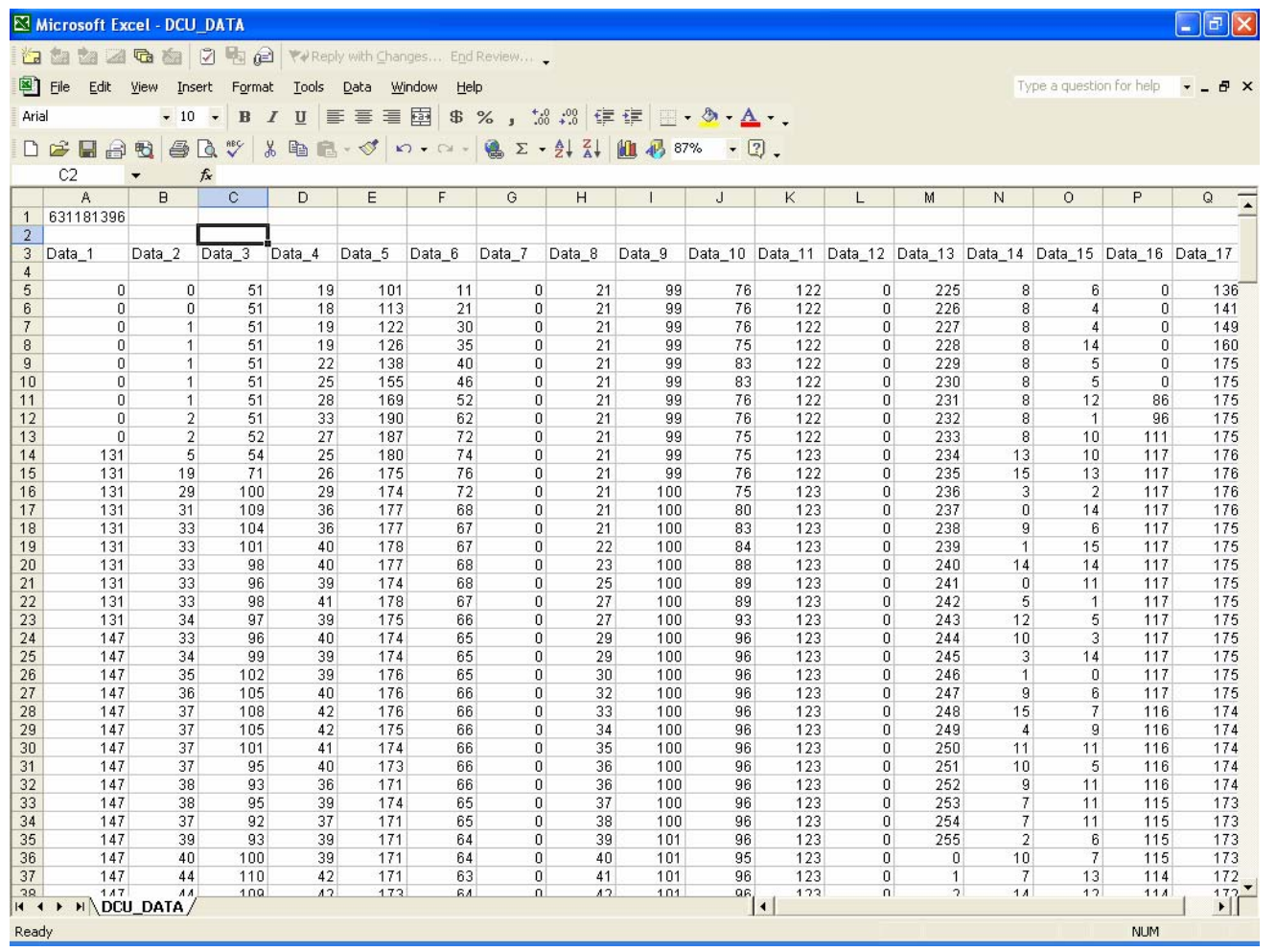

Figure 6.2 Machine data 
Since 8 out of 17 or 15 columns in a machine data file are used for the identification of roof geology, there is no need to import all data recorded in a machine data file into MRGIS in order to save the space for storing the data. Additionally, noise data is produced at the beginning and end of the hole and when the bit is plugged. MRGIS contains a procedure to remove noise data automatically.

Only the records having non-zero value in their columns are imported into MRGIS. A non-zero value indicates the DCU is controlling the drill. Hole mouth position is calculated from the first record with non-zero value in the first column and saved in the database of MRGIS. Before a record is imported into MRGIS, its bit position is calculated and compared with the bit position of the previous record, this record will be filtered out to ensure that every record for the same hole has a unique bit position.

To prevent a redundant machine data file from being imported into MRGIS, a checking procedure is also provided to ensure there is a unique set of machine data for each hole in MRGIS.

When drilling a hole in the roof, the operator of the roof bolter may stop drilling before the bit reaches the designed hole depth. This may be due to bit jam, clog, or other reasons. The same hole may be drilled again until its completion. This will produce two sets of machine data files for the same hole with different file names, one with uncompleted machine data and another with completed machine data. In order to distinguish the completed machine data file from uncompleted one, a checking procedure is provided in MRGIS to check the value of final bit position in a machine data file. Before a machine data file is imported into MRGIS, the user is asked to enter or select the designed hole depth. The designed hole depth is used to compare with the final bit position. If the final bit position is less than $0.95 \times$ designed hole depth, the machine data in the machine data file will be classified as uncompleted and is removed from the database. A message will pop up to remind the user that the machine data file being imported is invalid.

MRGIS is specifically designed for the purpose of identifying roof geology from roof drilling data recorded by J.H. Fletcher \& Co.'s roof bolter including DCU. it is 
compatible for the format of the machine data files produced by J.H. Fletcher \& Co.'s roof bolters and DCUs. If the user tries to import the data from a file with a format different from that of machine data file, an error will be produced and the importing process will be stopped. If one is not sure if a data file with correct format is being imported, the user can use preview to check the format of a data file manually although it may take longer time to import data (Figure 6.3).

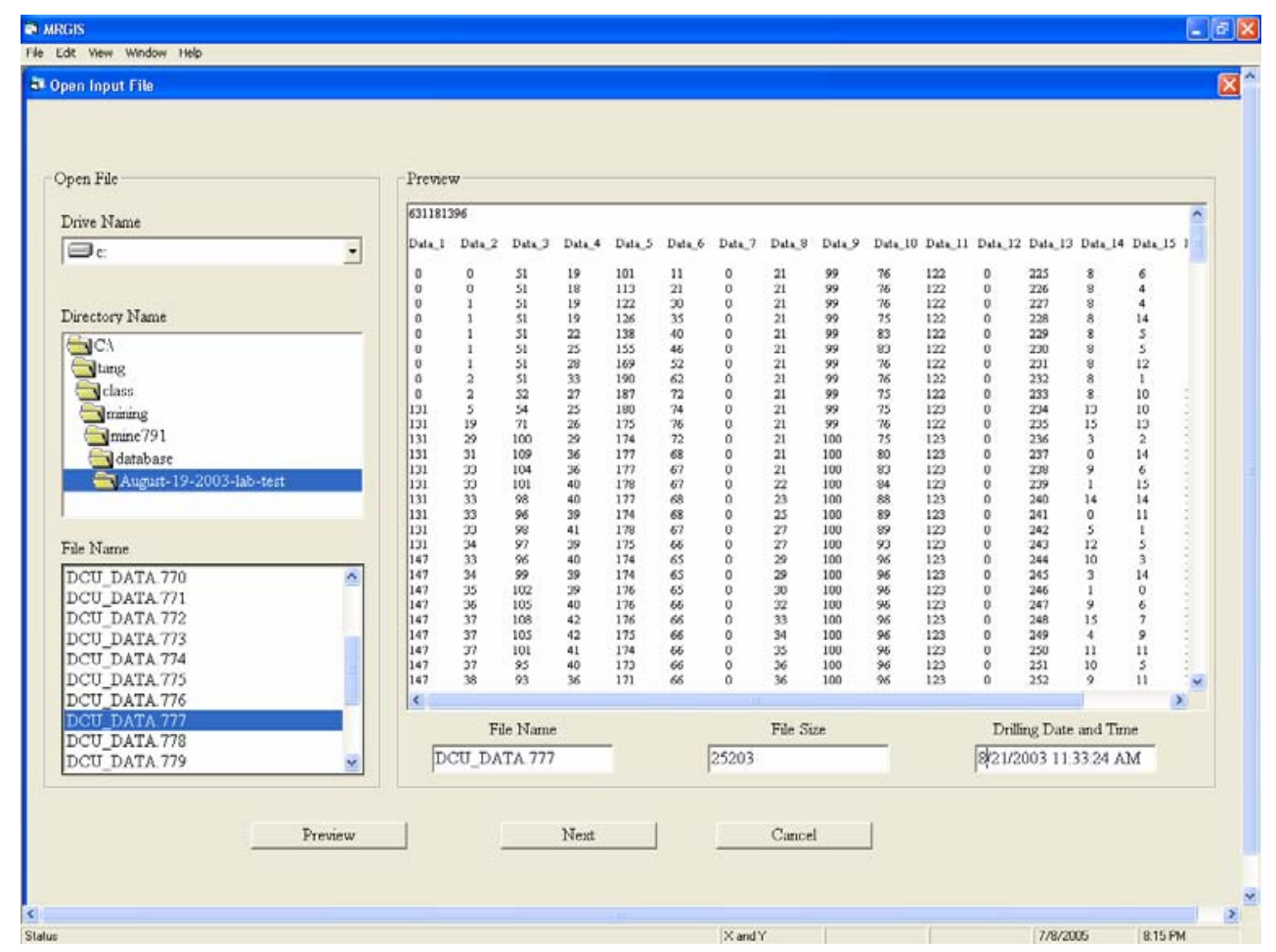

Figure 6.3 Interface for data importing

When a machine data file is imported, its name, size, data and time created are also automatically imported into the database of MRGIS, which can be used as searching criteria. The data and time created of a machine data file can also be used as the data and the time of finishing drilling the hole.

Roof geology data is directly related to specific location in underground. People who use geology data are interested in not only "What" but also "Where". To make roof geology data meaningful and useful, spatial data is necessary. Distinguishing itself from traditional geographic data which usually employs 2 dimensional (X and Y) coordinate 
system, roof geology data carries information associated with $\mathrm{Z}$ to represent location. Unfortunately, a machine data file contains only $\mathrm{Z}$ data (bit position above the roofline) but not $\mathrm{X}$ and $\mathrm{Y}$ information of a bolt hole. Although it is not elevation which is commonly used as $\mathrm{Z}$ value in Geographic Information System, bit position provides enough $\mathrm{Z}$ information from roof support design perspective because people are more interested in what geology it is at what location above the roof line when designing roof bolting. So there is no need to convert bit position into elevation. But without $\mathrm{X}$ and $\mathrm{Y}$ data, drilling parameters are still less useful for roof support designing since people often rely on the knowledge of the geology within a relatively larger area instead of at the point of interest or nearby. Manually calculating and entering $\mathrm{X}$ and $\mathrm{Y}$ data for every drilling hole is time-consuming and labor intensive since hundreds of bolting holes will be drilled each day in a mine. To solve this problem, a routine is being developed to automatically drive $\mathrm{X}$ and $\mathrm{Y}$ location information of the drilling holes based on the drilling time, drilling sequence, and entry orientation. It is indispensable to develop the system for measuring and recording the relative location of each hole automatically.

Conversion factors and information about the seam being mined also need to be entered or selected from database when importing a machine data file.

\subsection{Data Base Design}

One of the tasks of MRGIS is to store and manage drilling parameters. To accomplish this goal, a database is created in MRGIS.

A "database" is a collection of interrelated data specifically designed to be shared by multiple users. Data redundancy is controlled and a uniform approach is used for accessing and modifying data within a database. Using a database to store the data has many advantages. Most importantly, all of the data is stored together to prevent data files from being lost or being updated without authorization. Any required updating can be completed efficiently with data integrity being maintained.

In MRGIS, the database has been implemented as MS Access (.mdb) file. This approach has been taken in order to ensure that there are commercial tools to alter and access the contents of the database besides MRGIS. Also we have implemented the functionality and the User Interface for the database by means of a form for editing. 
Design philosophy of the database in MRGIS:

- Only the information about drilling holes, such as $\mathrm{X}$ and $\mathrm{Y}$, conversion factors, bit parameters, and seam name in which the hole is drilled, are stored in database in order to make the required storage volumes smaller and make data structure simple.

- Vector data model and 2D point data structure are selected to represent the location (X and $\mathrm{Y}$ ) of the collar of a drilling hole. Bit position $(\mathrm{Z})$ data are stored together with other machine data in a separate table from the table containing the location data of hole collars. This approach can avoid redundancy and make it easier to maintain the database.

- Only machine data are stored in database. The data derived or interpreted from machine data are calculated and stored in temporary tables only when being queried and will be deleted from the database once query is finished in order to save storage space and provide more flexibility for different sets of conversion factors and interpretation rules.

- Only the information about the collar of a drilling hole can be edited to keep data integrity.

- Database access is accomplished though ADO (ActiveX Data Object). ADO is the high-level data access architecture and the standard data access object model across Microsoft tools. MapObjects now supports ADO as a source of data for Table objects so a MapObjects Table object can be populated with data from a MS Access database (using the MS Jet 4.0 OLE DB Provider) and set up an AddRelate to join this information to a shapefile.

\subsection{Data Interpretation}

The core of MRGIS is its capability to allow the users to make use of roof drilling parameters to understand roof geology. What a user of MRGIS is interested in is the roof geology features interpolated from drilling parameters rather than drilling parameters 
themselves. So a procedure has been built in MRGIS to interpret the roof drilling parameters into roof geology features automatically based on interpretation rules.

As mentioned above, a number of researchers have developed the rules for interpolating the roof geology features based on roof drilling parameters. Each set of rules have their advantages and limitations. The methodology developed in this research is used to estimate roof geology such as the existence and location of void/fracture and the roof rock strength based on drilling parameters in MRGIS.

\subsection{Roof Geology Visualization}

In production environment, hundreds of bolting holes will be drilled everyday. Providing an easy and convenient method to query and display roof geology information resulted from the interpolation of roof drilling data is another key task of MRGIS. The best way to accomplish this task is to visualize it by using maps and figures. A number of researchers have reported their studies on visualization of geological data ${ }^{(14),(20)-(24)}$. But, they did not provide an efficient querying method.

In MRGIS, two roof geology visualization methods are provided. The first is $2 \mathrm{D}+1 \mathrm{D}$ as shown in Figure 6.4. The interface is divided into 2 parts. The left parts is $2 \mathrm{D}$ map which is used to display the location ( $\mathrm{X}$ and $\mathrm{Y}$ ) of drilling hole collar. The right part is $1 \mathrm{D}$ figure which is used to display geological features along $\mathrm{Z}$ direction of a drilling hole.

The following map tools are provided to help the user zoom in an area of interests:

- Zoom In

- Zoom Out

- Pan

- Full Extend

Also a ruler tool is provided to enable the user to measure the distance in map units on the 2D map. 
To facilitate finding a specific area, MRGIS supports AutoCad DXF file format so a DXF formatted mine map can be imported into MRGIS. This DXF file is used as a means to relate the location of drilling holes to the mine map.

Before an AutoCad mine map file is imported, some preparation work may be necessary. An AutoCad mine map file may contain multiple layers which represent multiple seams and the surface. Such a file needs to be divided into multiple files so that each file contains the information for only one seam.

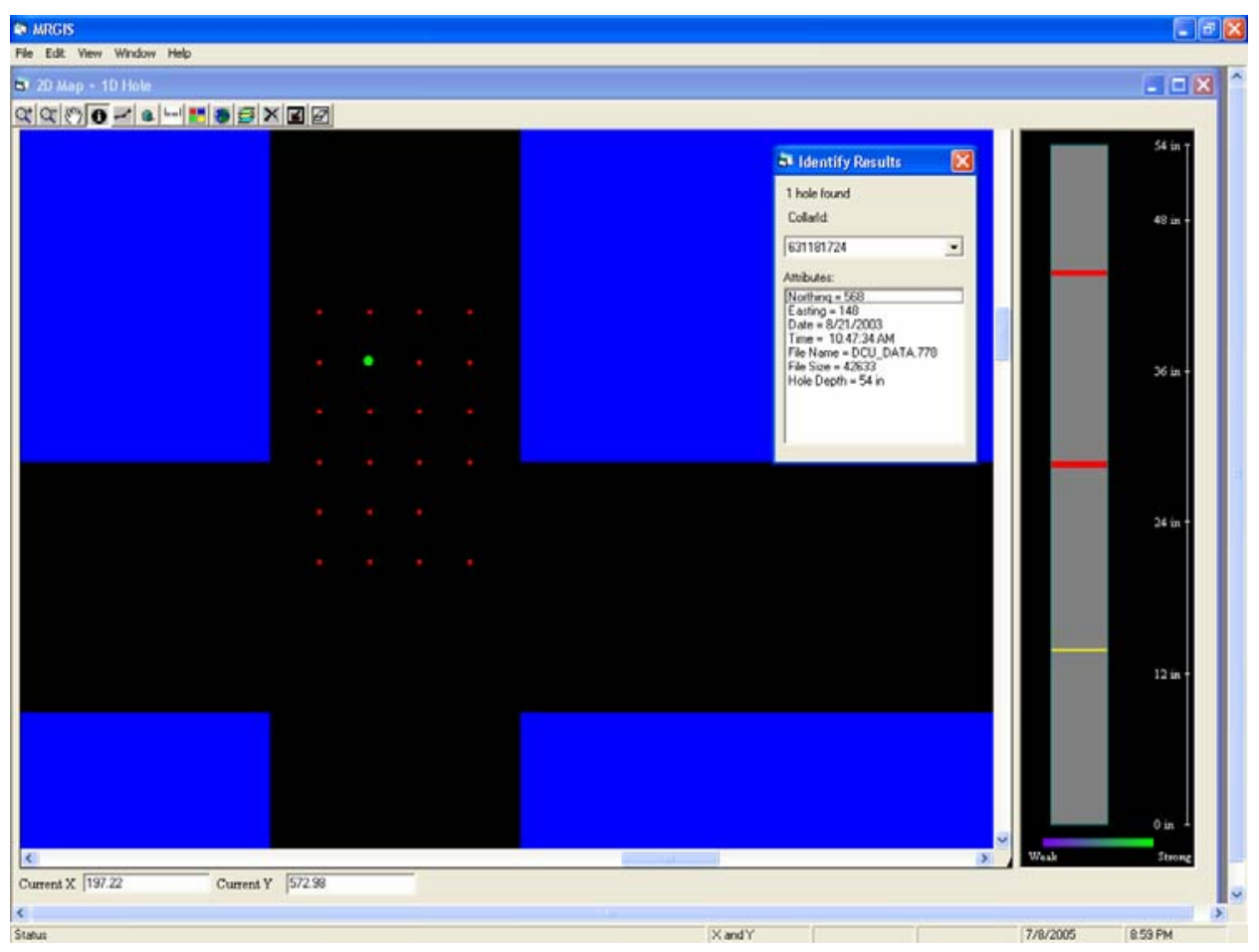

Figure 6.4 Roof geology visualization: 2D + 1D

When being imported into MRGIS, only line and area objects in an AutoCad mine map file are imported and point objects are filtered out so that all point objects in 2D map only represent the locations of drilling holes.

Correspondingly, the locations of drilling hole collars also relate to a certain seam. Considering the fact that a large number of machine data files will be generated and imported in MRGIS every day, a routine is provided to automatically produce drilling hole map for each seam when importing machine data files is finished. This approach can 
keep drilling hole map up to date. The user can also create a specific drilling hole map by using different searching criteria such as drilling date or hole length.

To find a drilling hole, the user must add at least one drilling hole map into 2D map. Adding a DXF mine map is not a must but can make it much easier to determine the location of a drilling hole.

The user can use the mouse to select a hole on 2D map. The selected hole will flash and then be highlighted. The interpretation results of drilling parameters of the selected hole will be displayed in 1D figure. A message box will pop up to display geological features at a selected position by clicking on 1D figure. Meanwhile a form will pop up to display the information about the collar of selected hole.

The second visualization method is $2 \mathrm{D}+2 \mathrm{D}$. It allows the user to select multiple holes at the same time and display the results of the interpretation (Figure 6.5). Similar to $2 \mathrm{D}+1 \mathrm{D}$, the interface is also divided into two parts. The left one is also used to display 2D map but uses a smaller display area. The user can use the mouse to draw a polyline on 2D map and the geological features of all hole on or close to the polyline will be displayed on the right side if the interface.

The third visualization method is $2 \mathrm{D}+3 \mathrm{D}$. This feature can display a $2 \mathrm{D}$ mine map and a 3D figure that represents the results of the interpretation of roof drill holes at the same time (Figure 6.6). It allows the users to do a "virtual walk" through the underground spaces to check out roof geological properties at different locations.

\subsection{Hardware}

Since large datasets are processed in MRGIS, a high performance computer is needed in order to make fast operations.

MRGIS was actually test on a PC with the following hardware:

- Pentium 4 processor $(2.0 \mathrm{GHz})$

- RAM 512 MB

- $\quad 17^{\prime}$ Monitor, $1,152 \times 864$ pixels and true color were used as video settings 


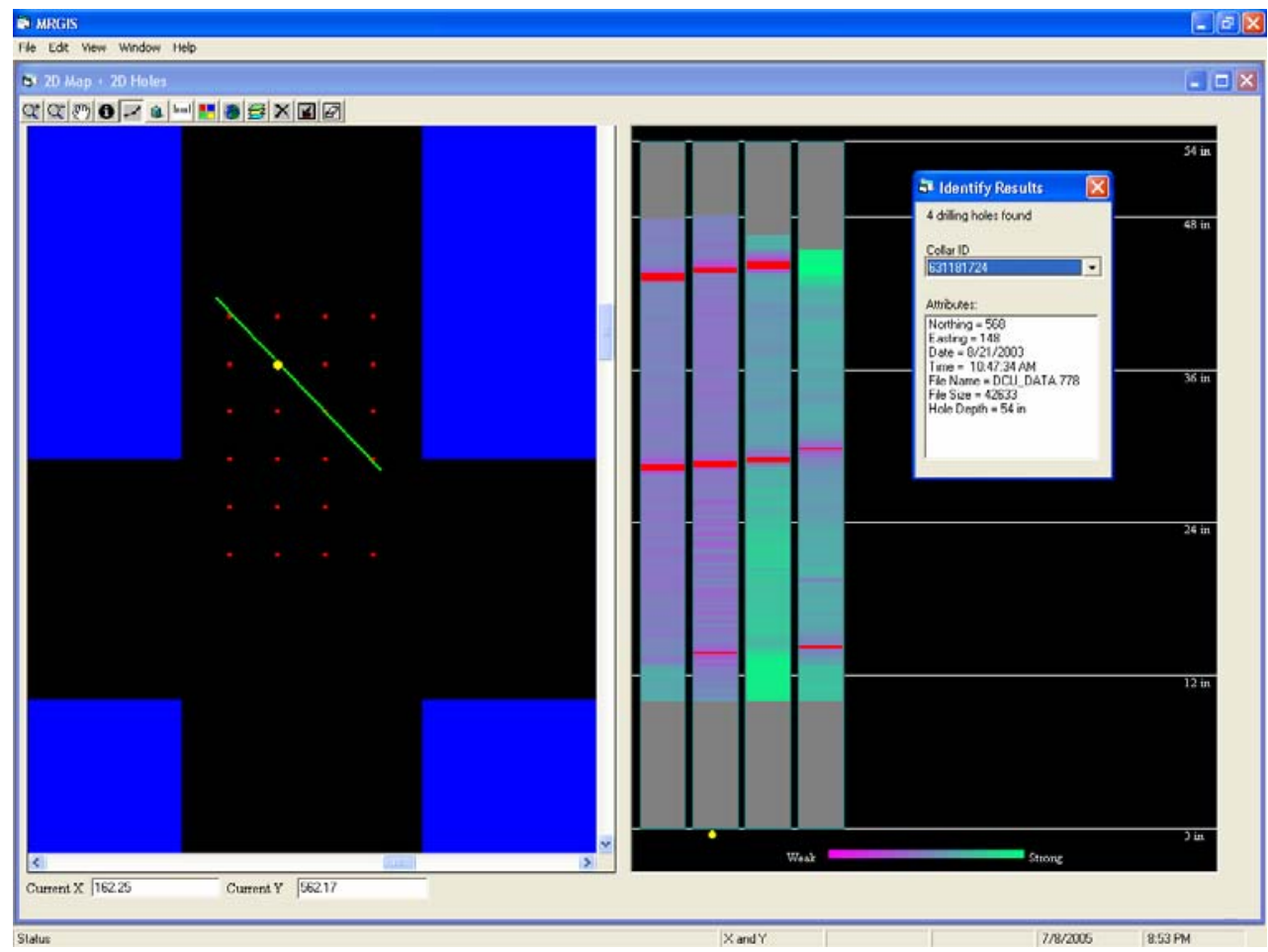

Figure 6.5 Roof geology visualization: $2 \mathrm{D}+2 \mathrm{D}$

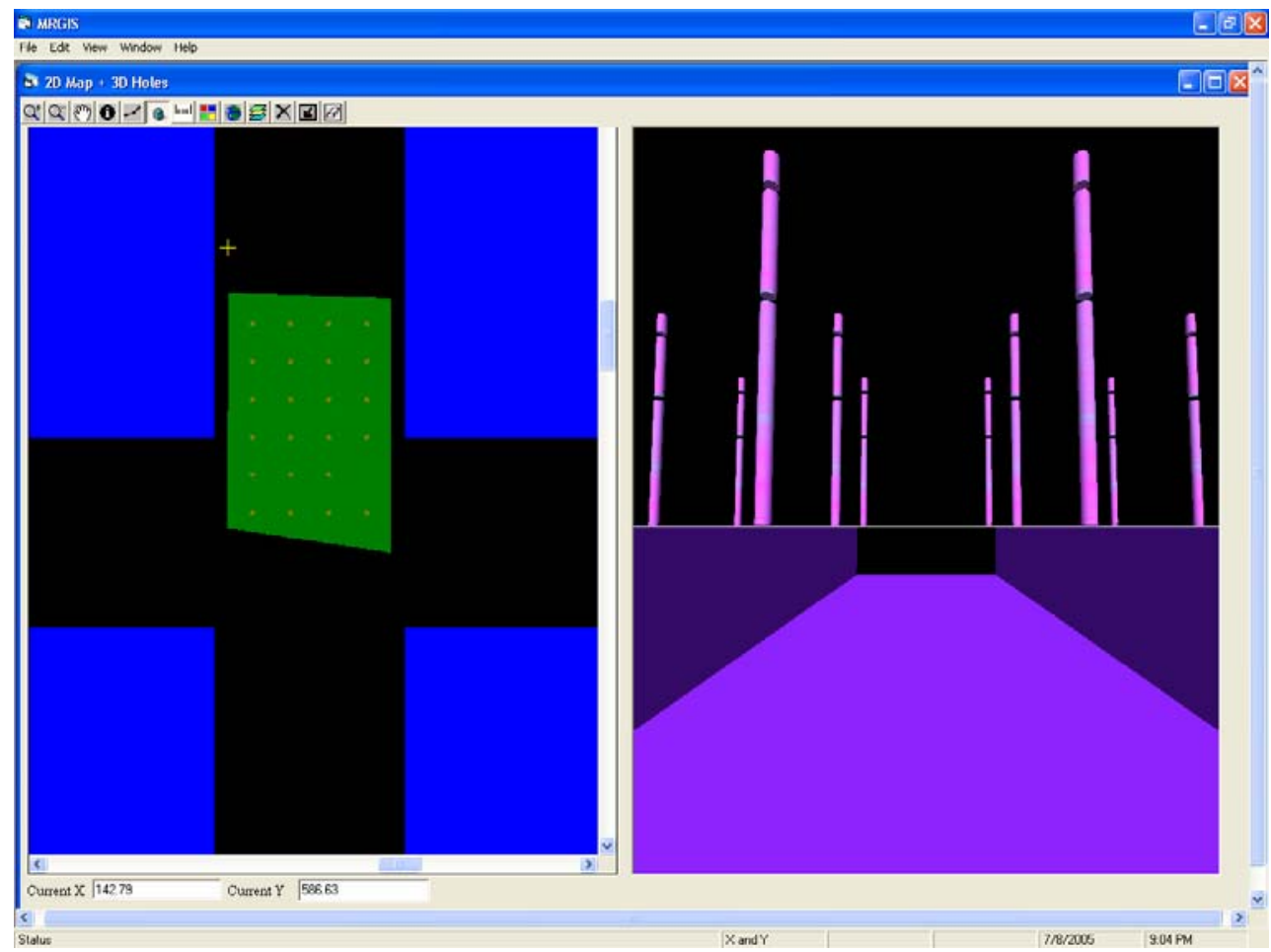

Figure 6.6 Roof geology visualization: $2 \mathrm{D}+3 \mathrm{D}$ 


\section{CHAPTER 7}

\section{CONCLUSIONS}

The objectives of this research are: (1) to develop a method for identifying the geological features in the mine roof strata based on the drilling parameters obtained during normal roof bolting operation, and (2) to develop a new software package, MRGIS (Mine Roof Geology Information System), to allow mine engineers to make use of the large amount of roof drilling parameters for roof support design.

From the results of a series of laboratory and underground tests, feed pressure is found to be a good indicator for identifying the voids/fractures and estimating the roof rock strength when both penetration rate and rotation rate were controlled or kept constant. Methods for determining quantitatively the location and the size of void/fracture and estimating the roof rock strength from the drilling parameters of roof bolter have been developed. In addition, characteristics of the machine, sensors, and drilling control algorithm, etc were also studied as to eliminate these effects from drilling data as much as possible. The following conclusions can be made:

\section{$\underline{\text { Void/Fracture Prediction }}$}

- The feed pressure trends of dropping to the level of drilling in the air when a void / fracture in rock is encountered can be used to detect the voids/fractures. This is the main criteria of void/fracture prediction. In addition, in order to enhance the prediction accuracy, a supplemental prediction criterion is developed considering the shape of feed pressure valley.

- From the results of lab and field tests, the prediction results show that a very high prediction percentage have been achieved for the 1/8-in or larger voids. But, a void/fracture of 1/16-in or smaller are difficult to predict by the system developed.

- The width of the plateau at the bottom of a feed pressure valley is much closer to the size of the void/fracture. However, the accuracy of predicting the void / fracture size using drilling parameters measured by the current data collecting system is too low to be acceptable for voids/fractures smaller than 1/2-in. 


\section{Estimation of Roof Rock Strength}

- Feed pressure is the most sensitive and reliable parameters when the strength of roof rock changes under the current system. In order to eliminate the machine effect, the net feed pressure is recommended for estimating rock strength instead of the feed pressure.

- Both penetration rate and rotation rate have obvious impact on the magnitude of the feed pressure.

- The strength of roof rock can be determined / classified based on the magnitude of feed pressure because it takes both the effects of penetration rate and rotation rate into account.

\section{Development of Data Visualization and Database Software (MRGIS)}

- A new software package, MRGIS (Mine Roof Geology Information System), has been developed to allow mine engineers to make use of the large amount of roof drilling parameters for roof support design. MRGIS consists of four modules: data importing and cleaning, data management, data interpretation, and data visualization. 


\section{CHAPTER 8}

\section{RECOMMENDATIONS FOR FUTURE RESEARCH}

Although a progress has been made in this research regarding understanding the relationship of the roof rock geology properties and drilling parameters, more work needs to be done in order to make the results of the research applicable in the production environment. The following topics are recommended for future research:

1. Conduct more drilling tests in the roof consisting of rock layers with different strengths to observe the behavior of drilling parameters in transition zones

2. Conduct more compensation runs to establish a model for estimate machine effects on rotation pressure

3. Develop a methodology to evaluate and estimate bit wear

4. Evaluate the effects of DCU control commands on drilling parameters

5. Conduct more field tests, especially in the roof with UCS ranging from 3500 psi to $8500 \mathrm{psi}$, to improve the classification of rock strength boundary planes for estimating rock strength

6. Develop a method to automatically record $\mathrm{X}$ and $\mathrm{Y}$ coordinates of a drilling hole 


\section{REFERENCES}

1. Freme, F., U.S. Coal Supply and demand: 2003 Review. Mining Engineering, Vol. 56, No.5, May 2004, pp 38-47

2. Coal Production and Number of Mines by State and Coal Rand, 2003, http//www.eia.doe.gov/coal/palge/acr/table6.html

3. Teale, R. The Concept of Specific Energy in Rock Drilling. Int. J. Rock Mech. Min. Sci. \& Geomech, 2, 1965, pp. 57-73.

4. Finfinger, G., Peng, S., Gu, Q., Wilson, G., An approach to Identifying Geological Properties from Roof Bolter Drilling Parameters. Proc. 19th Conf. On Ground Control in Mining. Ed.: S.S. Peng. West Virginia University, Morgantown, 2000. pp: $1-12$

5. Finfinger, G., Luo, Y., Peng, S., Wilson, G., Identificating of Lithologic Changes Using Drilling Parameters. Paper presented at 2002 SME Annual Meeting held at Phoenix, AZ, Pre-print 02-194 , 7 pp.

6. Luo, Y., Peng, S., Mirabile, B., Finfinger, G., Wilson, G., Estimating Rock Strengths Using Drilling Parameters During Roof bolting Operations - Progress Report. Proc. 21 st Conf. On Ground Control in Mining. Ed.: S.S. Peng. West Virginia University, Morgantown, 2002. pp: 288 - 294

7. Finfinger, G., (2003). A Methodology for Determining the Character of Mine Roof Rocks, PhD Dissertation, Dept. of Mining Engineering, West Virginia University, $214 \mathrm{pp}$.

8. Gu, Q., (2003). Geological Mapping of Entry Roof in Mines, PhD Dissertation, Dept. of Mining Engineering, West Virginia University, 190 pp.

9. Peng, S. S., Tang, D. X., Mirabile, B., Luo, Y., Wilson, G., Mine Roof Geology Information System. Proc. 22nd Conf. On Ground Control in Mining. Ed.: S.S. Peng. West Virginia University, Morgantown, 2003. pp: 127 - 135

10. Mirabile, B., (2003). Geologic Features Prediction Using Roof Bolter Drilling Parameters, Master Thesis, Dept. of Mining Engineering, West Virginia University, 84 pp. 
11. Mirabile, B., Peng, S. S., Luo, Y., Tang, D. X., Roof Bolter Drilling Parameters as a Tool for Strata Prediction. Paper presented at 2004 SME Annual Meeting held at Denver, CO.

12. Thomas, B., Wilson, G., Control Technology for Roof Drill Operators. Proc. 18th Conf. On Ground Control in Mining. Ed.: S.S. Peng. West Virginia University, Morgantown, 1999. pp: 216 - 221

13. Itakura K, Sato K, Deguchi G, Ichihara Y, Matsumoto H, Eguchi H. Development of a Roof Logging System by Rock Bolt Drilling. Trans of the Institution of Mining and Metallurgy, Vol. 106, A95-184, Section A, 1997, pp. A118-A123.

14. Itakura K, Sato K, Deguchi G, Ichihara Y, Matsumoto H. Visualization of Geostructure by Mechanical Data Logging of Rockbolt Drilling and Its Accuracy. Proceedings of the 20th International Conference on Ground Control in Mining, Morgantown, WV, 2001, pp. 184-190.

15. King, R, Hicks, M, Signer, S. Using Unsupervised Learning for feature Detection in a Coal Mine Roof. Engng Applic. Artif. Intell. Vol. 6. No. 6, 1993, pp. 565-573,

16. Quinlan, J.R., C4.5: Programming for machine learning. San Francisco: Morgan Kaufmann, 1993

17. Whittaker, B.N., Singh, R.N., Sun, G., (1992), Rock Fracture Mechanics - Principles, Design and Applications, Elsevier, pp. $413-414$

18. Mishnaevsky Jr., L., (1998), Rock Fragmentation and Optimization of Drilling Tools, Fracture of Rock (Advances in Fracture Mechanics Volume 5). Ed.: Aliabadi, M. H. pp. $168-170$

19. Clark, G. B. Princples of Rock Fragmentation, John Wiley \& Sons, Inc., 1987

20. Lees, J., "Geotouch: Software for Three and Four Dimensional GIS in the Earth Sciences", http://www.unc.edu/ lees/CANDG/geotouch_New.html.

21. Kudowor, A. and Taylor, G., "Borehole Data Management and Volume Calculation", http://www.comp.glam.ac.uk/pages/staff/getaylor/papers/gis ru k98.PDF,

22. Jacobsen, T. and Hansen, M., "Geological Modeling and Editing in GIS Environment", http://www.vandmodel.dk/hydropaper_bigfig.pdf. 
23. Weaver, S. and Donze, J., "Visualizing Environmental Data using Arc View and EQuIs", http://gis.esri.com/library/userconf/proc00/professional/papers/PAP717 /p717.htm.

24. Hilbring, D., "GEOPRO 3D - A Three Dimensional GIS Tool for the Visualization and Analysis of the Water Table and Hydrogeological Profiles", http://www-ipf.bauverm.uni-karlsruhe.de/Personen/hilbring/hilbringisprspaper

210901.doc 


\section{APPENDIX A \\ CONVERSION OF DRILLING PARAMETERS}

As mentioned in Chapter 4, all drilling parameters except the data of two feed position sensors are not converted from dimensionless machine data into engineering units in this research.

One may be interested in converting these drilling parameters into engineering units so that the drilling data collected in this research can be compared with those from other resources or other drilling data collection systems.

Well-accepted engineering units for drilling parameters are thrust, penetration rate, torque, and rotation rate. In the early stage of this project, the following equations were used for conversion ${ }^{(4-11)}$ :

$$
\begin{aligned}
& \text { Thrust }=12.25 \times \pi \times \mathrm{D}^{2} \div 4 \times \text { Data_} 2, \text { lbs } \\
& \text { where D is cylinder diameter, } 3 \text { in } \\
& \text { Penetration rate }=0.02691 \times \text { Data_3, in } / \mathrm{sec} \\
& \text { Torque }=18.01 \times 10.5 \times 0.91 \times \text { Data_ } 4 /(2 \times \pi), \text { lbs-in } \\
& \text { Rotation rate }=6.977 \times \text { Data_ } 6, \text { rpm }
\end{aligned}
$$

The above equations are $\mathrm{OK}$ for the purpose of feedback control using the horsepower control mode, which is developed by J.H. Fletcher \& Co. and adjusts penetration rate and rotation rate based on a target horsepower in order to improve overall drilling and bolting consistency. But their weaknesses are obvious if being used for comparison with the drilling data from other resources because the effects of the drilling machine on these measured drilling parameters are also included in calculation. To drill a hole in a given rock under a given drilling setting, the energy consumed for rock fragmentation is supposed to keep within a certain range if a same type of drill bit is used while the energy consumed by a drilling machine itself may vary due to different machine designs. Therefore machine effects should be excluded in order to make the 
comparison meaningful. Thus thrust should be defined as rock resistance to bit penetration while torque as the moment of rock resistance to bit rotation.

Based on the above definitions and the discussions about the effects of drilling settings and the oil temperature on drilling parameters in Charter 5, the previous conversion equations should be revised as follows:

Thrust $=12.25 \times \pi \times \mathrm{D}^{2} \div 4 \times$ Netfeedpressure, lbs where $\mathrm{D}$ is cylinder diameter, 3 in

Netfeedpressure is the calculated result using equation (5.4)

Penetration rate: using equations $(5.16 \sim 5.19)$

Torque $=18.01 \times 10.5 \times 0.91 \times($ Data_ $4-15) /(2 \times \pi)$, lbs-in

As discussed in Chapter 5, different drilling settings and oil temperatures have a large impact on the magnitude of the rotation pressure in compensation runs and a model needs to be developed to eliminate these effects. Before this model is available, it is recommended to use 15 as the estimation of machine effects. Please note that this estimation may result in a relatively large error because the rotation pressure is less sensitive to the change of the rock strength.

There is no need to revise the equation for the rotation rate because no machine effects on it were found out.

In addition, the oil temperature can be obtained from the following equation:

Oil temperature $=1.529 \times$ data $\_11-40, \mathrm{~F}^{\circ}$ 


\section{APPNDIX B}

\section{DRILLING PARAMETERS PLOTS}

\begin{tabular}{|c|c|}
\hline Data Num. & Figures \& Tables \\
\hline 52 & 5.43 \\
\hline 54 & 5.43 \\
\hline 103 & $5.37,5.38,5.39,5.40$ \\
\hline 106 & $5.23,5.26,5.34,5.35,5.37,5.38,5.39,5.40$ \\
\hline 109 & $5.37,5.38,5.39,5.40$ \\
\hline 112 & $5.37,5.38,5.39,5.40$ \\
\hline 116 & $5.23,5.26,5.34,5.35,5.37,5.38,5.39,5.40$ \\
\hline 120 & $5.37,5.38,5.39,5.40$ \\
\hline 123 & $5.37,5.38,5.39,5.40$ \\
\hline 137 & $5.37,5.38,5.39,5.40$ \\
\hline 140 & $5.37,5.38,5.39,5.40$ \\
\hline 144 & $5.23,5.26,5.34,5.35,5.37,5.38,5.39,5.40$ \\
\hline 147 & $5.37,5.38,5.39,5.40,5.51$ \\
\hline 148 & 5.51 \\
\hline 149 & 5.51 \\
\hline 151 & $5.36,5.37,5.38,5.39,5.40$ \\
\hline 154 & $5.23,5.34,5.35,5.36,5.37,5.38,5.39,5.40$ \\
\hline 157 & $5.36,5.37,5.38,5.39,5.40$ \\
\hline 161 & 5.36 \\
\hline 167 & $5.37,5.38,5.39,5.40$ \\
\hline 170 & 5.2 \\
\hline 191 & $5.37,5.38,5.39,5.40$ \\
\hline 198 & $5.23,5.26,5.34,5.35,5.37,5.38,5.39,5.40$ \\
\hline 201 & $5.37,5.38,5.39,5.40$ \\
\hline 210 & $5.37,5.38,5.39,5.40$ \\
\hline 266 & 5.46 \\
\hline 267 & 5.46 \\
\hline 268 & 5.46 \\
\hline 269 & 5.46 \\
\hline 270 & 5.46 \\
\hline 272 & 5.47 \\
\hline 273 & 5.47 \\
\hline 274 & 5.47 \\
\hline 275 & 5.47 \\
\hline 276 & 5.47 \\
\hline 307 & $5.37,5.38,5.39,5.40$ \\
\hline 312 & $5.37,5.38,5.39,5.40$ \\
\hline 316 & $5.37,5.38,5.39,5.40$ \\
\hline 320 & $5.37,5.38,5.39,5.40$ \\
\hline 324 & $5.37,5.38,5.39,5.40$ \\
\hline
\end{tabular}




\begin{tabular}{|c|c|}
\hline 337 & $5.37,5.38,5.39,5.40,5.49$ \\
\hline 338 & 5.49 \\
\hline 339 & 5.49 \\
\hline 341 & $5.37,5.38,5.39,5.40$ \\
\hline 349 & $5.37,5.38,5.39,5.40$ \\
\hline 353 & $5.37,5.38,5.39,5.40,5.50$ \\
\hline 354 & 5.50 \\
\hline 355 & 5.50 \\
\hline 357 & $5.37,5.38,5.39,5.40$ \\
\hline 371 & 5.53 \\
\hline 374 & 5.53 \\
\hline 376 & 5.53 \\
\hline 378 & 5.53 \\
\hline 380 & 5.53 \\
\hline 392 & $5.27,5.28,5.31$ \\
\hline 423 & $5.29,5.30,5.31$ \\
\hline 434 & $5.12,5.30$ \\
\hline 398 & $5.2,5.4,5.30$ \\
\hline 402 & $5.22,5.30,5.37,5.38,5.39,5.40$ \\
\hline 403 & $5.30,5.37,5.38,5.39,5.40$ \\
\hline 404 & $5.30,5.37,5.38,5.39,5.40,5.52$ \\
\hline 405 & $5.30,5.37,5.38,5.39,5.40,5.52$ \\
\hline 406 & $5.30,5.37,5.38,5.39,5.40$ \\
\hline 408 & $5.30,5.37,5.38,5.39,5.40$ \\
\hline 409 & $5.30,5.37,5.38,5.39,5.40$ \\
\hline 410 & $5.30,5.37,5.38,5.39,5.40$ \\
\hline 411 & $5.30,5.37,5.38,5.39,5.40$ \\
\hline 414 & $5.30,5.37,5.38,5.39,5.40$ \\
\hline 415 & $5.30,5.37,5.38,5.39,5.40$ \\
\hline 416 & $5.30,5.37,5.38,5.39,5.40$ \\
\hline 417 & $5.30,5.37,5.38,5.39,5.40$ \\
\hline 433 & $5.12,5.18,5.30$ \\
\hline 434 & $5.12,5.30$ \\
\hline 439 & $5.30,5.37,5.38,5.39,5.40$ \\
\hline 440 & $5.30,5.37,5.38,5.39,5.40$ \\
\hline 441 & $5.30,5.37,5.38,5.39,5.40$ \\
\hline 442 & $5.4,5.18,5.23,5.24,5.25,5.26,5.30,5.34,5.35,5.37,5.38,5.39,5.40$ \\
\hline 443 & $5.22,5.23,5.26,5.30,5.33,5.35,5.37,5.38,5.39,5.40$ \\
\hline 444 & $5.23,5.26,5.30,5.33,5.35,5.37,5.38,5.39,5.40$ \\
\hline 445 & $5.22,5.23,5.30,5.37,5.38,5.39,5.40$ \\
\hline 446 & $5.18,5.23,5.30,5.37,5.38,5.39,5.40$ \\
\hline 447 & 5.53 \\
\hline 448 & 5.53 \\
\hline 449 & 5.53 \\
\hline 450 & 5.53 \\
\hline 452 & 5.53 \\
\hline 458 & $5.27,5.30$ \\
\hline
\end{tabular}




\begin{tabular}{|c|c|}
\hline 472 & $5.30,5.41,5.56$ \\
\hline 474 & $5.30,5.41,5.56$ \\
\hline 477 & $5.30,5.41,5.56$ \\
\hline 482 & $5.30,5.41,5.56$ \\
\hline 484 & $5.30,5.41,5.56$ \\
\hline 488 & $5.30,5.41,5.56$ \\
\hline 490 & $5.30,5.41,5.56$ \\
\hline 492 & $5.30,5.41,5.56$ \\
\hline 494 & $5.30,5.41,5.56$ \\
\hline 498 & $5.30,5.41,5.56$ \\
\hline 501 & $5.30,5.41,5.56$ \\
\hline 503 & $5.30,5.56$ \\
\hline 519 & $5.30,5.33,5.41$ \\
\hline 523 & $5.30,5.33,5.41$ \\
\hline 525 & $5.30,5.33,5.41$ \\
\hline 527 & $5.30,5.33,5.41$ \\
\hline 529 & $5.30,5.33,5.41$ \\
\hline 531 & $5.30,5.31,5.41$ \\
\hline 664 & $5.7,5.8,5.30$ \\
\hline 665 & $5.7,5.8,5.30,5.32$ \\
\hline 666 & $5.7,5.8,5.30$ \\
\hline 667 & $5.7,5.8,5.30$ \\
\hline 668 & $5.7,5.8,5.30$ \\
\hline 669 & $5.7,5.30$ \\
\hline 670 & $5.6,5.7,5.8,5.11,5.30$ \\
\hline 671 & $5.11,5.30$ \\
\hline 701 & $5.7,5.30,5.40$ \\
\hline 750 & $5.5,5.7,5.10,5.30$ \\
\hline 751 & $5.5,5.7,5.11,5.13,5.30$ \\
\hline 758 & $5.5,5.7,5.10,5.30$ \\
\hline 760 & $5.5,5.7,5.10,5.30$ \\
\hline 769 & $5.6,5.7,5.11,5.30$ \\
\hline 770 & $5.11,5.30,5.40$ \\
\hline 820 & $5.7,5.30,5.32$ \\
\hline
\end{tabular}




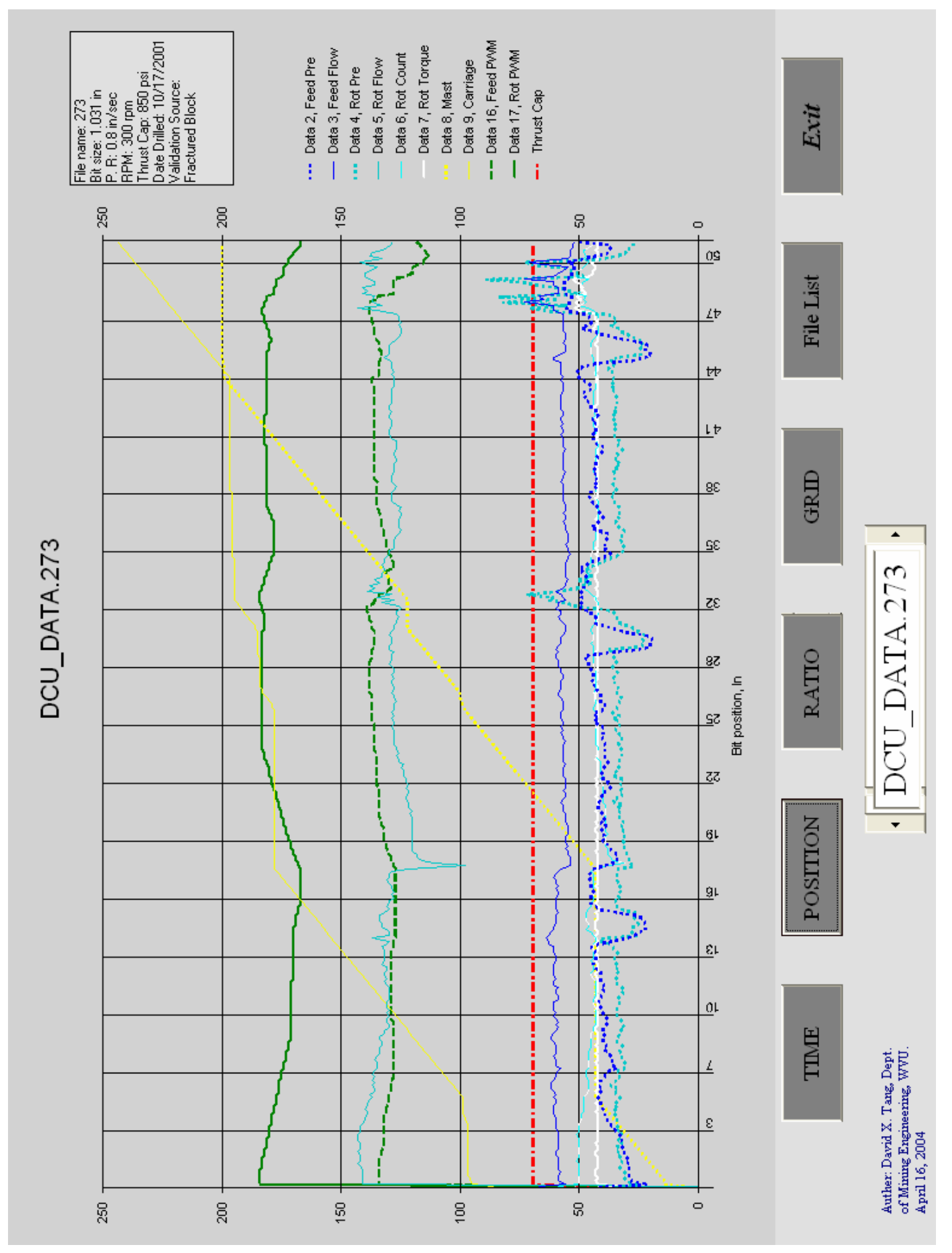




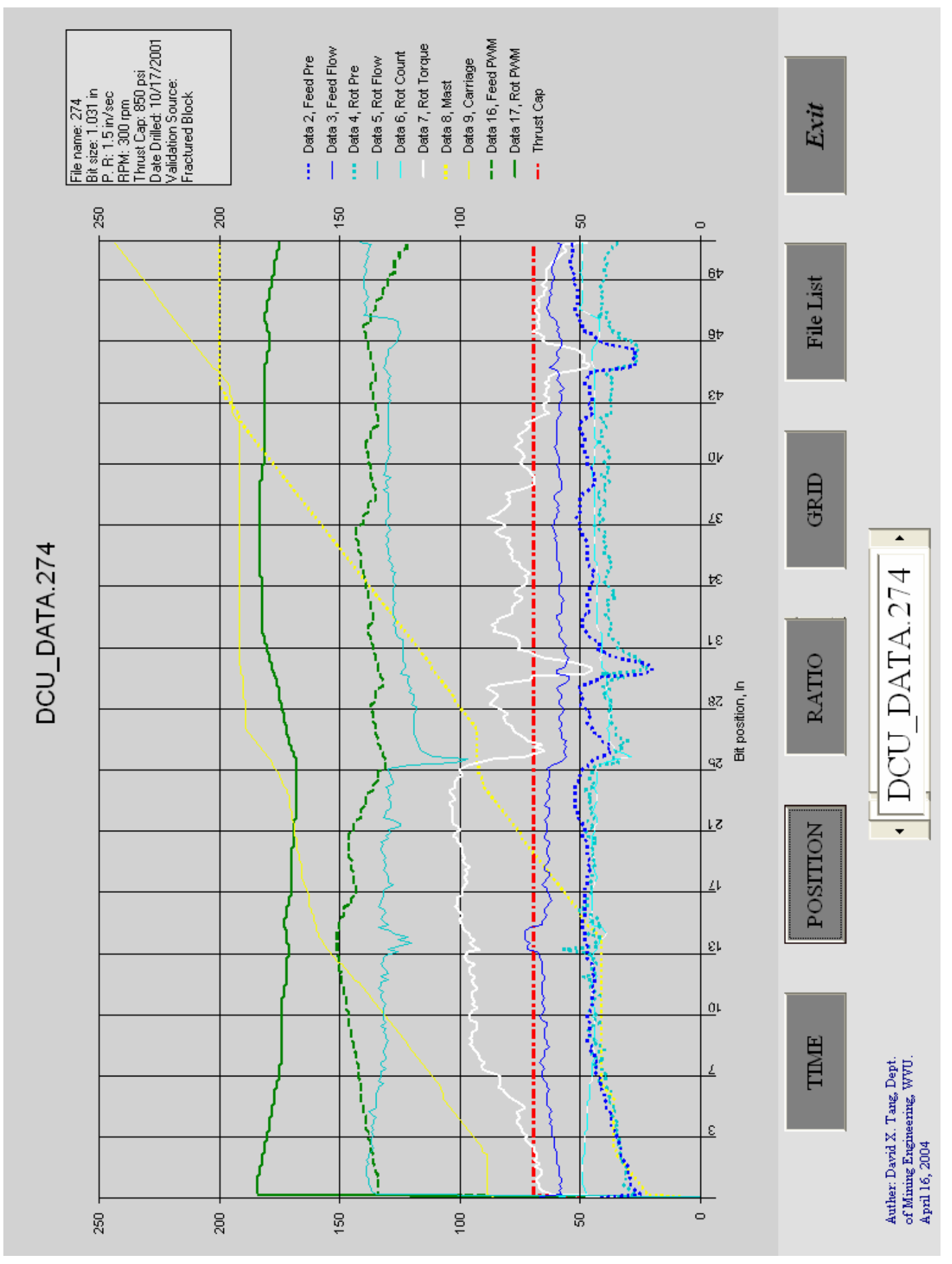




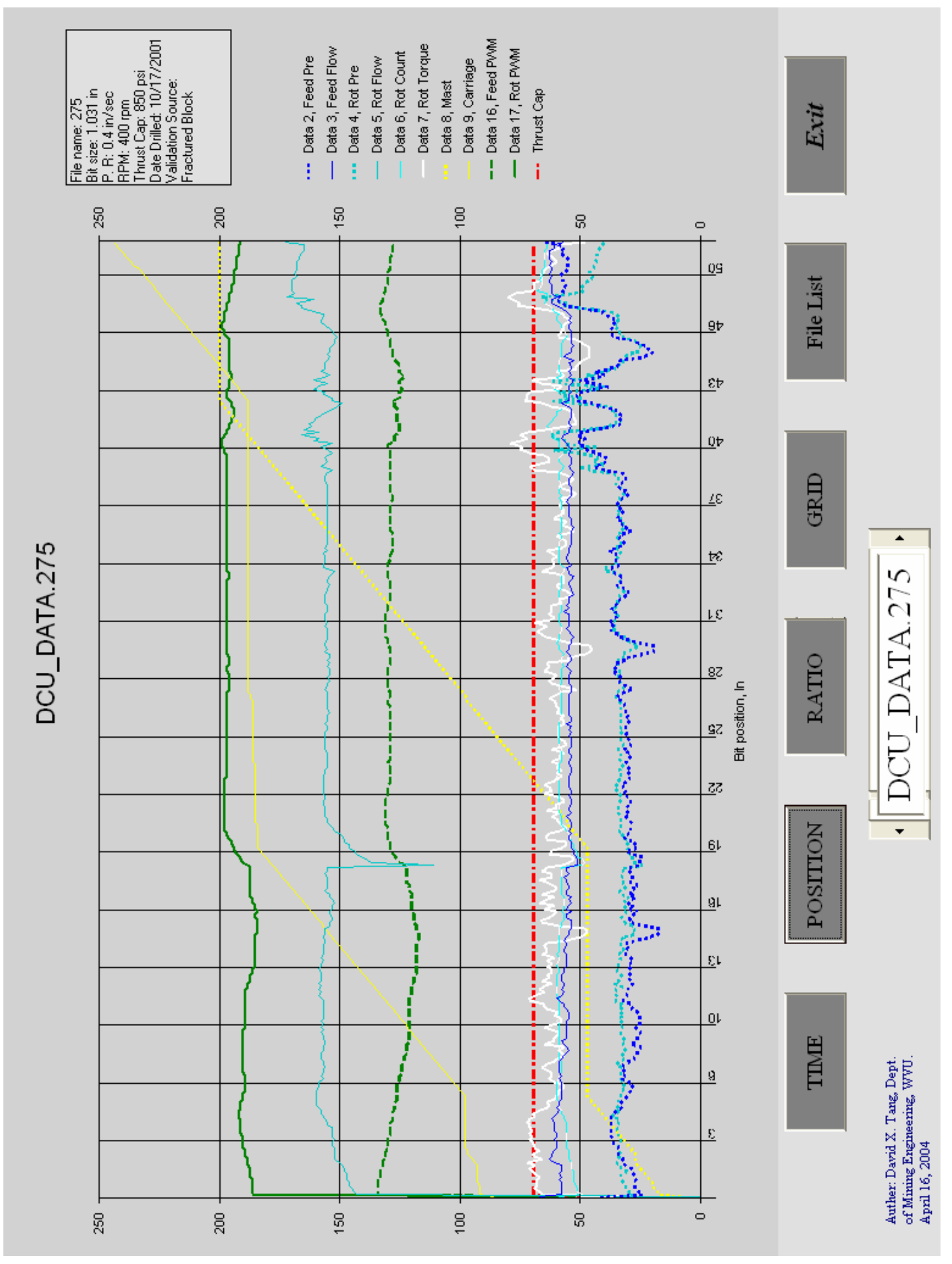




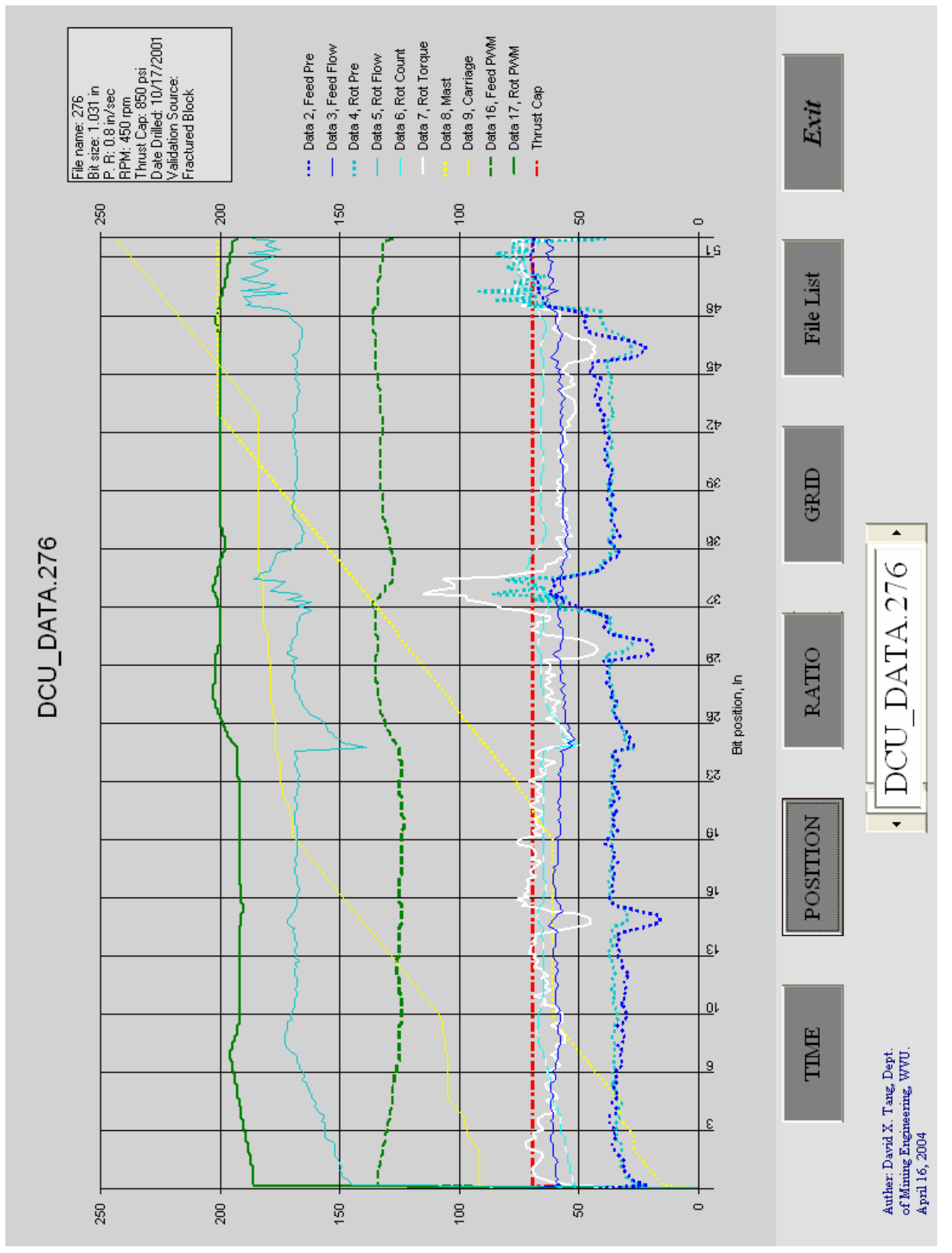




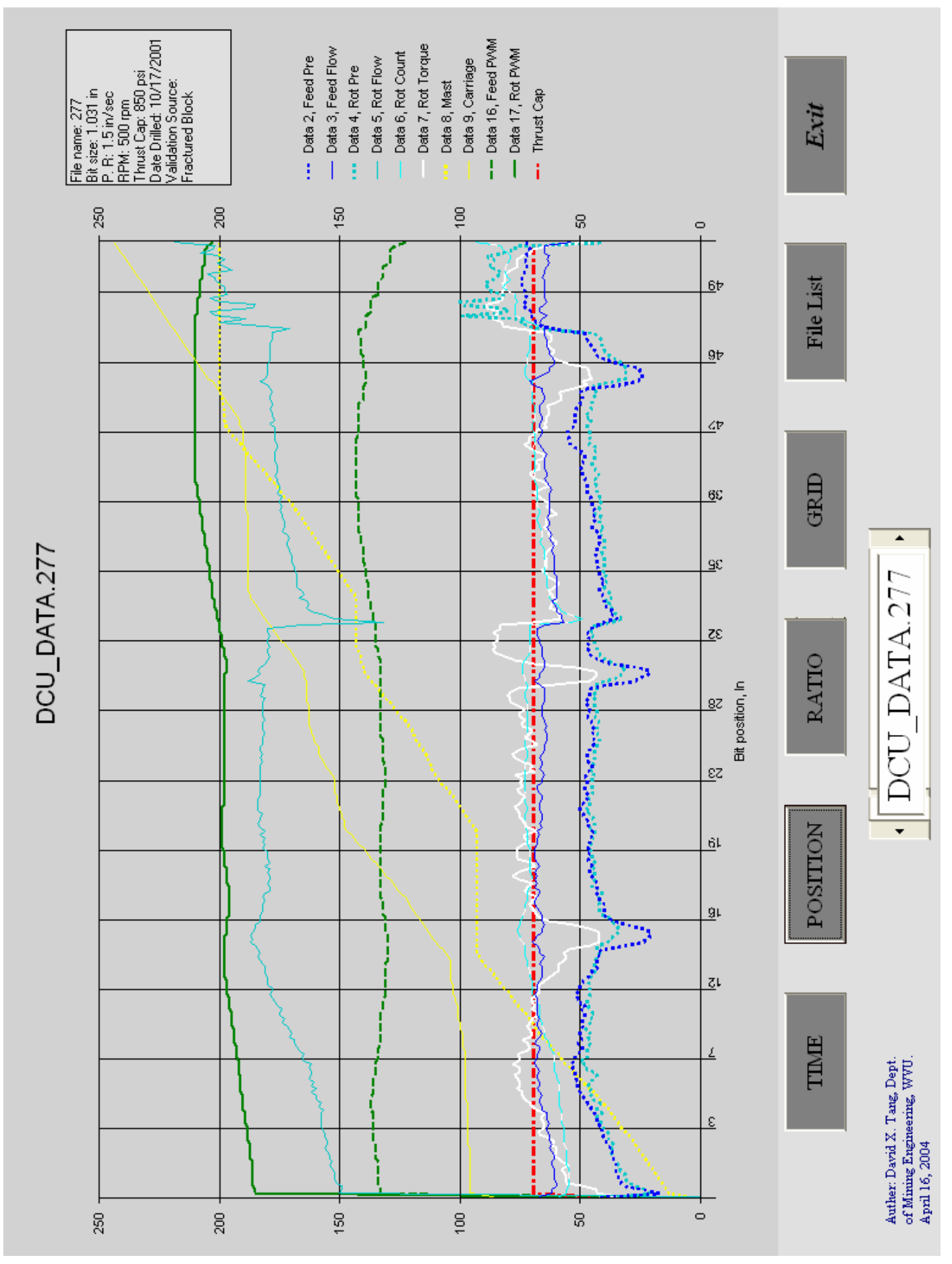




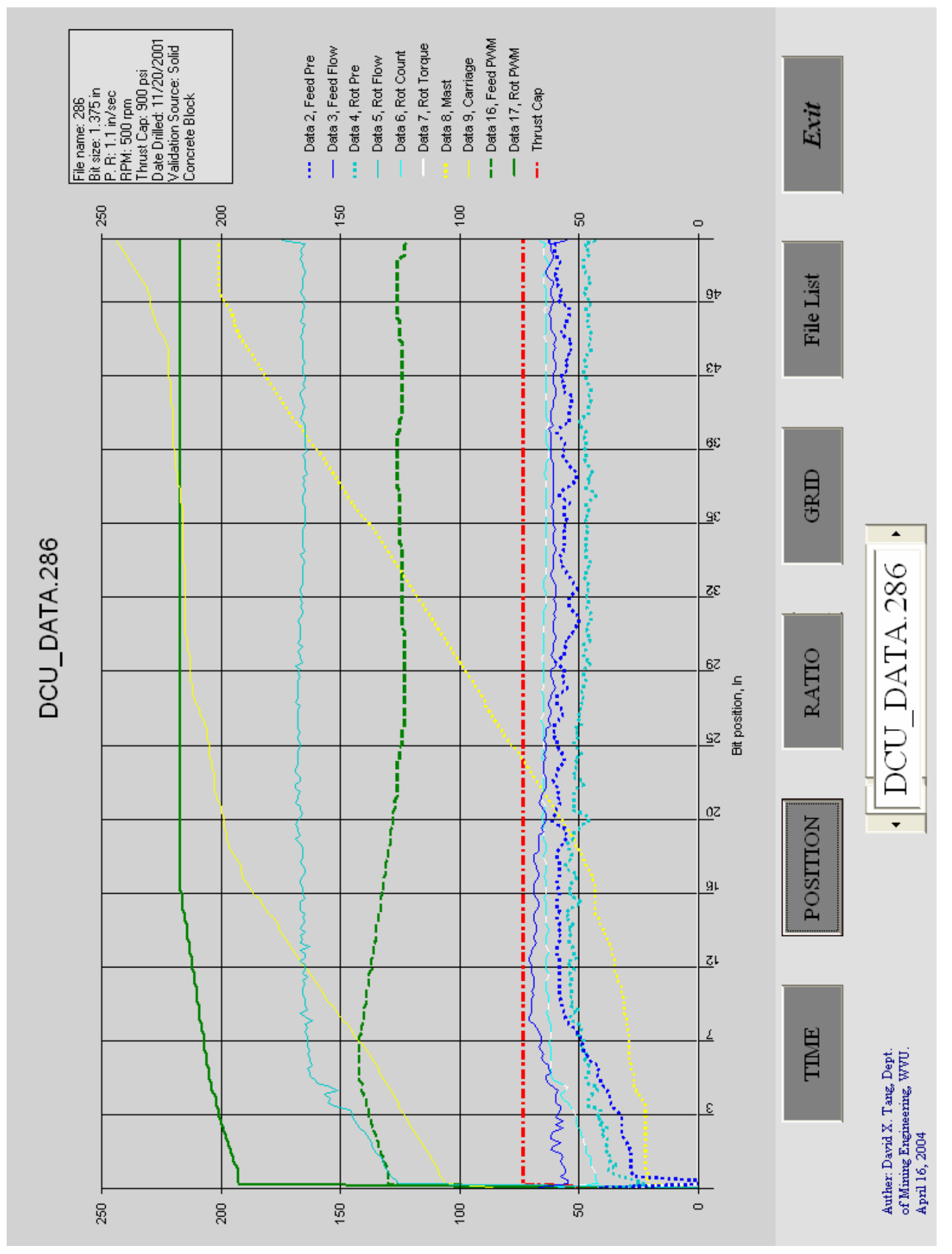




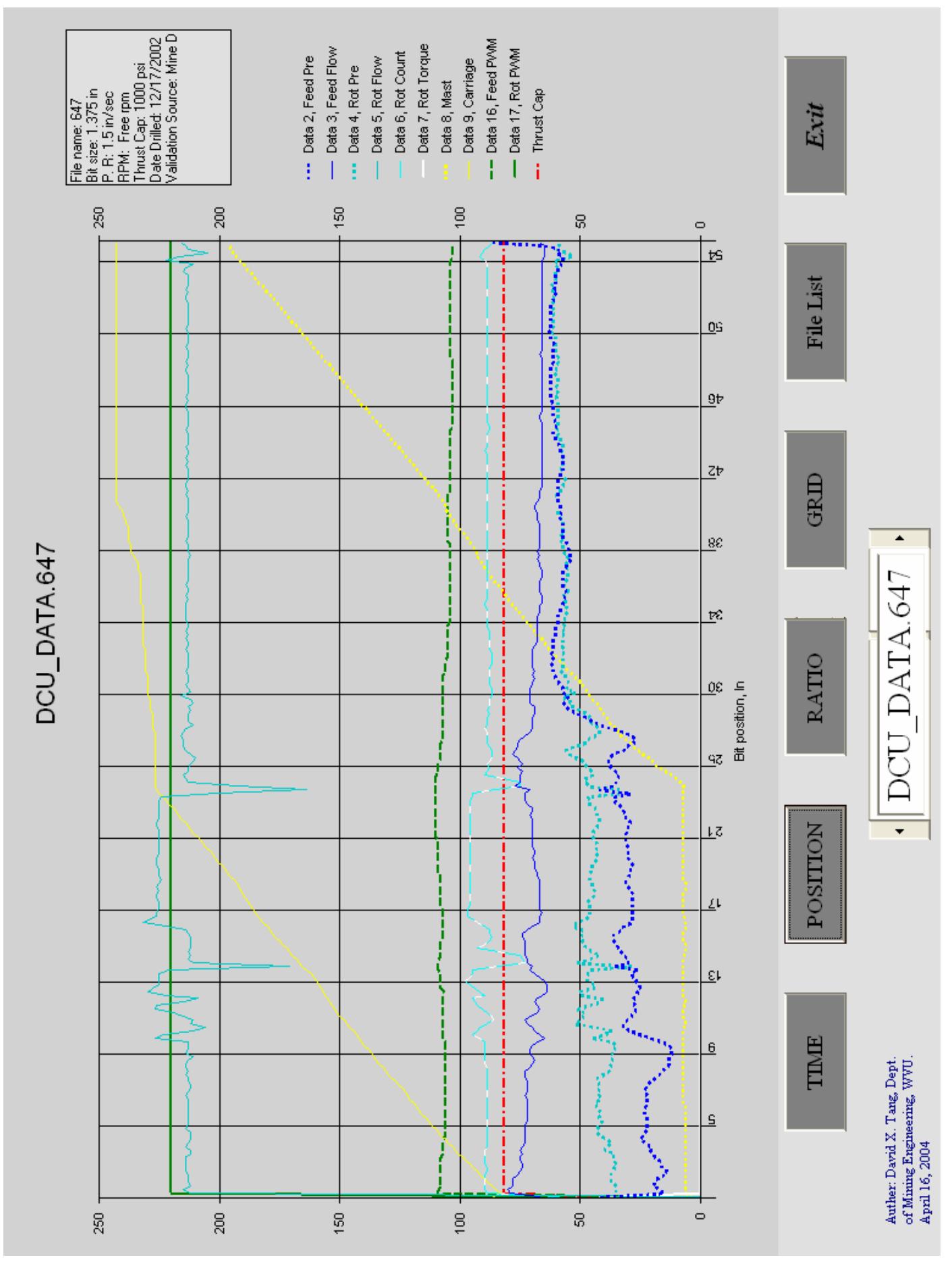




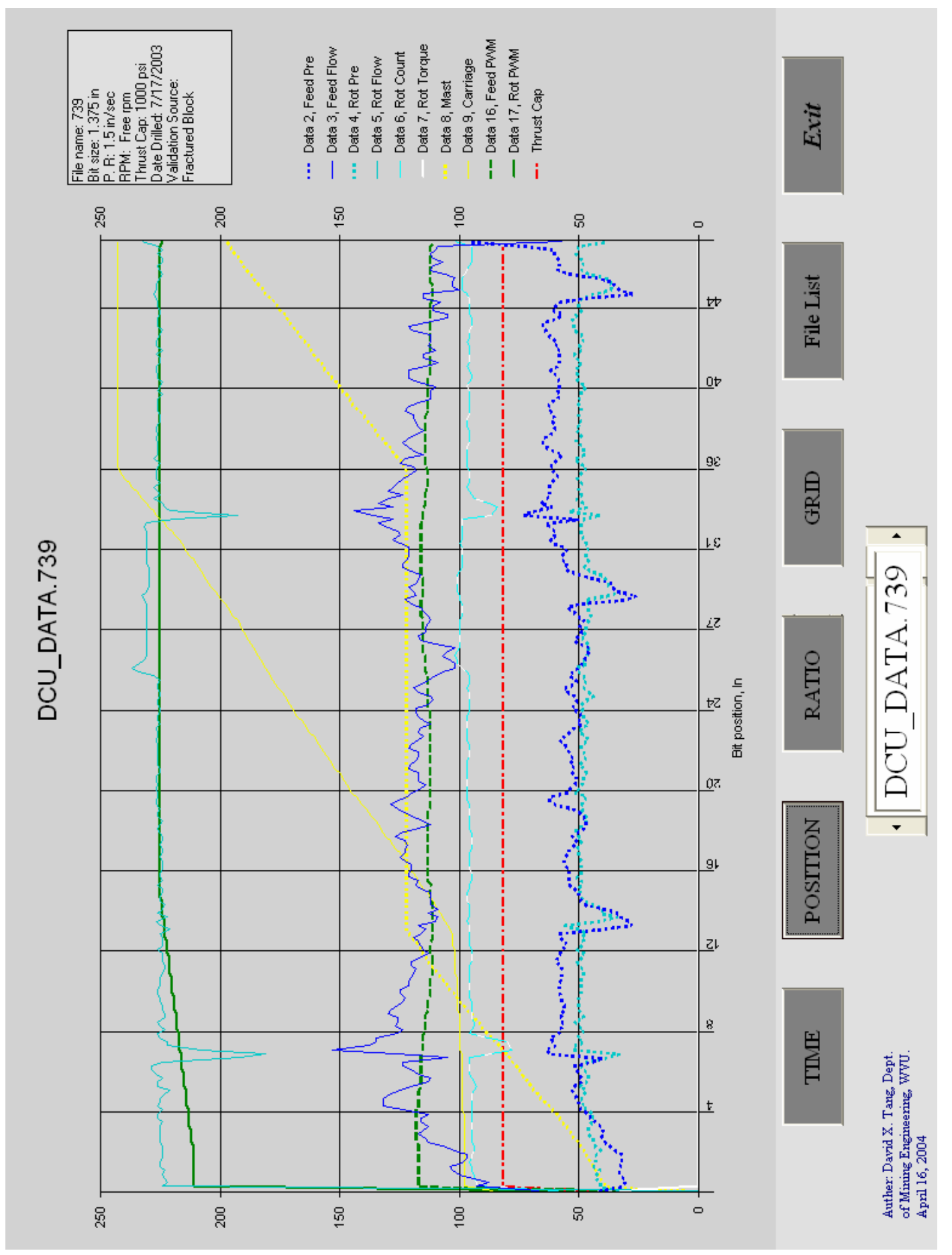




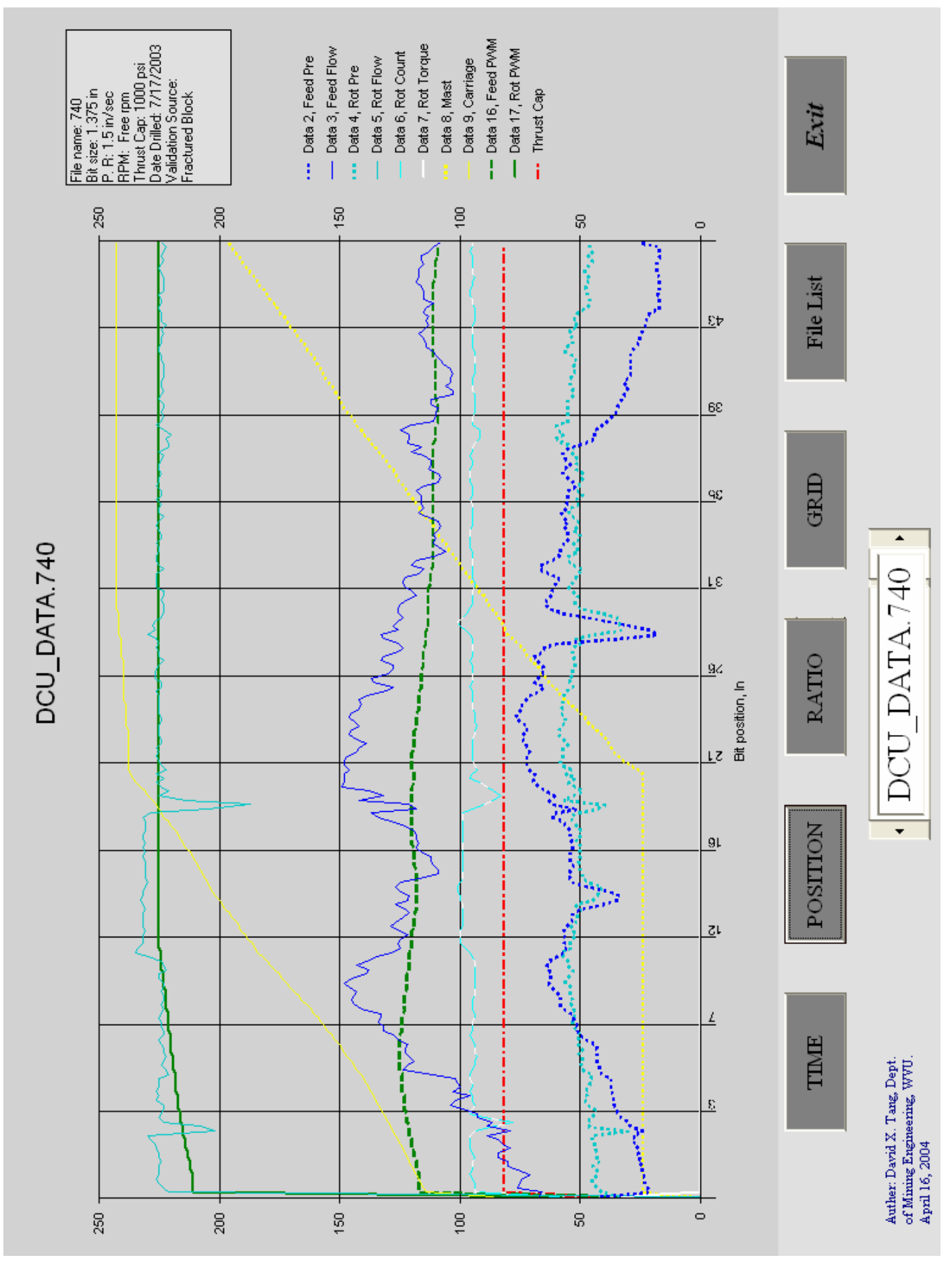




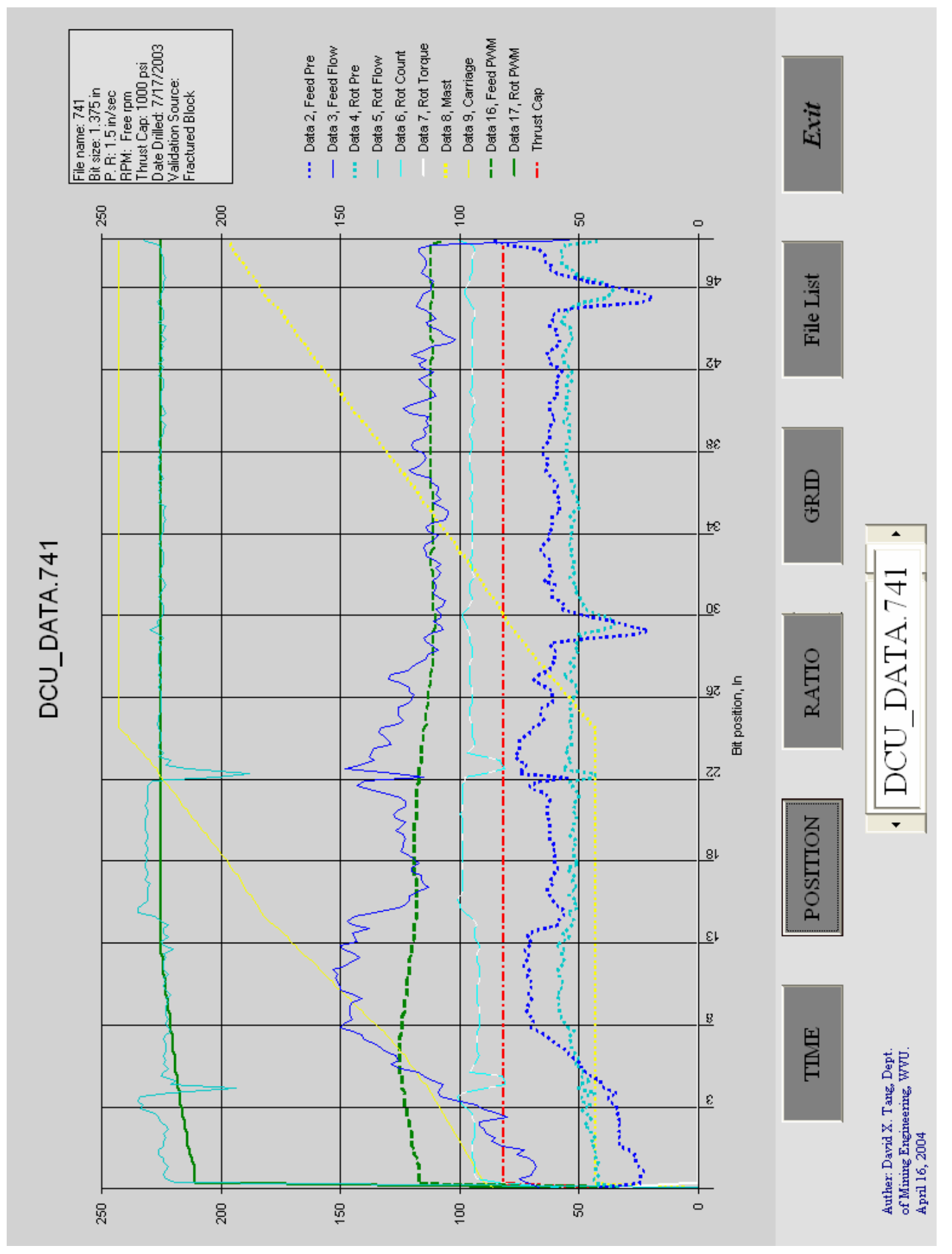




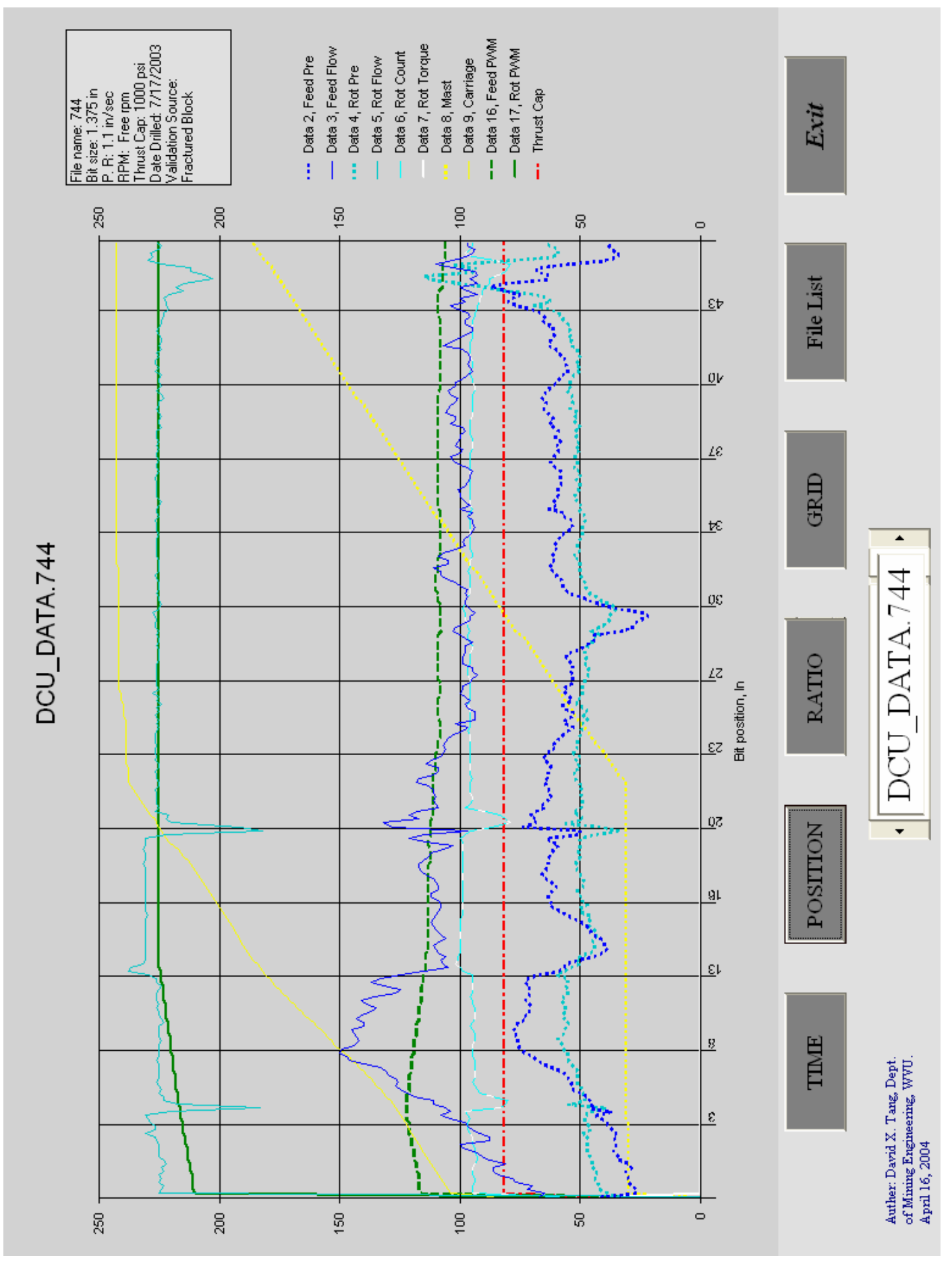




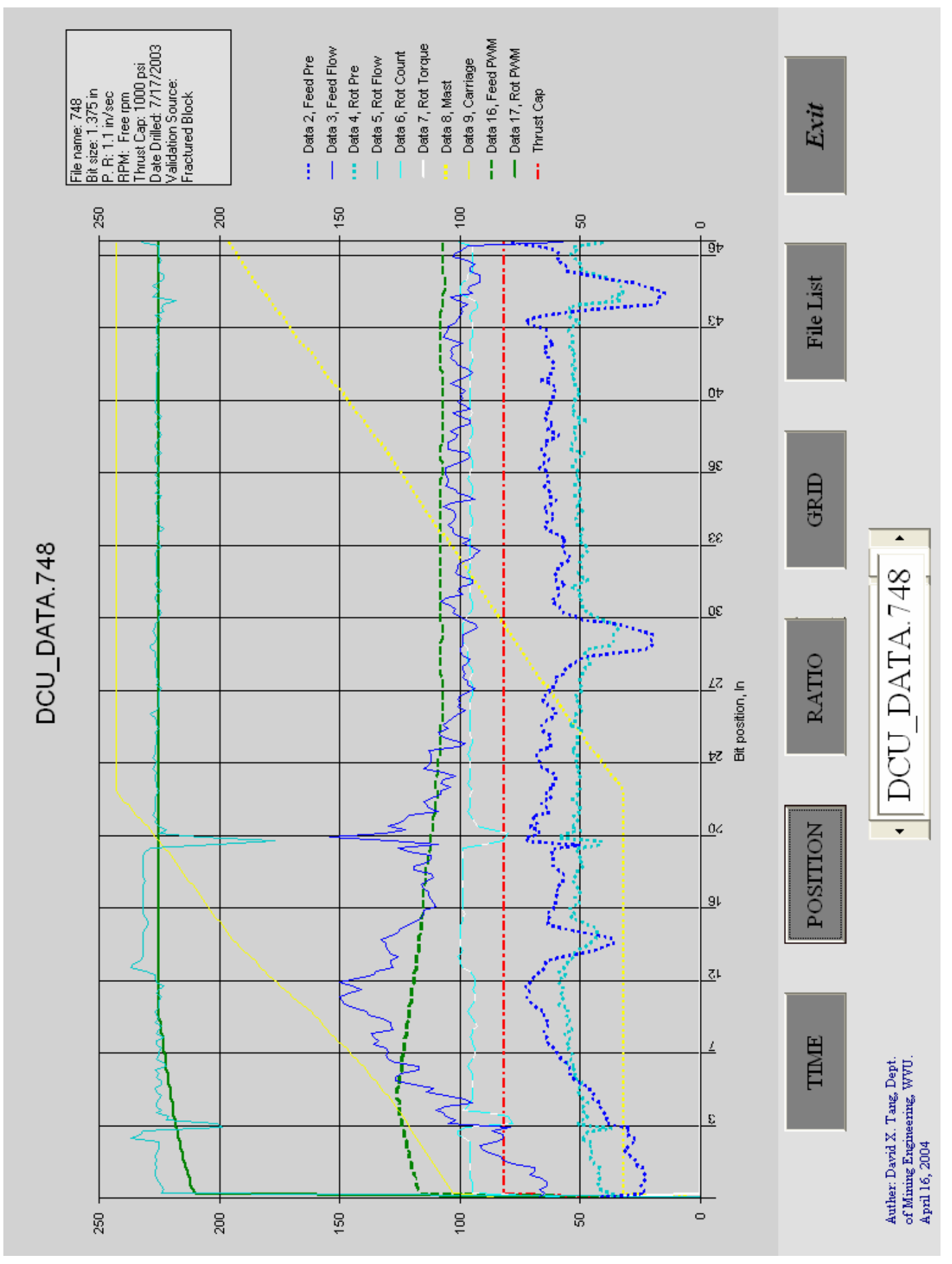




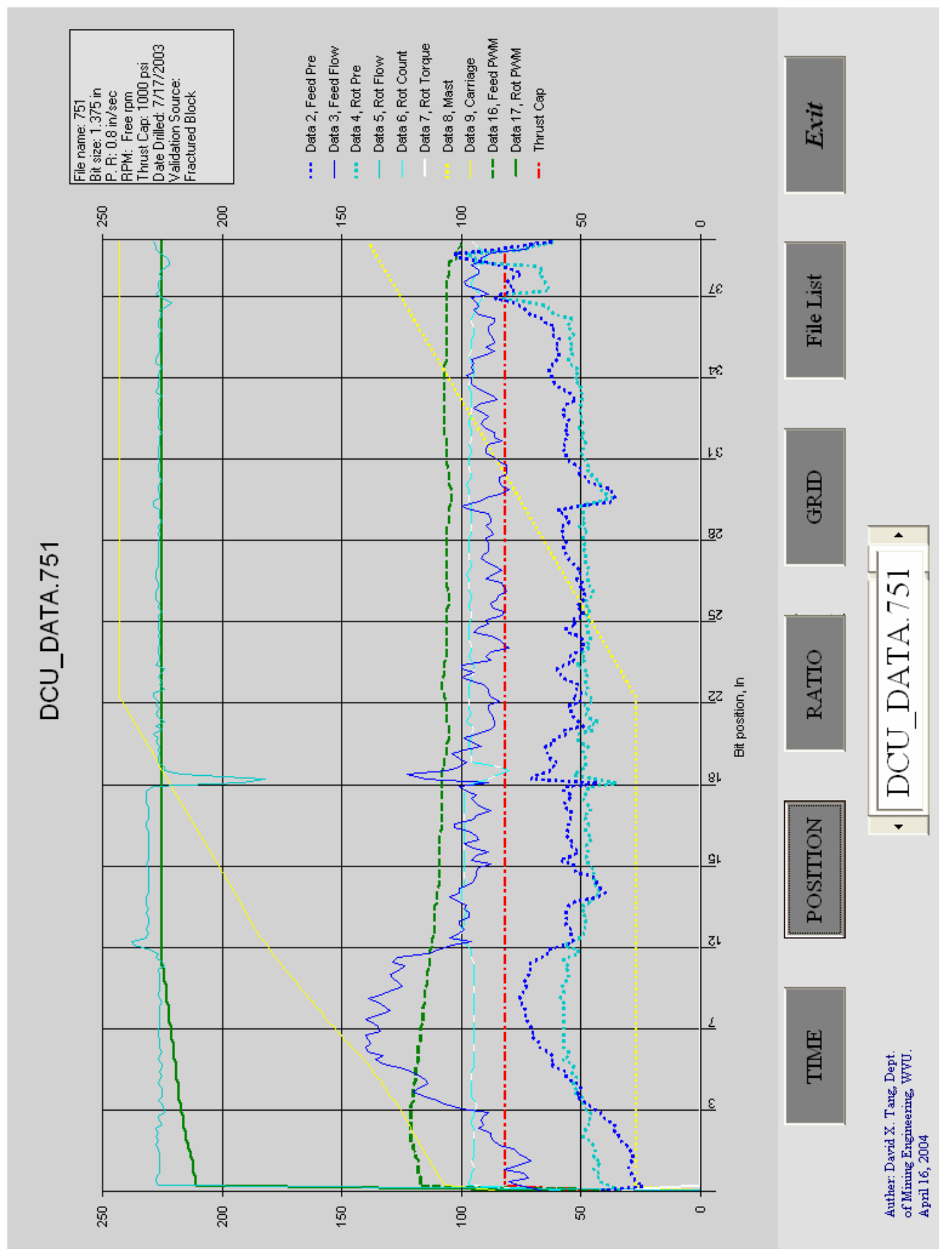




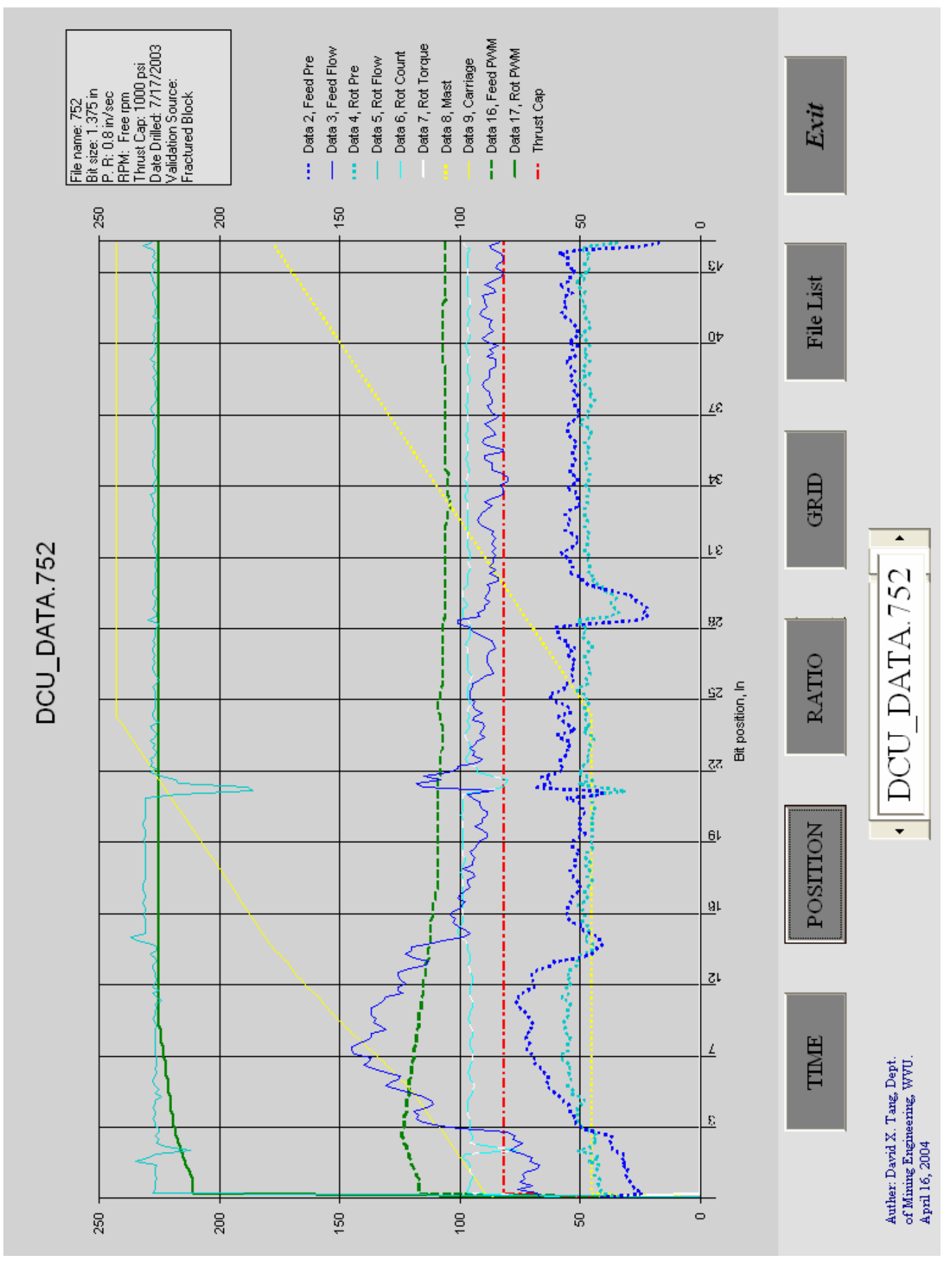




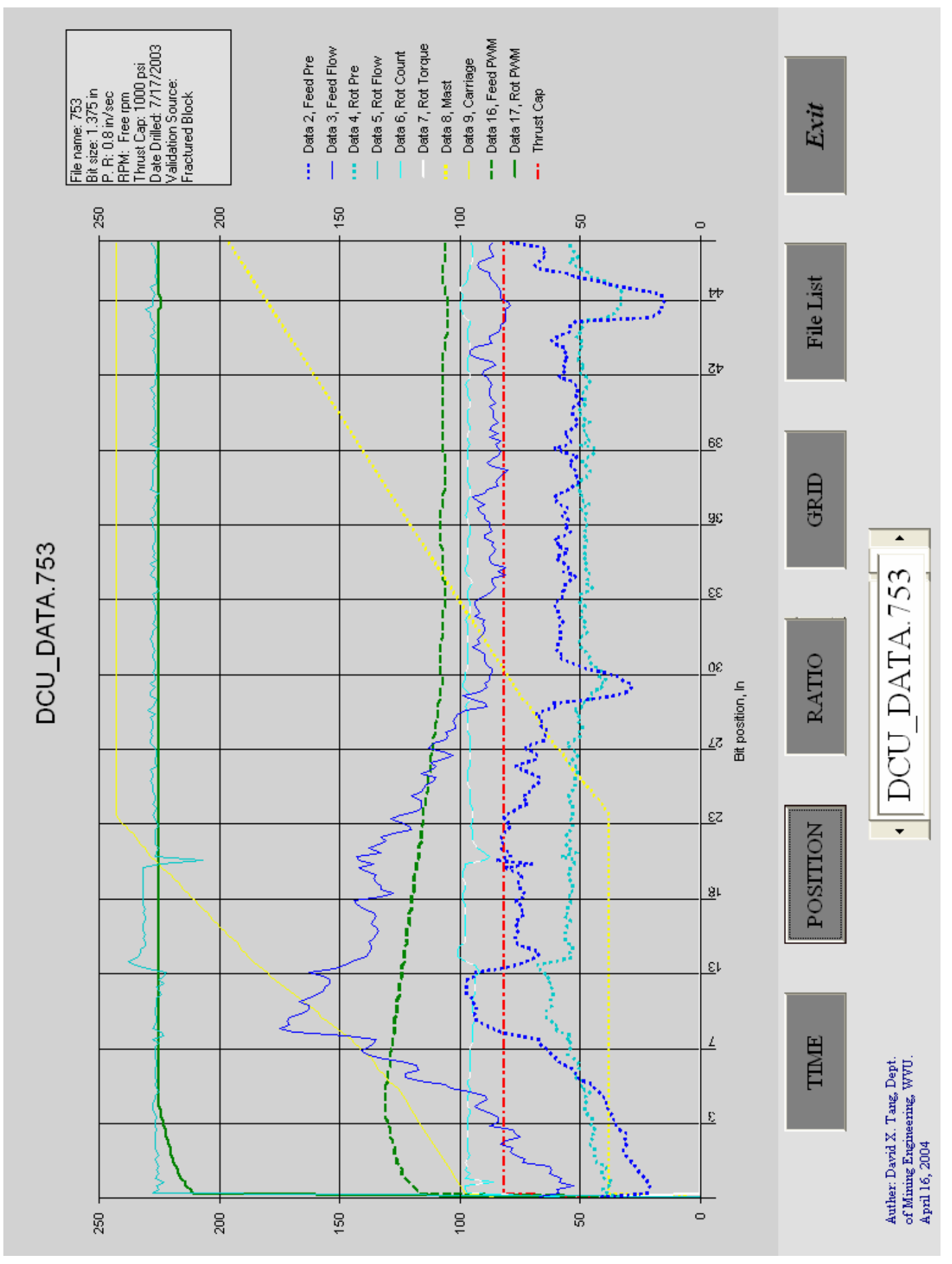




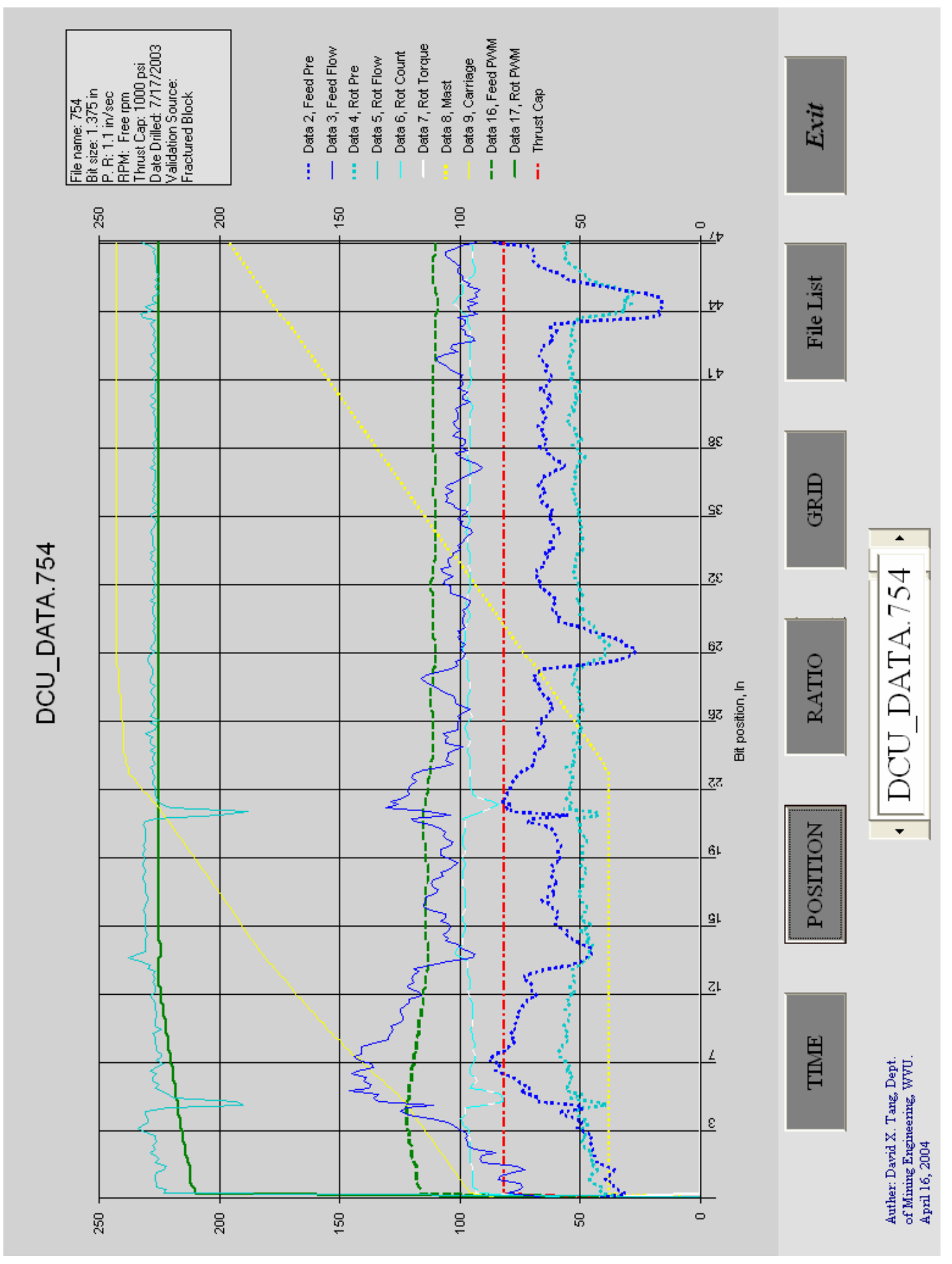




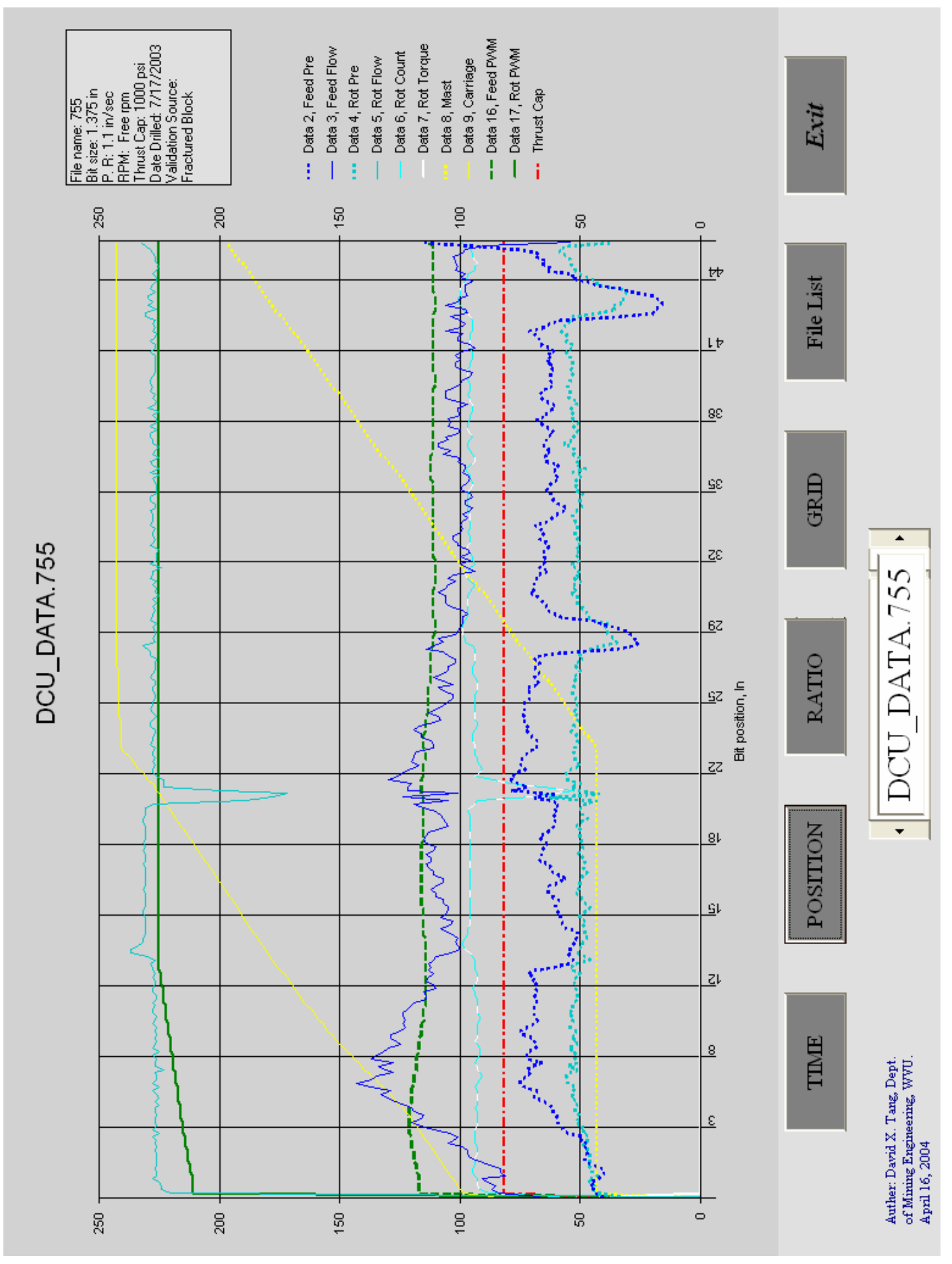




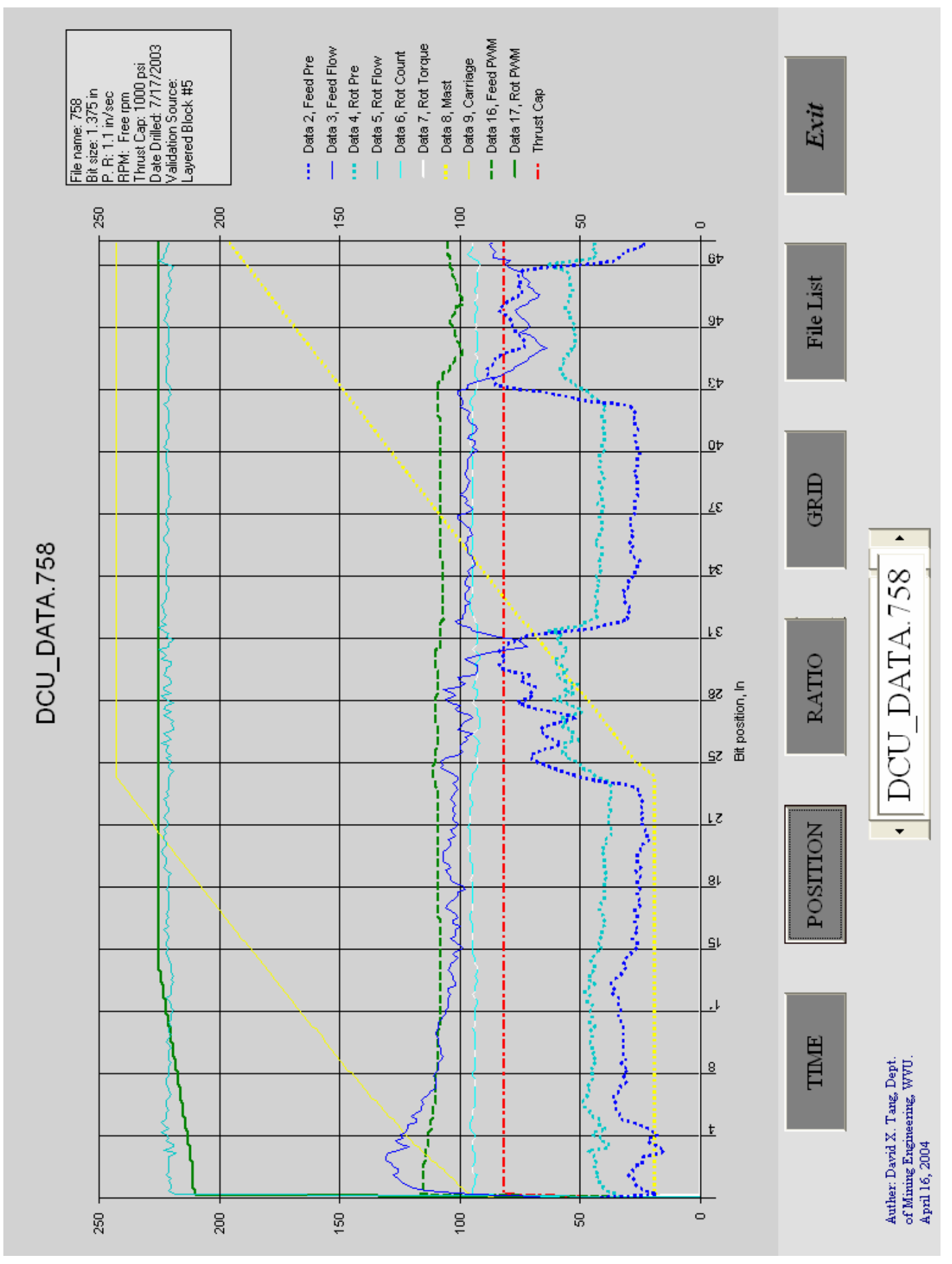




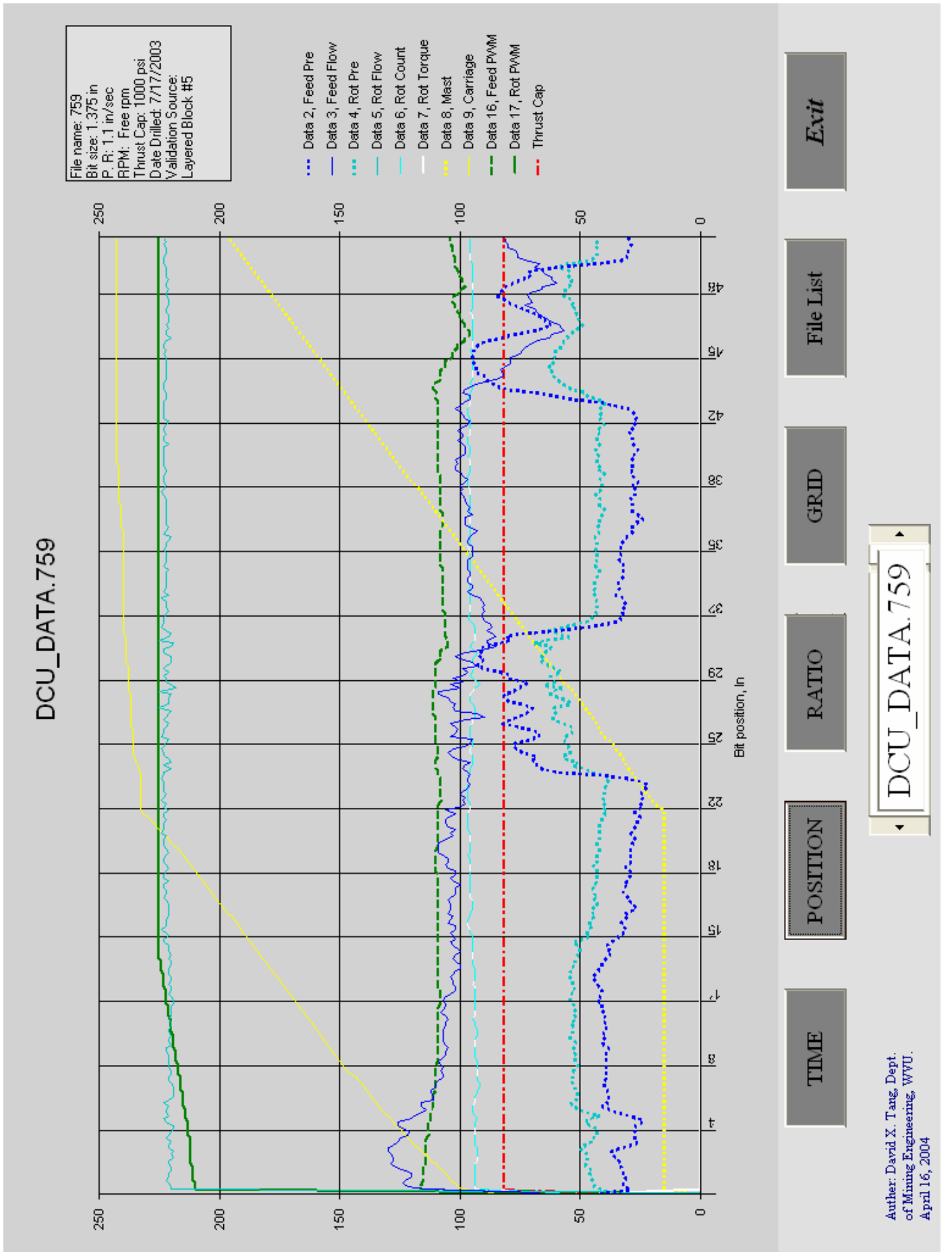




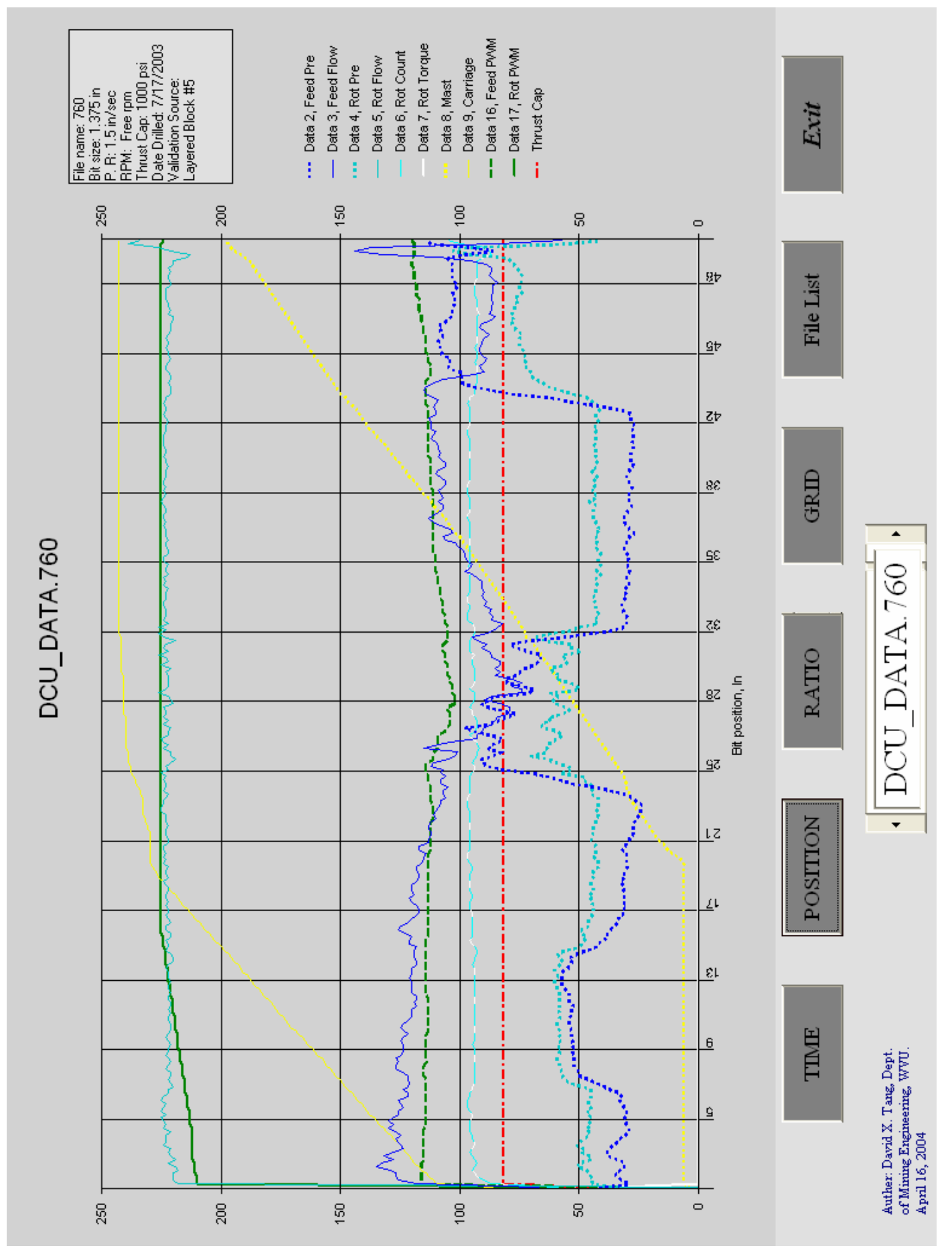




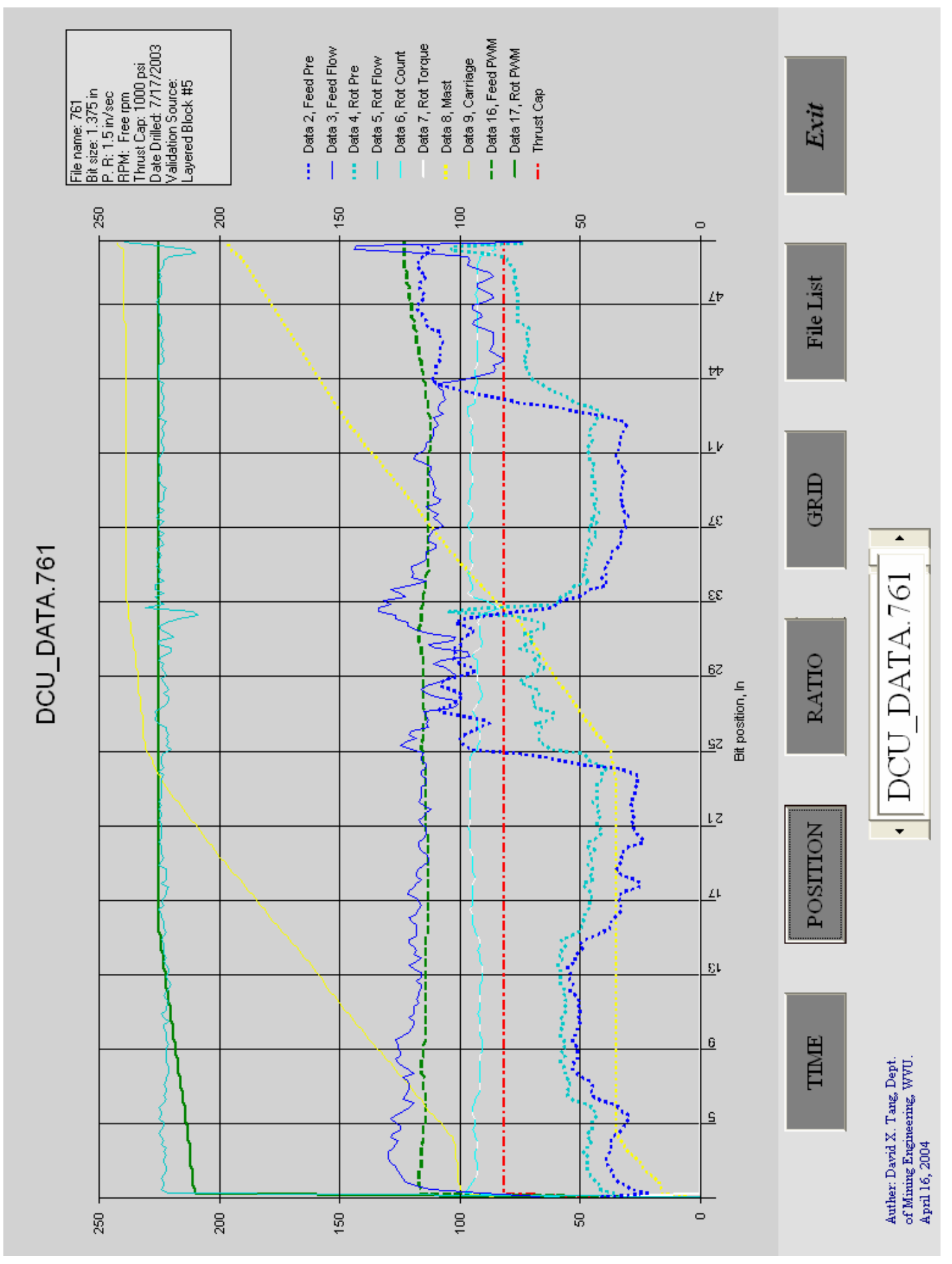




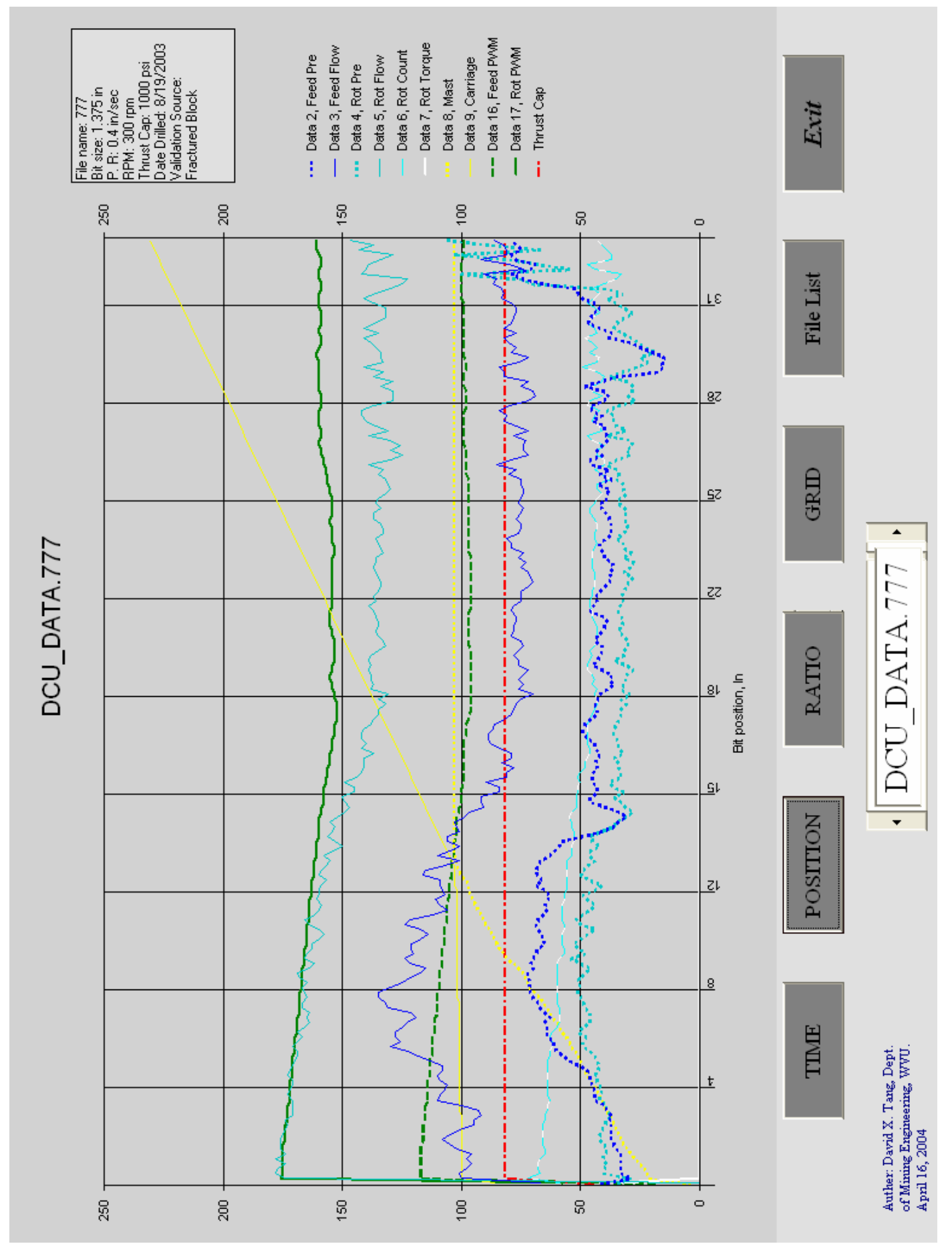




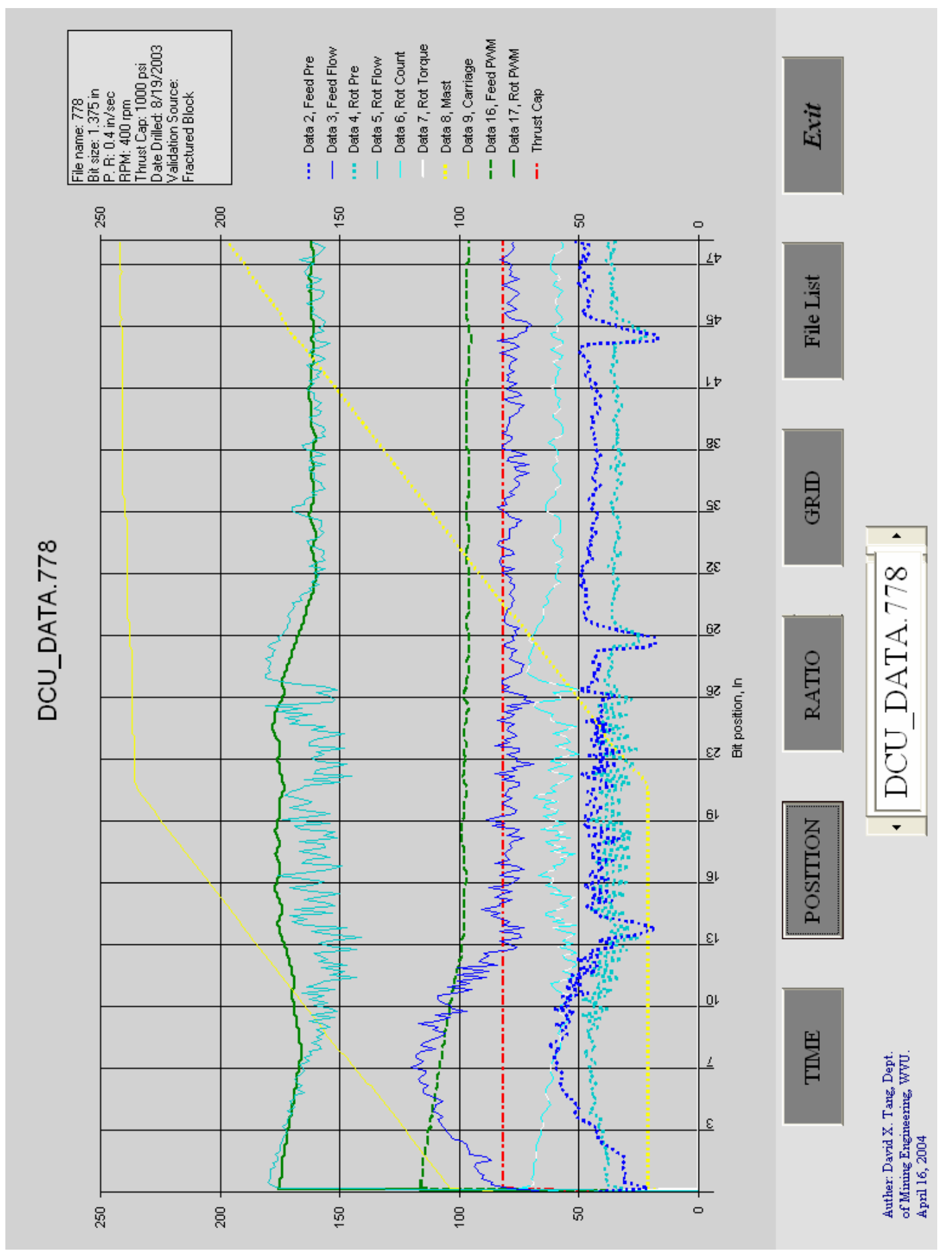




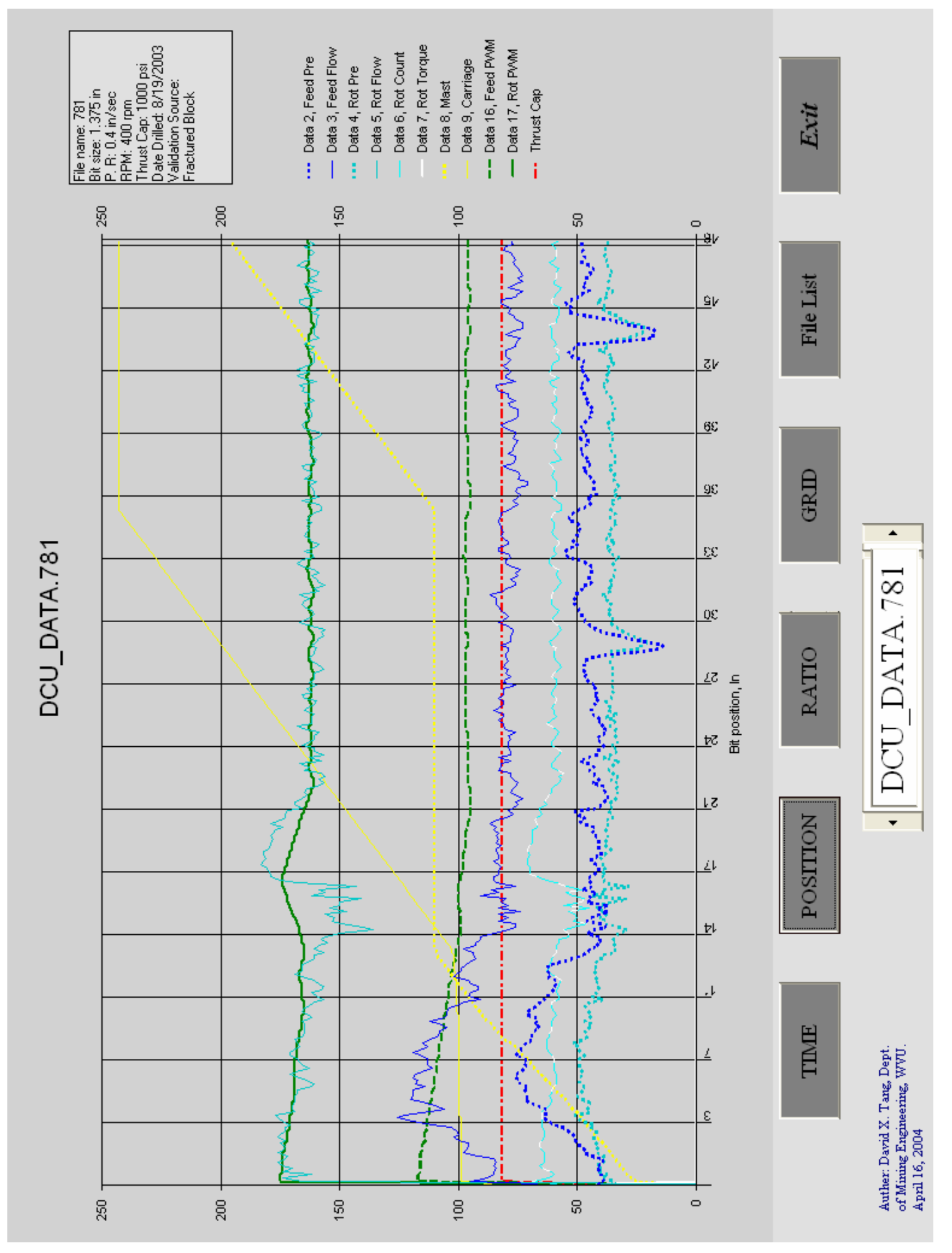




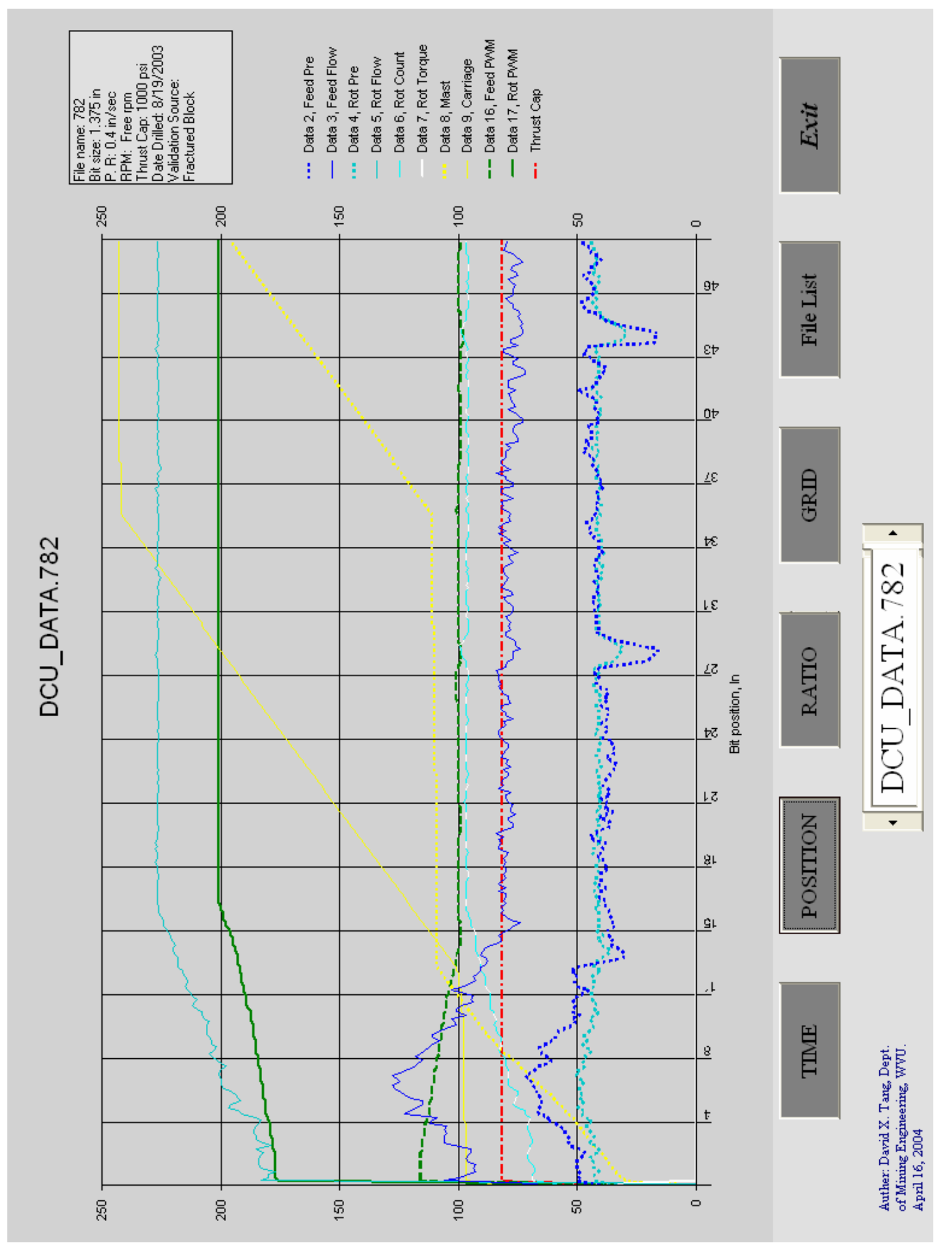




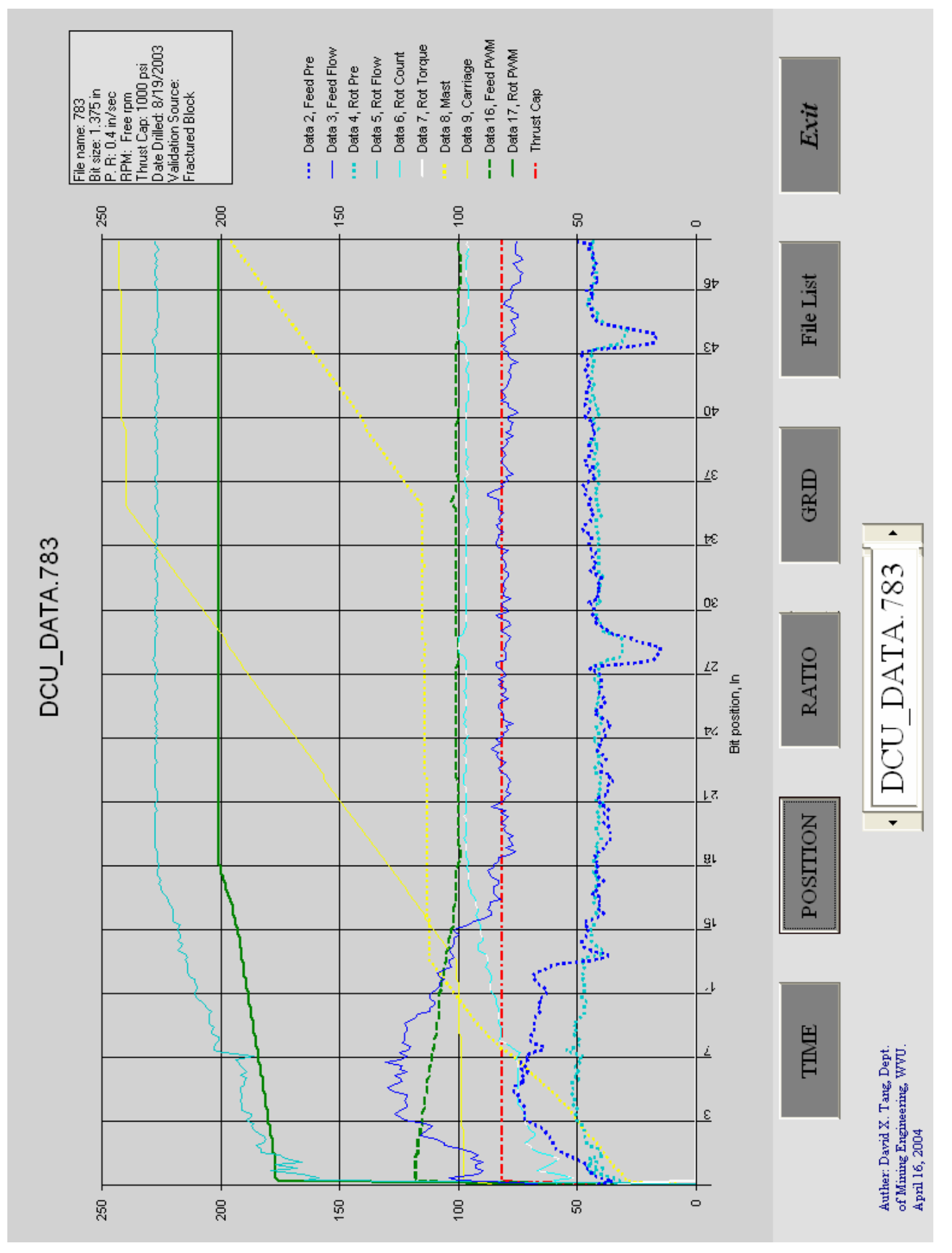




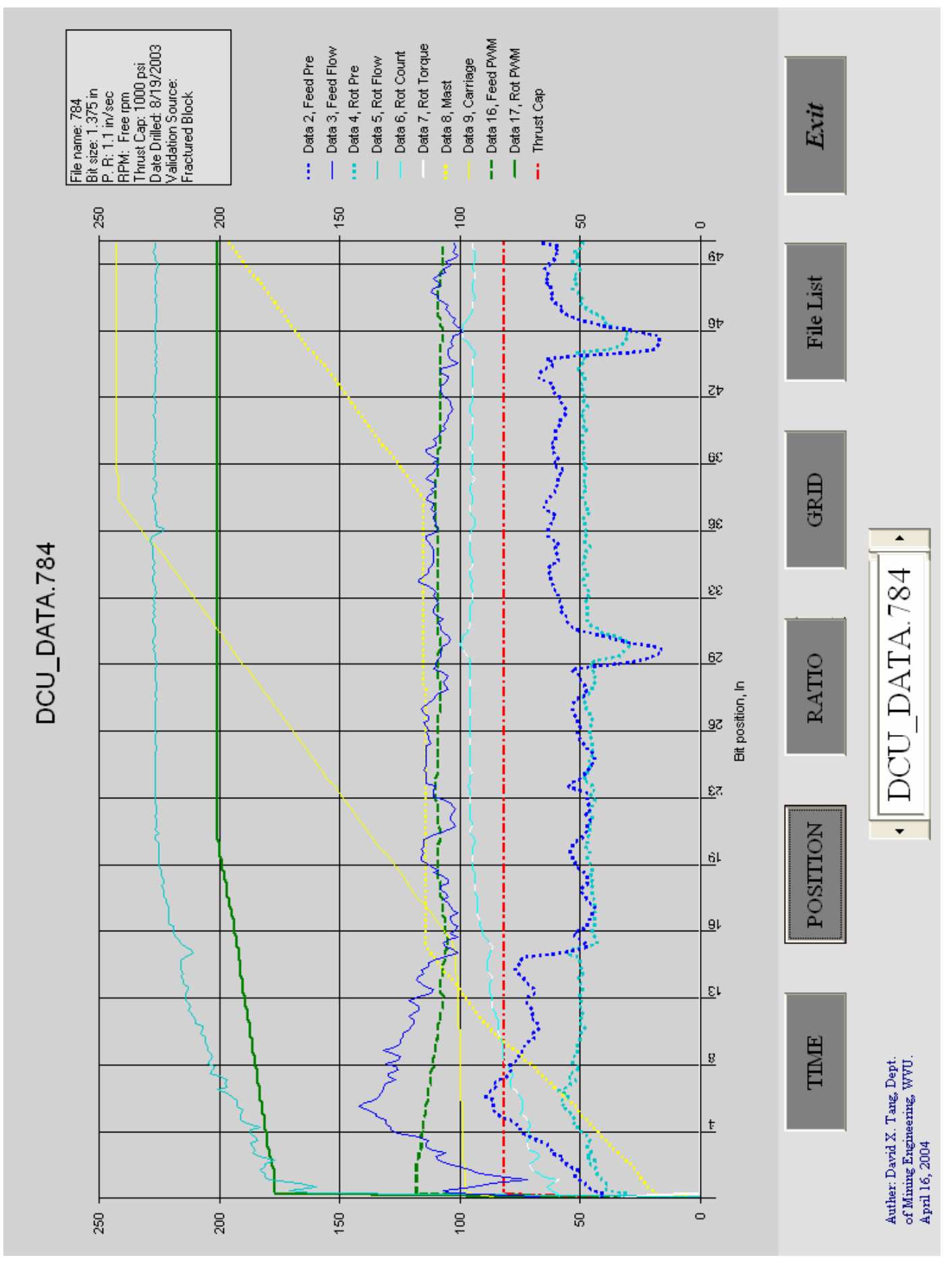




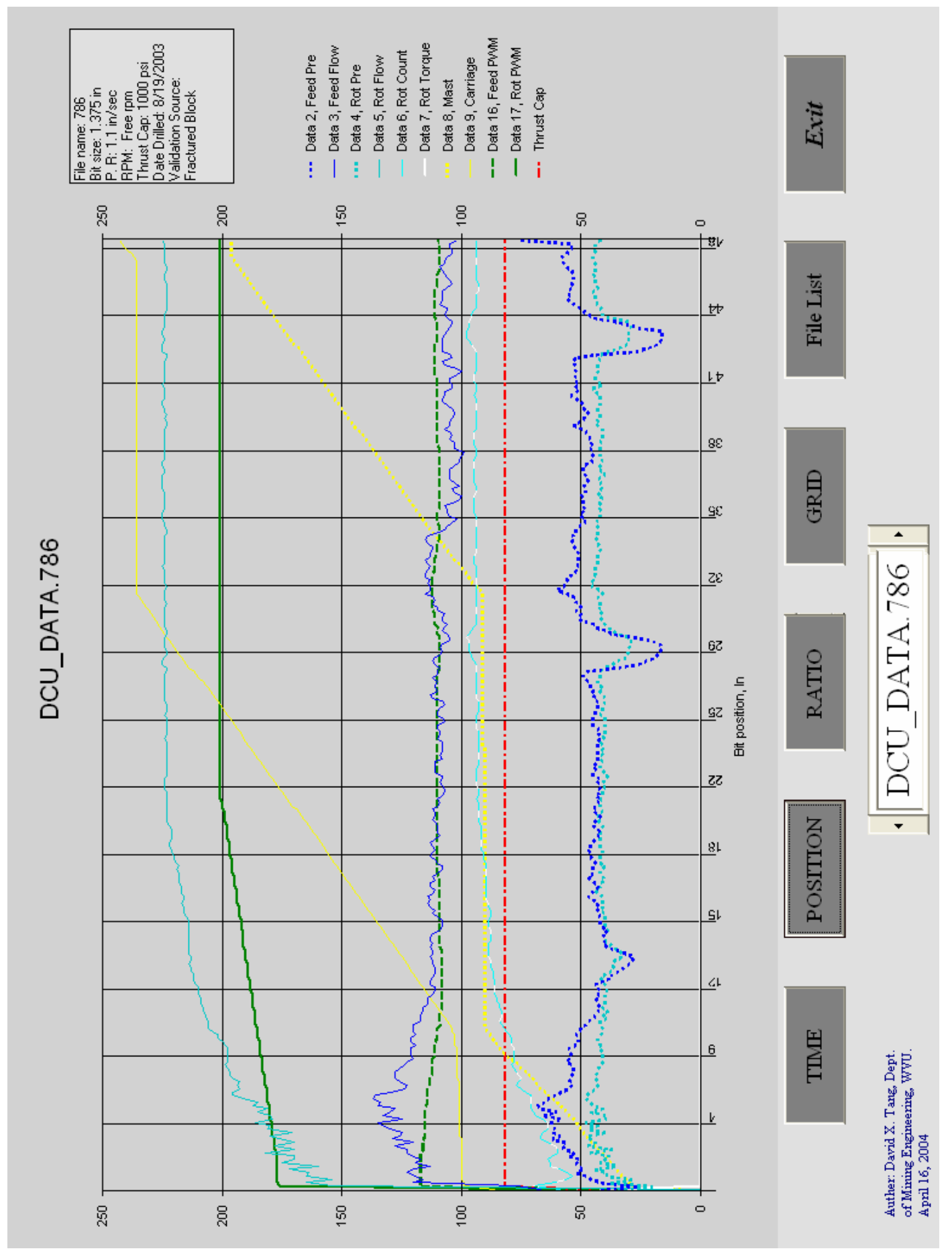




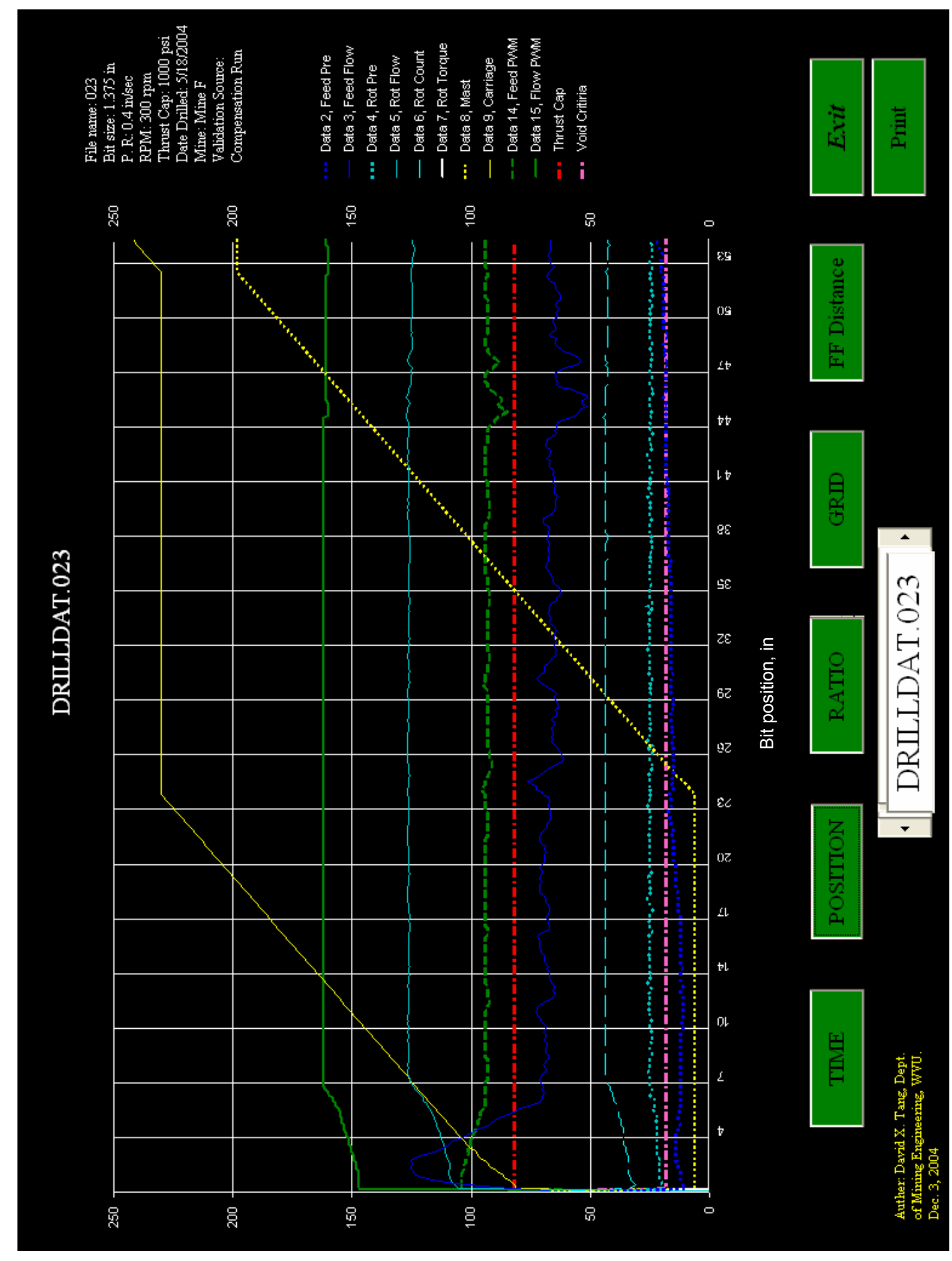




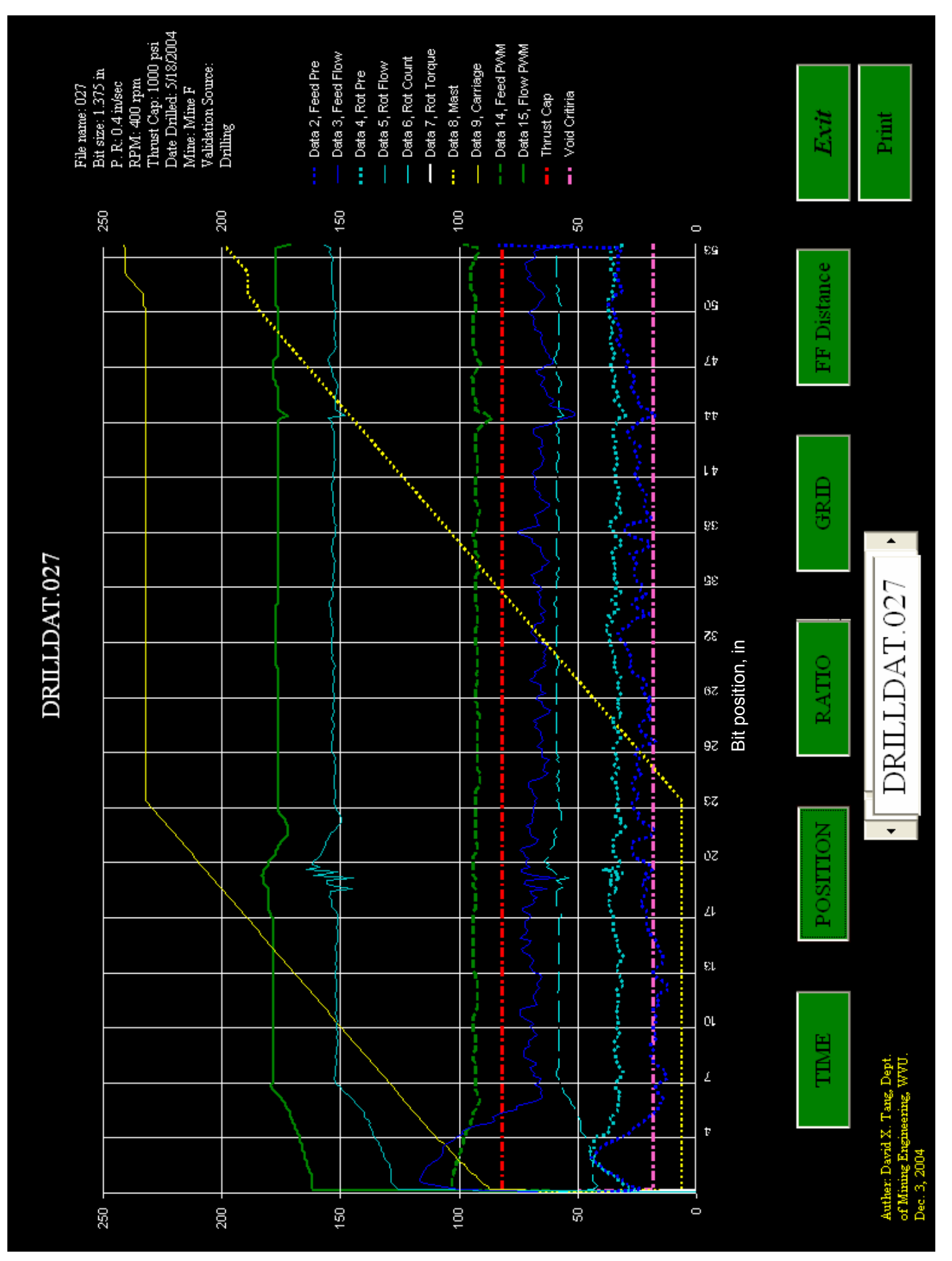




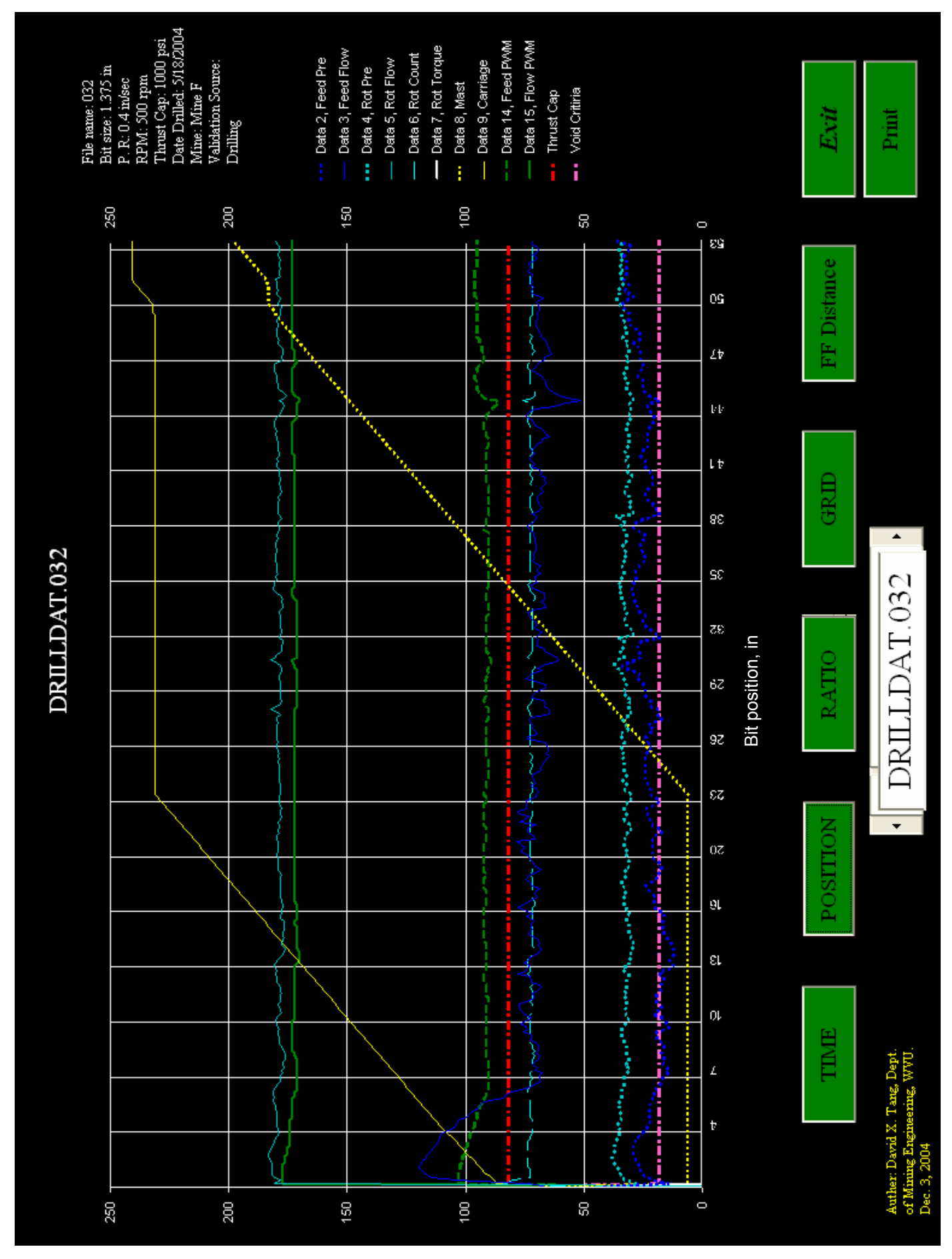




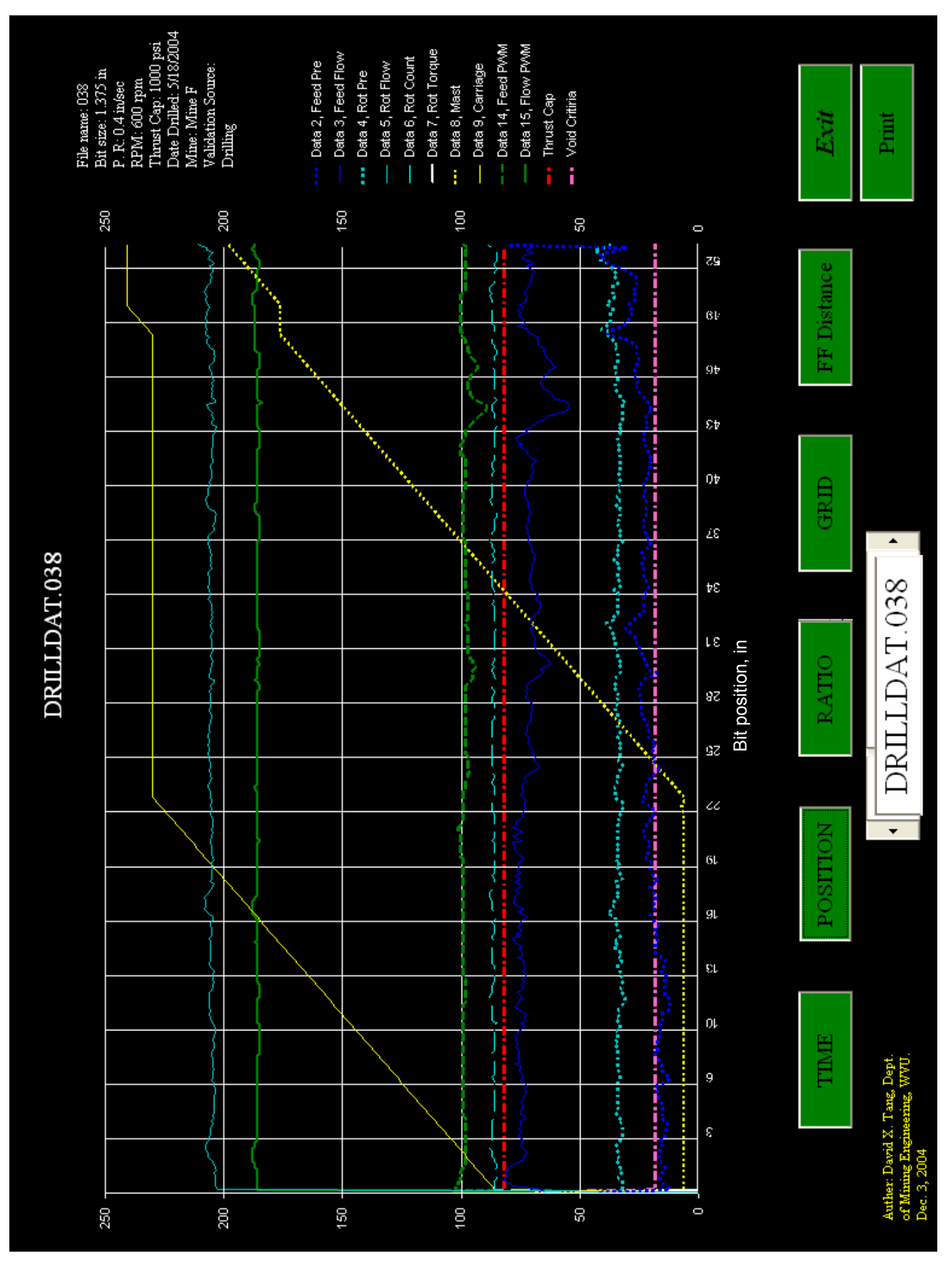




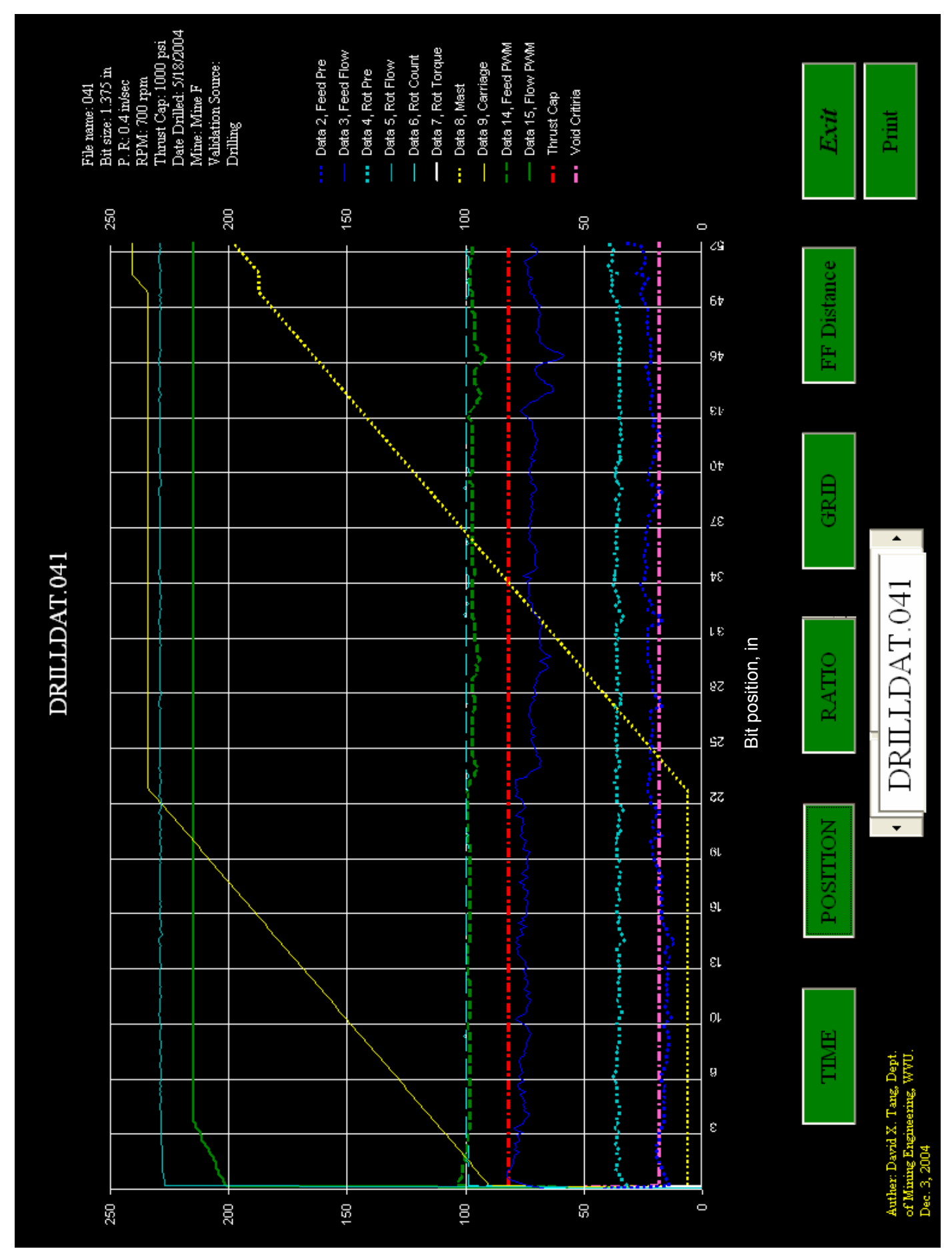




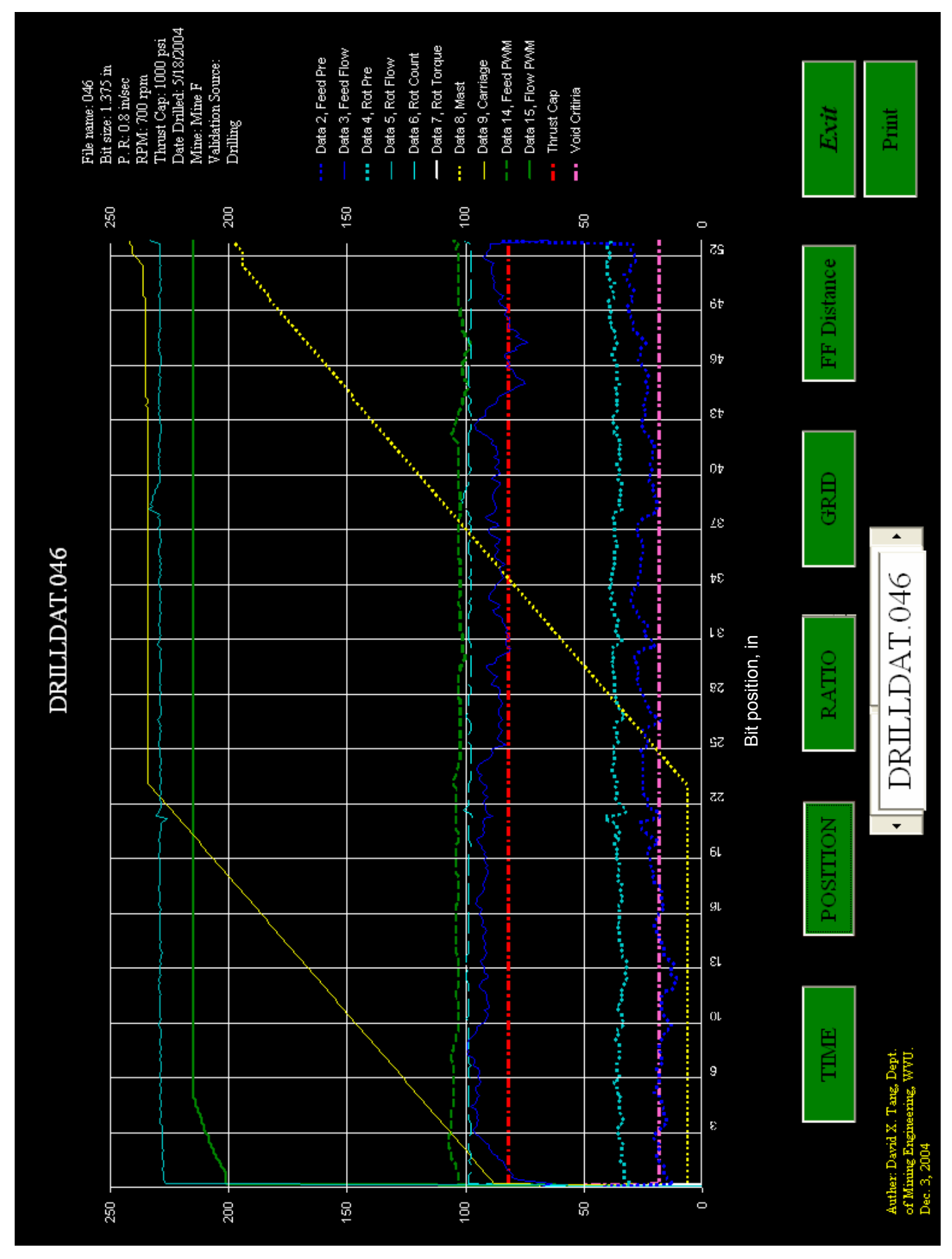




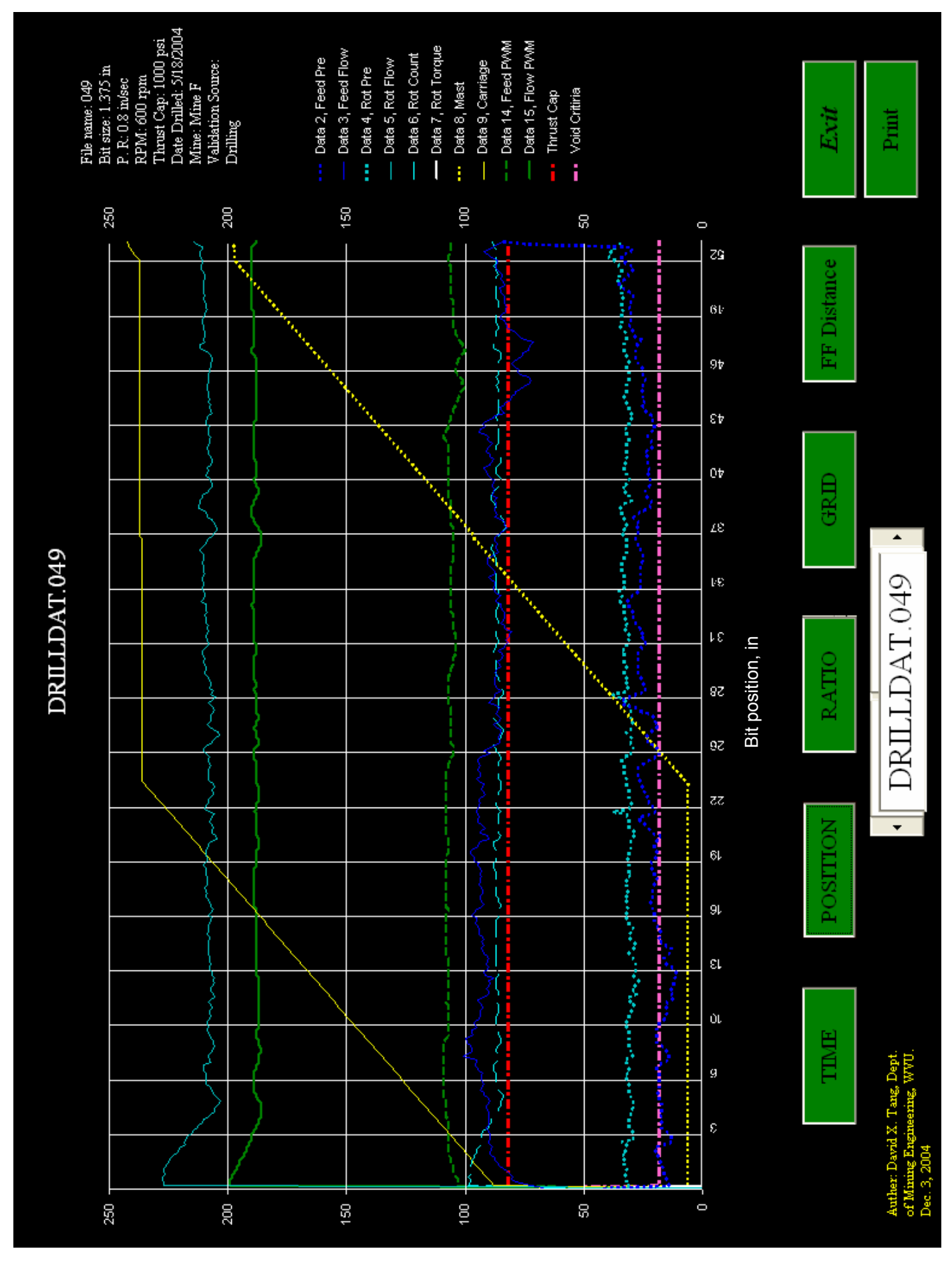




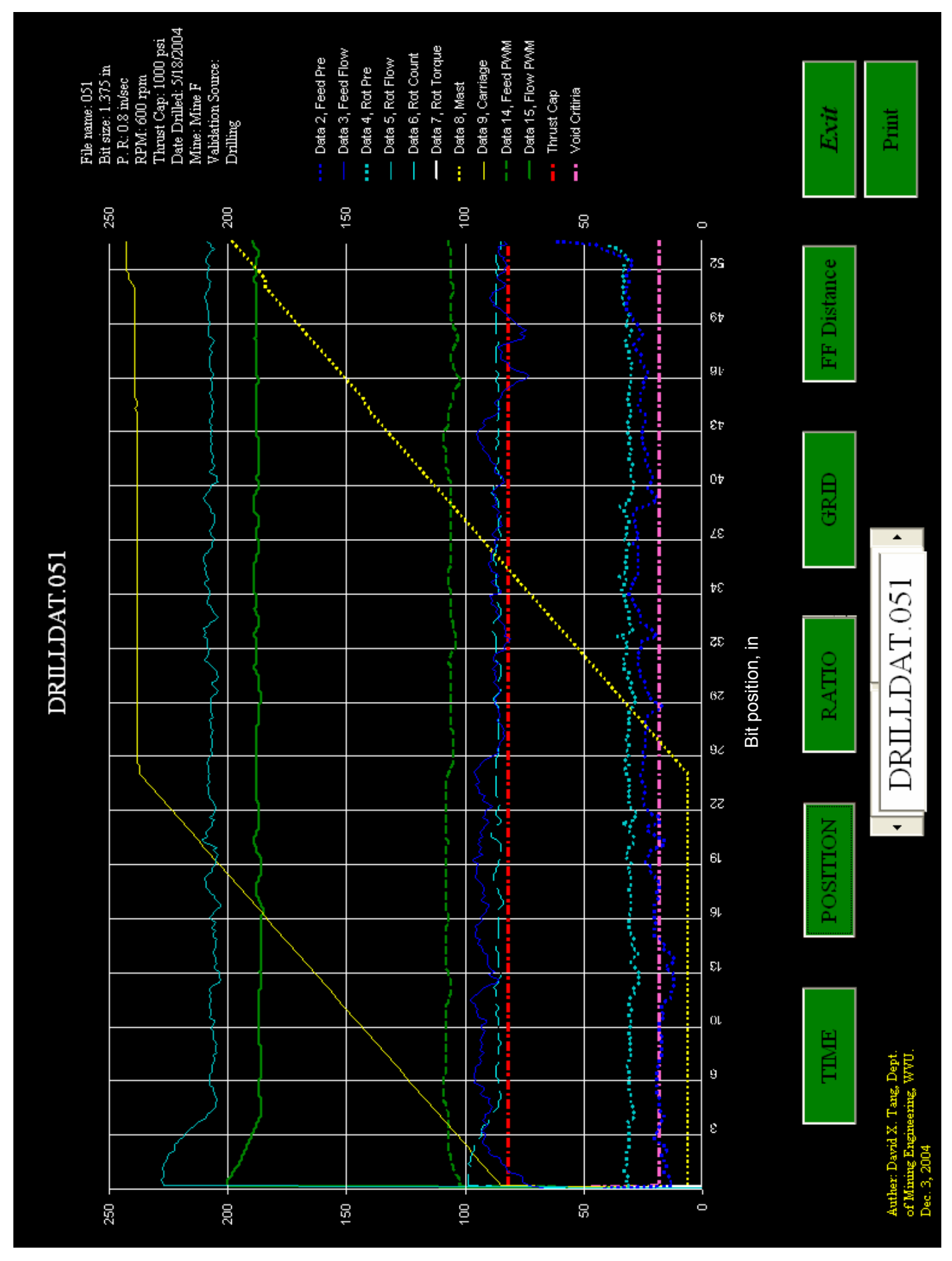




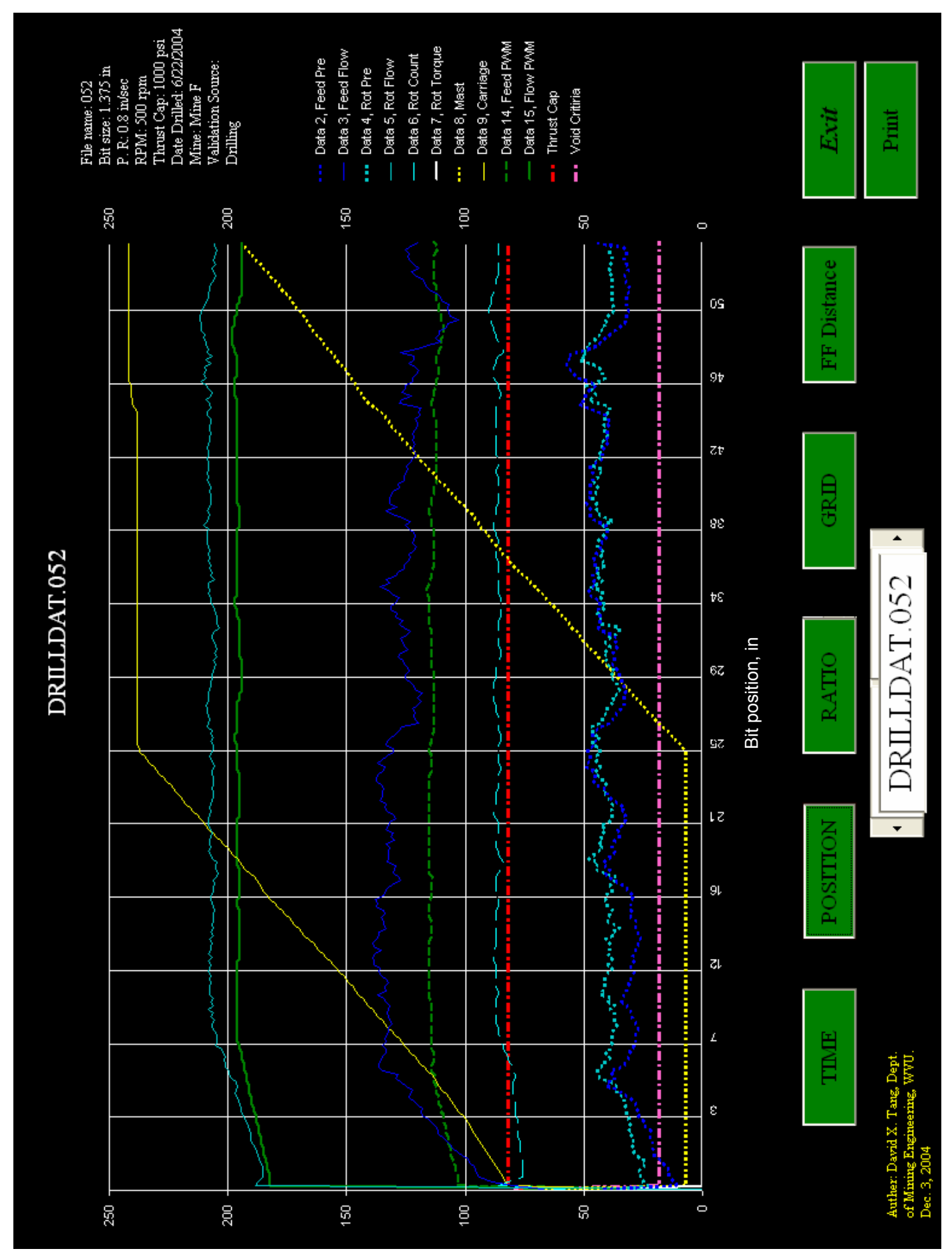




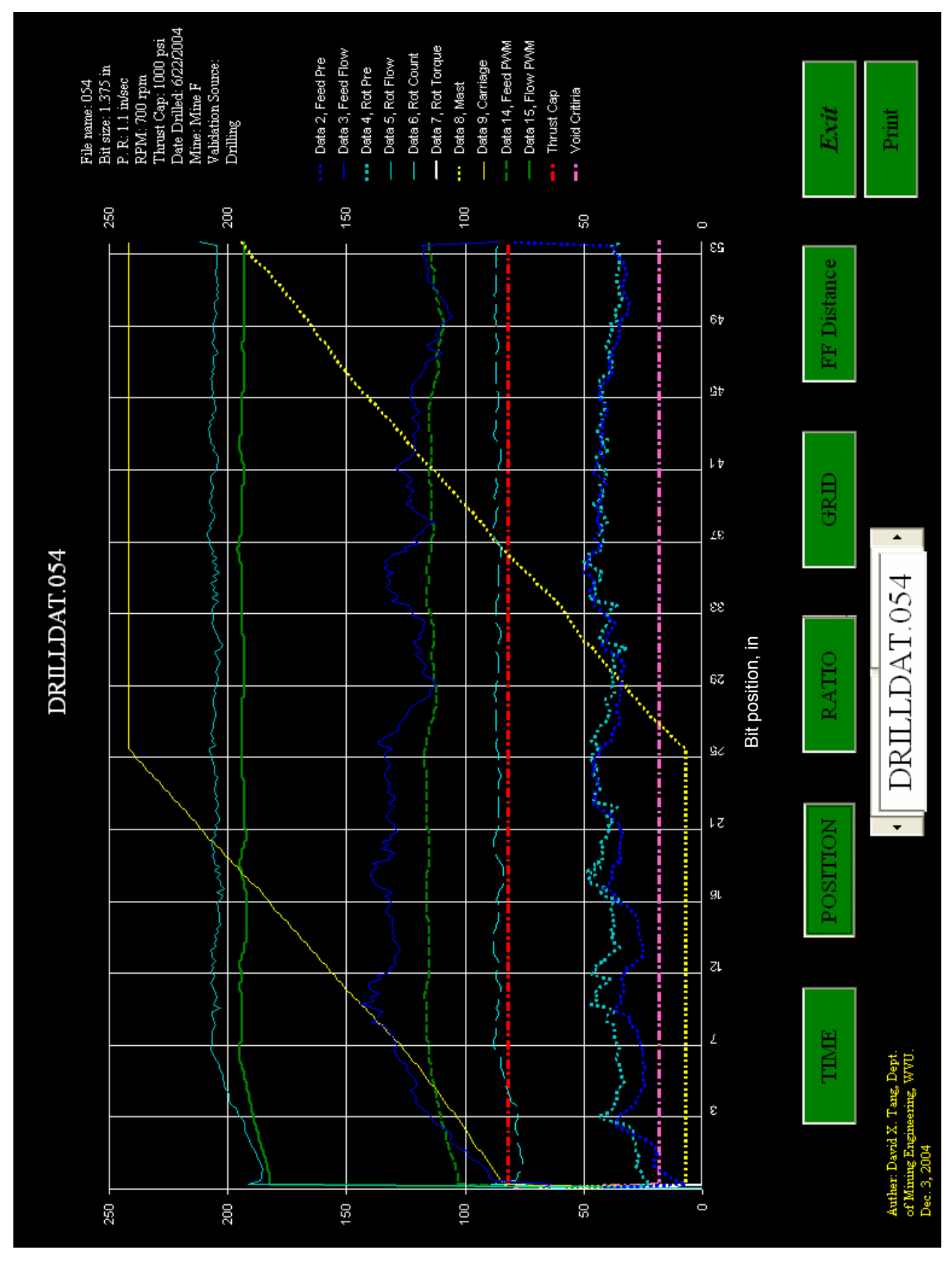




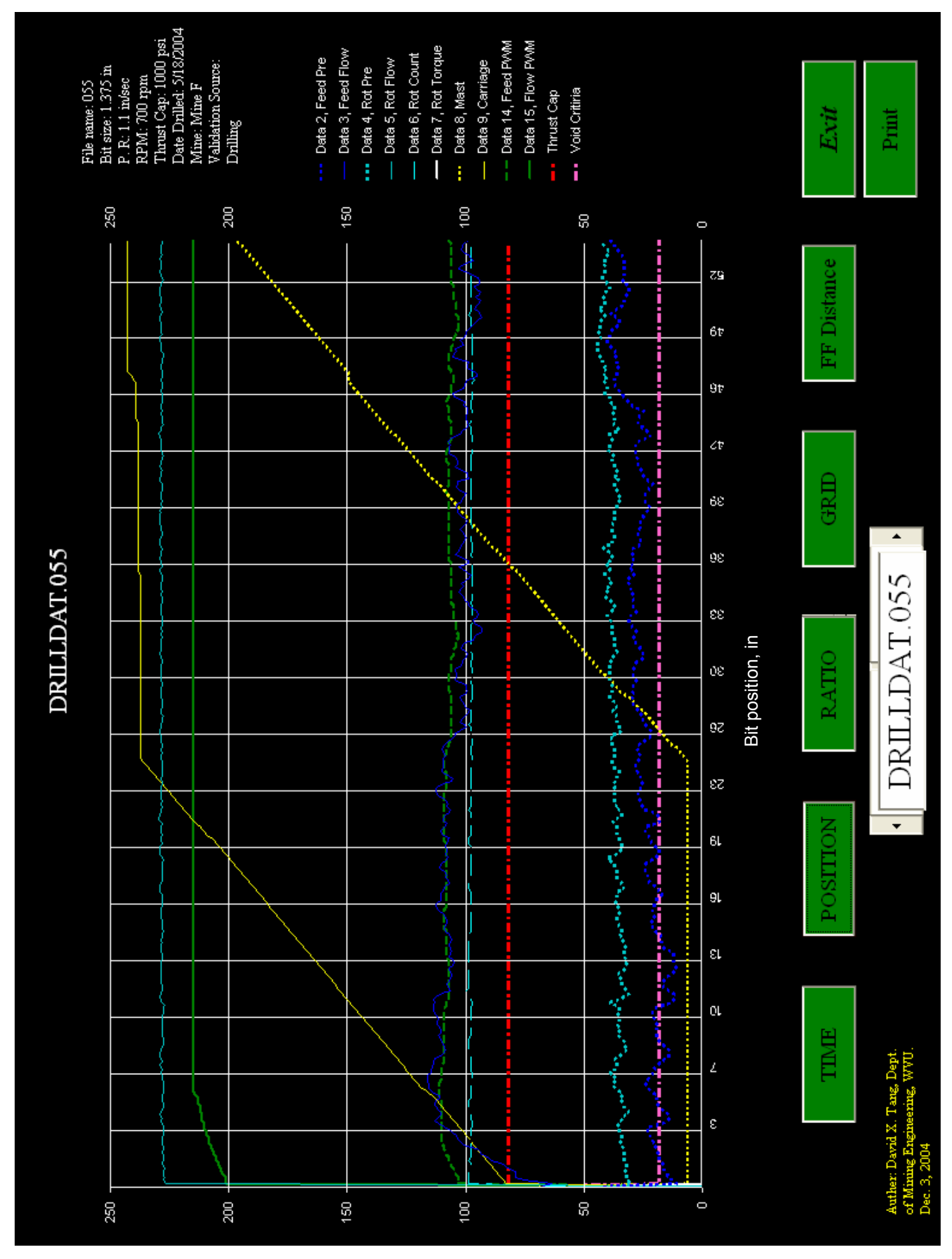




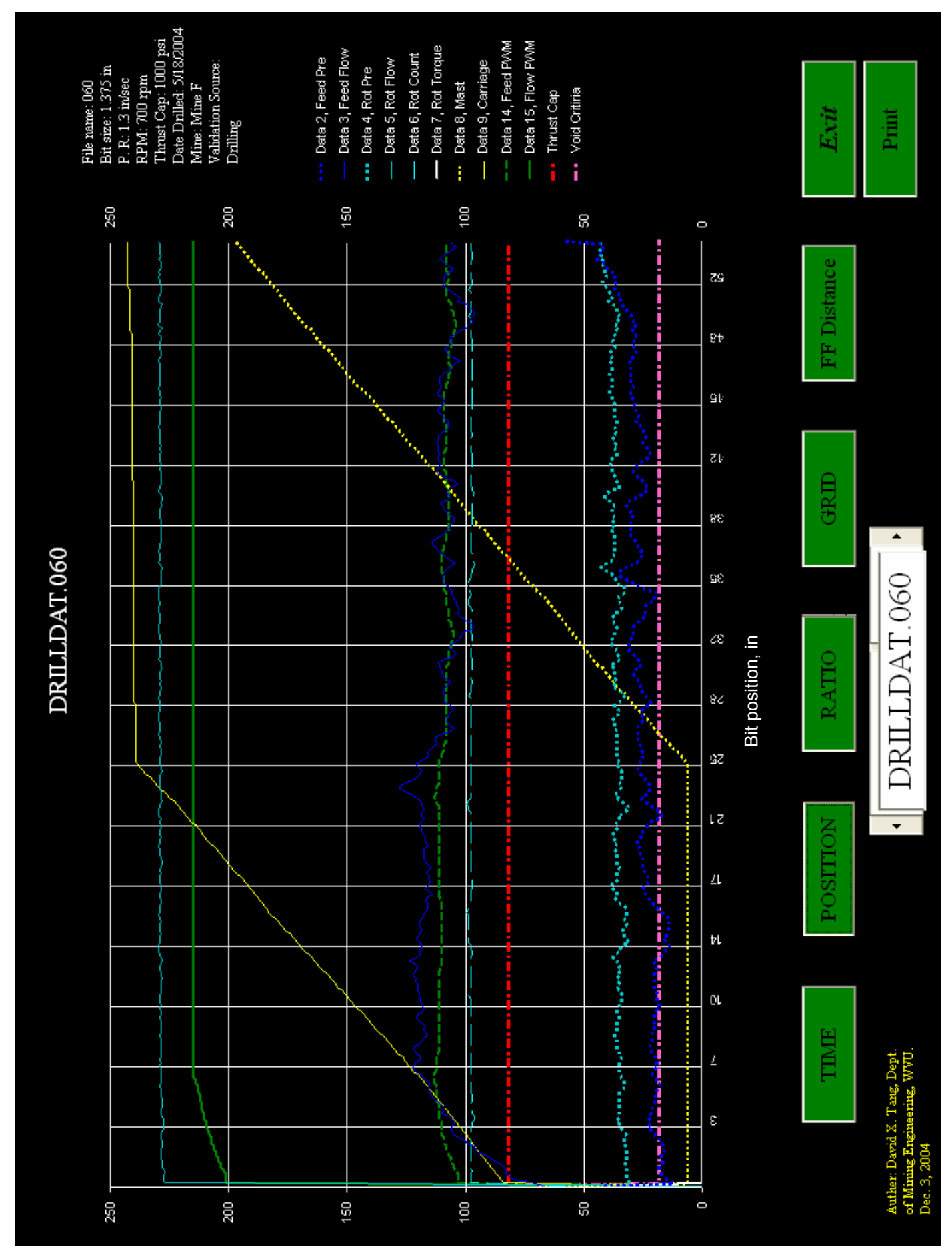




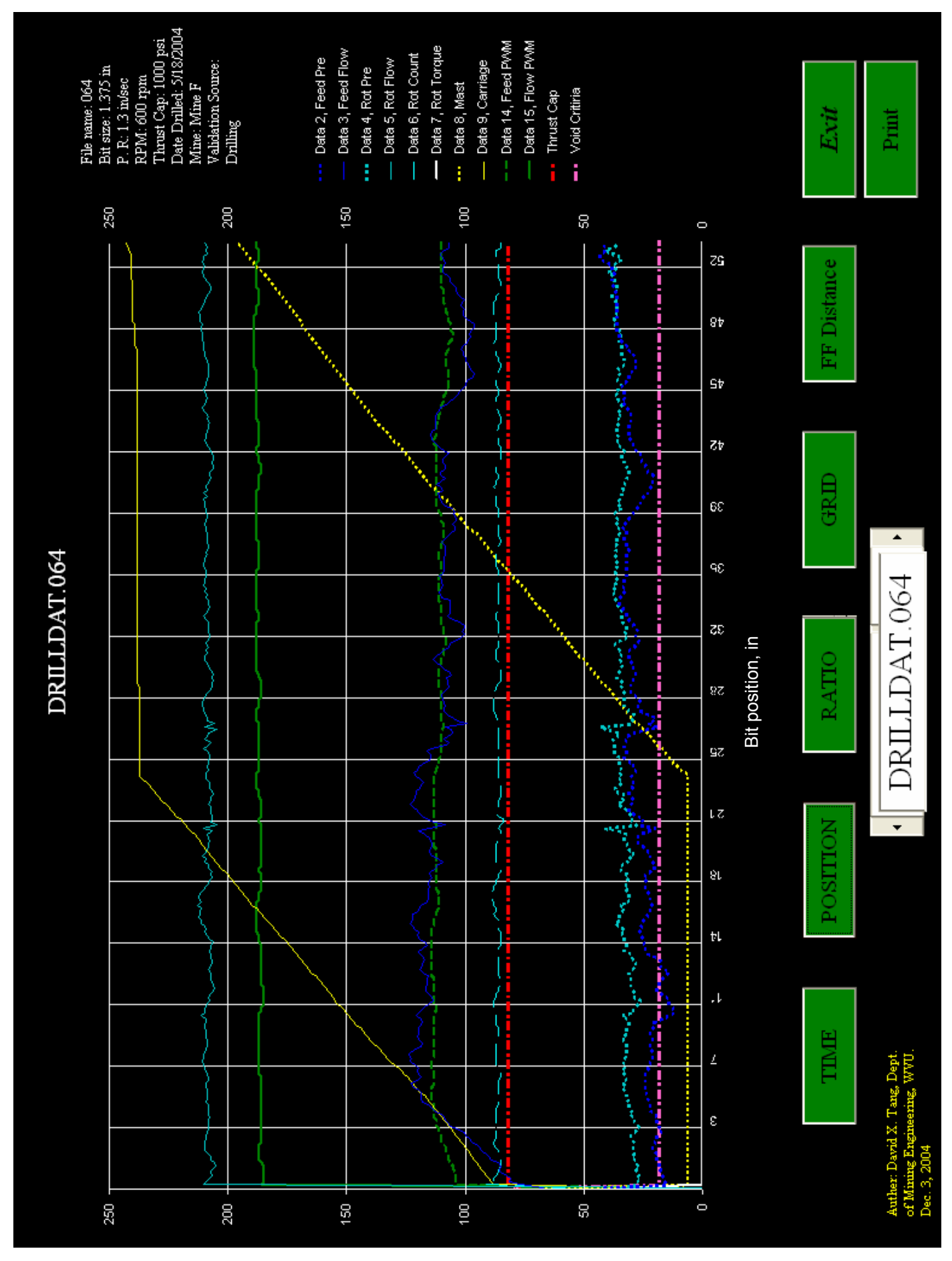




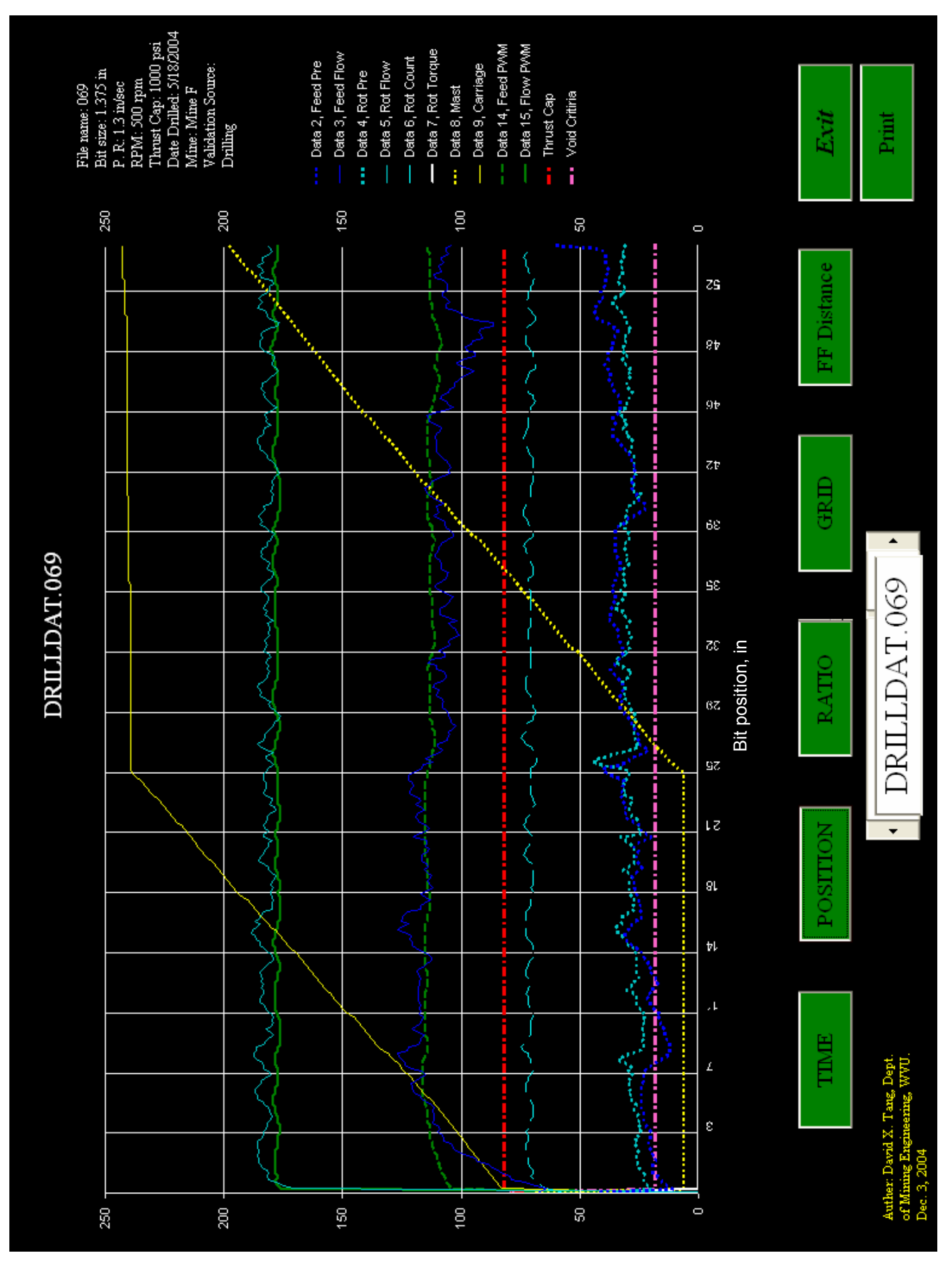




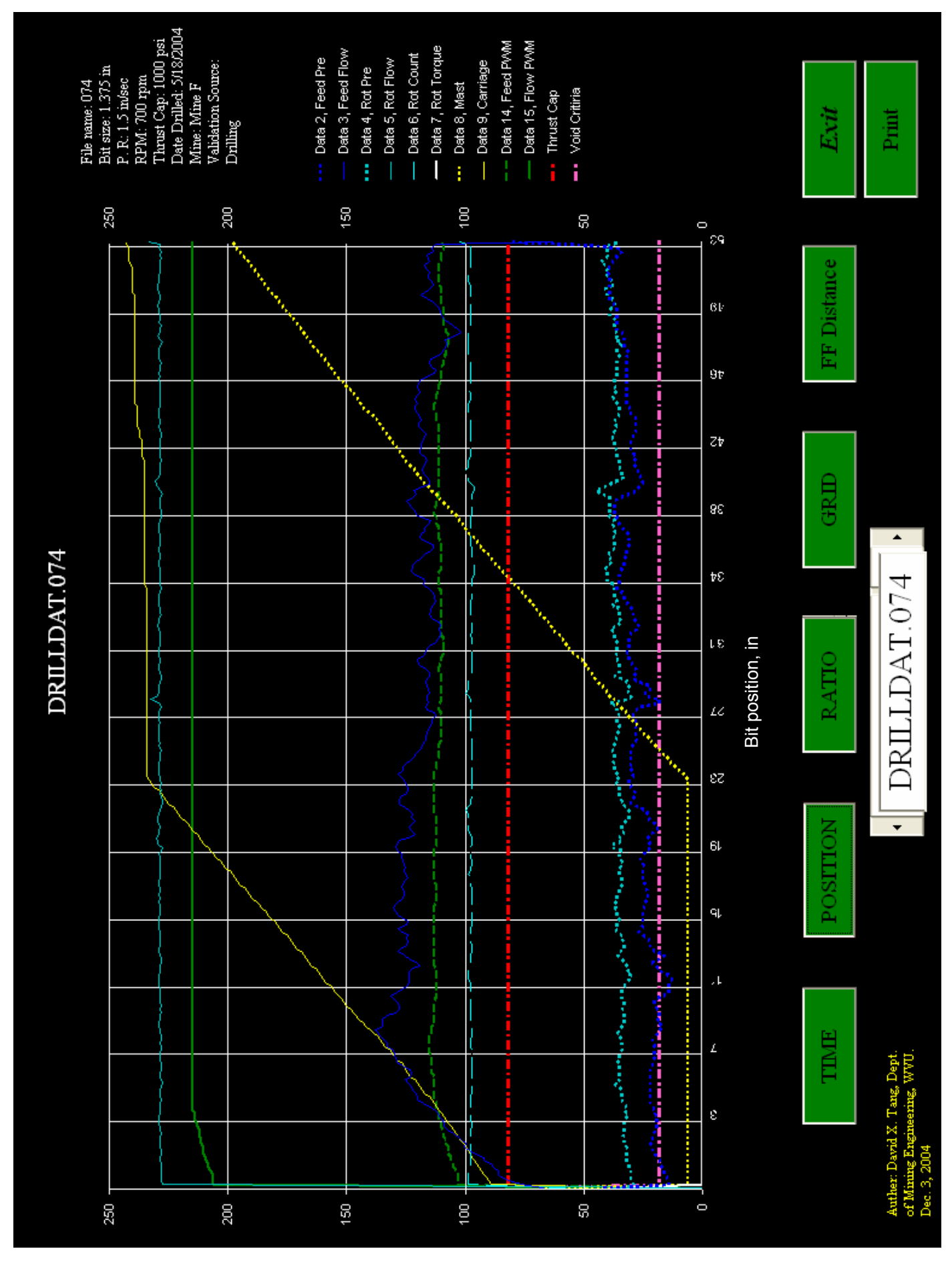




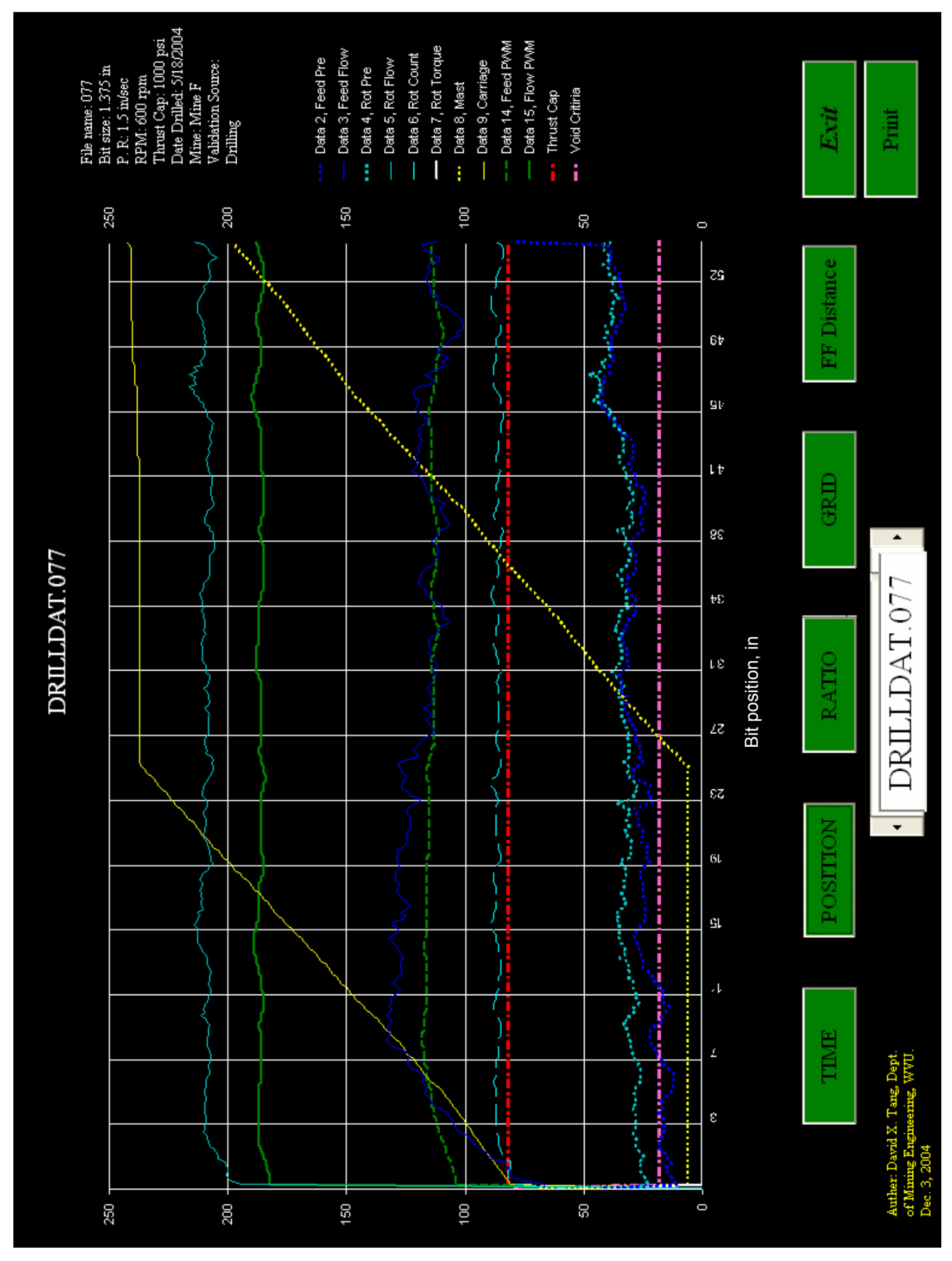




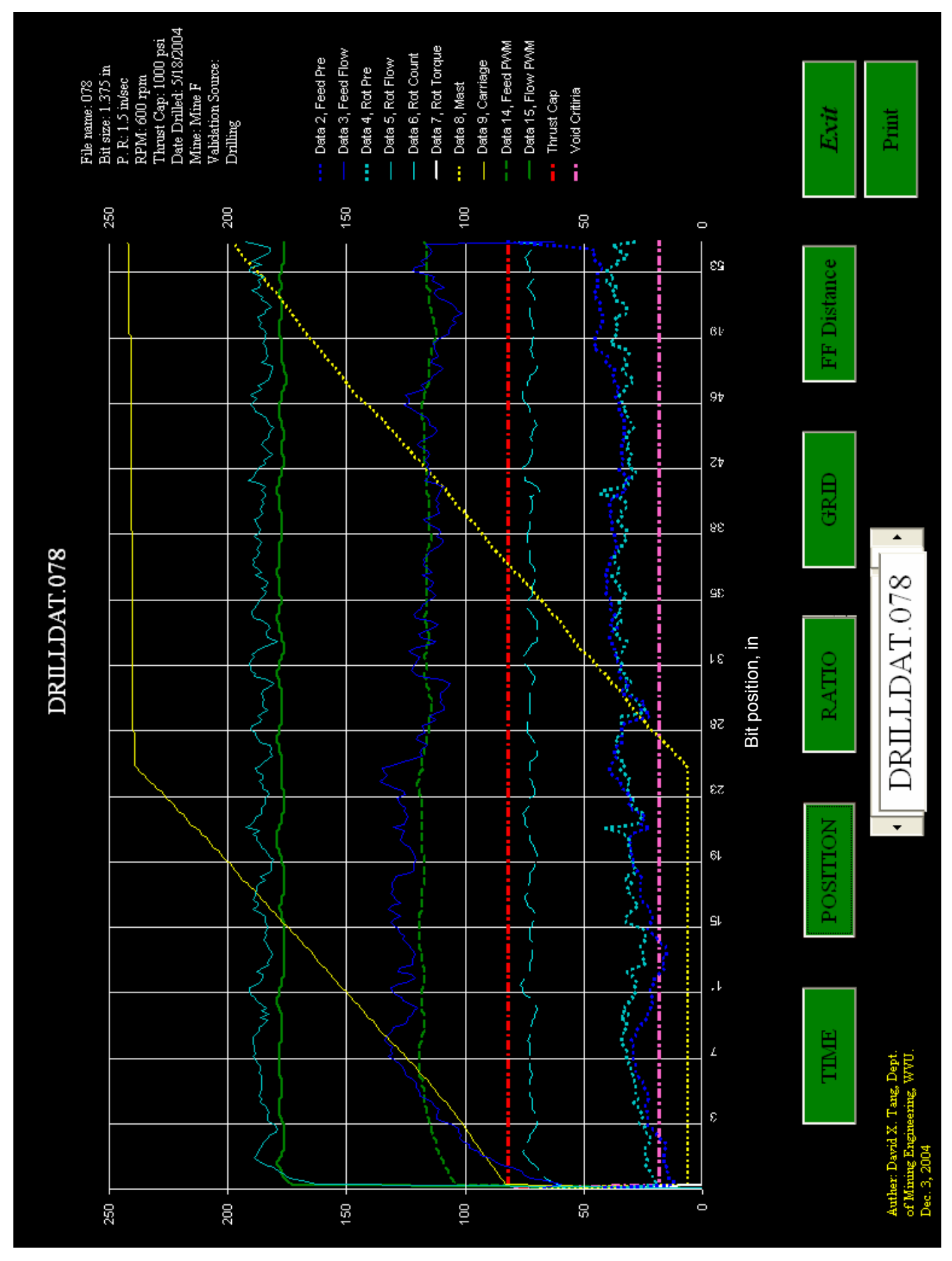




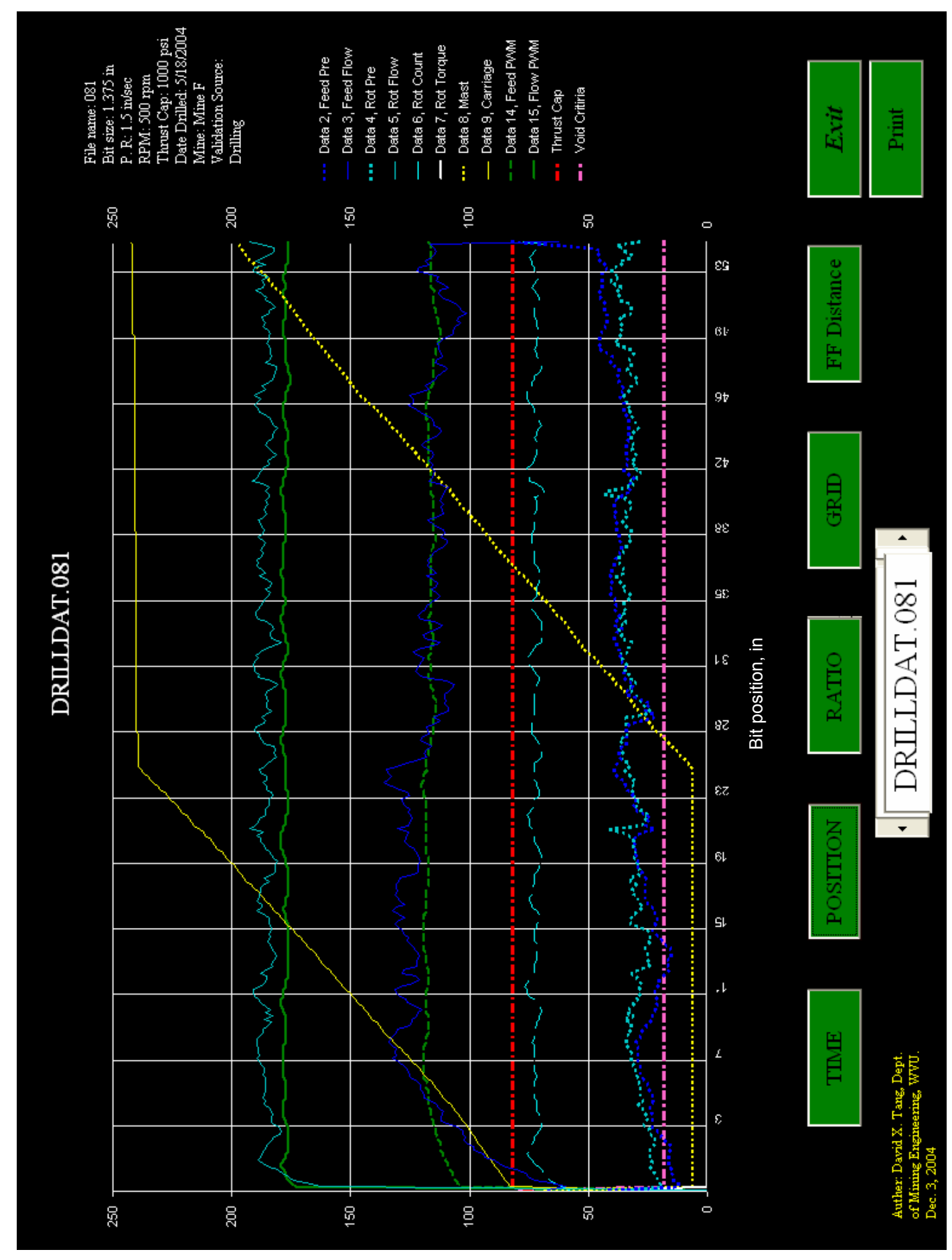




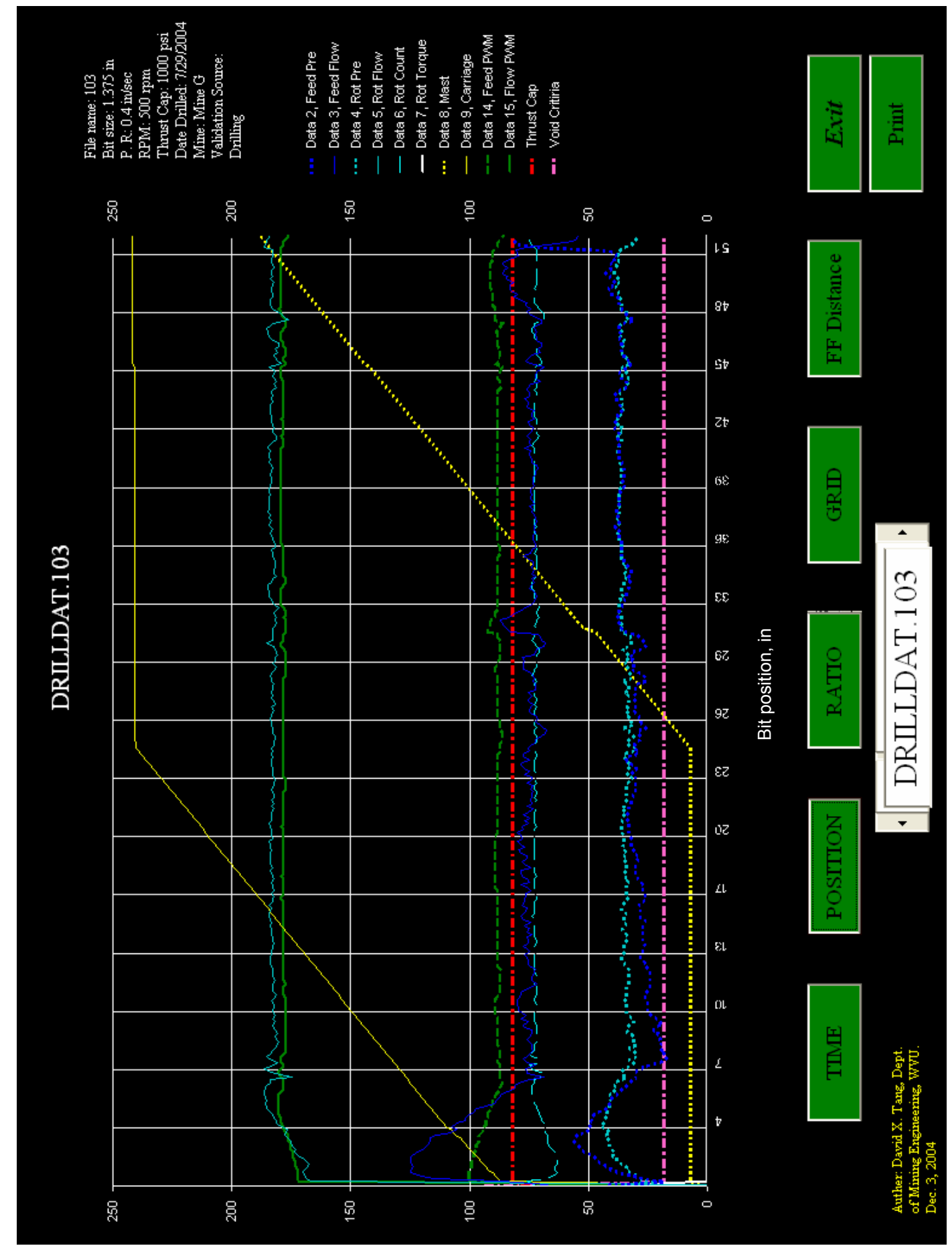




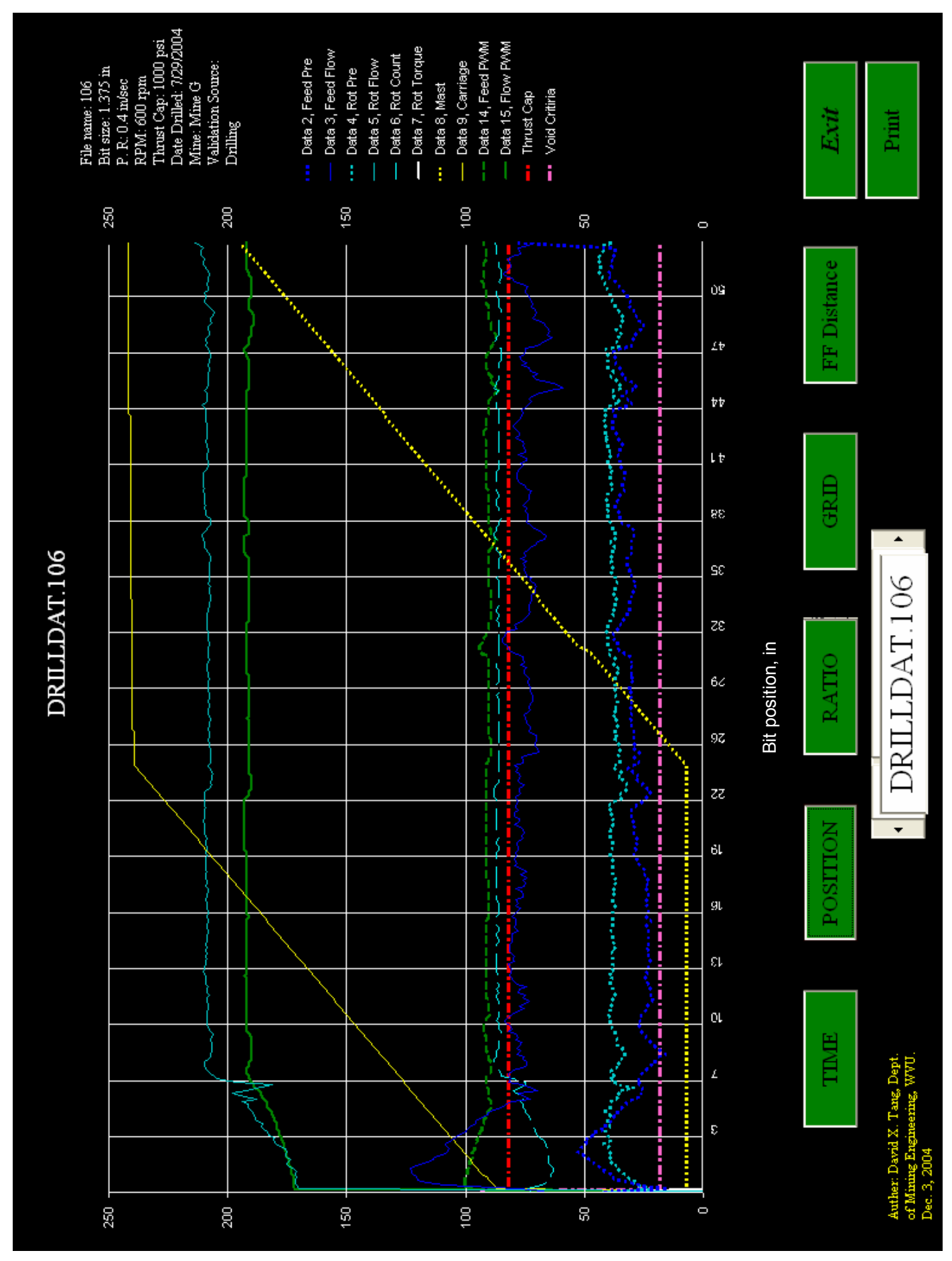




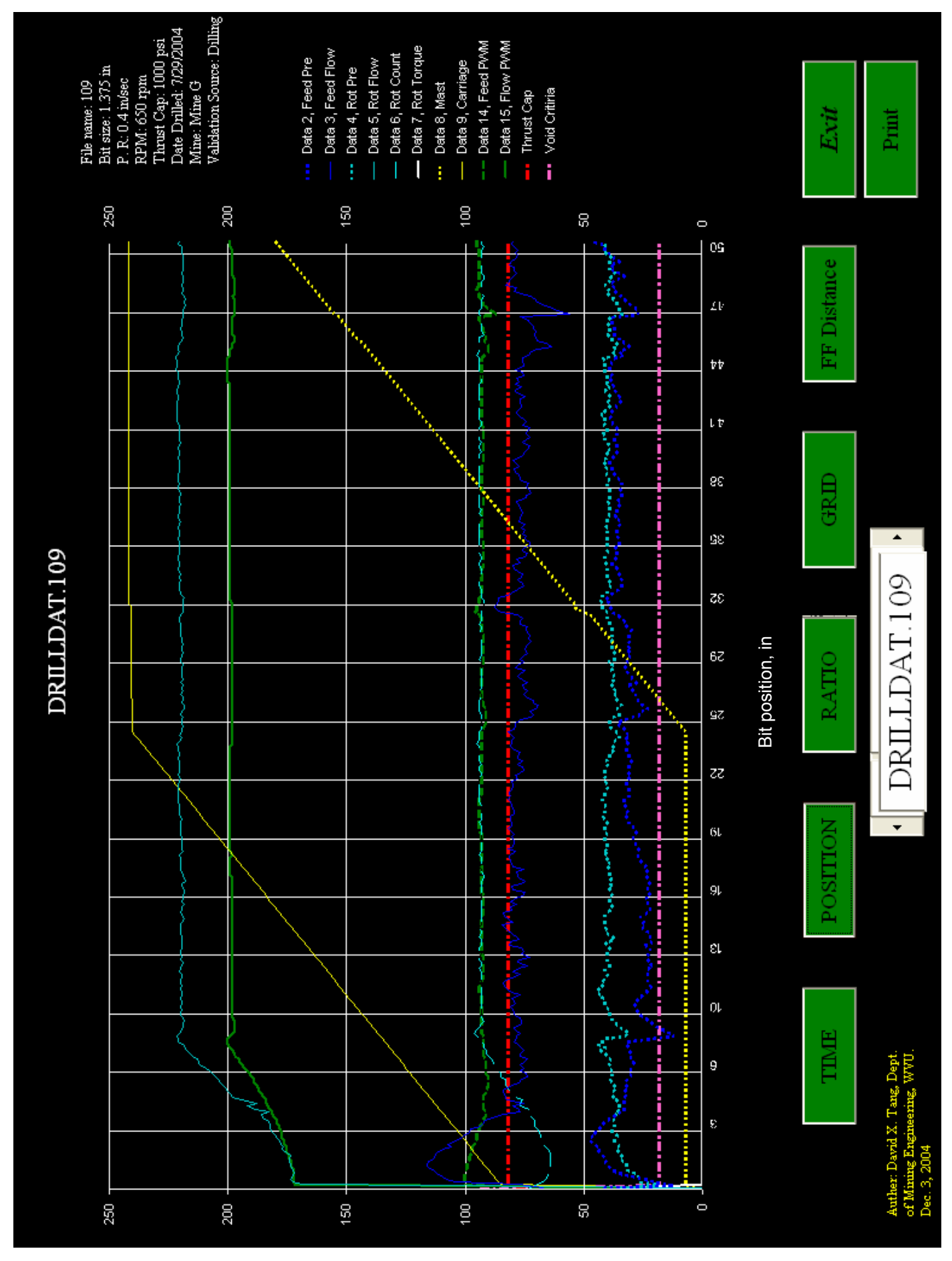




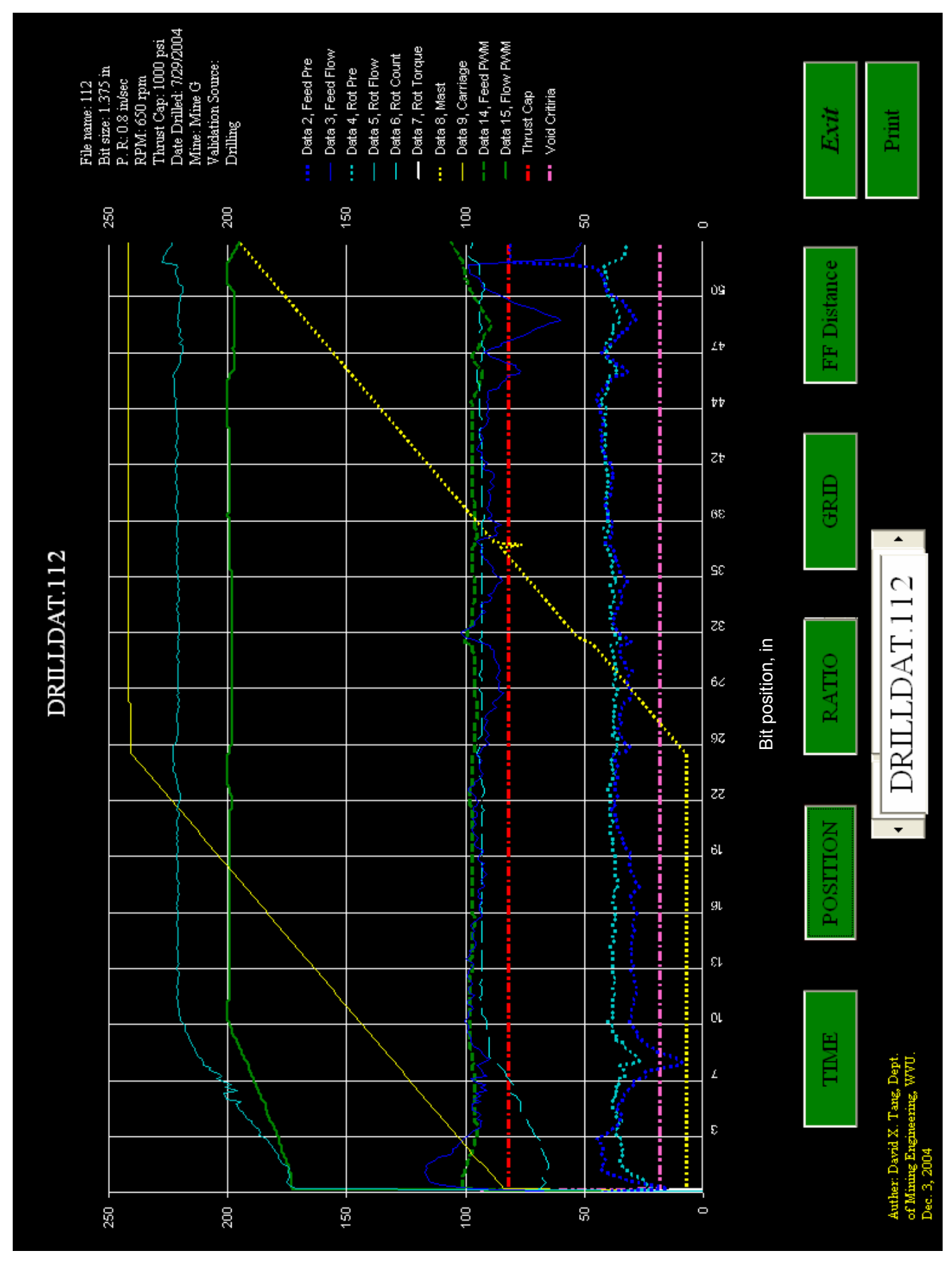




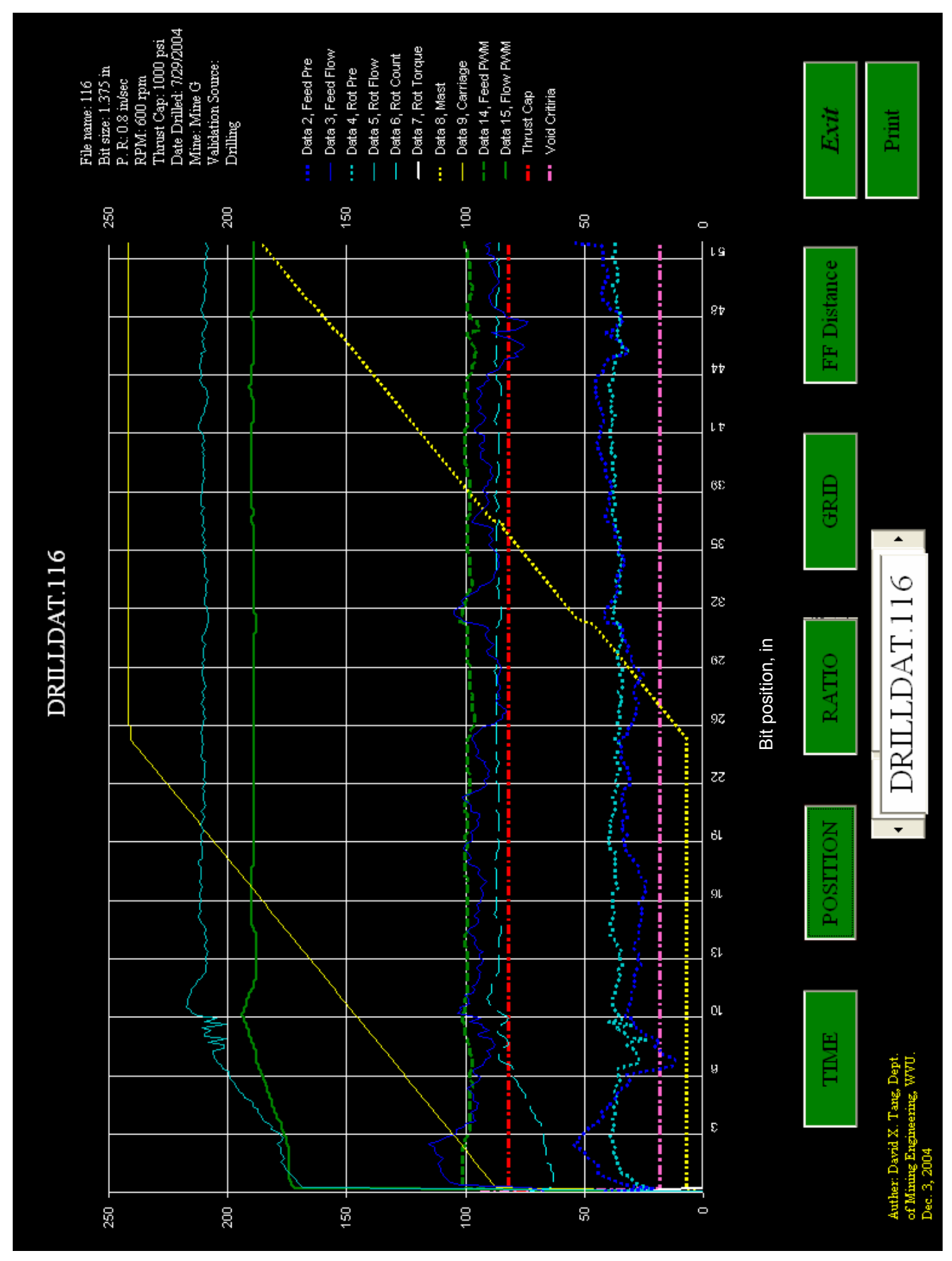




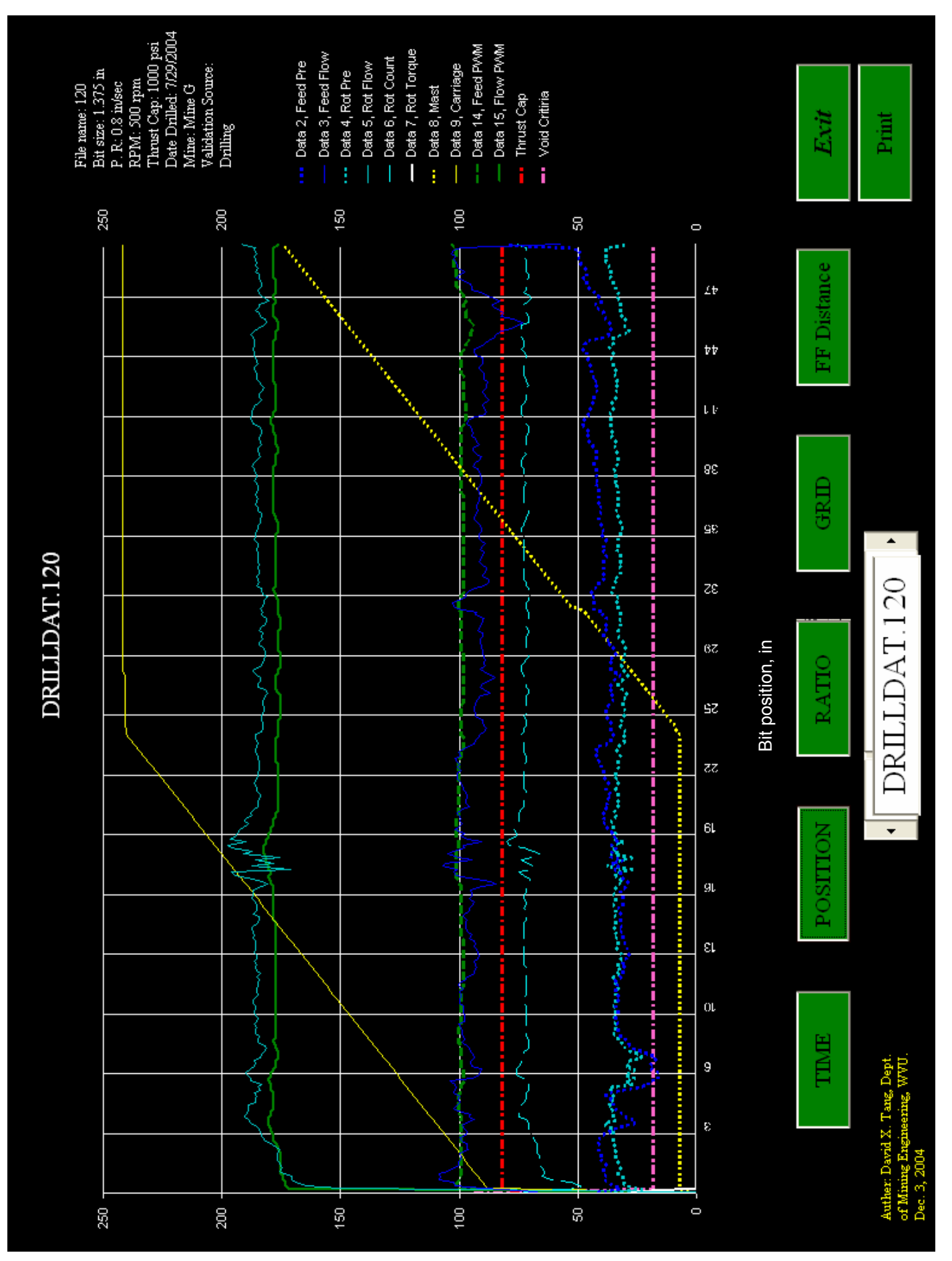




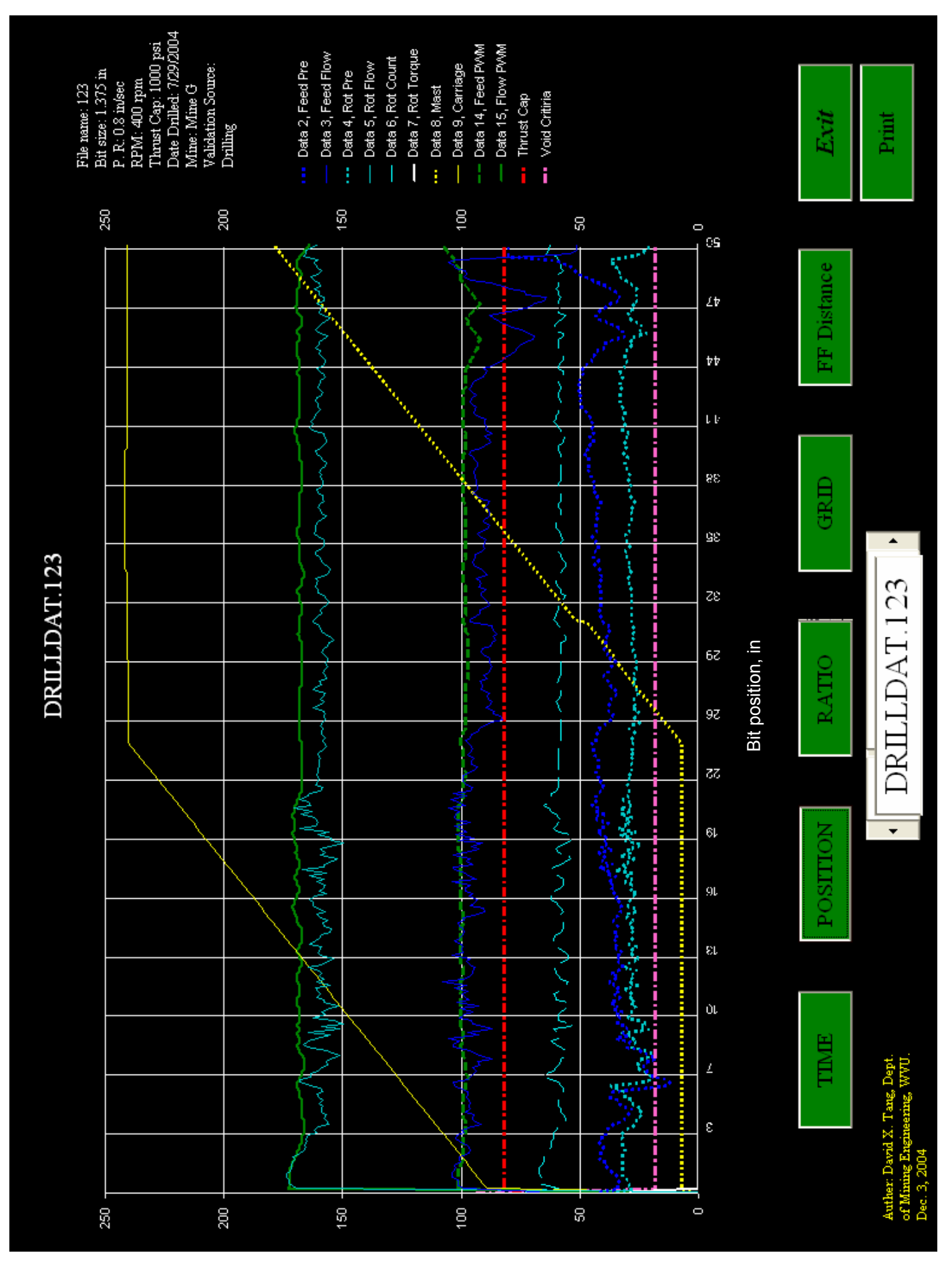




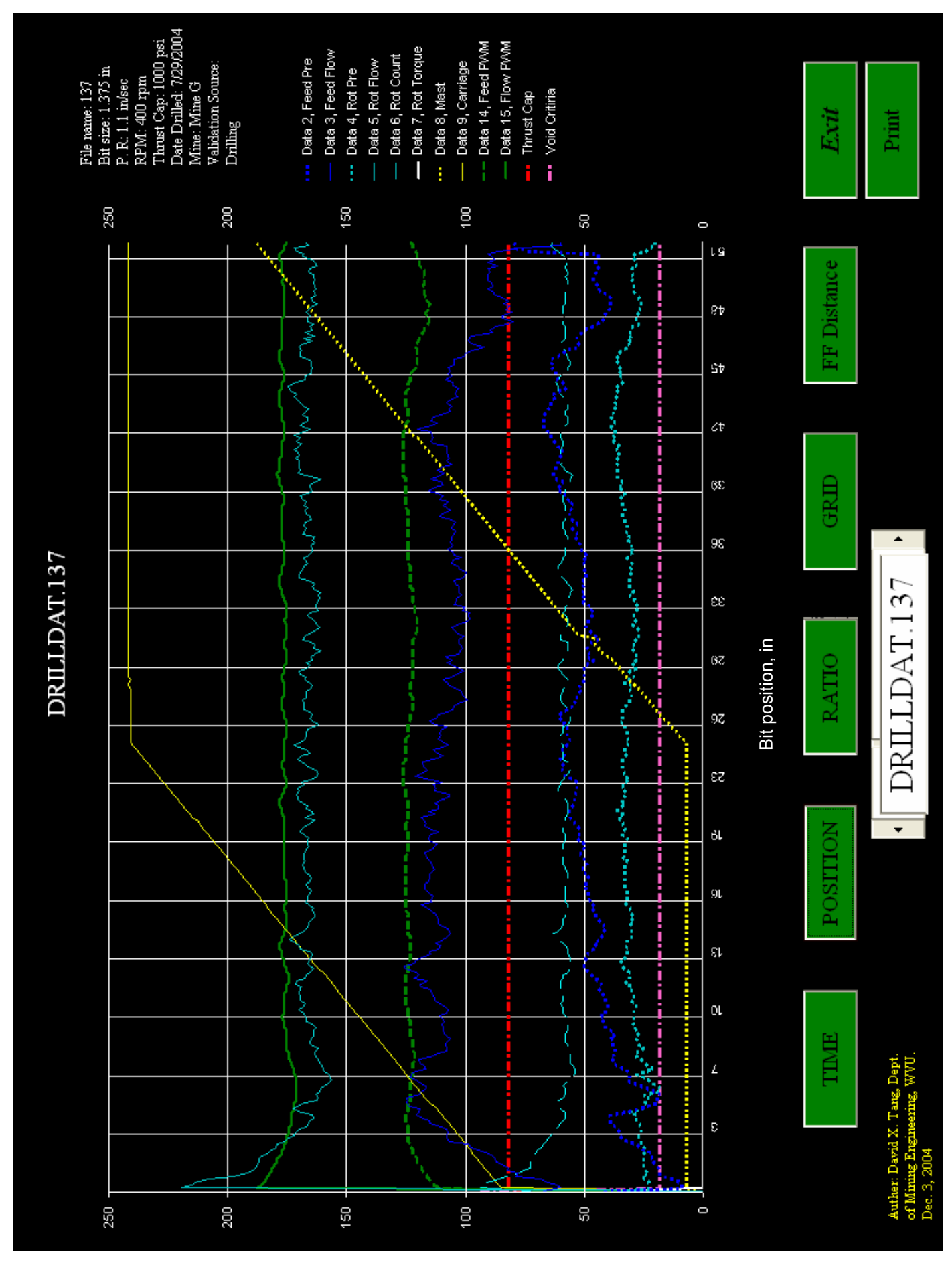




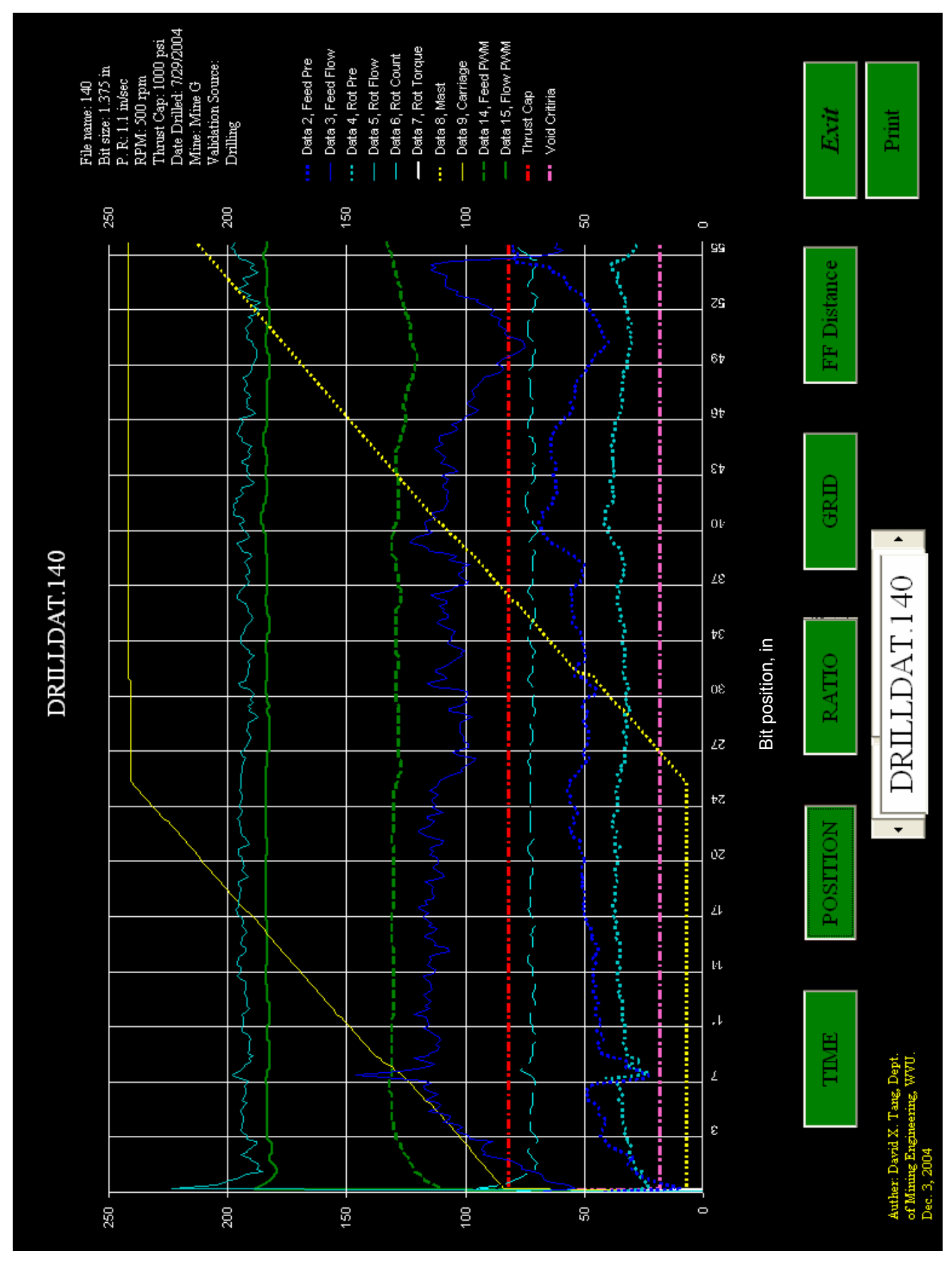




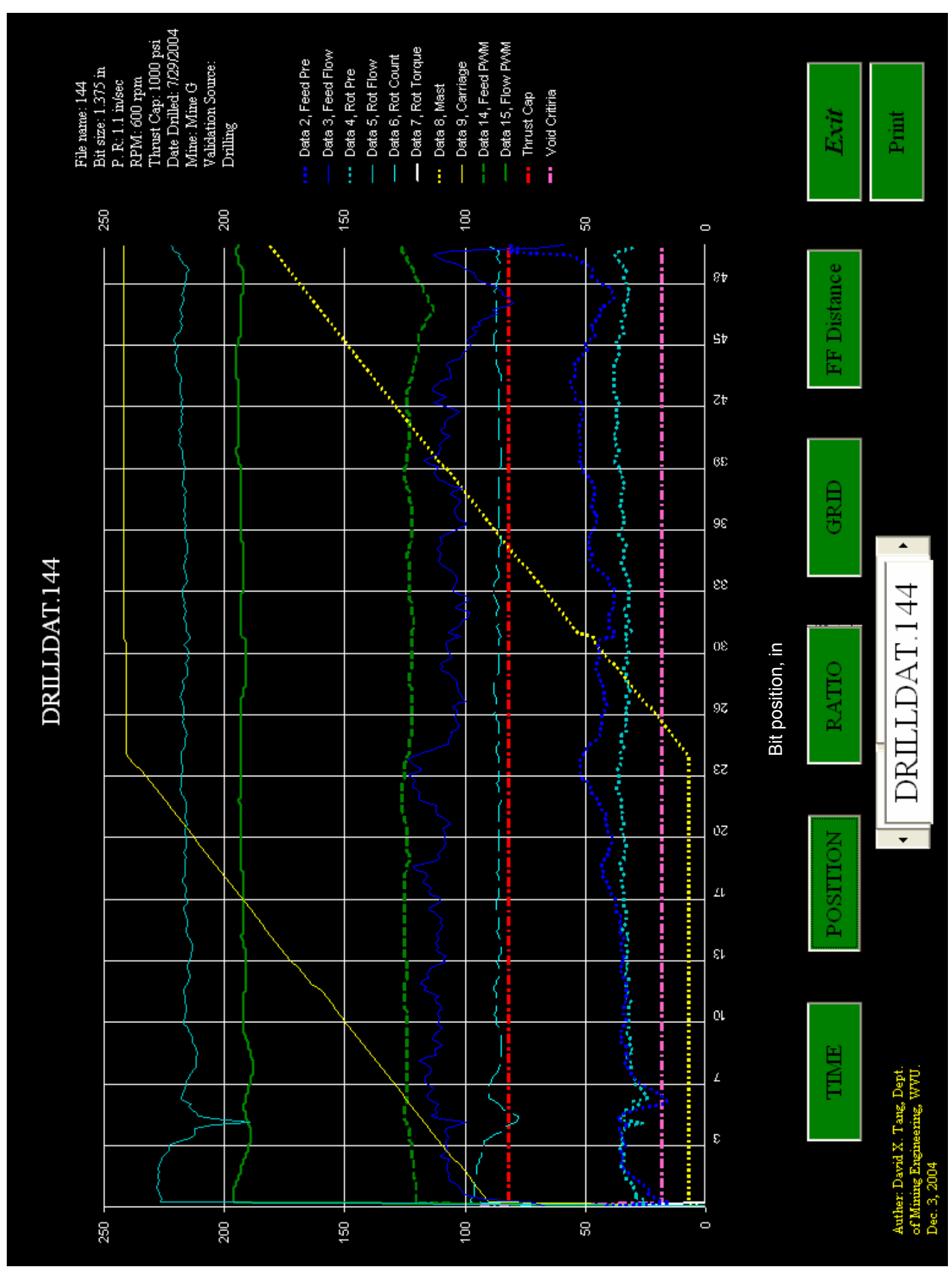




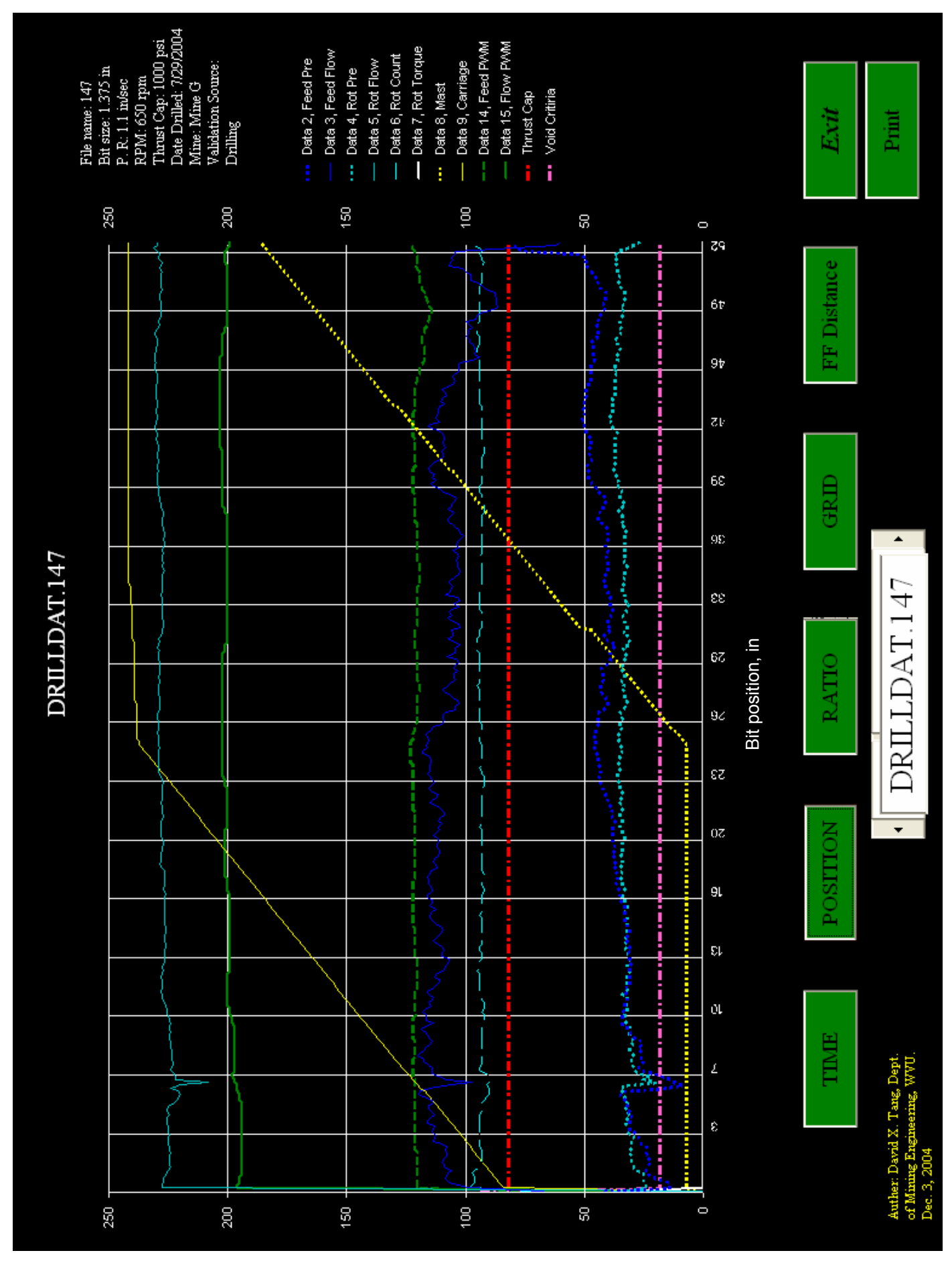




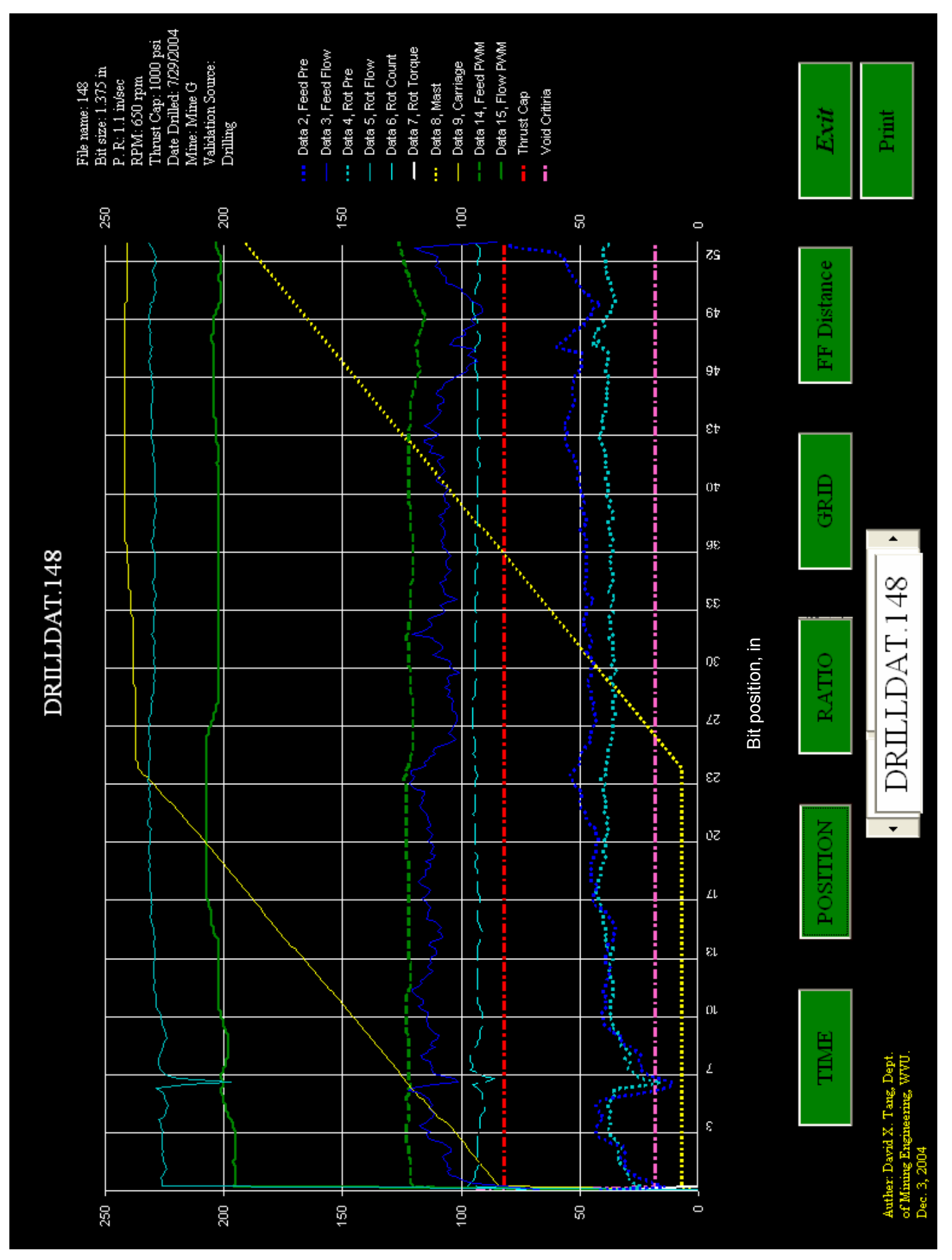




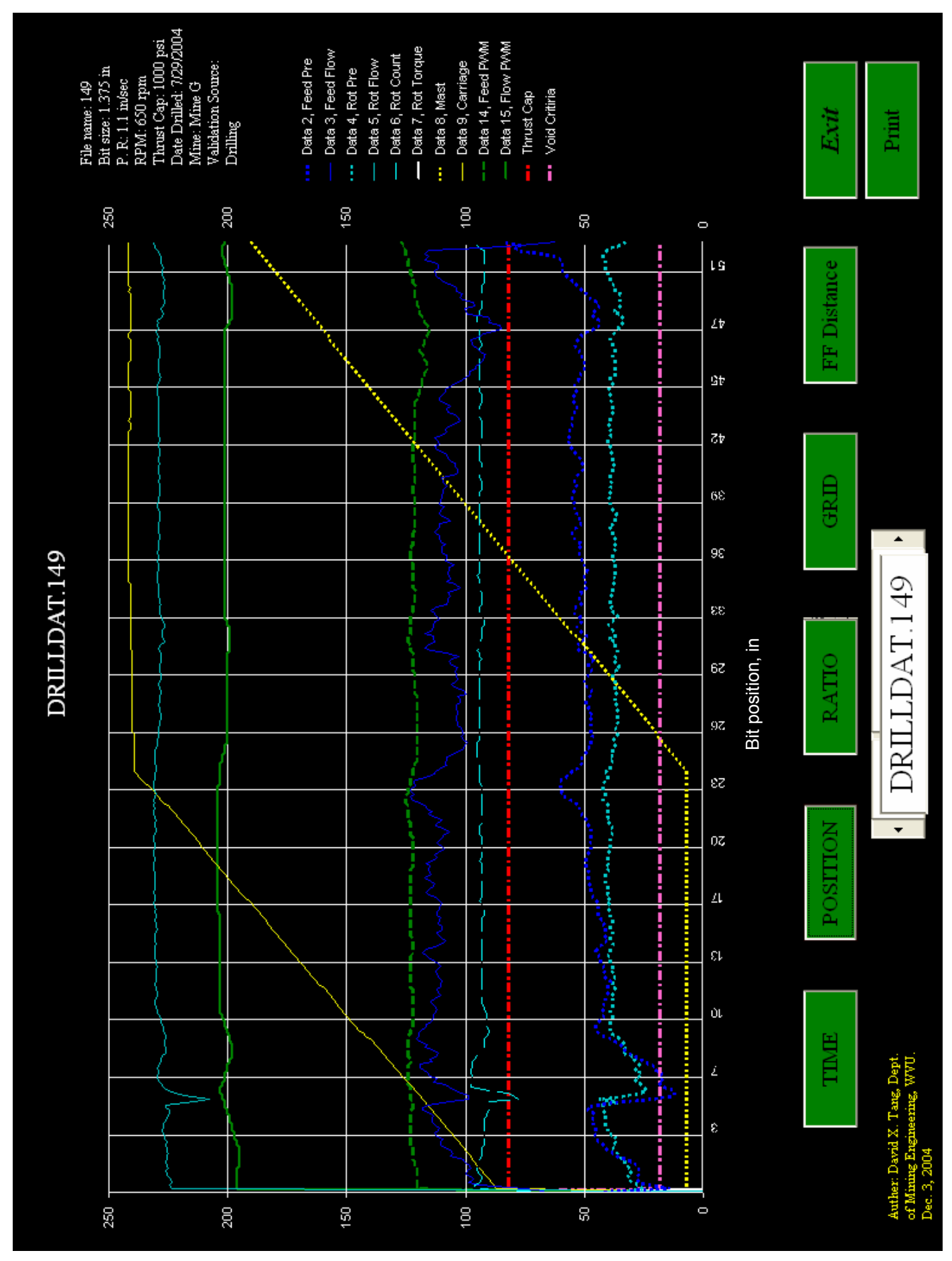




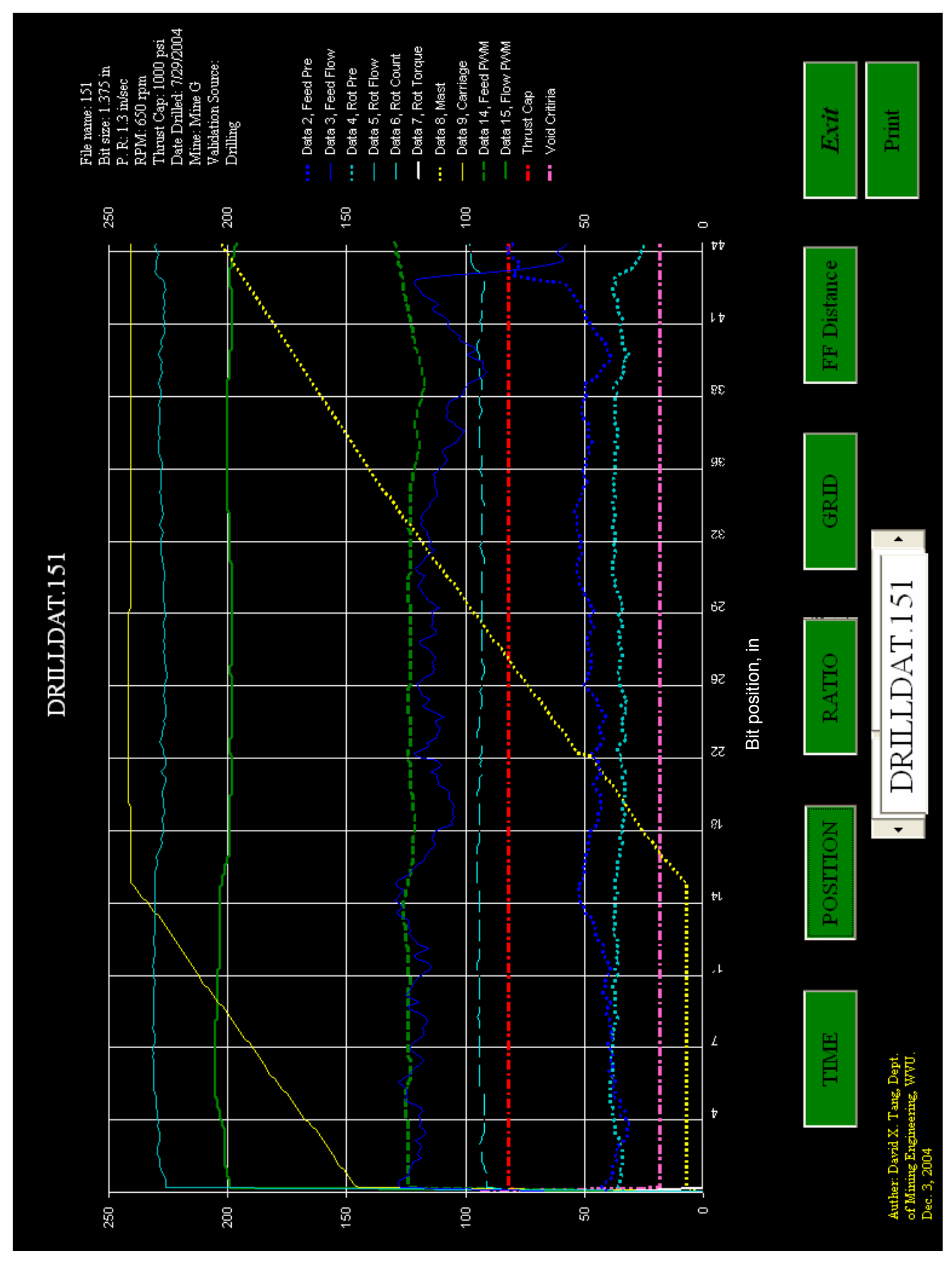




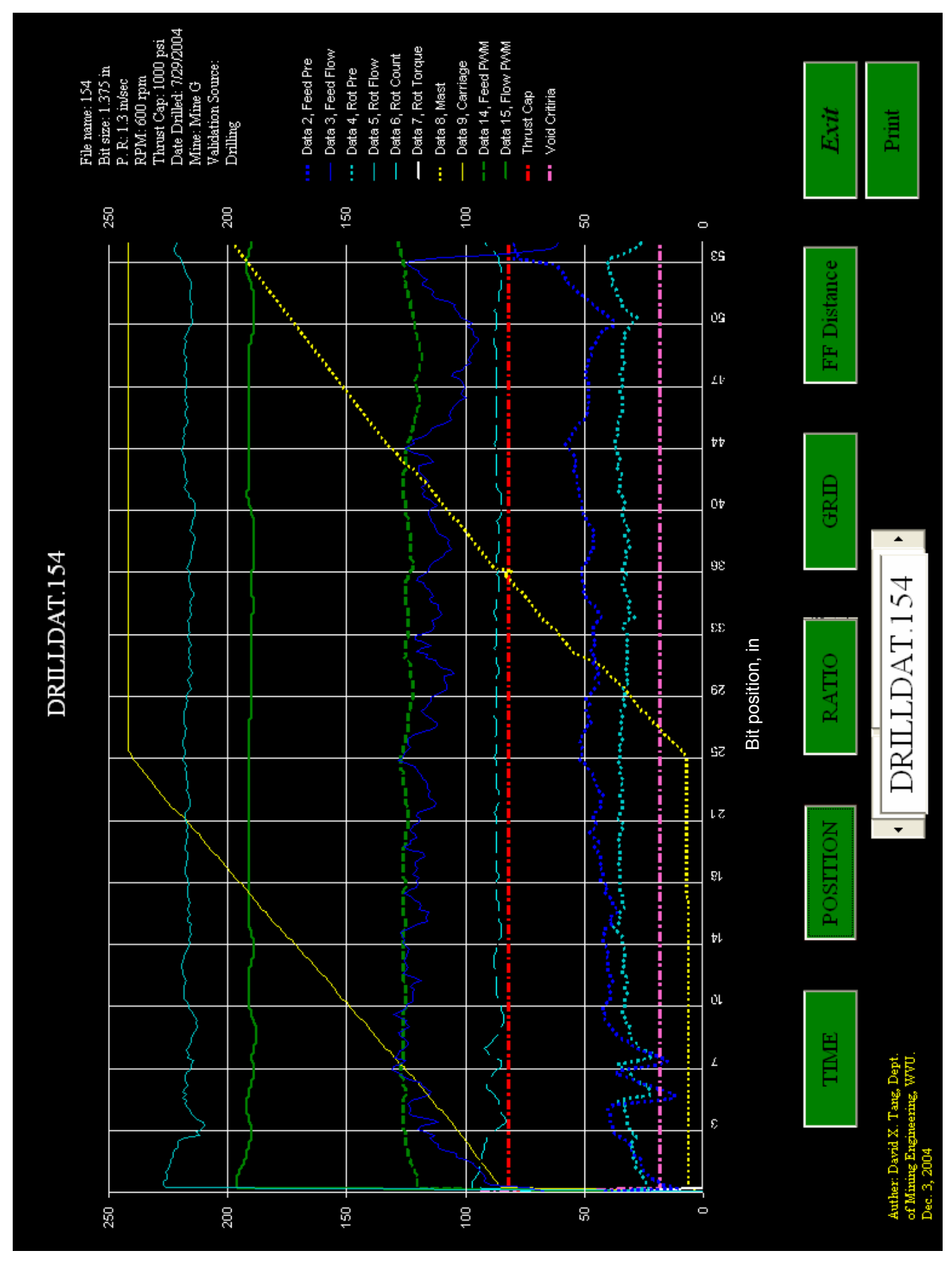




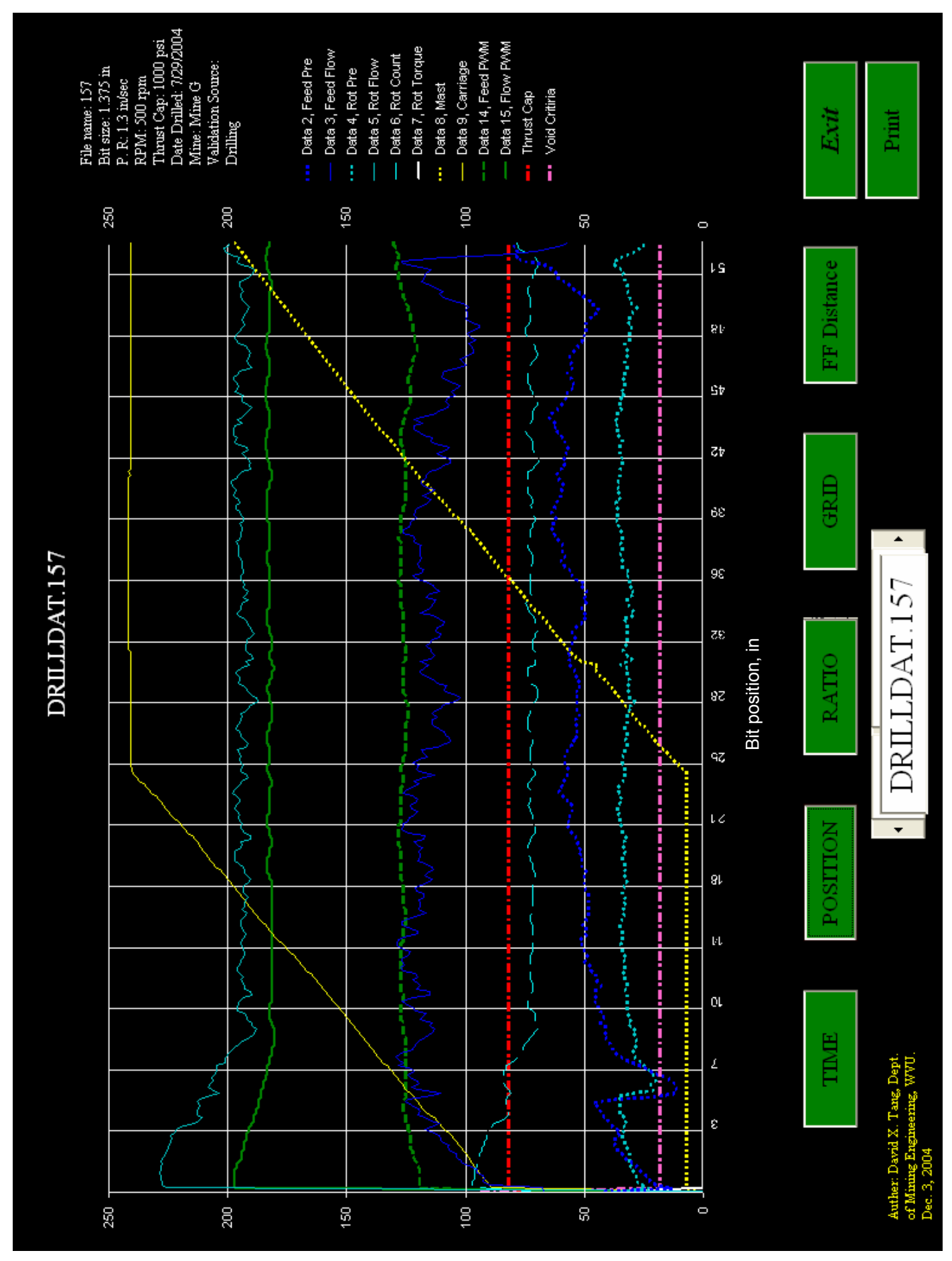




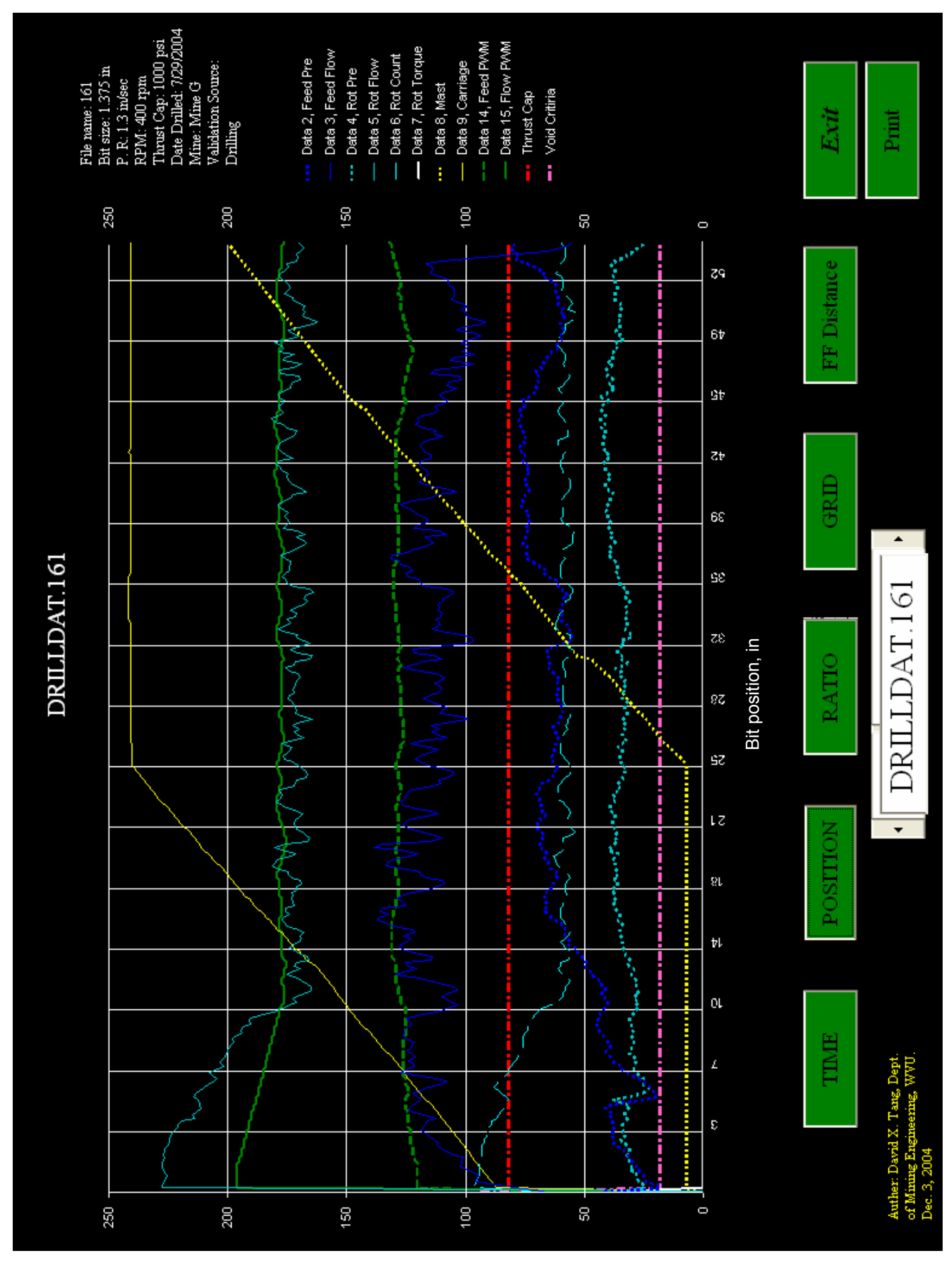




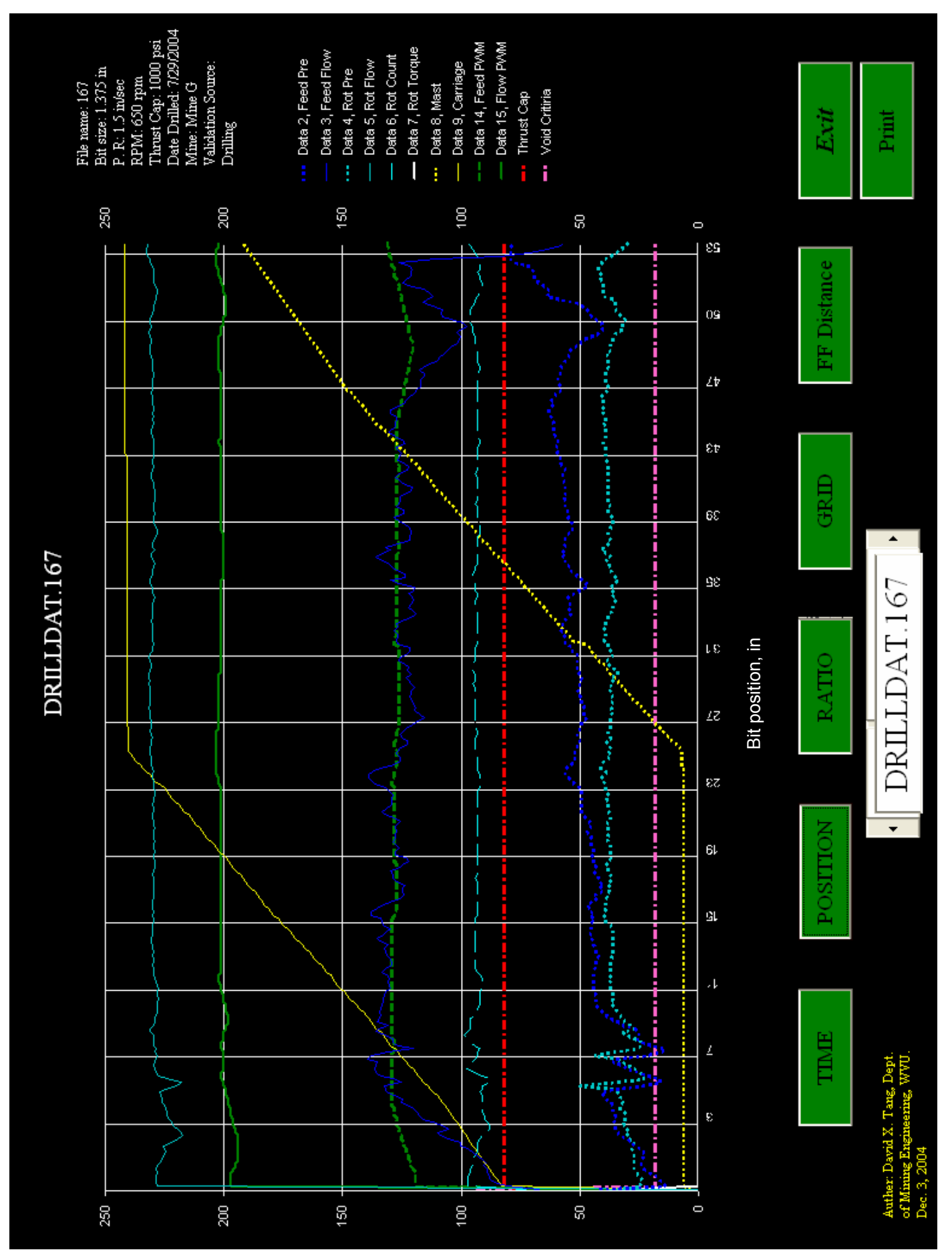




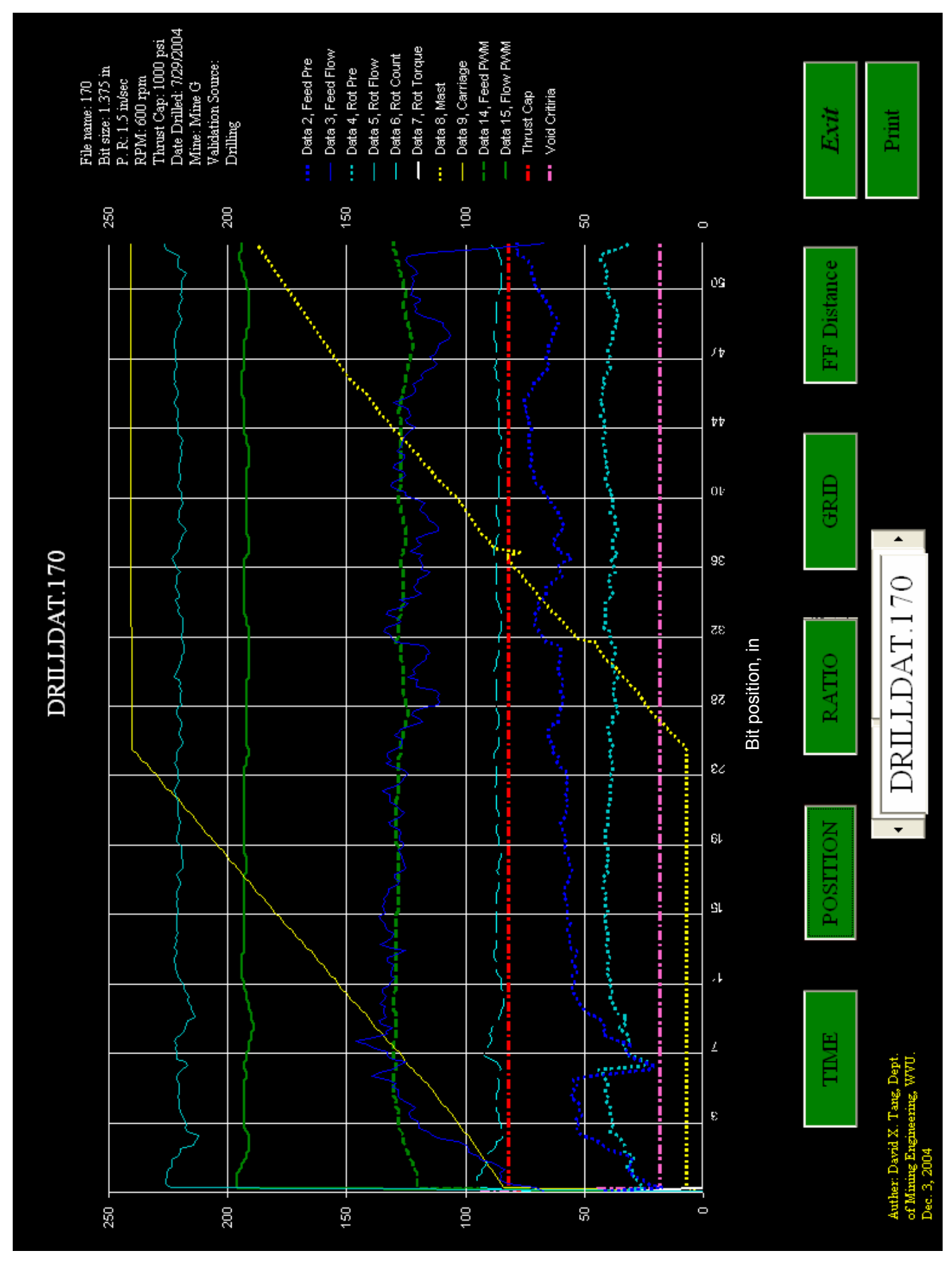




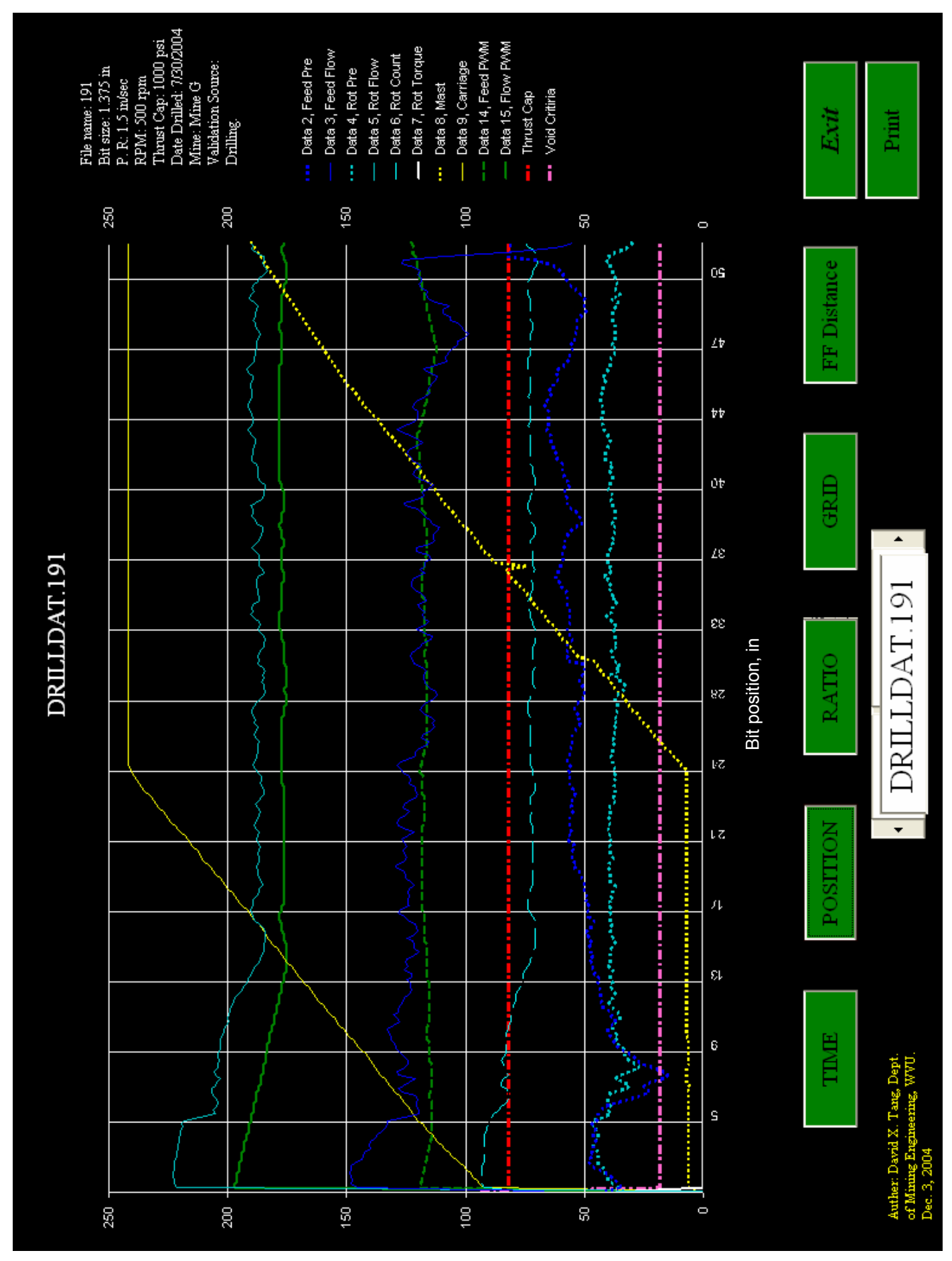




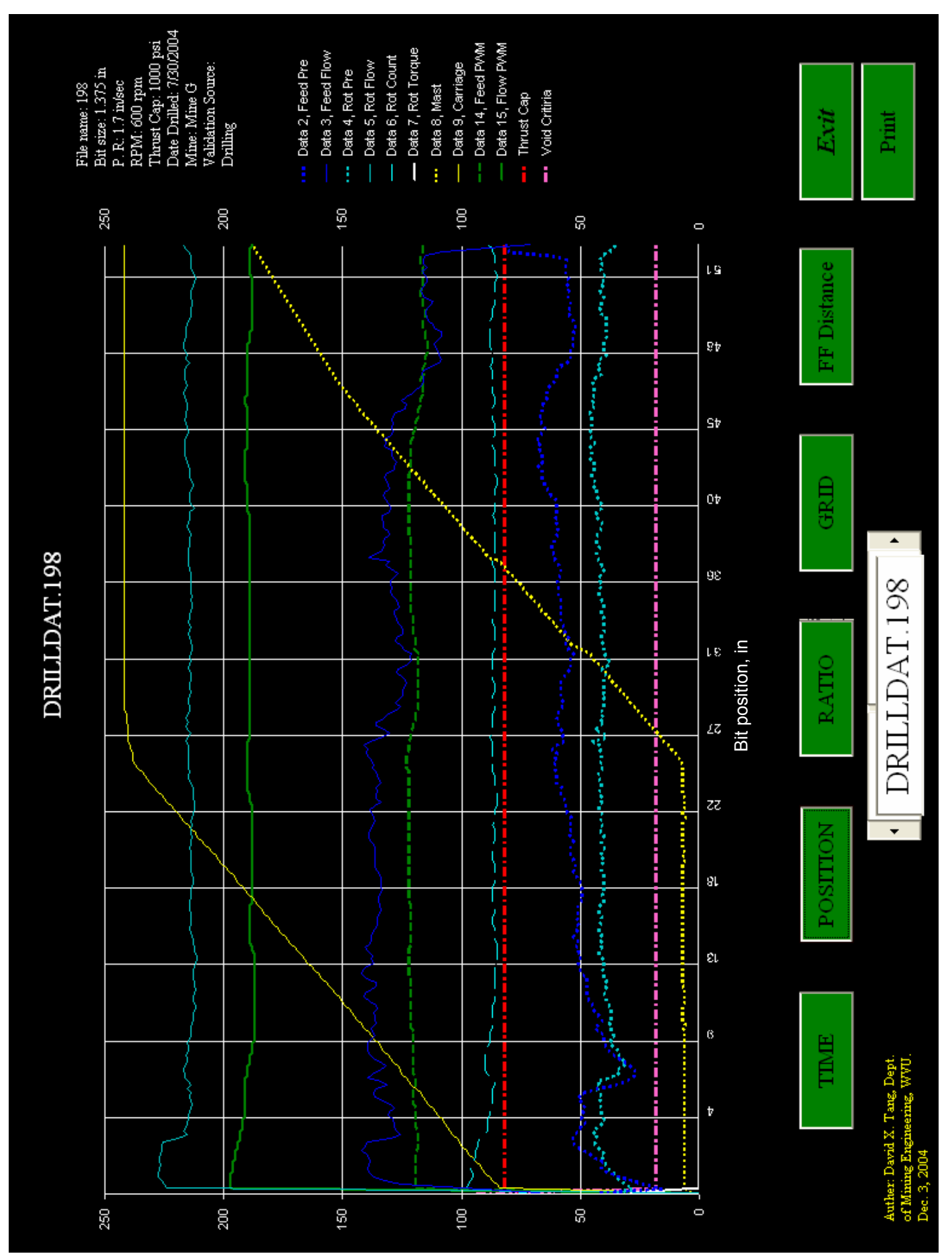




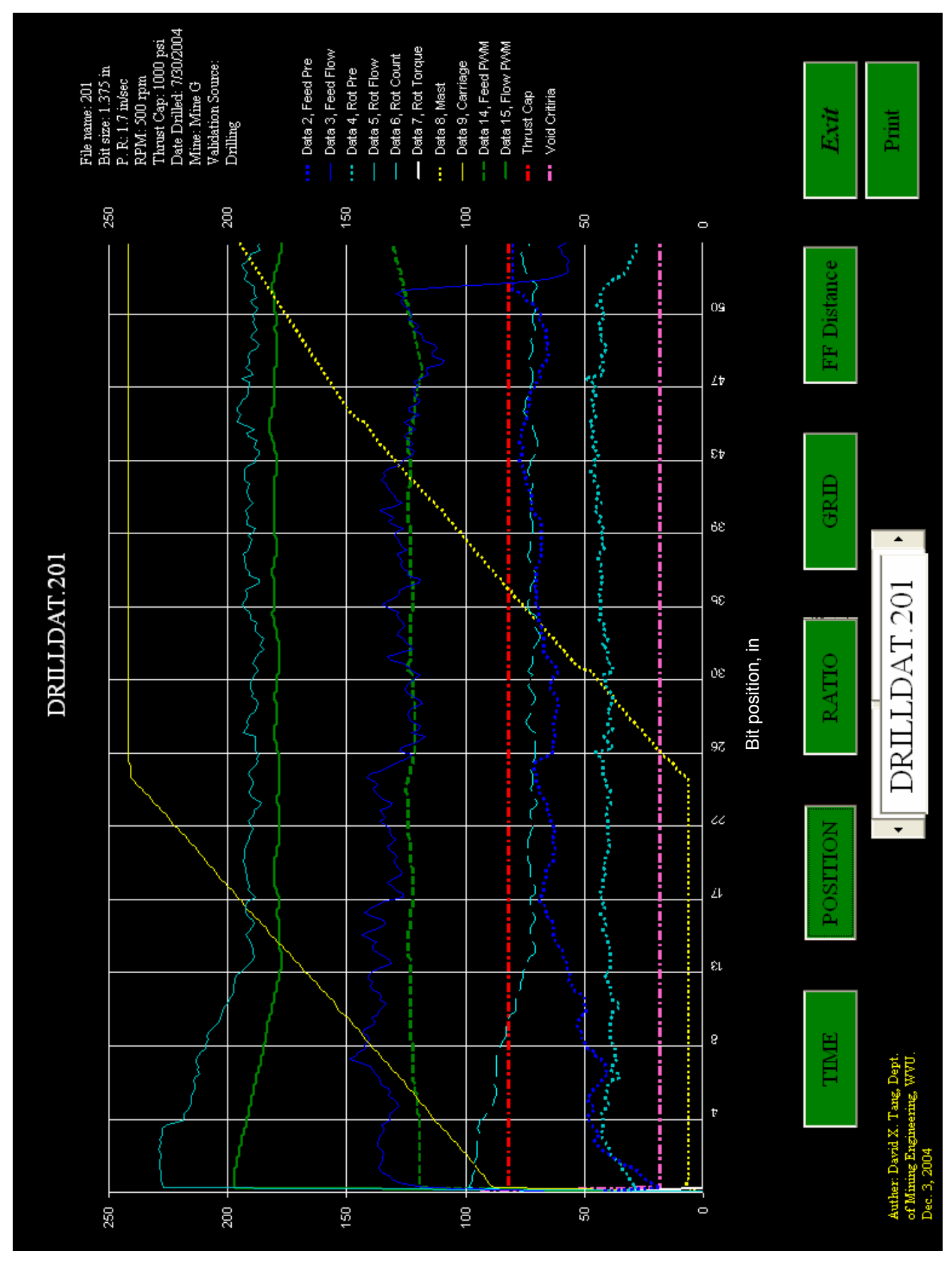




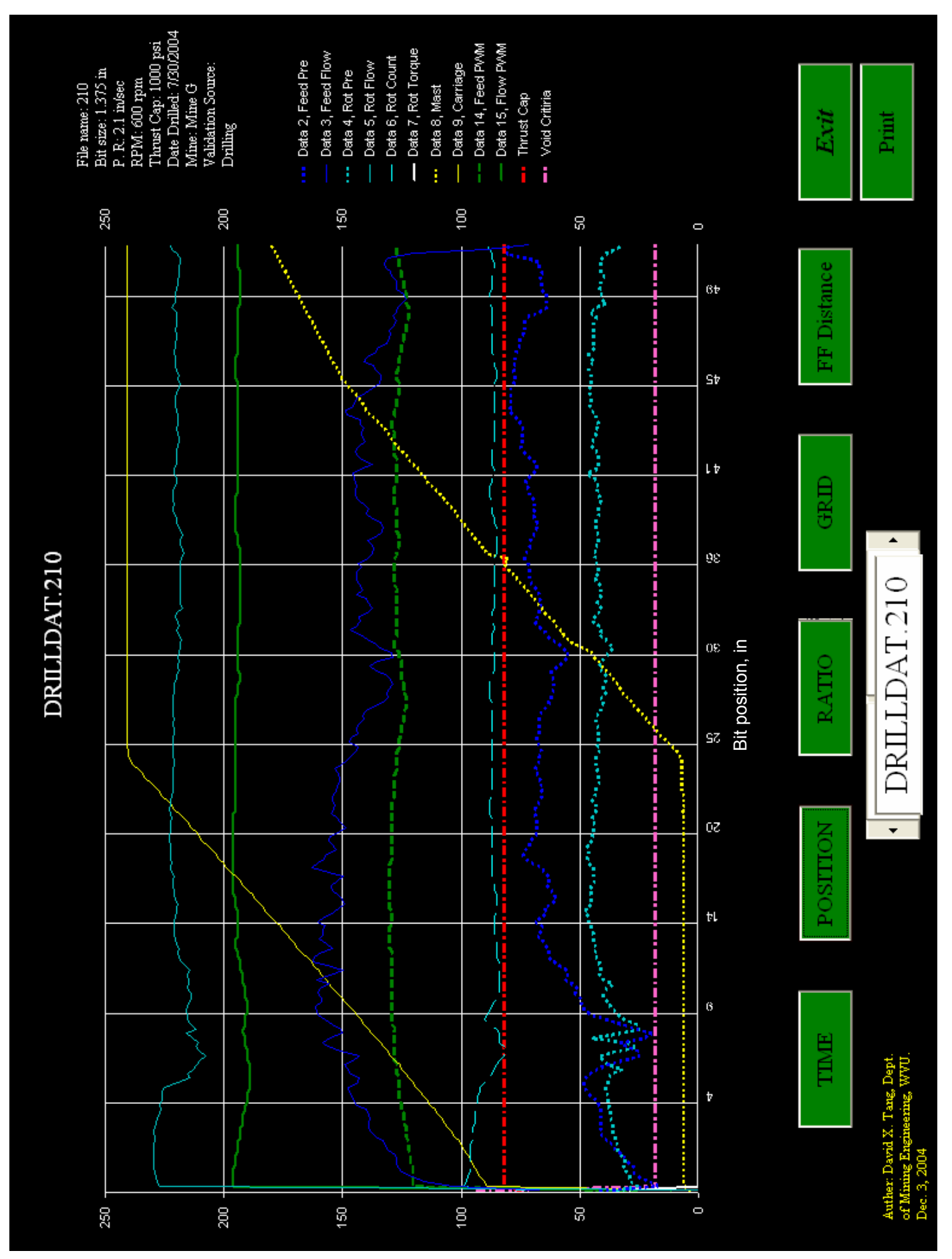




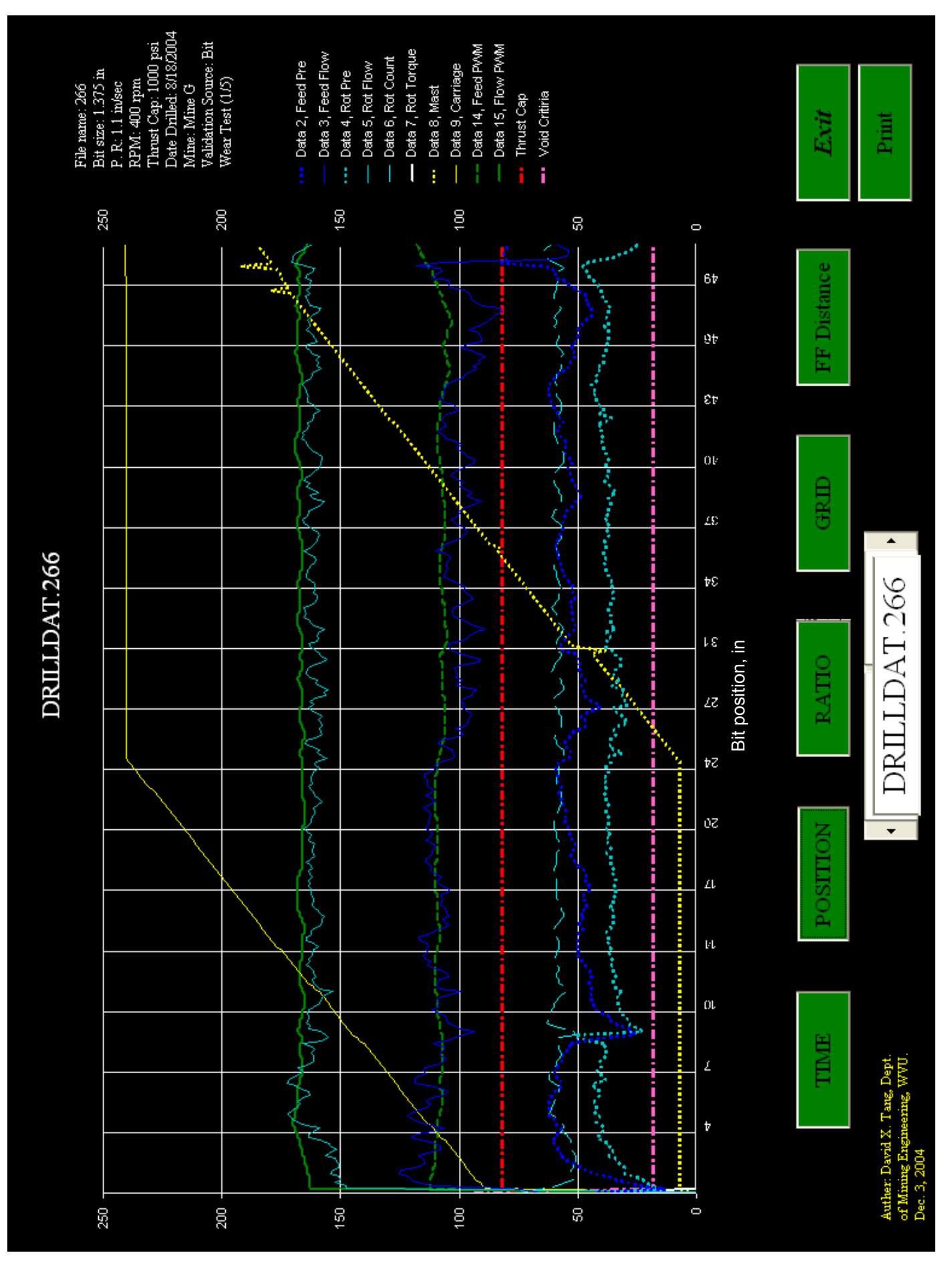




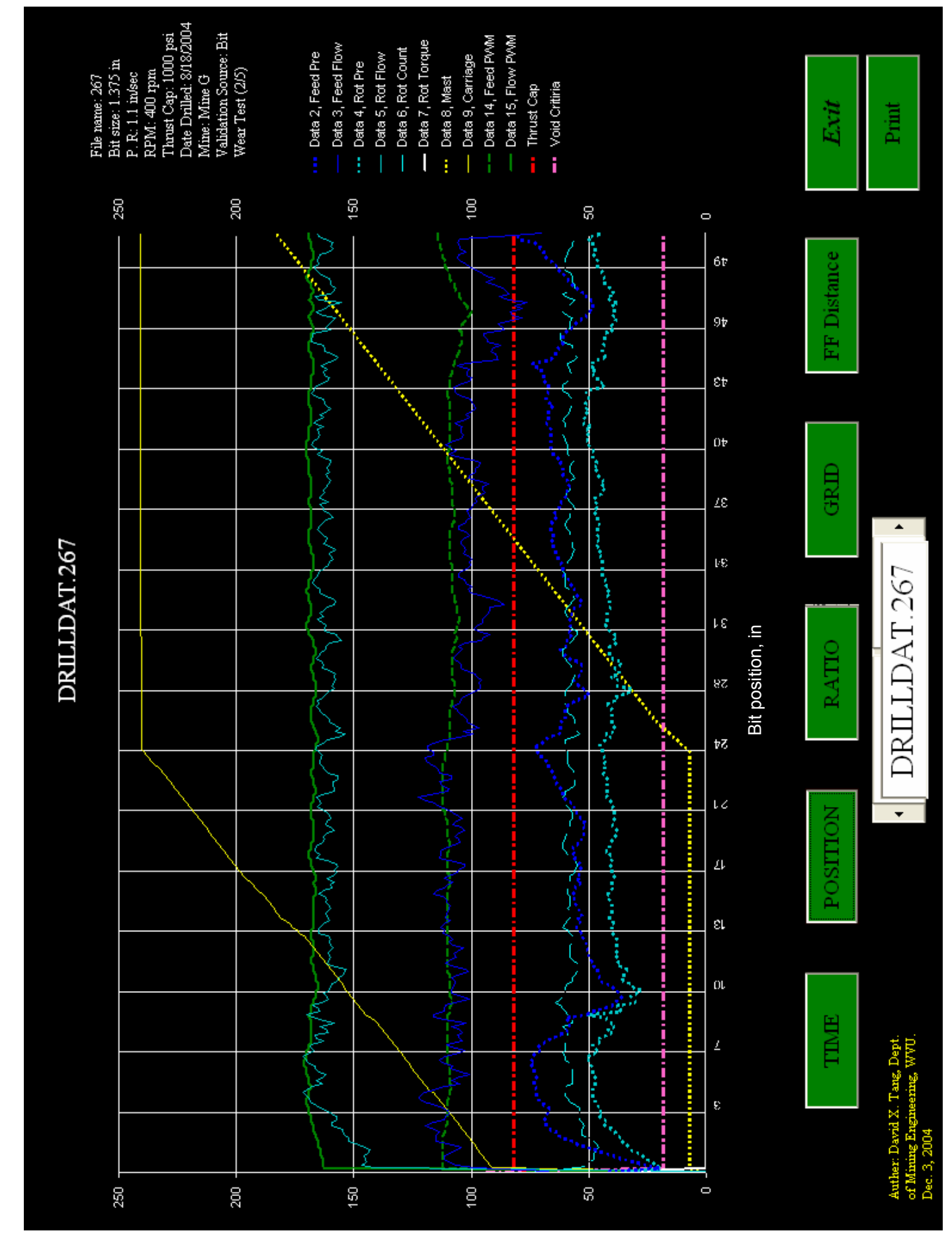




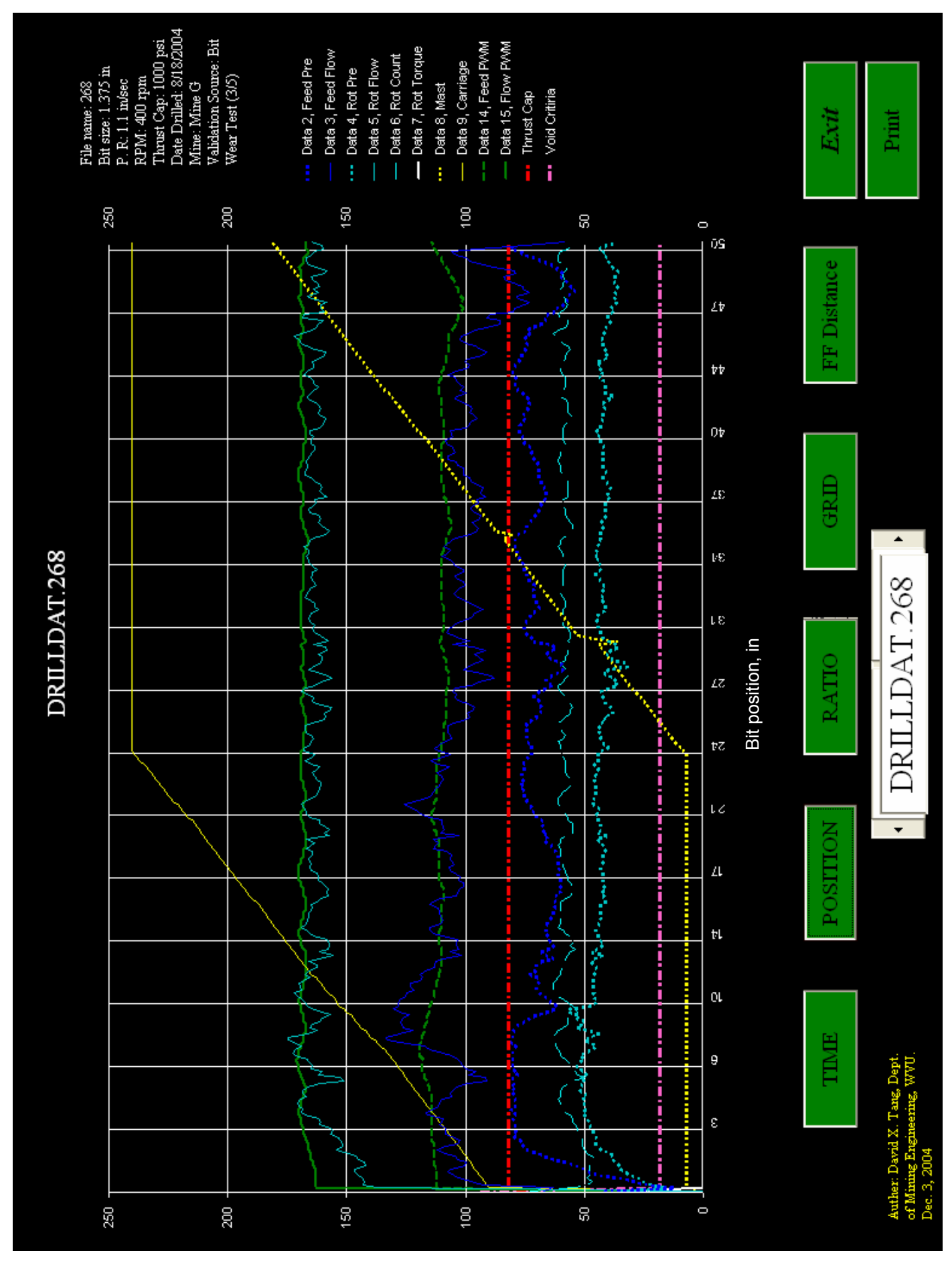




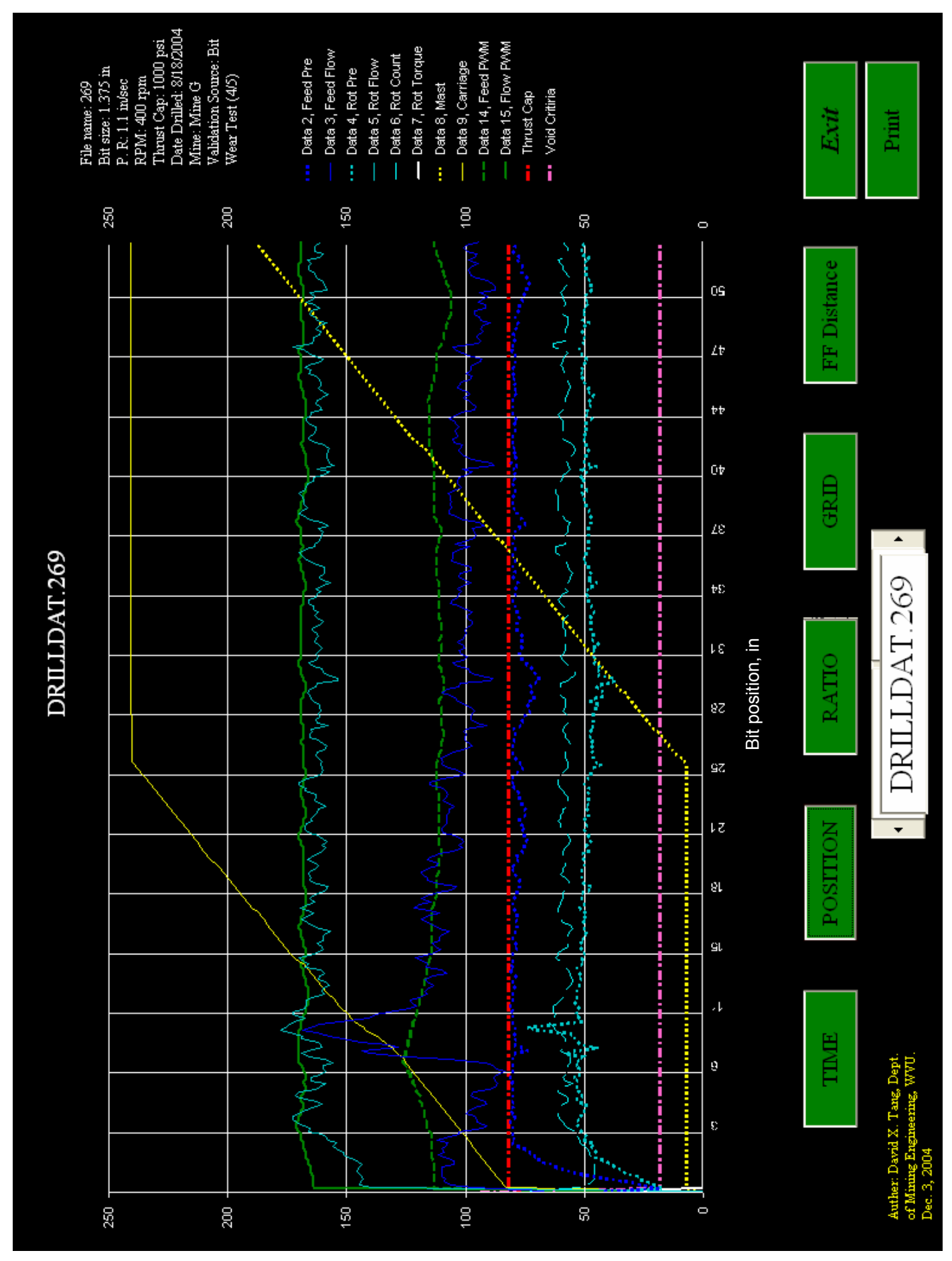




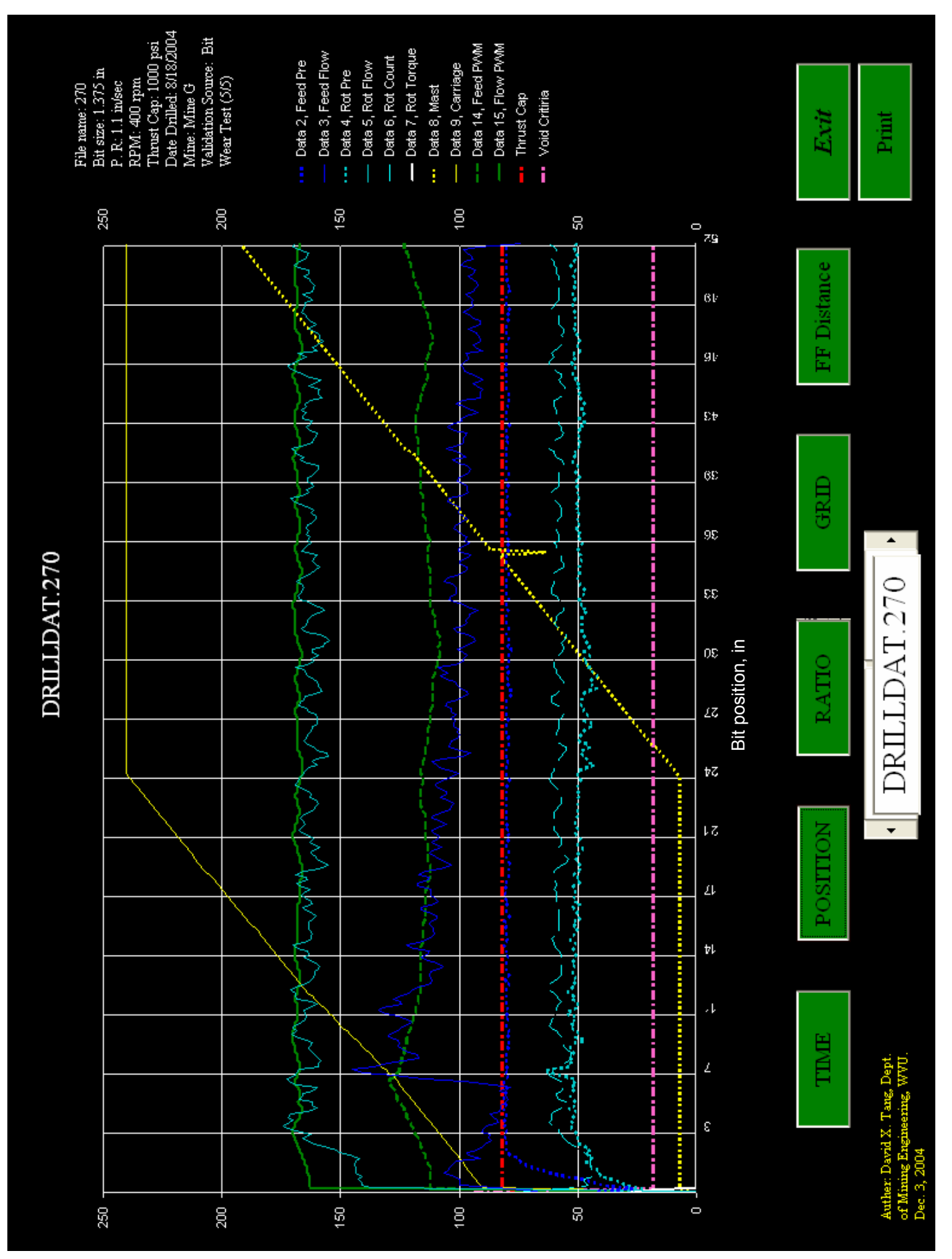




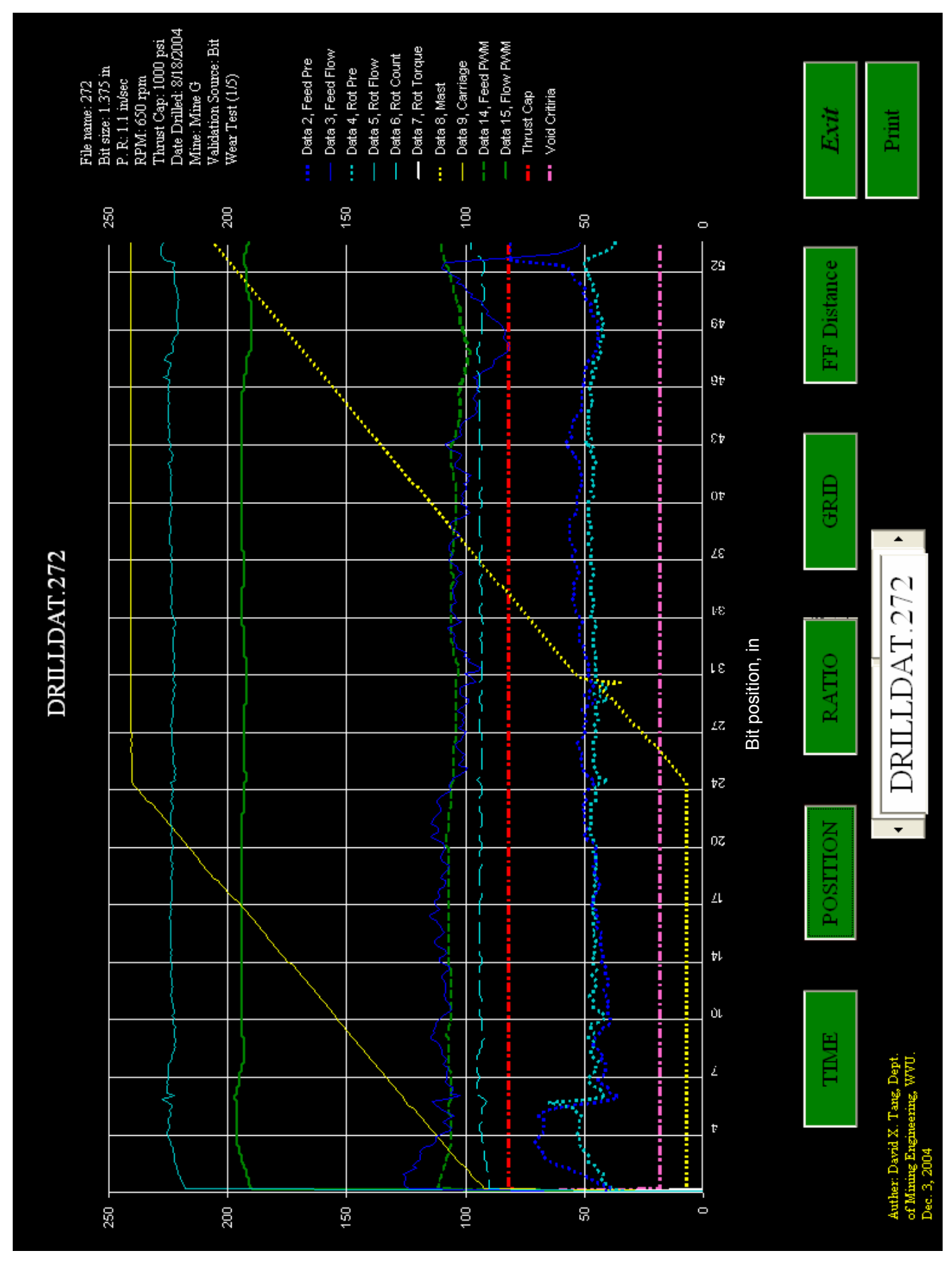




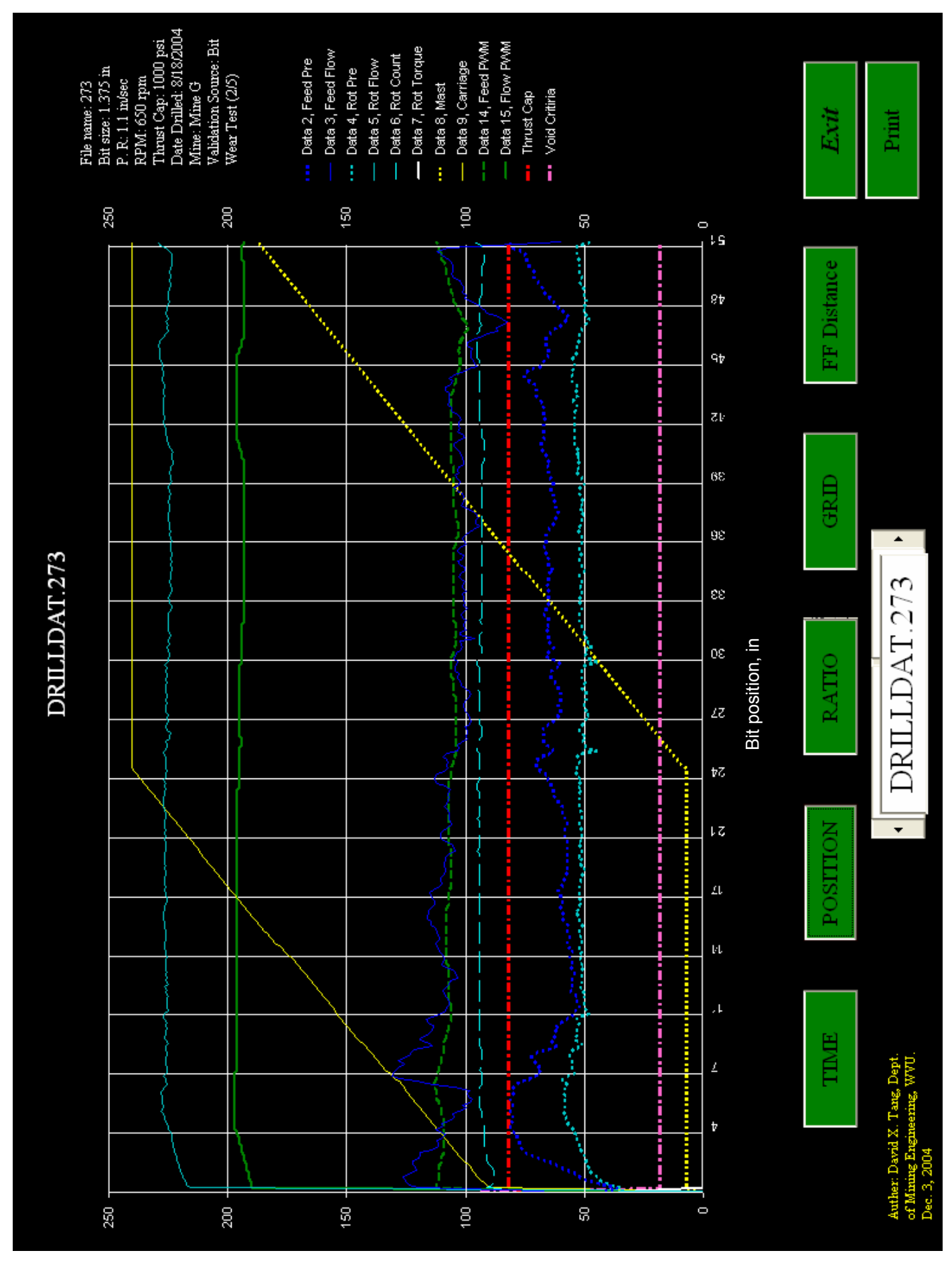




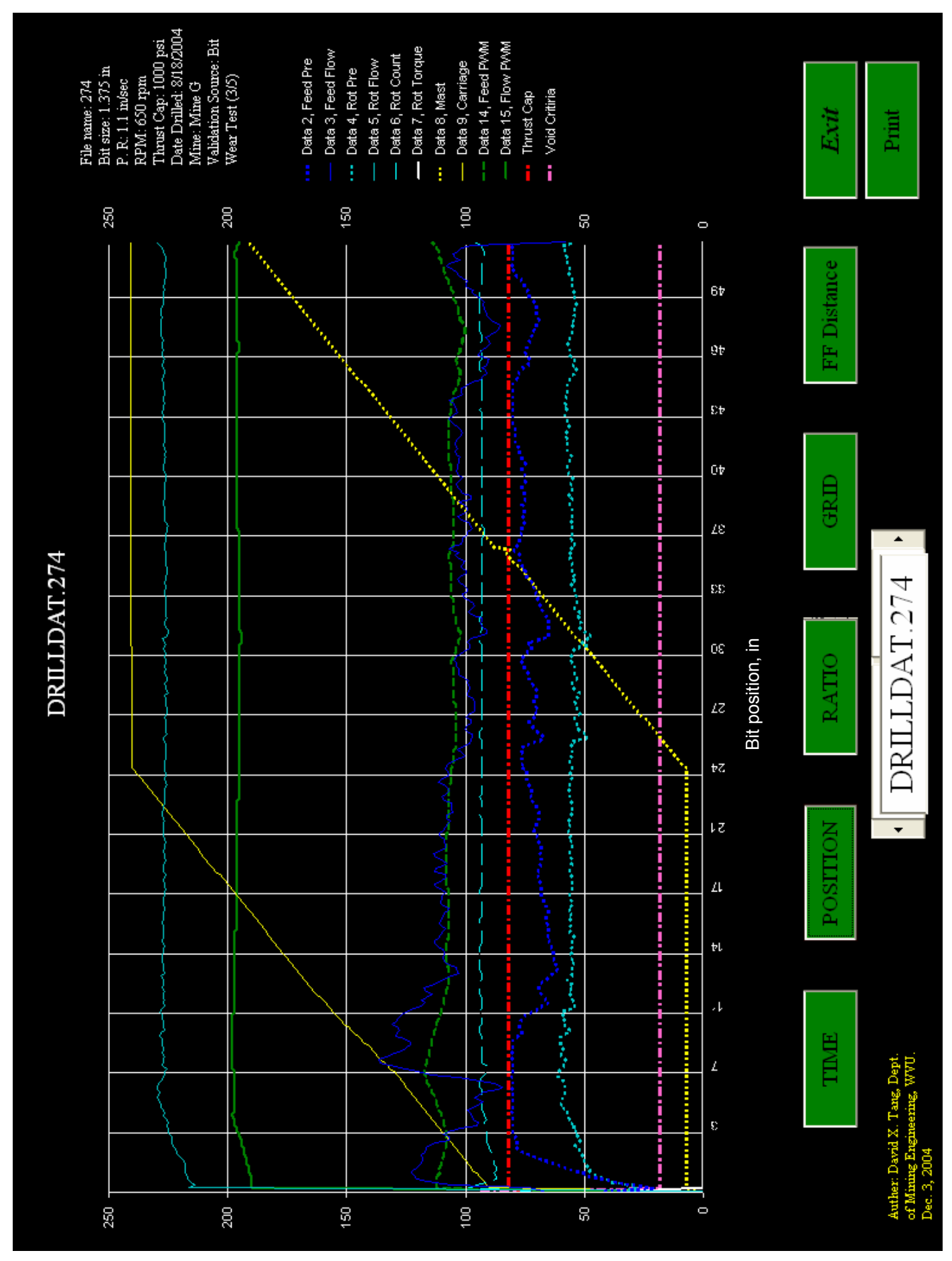




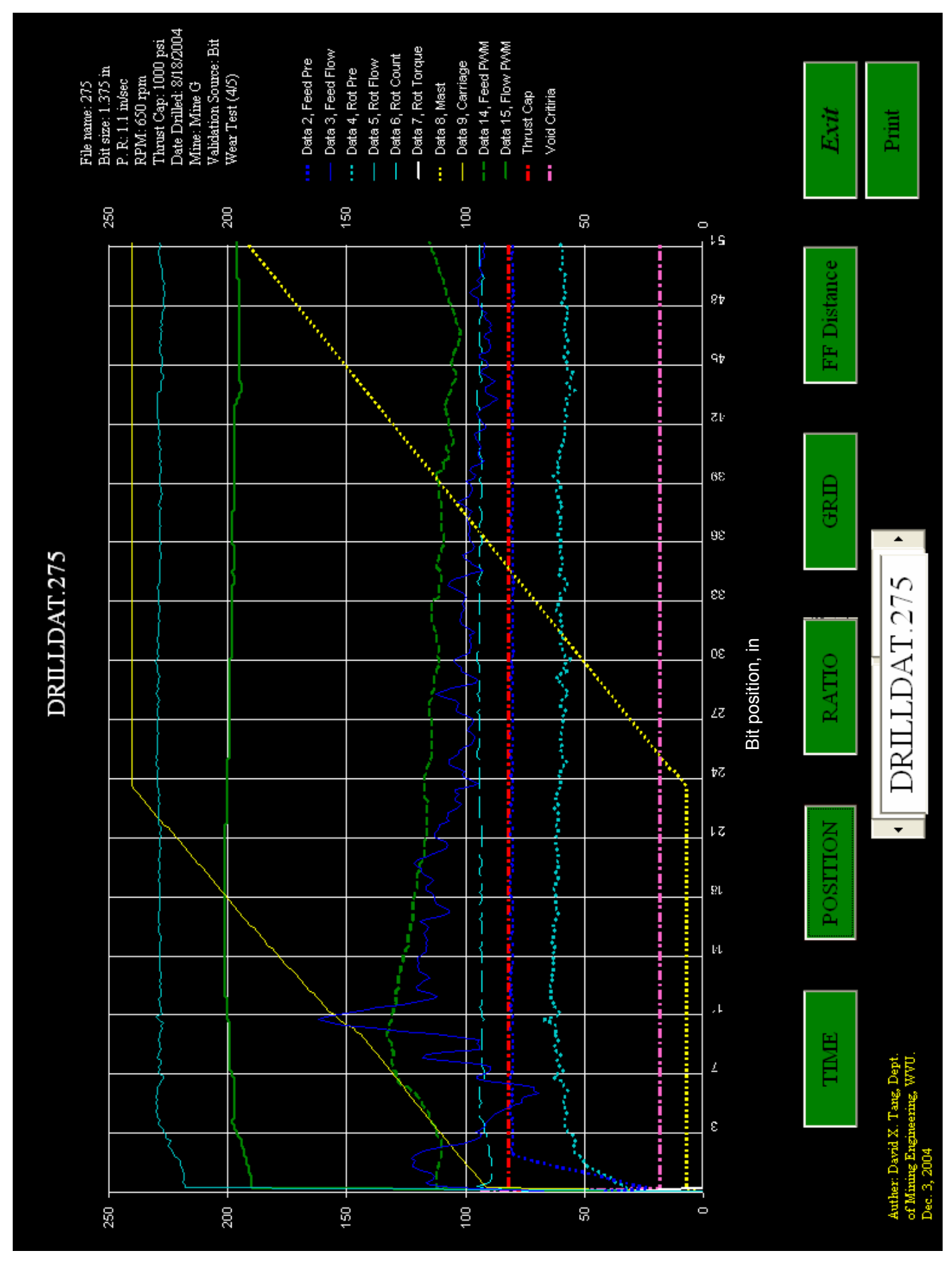




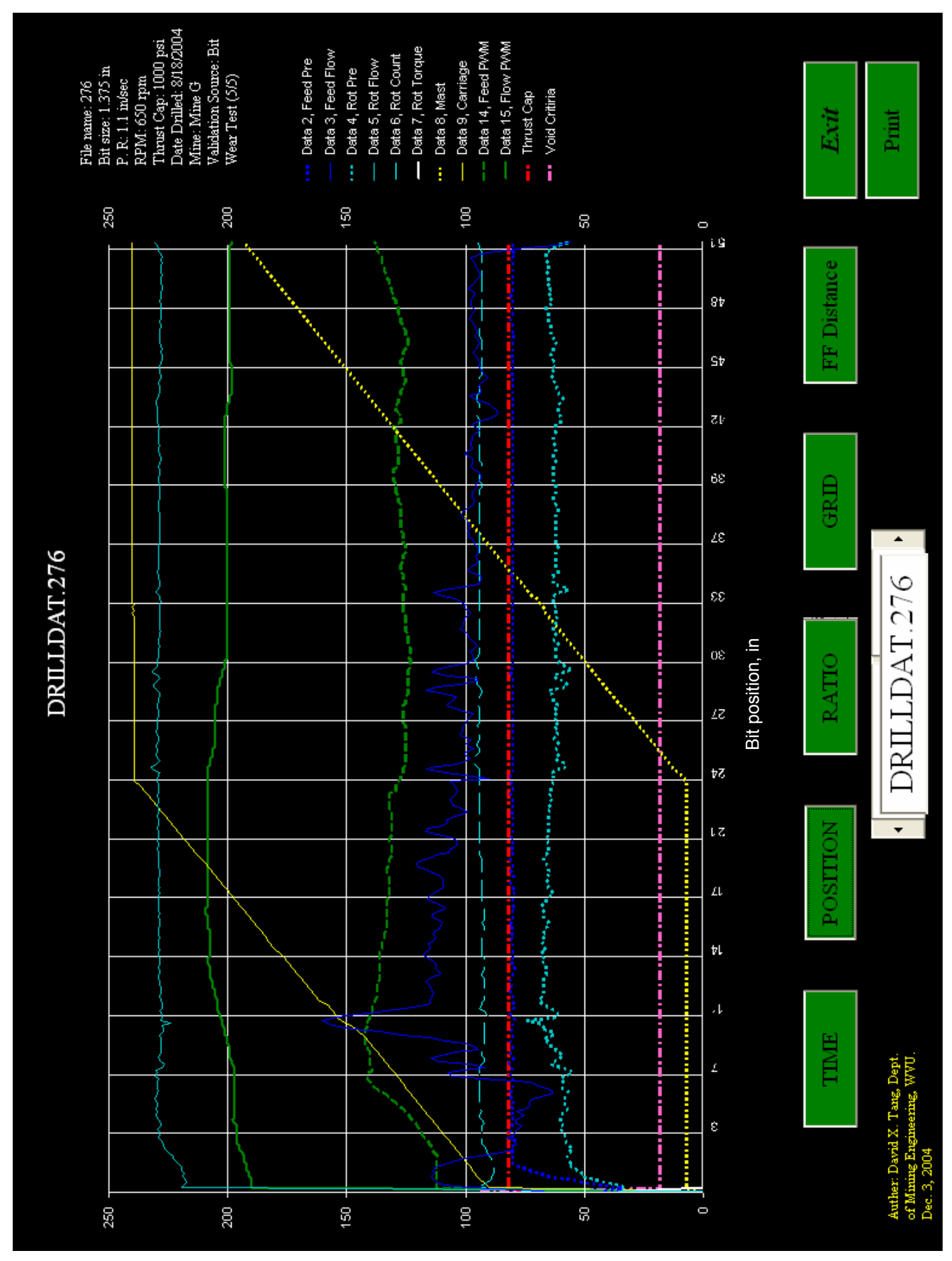




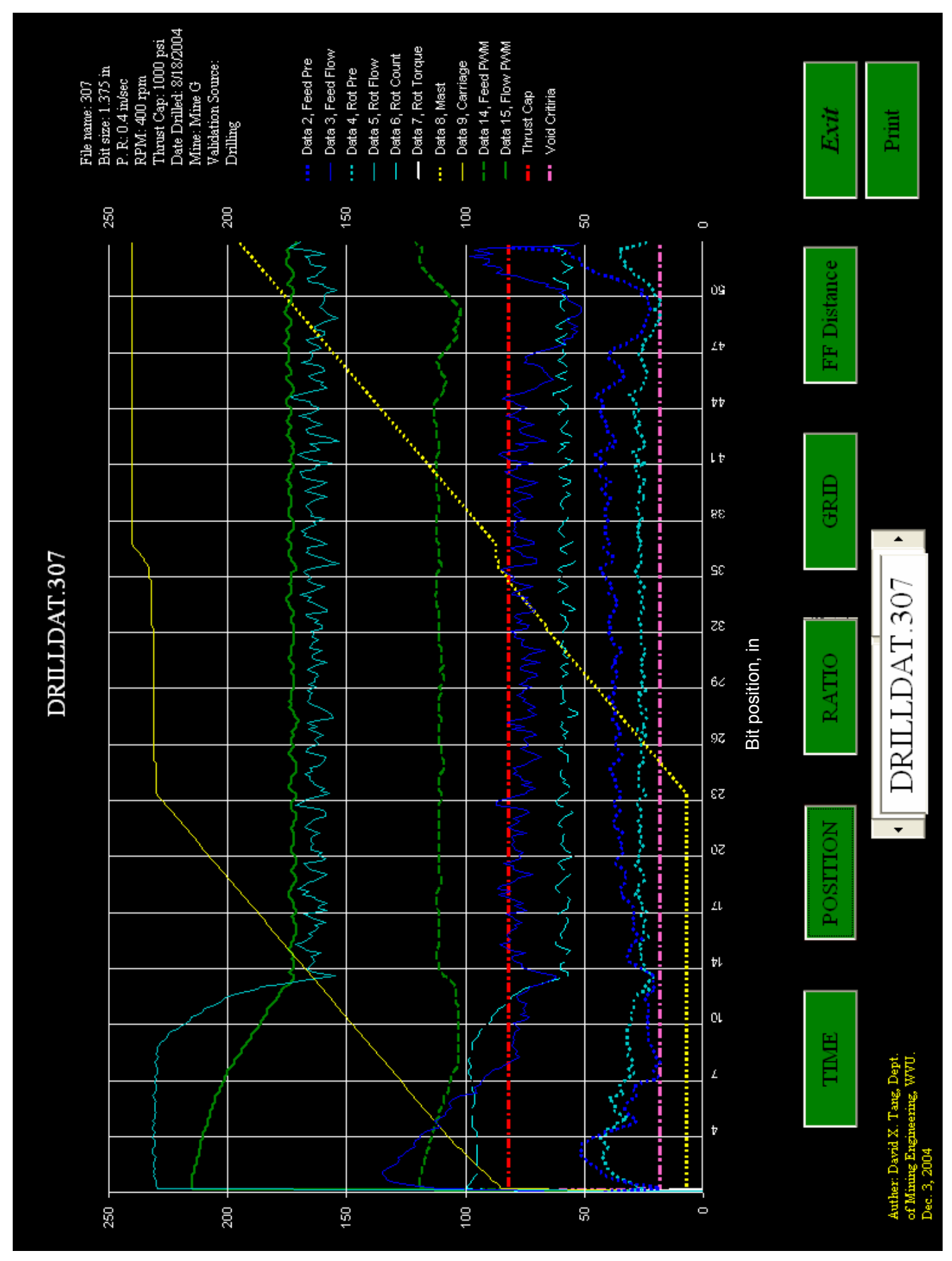




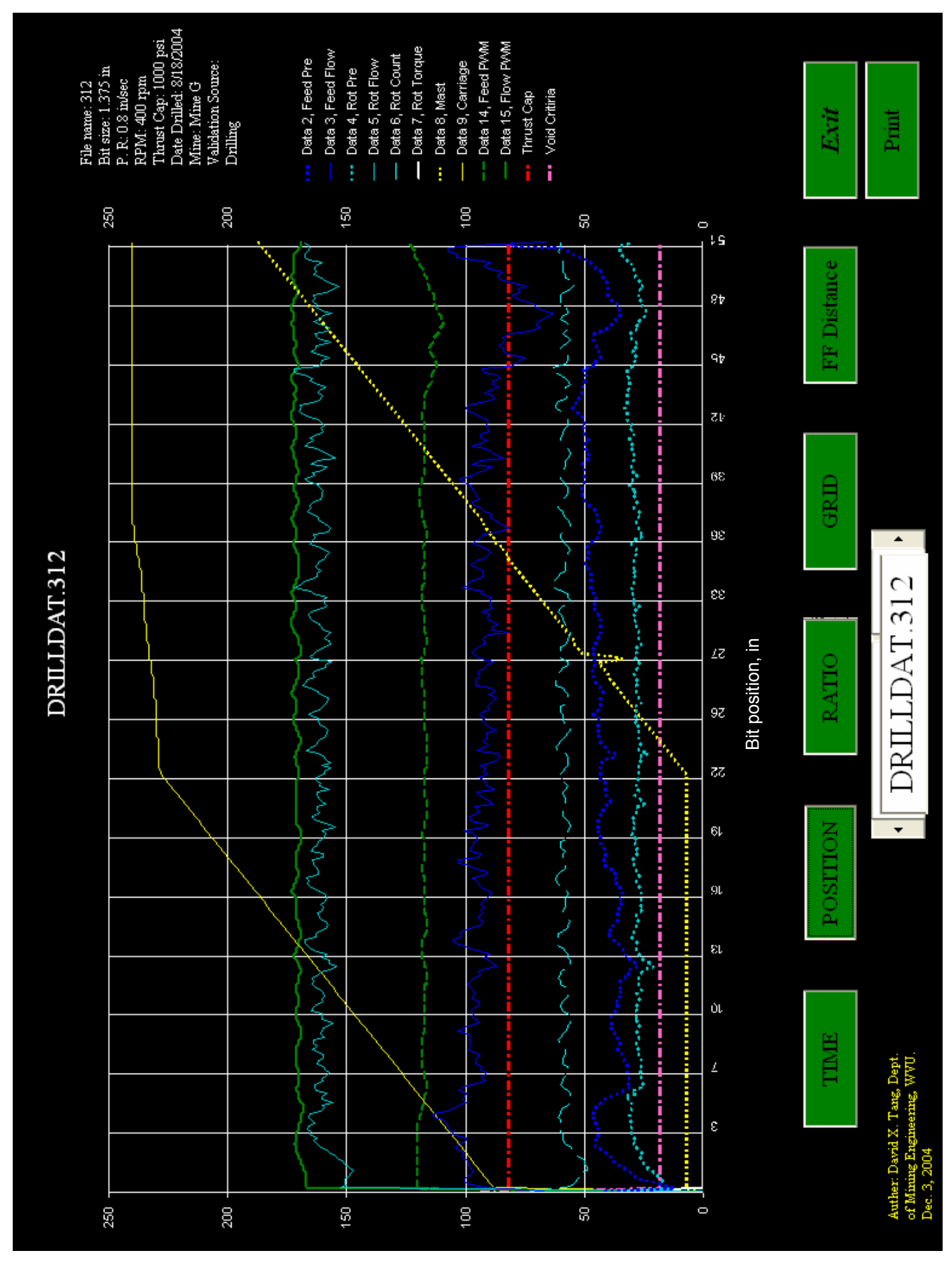




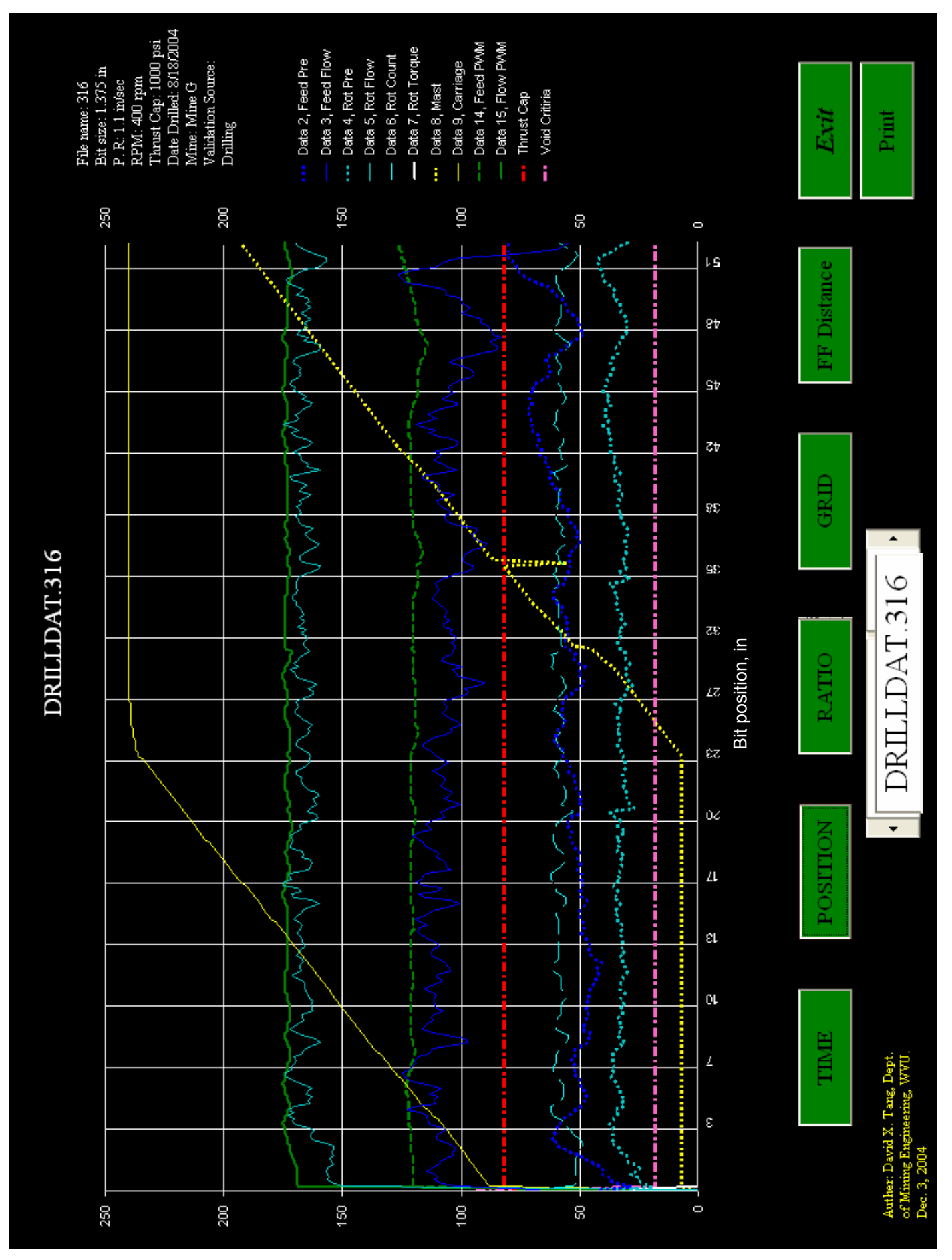




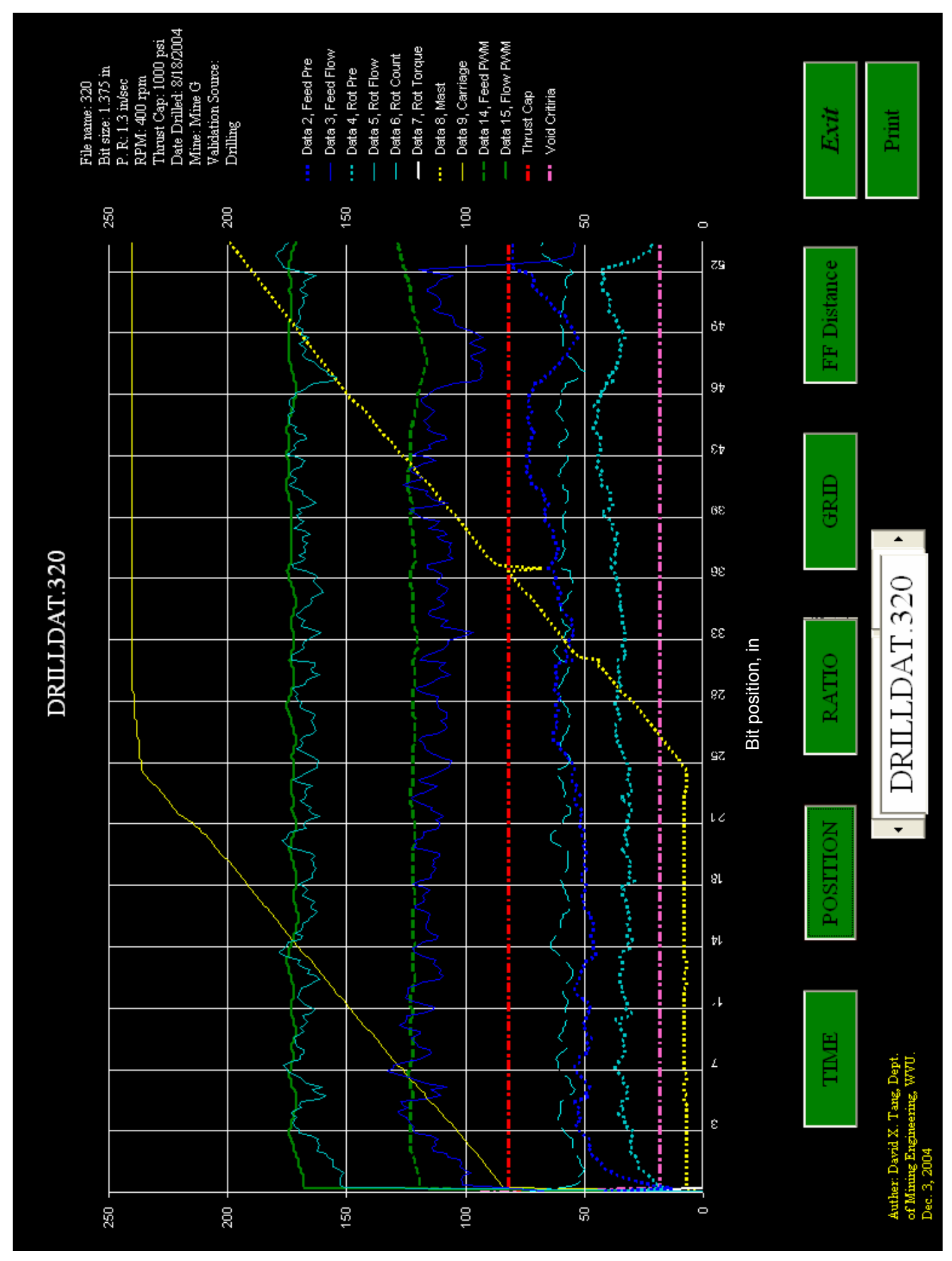




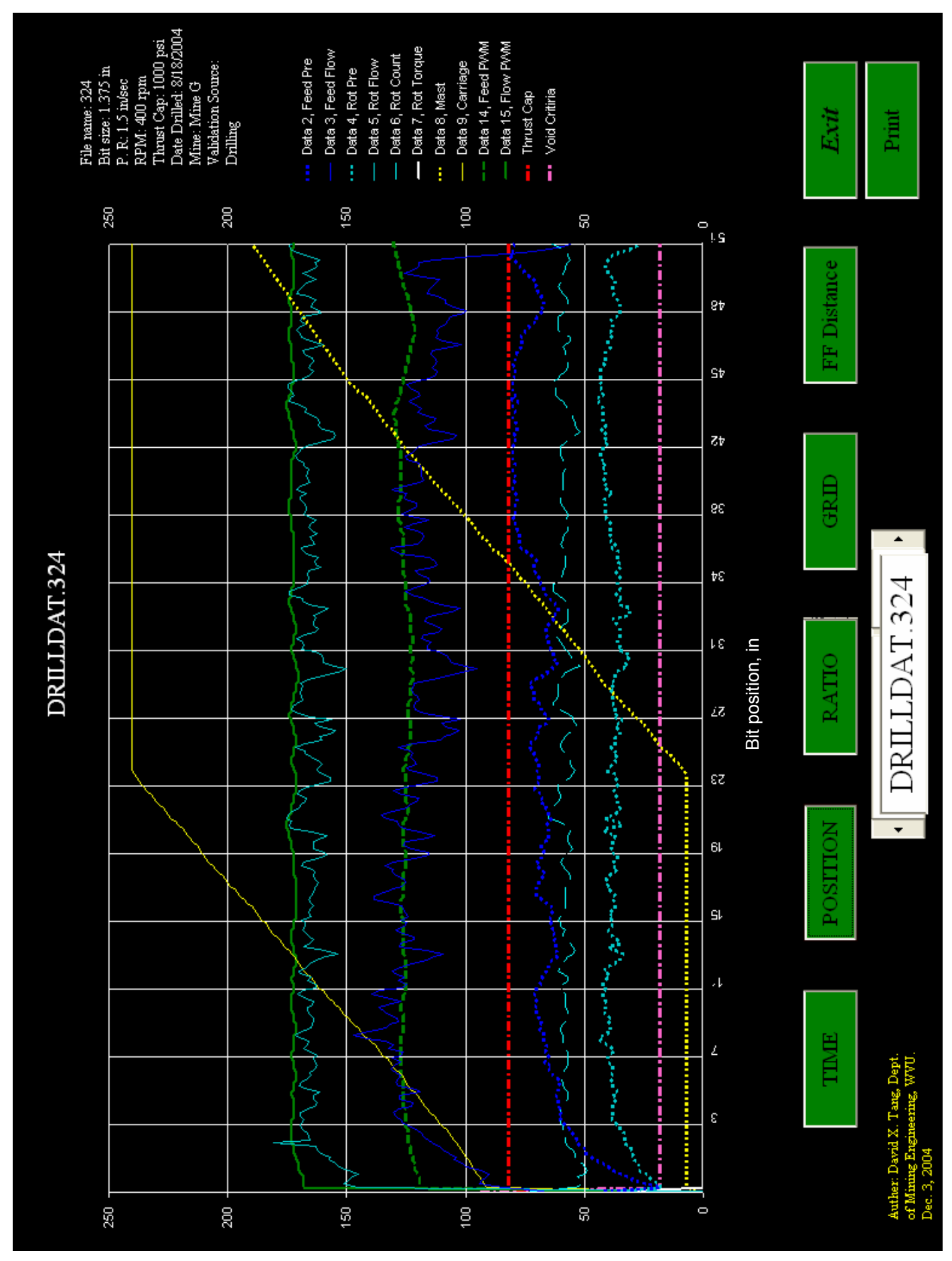




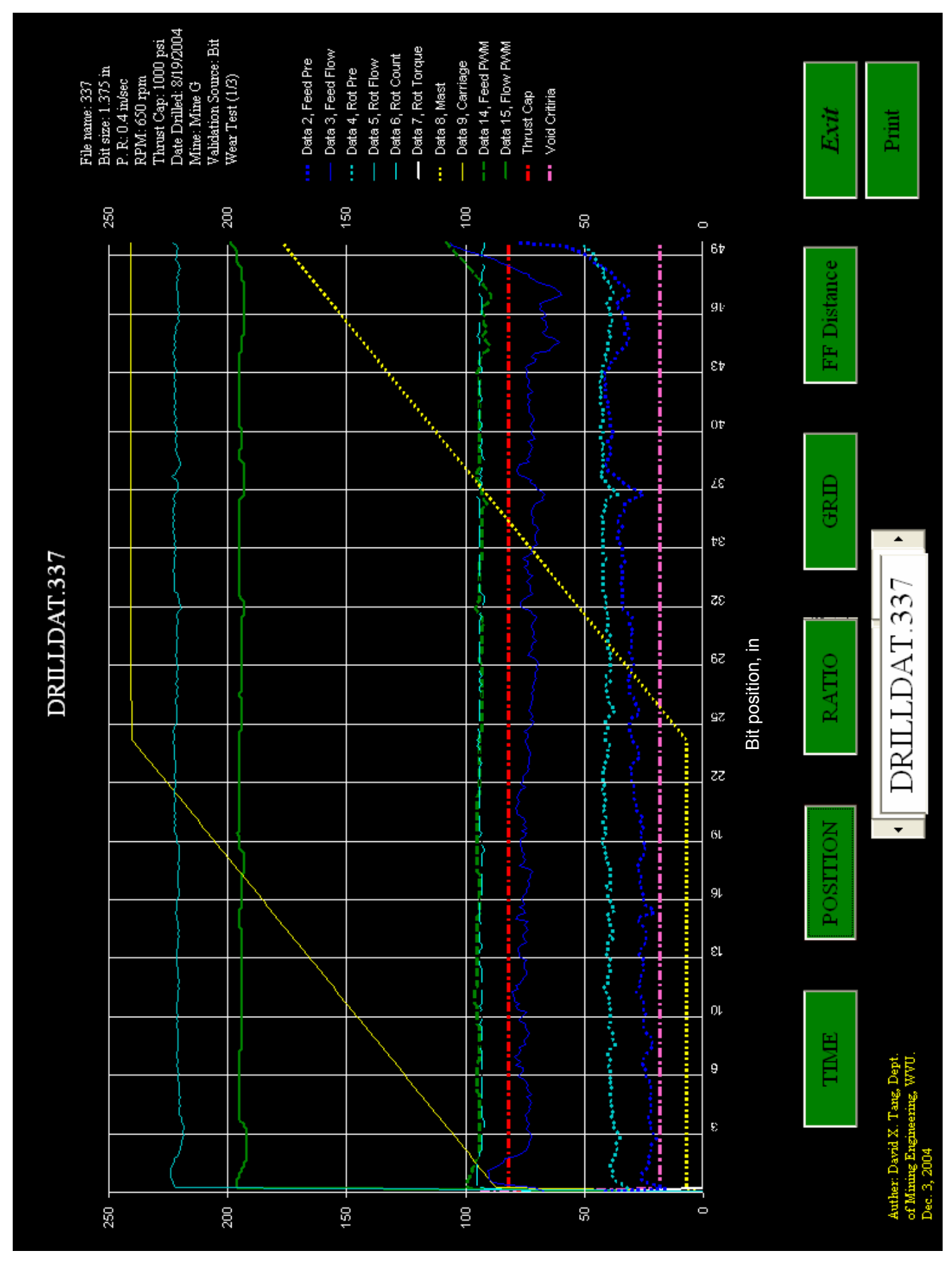




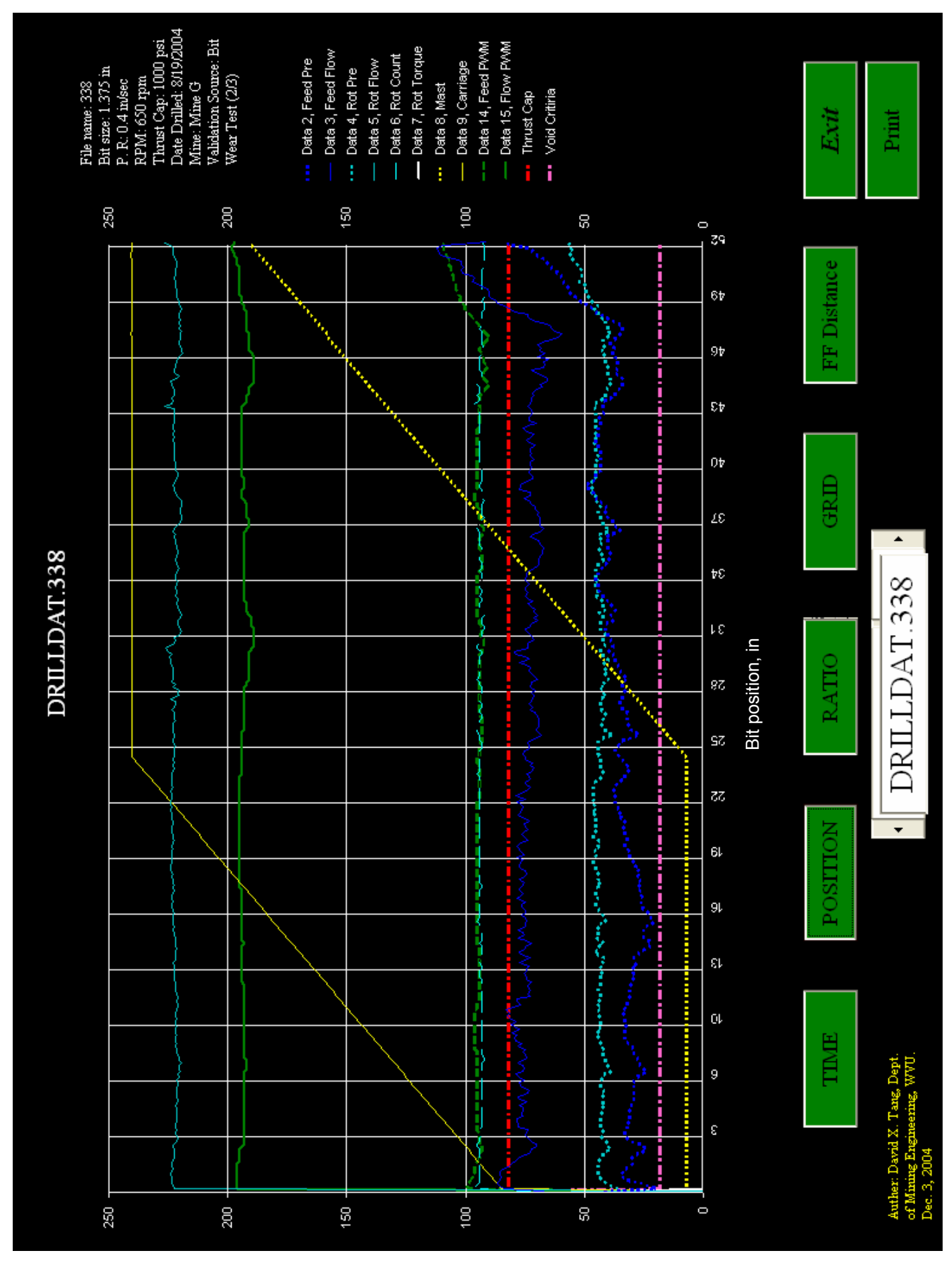




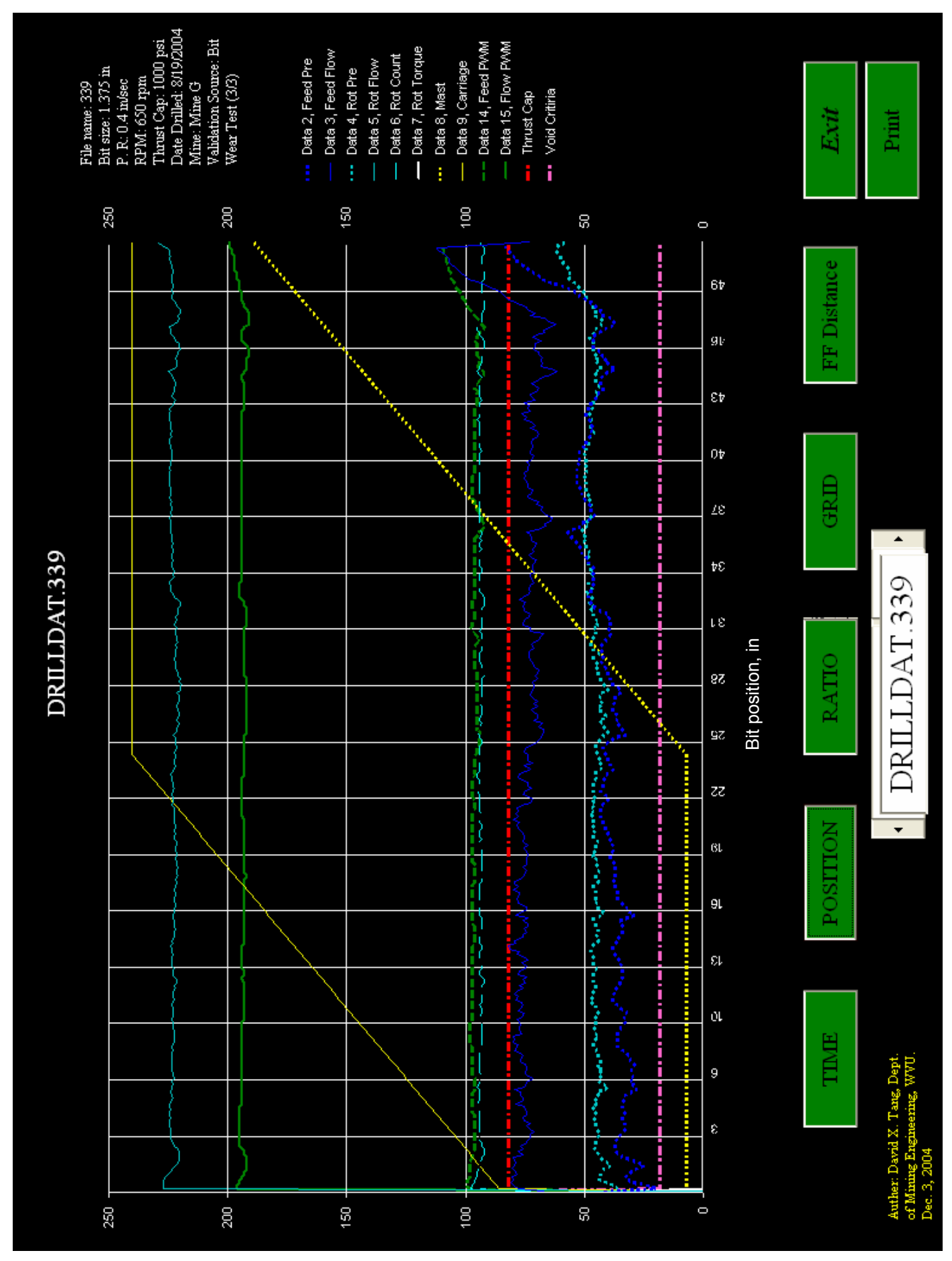




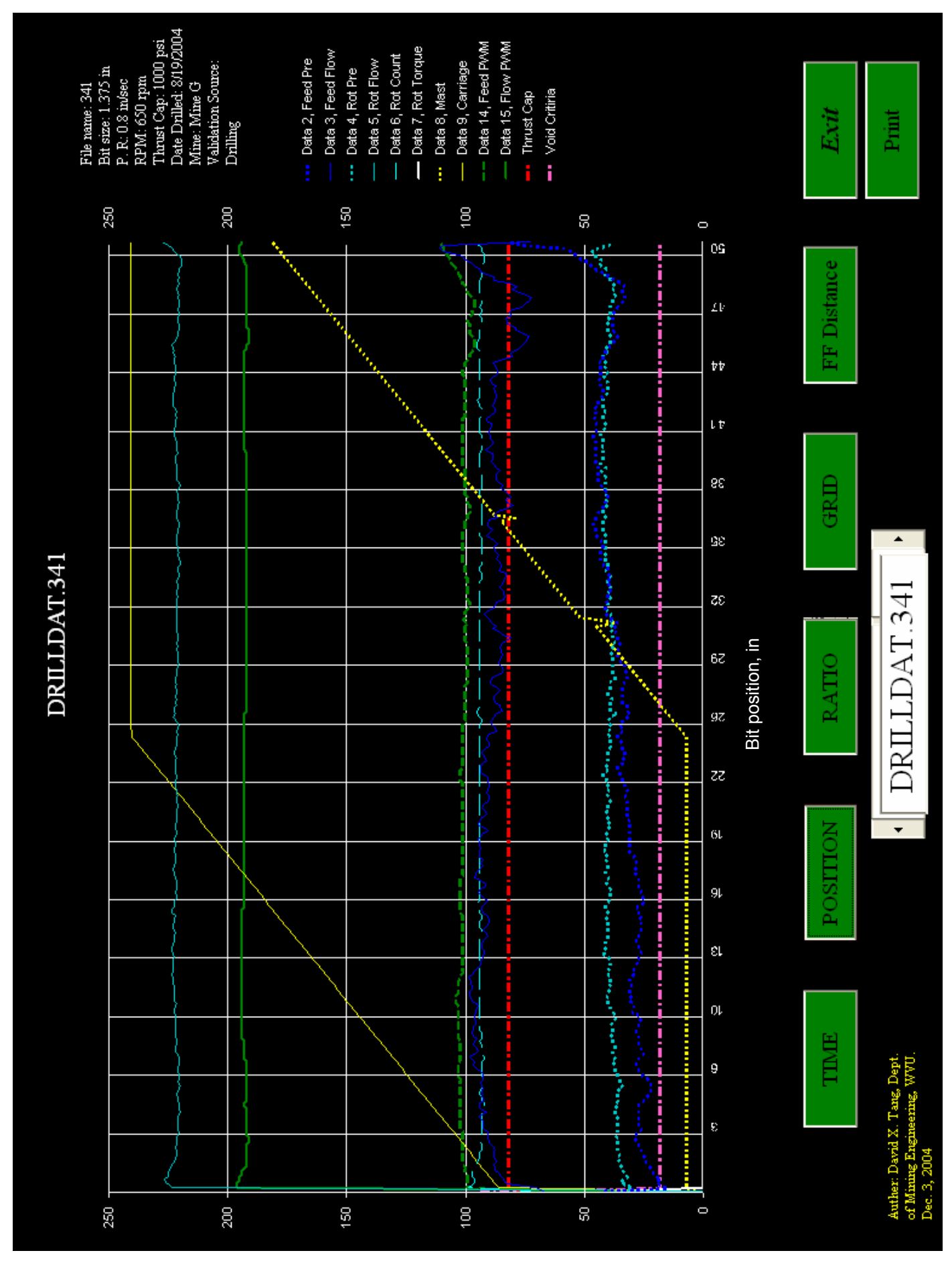




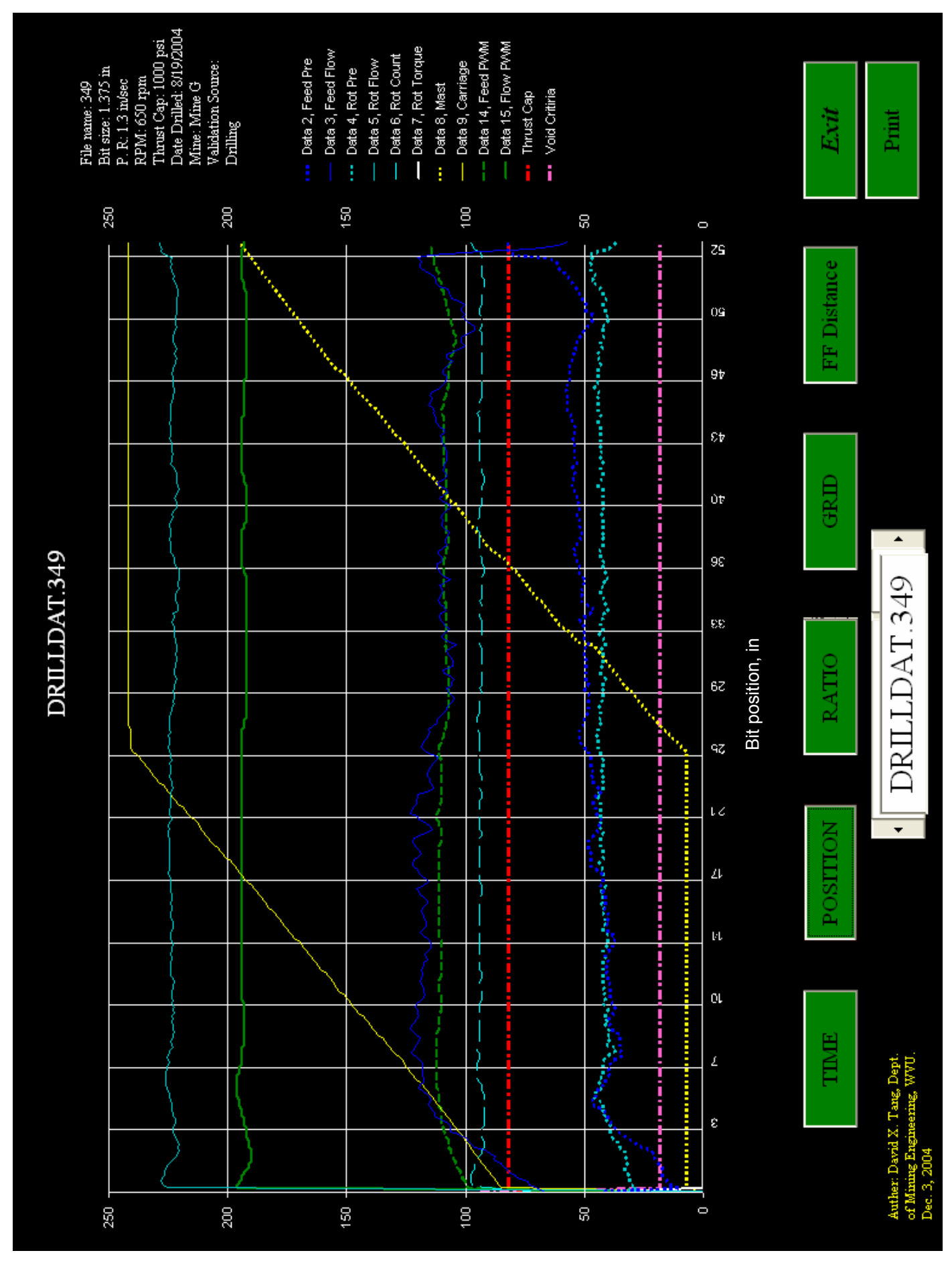




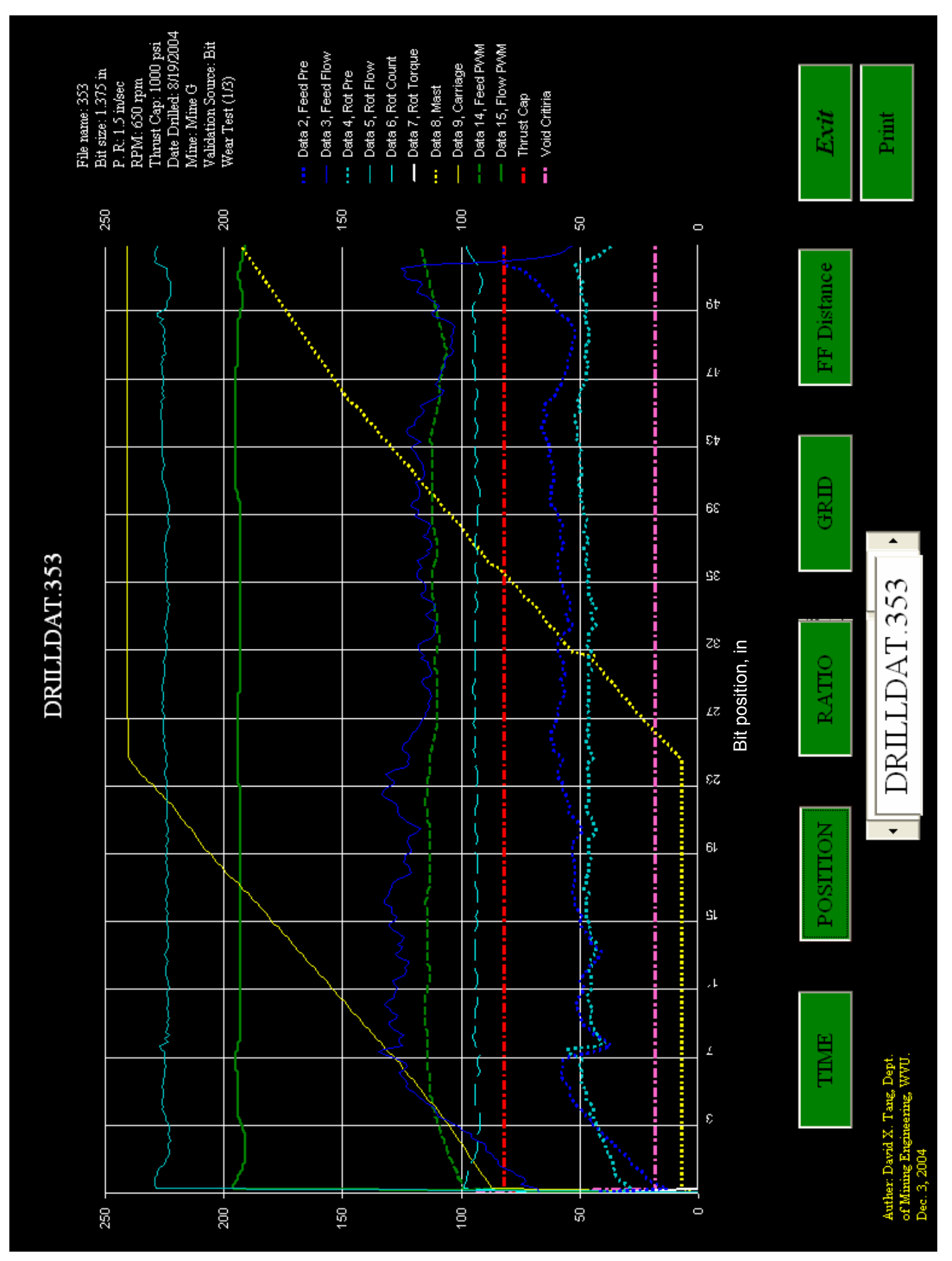




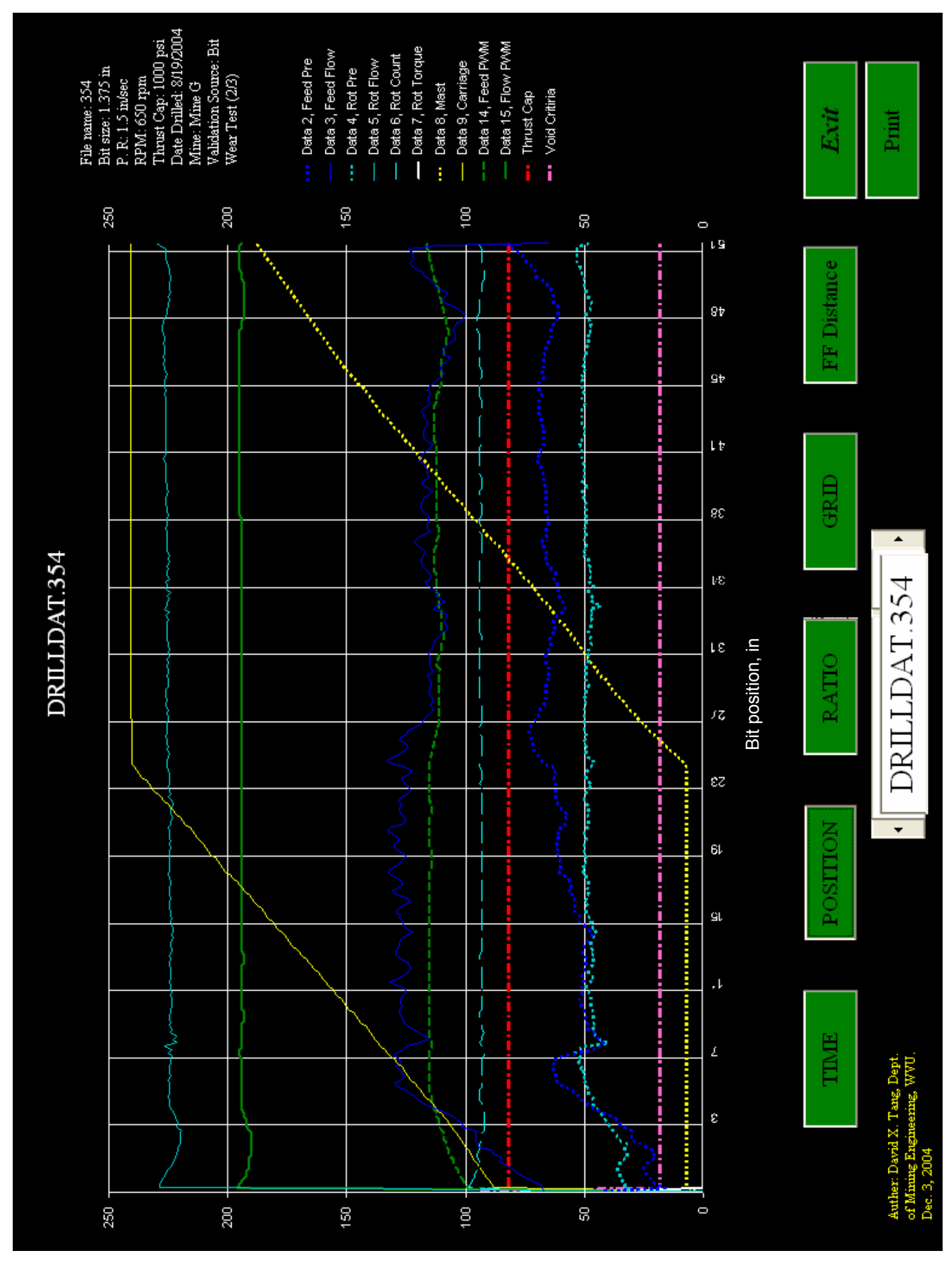




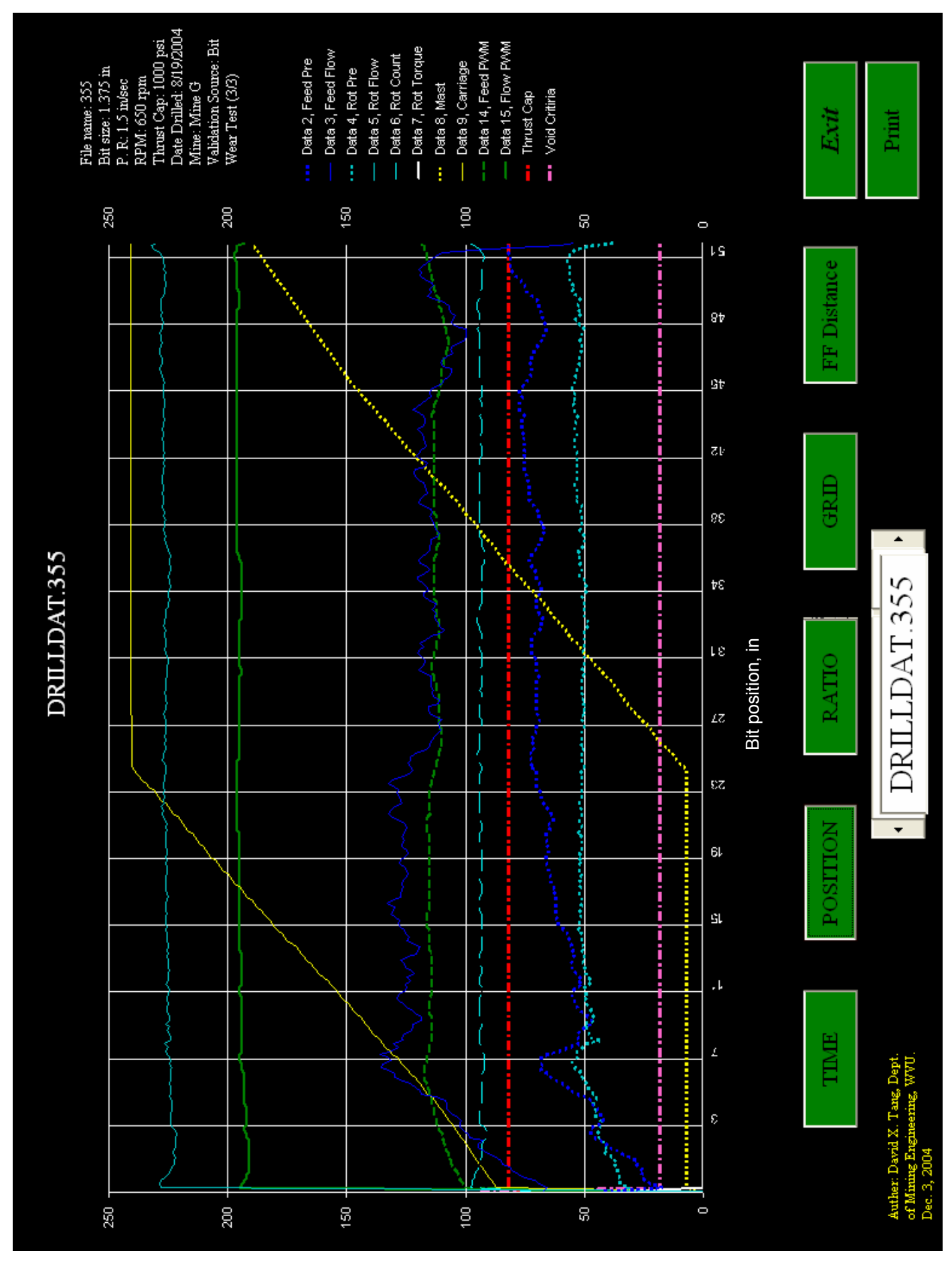




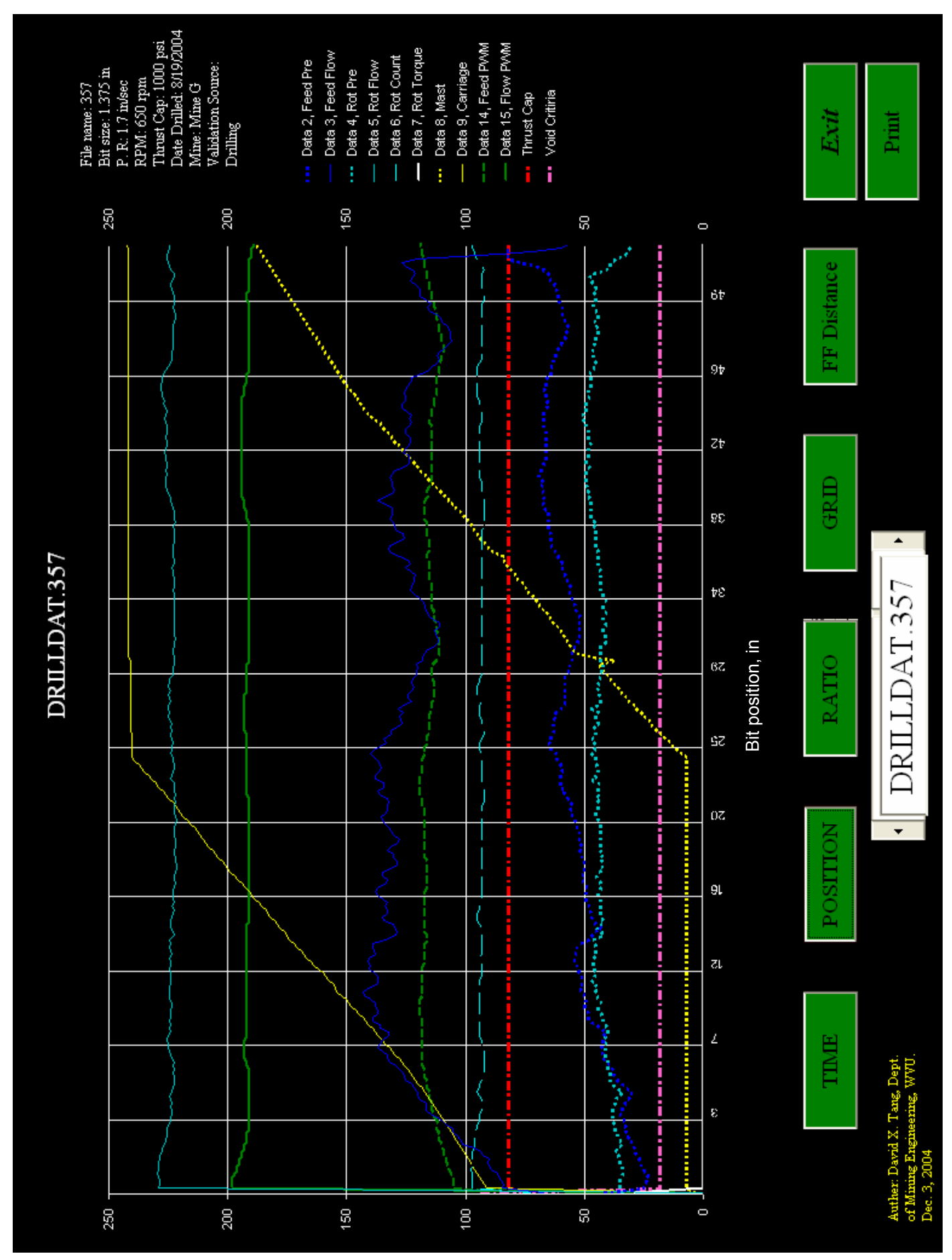




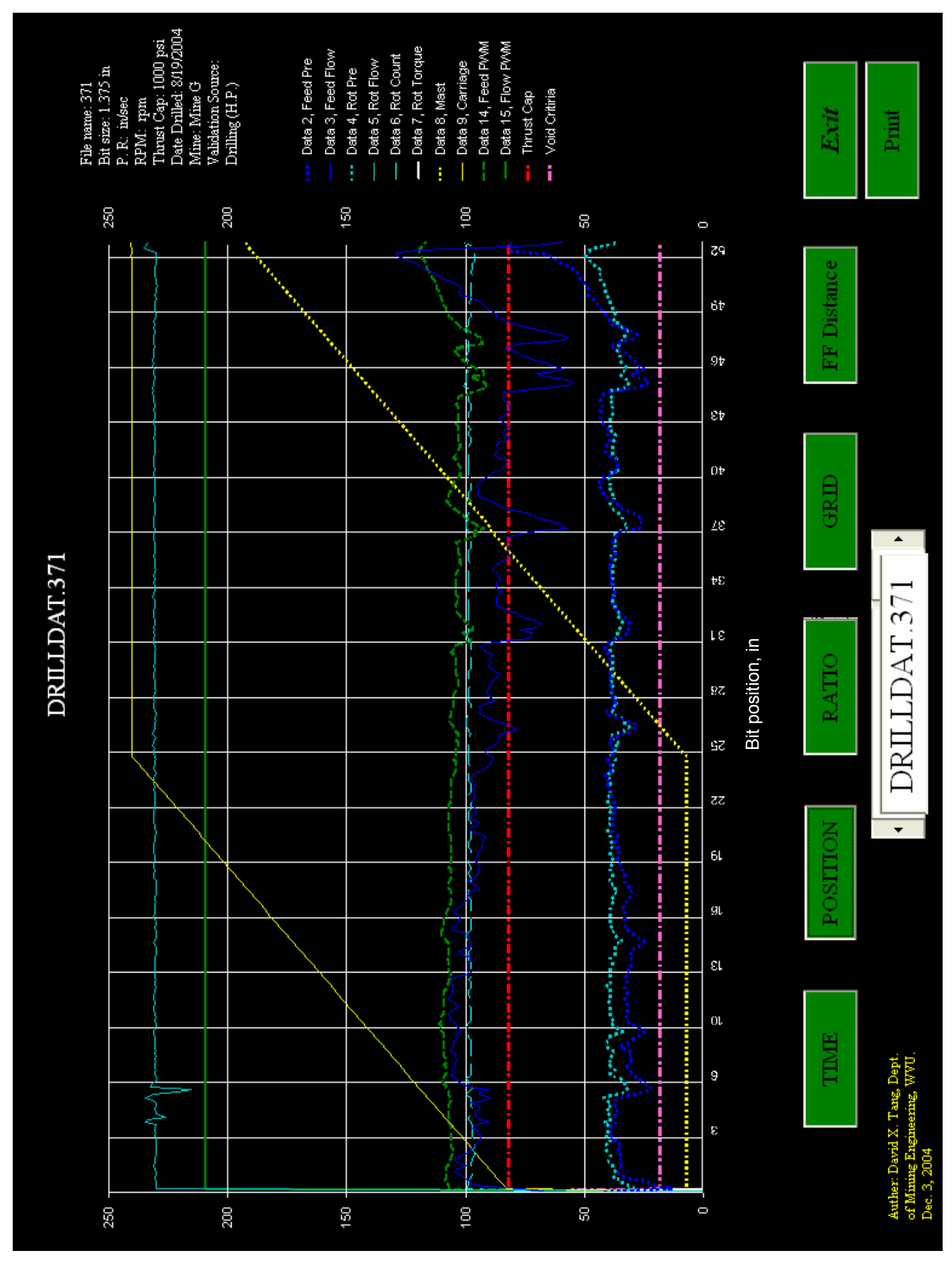




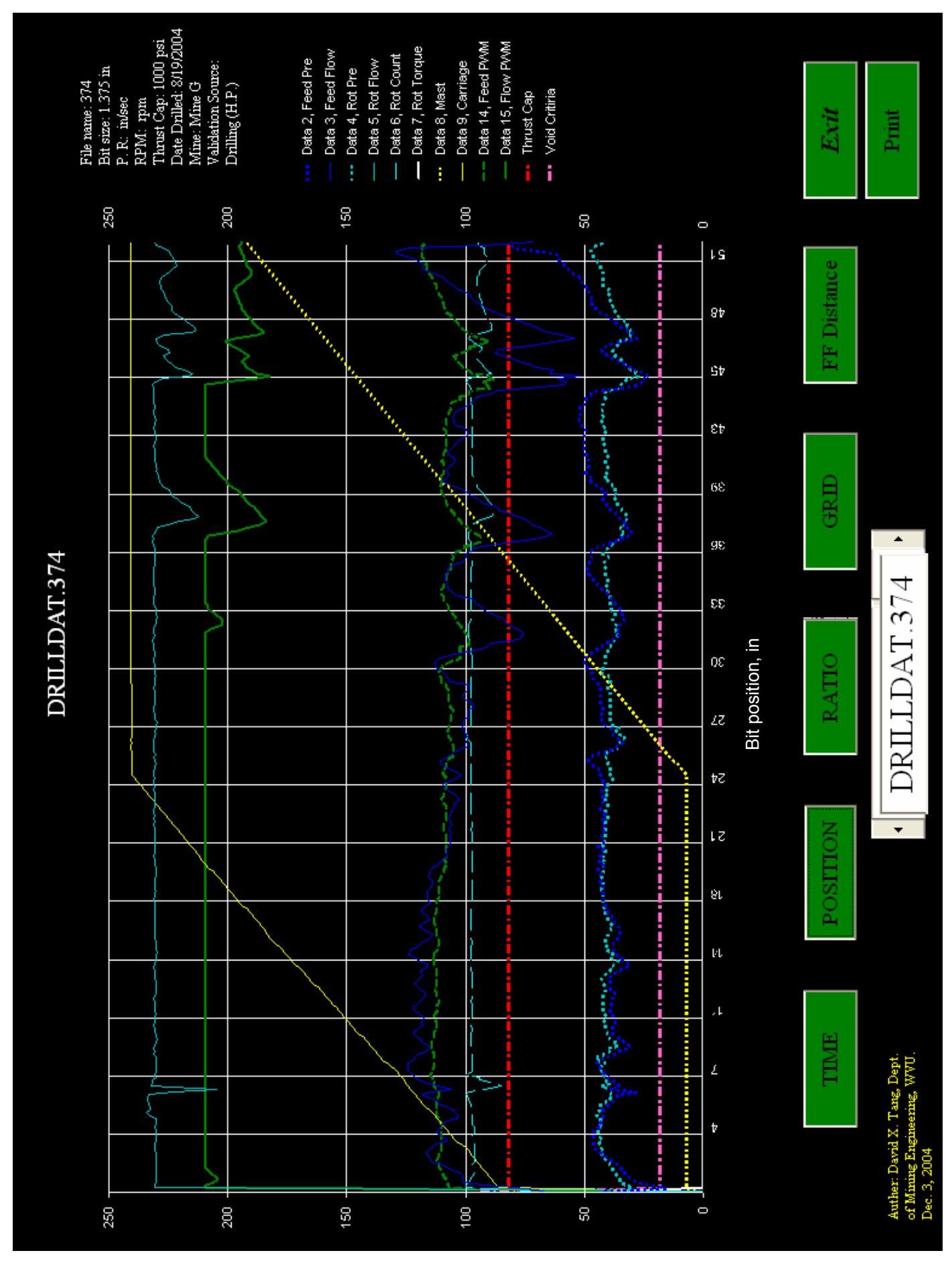




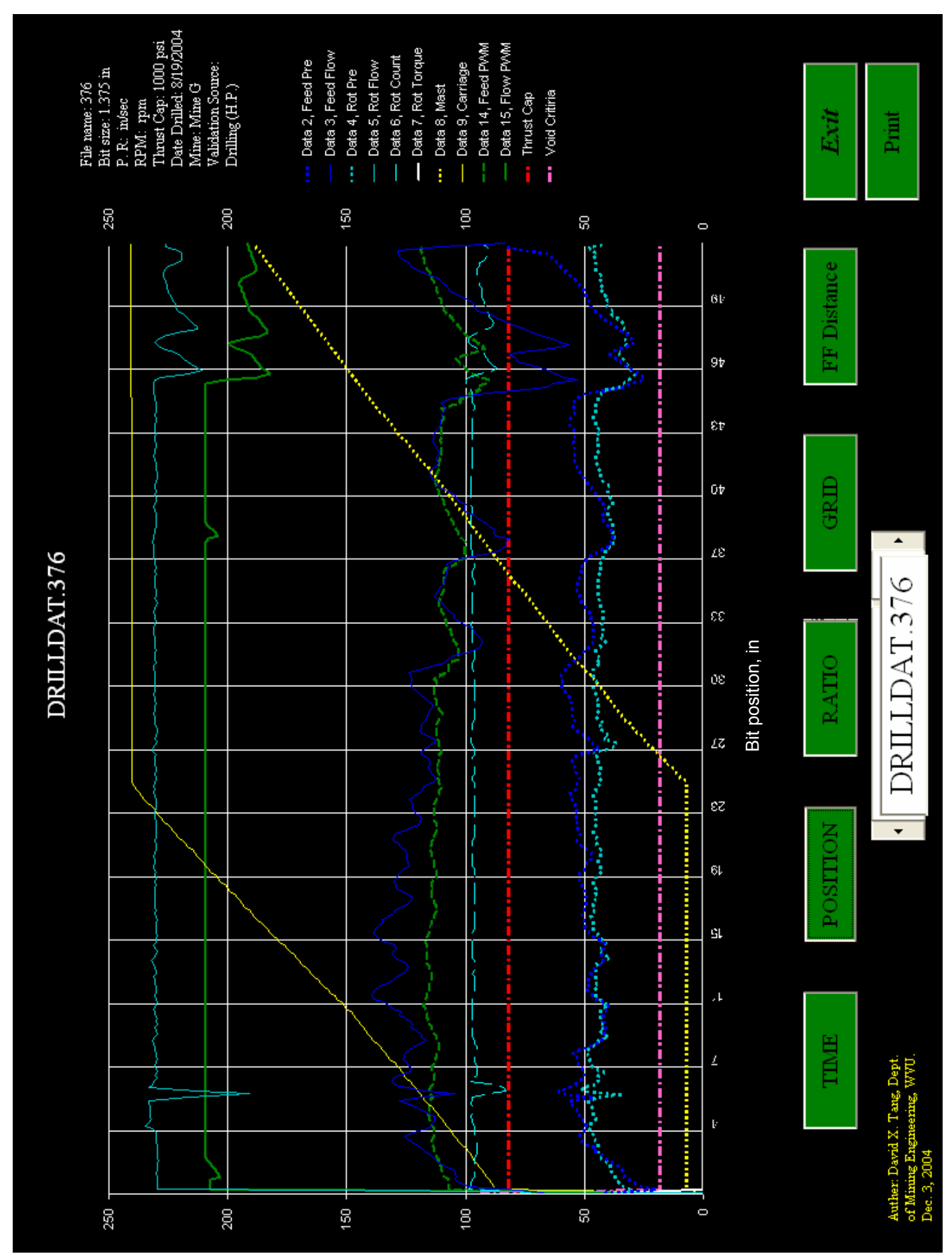




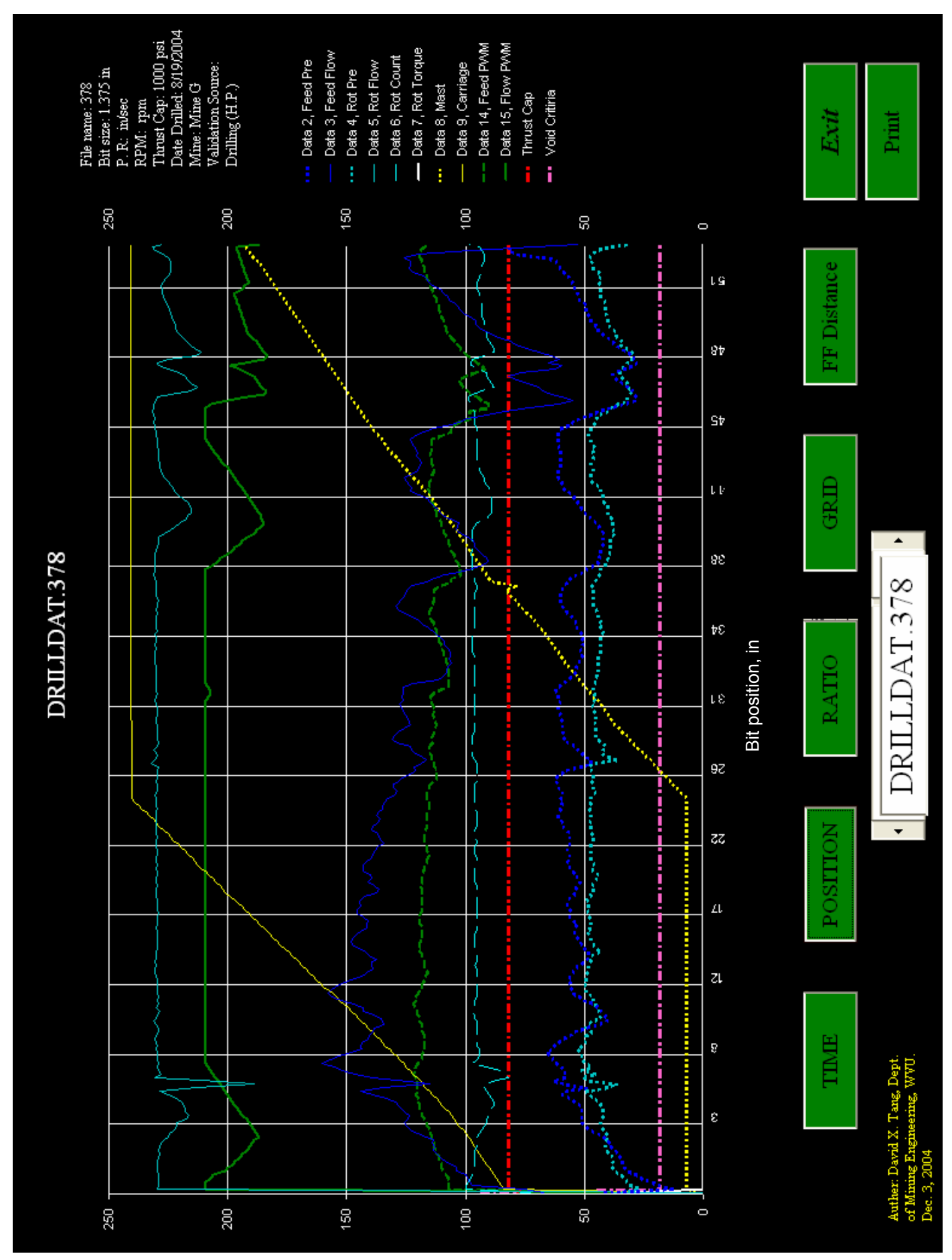




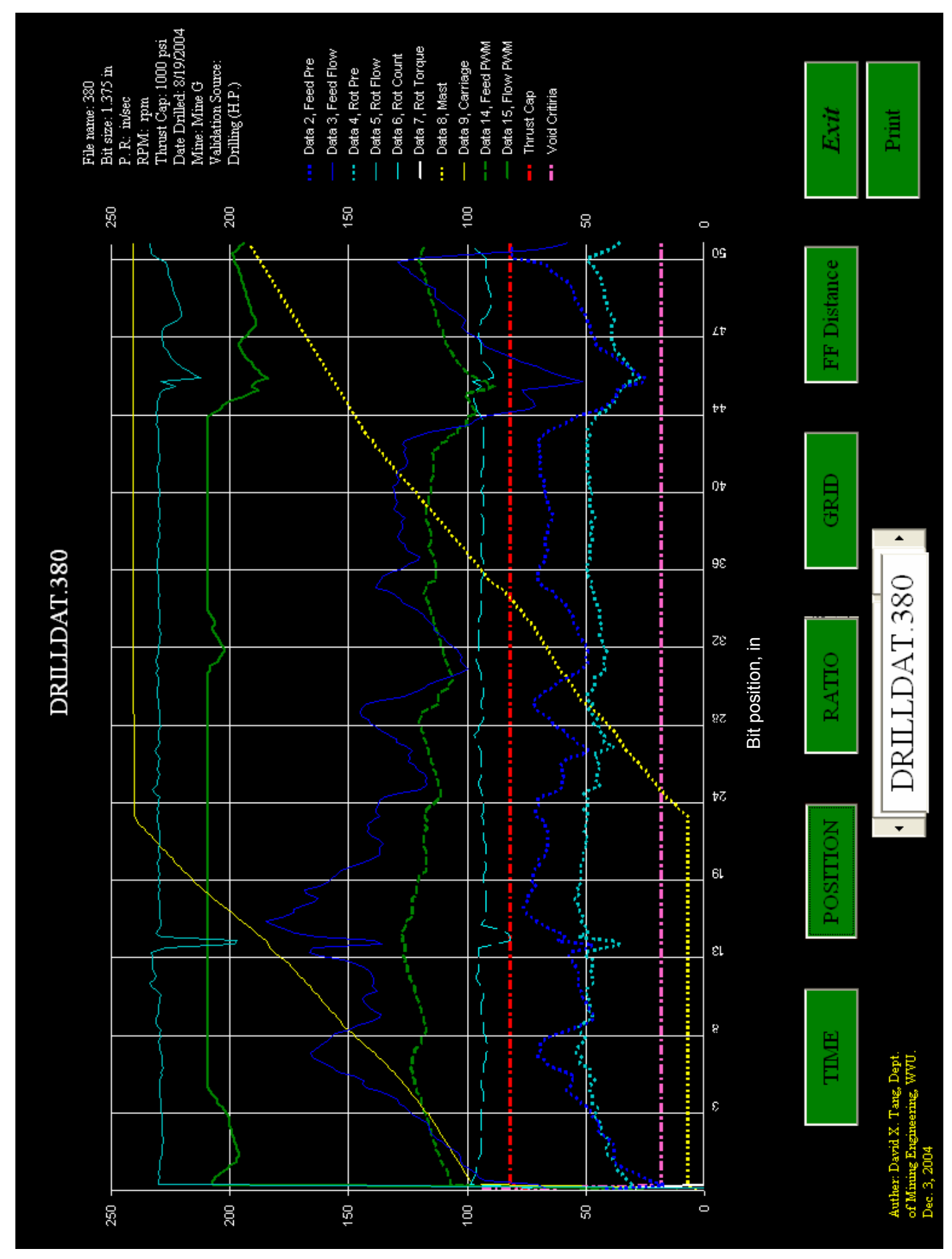




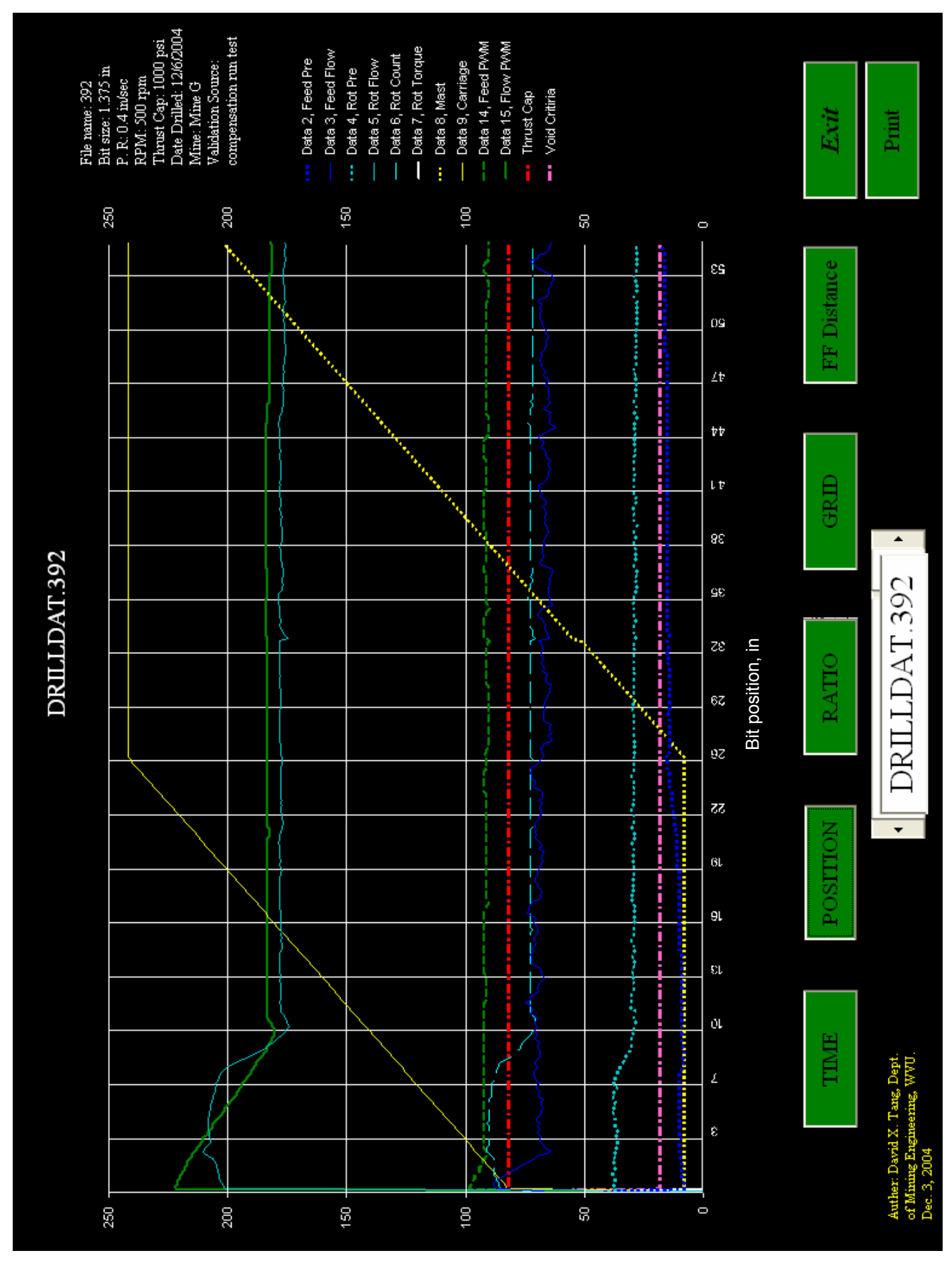




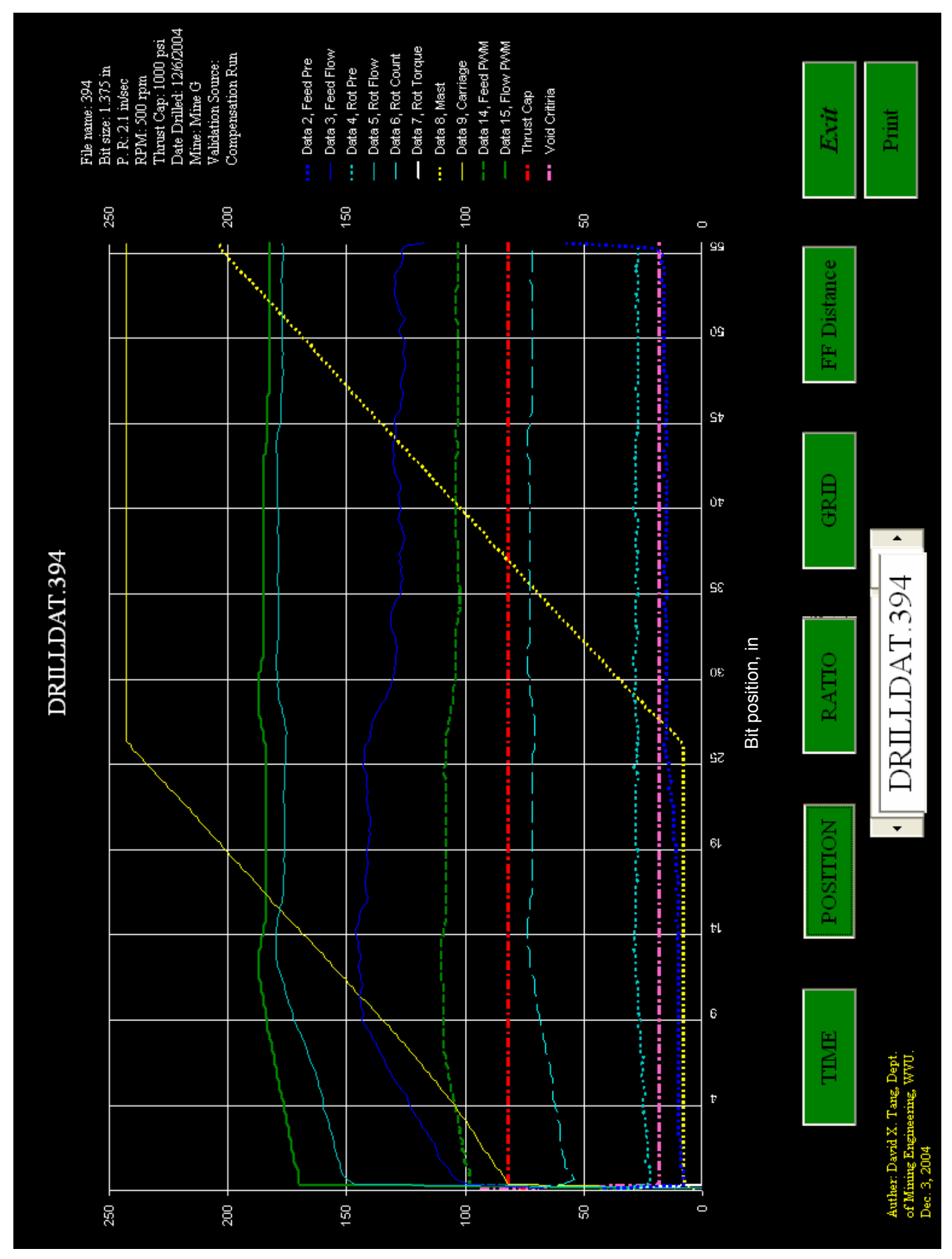




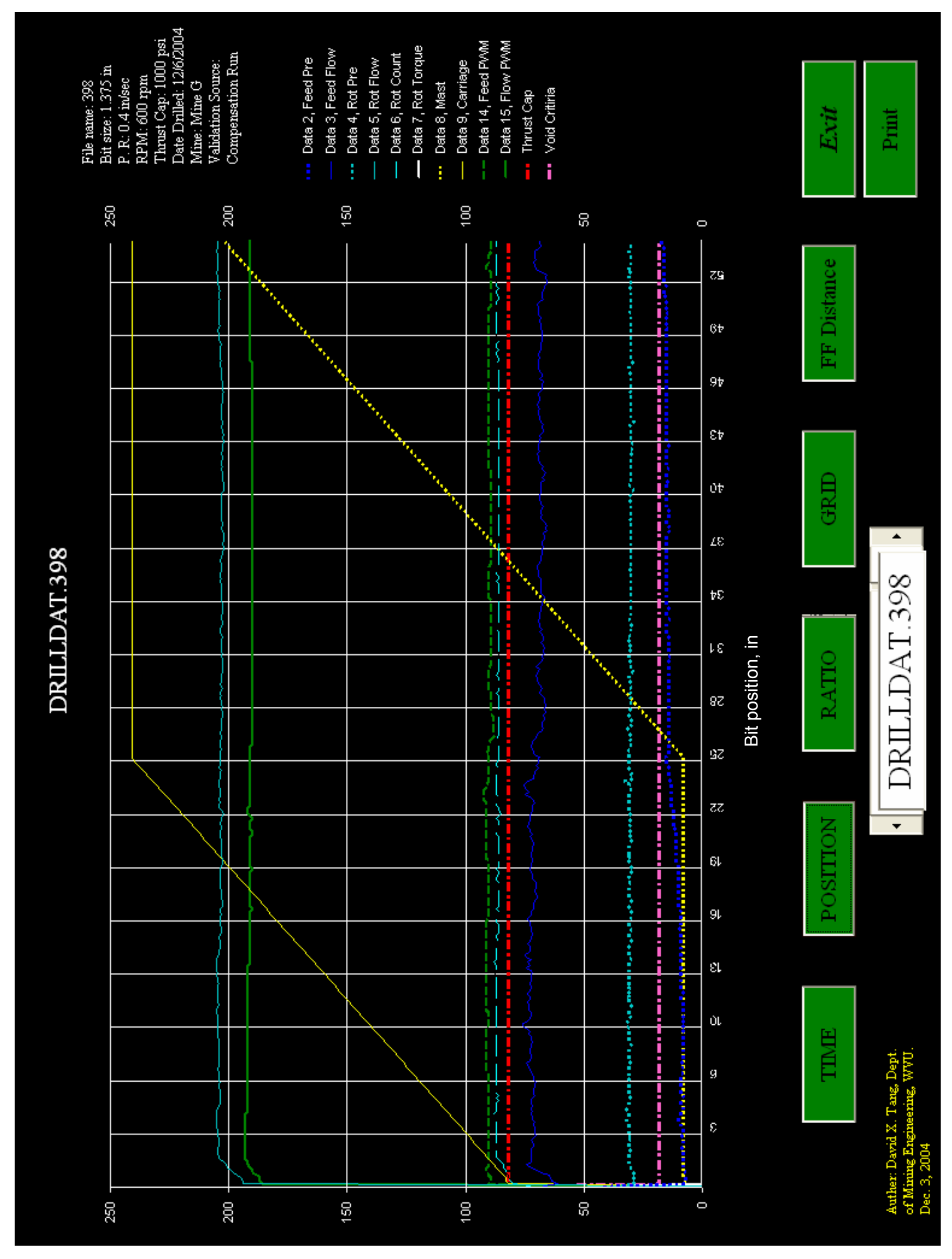




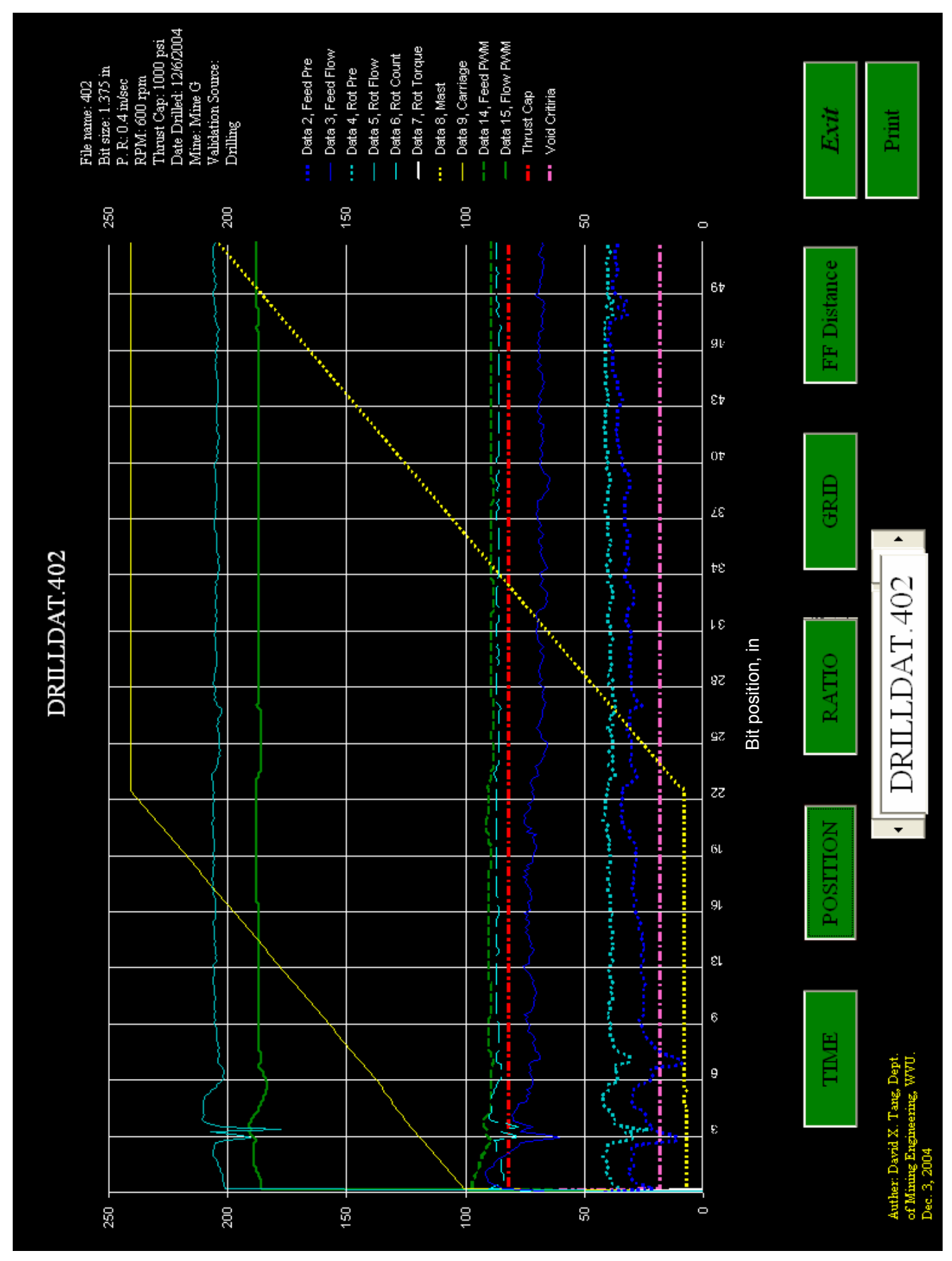




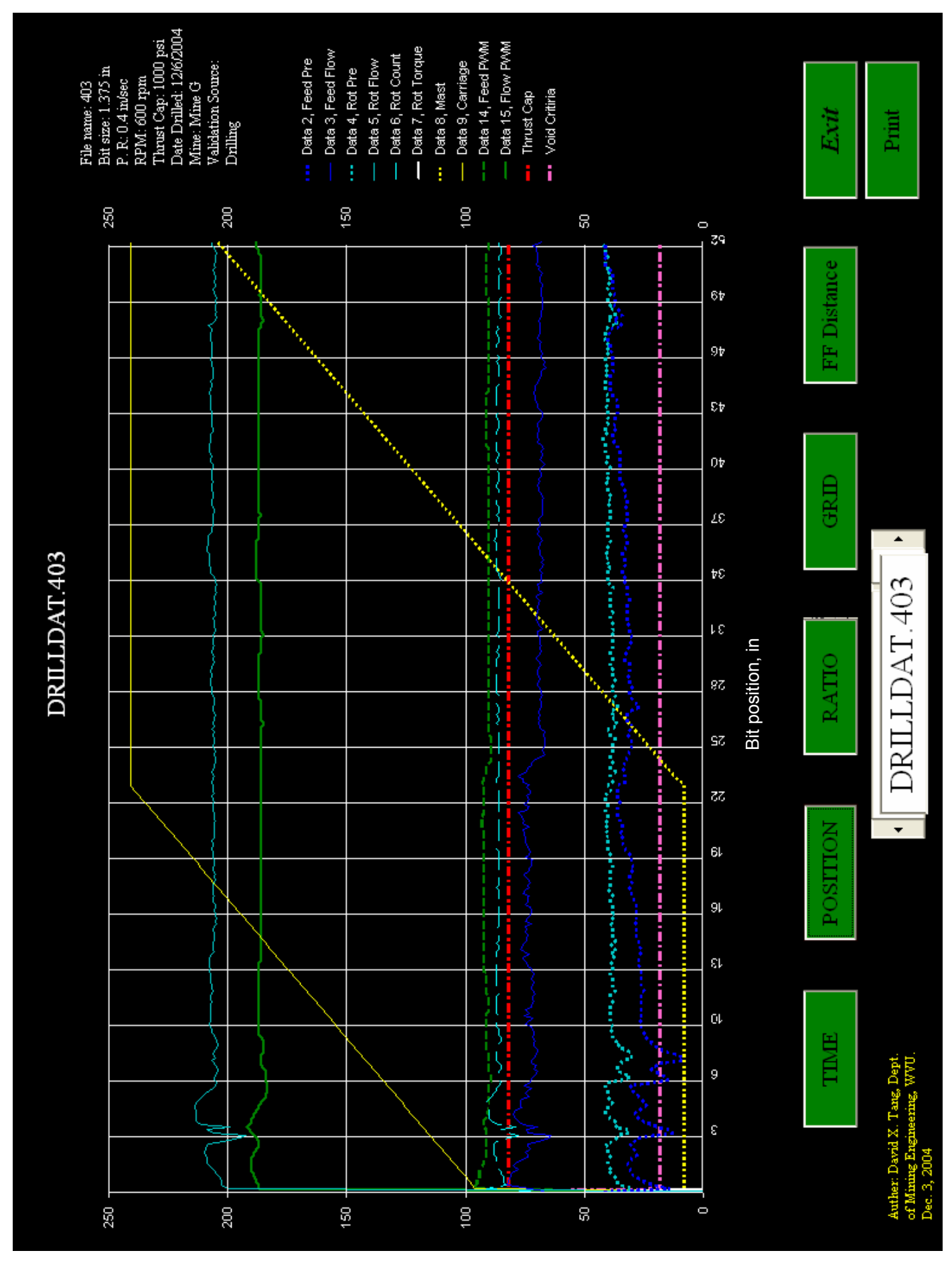




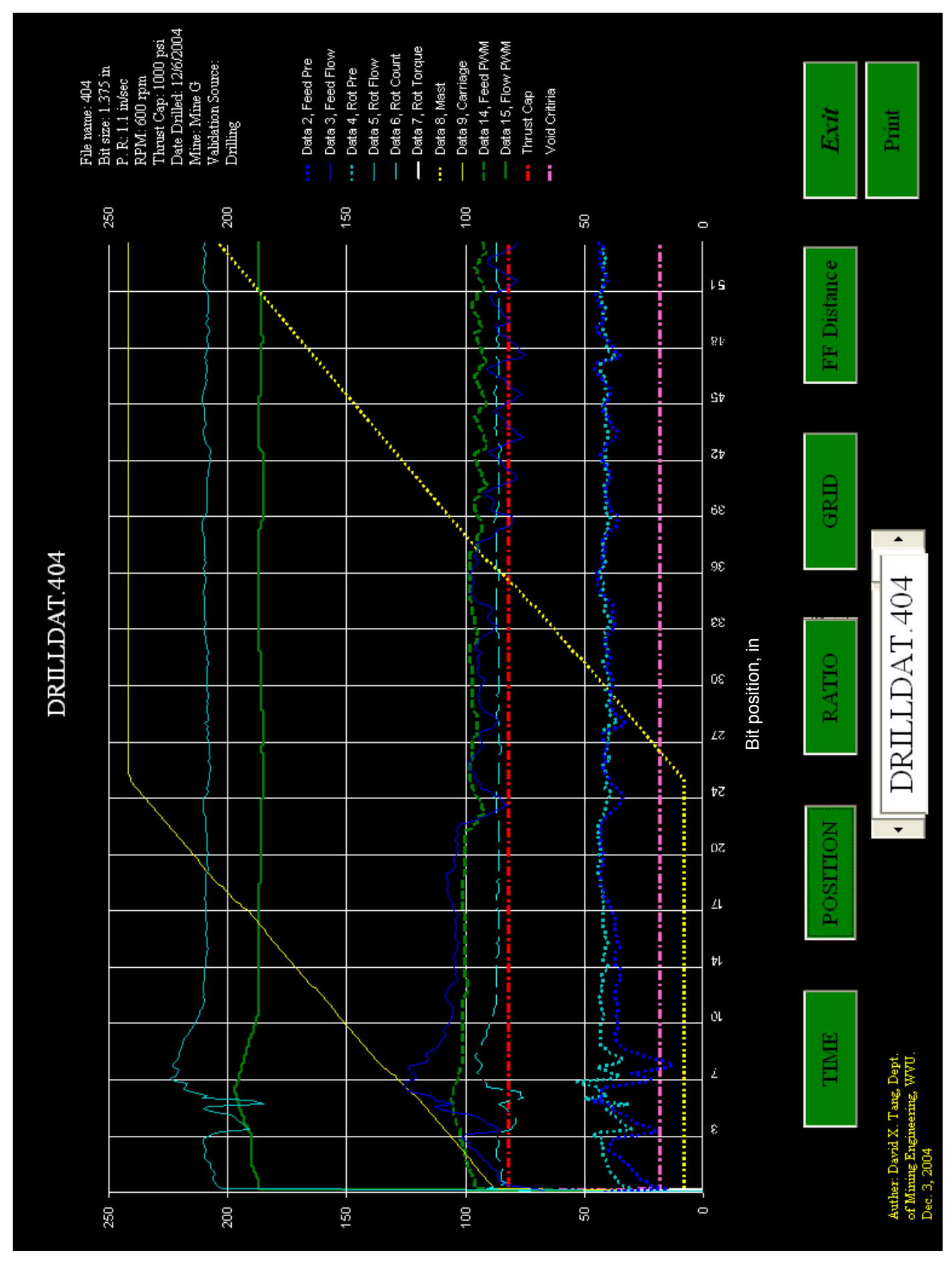




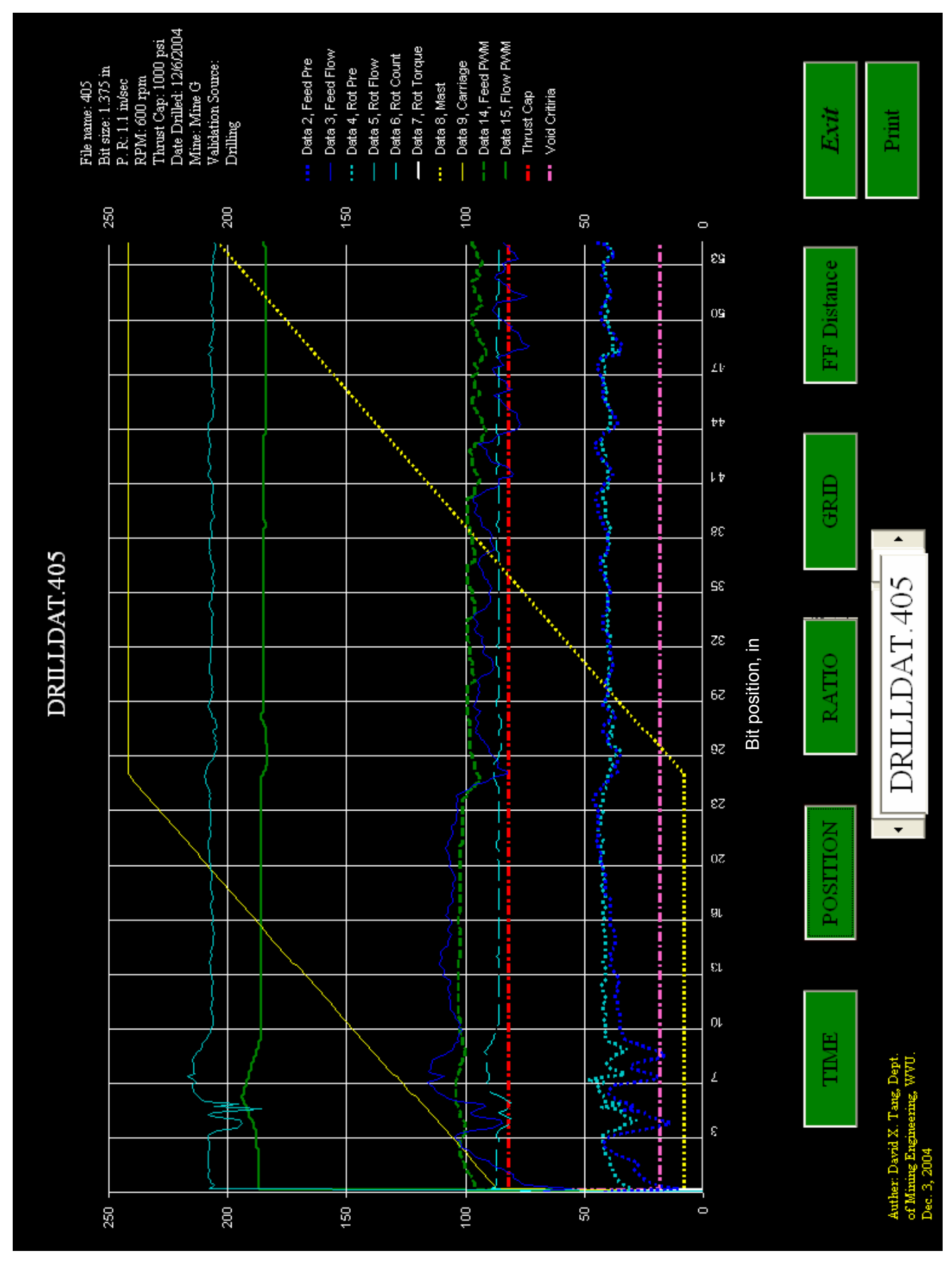




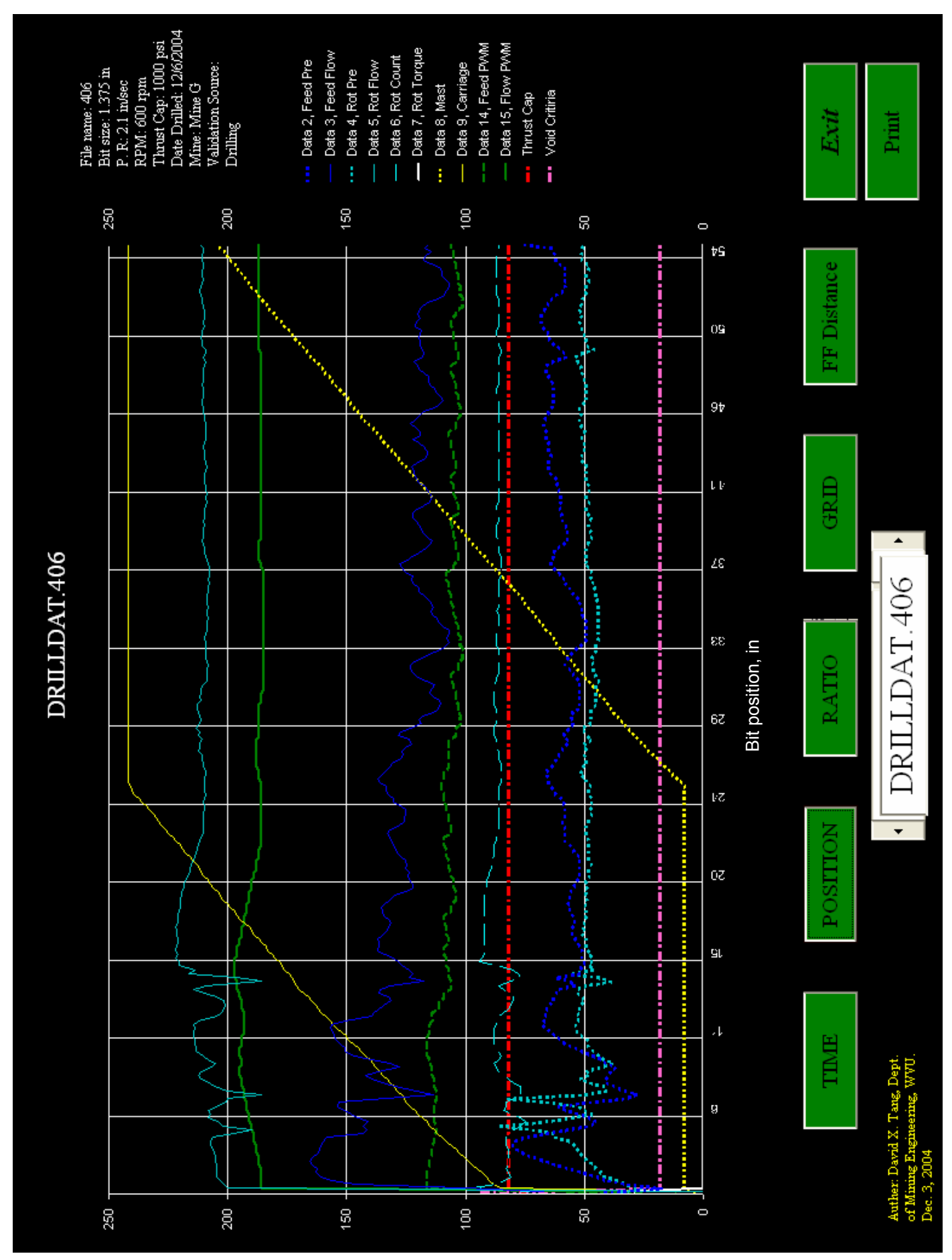




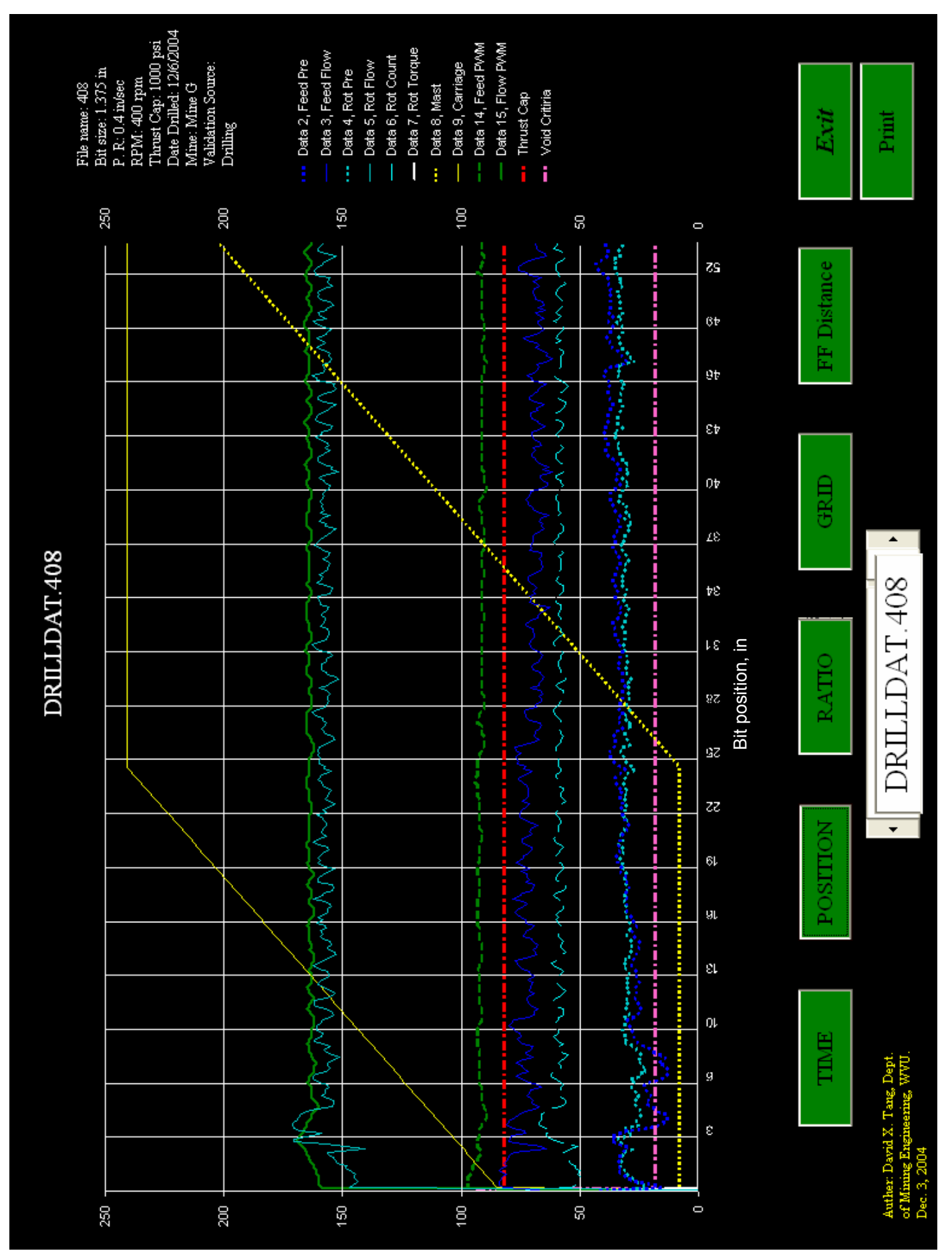




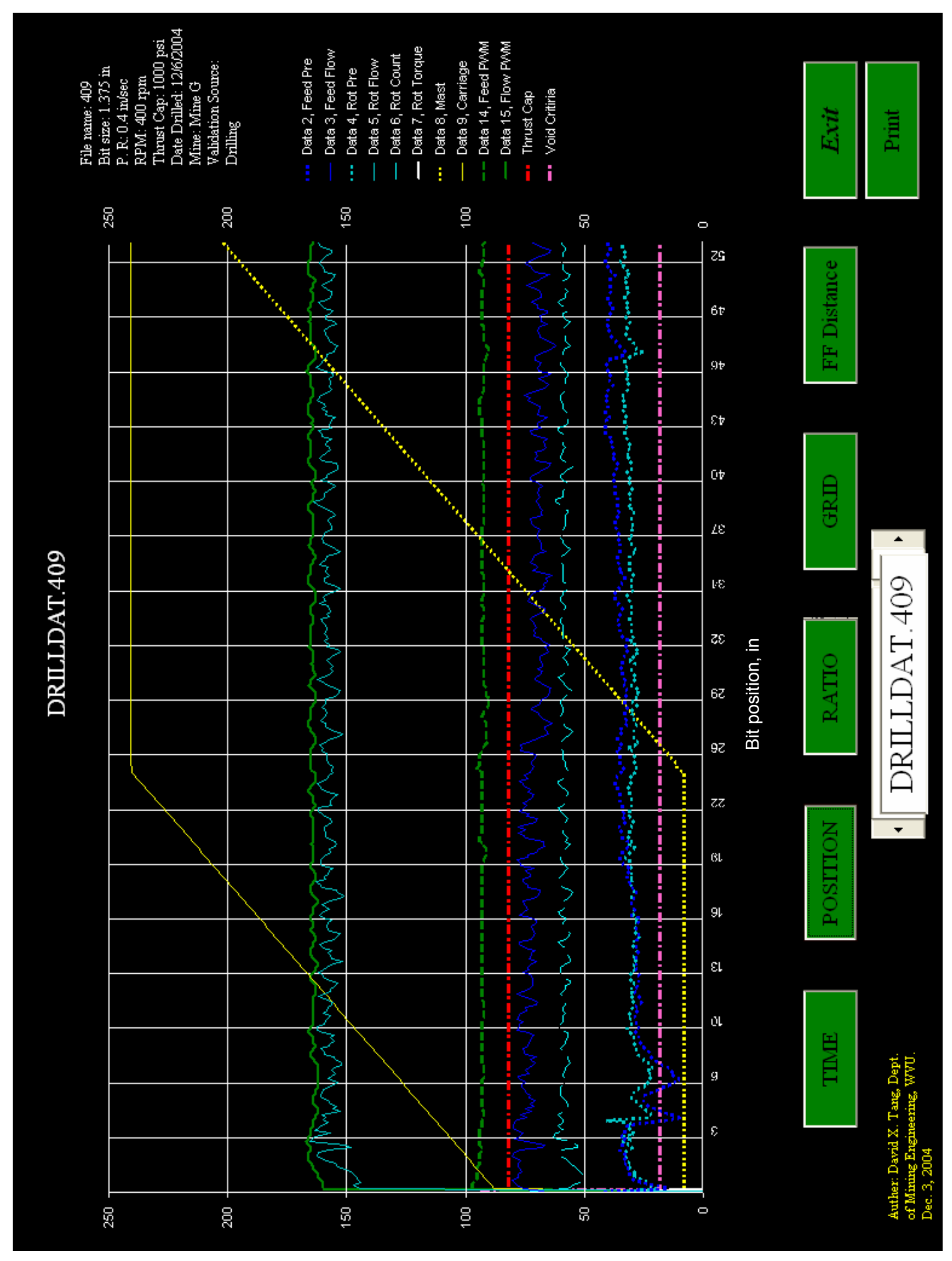




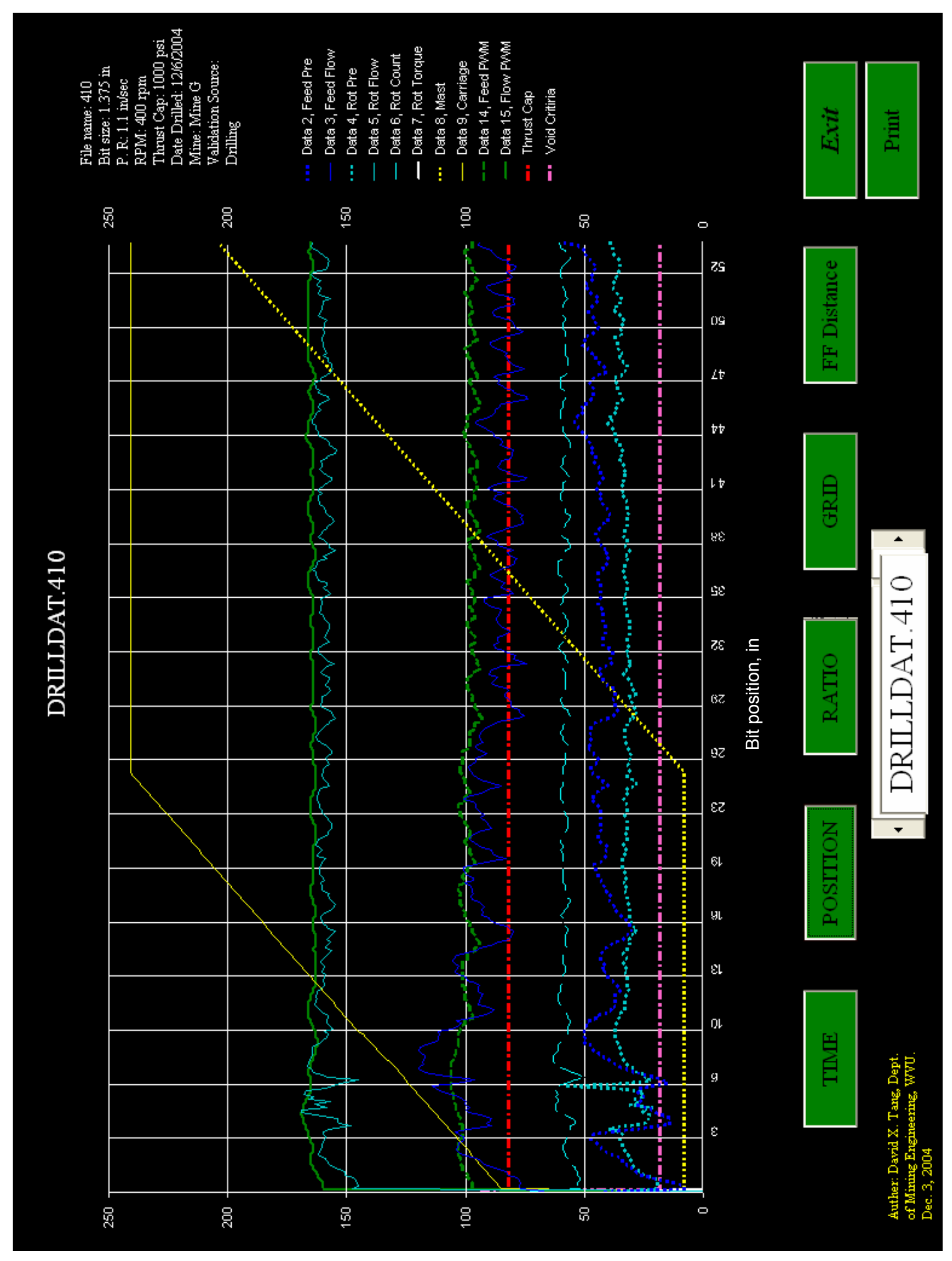




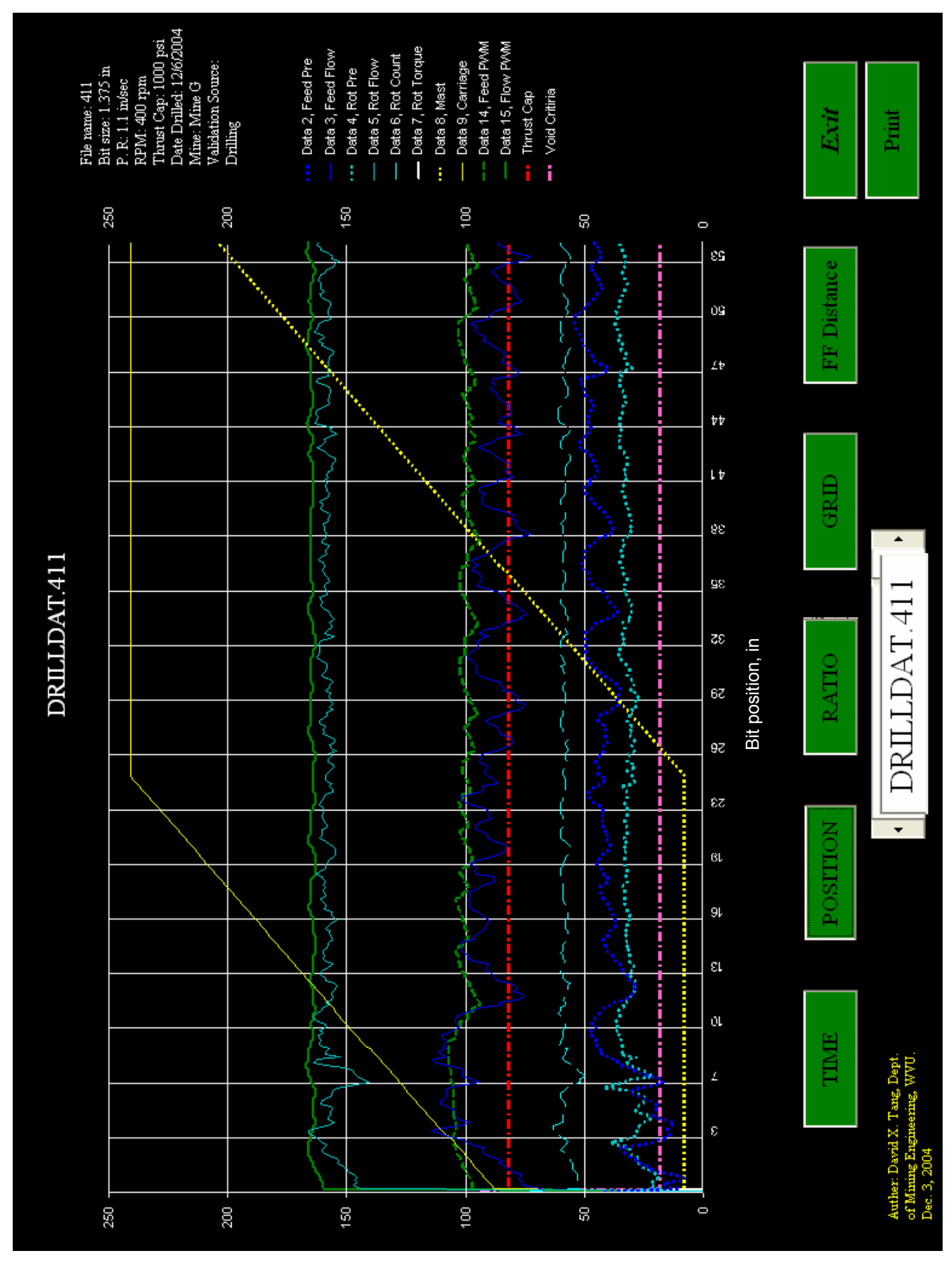




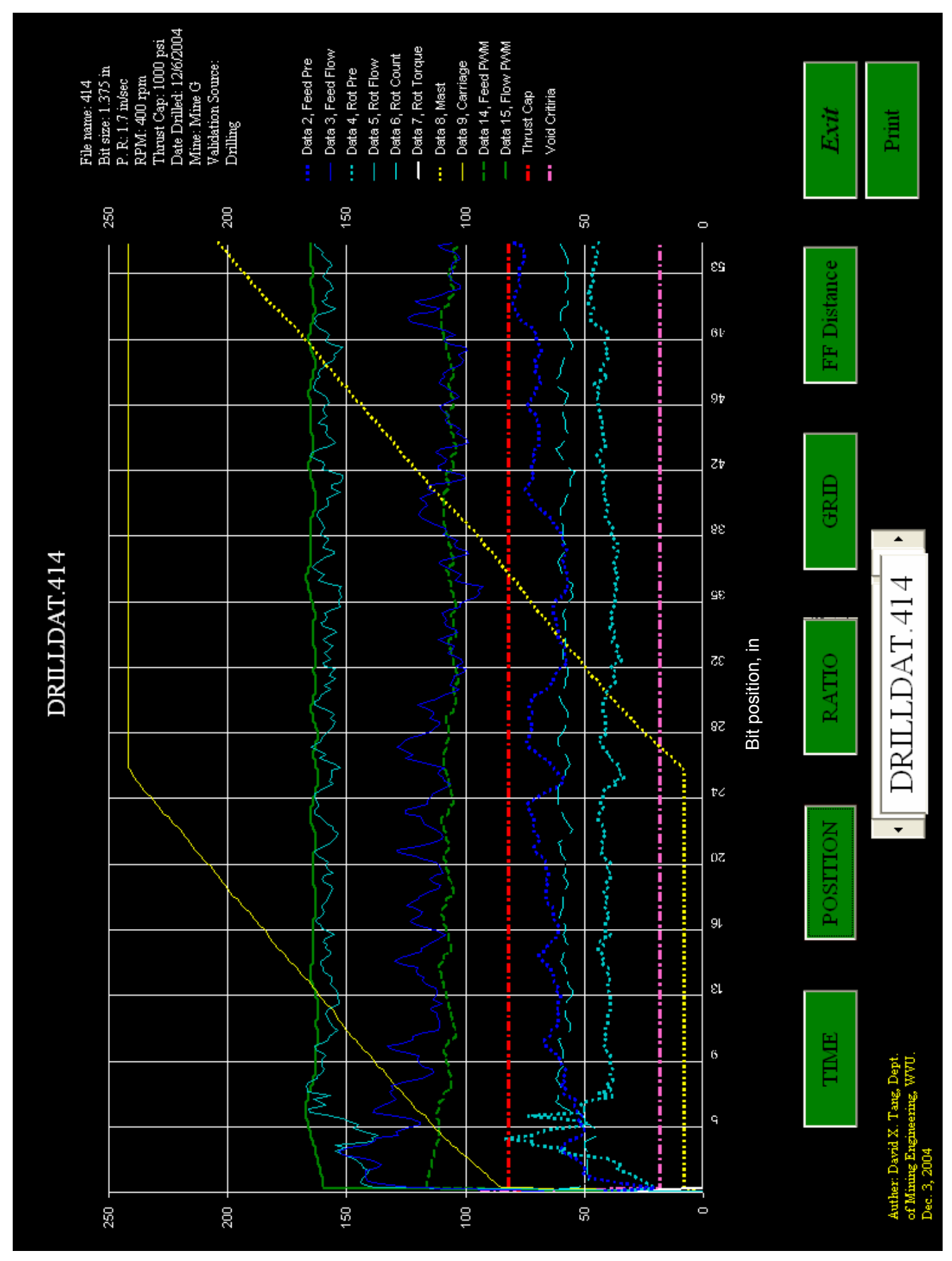




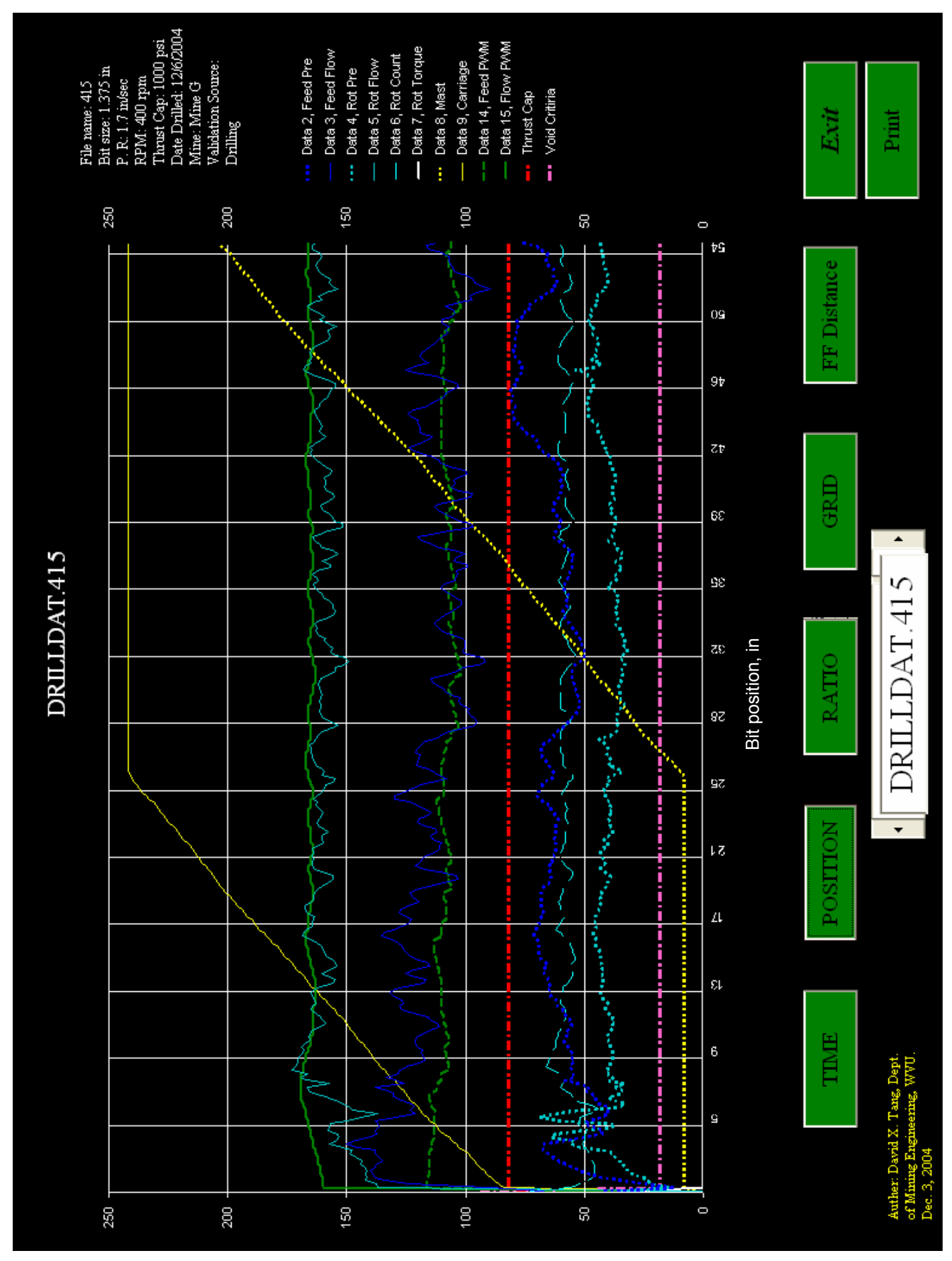




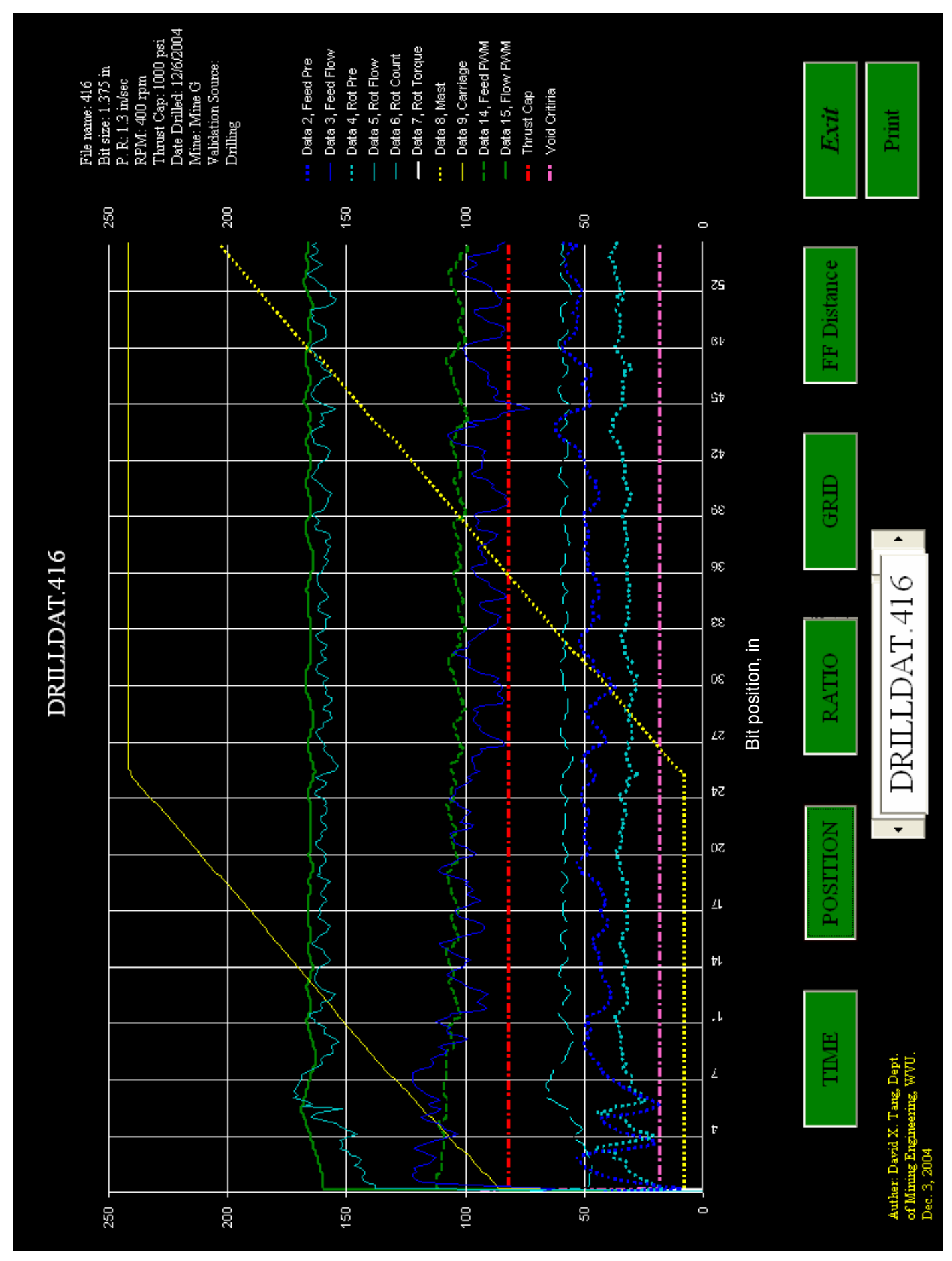




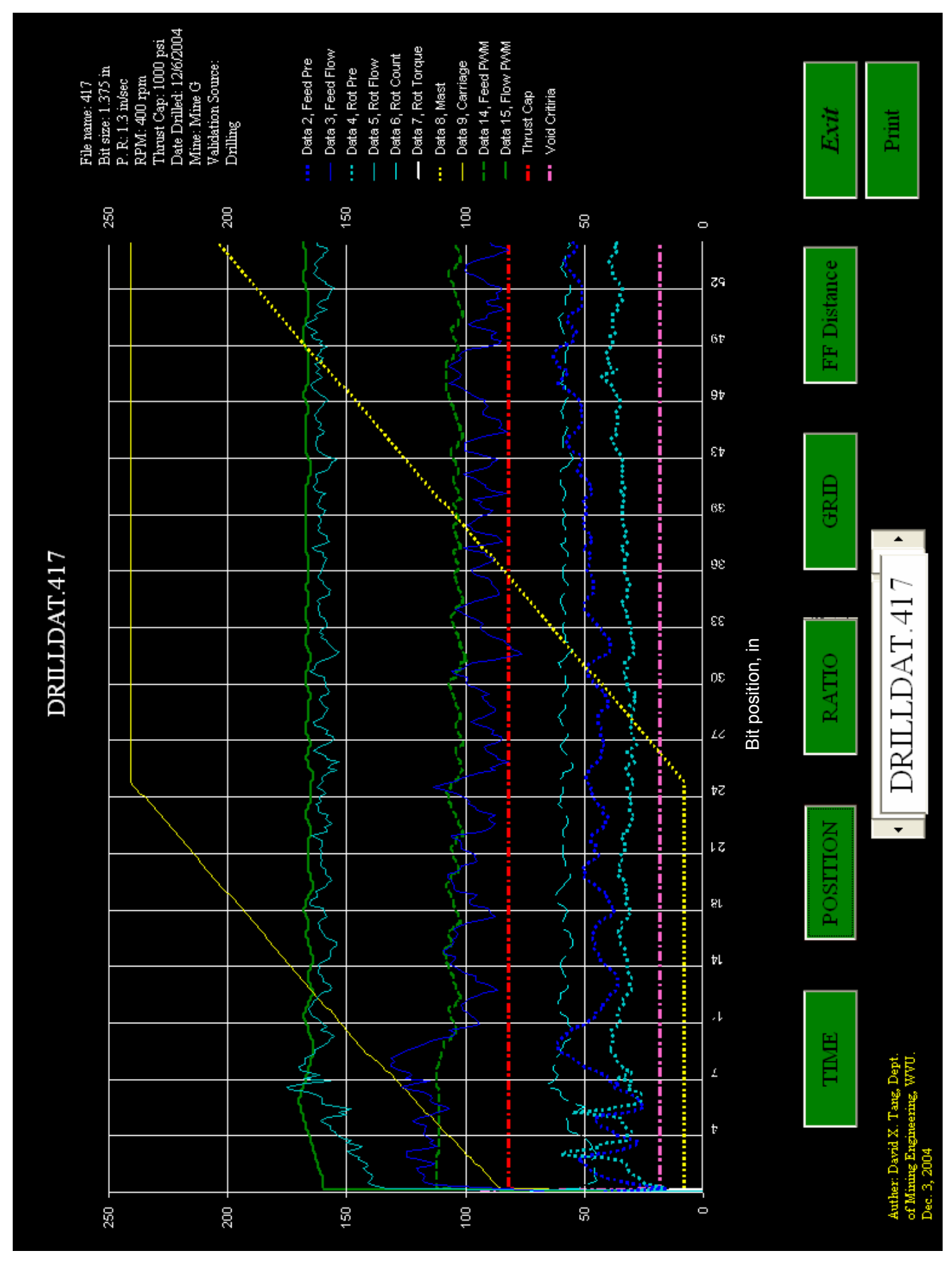




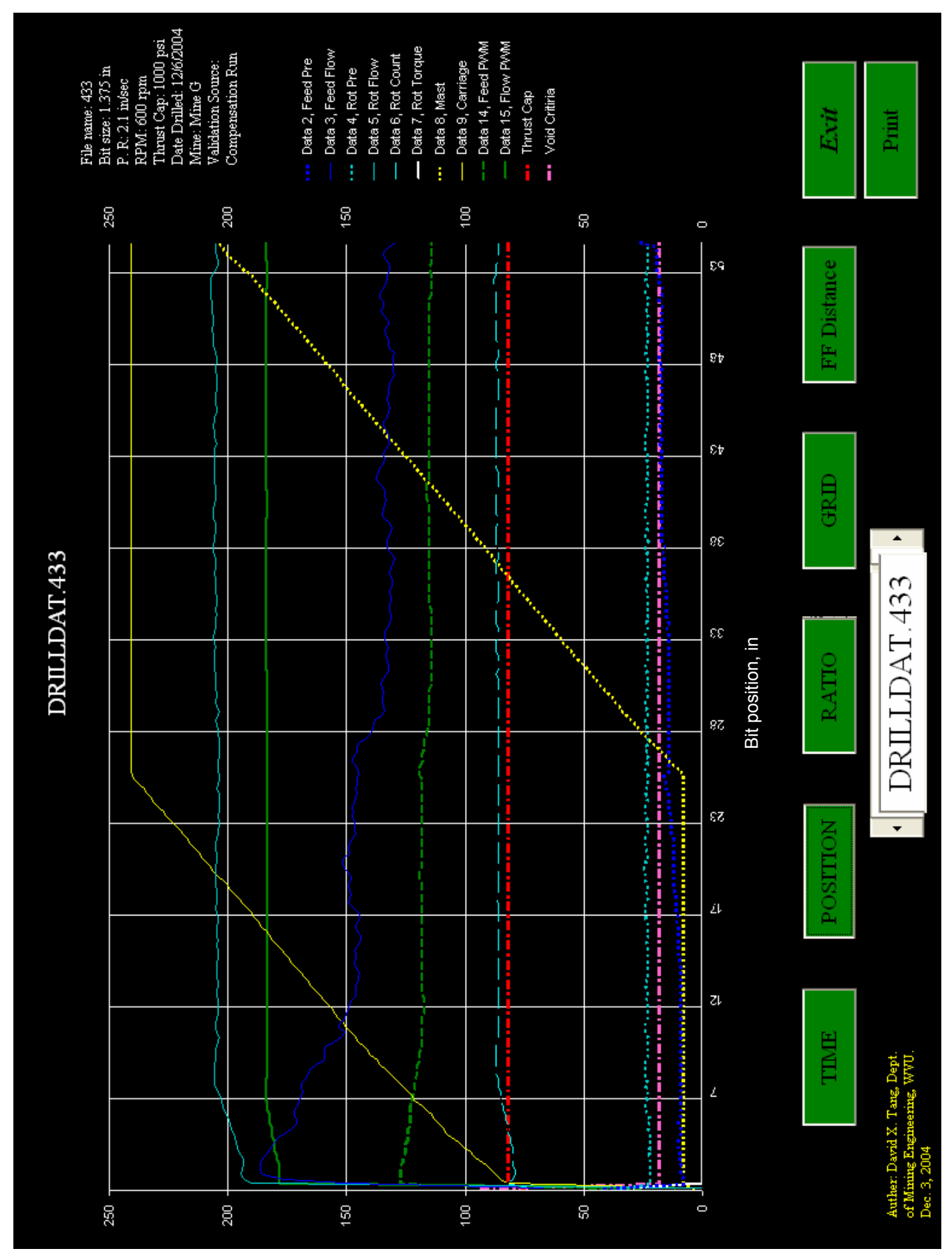




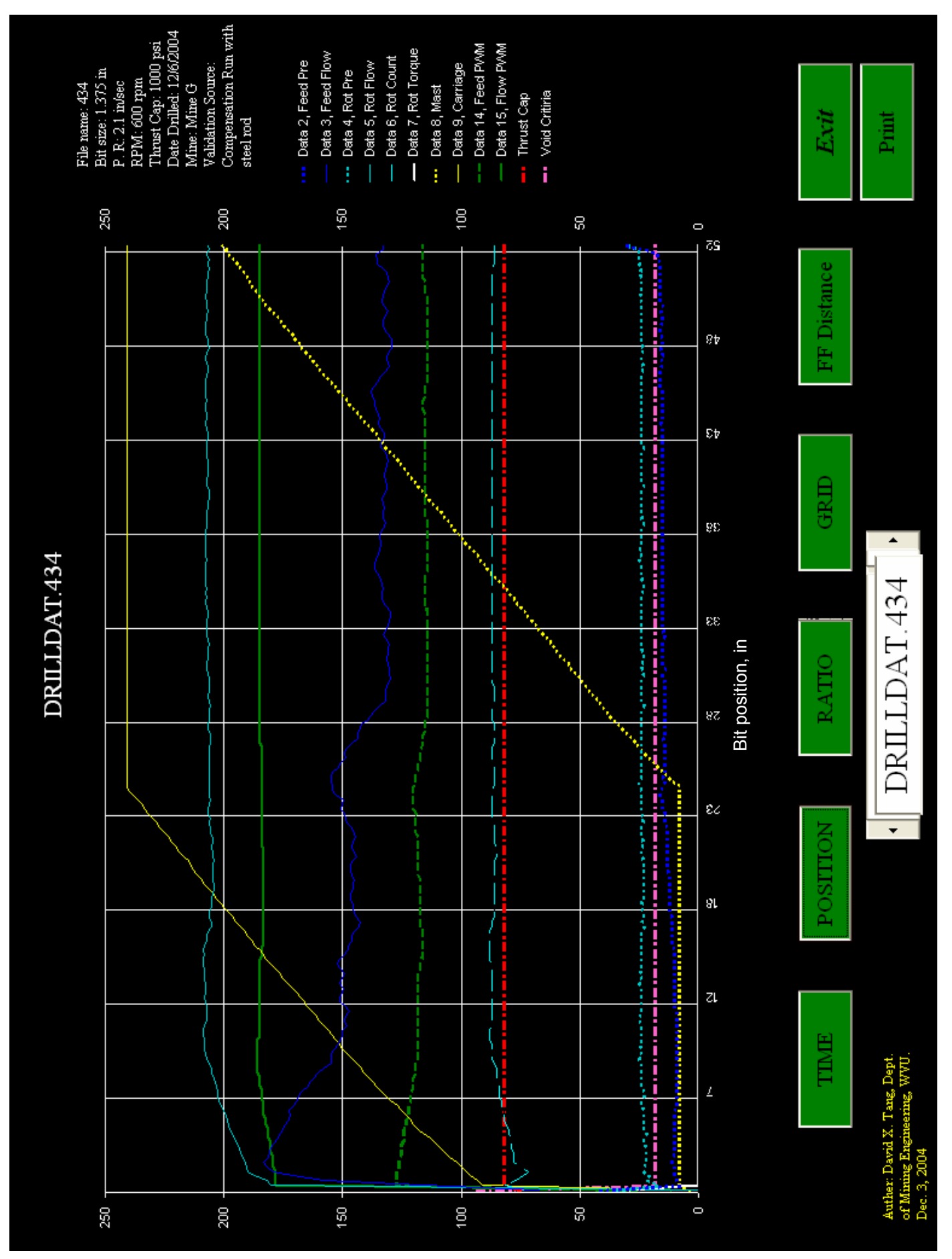




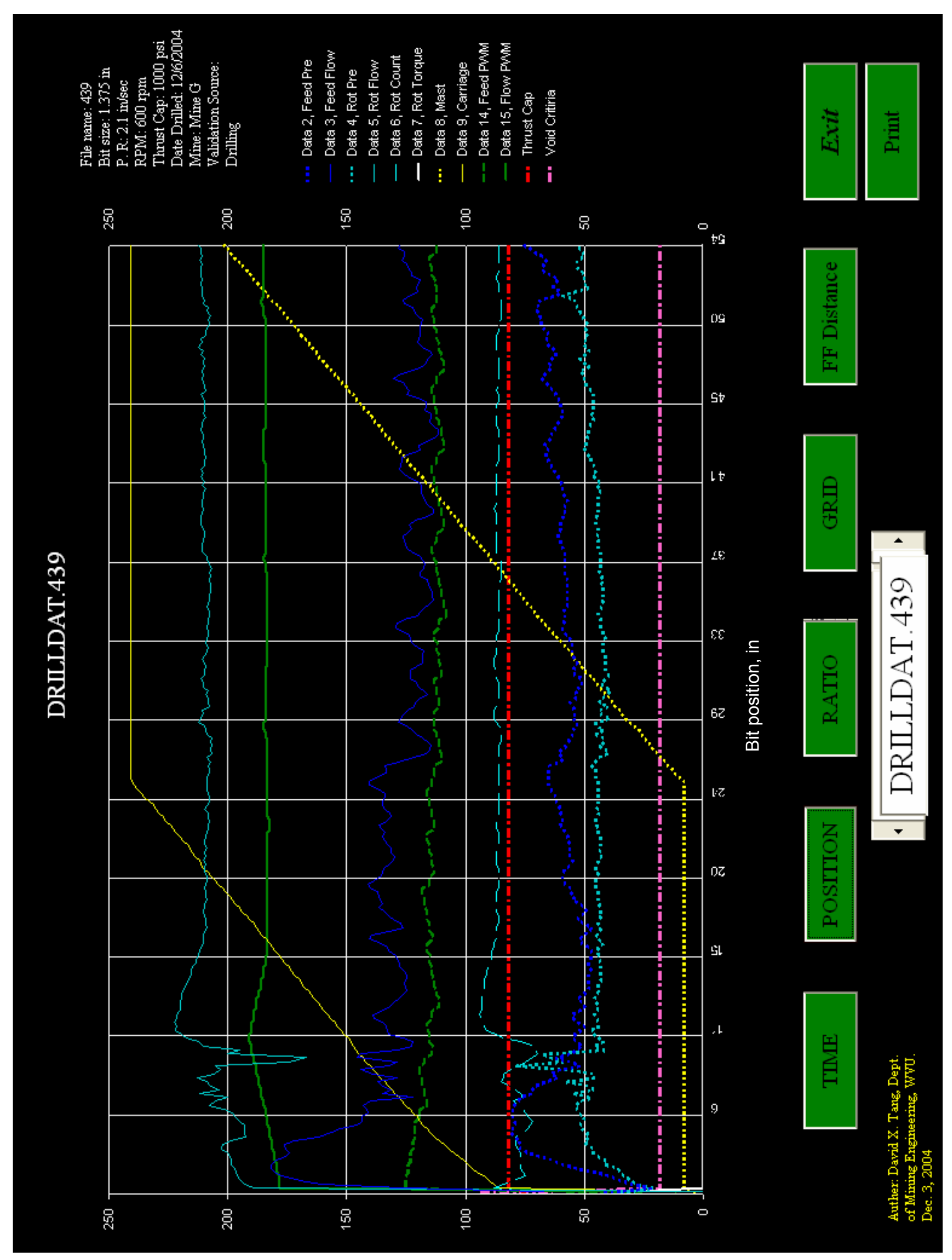




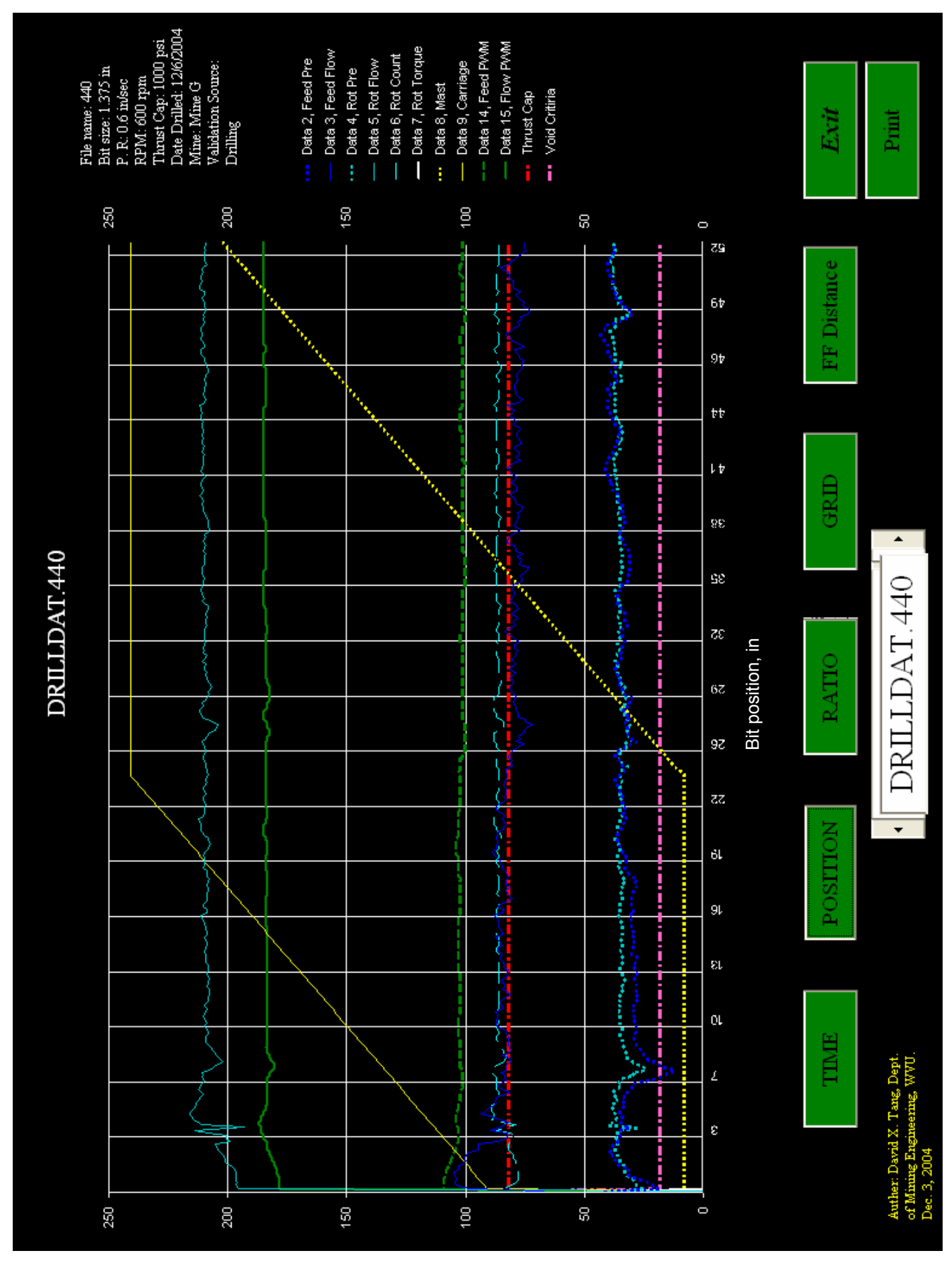




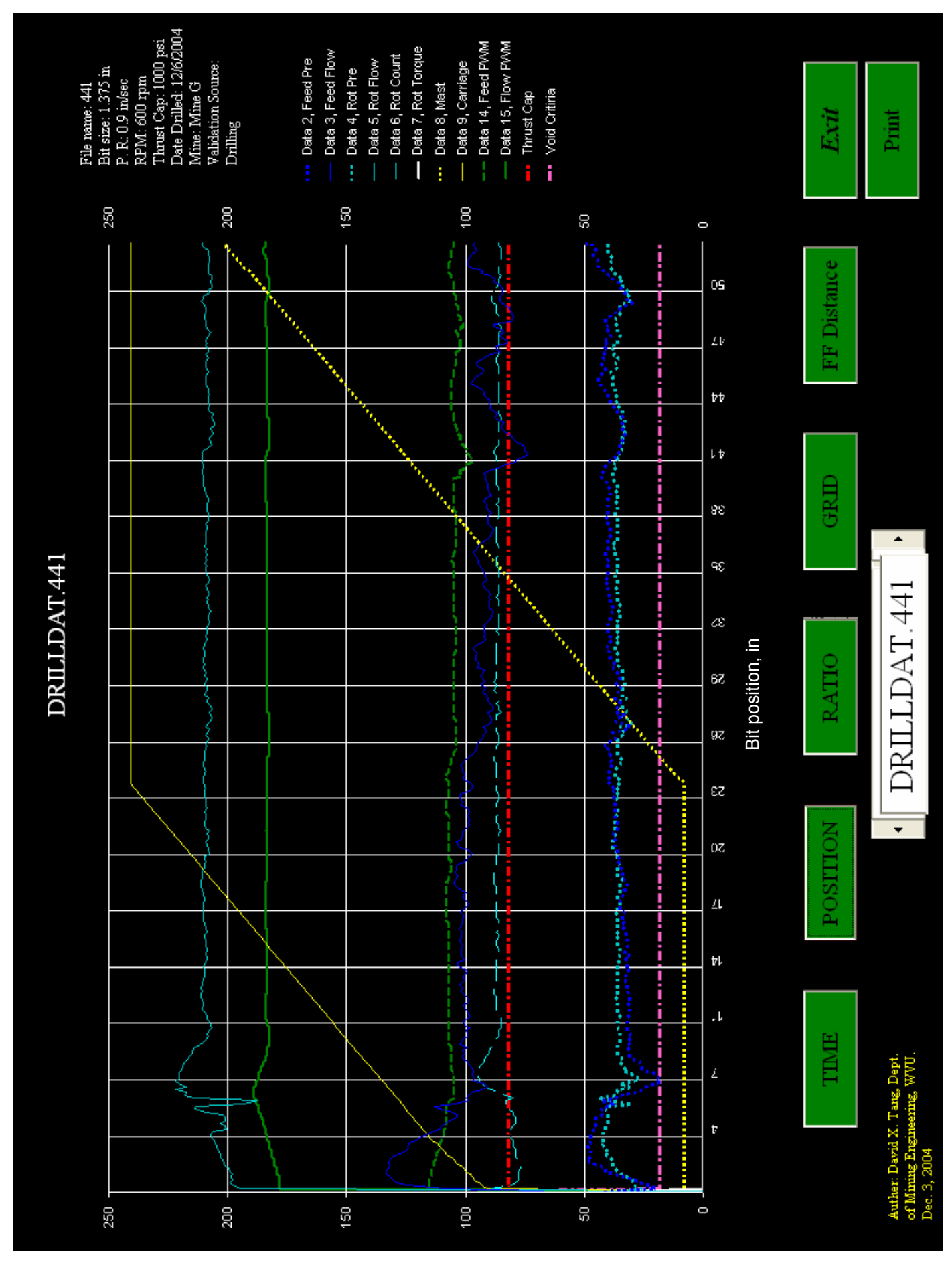




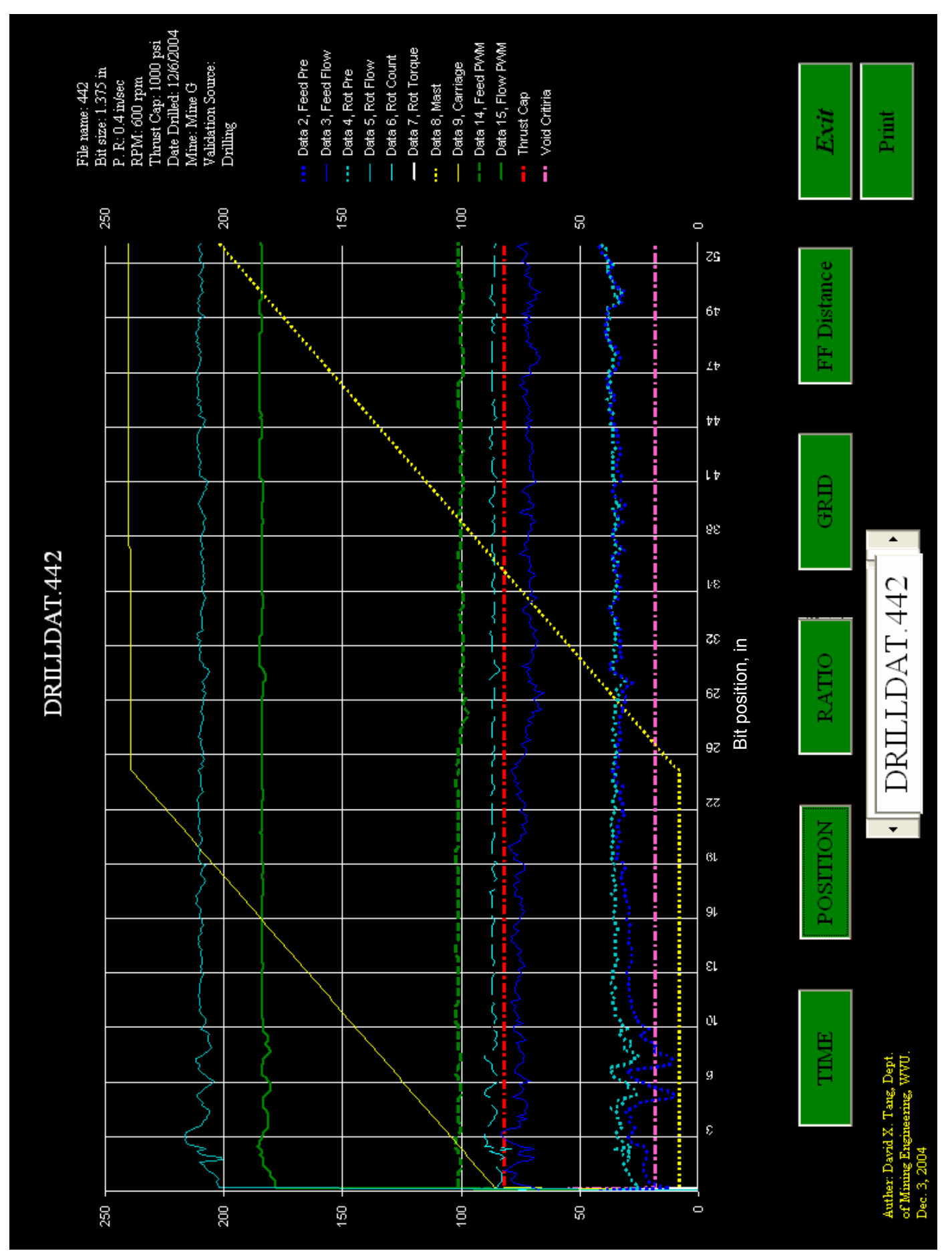




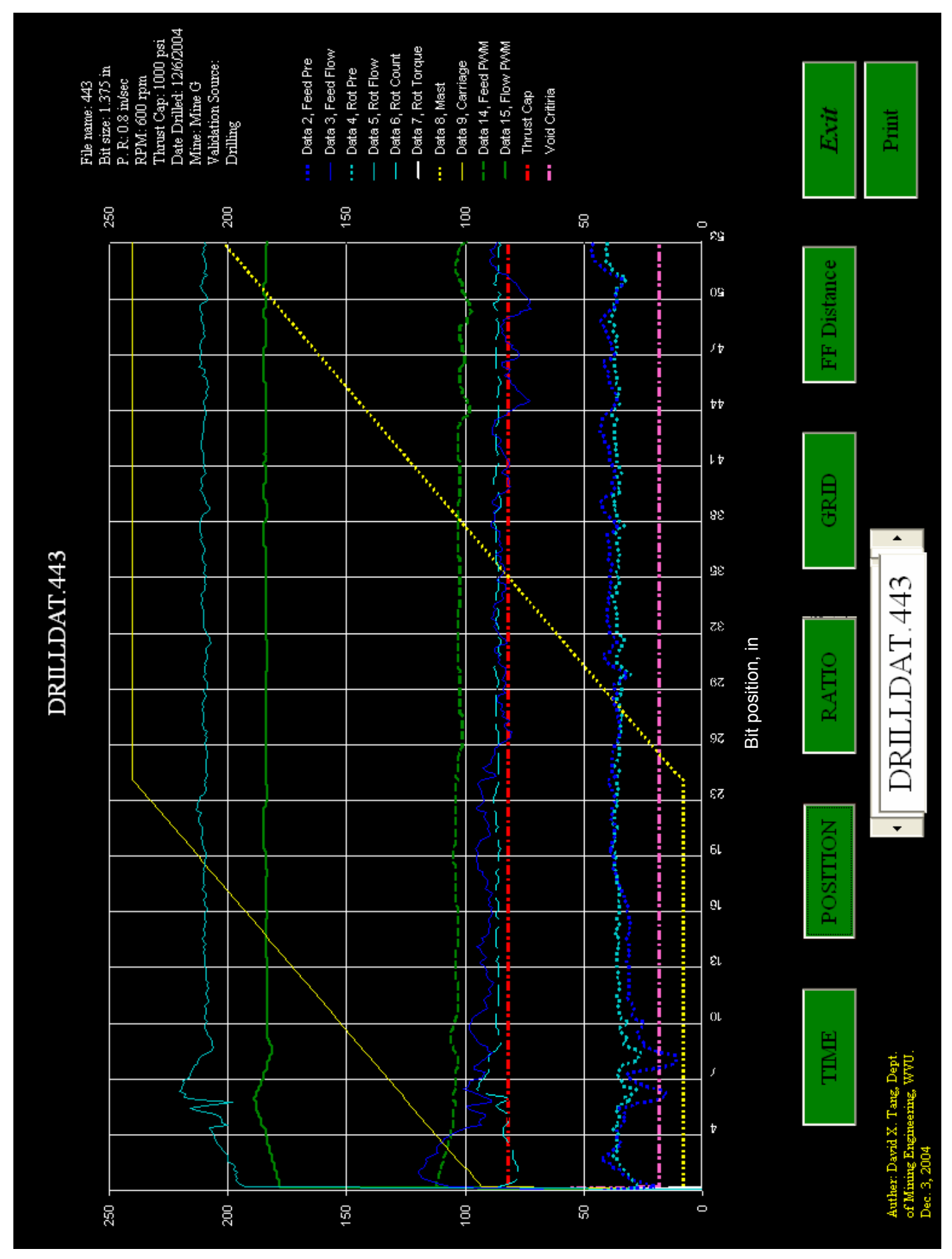




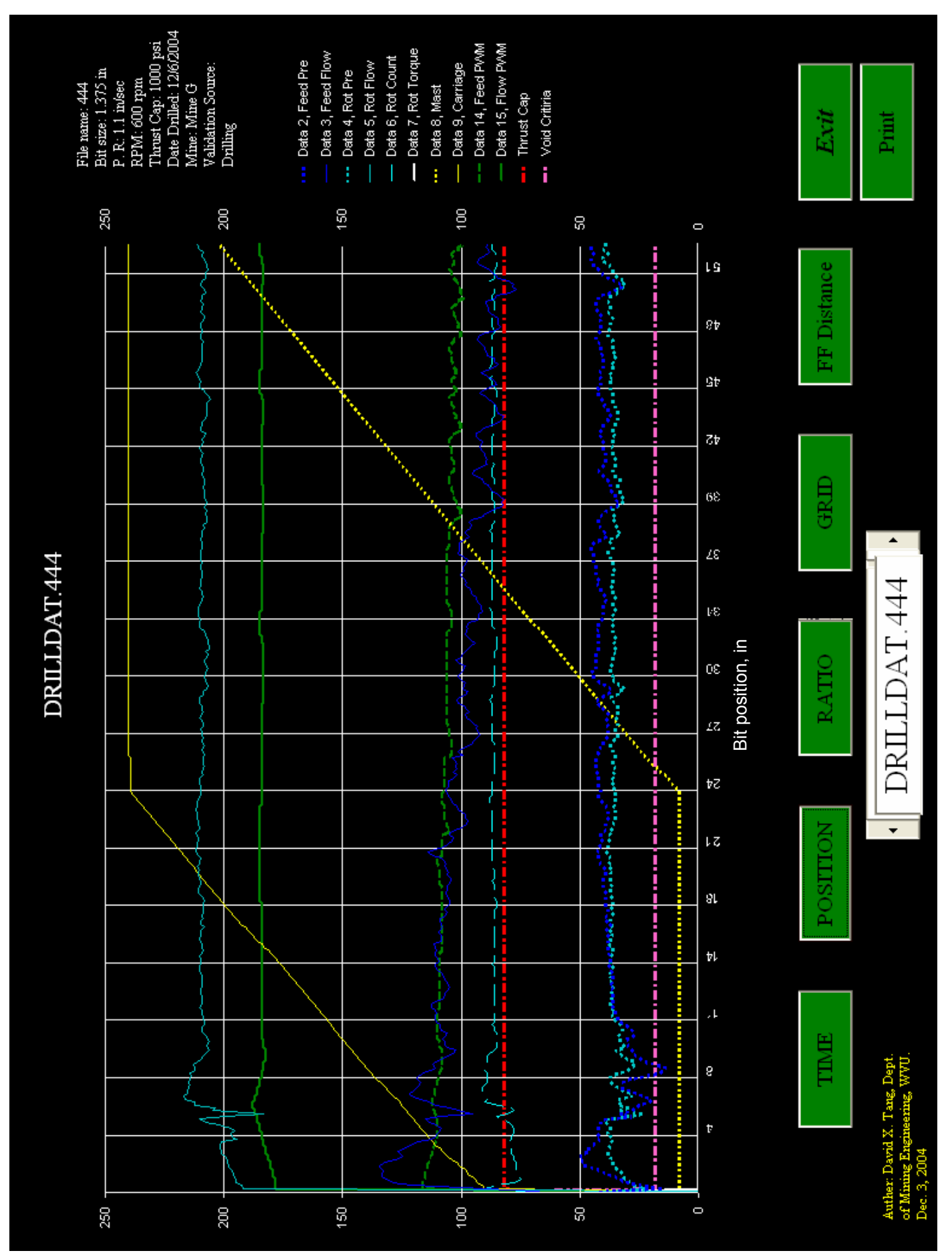




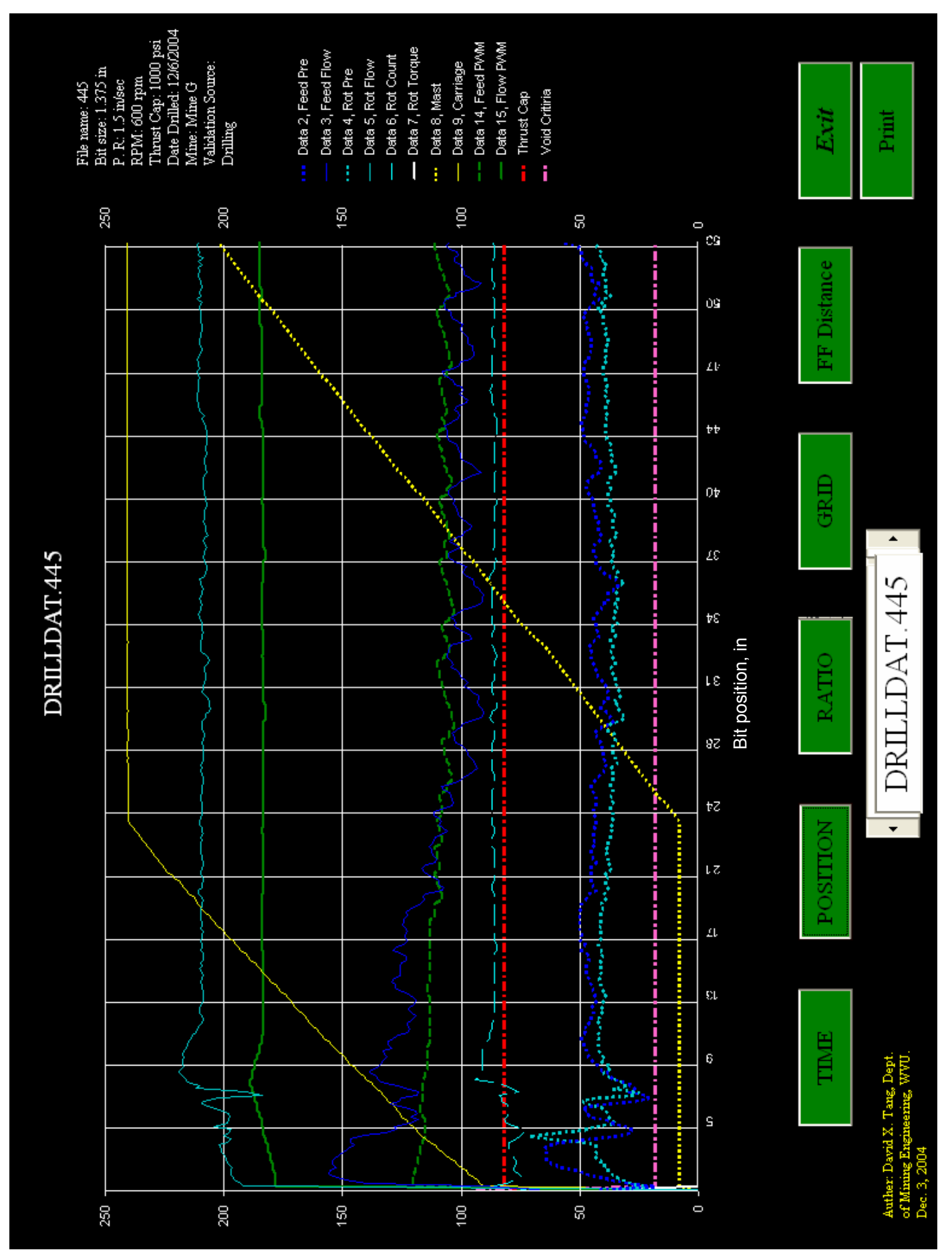




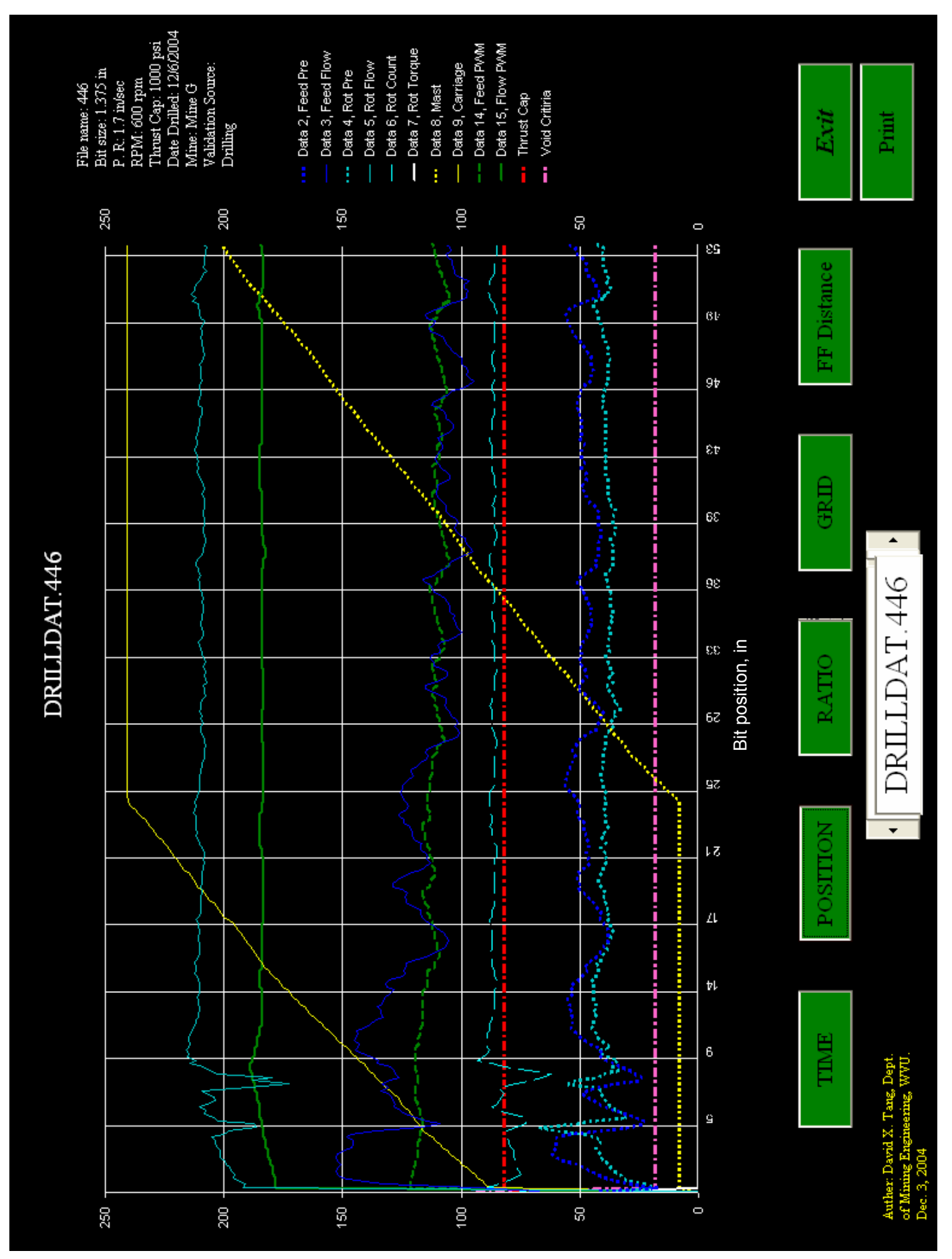




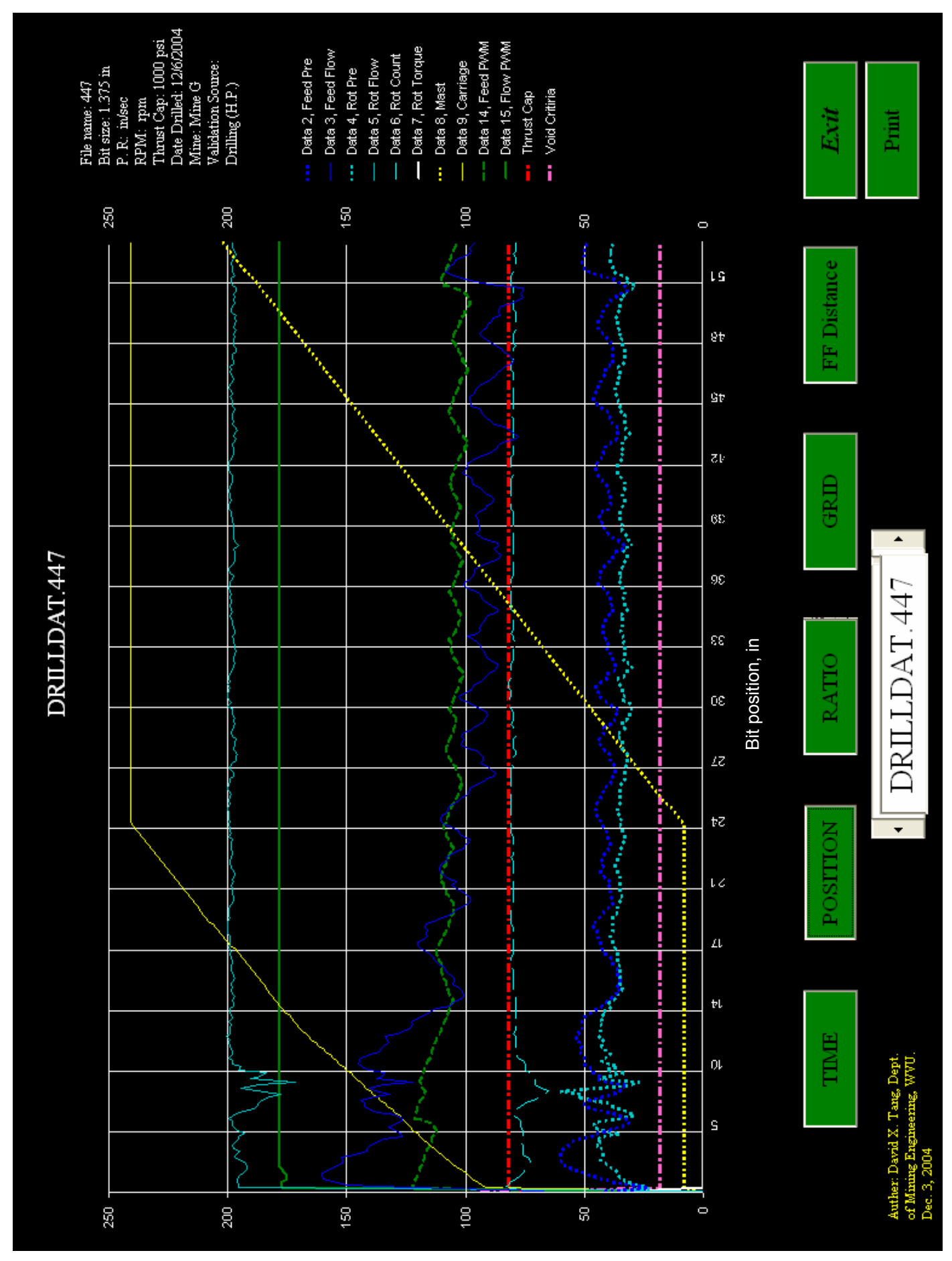




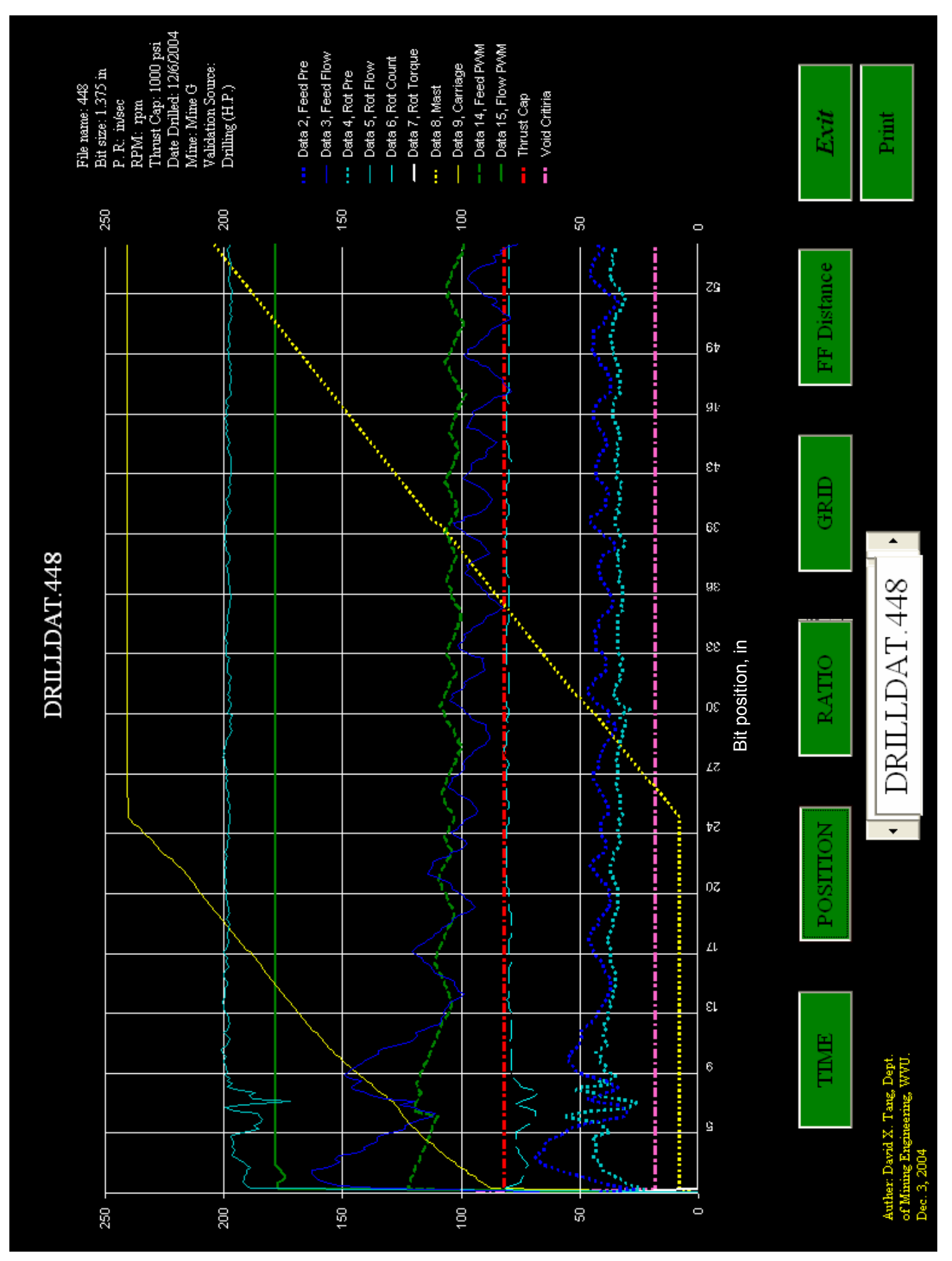




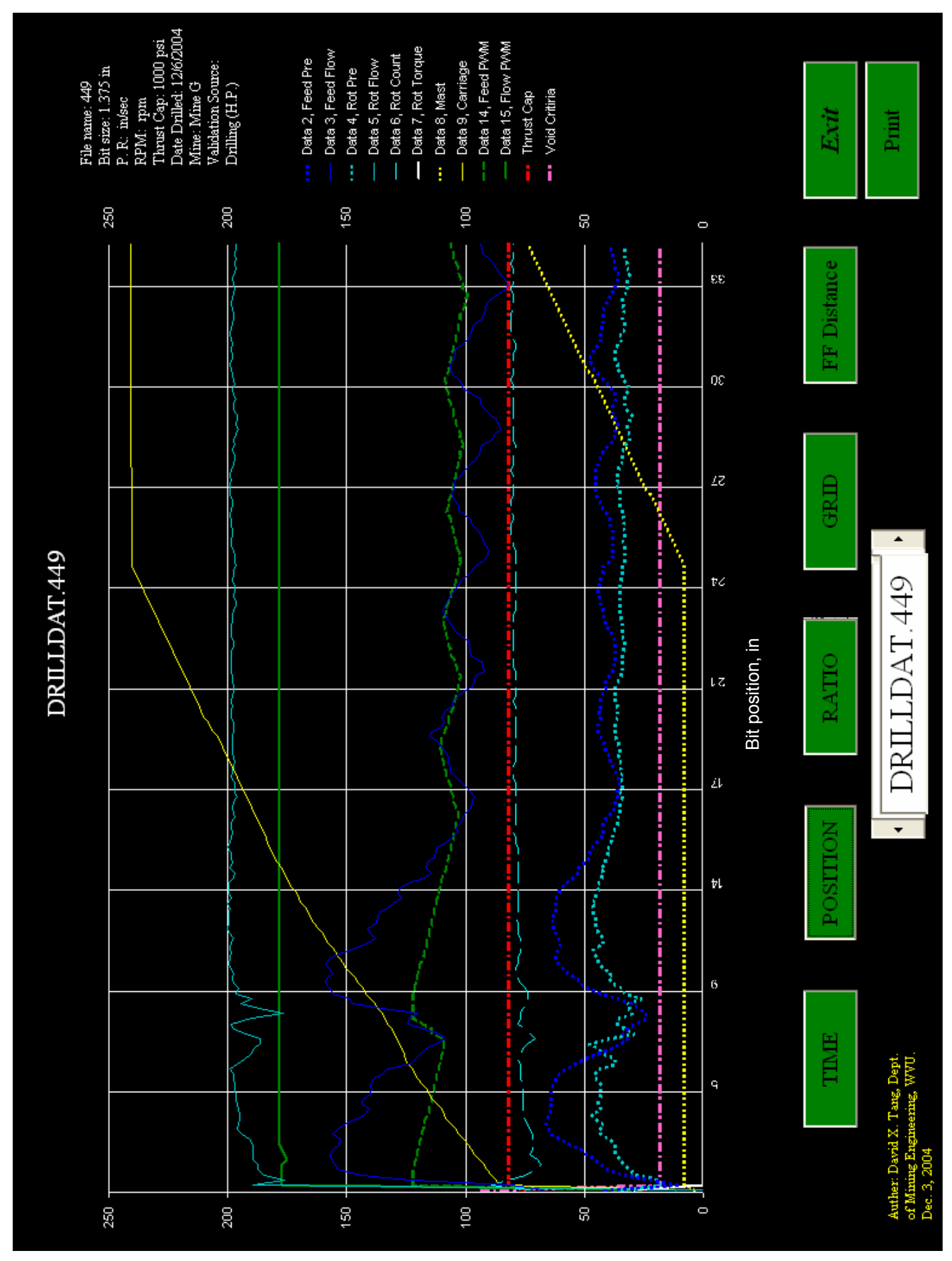




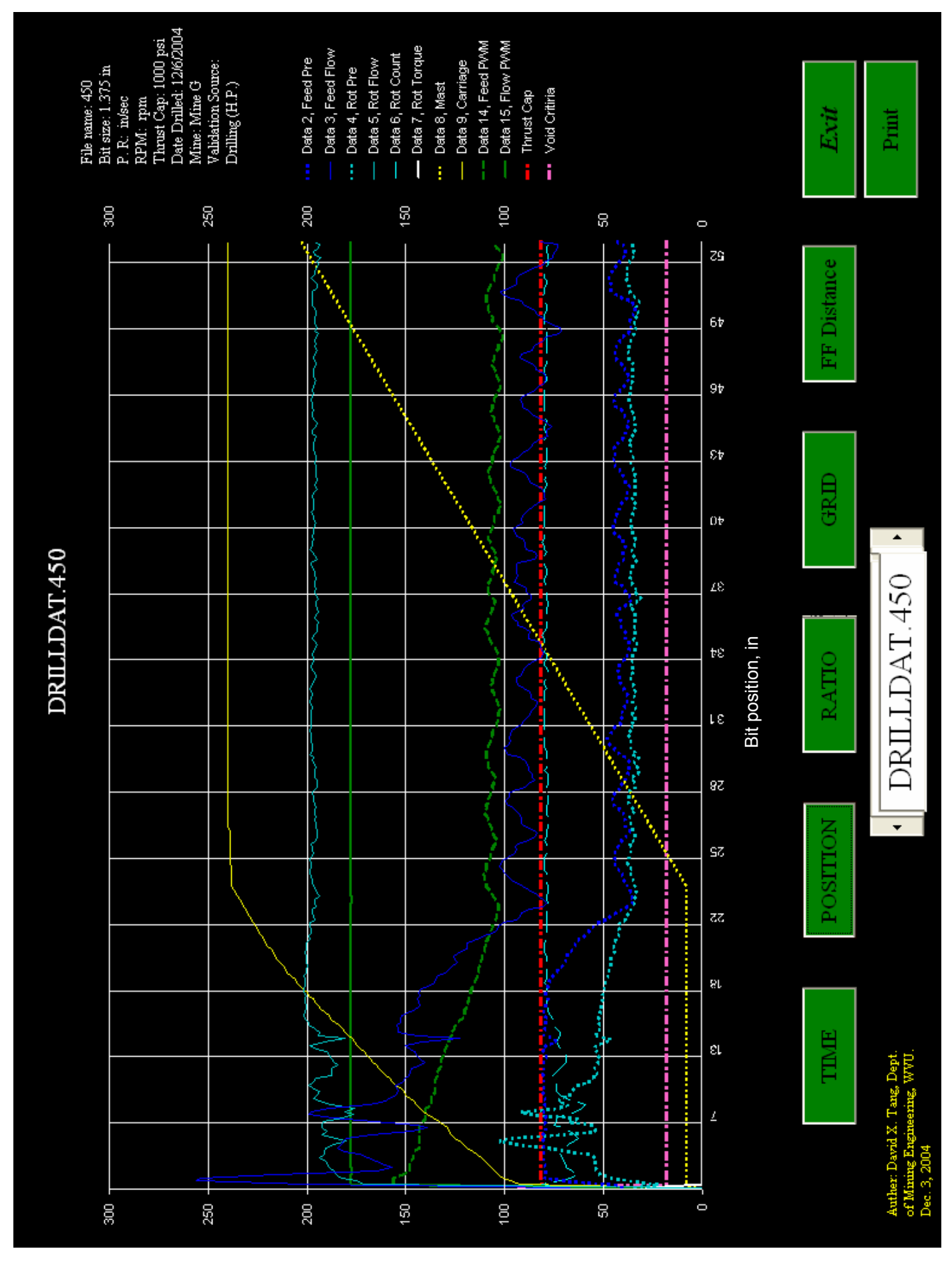




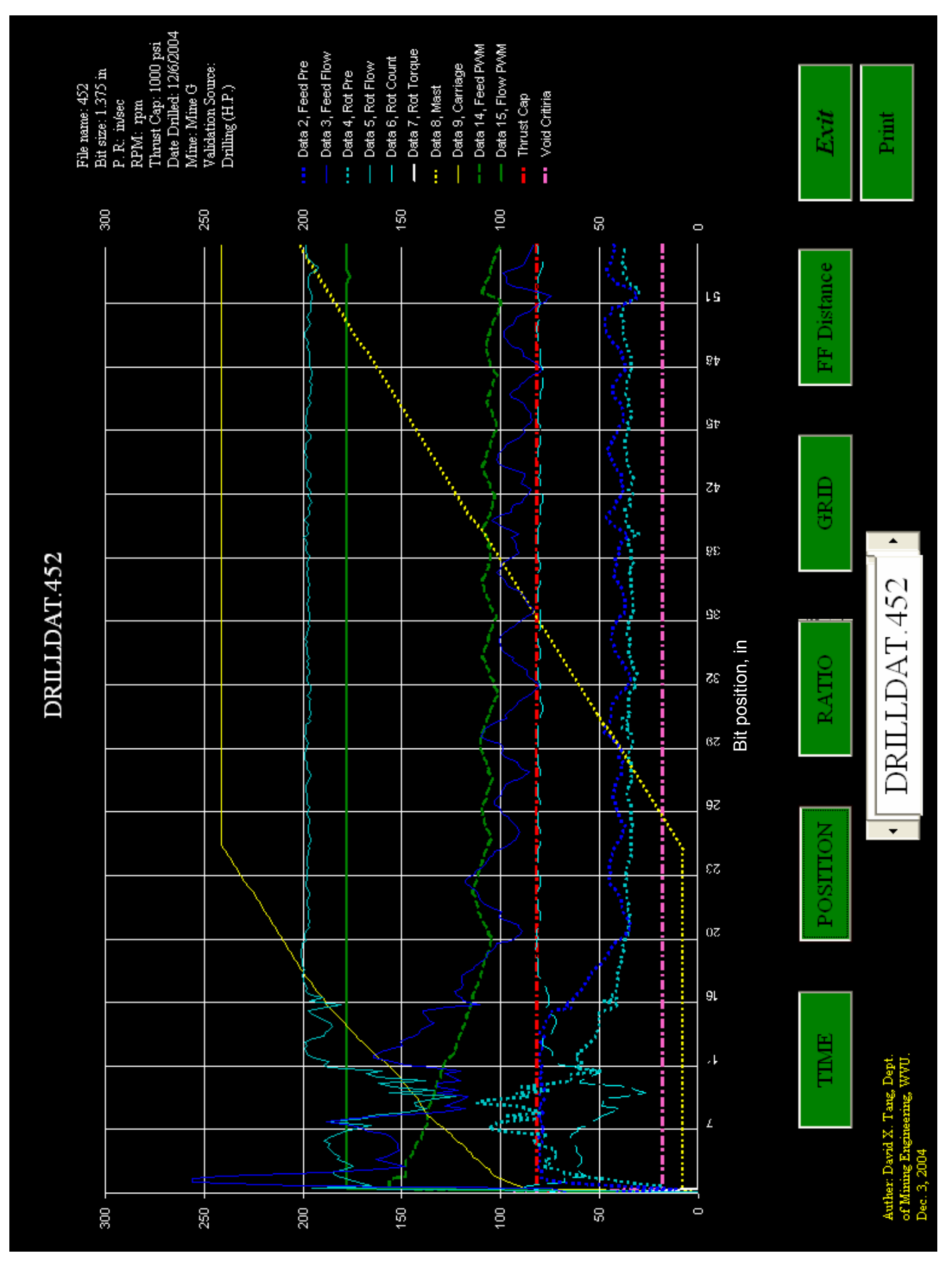




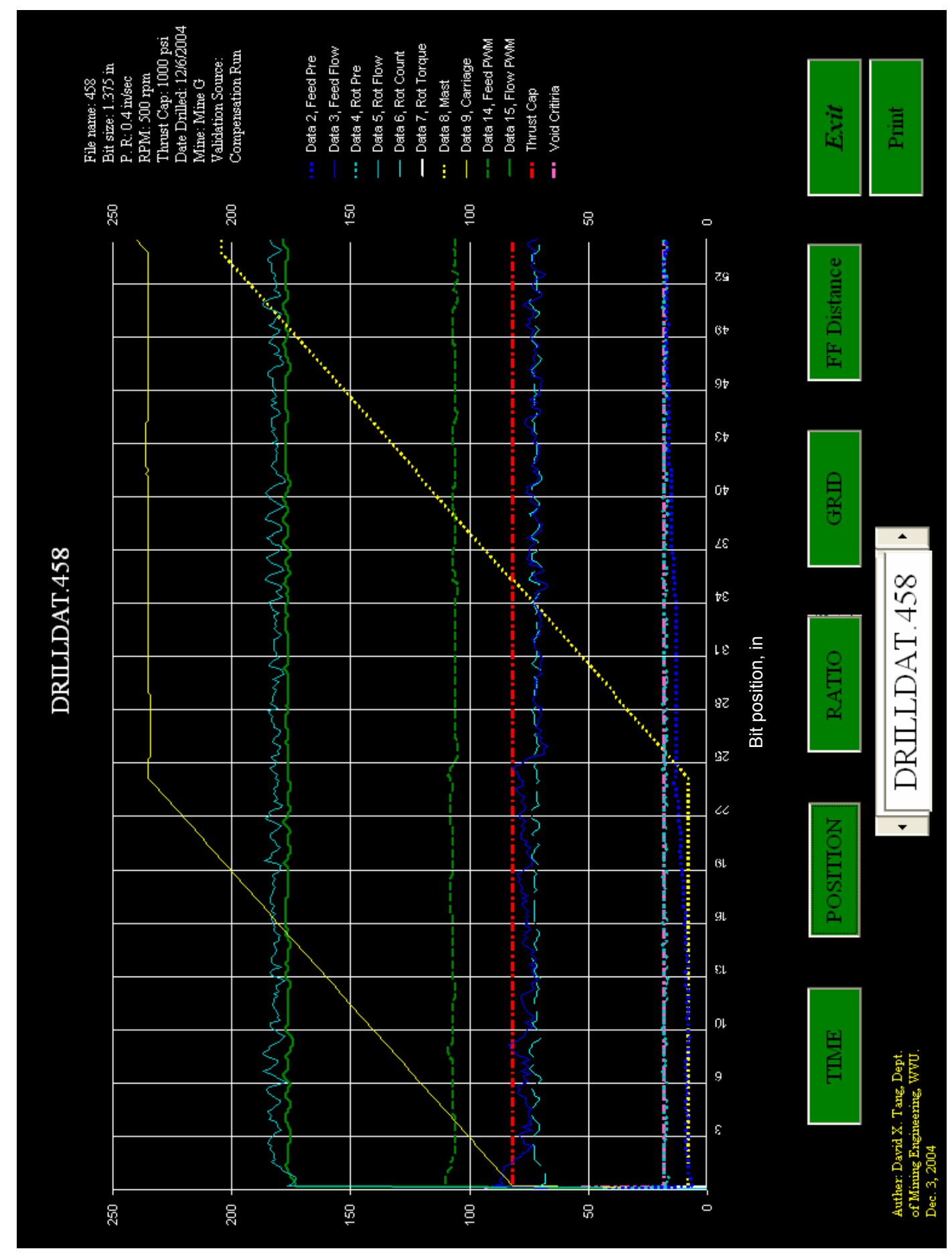




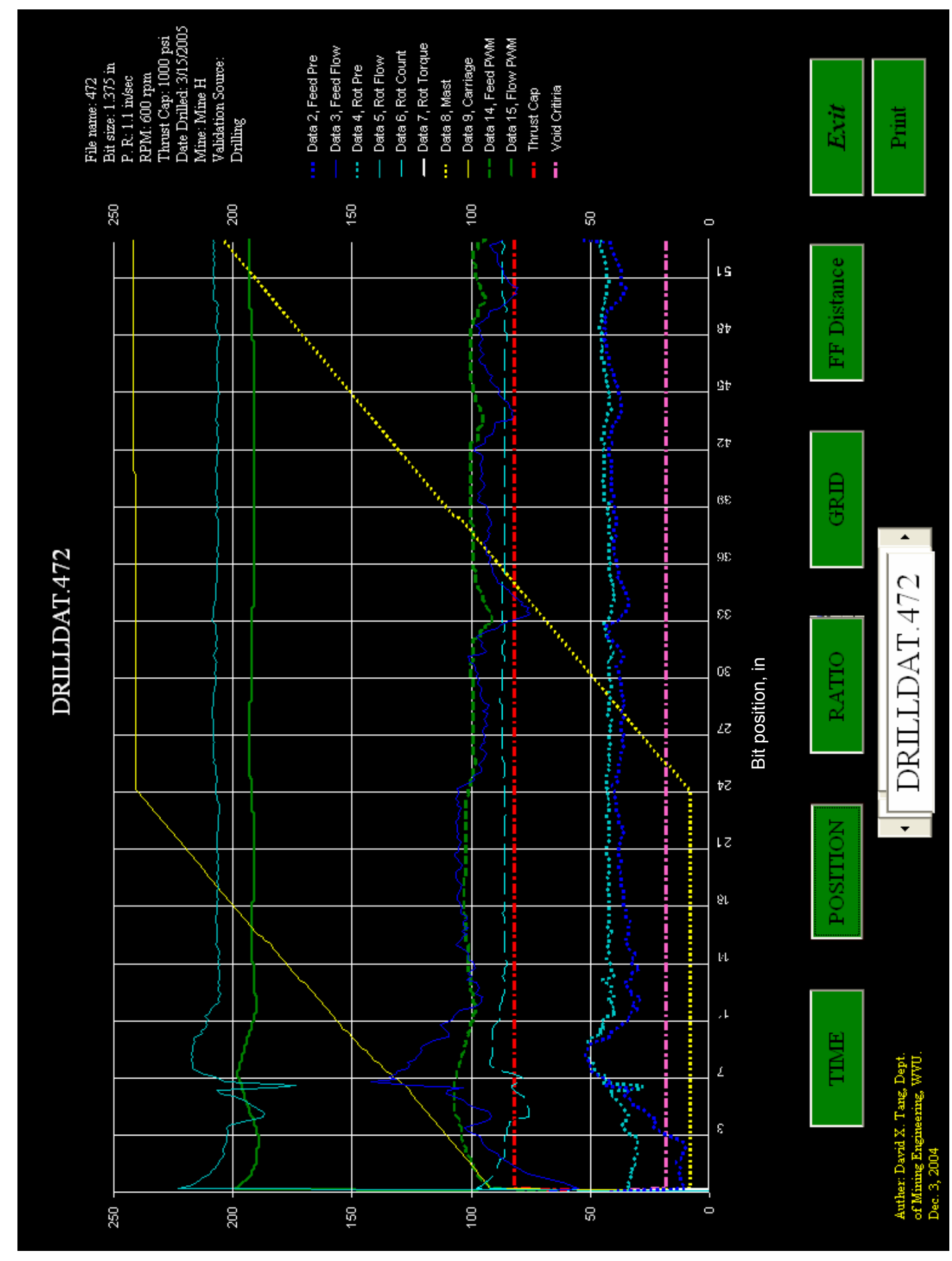




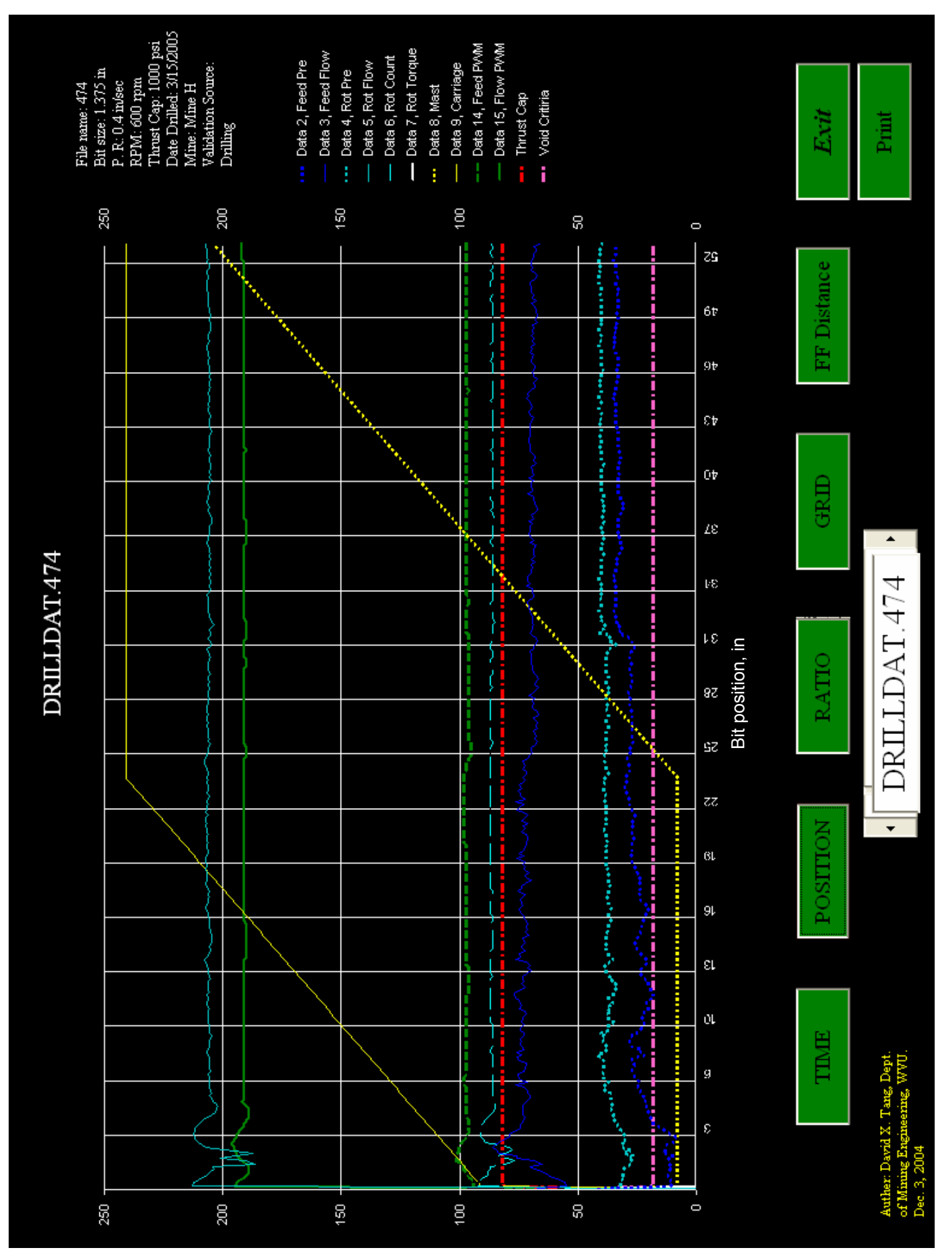




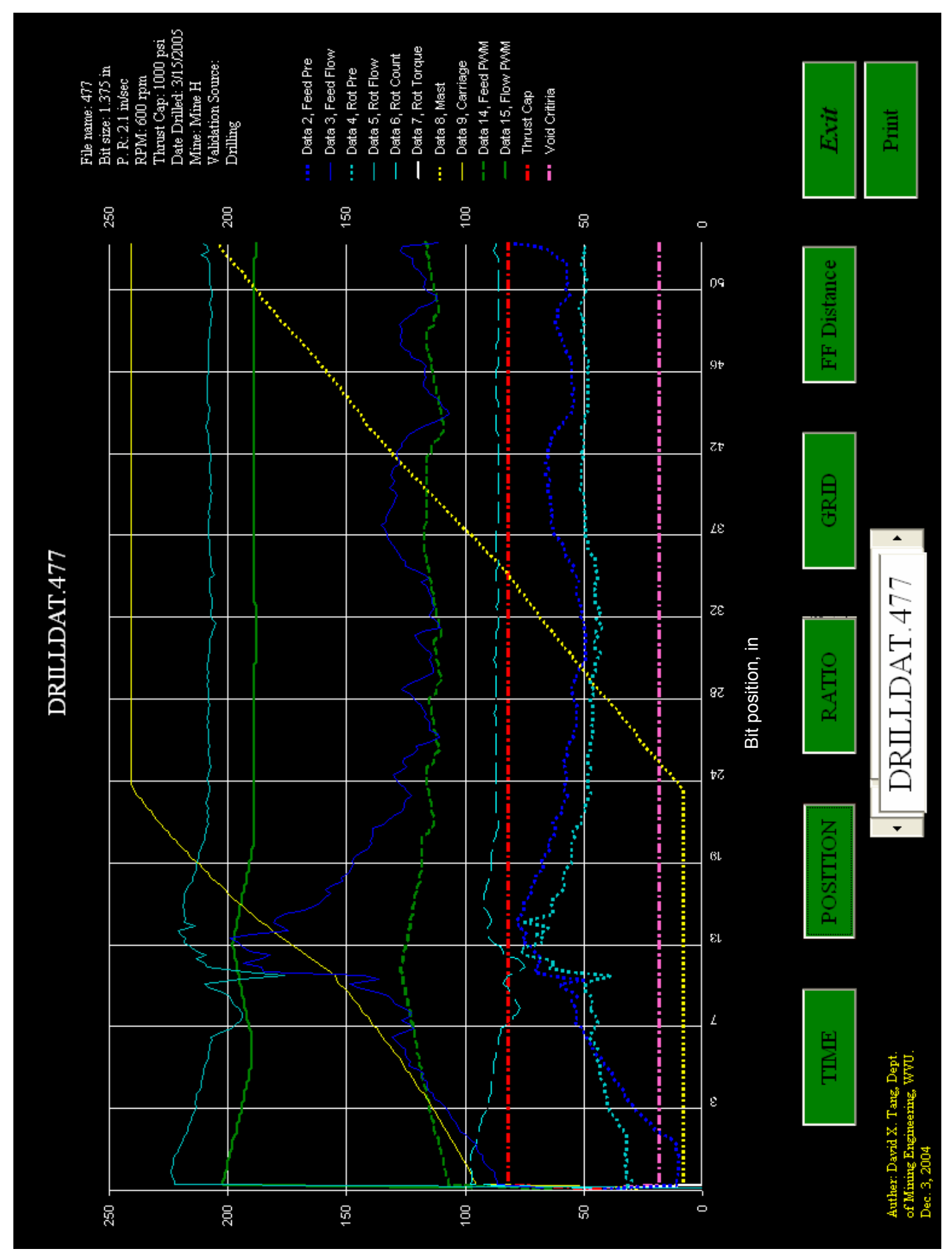




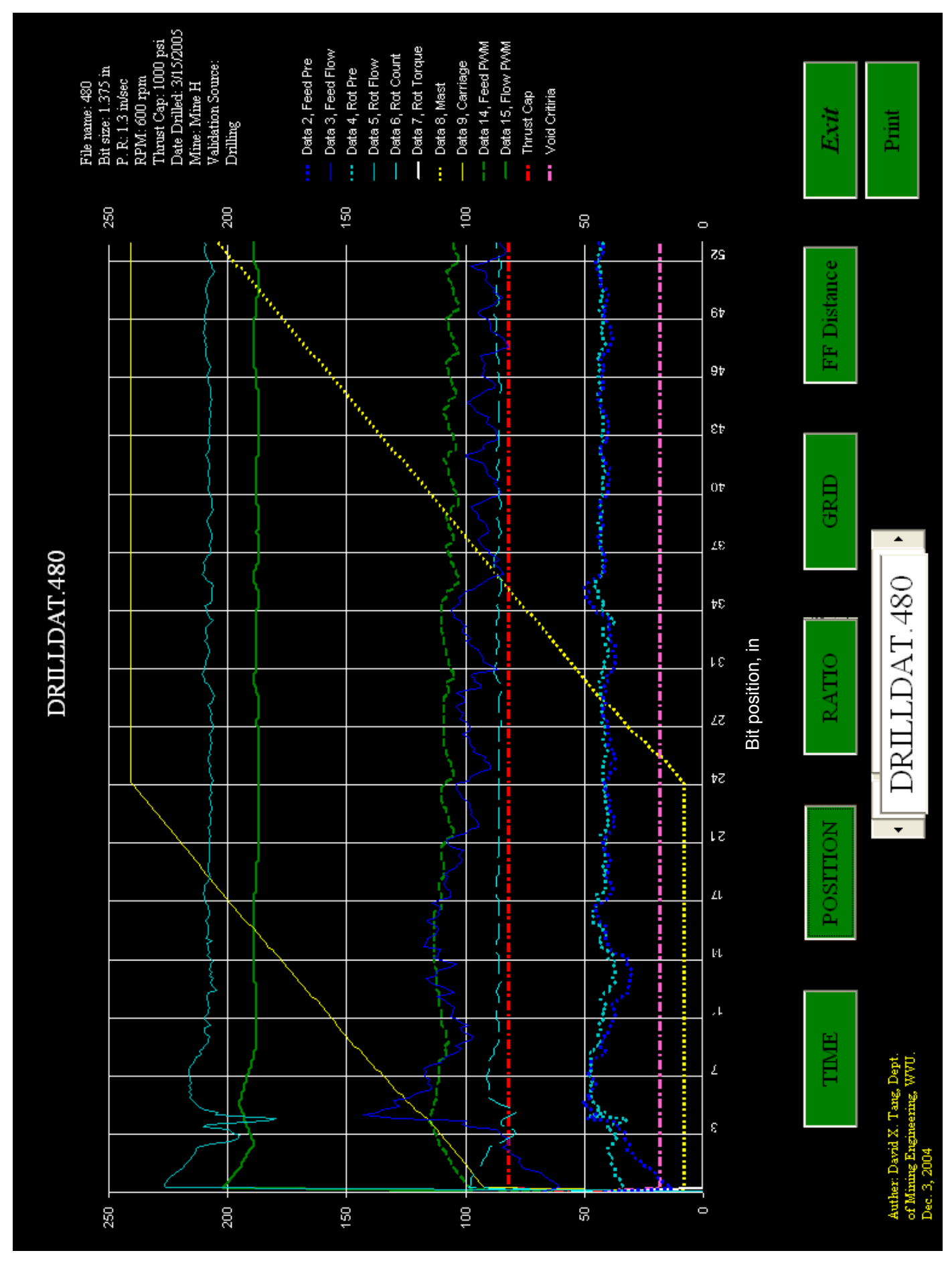




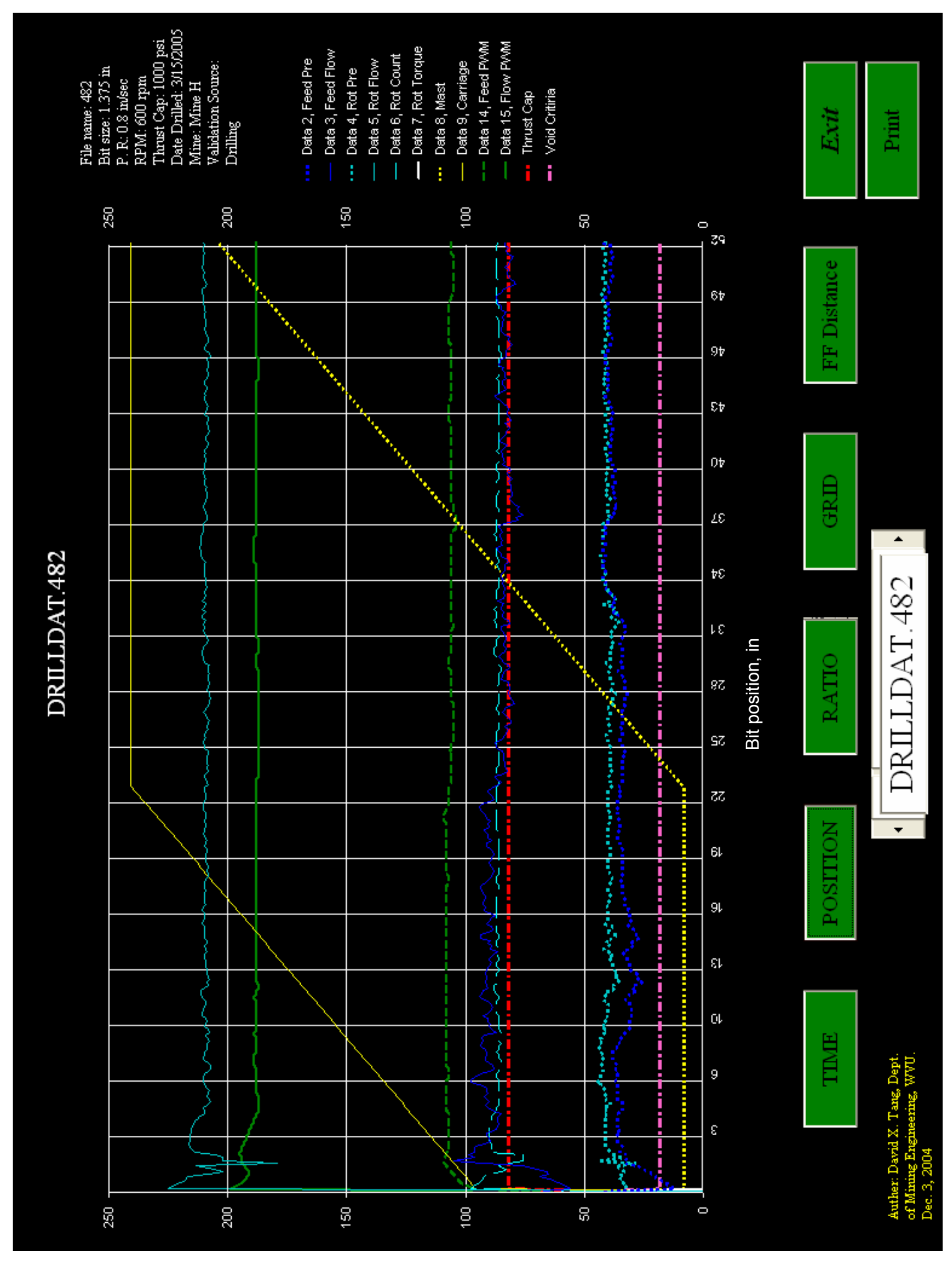




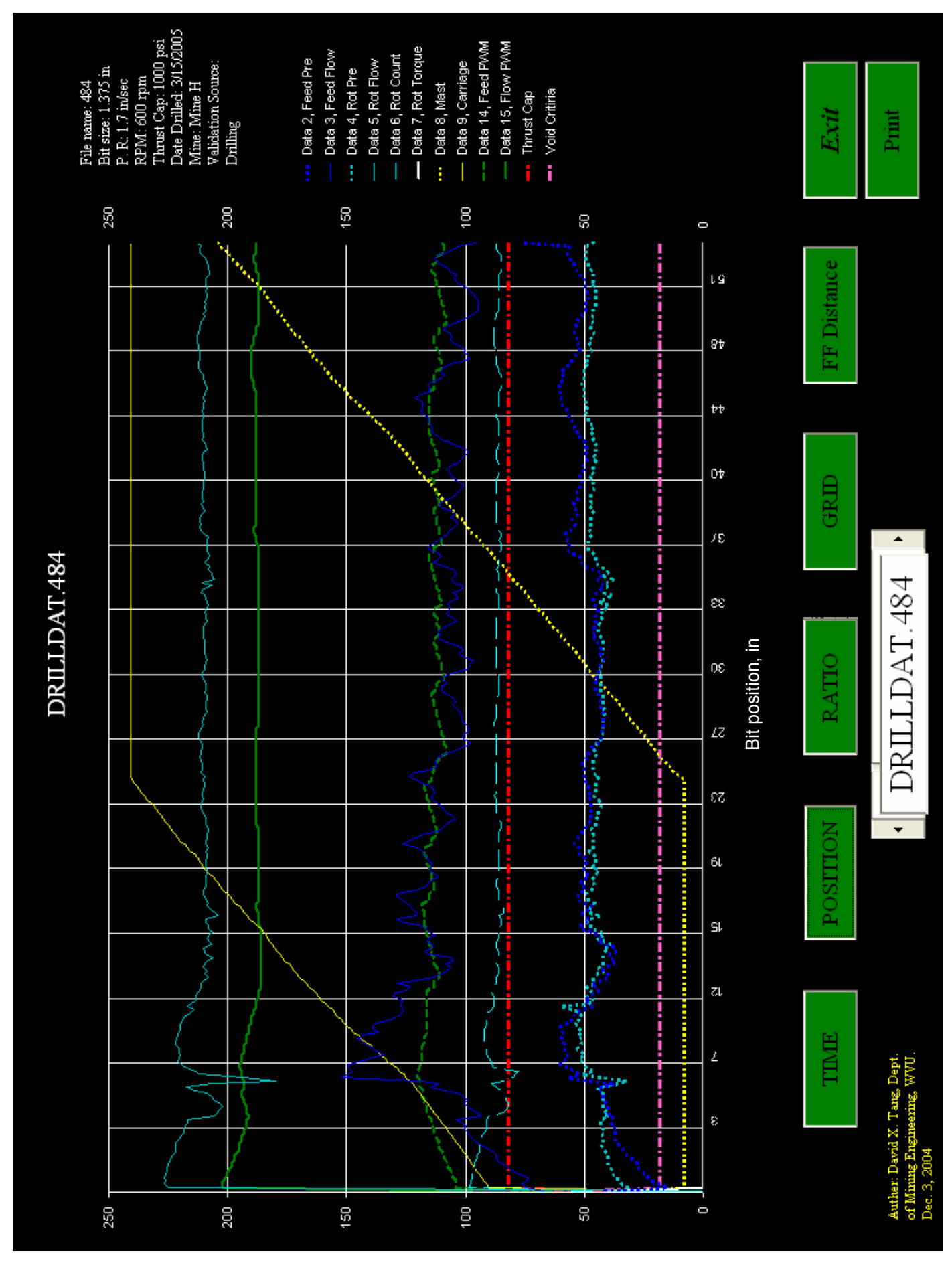




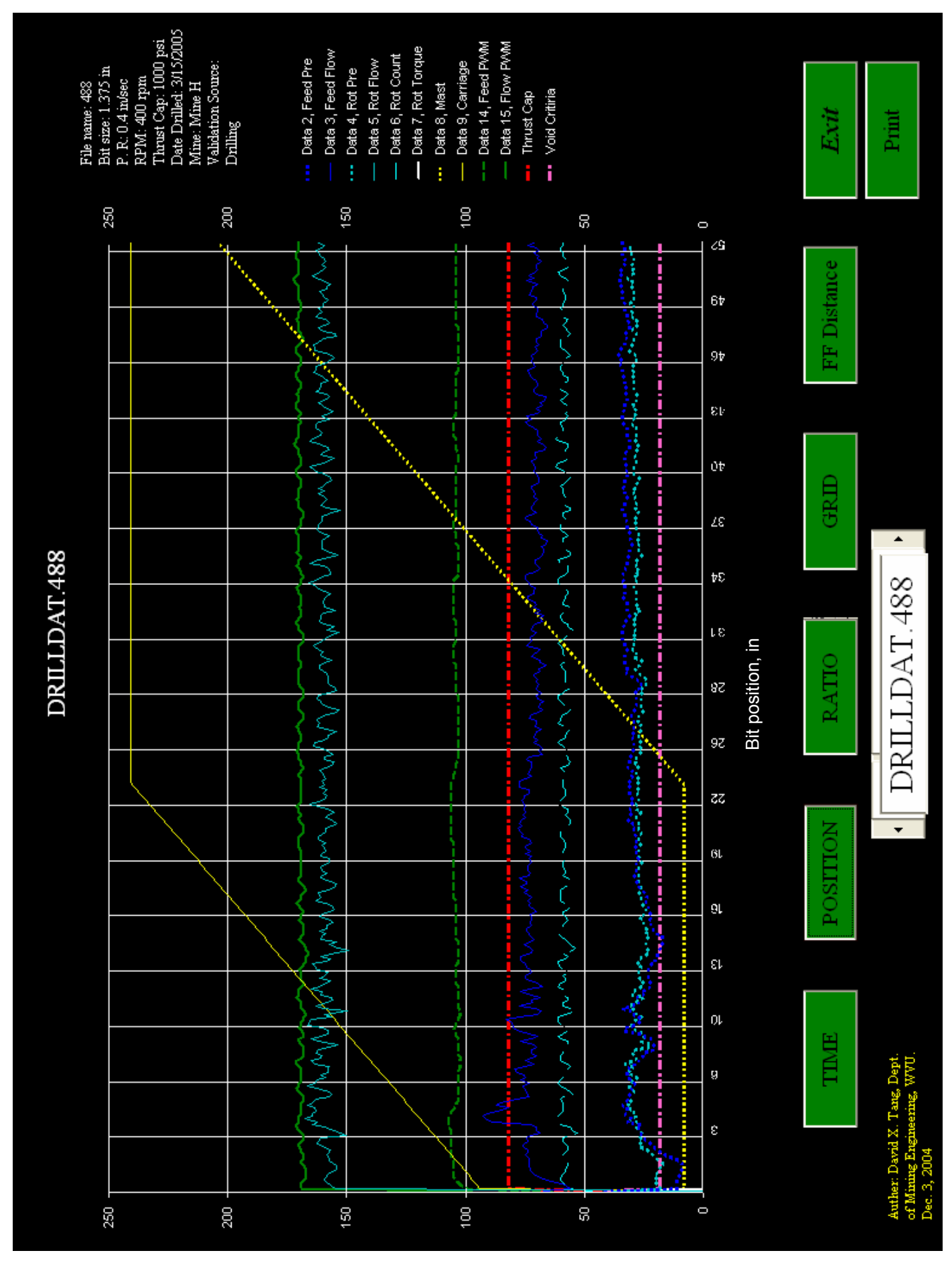




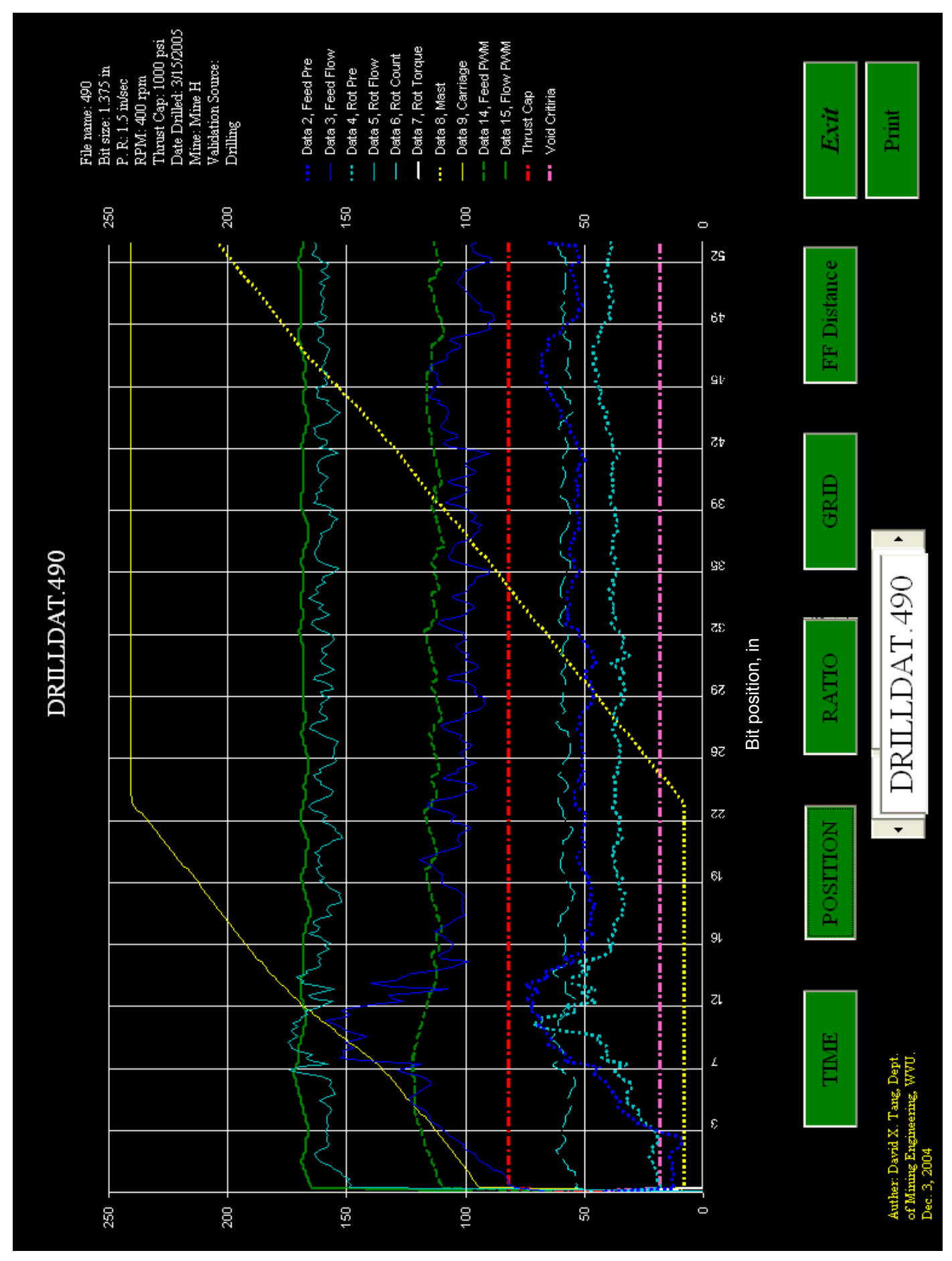




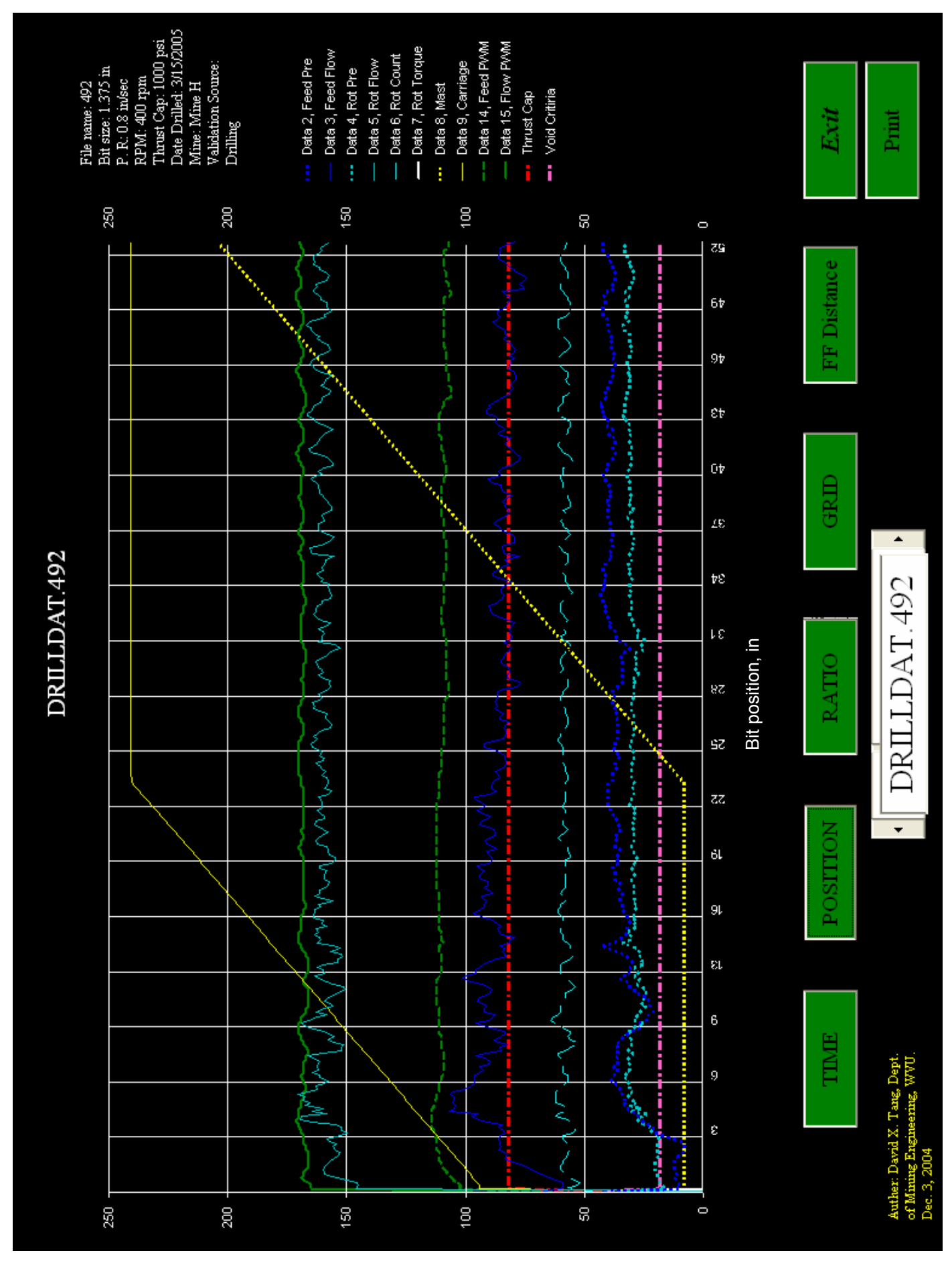




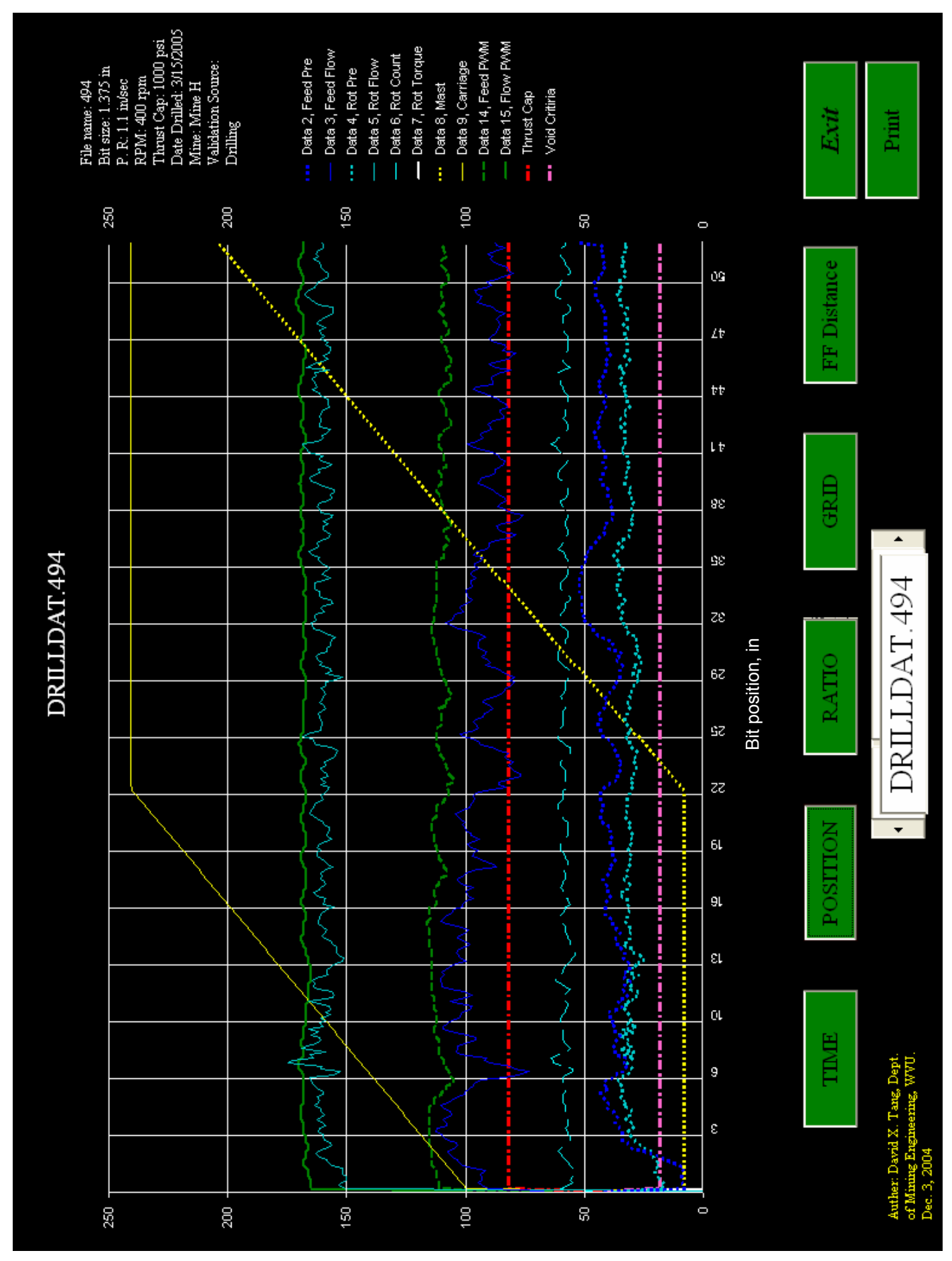




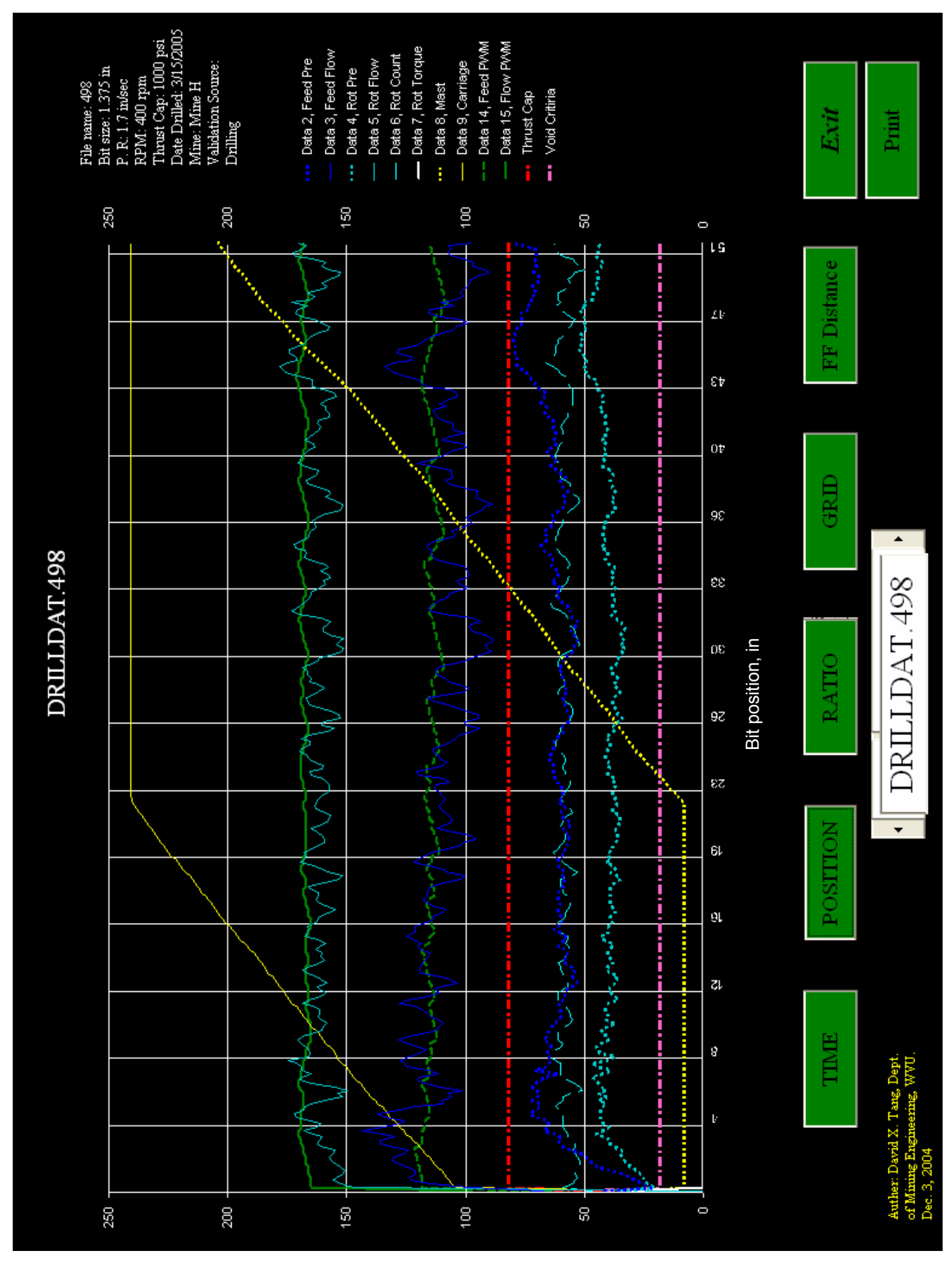




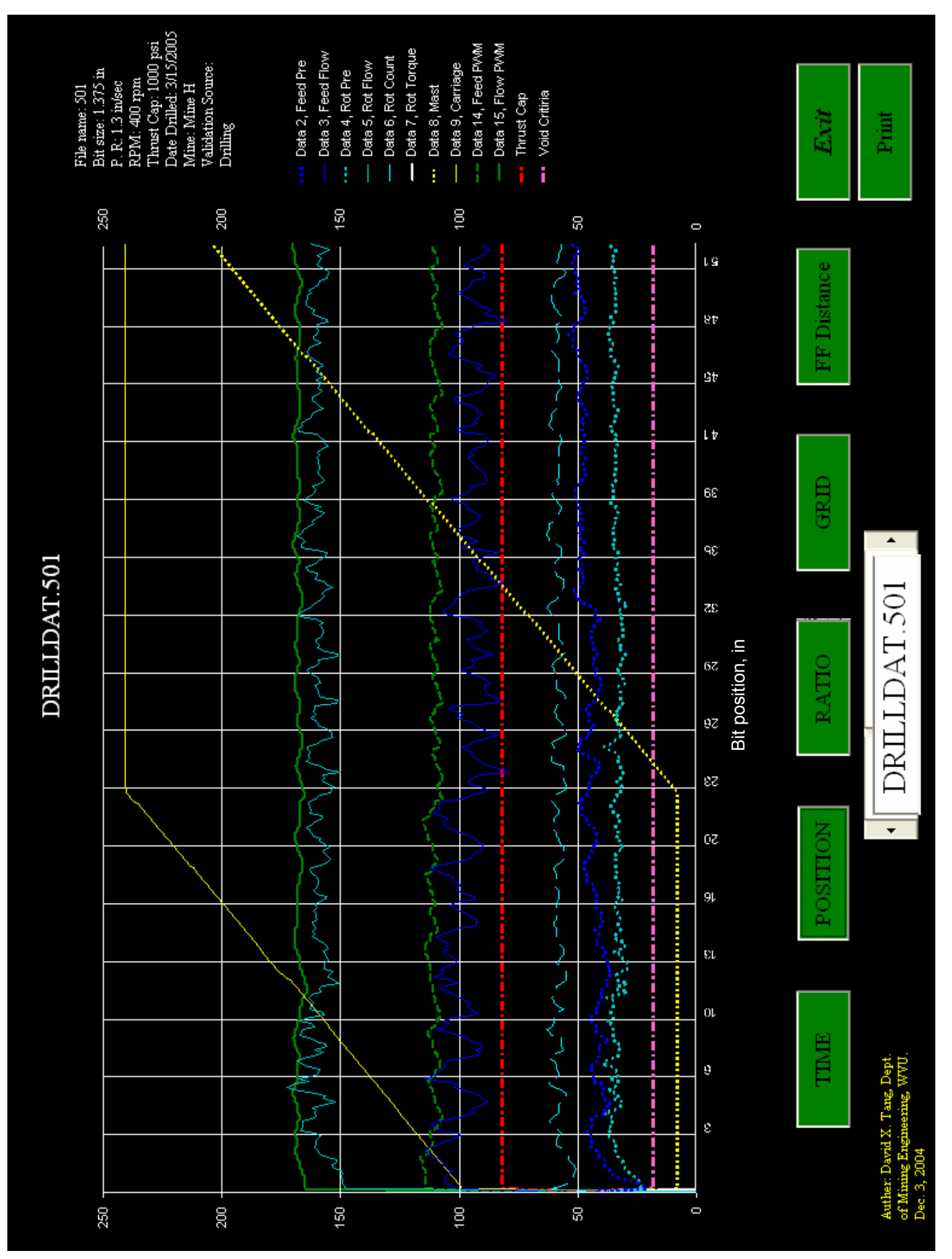




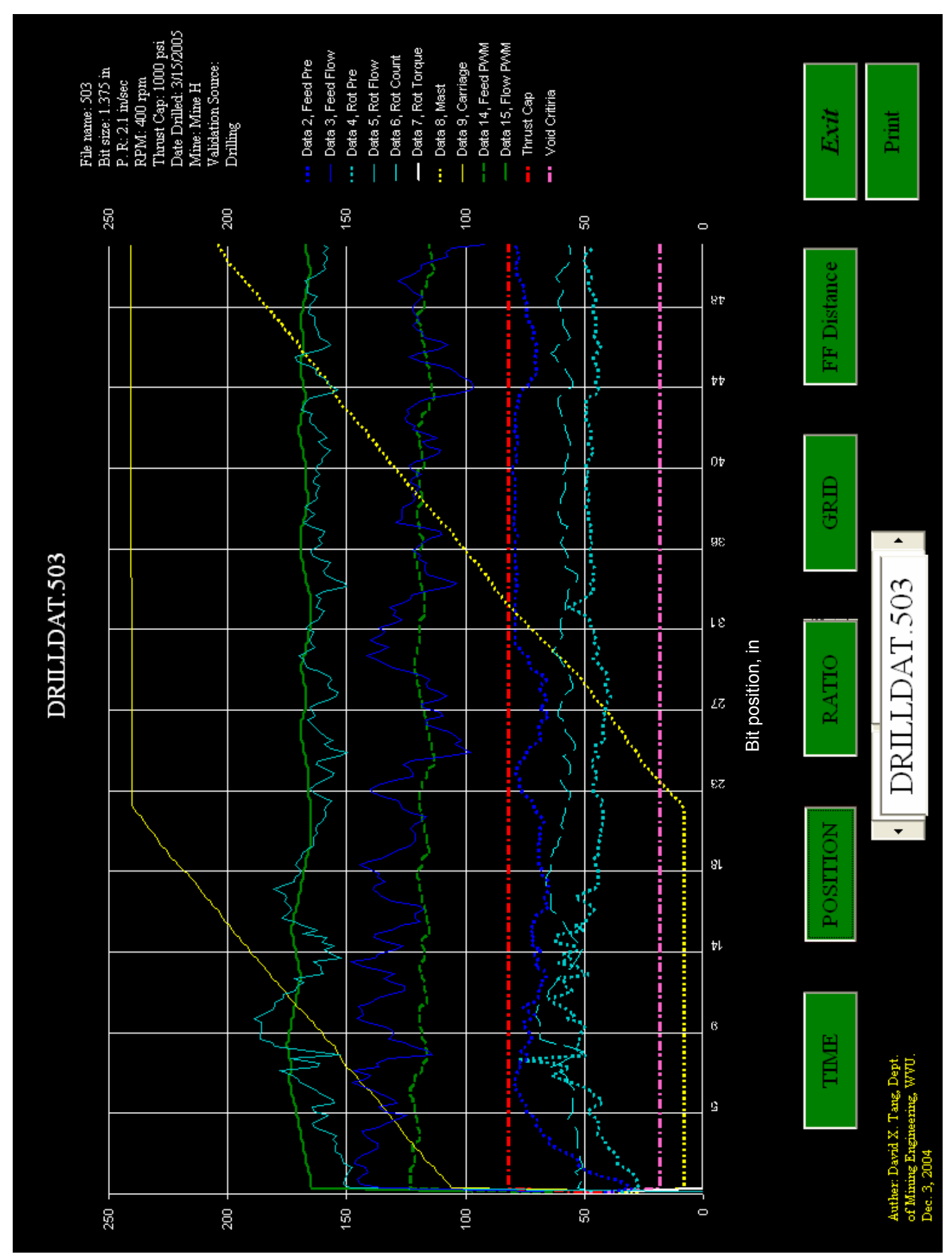




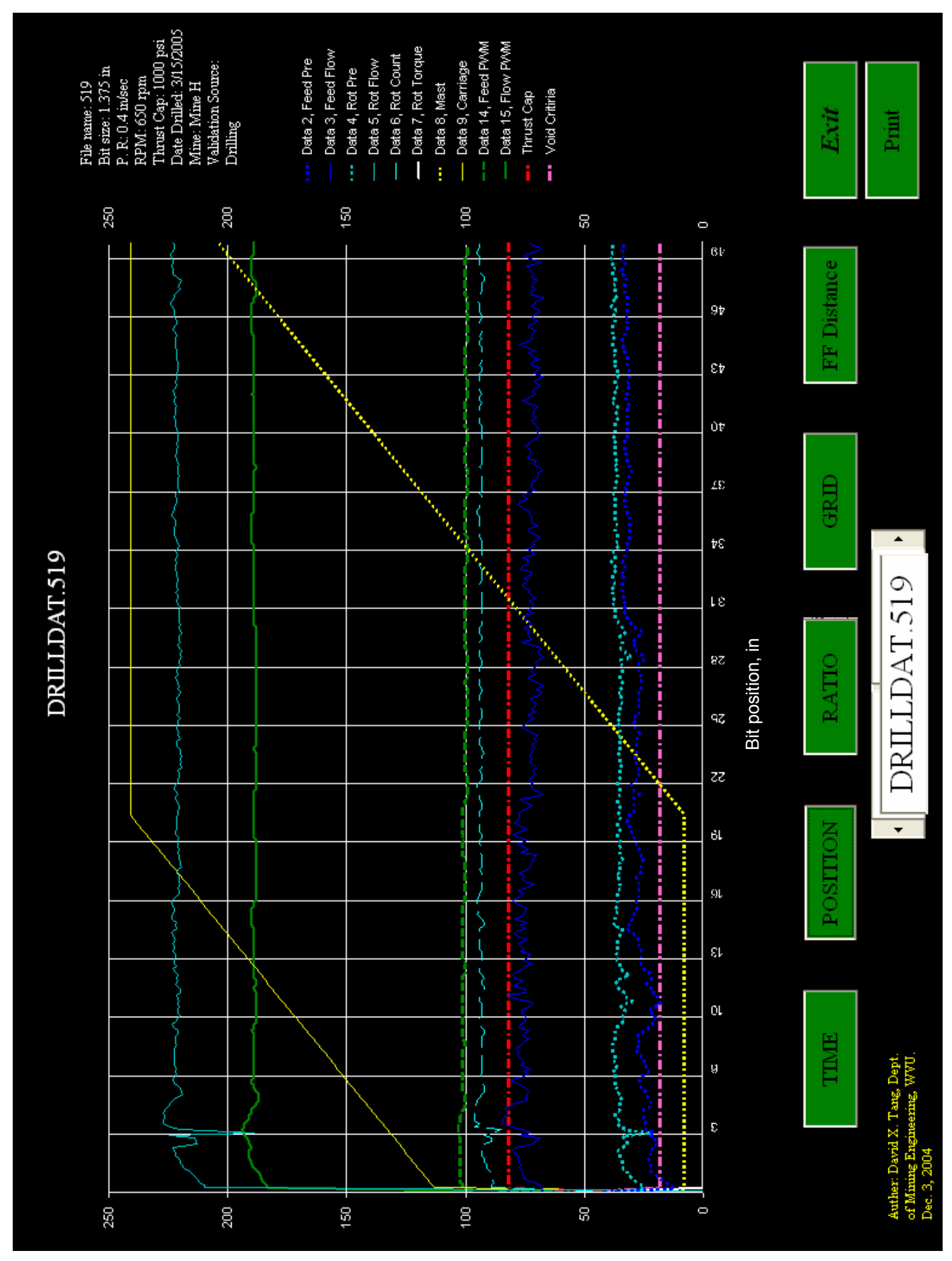




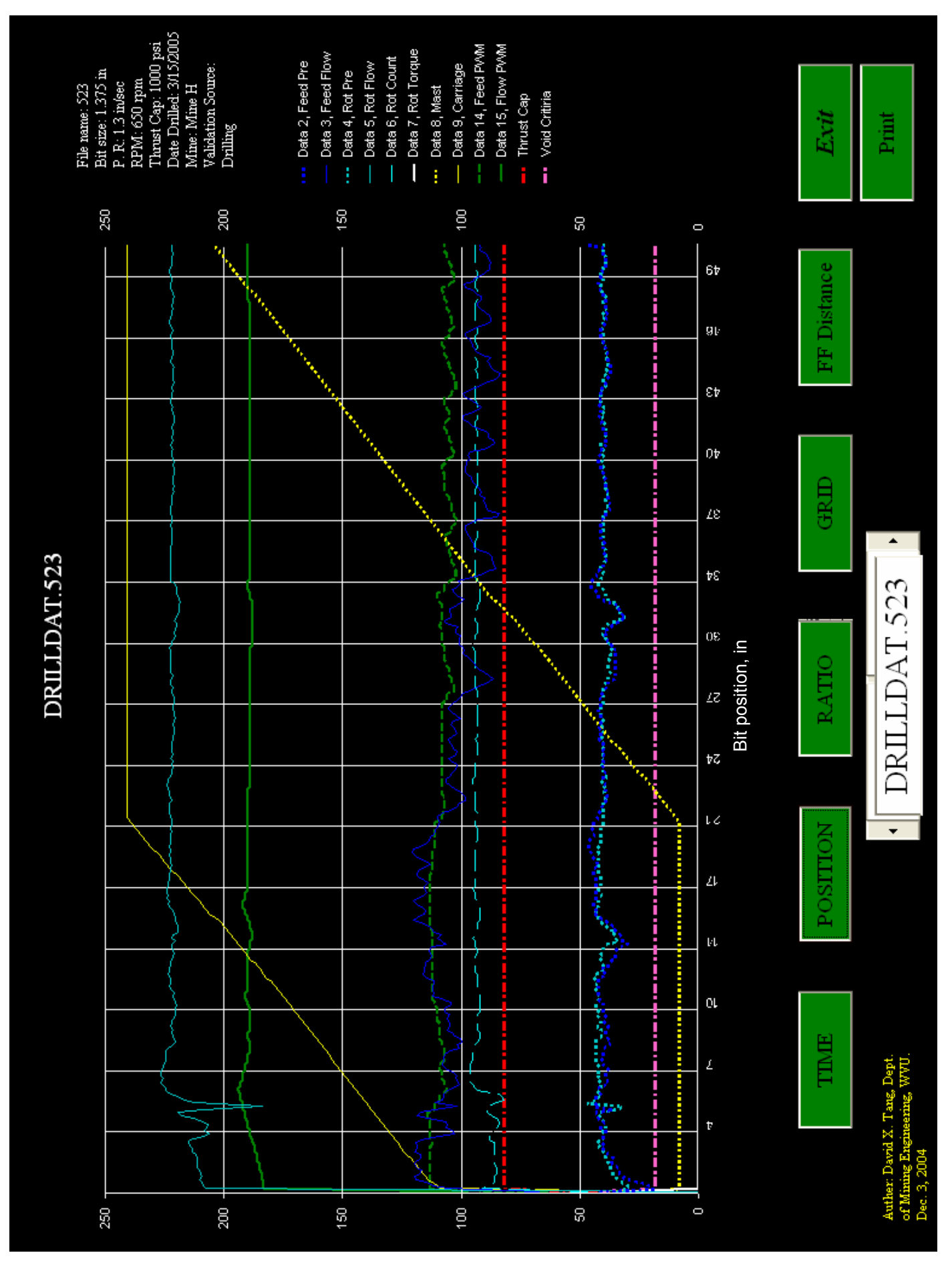




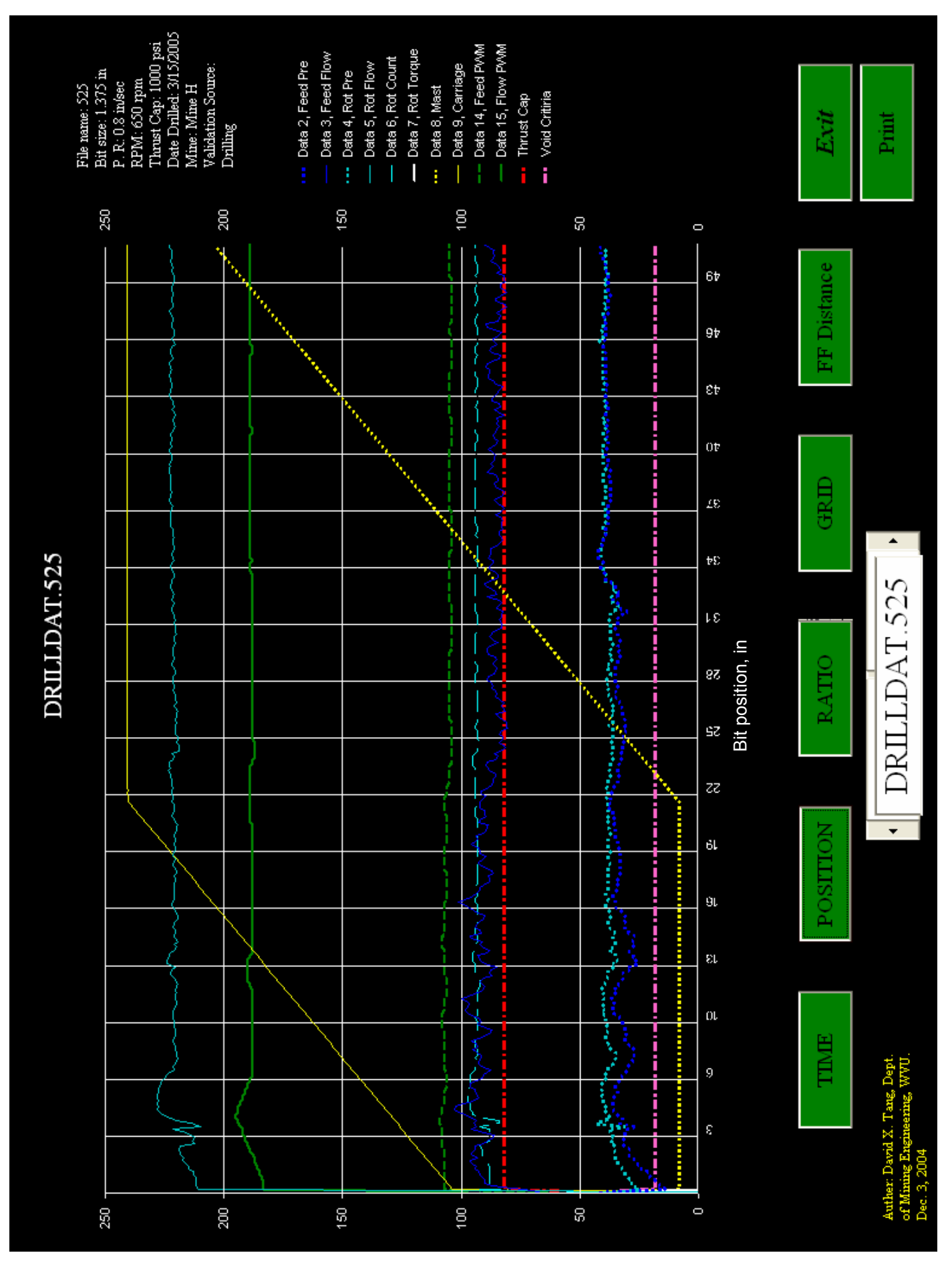




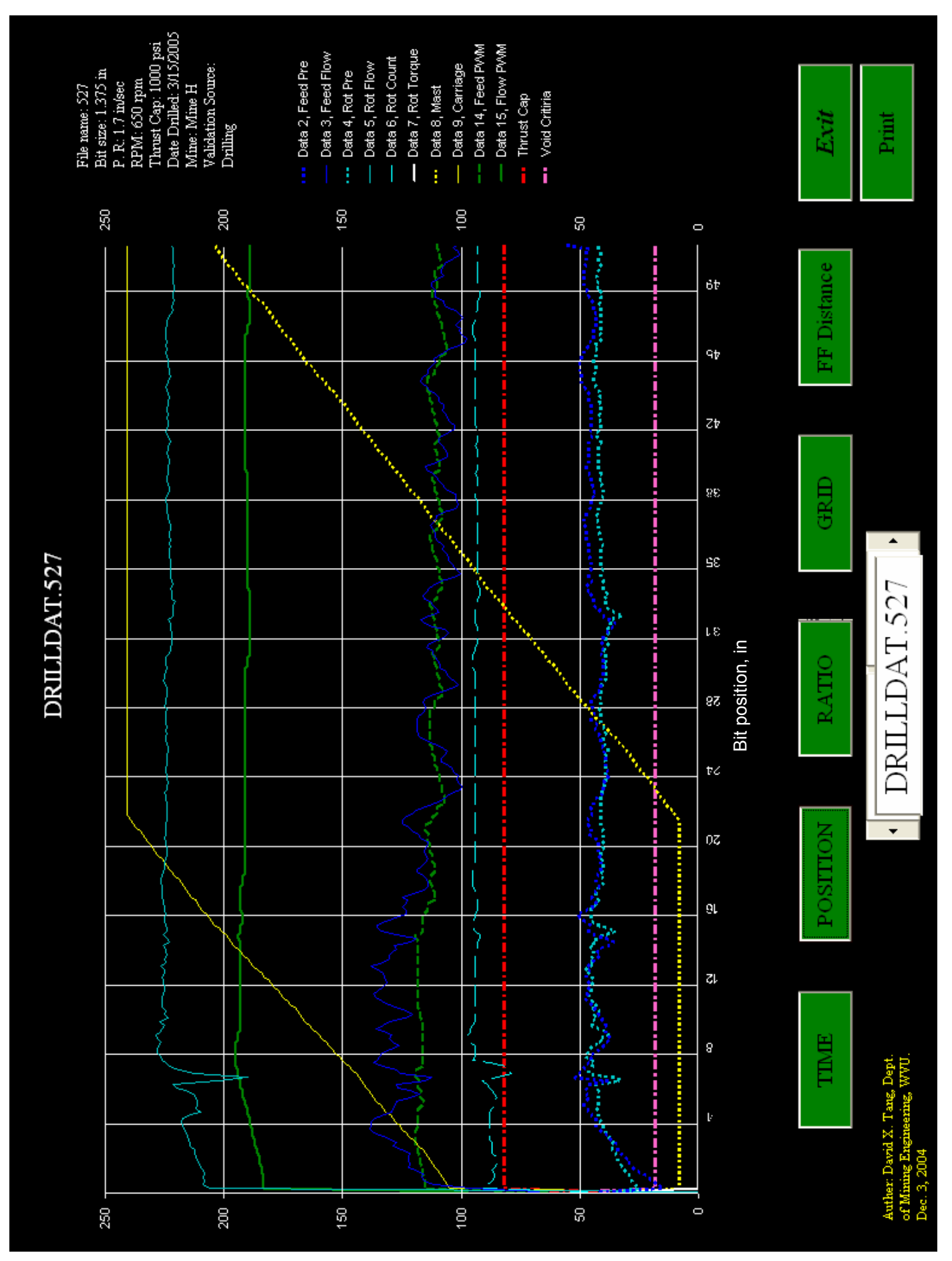




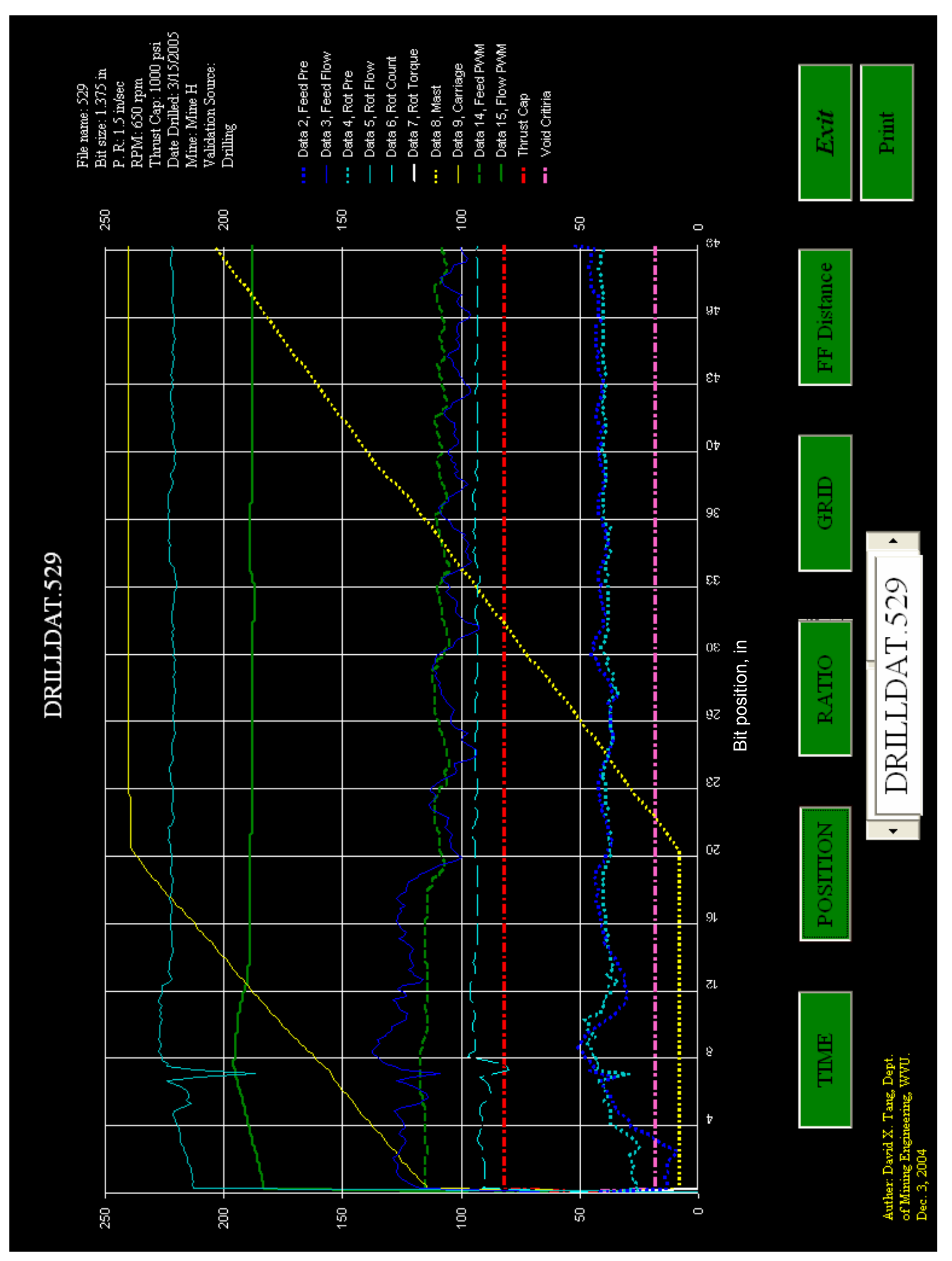




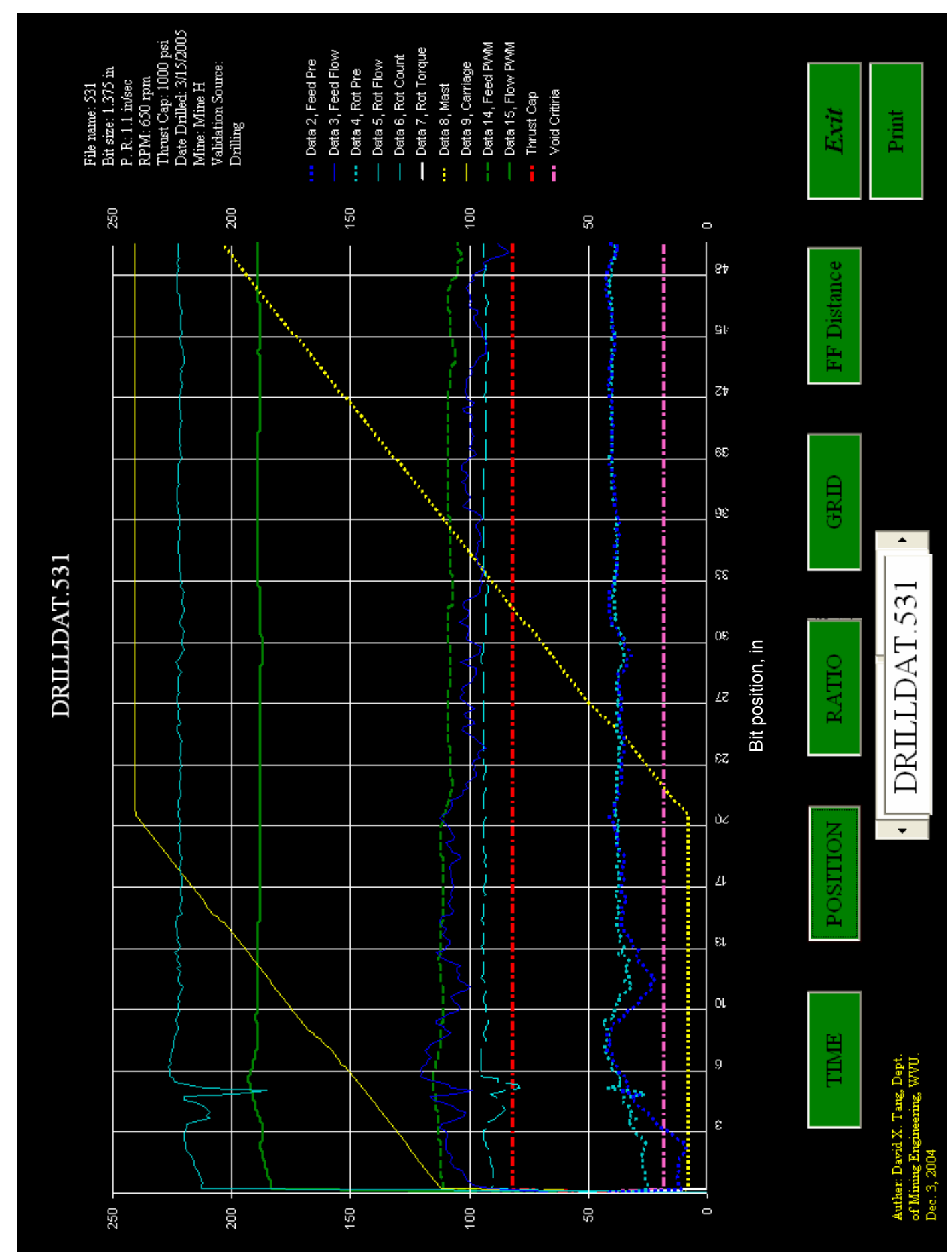




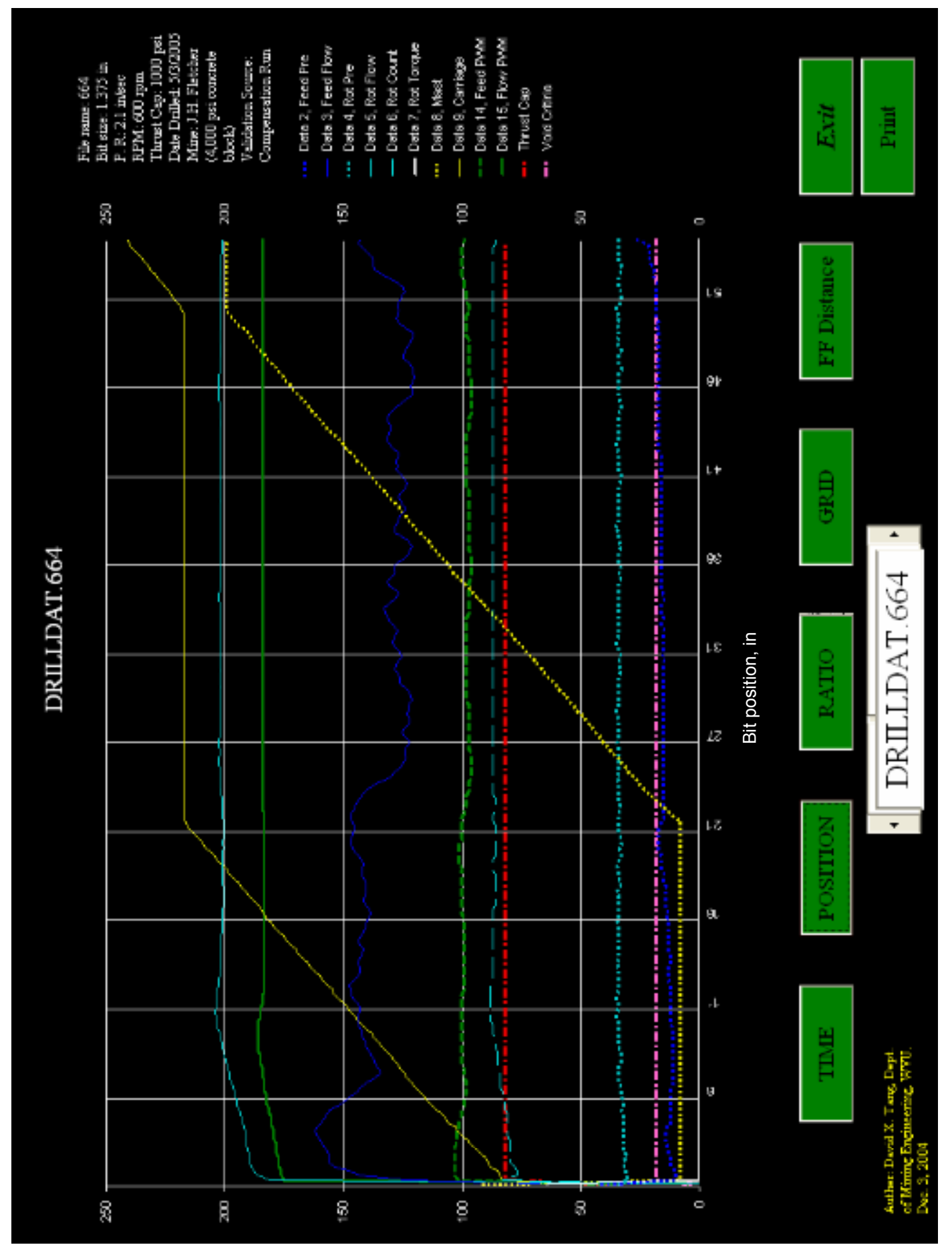




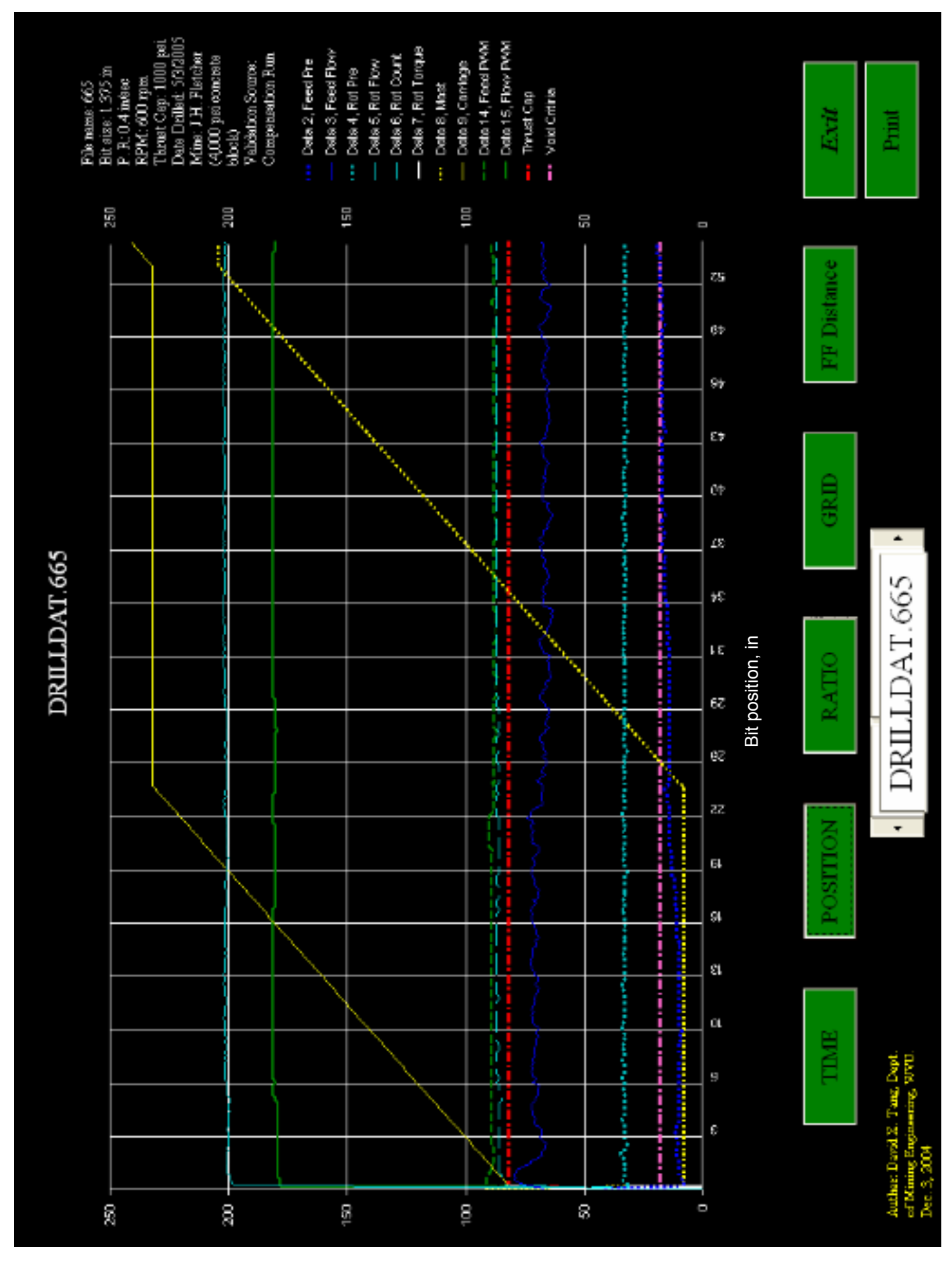




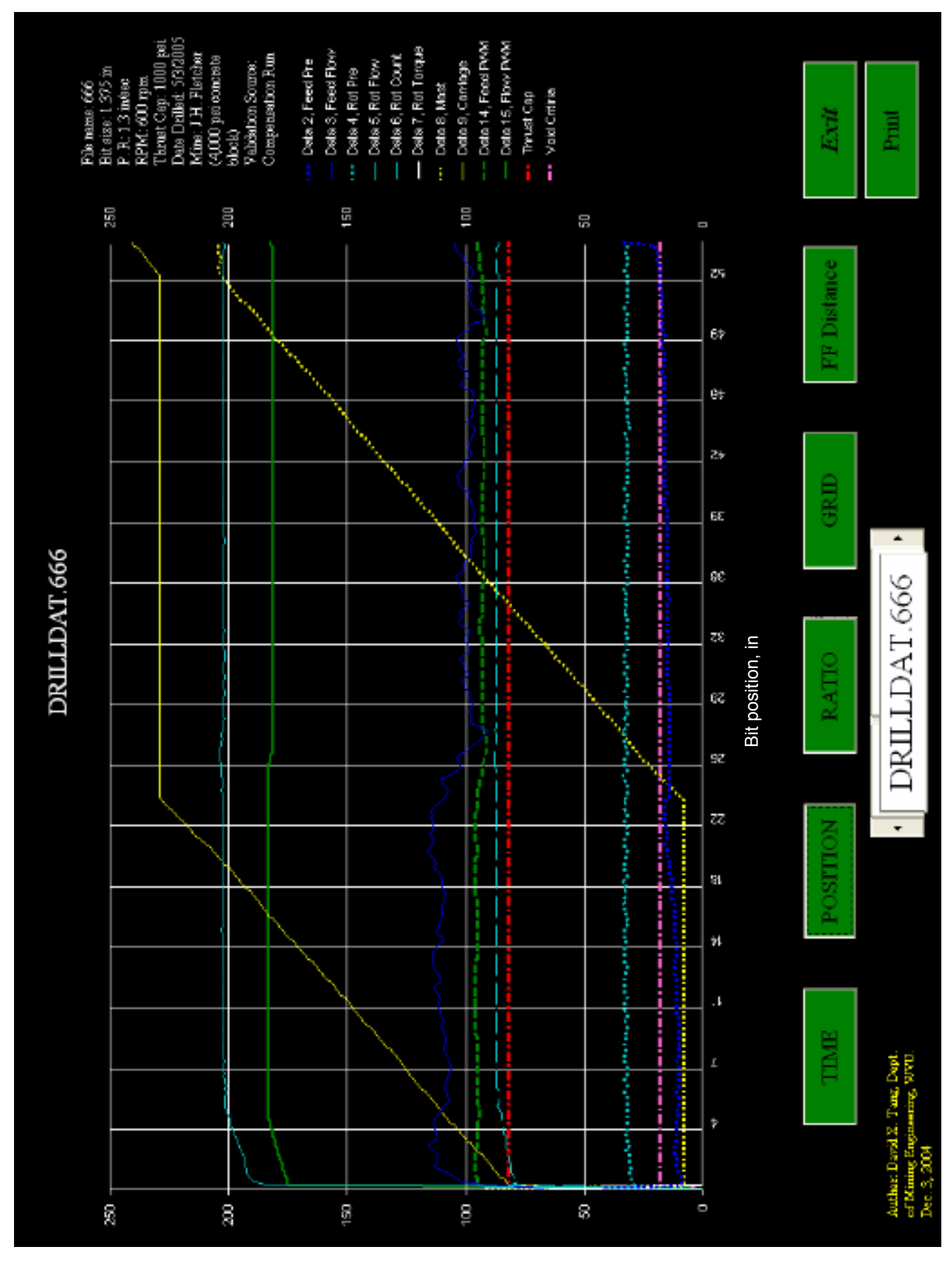




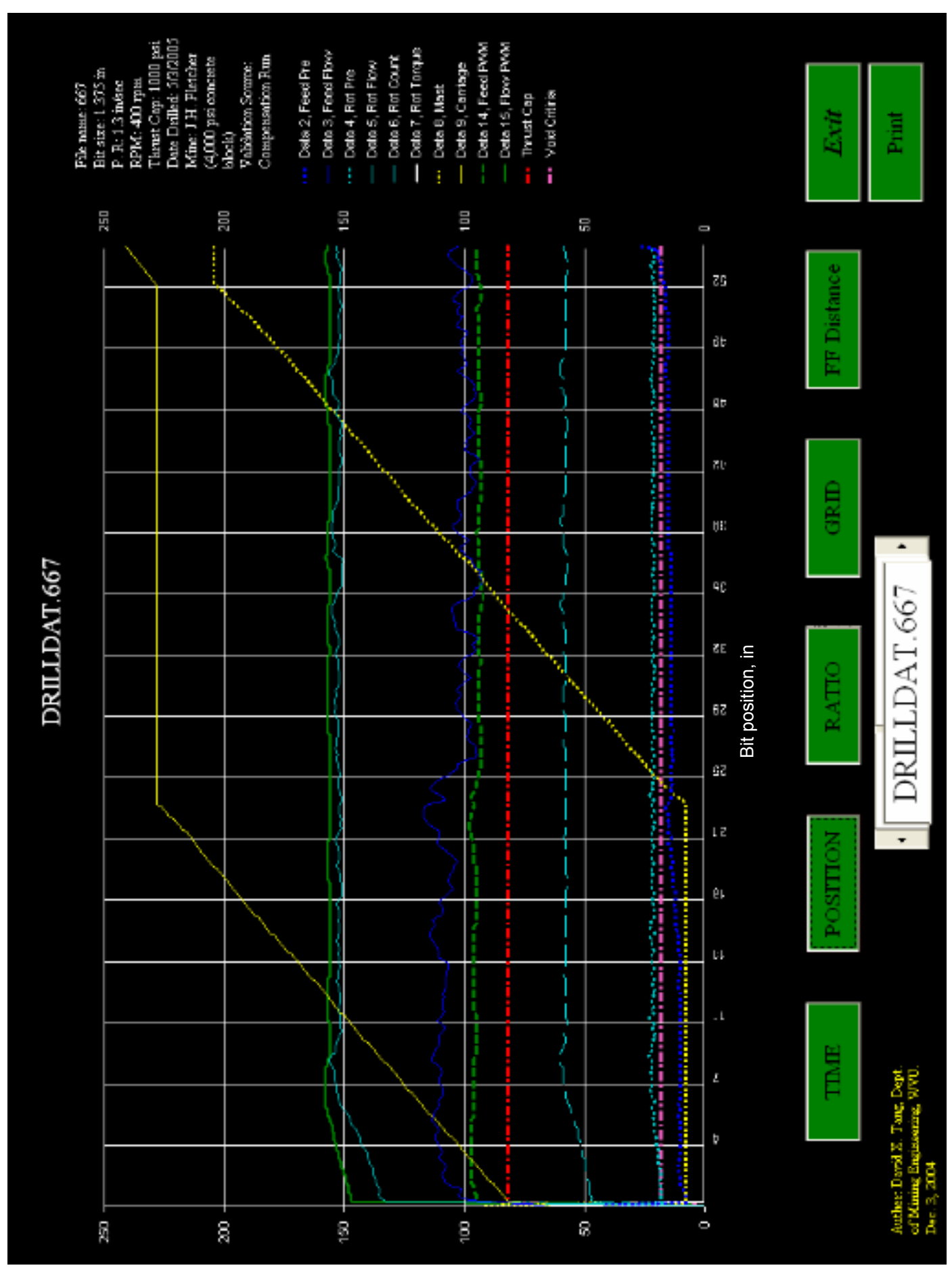




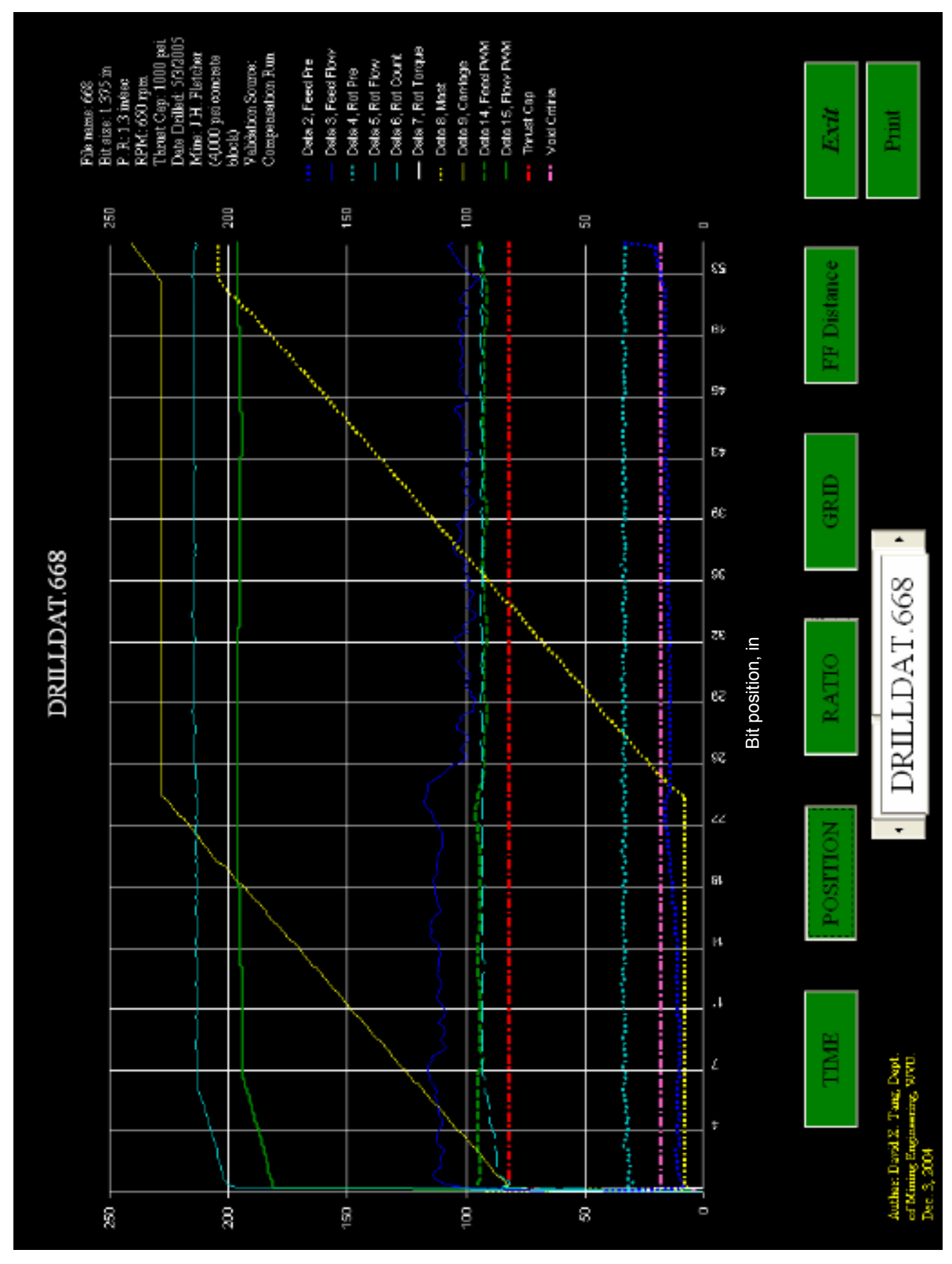




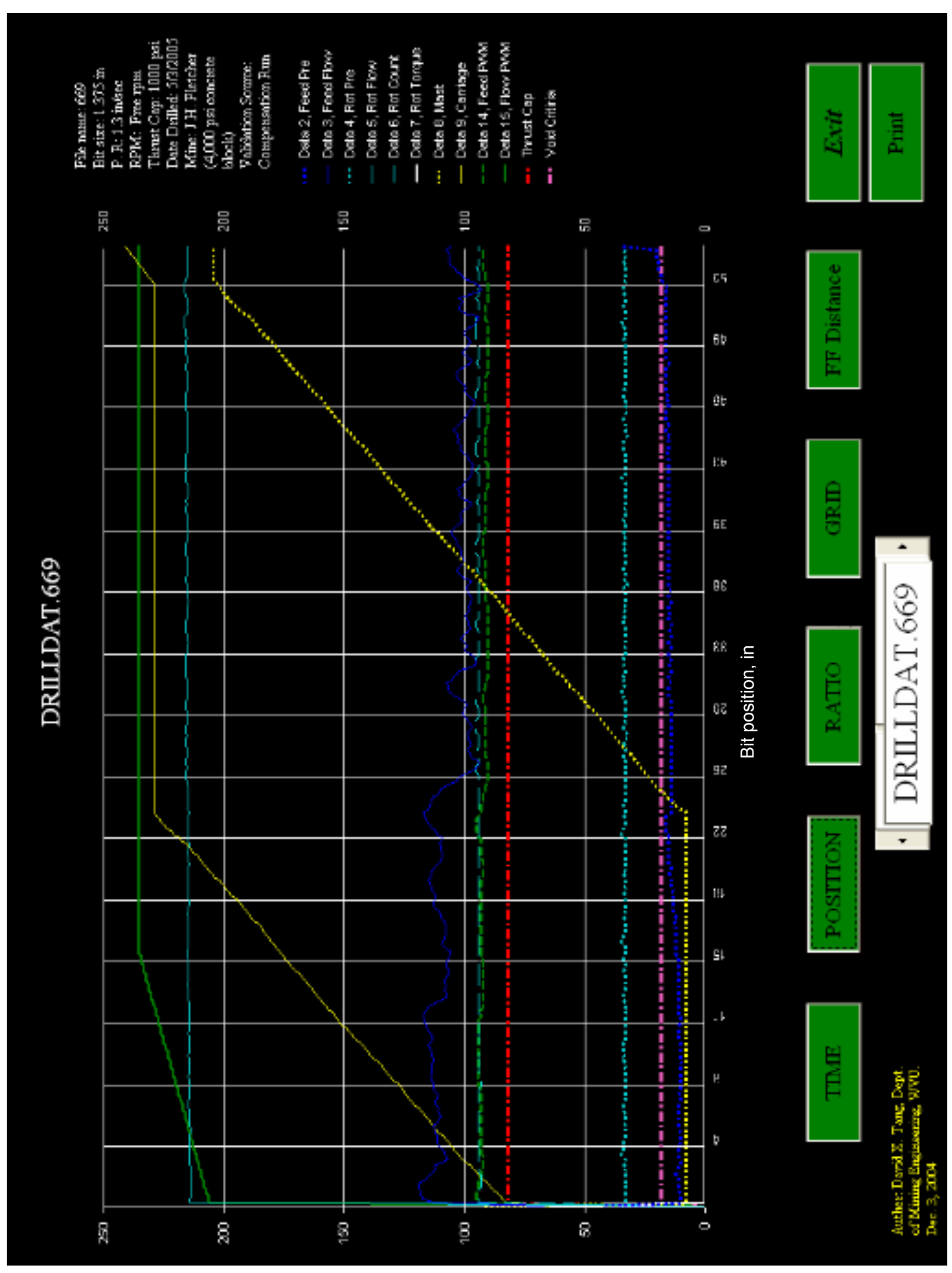




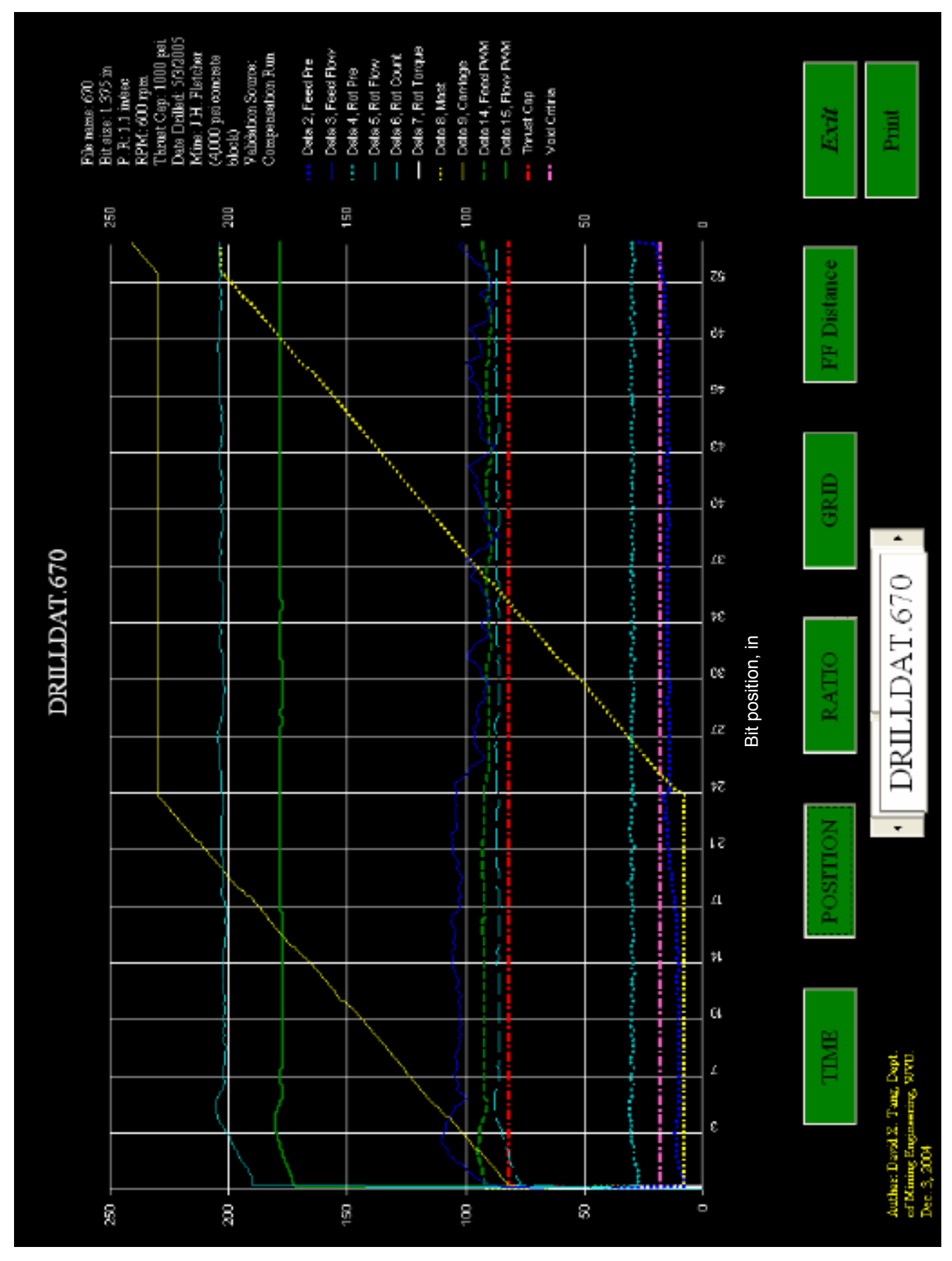




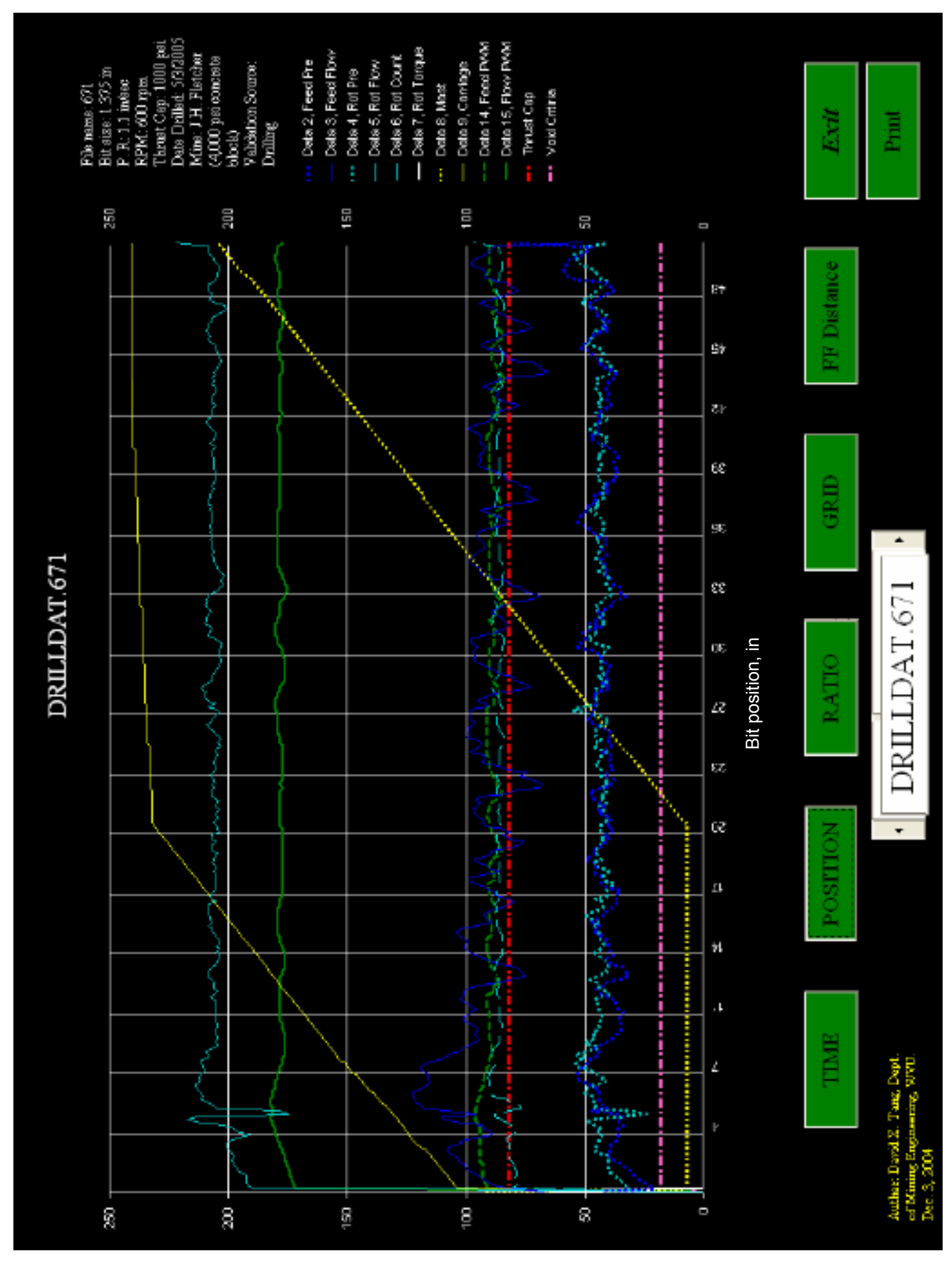




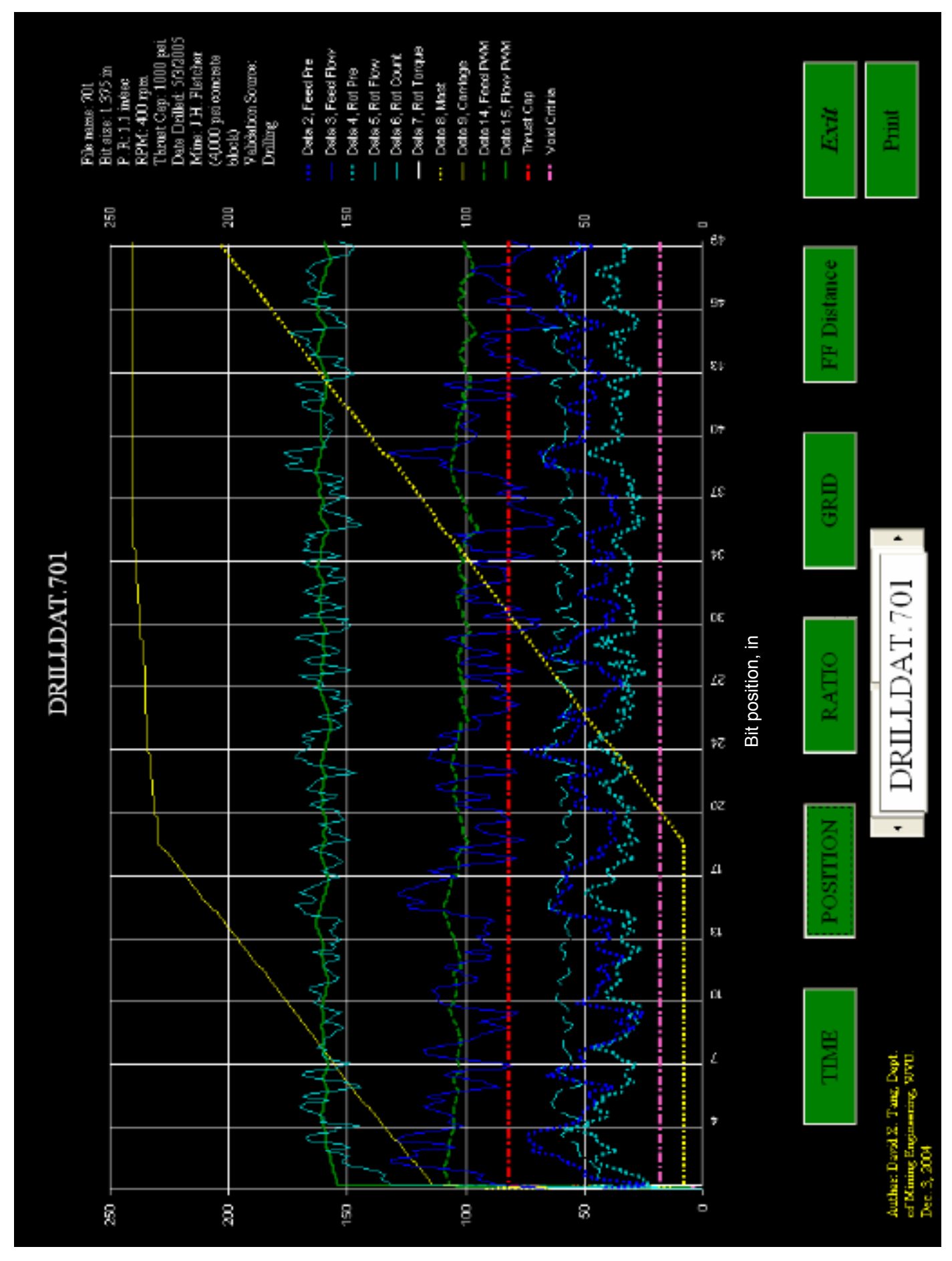




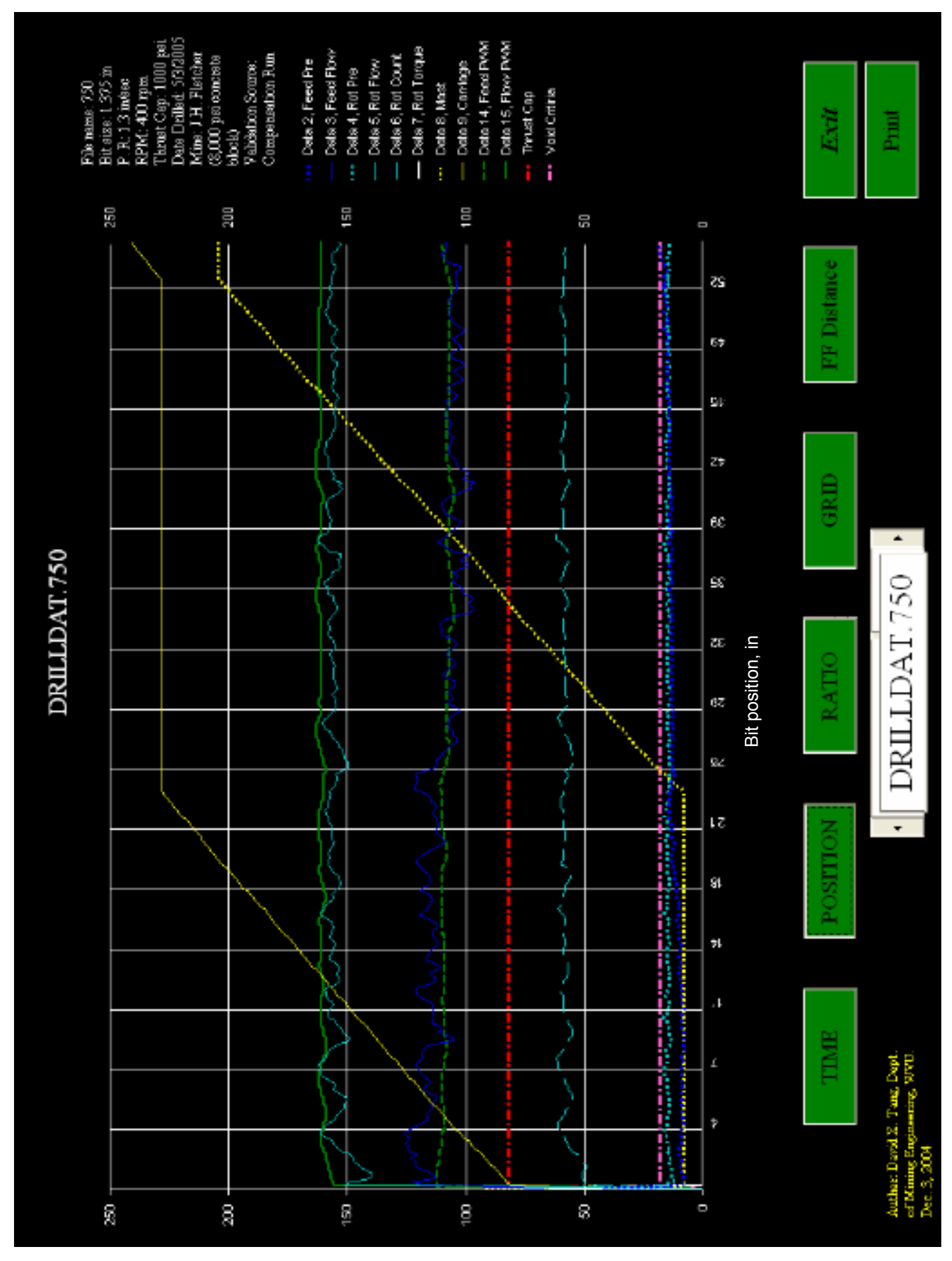




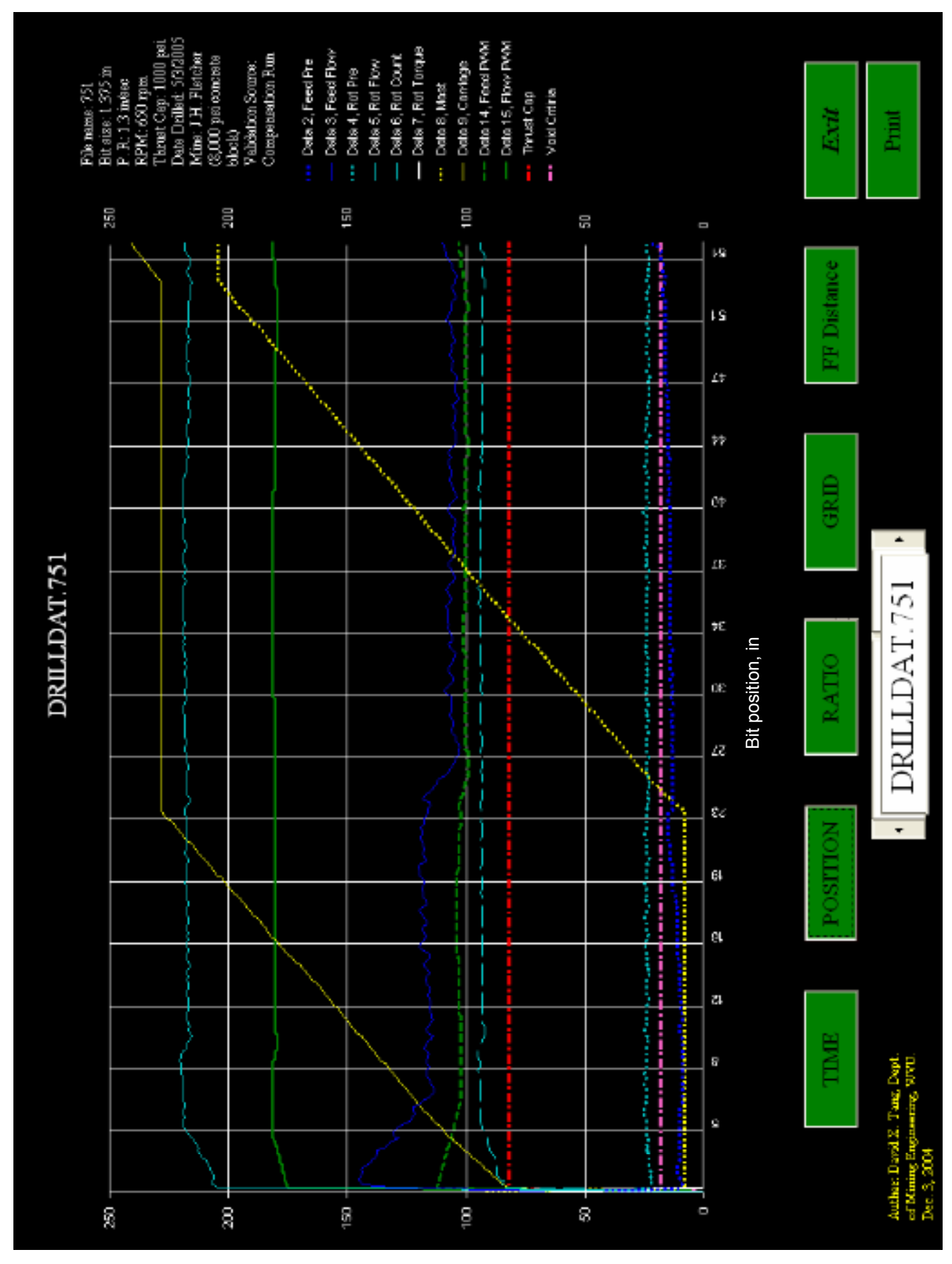




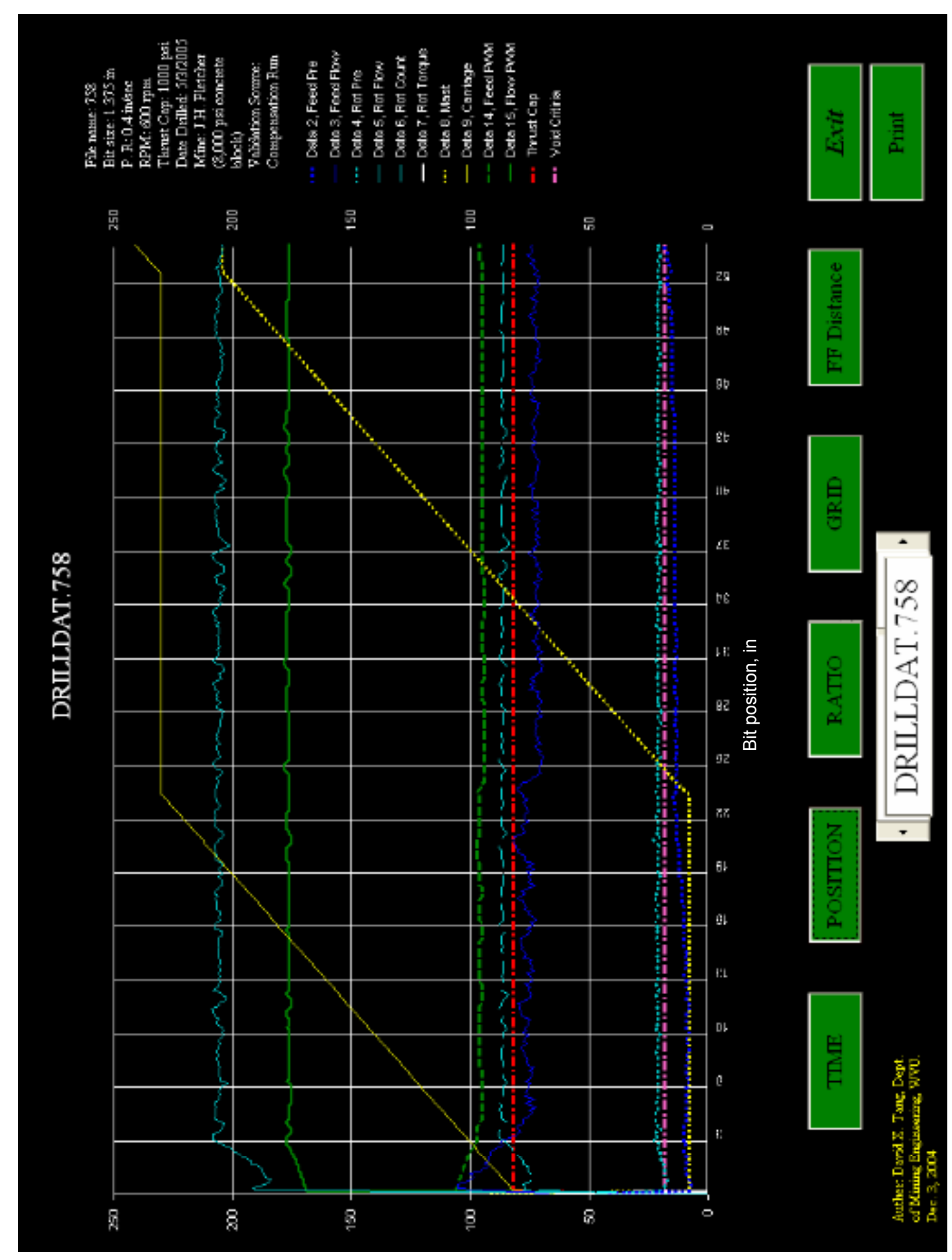




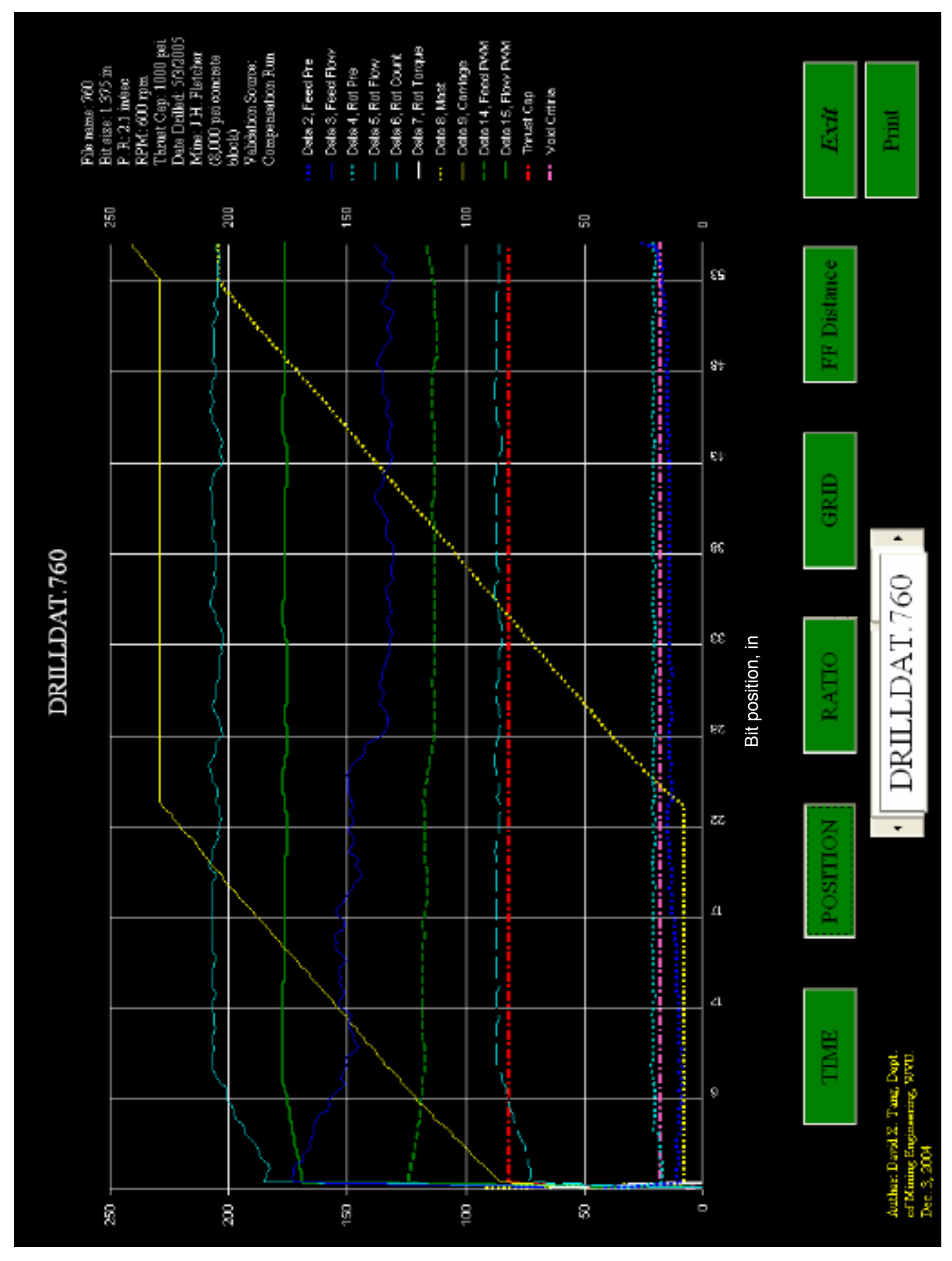




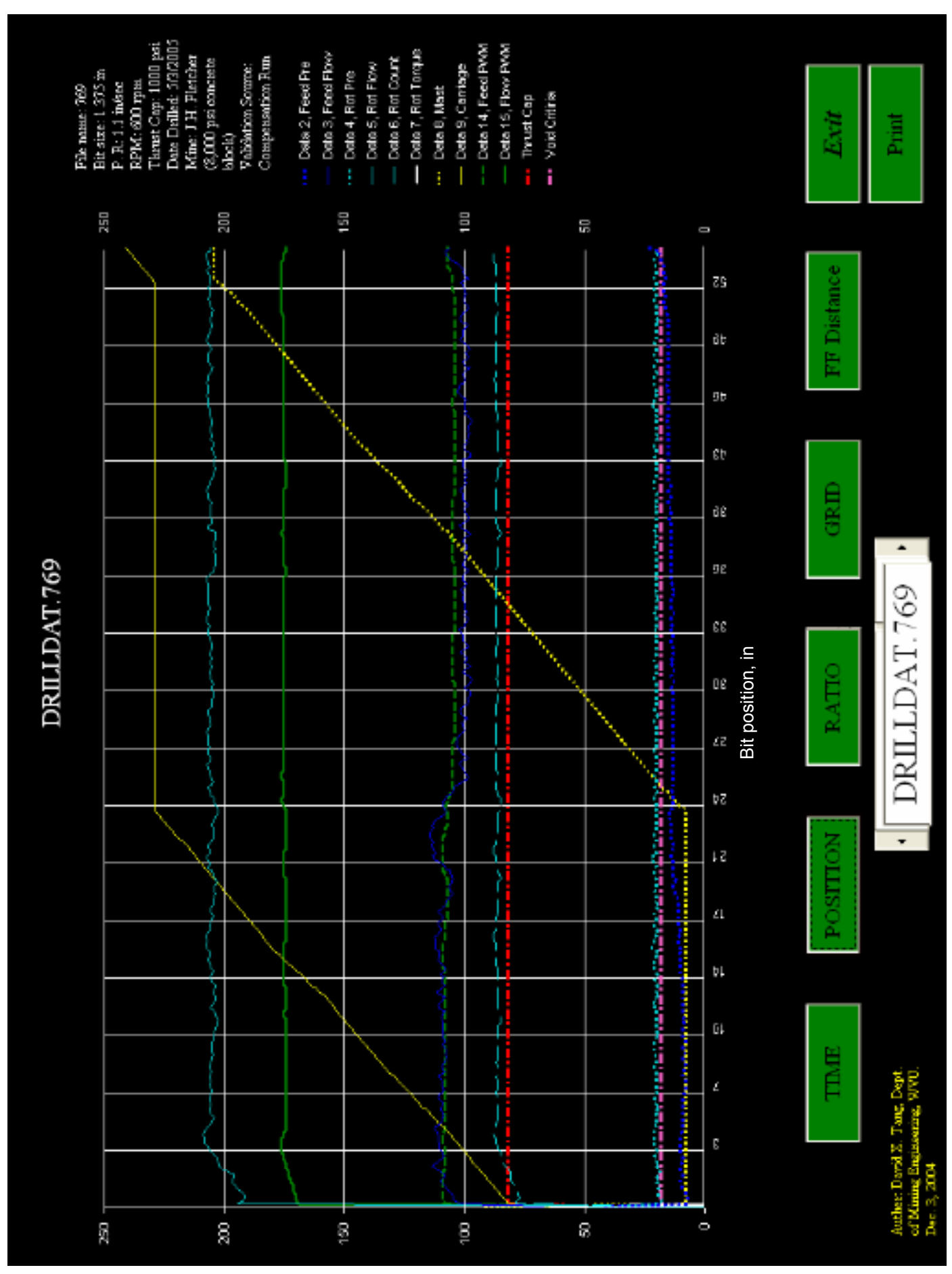




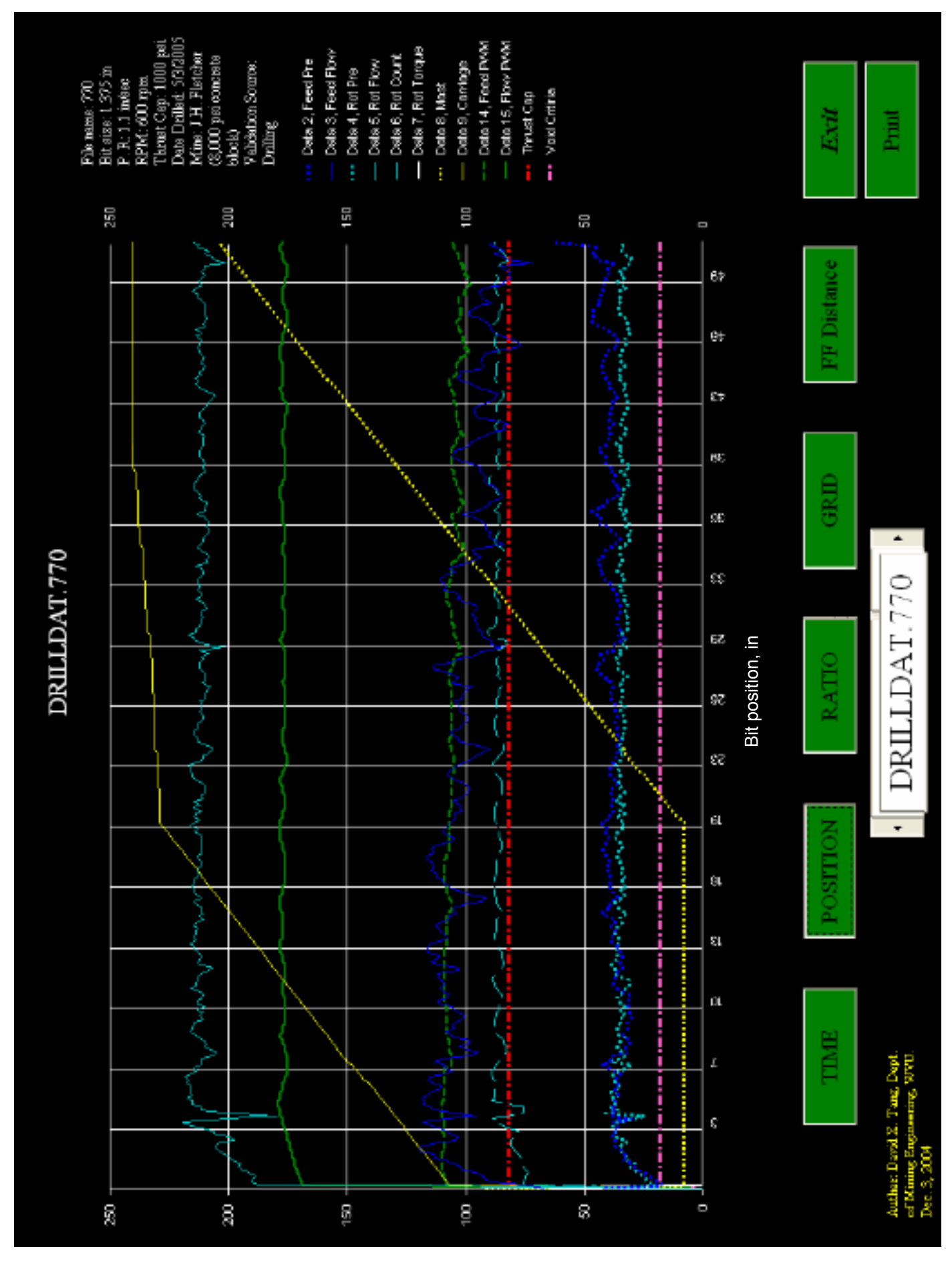




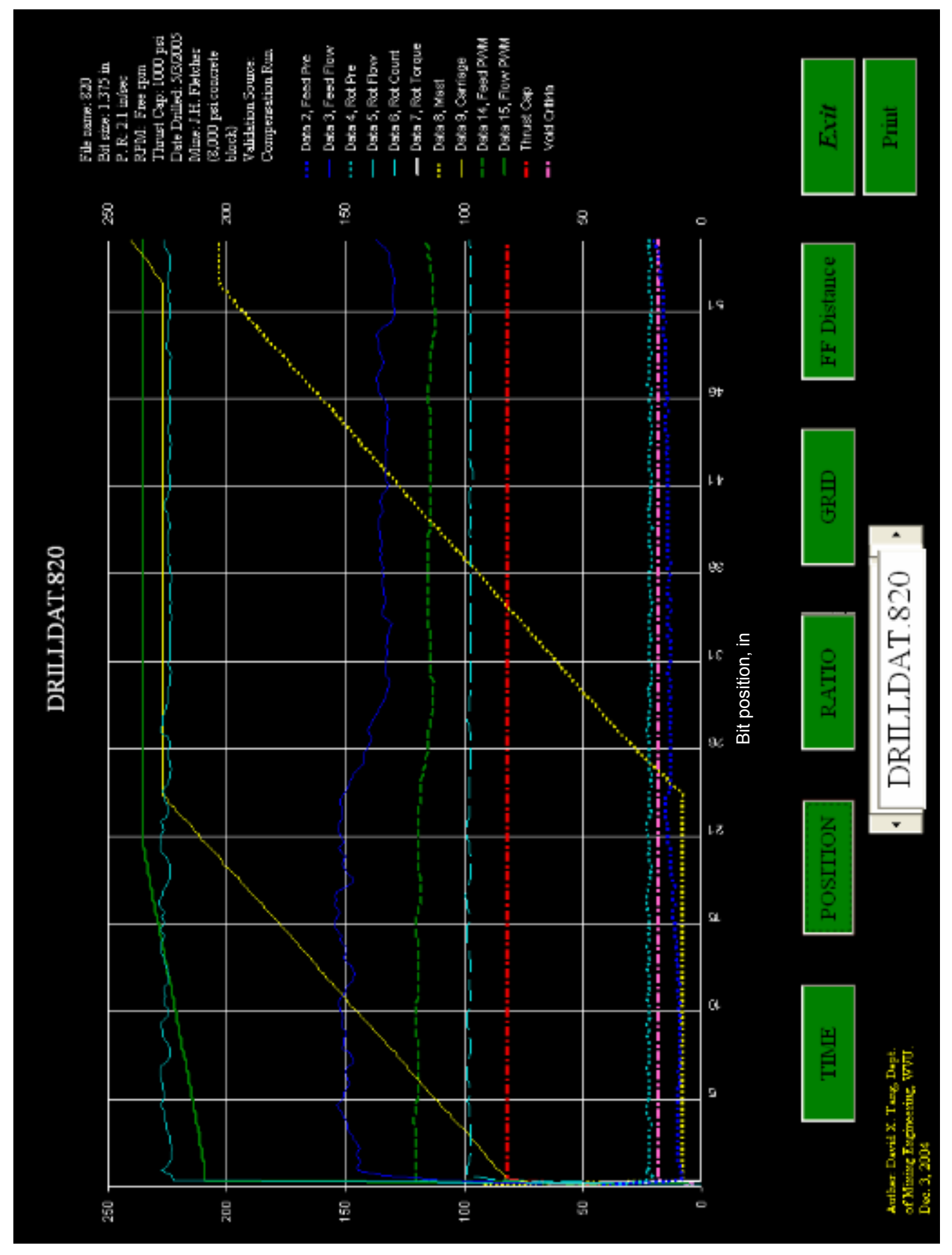


APPENDIX C

PHOTOS AND DRILLING PARAMETERS PLOTS OF BIT WEAR TESTS 

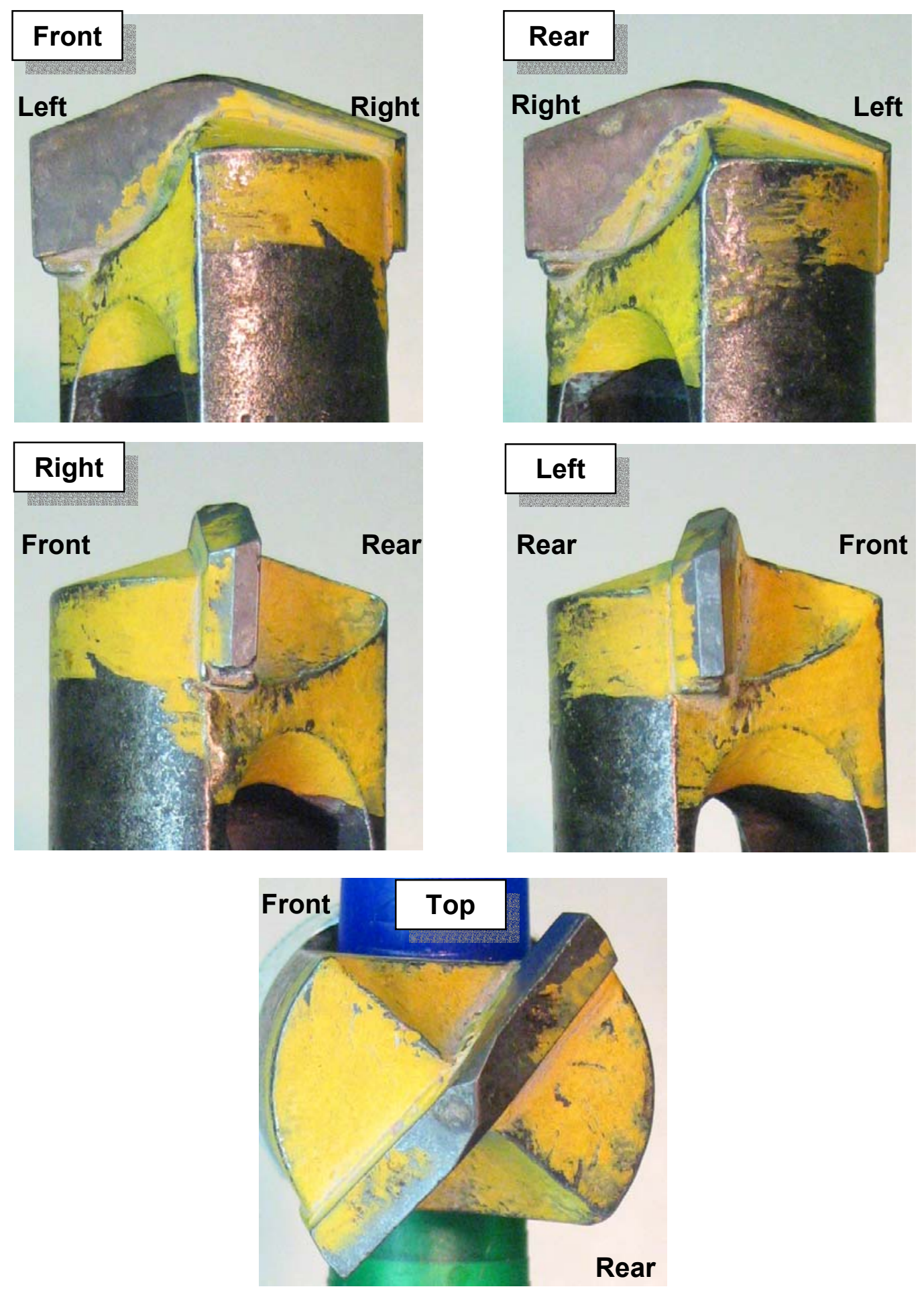

\begin{tabular}{|c|c|c|c|c|c|c|}
\hline \multicolumn{3}{|c|}{ Controlled Values } & \multirow{2}{*}{ Mine } & \multicolumn{2}{c|}{ Roof Rock } & \begin{tabular}{c} 
Number of \\
\cline { 4 - 5 } \\
\cline { 1 - 2 } Penetration Rated holes
\end{tabular} \\
\hline $\begin{array}{c}0.4,1.1,1.5, \text { and } \\
1.7 \mathrm{in} / \mathrm{sec}\end{array}$ & $\begin{array}{c}500 \text { and } 600 \\
\mathrm{rpm}\end{array}$ & $1,000 \mathrm{psi}$ & Mine F & Shale & $<3,400 \mathrm{psi}$ & 17 holes \\
\hline
\end{tabular}




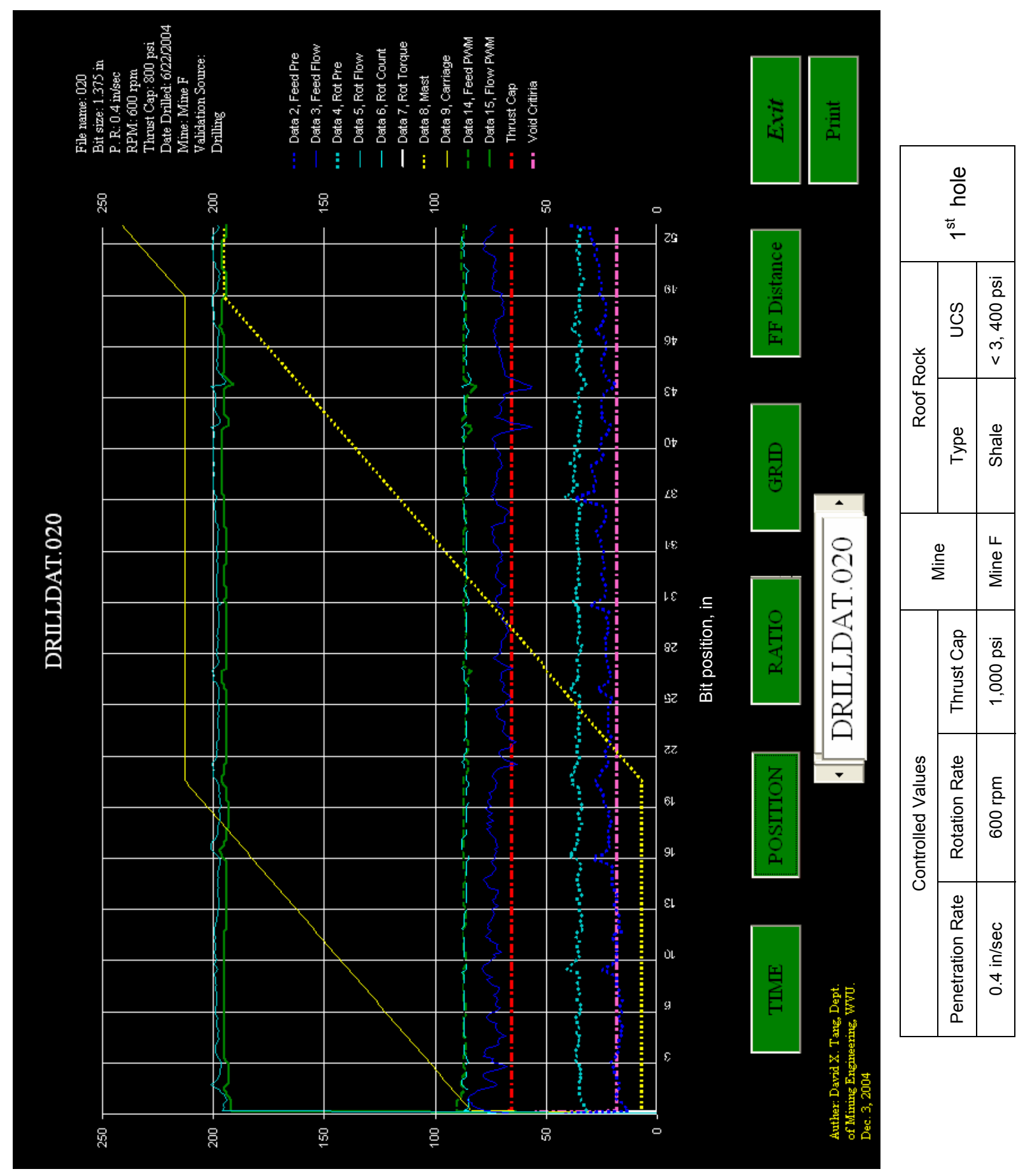




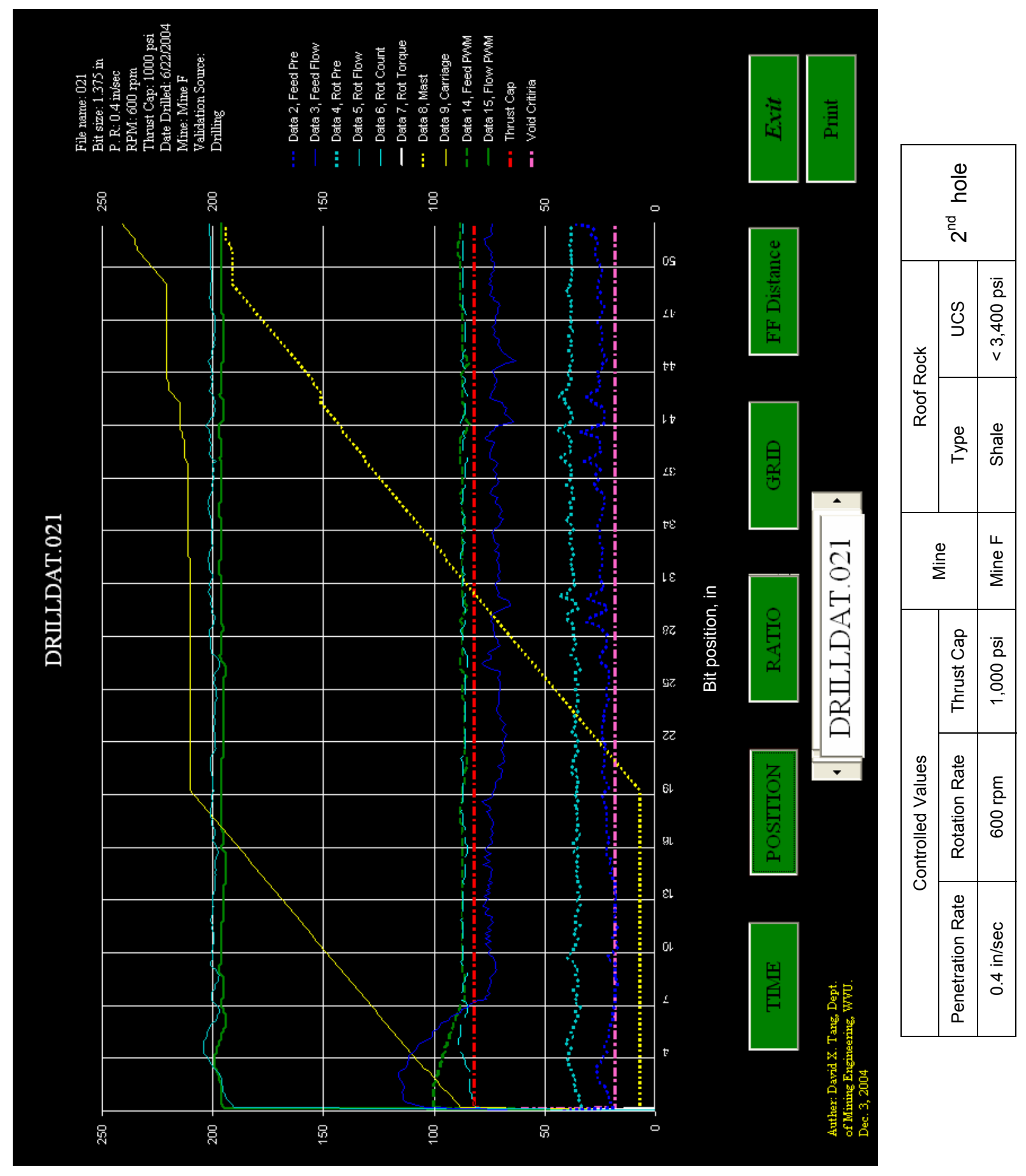




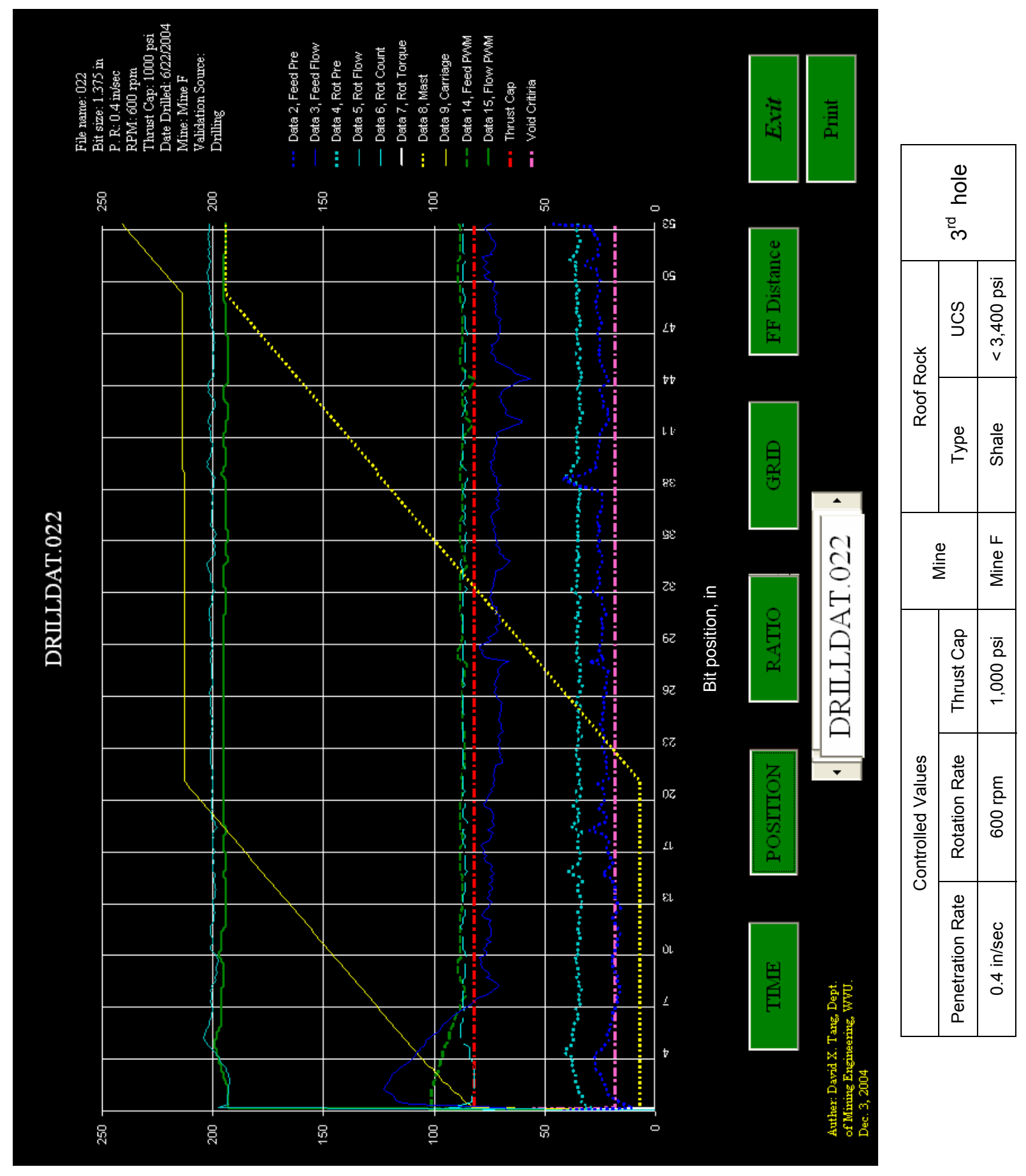




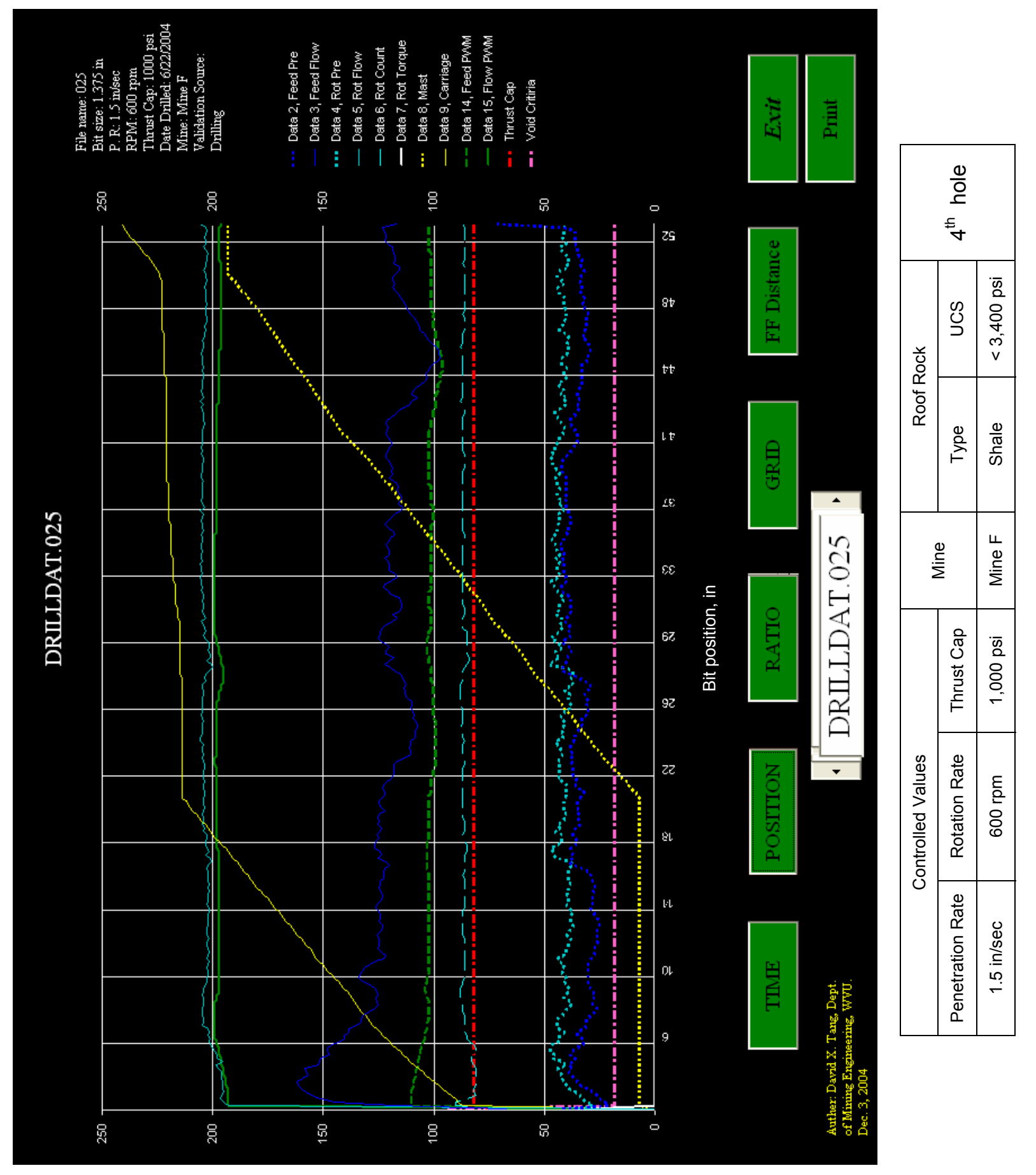




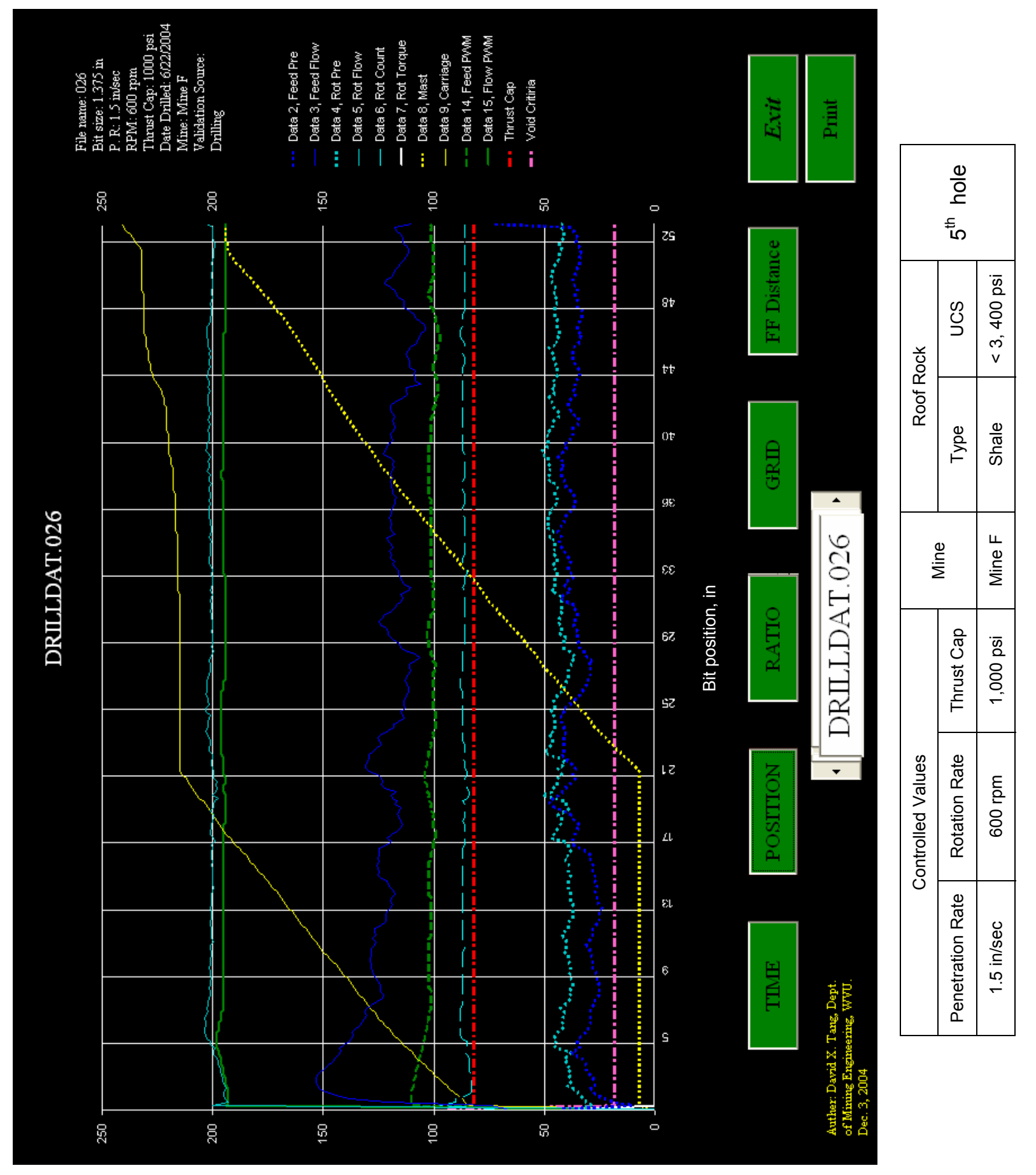




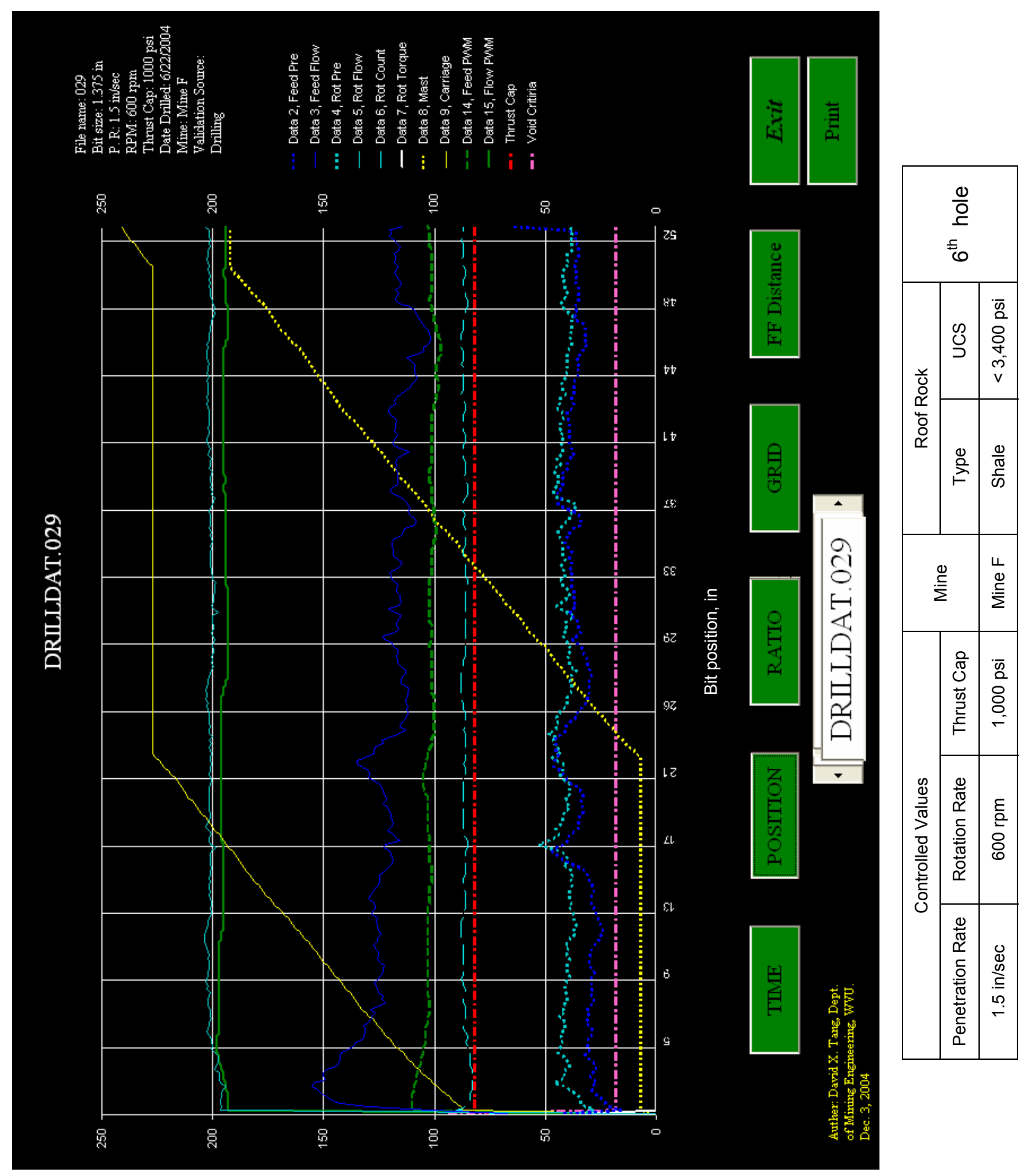




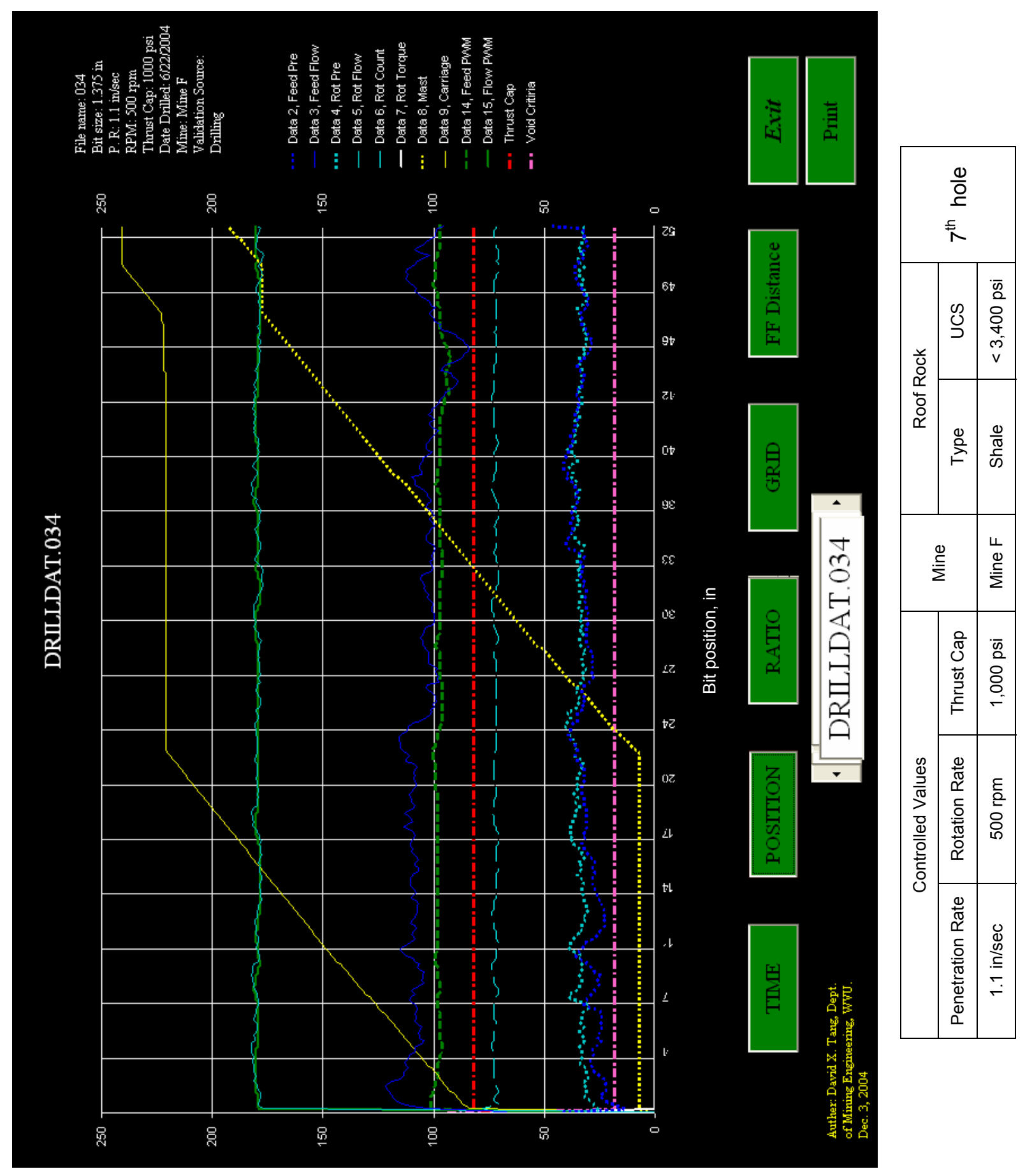




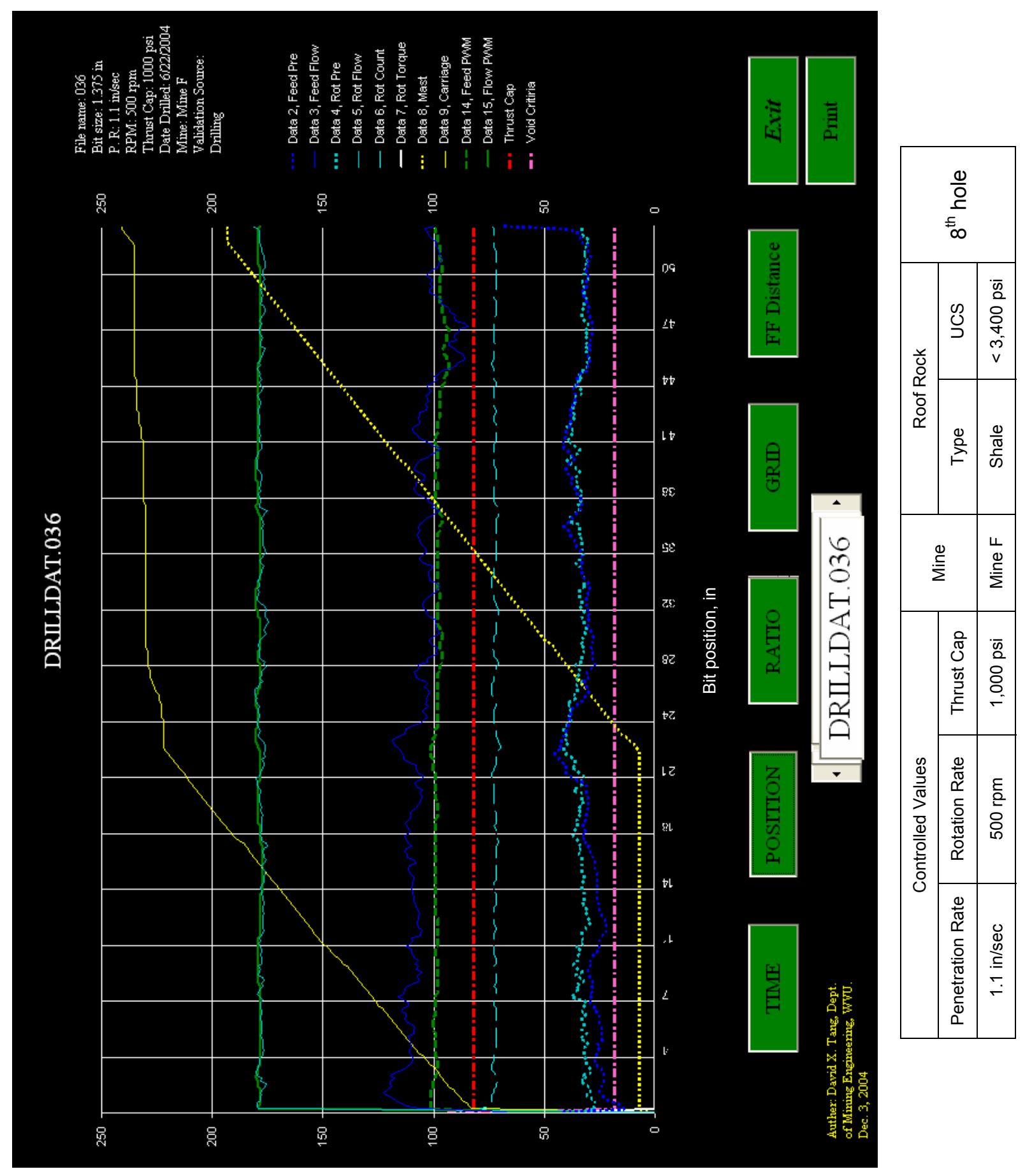




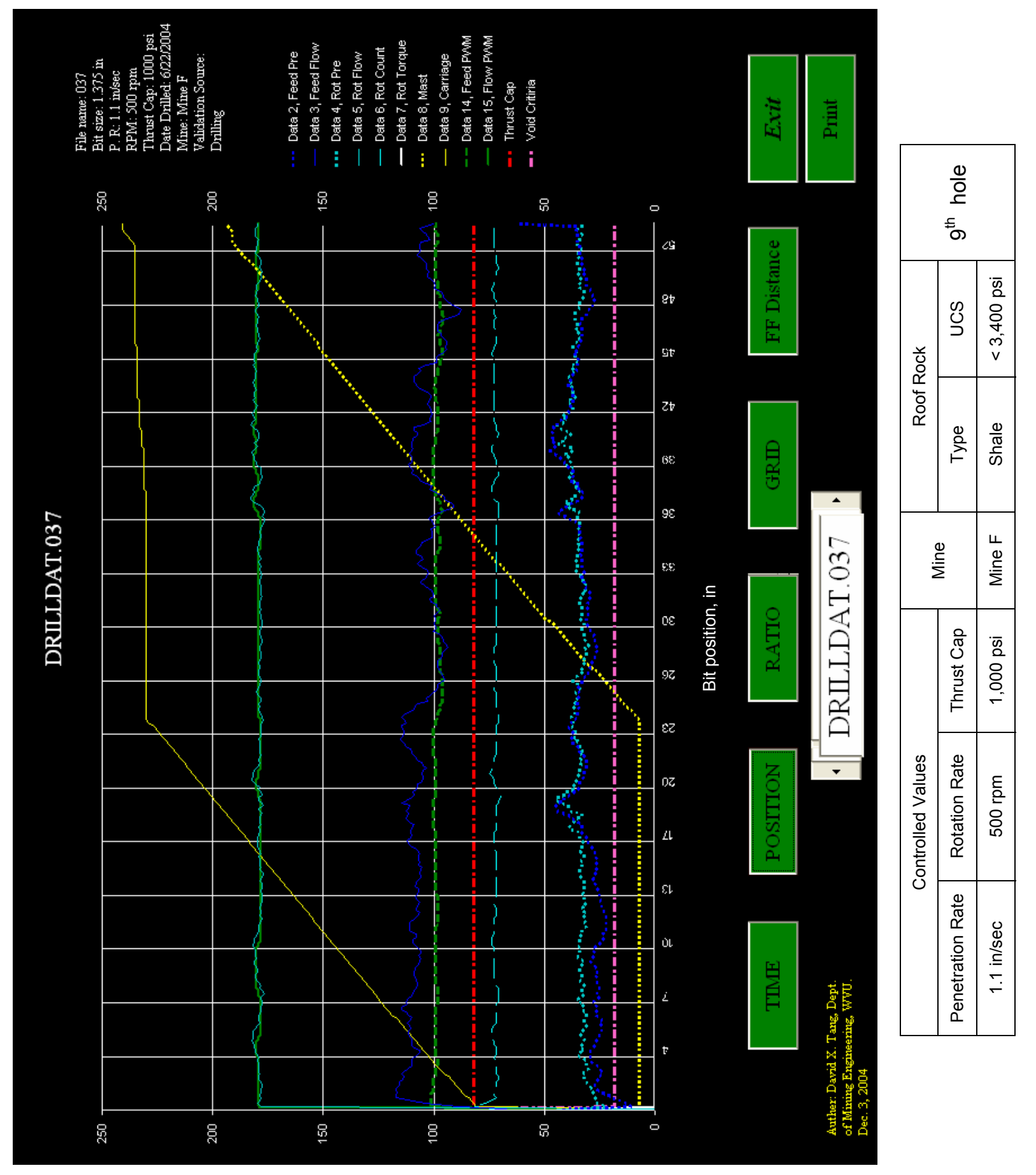




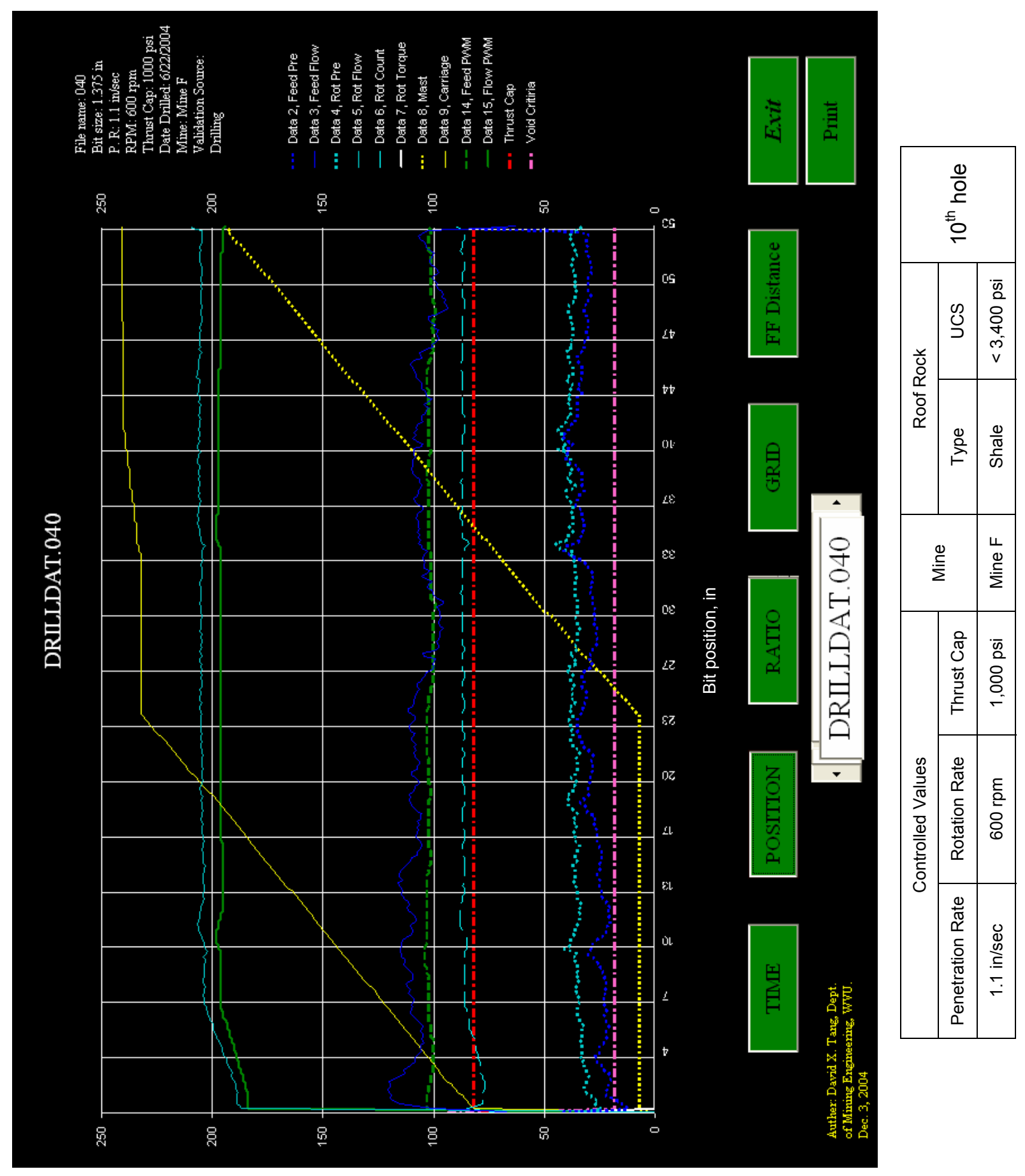




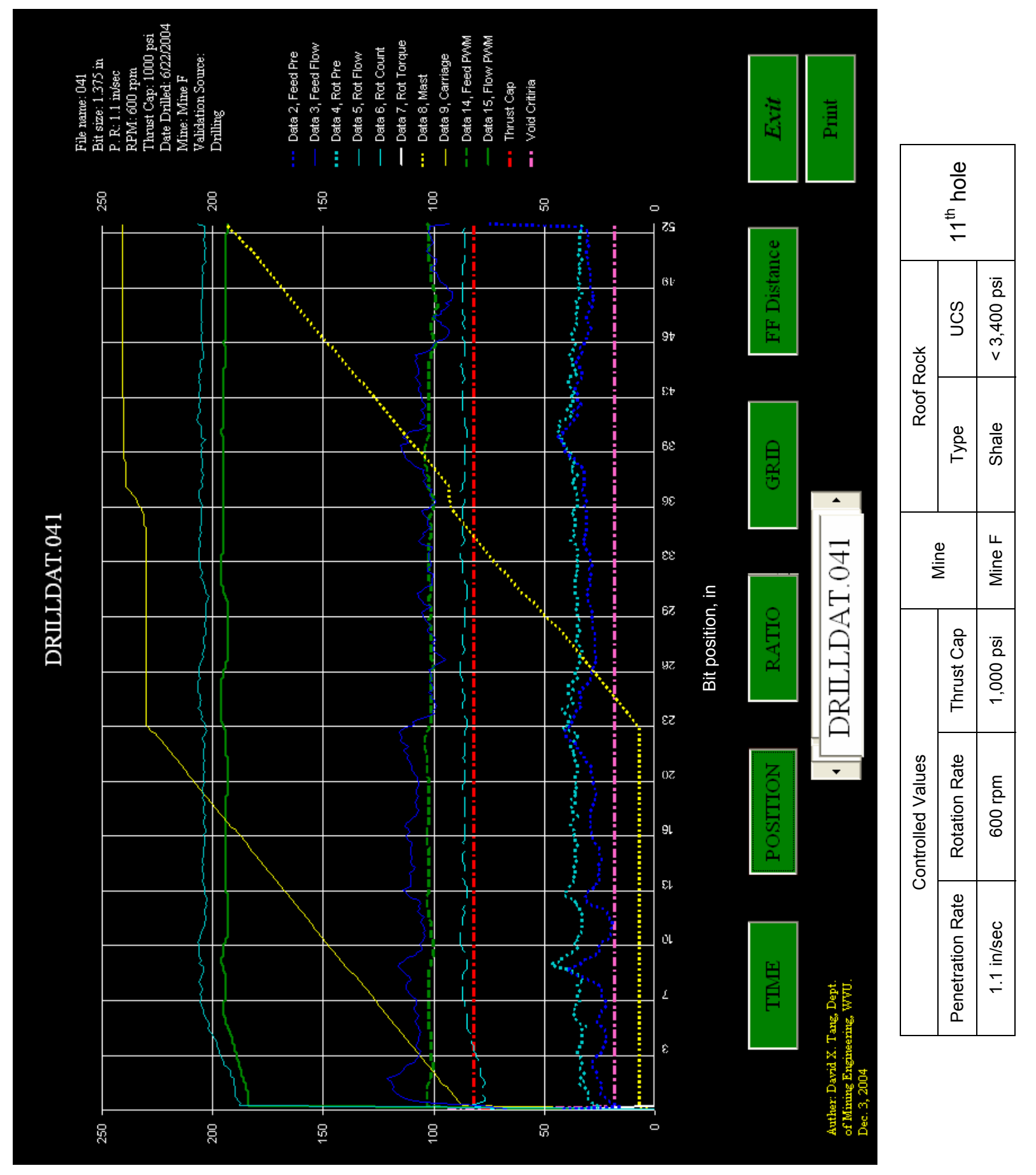




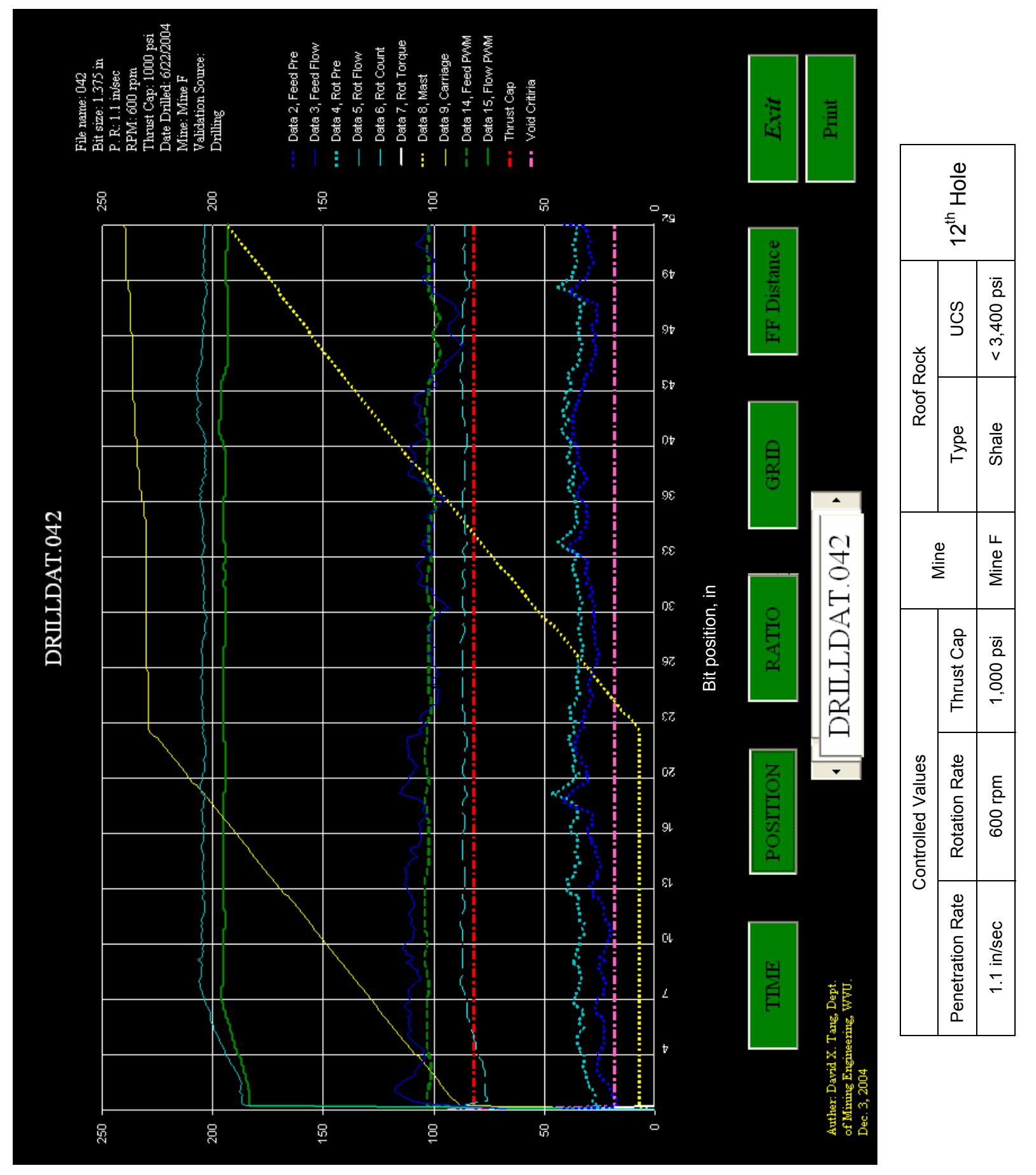




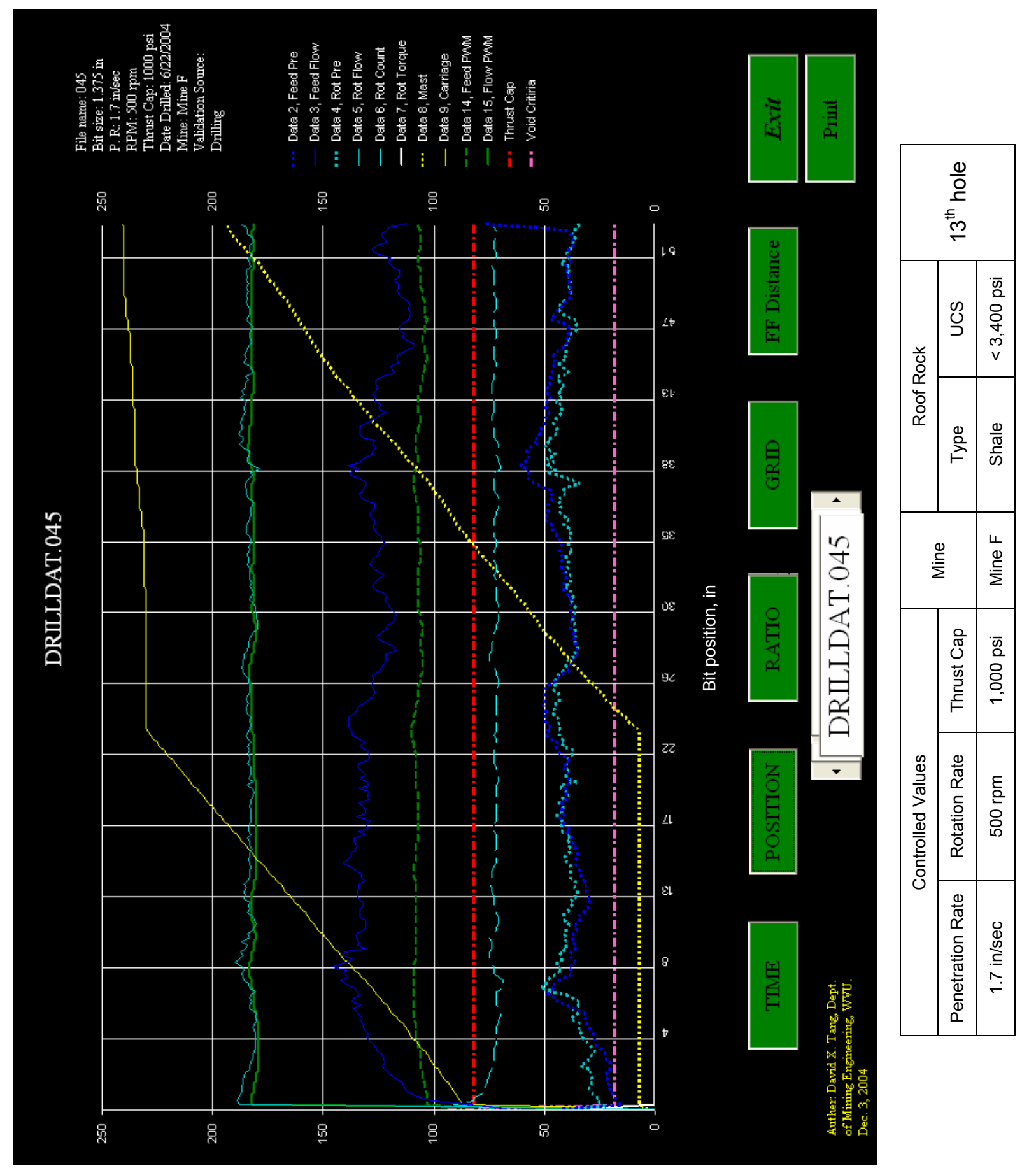




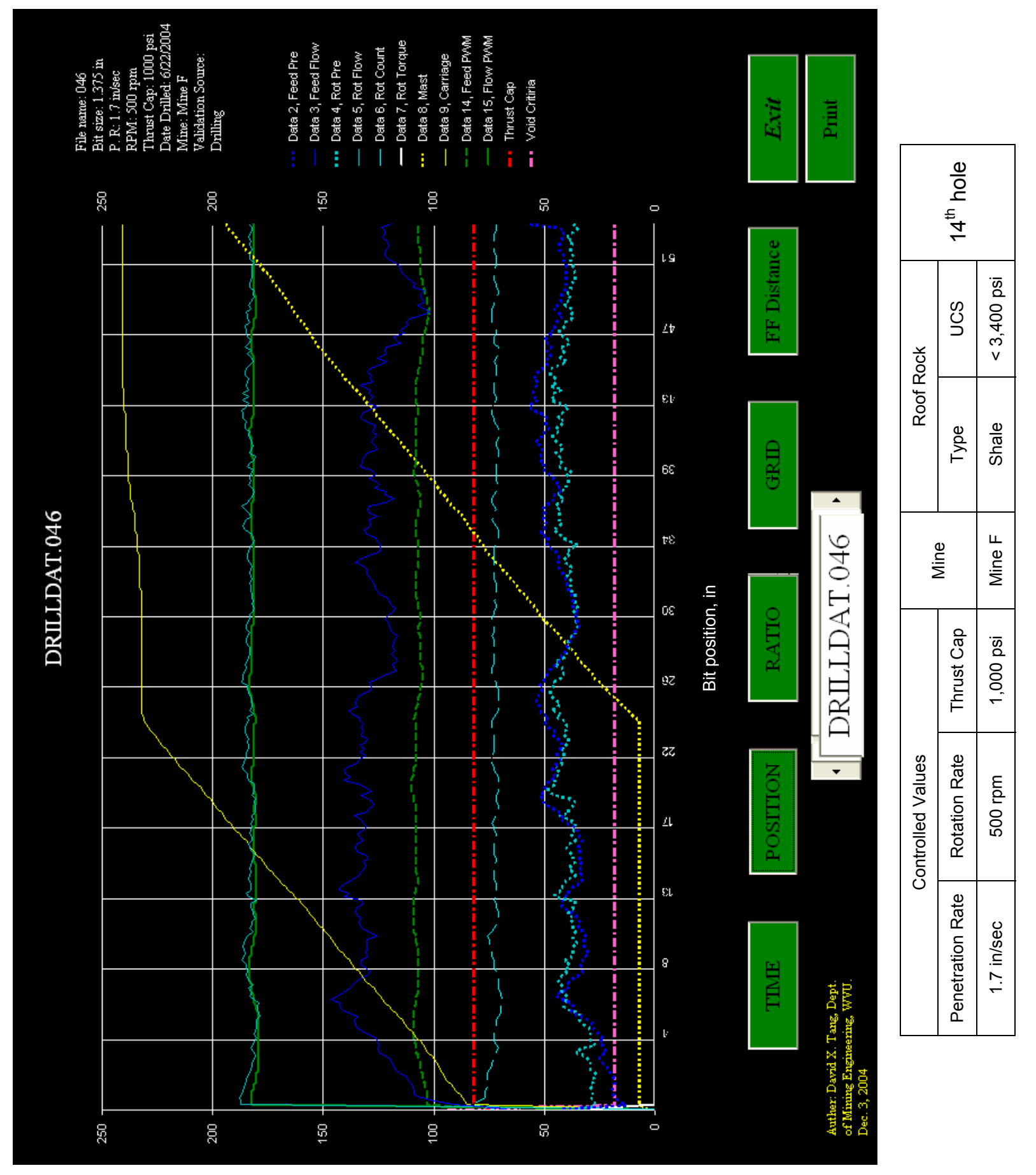




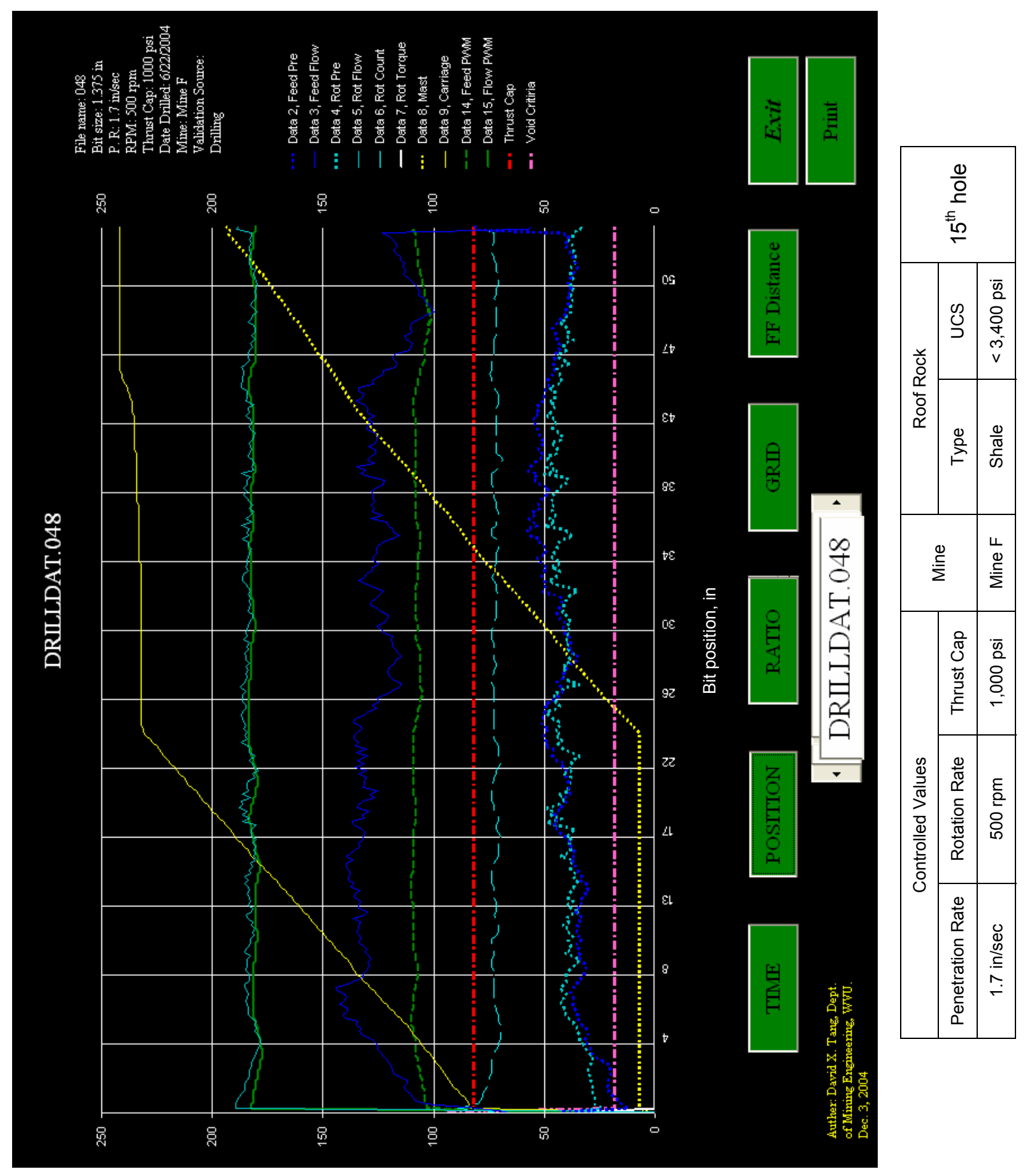




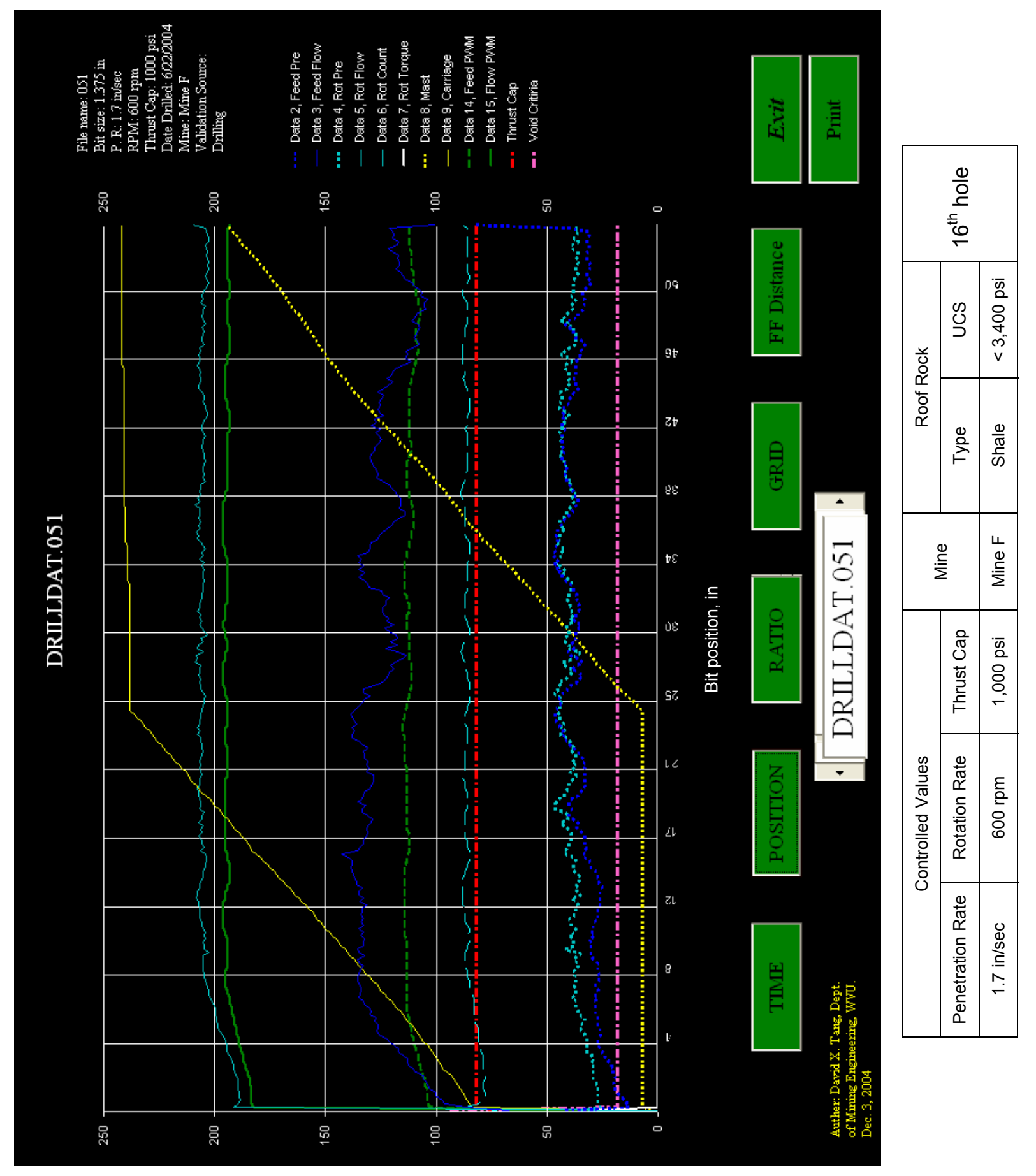




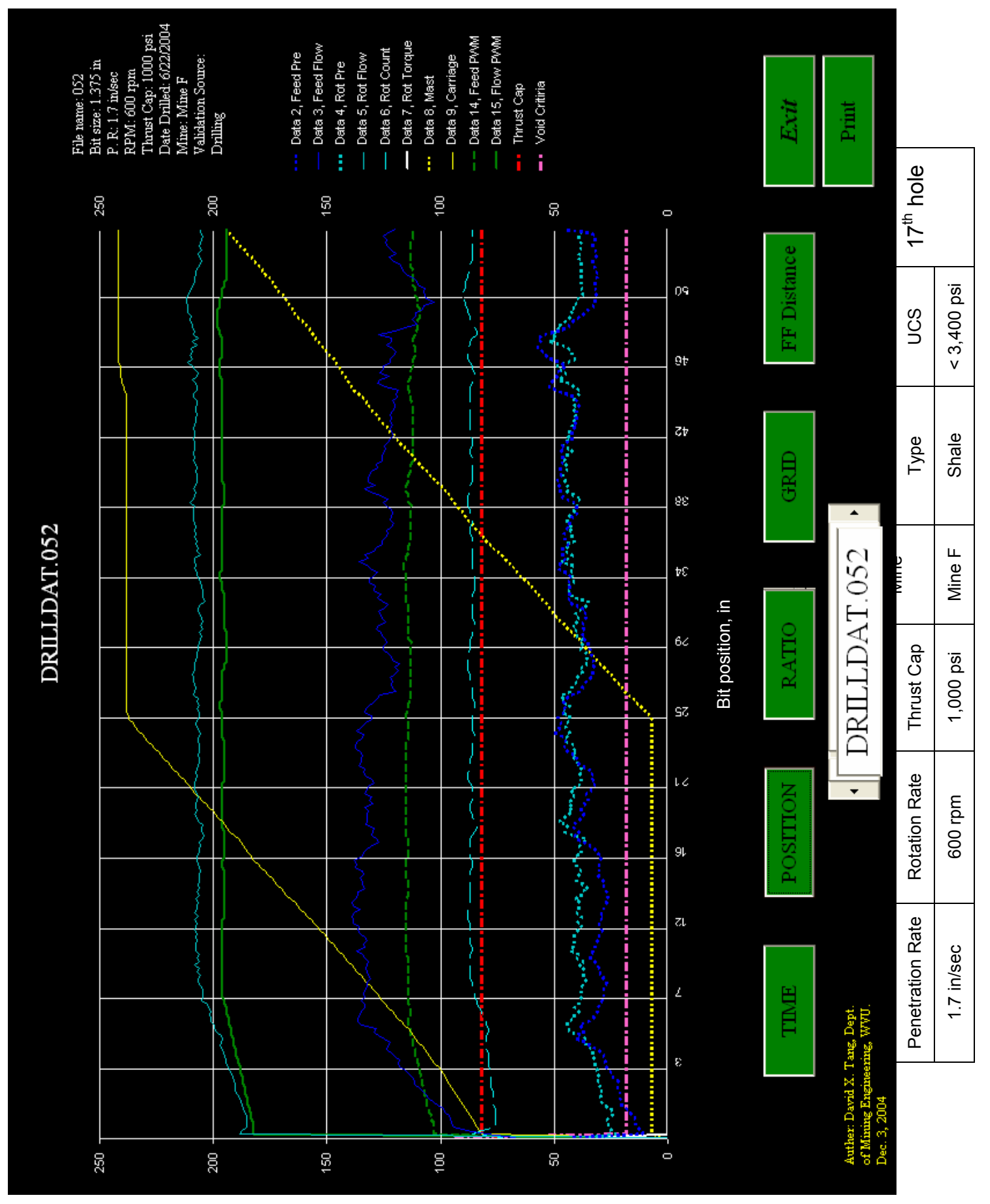



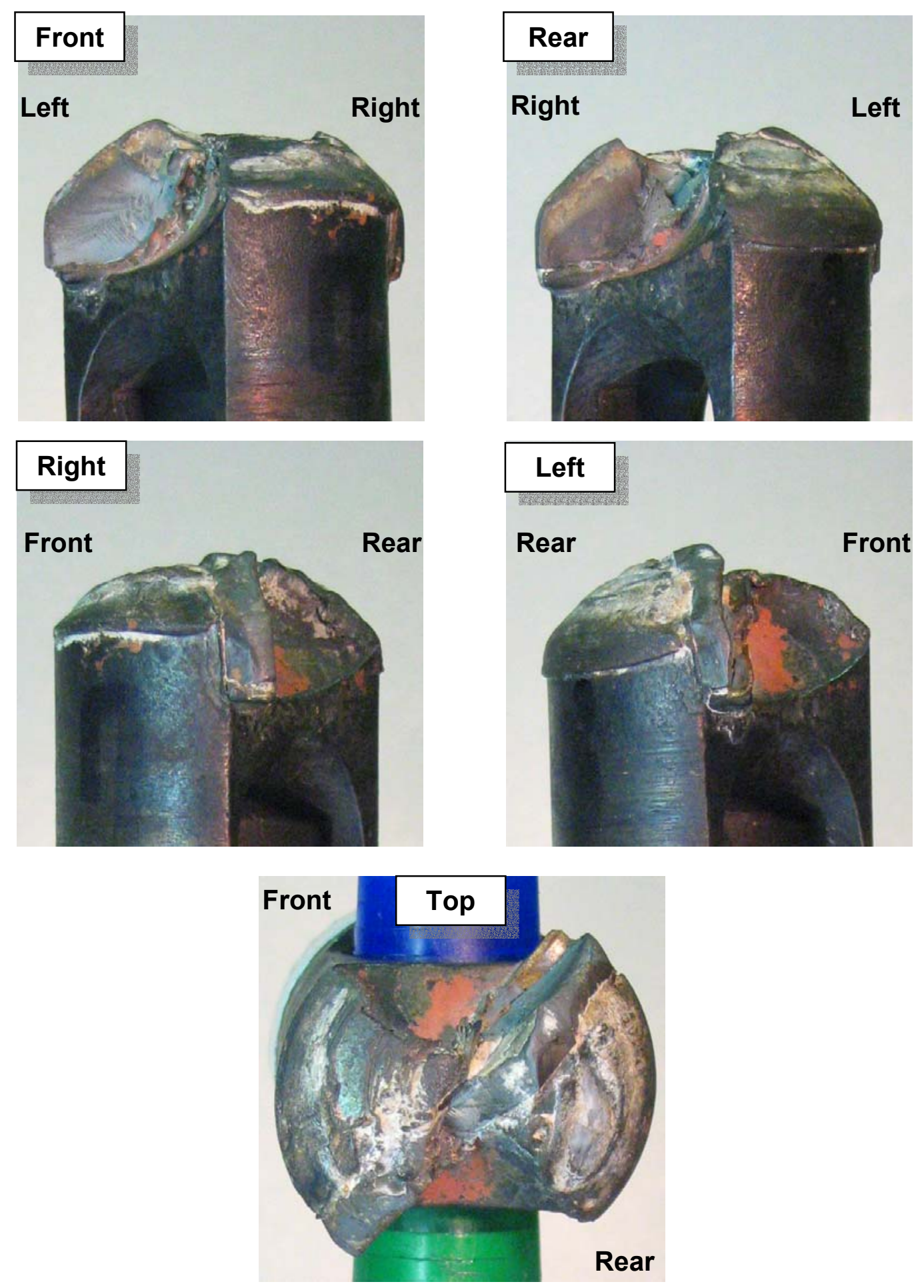

\begin{tabular}{|c|c|c|c|c|c|c|}
\hline \multicolumn{3}{|c|}{ Controlled Values } & \multirow{2}{*}{ Mine } & \multicolumn{2}{c|}{ Roof Rock } & Number of \\
\cline { 1 - 2 } & Drilled holes \\
\hline Penetration Rate & Rotation Rate & Thrust Cap & & Type & UCS & 7 holes \\
\hline $1.1 \mathrm{in} / \mathrm{sec}$ & $650 \mathrm{rpm}$ & $1,000 \mathrm{psi}$ & Mine G & Sandstone & $9,392 \mathrm{psi}$ & 7 \\
\hline
\end{tabular}




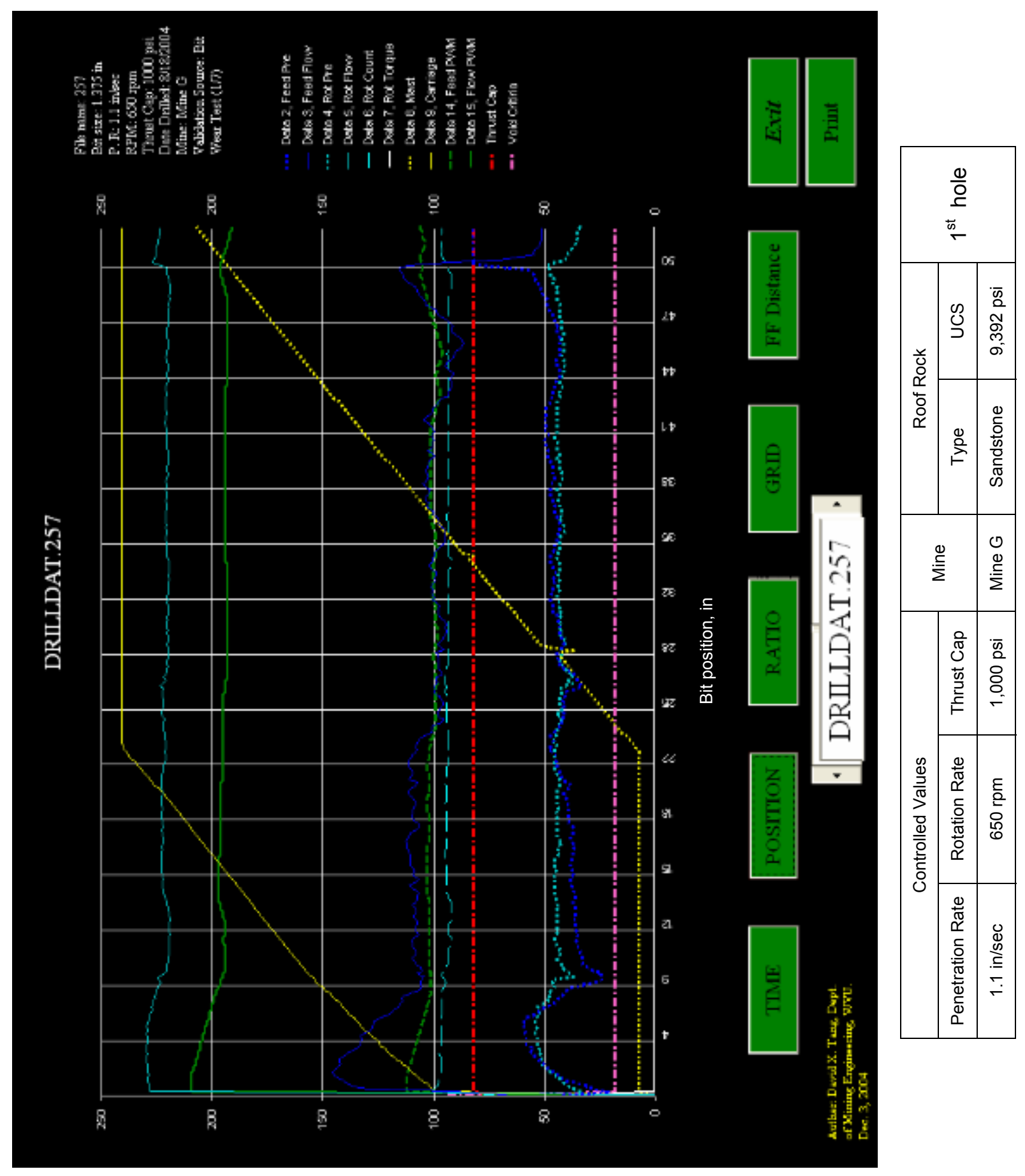




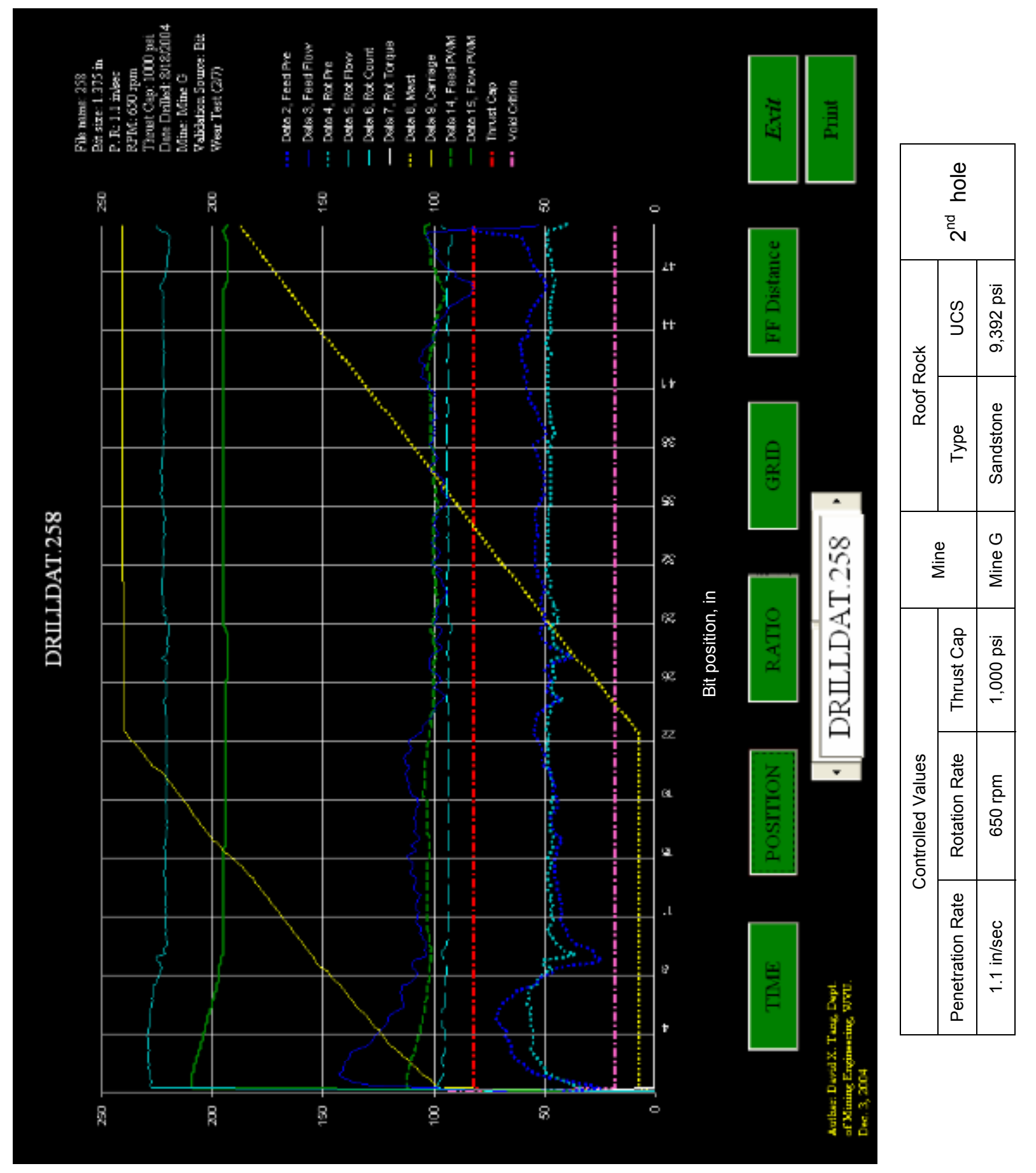




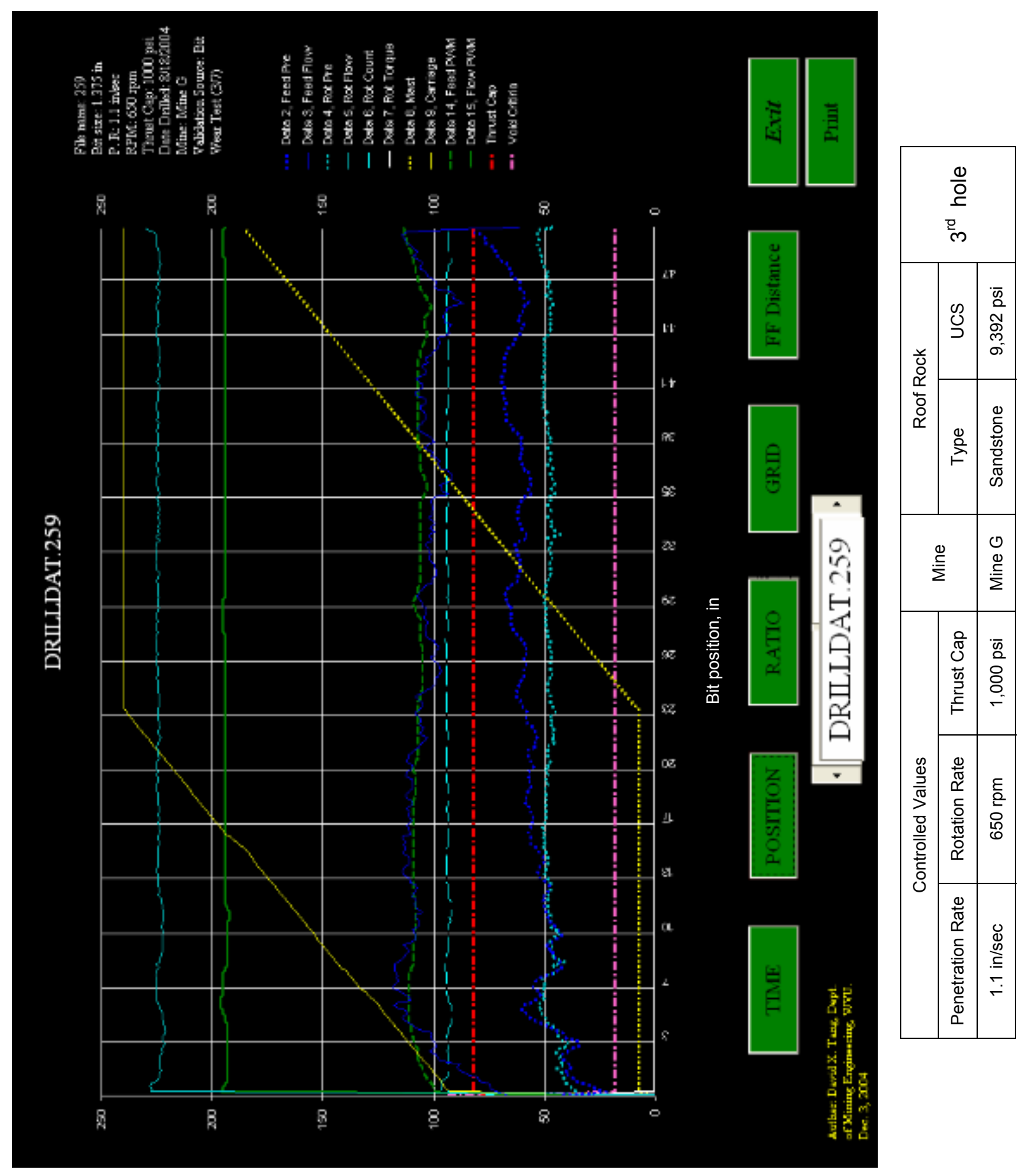




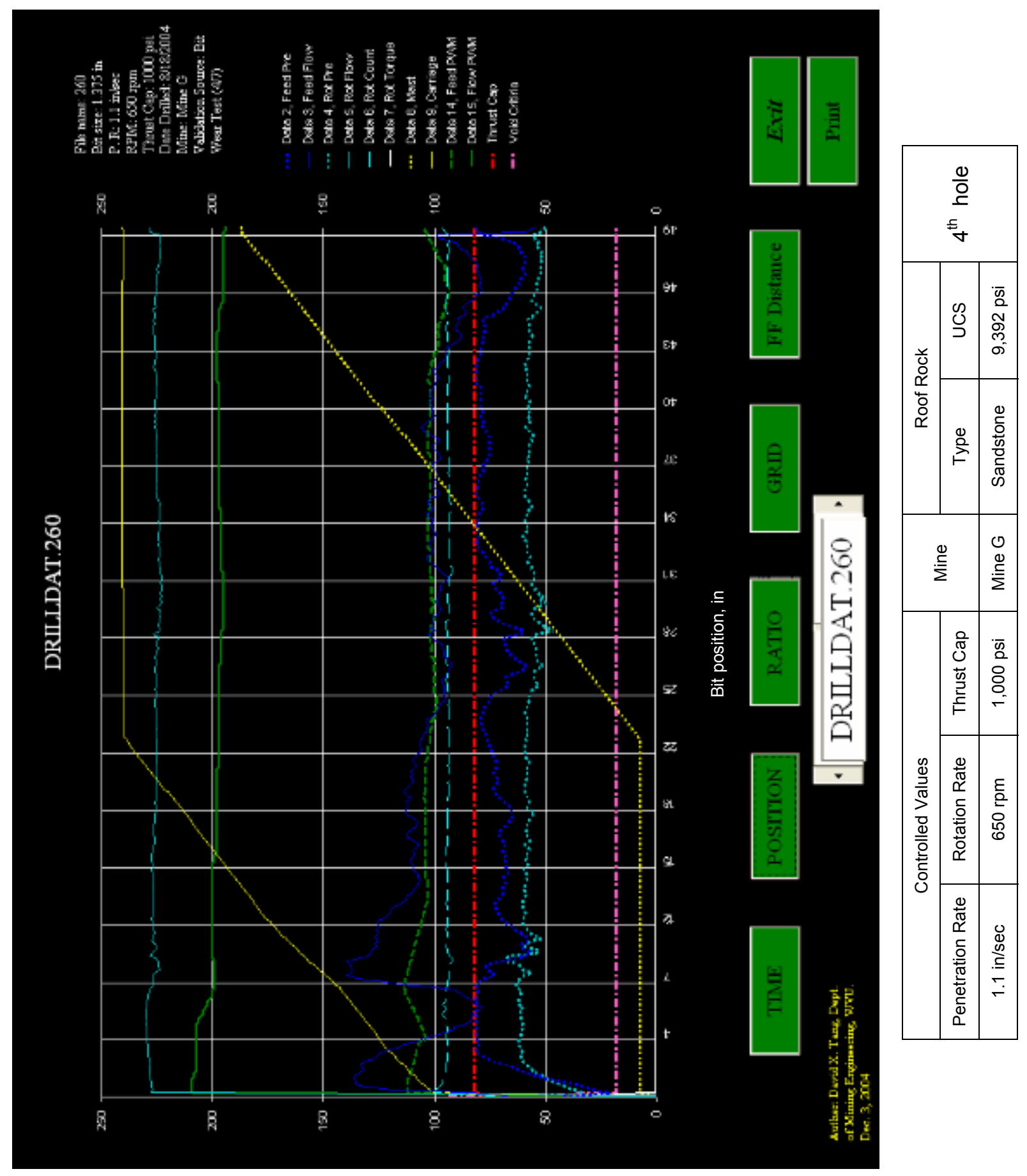




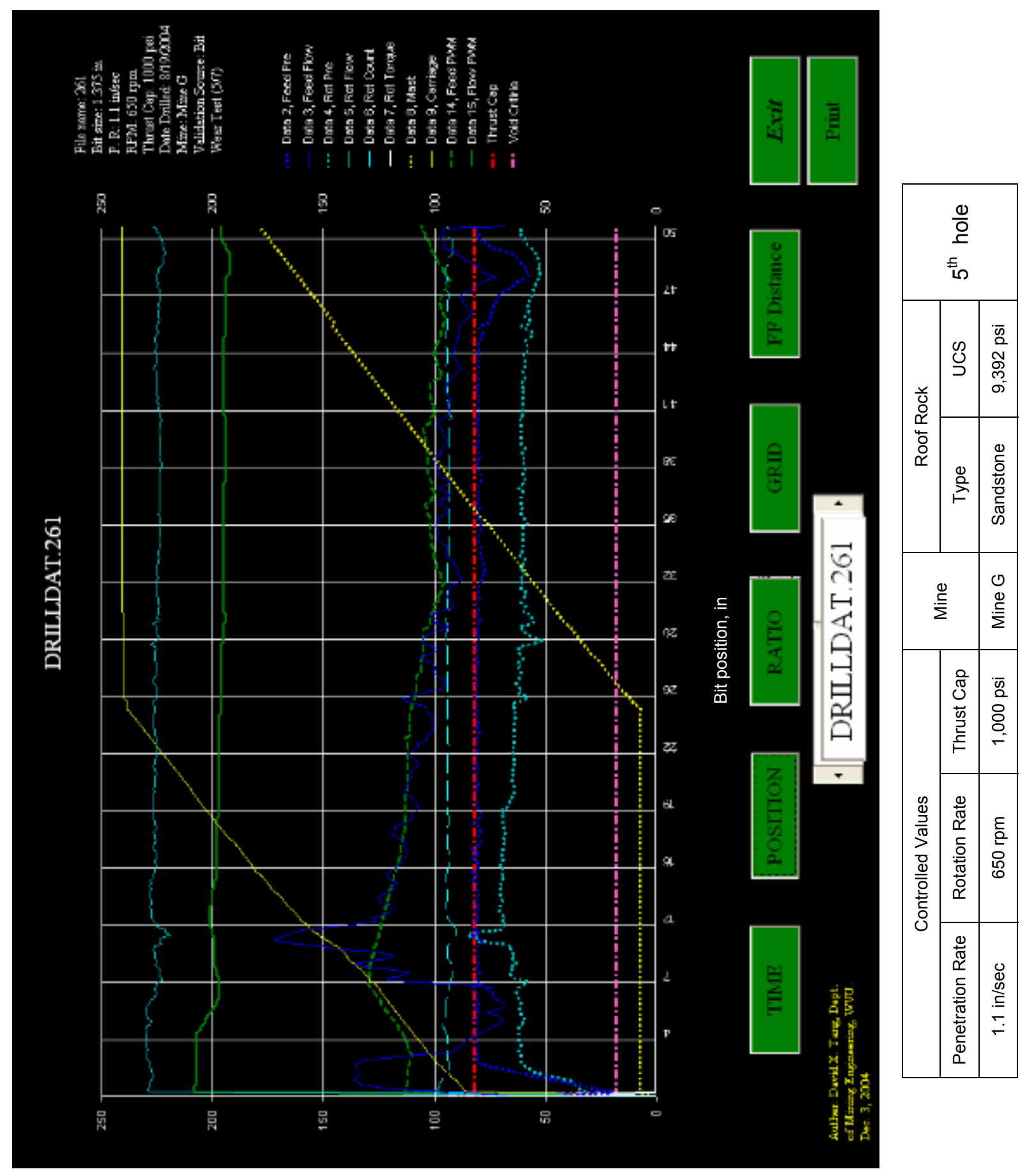




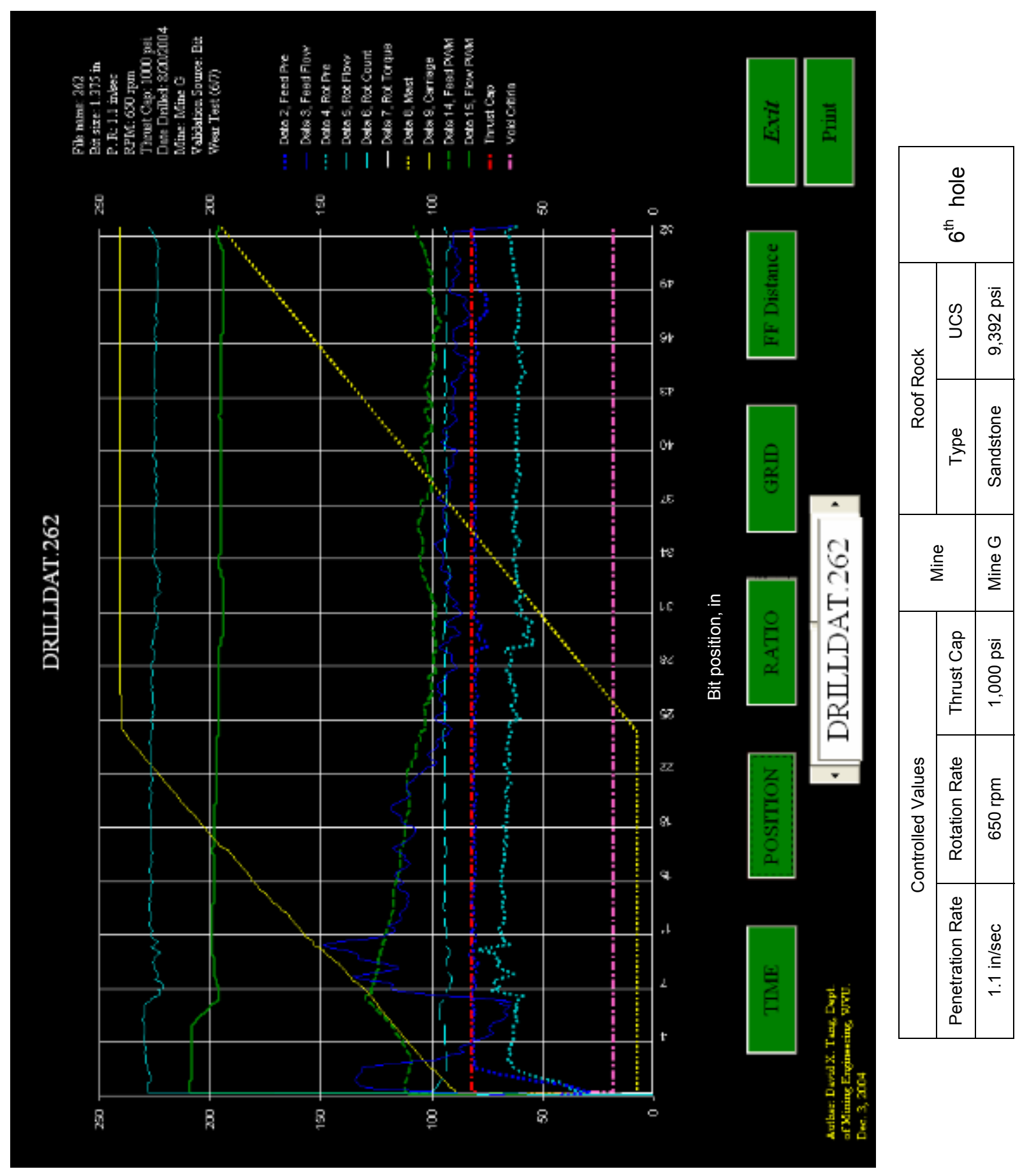




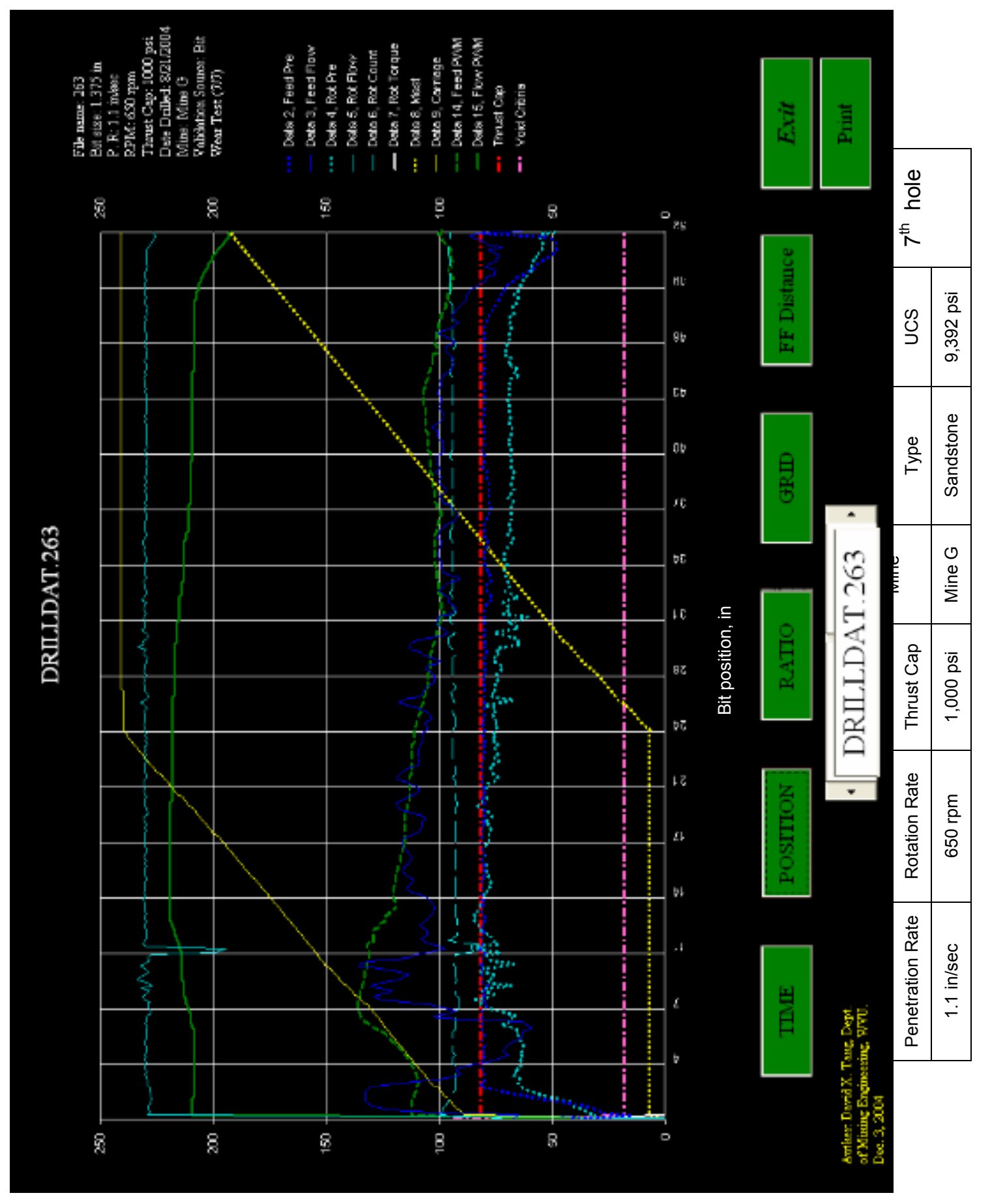



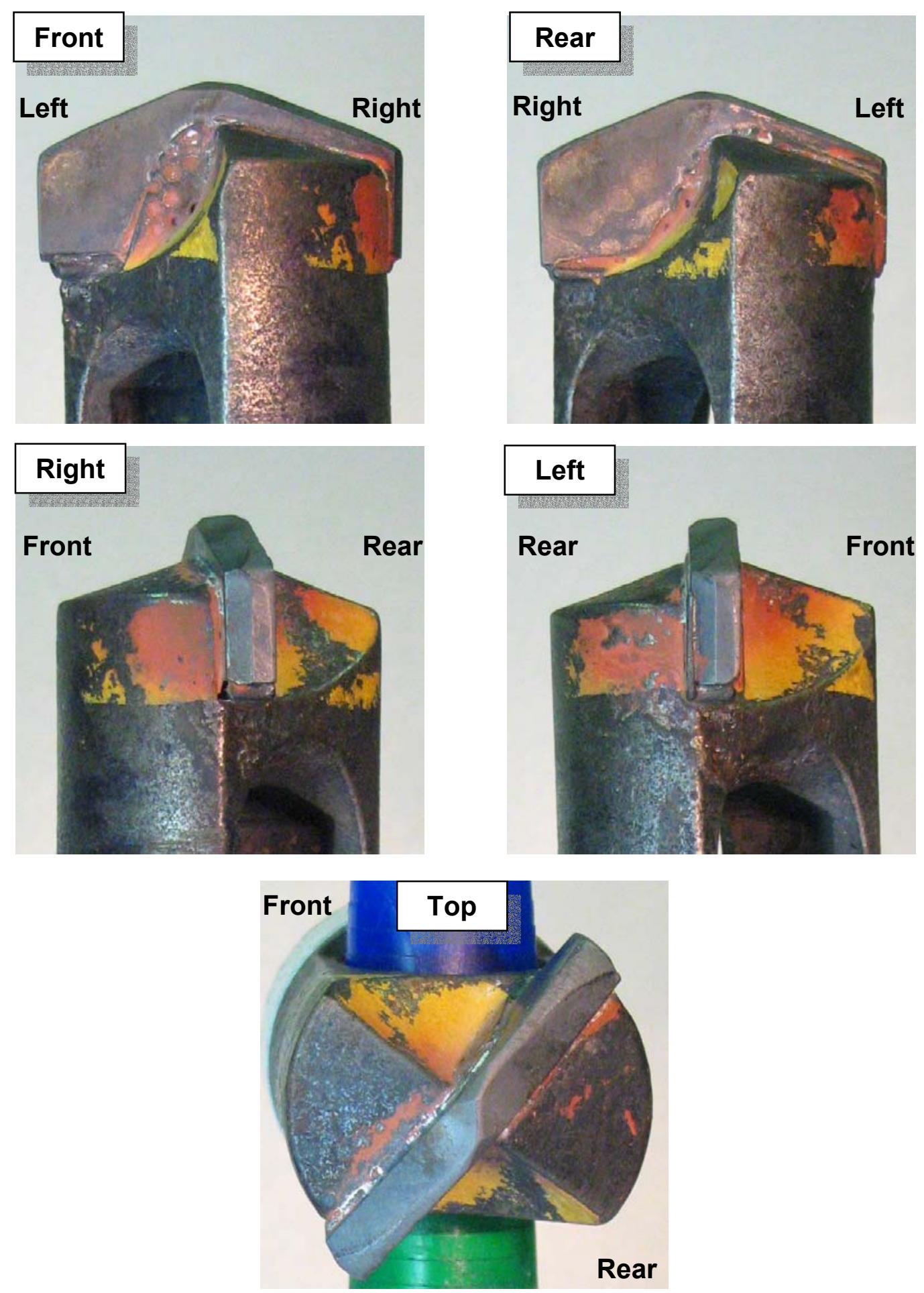

\begin{tabular}{|c|c|c|c|c|c|c|}
\hline \multicolumn{3}{|c|}{ Controlled Values } & \multirow{2}{*}{ Mine } & \multicolumn{2}{|c|}{ Roof Rock } & \multirow{2}{*}{$\begin{array}{c}\text { Number of } \\
\text { Drilled holes }\end{array}$} \\
\hline Penetration Rate & Rotation Rate & Thrust Cap & & Type & UCS & \\
\hline $1.1 \mathrm{in} / \mathrm{sec}$ & $400 \mathrm{rpm}$ & 1,000 psi & Mine G & Sandstone & $9,392 \mathrm{psi}$ & 5 holes \\
\hline
\end{tabular}




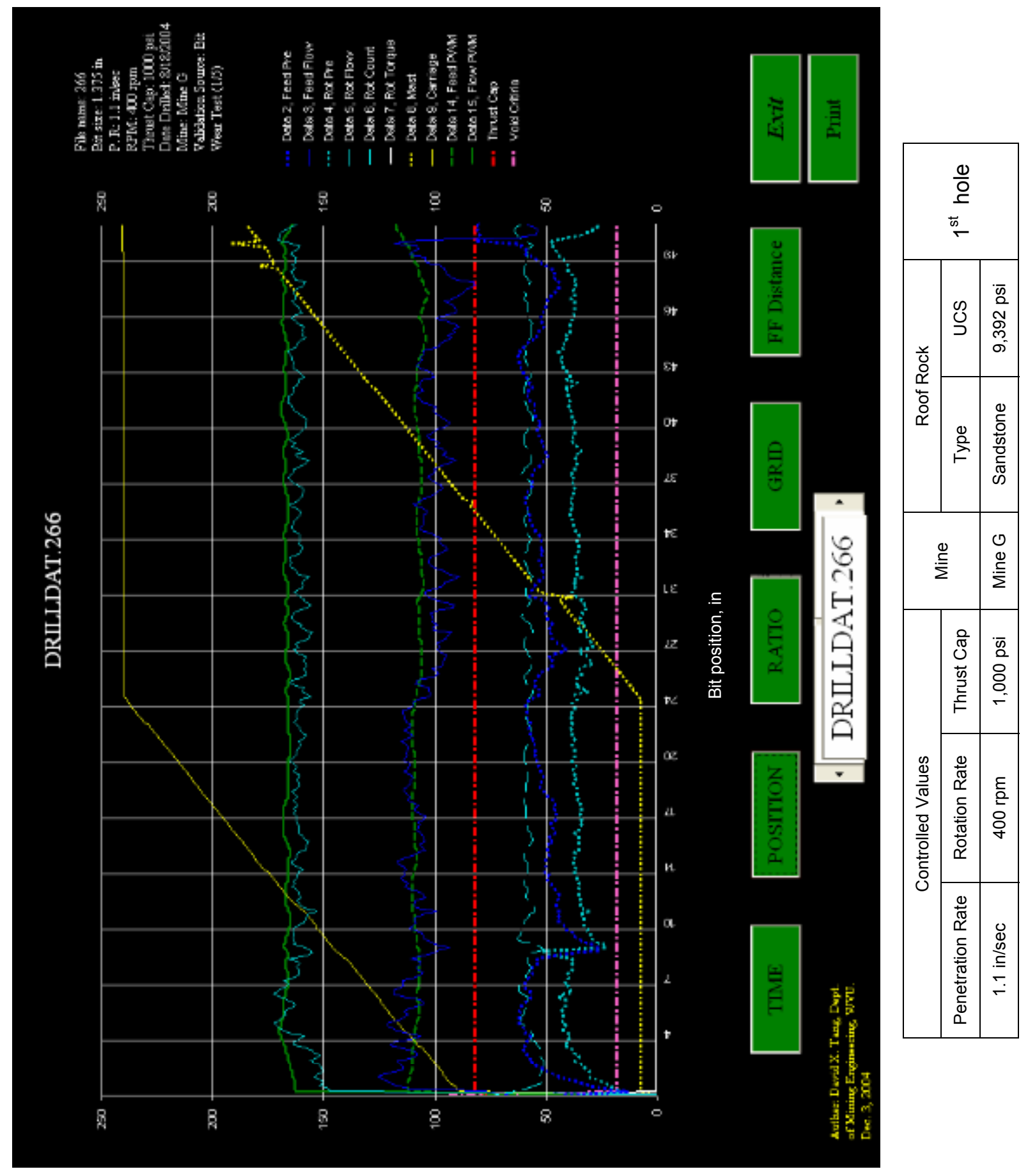




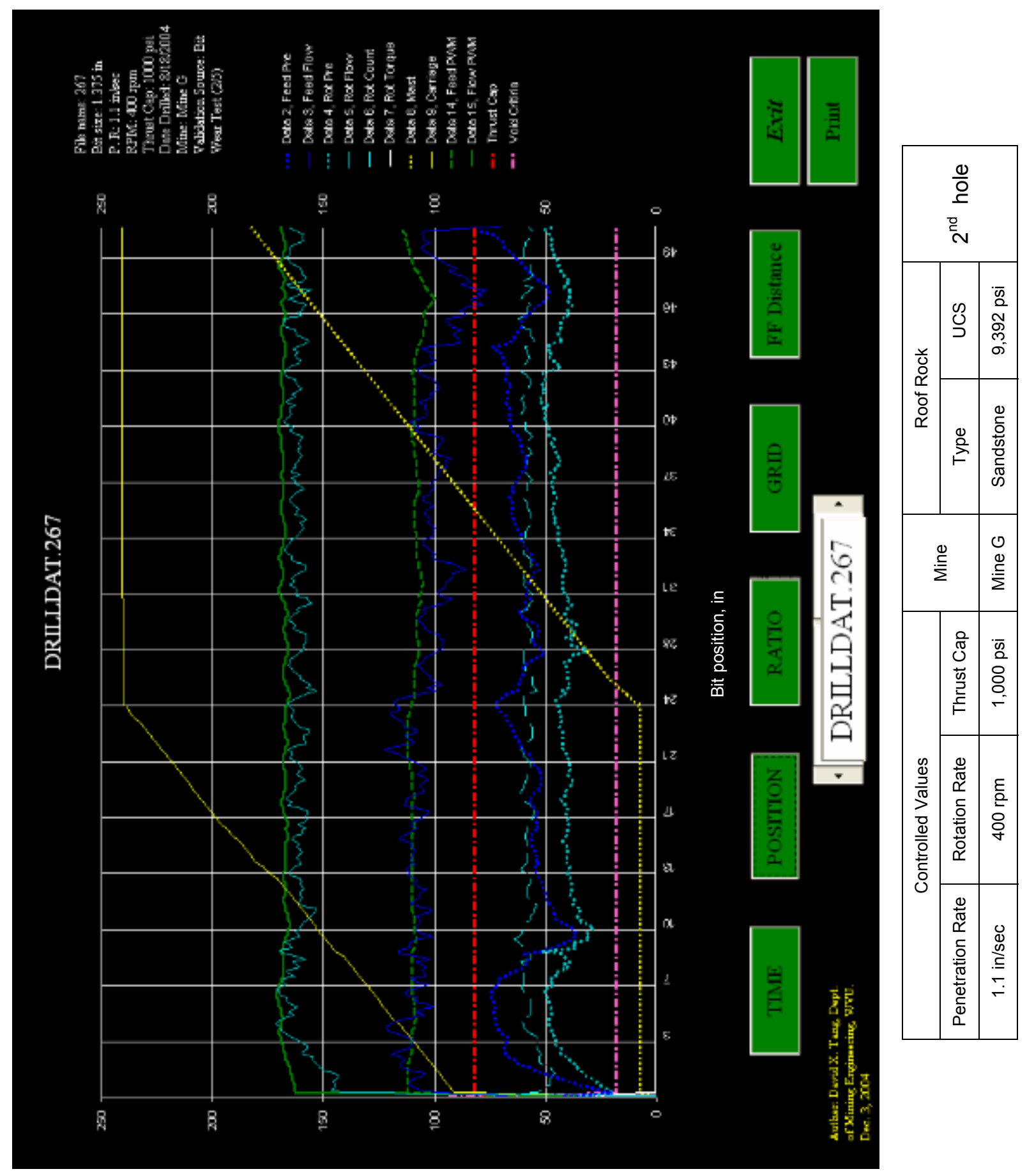




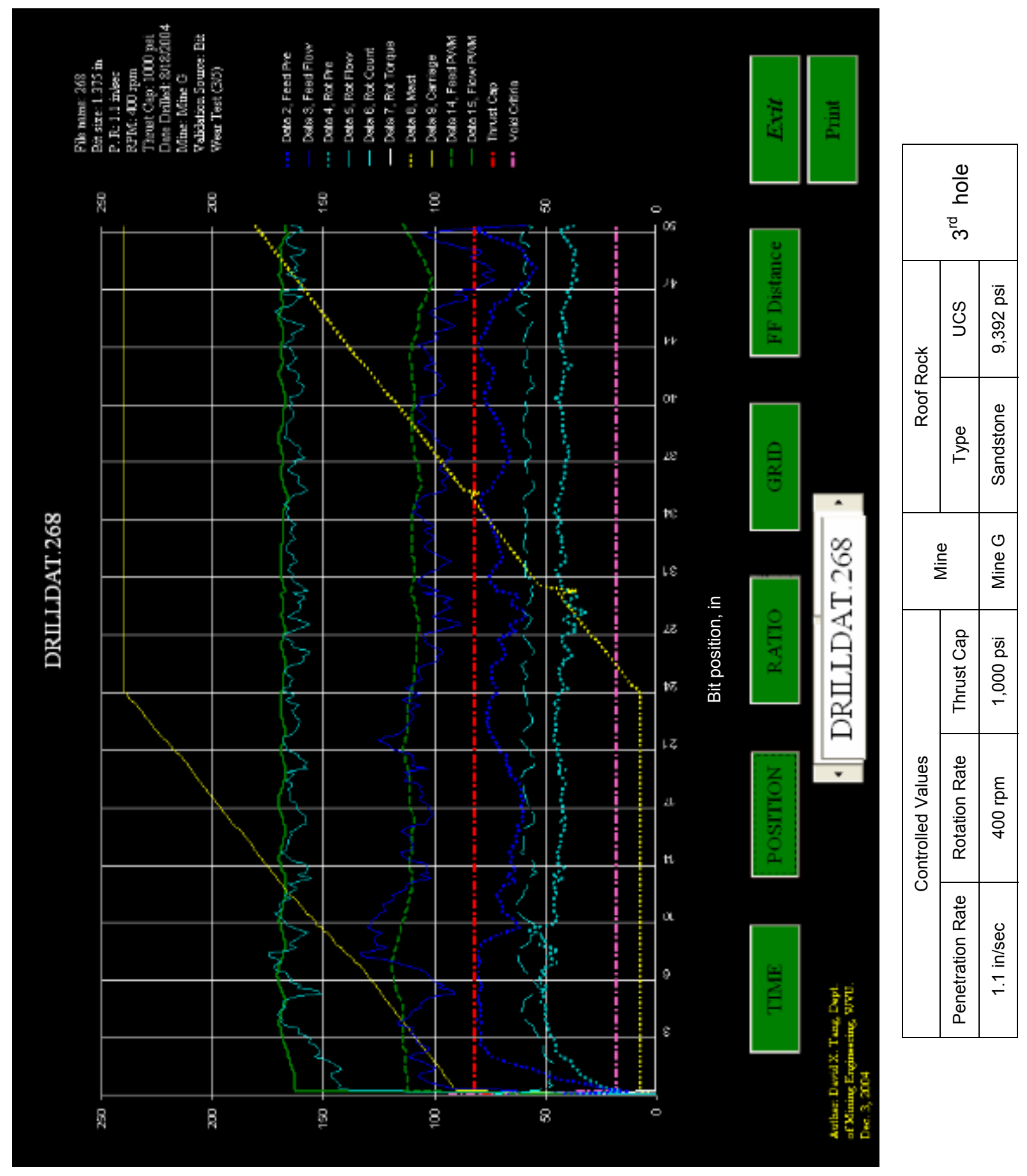




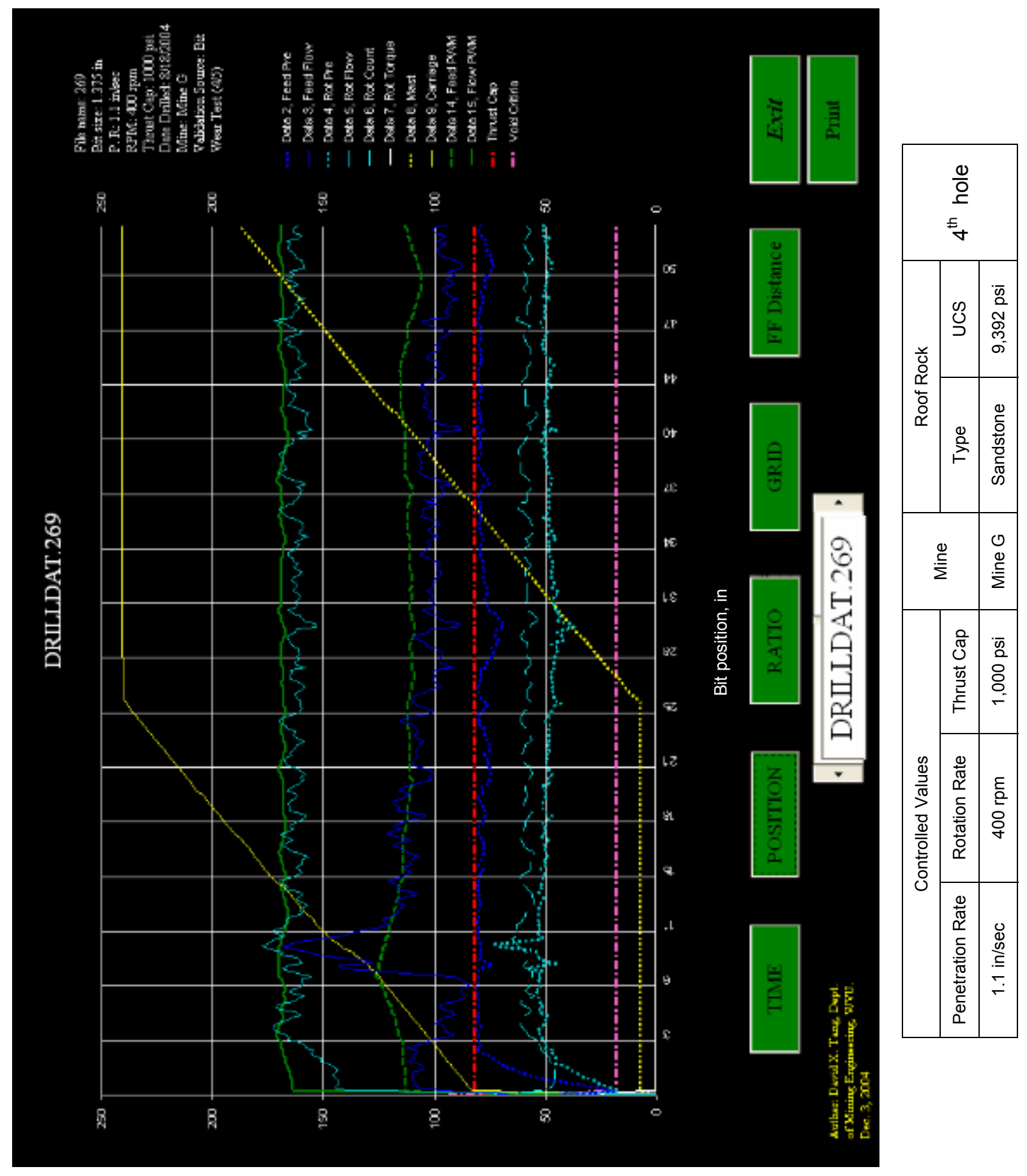




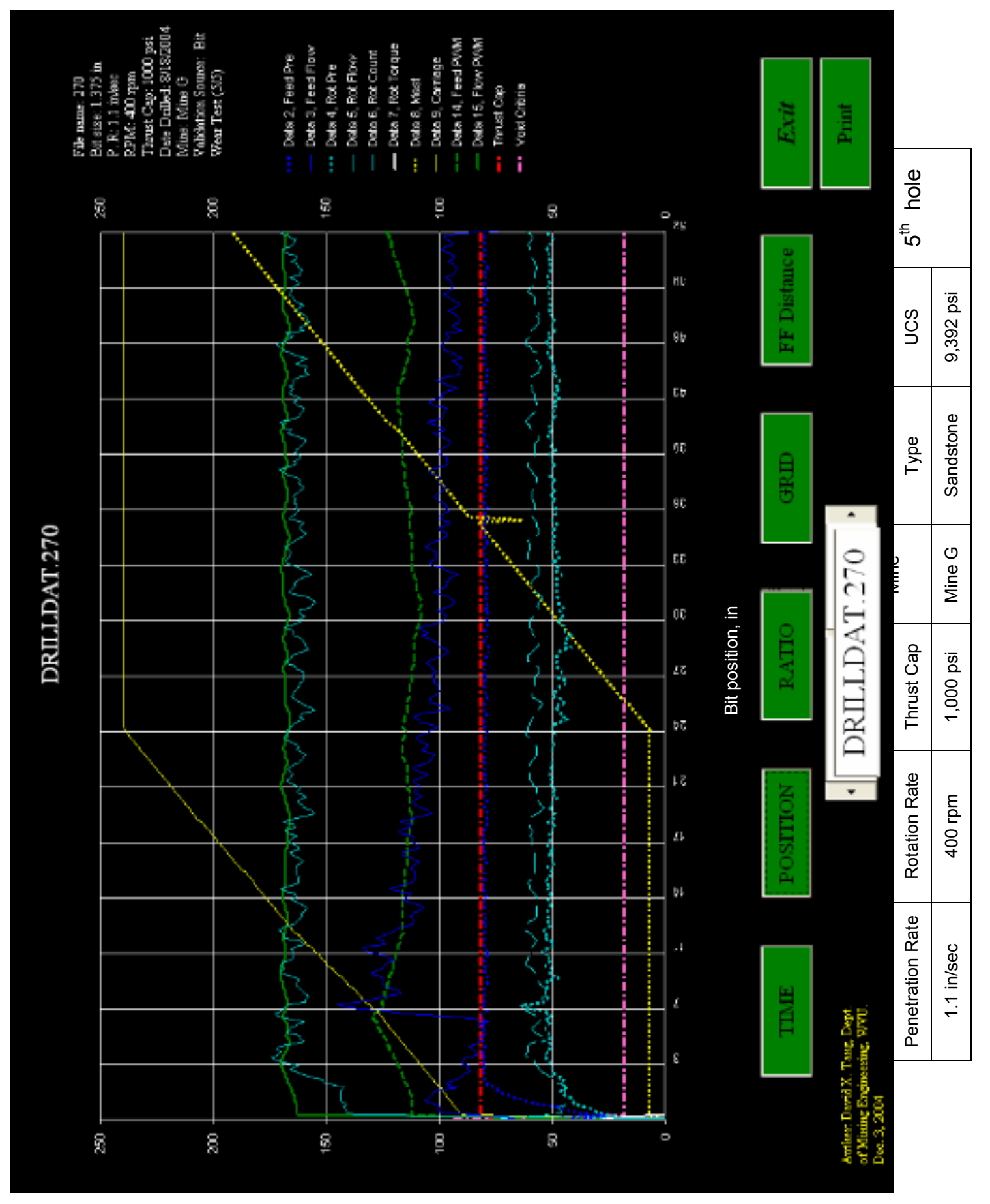



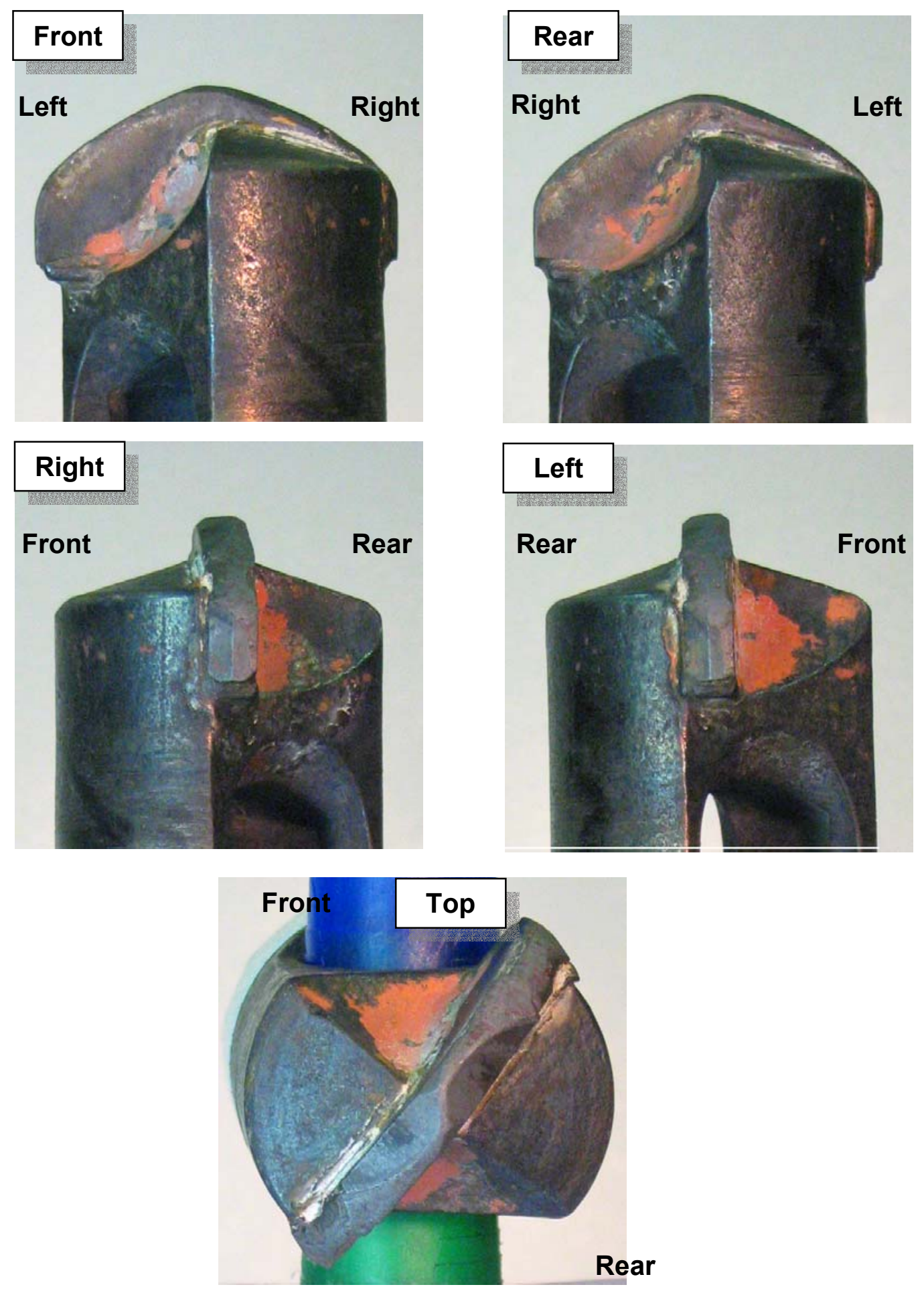

\begin{tabular}{|c|c|c|c|c|c|c|}
\hline \multicolumn{3}{|c|}{ Controlled Values } & \multirow{2}{*}{ Mine } & \multicolumn{2}{|c|}{ Roof Rock } & \multirow{2}{*}{$\begin{array}{c}\text { Number of } \\
\text { Drilled holes }\end{array}$} \\
\hline Penetration Rate & Rotation Rate & Thrust Cap & & Type & UCS & \\
\hline $1.1 \mathrm{in} / \mathrm{sec}$ & $650 \mathrm{rpm}$ & 1,000 psi & Mine G & Sandstone & $9,392 \mathrm{psi}$ & 5 holes \\
\hline
\end{tabular}




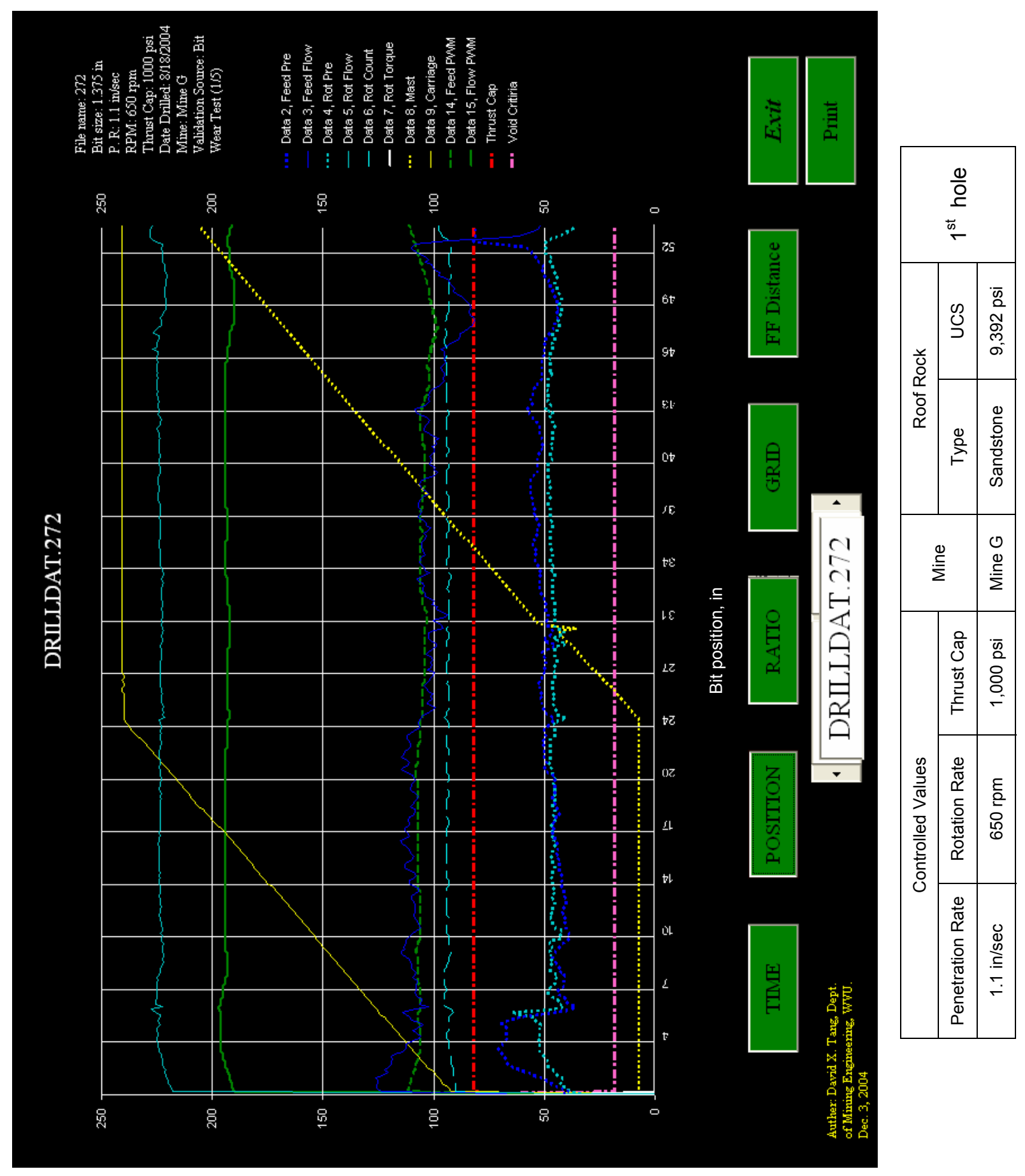




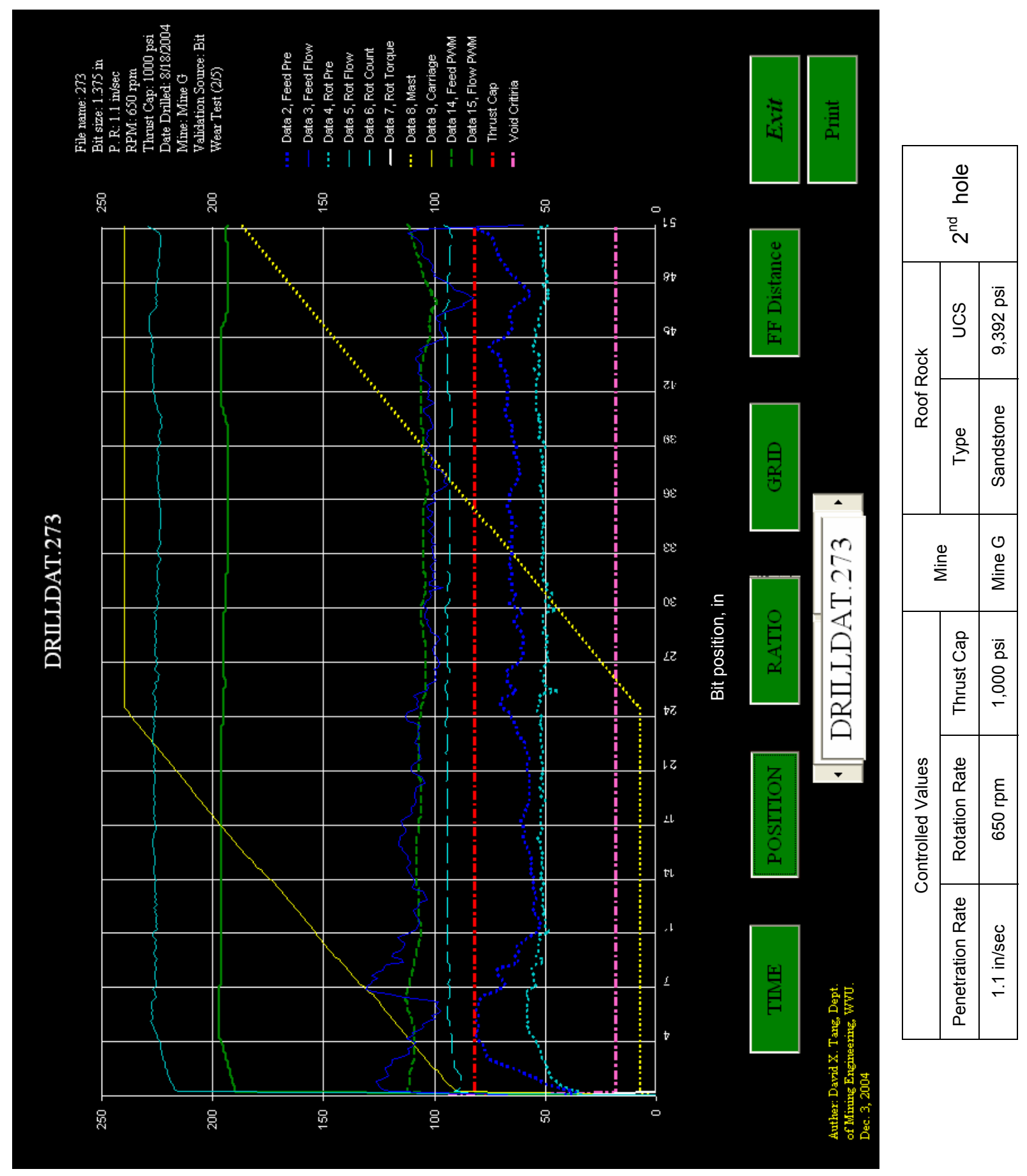




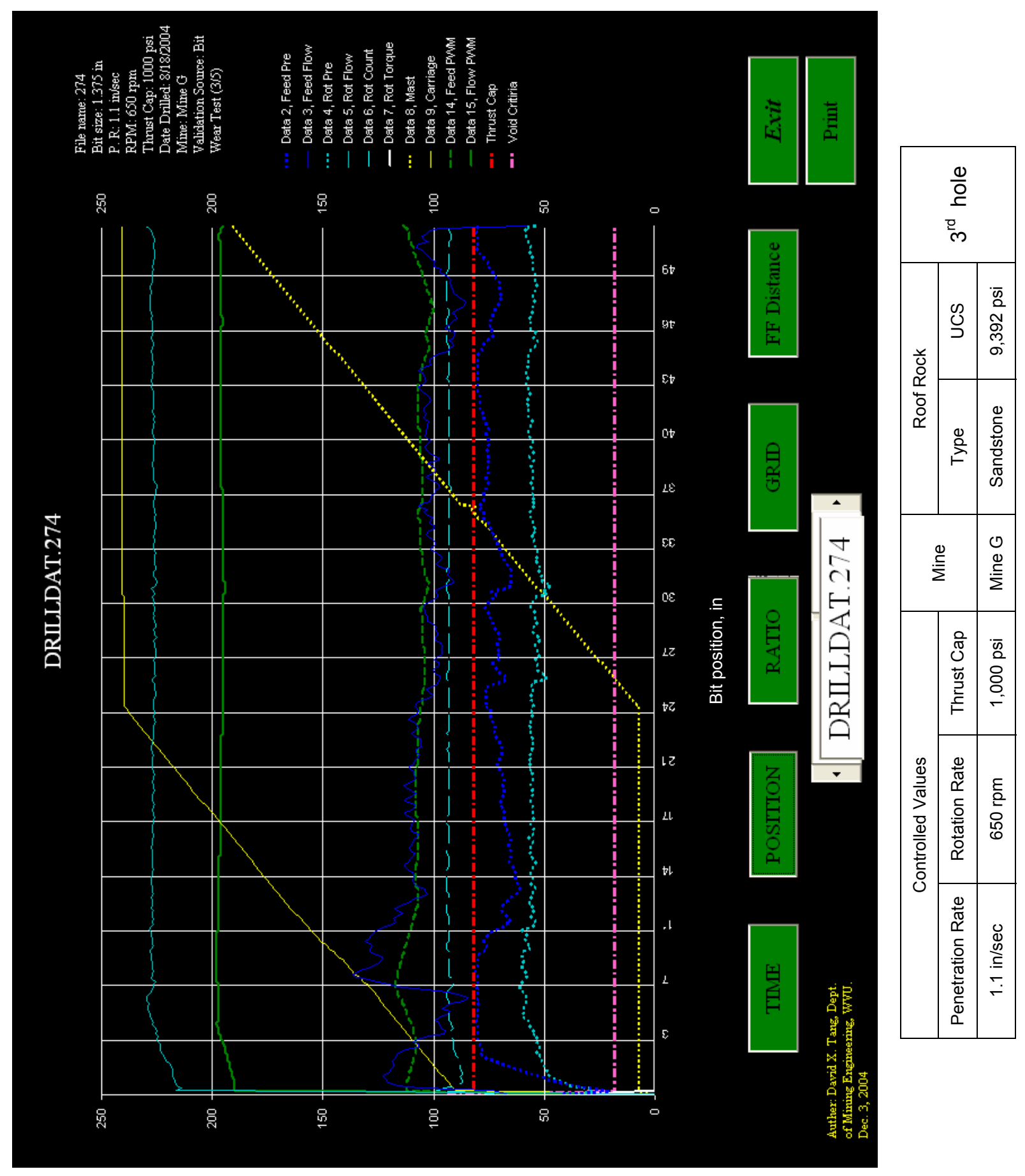




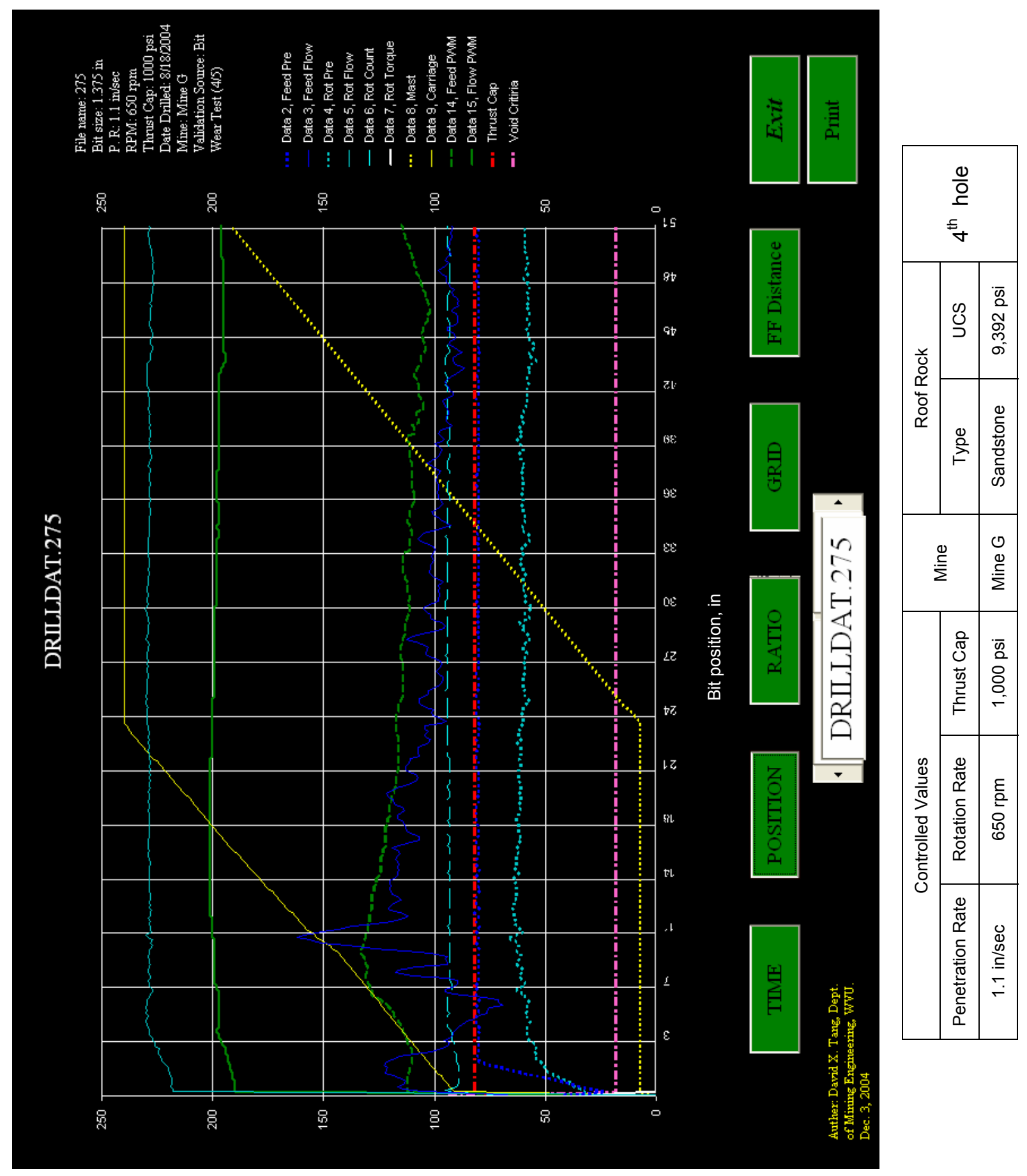




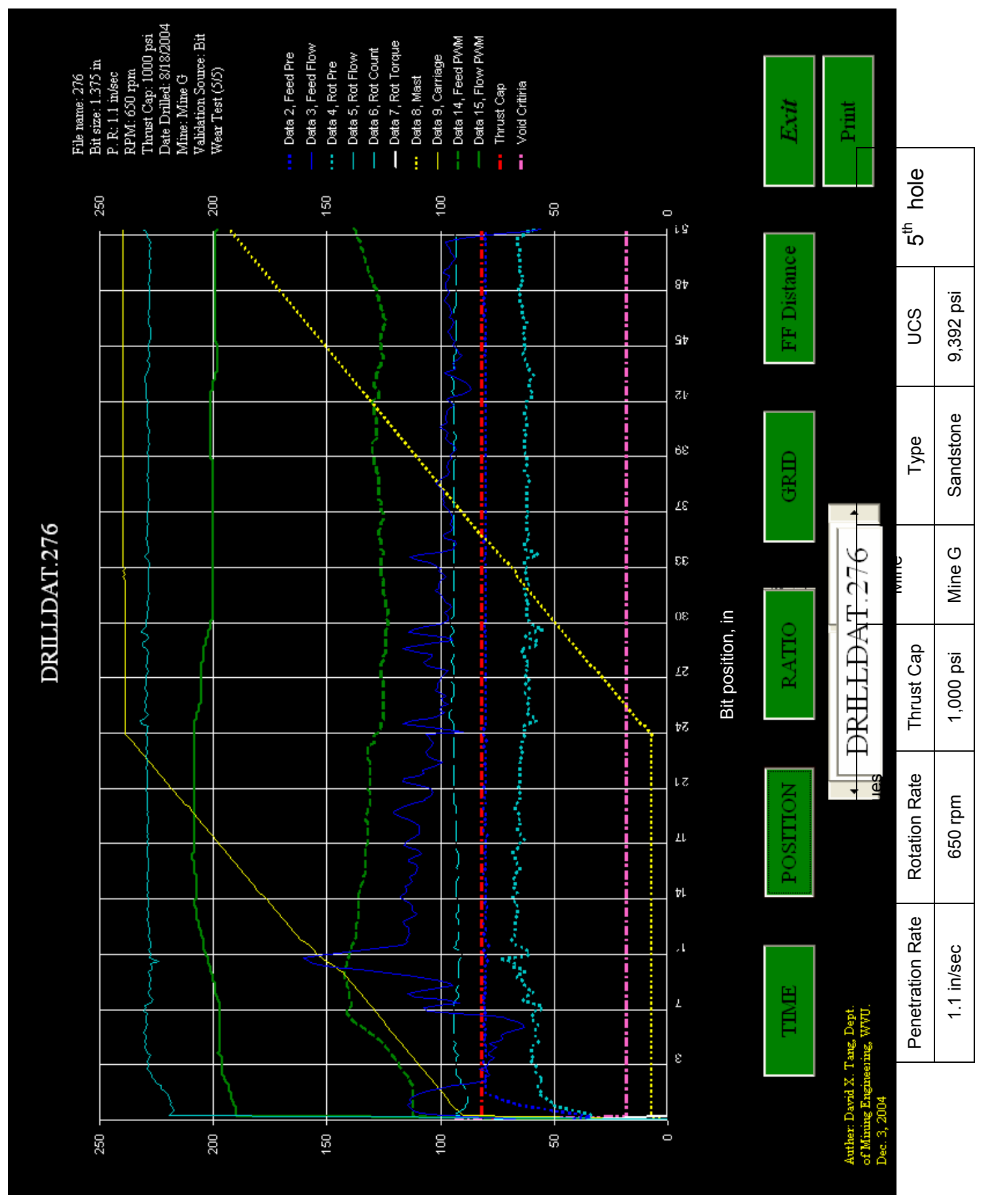



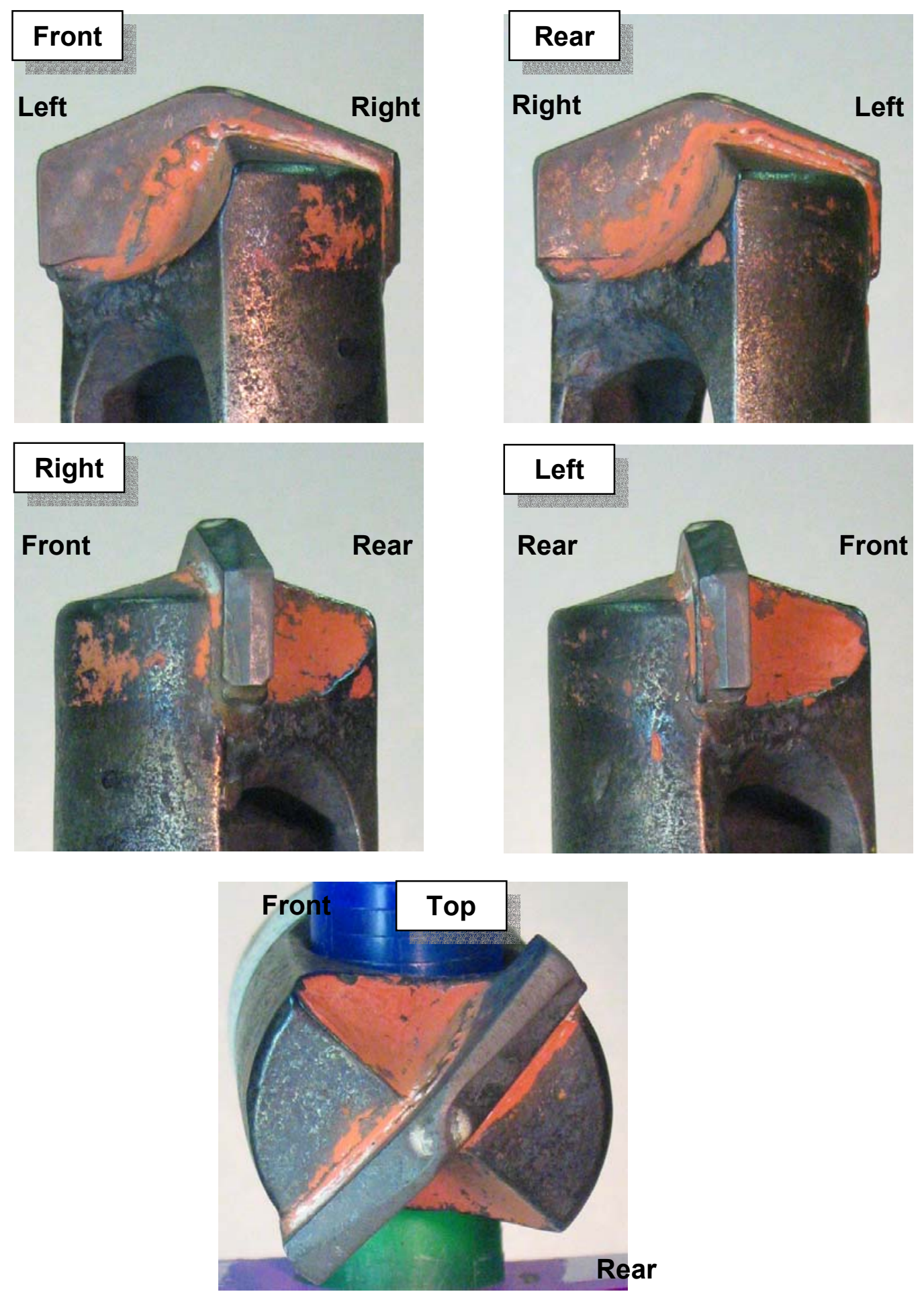

\begin{tabular}{|c|c|c|c|c|c|c|}
\hline \multicolumn{3}{|c|}{ Controlled Values } & \multirow{2}{*}{ Mine } & \multicolumn{2}{|c|}{ Roof Rock } & \multirow{2}{*}{$\begin{array}{c}\text { Number of } \\
\text { Drilled holes }\end{array}$} \\
\hline Penetration Rate & Rotation Rate & Thrust Cap & & Type & UCS & \\
\hline $0.4 \mathrm{in} / \mathrm{sec}$ & $650 \mathrm{rpm}$ & 1,000 psi & Mine G & Sandstone & $9,392 \mathrm{psi}$ & 3 holes \\
\hline
\end{tabular}




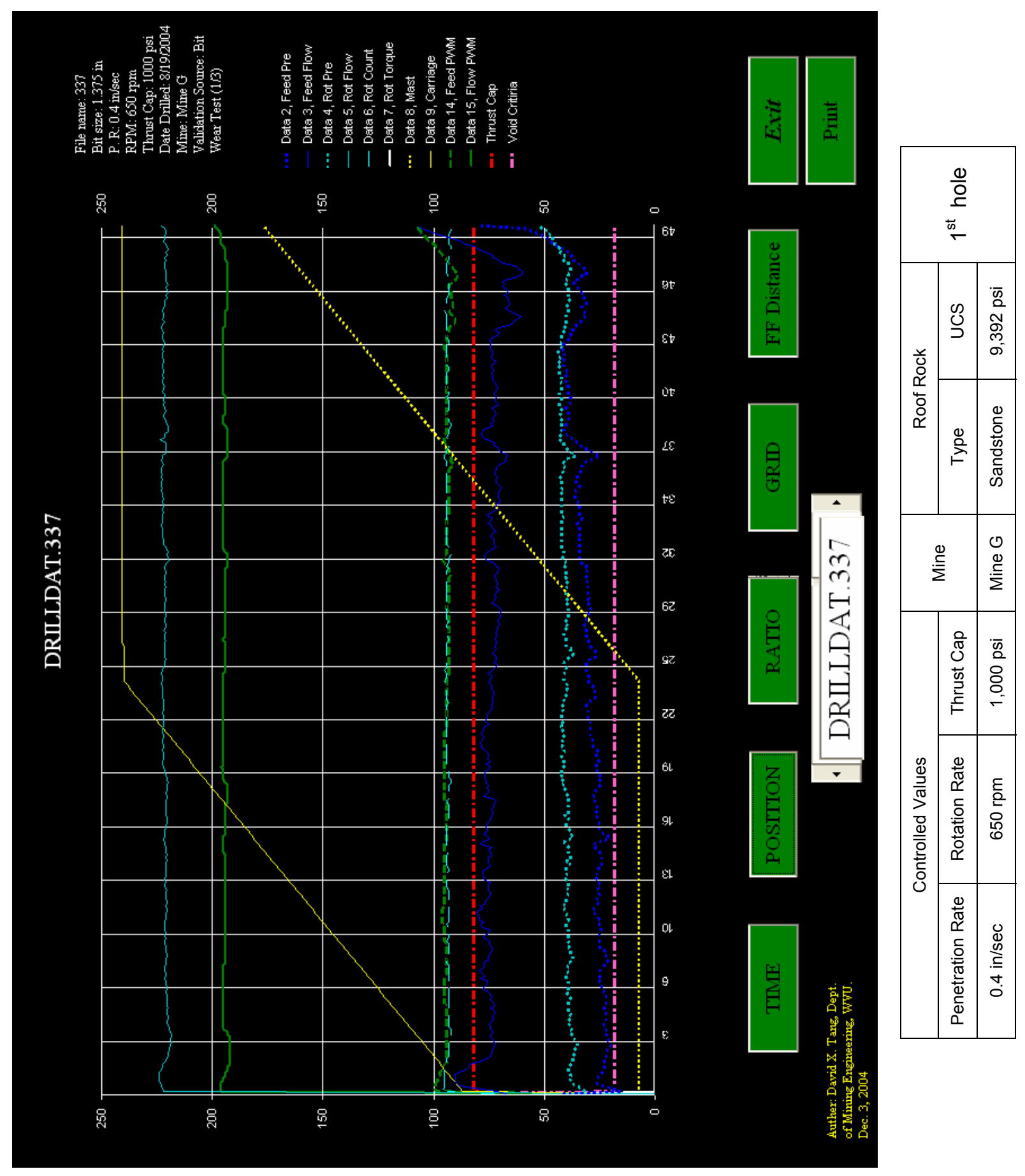




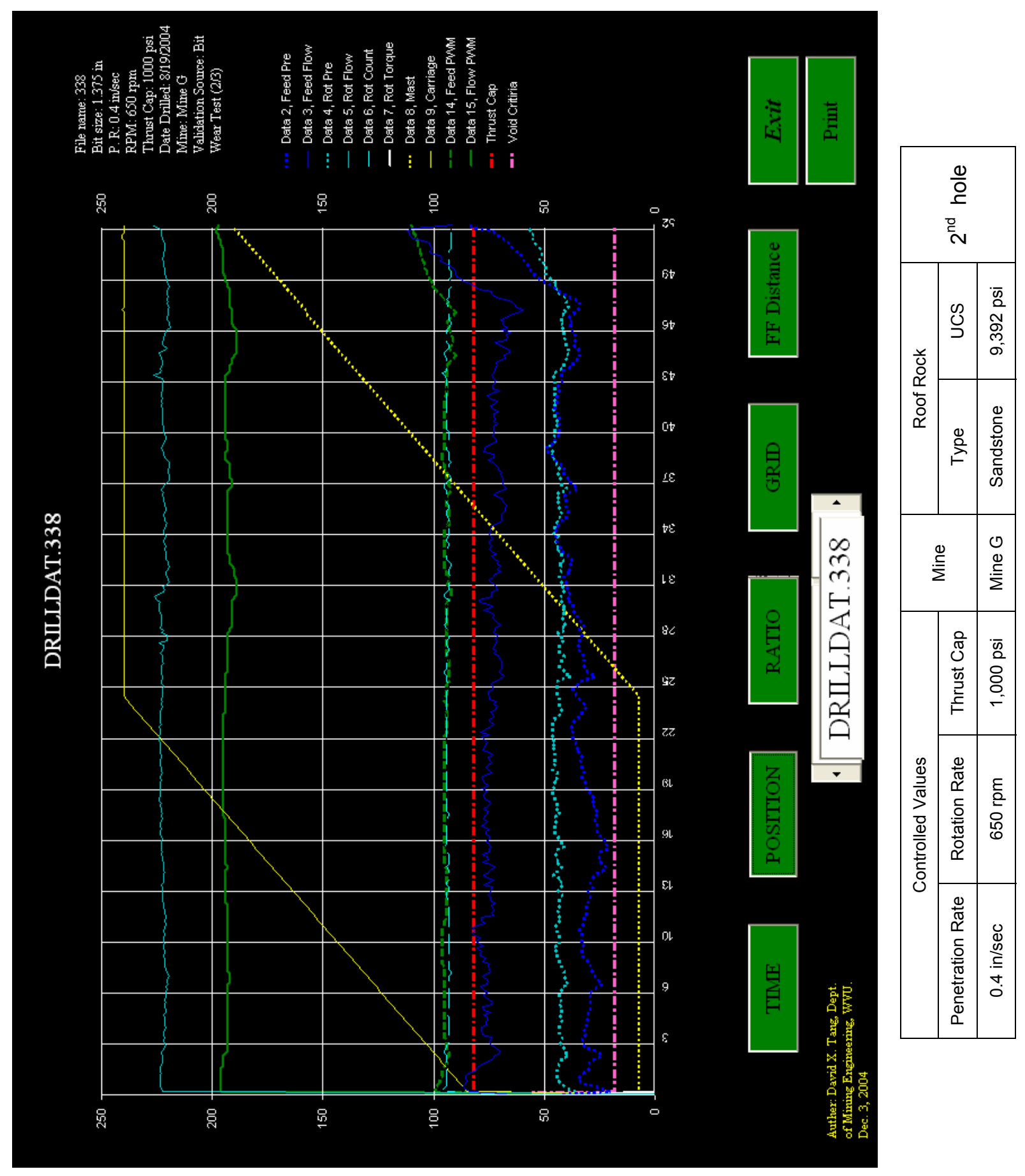




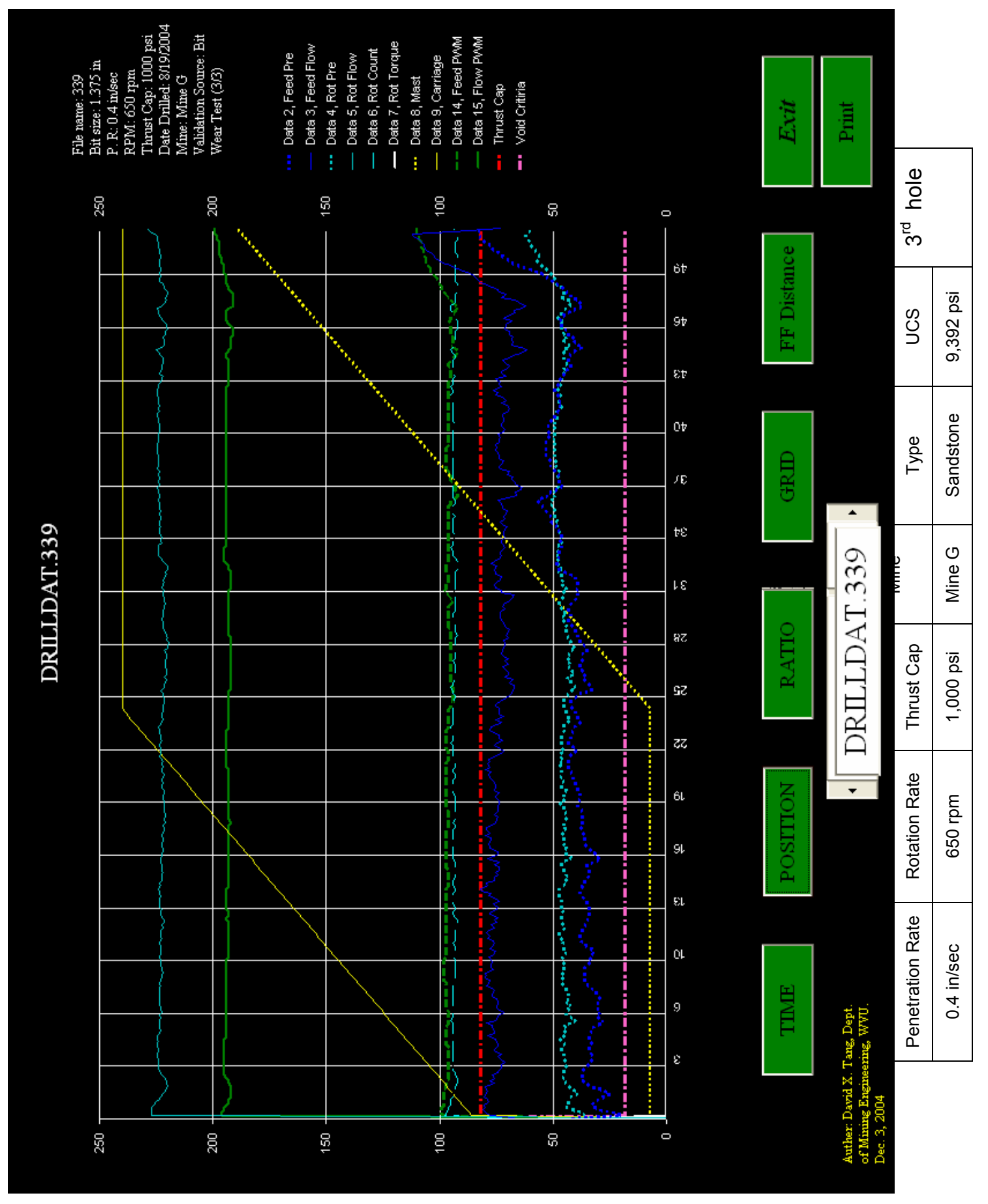



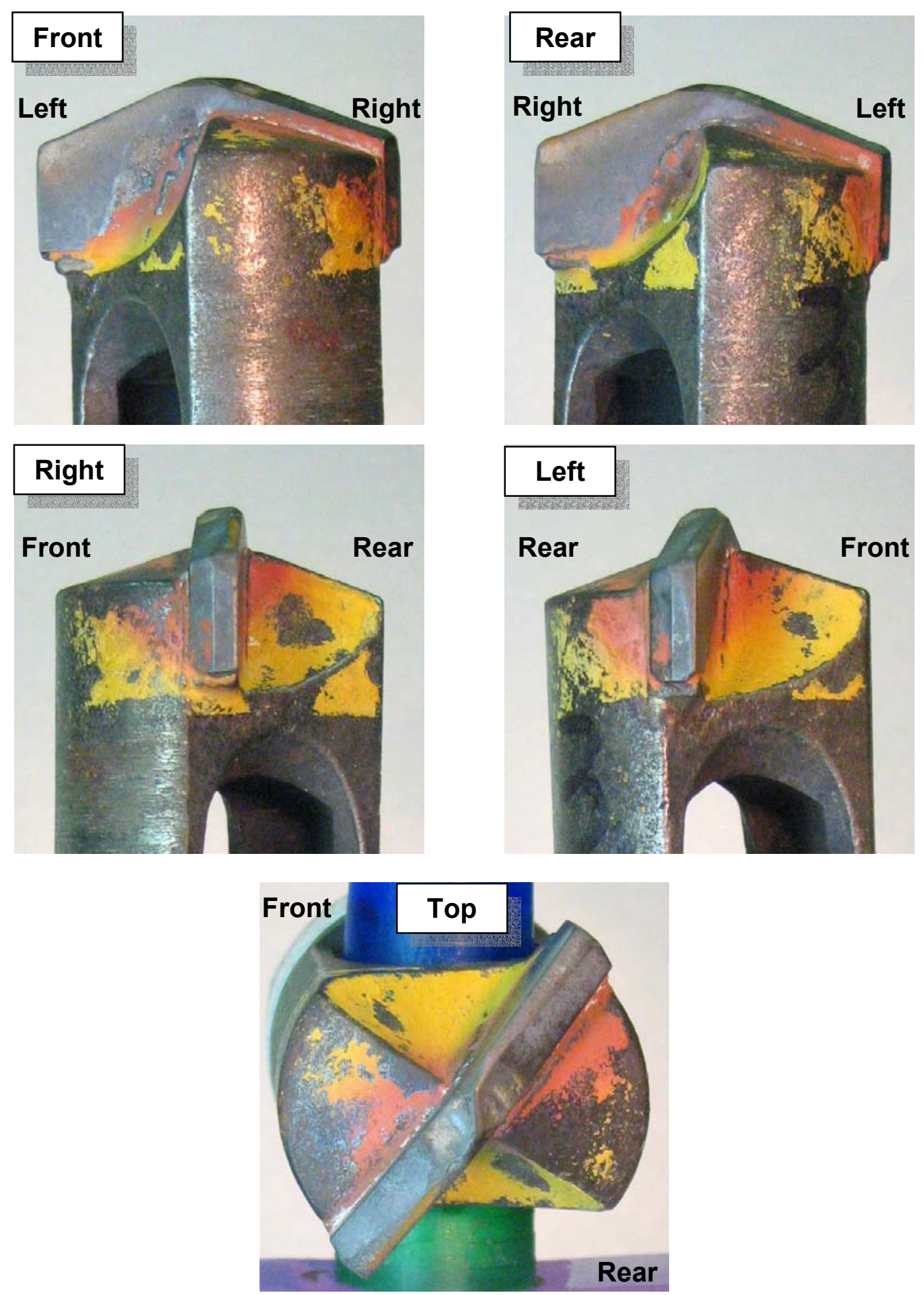

\begin{tabular}{|c|c|c|c|c|c|c|}
\hline \multicolumn{3}{|c|}{ Controlled Values } & \multirow{2}{*}{ Mine } & \multicolumn{2}{c|}{ Roof Rock } & Number of \\
\cline { 1 - 2 } & Drilled holes \\
\hline Penetration Rate & Rotation Rate & Thrust Cap & & Type & UCS & 3 holes \\
\hline $1.5 \mathrm{in} / \mathrm{sec}$ & $650 \mathrm{rpm}$ & $1,000 \mathrm{psi}$ & Mine G & Sandstone & $9,392 \mathrm{psi}$ & 3 \\
\hline
\end{tabular}




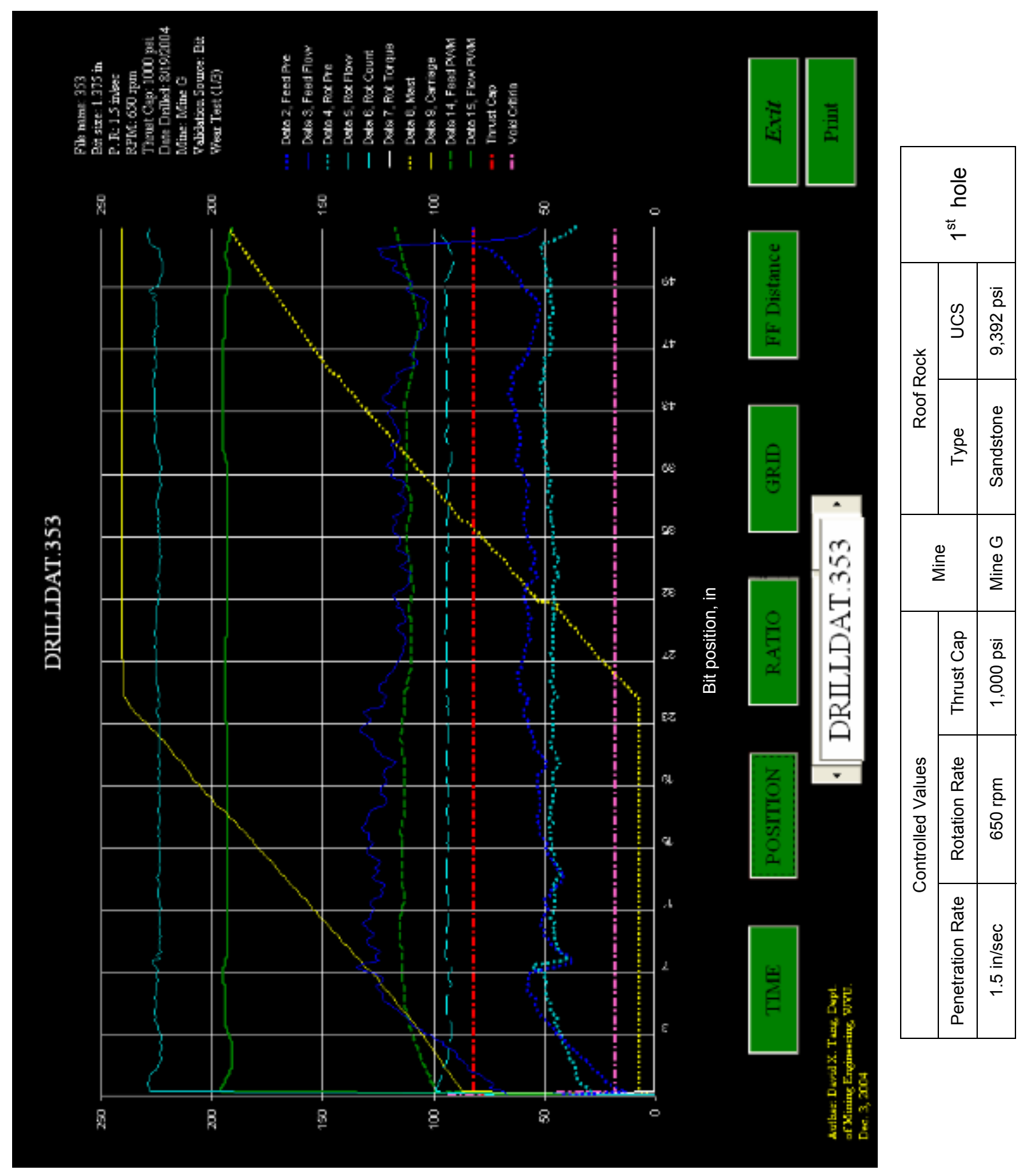




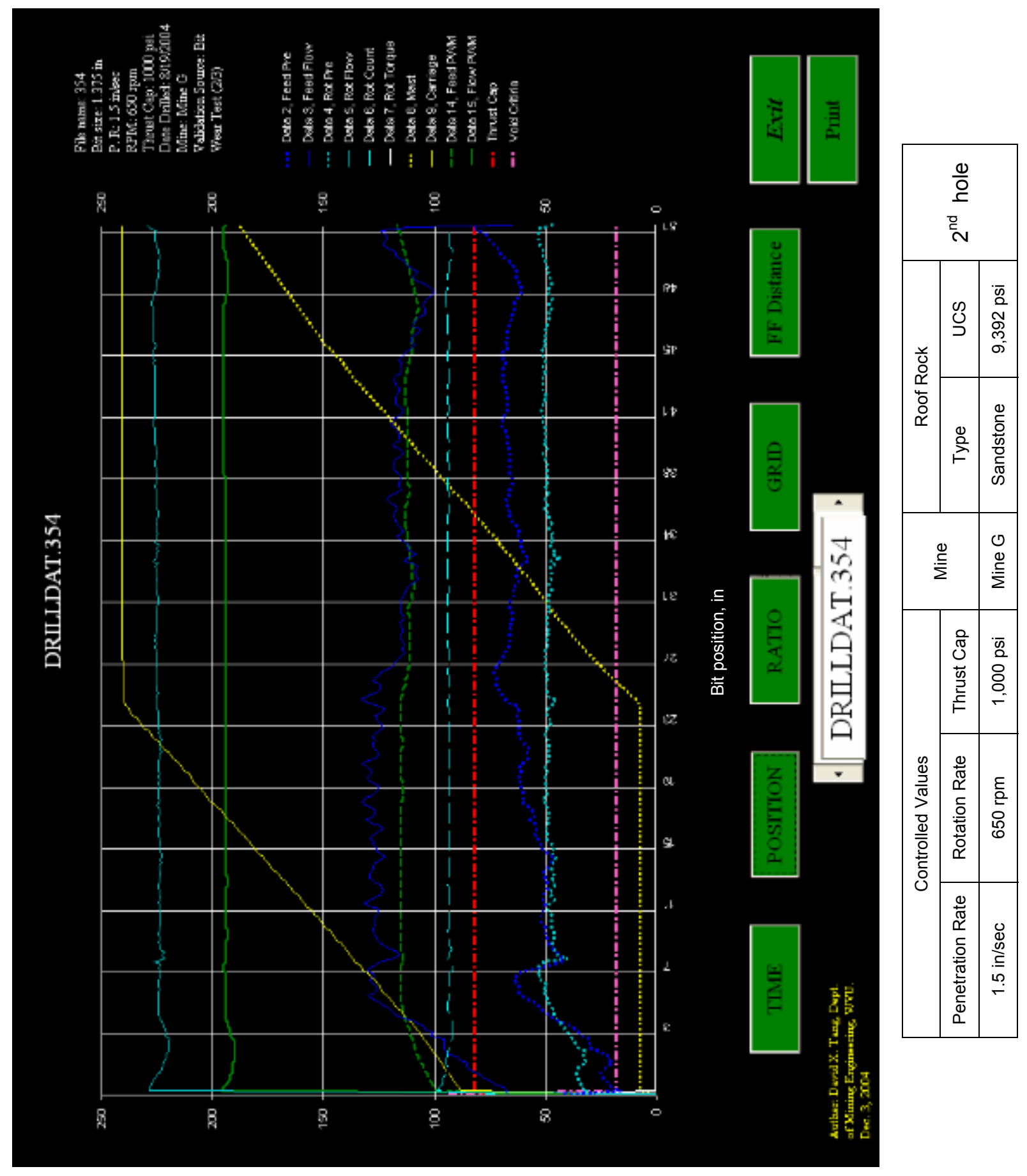




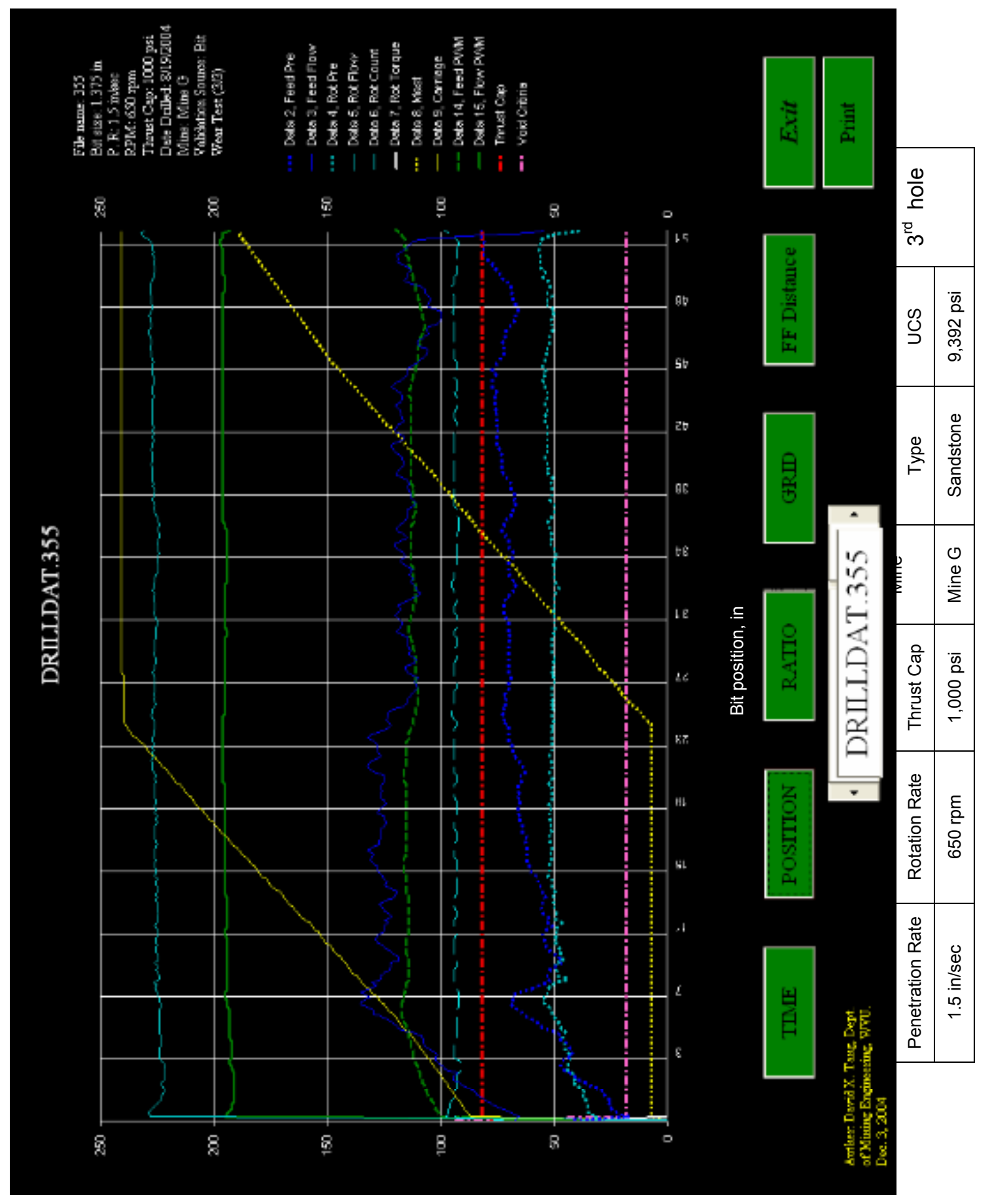



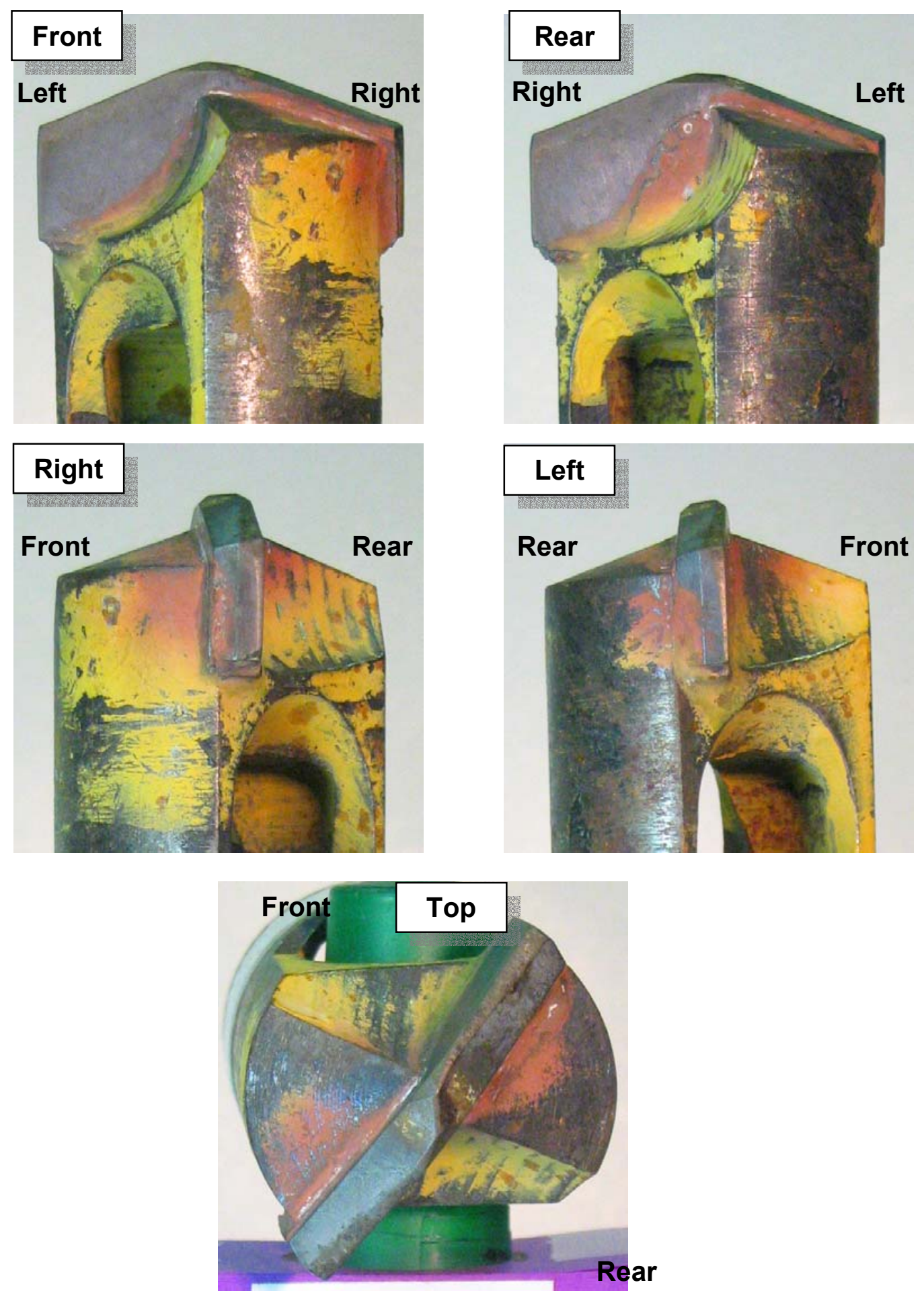

\begin{tabular}{|c|c|c|c|c|c|c|}
\hline \multicolumn{3}{|c|}{ Controlled Values } & \multirow{2}{*}{ Mine } & \multicolumn{2}{|c|}{ Roof Rock } & \multirow{2}{*}{$\begin{array}{c}\text { Number of } \\
\text { Drilled holes }\end{array}$} \\
\hline Penetration Rate & Rotation Rate & Thrust Cap & & Type & UCS & \\
\hline $1.1 \mathrm{in} / \mathrm{sec}$ & 650 rpm & 1,000 psi & Mine G & Sandstone & $9,392 \mathrm{psi}$ & 3 holes \\
\hline
\end{tabular}




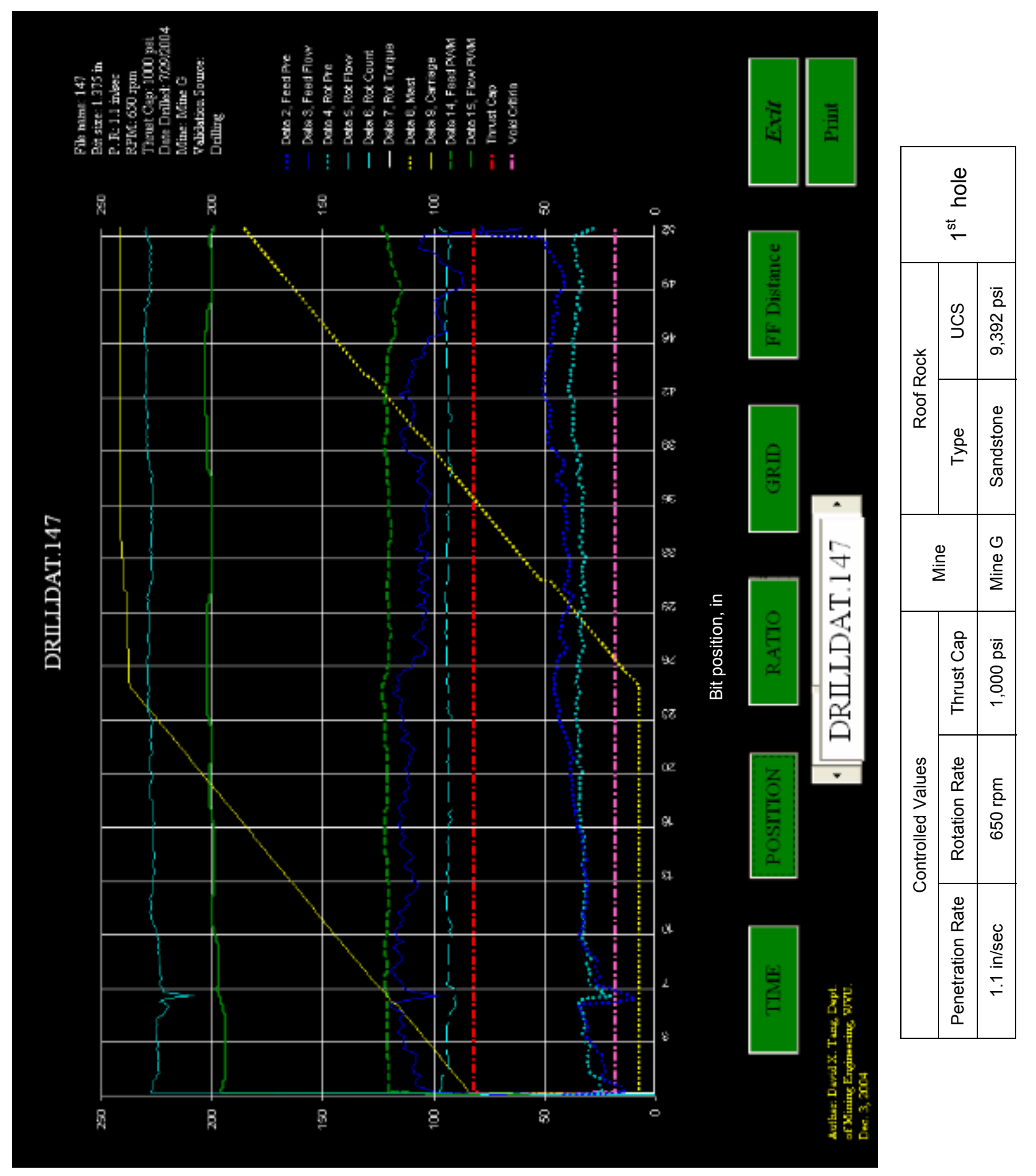




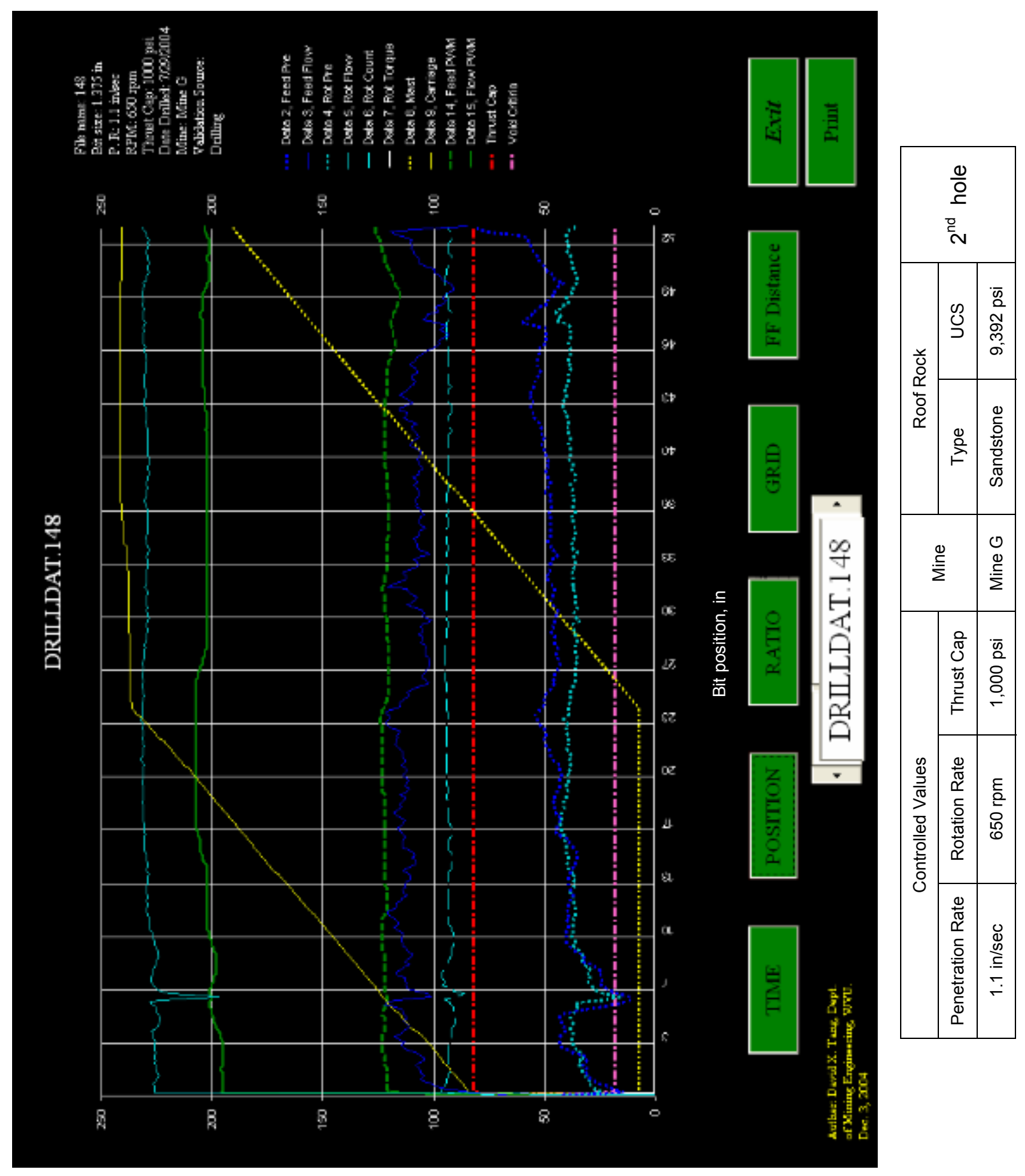




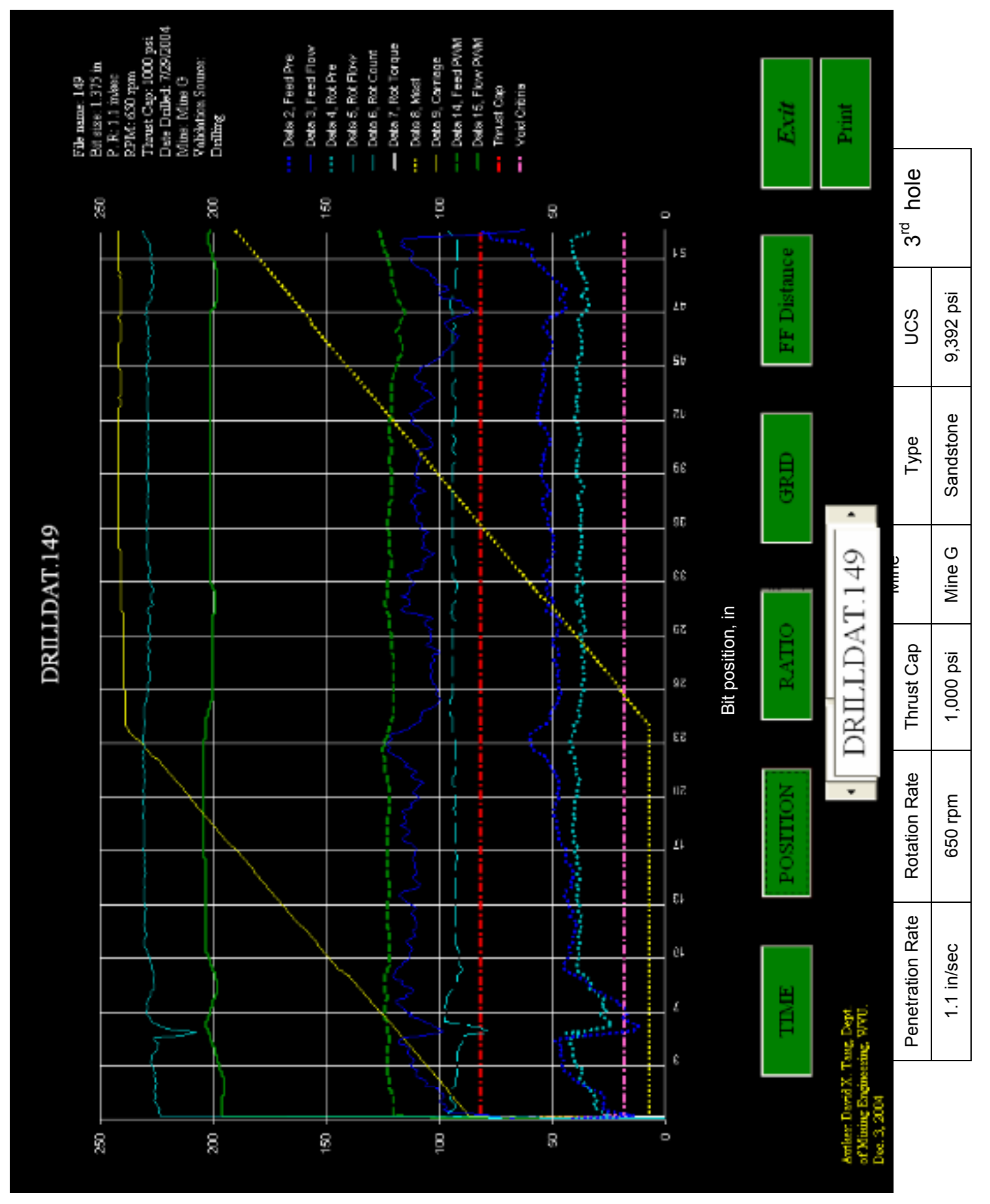




\section{VITA}

\section{(David) Xianguang Tang}

\section{Education}

Fuxin Coal Mining Institute, China, BS, 1982

West Virginia University, USA, MS, 2000

West Virginia University, USA, 2000 - present

\section{Professional Experience}

Fuxin Coal Mining Institute, China, Assistant Professor, 1982-1985

Shenyang Coal Mining Research Institute, China, Assistant Mining Engineer, 1985-1987

Shenyang Coal Mining Research Institute, China, Mining Engineer, 1987-1993

Shenyang Coal Mining Research Institute, China, Senior Mining Engineer, 1993-1998

DBT America Inc., USA, Sales Director, 2005 - present

\section{Selected Publications}

1. Peng, S. S., Tang, D. X., Mirabile, B., Luo, Y., Wilson, G., Mine Roof Geology Information System. Proc. 22nd Conf. On Ground Control in Mining. Ed.: S.S. Peng. West Virginia University, Morgantown, 2003. pp: 127 - 135

2. Tang, D. X., Peng, S. S., Luo, Y., and Wilson, G., (2004), "Void Prediction in Mine Roof Geology Information System (MRGIS)", Proceeding of 2004 SME Annual Meeting, February, Preprint No. 04-148, 7 pp.

3. Mirabile, B., Peng, S. S., Luo, Y., Tang, D. X., Roof Bolter Drilling Parameters as a Tool for Strata Prediction. Paper presented at 2004 SME Annual Meeting held at Denver, CO. 
4. Peng, S. S., Sasaoka, T., Tang, D. X., Luo, Y., Wilson, G., Mine Roof Geology Information System, Coal Age, May 2005, pp: 44 - 49

5. Collins, C., Wilson, G., Peng, S. S., Tang, D. X., Field Testing of a Real Time Roof Mapping Drilling Display System in a Linestone Mine. Proc. 23rd Conf. On Ground Control in Mining. Ed.: S.S. Peng. West Virginia University, Morgantown, 2004. pp: $48-55$

6. Peng, S. S., Tang, D. X., Takashi, S., Luo, Y., Finfiger, G., Wilson, G., A Method for Quantitative Void/Fracture Detection and Estimation of Rock Strength for Underground Mine Roof Proc. 24th Conf. On Ground Control in Mining. Ed.: S.S. Peng. West Virginia University, Morgantown, 2005. pp: 187 - 195

7. Sasaoka,T., Peng, S.S., Tang, D. X., Wilson, W., Estimation of roof strata strength in Mine Roof Geology Information System (MRGIS). Paper presented at 2006 SME Annual Meeting held at St. Louis, MO. Preprint No. 06 - 09 